\title{
Crystalline, membrane-embedded, and fibrillar proteins investigated by solid-state NMR spectroscopy
}

\author{
Dissertation \\ zur Erlangung des Doktorgrades \\ der Mathematisch-Naturwissenschaftlichen Fakultäten \\ der Georg-August-Universität zu Göttingen
}

vorgelegt von

Robert Schneider

aus München

Göttingen 2009 
D7

Referent: Prof. Dr. Konrad Samwer

Korreferent: Prof. Dr. Christian Griesinger

Tag der mündlichen Prüfung: 30.01.2009 


\section{Abstract}

This thesis describes research on atomic structure and molecular dynamics of biomolecules in the solid phase using solid-state nuclear magnetic resonance spectroscopy. A method for detecting residue-specific mobility using double-quantum $\left({ }^{13} \mathrm{C},{ }^{13} \mathrm{C}\right)$ spectroscopy was developed on a small model system and applied to the 76-residue protein ubiquitin. Together with a structure calculation employing an automated algorithm, results allowed to relate preparation-dependent chemical-shift changes in this protein to local differences in mobility or conformation. Work on the potassium channel KcsA-Kv1.3 in lipid bilayers led to resonance assignments for $59 \%$ of its residues. Secondary structure of its pore domain was found to closely resemble that of the parent KcsA channel, but differences became apparent with respect to KcsA preparations in micelles, highlighting the influence of the membrane environment. In close reference to functional experiments, activation and inactivation gating in KcsA-Kv1.3 and their dependence on $\mathrm{pH}$ and potassium concentration were investigated. At acidic pH, KcsA-Kv1.3 was found to reside in an open-inactivated conformation. It is characterized by bent inner transmembrane helices, corresponding to an opened activation gate, and by a nonconductive selectivity filter conformation, i.e. a closed inactivation gate. The open-inactivated state of KcsA-Kv1.3 was demonstrated to be correlated with protonation of specific glutamate residues. It is also strongly influenced by the potassium concentration. Potassium affects the conformation of both activation and inactivation gates via binding to the selectivity filter, providing a mechanism for gate coupling and coordinated gating transitions. Finally, fibrillar aggregates formed by disease-relevant polyglutamine peptides were investigated structurally. Experimental data argue against water-filled $\beta$-helical models and are best explained by an antiparallel, superpleated cross- $\beta$ structure with a polar zipper arrangement of tightly interdigitated, hydrogen-bonded sidechains. 


\section{Kurzzusammenfassung}

Die vorliegende Arbeit beschreibt Untersuchungen von atomarer Struktur und molekularer Dynamik von Biomolekülen in fester Phase mittels kernmagnetischer Resonanzspektroskopie. An einem kleinen Modellsystem wurde eine Methode zur Messung lokaler molekularer Mobilität entwickelt, die auf $\left({ }^{13} \mathrm{C},{ }^{13} \mathrm{C}\right)$-Doppelquantenspektroskopie basiert. Diese Methode wurde auf das 76-Reste-Protein Ubiquitin angewendet. Mit den Ergebnissen dieser Messungen sowie einer automatisierten Strukturrechnung konnte gezeigt werden, dass präparationsabhängige Änderungen in den chemischen Verschiebungen dieses Proteins vorwiegend in Regionen auftreten, in denen sich Konformation oder Dynamik zwischen den Probenpräparationen unterscheiden. Weiterhin wurde der Kalium-Ionenkanal KcsA-Kv1.3 in einer Lipidmembranumgebung spektroskopisch untersucht. Für 59\% der Aminosäurereste dieses Proteins konnten Resonanzen zugeordnet werden. Es zeigte sich, dass die Sekundärstruktur im Transmembranbereich dieses Kanals derjenigen des nahe verwandten Proteins KcsA sehr ähnlich ist. Im Vergleich zu KcsA-Präparationen in Micellen wurden jedoch Unterschiede gefunden, was den Einfluss der Membranumgebung auf das Protein verdeutlicht. Die Vorgänge der Aktivierung und Inaktivierung dieses Ionenkanals sowie ihre Abhängigkeit von pH-Wert und Kaliumkonzentration wurden untersucht, wobei auf elektrophysiologische Experimente zur Funktion des Kanals Bezug genommen wurde. Bei saurem pH zeigten die spektroskopischen Daten eine offen-inaktivierte Konformation von KcsA-Kv1.3. In diesem Zustand liegen die inneren Transmembran-Helices gekrümmt vor, wodurch das Aktivierungs-Gate geöffnet wird, und der Selektivitätsfilter nimmt eine nichtleitende Konformation ein, was einem geschlossenen Inaktivierungs-Gate entspricht. Es wurde gezeigt, dass der offen-inaktivierte Zustand von KcsA-Kv1.3 mit der Protonierung bestimmter Glutamat-Reste korreliert ist und außerdem stark von der Kaliumkonzentration abhängt. Kalium beeinflusst die Konformation sowohl des Aktivierungs- als auch des Inaktivierungs-Gate über seine Bindung an den Selektivitätsfilter. Dieser Mechanismus koppelt die beiden Proteinbereiche und ermöglicht koordinierte Konformationsänderungen 
zum Öffnen und Schließen des Kanals. Schließlich wurden krankheitsrelevante fibrilläre Aggregate aus Polyglutamin-Peptiden strukturell untersucht. Die experimentellen Daten sprechen gegen eine wassergefüllte, $\beta$-helikale Struktur und lassen sich durch antiparallele, übereinander angeordnete $\beta$-Faltblätter mit eng verzahnten, durch Wasserstoffbrücken verbundenen Seitenketten erklären. 


\section{Related Publications}

Parts of the work presented in this thesis are based on the following publications or manuscripts. I am very grateful to all co-authors of these papers, as well as the people acknowledged in them, for the stimulating and fruitful cooperations.

- Christian Ader*, Robert Schneider*, Sönke Hornig, Phanindra Velisetty, Erica M. Wilson, Adam Lange, Karin Giller, Iris Ohmert, Marie-France Martin-Eauclaire, Dirk Trauner, Stefan Becker, Olaf Pongs, and Marc Baldus. A structural link between inactivation and block of a $\mathrm{K}^{+}$channel. Nature Structural and Molecular Biology, 15:605-612, 2008. (*equally contributing authors) [1]

- Robert Schneider, Christian Ader, Adam Lange, Karin Giller, Sönke Hornig, Olaf Pongs, Stefan Becker, and Marc Baldus. Solid-state NMR spectroscopy applied to a chimeric potassium channel in lipid bilayers. Journal of the American Chemical Society, 130:7427-7435, 2008. [2]

- Christian Ader*, Robert Schneider*, Sönke Hornig, Phanindra Velisetty, Vitya Vardanyan, Karin Giller, Iris Ohmert, Stefan Becker, Olaf Pongs, and Marc Baldus. Coupling of activation and inactivation gate in a $\mathrm{K}^{+}$channel: potassium and ligand sensitivity. EMBO Journal, accepted for publication, 2009. (*equally contributing authors)

- Robert Schneider et al. Probing molecular motion by double-quantum $\left({ }^{13} \mathrm{C},{ }^{13} \mathrm{C}\right)$ solid-state NMR spectroscopy: Application to ubiquitin. In preparation.

- Robert Schneider et al. Structural characterization of polyglutamine fibrils by solidstate NMR spectroscopy. In preparation. 
In addition, I was involved in cooperations leading to the following publications or manuscripts. I wish to express my kindest regards also to all people involved in these studies for the productive collaborations.

- Christian Ader, Robert Schneider, Karsten Seidel, Manuel Etzkorn, Stefan Becker, and Marc Baldus. Structural rearrangements of membrane proteins probed by wateredited solid-state NMR spectroscopy. Journal of the American Chemical Society, 131:170-176, 2009. [3]

- Ulrich Zachariae, Robert Schneider, Phanindra Velisetty, Adam Lange, Daniel Seeliger, Sören Wacker, Yasmin Karimi-Nejad, Gert Vriend, Stefan Becker, Olaf Pongs, Marc Baldus, and Bert L. de Groot. The molecular mechanism of toxin-induced conformational changes in a potassium channel: relation to C-type inactivation. Structure, 16:747-754, 2008. [4]

- Jegannath Korukottu, Robert Schneider, Vinesh Vijayan, Adam Lange, Olaf Pongs, Stefan Becker, Marc Baldus, and Markus Zweckstetter. High-resolution 3D structure determination of kaliotoxin by solid-state NMR spectroscopy. PLoS ONE, 3:e2359, 2008. [5]

- Christian Ader, Robert Schneider, Karsten Seidel, Manuel Etzkorn, and Marc Baldus. Magic-angle-spinning NMR spectroscopy applied to small molecules and peptides in lipid bilayers. Biochemical Society Transactions, 35:991-995, 2007. [6]

- Karsten Seidel, Manuel Etzkorn, Robert Schneider, Christian Ader, and Marc Baldus. Comparative analysis of NMR chemical shift predictions for proteins in the solid phase. Solid State Nuclear Magnetic Resonance, 35:235-242, 2009. [7]

- Jegannath Korukottu et al. Conformational plasticity in ion channel recognition of a peptide toxin. In preparation. 


\section{Acknowledgements}

I am very grateful and indebted to:

Prof. Dr. Konrad Samwer of the University of Göttingen for supervising my thesis on behalf of the Department of Physics, as well as for his approachability, open mind, and support of my work.

Prof. Dr. Christian Griesinger of the Max-Planck-Institute for Biophysical Chemistry (MPIBPC), for providing an outstanding research environment at his Department for NMR-based Structural Biology and for his continued support of my thesis projects.

Prof. Dr. Marc Baldus, now at Utrecht University's Bijvoet Center for Biomolecular Research in the Netherlands, for his supervision of my scientific work, the diverse, interesting, and fruitful projects I could work on, and for all I was able to learn about NMR, structural biology, and many other topics during my time in his group.

Gitta Angerstein for her indispensable help and support in the lab and in all matters of daily life and work at the institute, as well as for her cheerful spirit. All current and former members of the solid-state NMR group for their assistance and cooperation, for helping me learn the ropes of solid-state NMR in the beginning, and for a friendly working atmosphere. In particular, Christian Ader for the very productive and stimulating cooperation on the ion channel project; without him, it would not have advanced as much as it has. Dr. Adam Lange for the collaboration on the ion channel project during his postdoctoral time, as well as for allowing me to finish my thesis in what is now his group. Dr. Karsten Seidel for his help in many aspects of my work and for providing a $\mathrm{LAT}_{\mathrm{E}} \mathrm{X}$ template which was of invaluable help for writing this thesis. Dr. Ovidiu Andronesi, Prof. Dr. Henrike Heise, and Dr. Colan Hughes for their unique support, teaching, and cooperation. Dr. Manuel Etzkorn, Dr. Henrik Müller, Dr. Itzam de Gortari, Dr. Vinesh Vijayan, Venita Daebel, Dr. Ashutosh Kumar, and Deepak Nand for manifold cooperation and for being or having been great colleagues.

Dr. Stefan Becker and Karin Giller at the MPI-BPC for their excellent molecular biology work and expert preparation especially of ion channel samples, as well as Dr. Volker Klaukien and Gerhard Wolf (MPI-BPC) for large quantities of polyglutamine peptides.

All other members of the MPI-BPC NMR-based Structural Biology department for cooperation, discussions, and a pleasant working atmosphere, especially Dr. Florian Odronitz for his programming expertise and help; Dr. Nils Lakomek for providing ubiquitin order parameters; 
Dr. Dirk Bockelmann for excellent IT support and infrastructure; and Siegrid Silberer for taking expert care of organizational matters.

Prof. Dr. Olaf Pongs and his students Sönke Hornig and Phanindra Velisetty at the University of Hamburg for their invaluable contributions to the ion channel project.

Dr. Ulrich Zachariae, Dr. Bert de Groot, and Prof. Dr. Helmut Grubmüller of the Theoretical and Computational Biophysics department at the MPI-BPC for the enjoyable and fruitful collaboration on kaliotoxin binding to KcsA-Kv1.3, and for allowing me to do a project week on molecular dynamics simulations in the department.

Dr. Jegannath Korukottu and Prof. Dr. Markus Zweckstetter, also in the NMR-based Structural Biology department of the MPI-BPC, for the collaboration in the high-resolution kaliotoxin structure determination project, where Dr. Korukottu also provided great help for my own efforts at automated structure calculation.

Miria Schumacher and Prof. Dr. Martin Engelhard at the Max-Planck-Institute for Molecular Physiology in Dortmund for the polyglutamine cooperation and sample preparation.

Dr. Dietmar Riedel at the MPI-BPC for many electron micrographs of polyglutamine samples.

Prof. Dr. Ad Bax (National Institutes of Health, Bethesda, Maryland, USA) and Prof. Dr. Roland Riek (ETH Zurich, Switzerland) for providing KcsA resonance assignments.

The DFG graduate school "Spectroscopy and Dynamics of Molecular Coils and Aggregates" for a Ph.D. stipend and travel funding, as well as its speaker Prof. Dr. Martin Suhm and Josée Kupferschmid for continued help and support; the Max Planck Society for further funding; and the 5th Alpine Conference on Solid-State NMR 2007 in Chamonix, France, for giving me the opportunity to present my work in a talk.

Prof. Dr. Maximilian Riesenhuber and Prof. Dr. Tomaso Poggio for allowing me to conduct my master's thesis research at the Massachusetts Institute of Technology, a time from which I could profit a lot.

The late Prof. Dr. Gerhard Neuweiler, former head of the Institute for Zoology and Neurobiology at the University of Munich. A true gentleman and scholar, he always had time and interest even for the freshman's questions, and he inspired me to pursue my own winding path across different branches of science. I have always viewed him as a bright example of what it means to be a scientist.

Last but definitely not least, my parents Prof. Dr. Ernst and Ursula Schneider for their neverending support and inexplicable trust in all my endeavors, and all my dear friends in Göttingen, Munich, and elsewhere in the world for being there for me in good and bad times, keeping me grounded, and occasionally reminding me of the non-scientific joys in life. 


\section{Contents}

$\begin{array}{lll}\text { Abstract } & \text { iii }\end{array}$

$\begin{array}{lll}\text { Kurzzusammenfassung } & \text { v }\end{array}$

Related Publications vii

$\begin{array}{ll}\text { Acknowledgements } & \text { ix }\end{array}$

Contents $\quad$ xi

I Basic principles and methods $\quad 1$

1 Introduction 3

2 Elementary theory of NMR spectroscopy $\quad 9$

2.1 Summary ............................. 9

2.2 Nuclear magnetic resonance . . . . . . . . . . . . . . . . . . . . . 9

2.3 Quantum-mechanical background . . . . . . . . . . . . . . . . . . . 12

2.3.1 Density matrix formalism . . . . . . . . . . . . . . 12

2.3.2 Time evolution . . . . . . . . . . . . . . . . . . 13

2.3.3 Form of the NMR density matrix and Hamiltonian . . . . . . . . 13

2.3.4 Rotating frame transformation . . . . . . . . . . . . . . 14

2.4 NMR interactions . . . . . . . . . . . . . . . . . . . . . . . . . . . . . 14

2.4 .1 Chemical shielding . . . . . . . . . . . . . . . . . . 15

2.4 .2 Interaction with r.f. fields . . . . . . . . . . . . . 15

2.4 .3 Dipolar coupling . . . . . . . . . . . . . . . . 16

2.4 .4 J coupling . . . . . . . . . . . . . . . . . . 16 
3 Solid-state NMR $\quad 17$

3.1 Summary . . . . . . . . . . . . . . . . . . . . . . . 17

3.2 Anisotropic interactions . . . . . . . . . . . . . . . 17

3.3 Tensors and rotations $\ldots \ldots \ldots \ldots \ldots$

3.4 Magic angle spinning . . . . . . . . . . . . . . . . . . . . . . 19

3.5 Decoupling and recoupling . . . . . . . . . . . . . . . 20

3.6 Average Hamiltonian theory . . . . . . . . . . . . . . . . . . 22

4 Experimental techniques $\quad 23$

4.1 Summary . . . . . . . . . . . . . . . . . . . . . . . 23

4.2 Isotope labeling . . . . . . . . . . . . . . . . . . . . 23

4.3 Spectroscopic techniques . . . . . . . . . . . . . . . . . . 24

4.3 .1 Cross-polarization . . . . . . . . . . . . . . . . 24

4.3.2 Correlations for sequential assignment . . . . . . . . . . . 25

4.3 .3 Double-quantum recoupling . . . . . . . . . . . . . . 28

4.3.4 Through-space correlations . . . . . . . . . . . . . . . 29

4.3.5 Water-edited experiments . . . . . . . . . . . . . . 32

4.4 Computational Methods . . . . . . . . . . . . . . . . . . . . 33

4.4 .1 Spin system simulations . . . . . . . . . . . . . . 33

4.4.2 Chemical shift and secondary structure prediction . . . . . . . . 34

$4.4 .3 \quad$ Structure calculation . . . . . . . . . . . . . . . . . . . . 35

$\begin{array}{lll}\text { II Molecular mobility } & 37\end{array}$

5 Mobility detected by double-quantum ssNMR 39

5.1 Summary . . . . . . . . . . . . . . . . . . . 39

5.2 Introduction . . . . . . . . . . . . . . . . . . . 39

5.3 Experimental methods . . . . . . . . . . . . . . . . . . 41

5.4 Simulations . . . . . . . . . . . . . . . . . . 41

5.4 .1 Theory ........................... 41

5.4 .2 Tests . . . . . . . . . . . . . . . . . 43

5.4 .3 Data fitting . . . . . . . . . . . . . . . . . . 47

5.5 Results and Discussion . . . . . . . . . . . . . . . . . . . 48

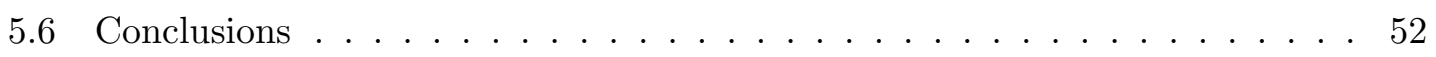


6 Structure and dynamics of microcrystalline ubiquitin $\quad 53$

6.1 Summary . . . . . . . . . . . . . . . . . . . . . . 53

6.2 Introduction . . . . . . . . . . . . . . . . . . 54

6.3 Materials and Methods . . . . . . . . . . . . . . . . . . 55

6.3 .1 Sample preparation . . . . . . . . . . . . 55

$6.3 .2 \quad$ Solid-state NMR . . . . . . . . . . . . . . . . . . . 55

6.3.3 CHHC and chemical shift analysis . . . . . . . . . . 56

6.3 .4 Analysis of dynamics . . . . . . . . . . . . . . . . . 5 56

6.3.5 Structure calculation . . . . . . . . . . . . . . . . 58

6.4 Results . . . . . . . . . . . . . . . . . . . . . . 59

6.4 .1 Analysis of CHHC spectra . . . . . . . . . . . . . 59

6.4 .2 Residue-specific dynamics in ubiquitin . . . . . . . . . . . . 61

6.4.3 Correlation of mobility with shift changes and peak loss . . . . . . 66

6.4 .4 Structure calculation of Ubi-P f . . . . . . . . . . . . . 68

6.5 Discussion . . . . . . . . . . . . . . . . . . 70

$\begin{array}{lll}\text { III The potassium channel KcsA-Kv1.3 } & 75\end{array}$

7 Assignment and structural analysis of KcsA-Kv1.3 $\quad 77$

7.1 Summary . . . . . . . . . . . . . . . . . . . . . 77

7.2 Introduction . . . . . . . . . . . . . . . . . . . 77

7.3 Materials and Methods . . . . . . . . . . . . . . . . . . . . . 79

7.3.1 Expression and purification of KcsA-Kv1.3 . . . . . . . . . . 79

7.3.2 Solid-state NMR experiments and analysis . . . . . . . . . . . . . 79

7.3.3 Generation of the KcsA-Kv1.3 structural model . . . . . . . . . . . . 80

7.4 Results and Discussion . . . . . . . . . . . . . . . . . . 80

7.4.1 2D and 3D ssNMR correlation experiments on KcsA-Kv1.3 . . . 80

7.4 .2 Structural analysis . . . . . . . . . . . . . . . . . 85

7.4 .3 Molecular mobility in KcsA-Kv1.3 . . . . . . . . . . . . . . . . 91

7.5 Conclusions . . . . . . . . . . . . . . . . . . . . 93

8 Gating and inactivation in KcsA-Kv1.3 95

8.1 Summary . . . . . . . . . . . . . . . . . . . . . 95

8.2 Introduction . . . . . . . . . . . . . . . . . . 95 
8.3 Materials and Methods . . . . . . . . . . . . . . . . . 97

8.3 .1 Sample preparation . . . . . . . . . . . . . . 97

8.3 .2 Solid-state NMR . . . . . . . . . . . . . . . . . . . 97

8.3.3 Structural analysis . . . . . . . . . . . . . . . . . . . . . . . 98

8.3 .4 Electrophysiology . . . . . . . . . . . . . . . . . . . . 99

8.4 Results . . . . . . . . . . . . . . . . . . . . . . . . 99

$8.4 .1 \quad$ KcsA-Kv1.3 at acidic $\mathrm{pH} \ldots \ldots \ldots 9$

8.4 .2 Structural analysis . . . . . . . . . . . . . . . . . . 102

8.4.3 Comparison to porphyrin binding . . . . . . . . . . . . . . . 105

8.5 Discussion . . . . . . . . . . . . . . . . . . . . . 106

9 Effects of potassium on activation and inactivation gating 111

9.1 Summary . . . . . . . . . . . . . . . . . . . . . . . . . . . 111

9.2 Introduction . . . . . . . . . . . . . . . . . . . . . . 112

9.3 Materials and Methods . . . . . . . . . . . . . . . . . . . . . 113

9.3.1 Sample preparation . . . . . . . . . . . . . . . 113

9.3.2 Solid-state NMR . . . . . . . . . . . . . . . . . . . . . . . 114

9.3.3 Electrophysiology . . . . . . . . . . . . . . . . . . . . . . . . . . 114

9.4 Results . . . . . . . . . . . . . . . . . . . . . . . . . . . . 114

9.4.1 Potassium sensitivity of KcsA-Kv1.3 conformation . . . . . . . . 114

9.4.2 Correlation of glutamate protonation with activation gating . . . . . 117

9.4.3 Coupling of activation and inactivation gates . . . . . . . . . 120

9.4.4 Modulation of gating by internal and external potassium . . . . . . 121

9.4.5 Ligand binding unmasks gate coupling . . . . . . . . . . . . . . 123

9.5 Discussion . . . . . . . . . . . . . . . . . . . . . . . . . 124

$\begin{array}{ll}\text { IV Fibrillar aggregates } & 131\end{array}$

10 Structural characterization of polyglutamine fibrils 133

10.1 Summary . . . . . . . . . . . . . . . . . . . . . . 133

10.2 Introduction . . . . . . . . . . . . . . . . . . . . . 133

10.3 Materials and Methods . . . . . . . . . . . . . . . . . . . . . 135

10.3.1 Peptide synthesis, expression and fibrillization . . . . . . . . 135

10.3.2 Solid-state NMR . . . . . . . . . . . . . . . . 136 
10.3.3 Analysis of water-edited buildup experiments . . . . . . . . . . 137

10.3.4 Generation of structural models . . . . . . . . . . . . . . . . 137

10.4 Results . . . . . . . . . . . . . . . . . . . . . . . 138

10.4.1 Electron microscopy . . . . . . . . . . . . . . . . 138

10.4.2 Overall fibril organization . . . . . . . . . . . . . . 140

10.4.3 Residue-specific ssNMR analysis . . . . . . . . . . . . . . . . . 142

10.4.4 Structural significance of the different shift populations . . . . . . 146

10.4.5 Through-space correlations . . . . . . . . . . . . . . . . . . . 149

10.5 Discussion . . . . . . . . . . . . . . . . . . . . . 153

$\begin{array}{lll}\text { V Summary } & 161\end{array}$

11 Summary and conclusions 163

$\begin{array}{llr}\text { VI Appendix } & 167\end{array}$

A Mobility by double-quantum ssNMR (Supplement) 169

$\begin{array}{ll}\text { B Structure and dynamics of ubiquitin (Supplement) } & 181\end{array}$

C Assignment and structural analysis of KcsA-Kv1.3 (Supplement) 189

D Gating and inactivation in KcsA-Kv1.3 (Supplement) 201

E Effects of potassium on KcsA-Kv1.3 gating (Supplement) 215

$\begin{array}{lrr}\text { F Polyglutamine fibrils (Supplement) } & \mathbf{2 2 1}\end{array}$

$\begin{array}{lr}\text { List of Symbols and Abbreviations } & 229\end{array}$

$\begin{array}{ll}\text { Bibliography } & 233\end{array}$

$\begin{array}{ll}\text { Lebenslauf } & \mathbf{2 6 1}\end{array}$ 


\section{Part I}

Basic principles and methods 



\section{Chapter 1}

\section{Introduction}

'If you want to understand function, study structure.'

-Francis Crick [8]

Structural biology has made enormous contributions towards advancing our understanding of the function of biological systems. As Watson and Crick wrote in the famous last sentence of their seminal paper describing the DNA double helix, this structure directly suggested a possible mechanism for replication of the genetic material [9]. Similarly, three-dimensional (3D) structures of proteins have revealed mechanistic details about this extremely diverse class of biological macromolecules that could not be attained otherwise, such as the mechanism of ion selectivity in potassium channels [10]. As basic building blocks of cellular structure and function in all domains of life, proteins have received particular attention by structural biology, leading to a total number of over 50,000 protein structures currently deposited in the Protein Data Bank (PDB, http://www.pdb.org/) [11].

While considerable progress has been made in the fields of protein structure prediction and molecular dynamics simulation of protein folding reactions (for recent reviews, see e.g. refs. $[12,13,14,15])$, a general approach to predicting structure from sequence is still elusive due to the the sheer complexity of the protein folding problem and the typical time scales involved. Thus, experimental structural biology is essential for obtaining structural information on proteins. The two methods of choice for obtaining atomic-resolution protein structures are X-ray crystallography and nuclear magnetic resonance (NMR) spectroscopy. While the former has contributed the majority of protein structures known today $(\sim$ 43,500 ), the latter technique allows to study proteins in a native-like environment and to obtain information on their dynamic behavior as well, which is often crucial for protein function [16]. 
Important classes or states of proteins are difficult to study with standard techniques, however. Membrane proteins, on the one hand, constitute about $30 \%$ of all proteins encoded for by the genome [17] (Fig. 1.1 a). They are crucial for processes such as protein synthesis, transport, signal transduction, or electrical signalling in the nervous system, and they represent a majority of pharmaceutical drug targets [18]. However, with currently only 412 structures of 178 unique membrane proteins known, they are drastically underrepresented in the $\mathrm{PDB}^{1}[19]$. This is chiefly due to their amphiphilic nature, which makes both growth of diffraction-quality crystals for X-ray studies and solubilization for solution-state NMR methods highly challenging. Nevertheless, recent

a

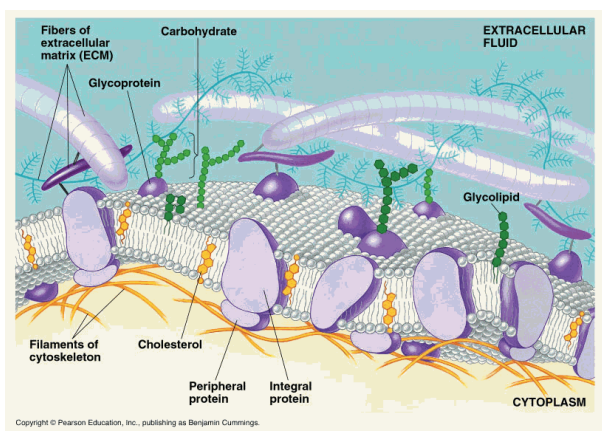

b

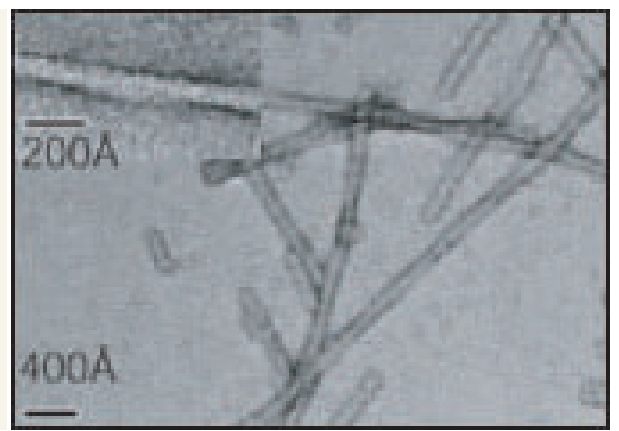

Figure 1.1: (a) Cartoon of the cellular membrane with membrane and other associated proteins. Taken from http://kentsimmons.uwinnipeg.ca/cm1504/plasmamembrane.htm. (b) Electron micrograph of amyloid fibrils formed by the protein $\alpha$-synuclein. Taken from ref. [20].

years have seen remarkable progress in this field. Antibody binding or the presence of lipids in the crystallization setup have in many cases enabled crystal growth from membrane proteins $[21,22,23,24]$, while detergent micelles or bicelles can be used to mimick the membrane environment in solution-state NMR experiments [25, 26, 27, 28]. However, protein functionality is often strongly linked to the surrounding bilayer environment $[29$, $30,31,32]$. Effects of molecular packing in crystals $[33,34]$ or of micelle curvature [35, 36] can additionally compromise the relation of NMR or crystallography data to native membrane protein structure and function.

Ion channels constitute a special class of membrane proteins involved in regulating ion flow and concentration, transmembrane voltage, and action potentials responsible for

\footnotetext{
${ }^{1}$ For lists of membrane proteins of known structure determined by X-ray crystallography or NMR, see: http://blanco.biomol.uci.edu/Membrane_Proteins_xtal.html and http://www.drorlist. com/nmr/MPNMR.html.
} 
information transmission in neurons [37]. As such, they can exist in different states. Apart from open and closed states of the ion-conducting pore, inactivated states exist which are related to, e.g., refractory periods of transmembrane voltage that limit the maximum rate of action potentials produced by an excitable cell [38] (Fig. 1.2). Ion channels have been

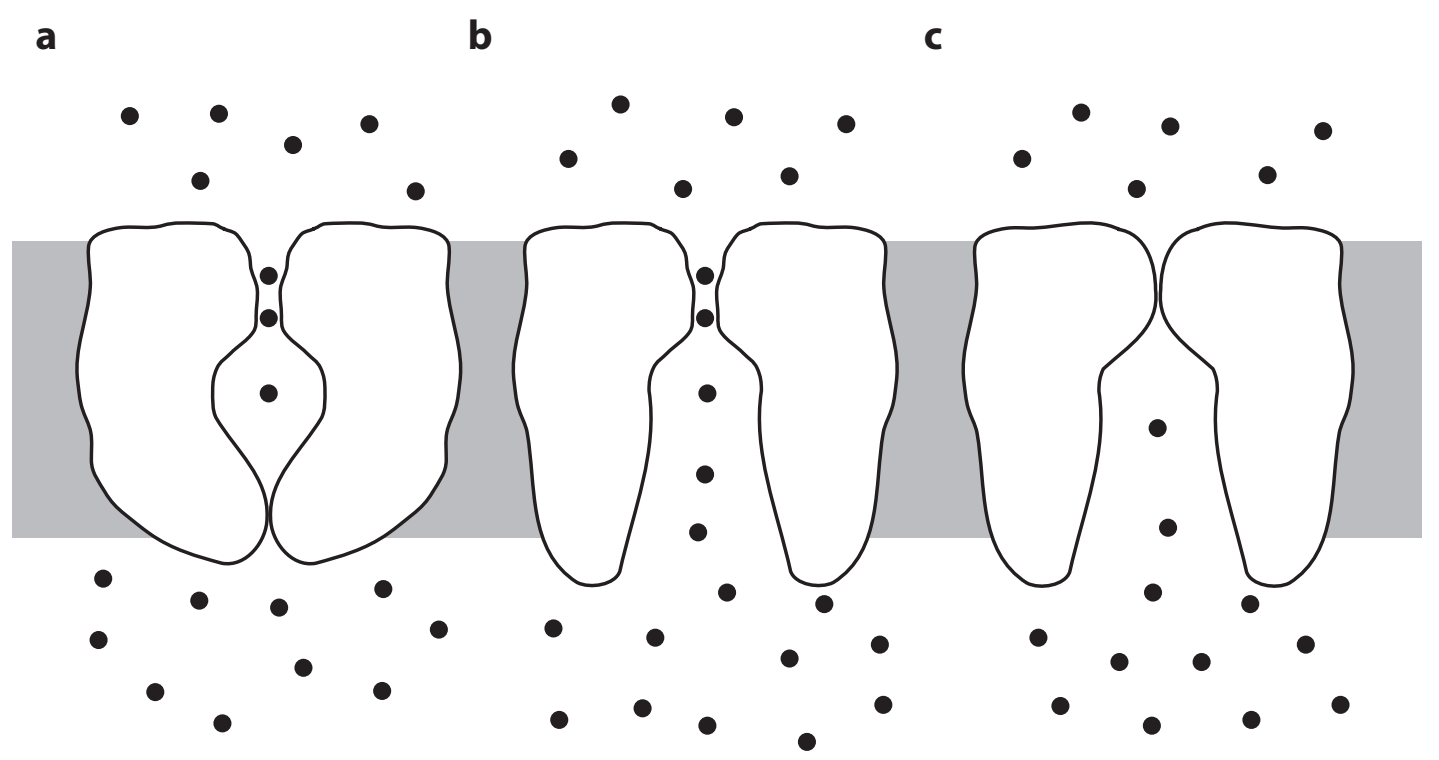

Figure 1.2: Sketch of an ion channel protein in different states in the lipid membrane (grey). Black circles represent ions. (a) closed, (b) open, (c) inactivated state.

intensely studied by functional means [37, 39], and several crystal structures exist (see, e.g., refs $[24,40,41])$. However, still much information especially on the structural basis of these different states and the transitions between them is lacking, and it would be highly desirable to study such processes under native or close to native conditions.

On the other hand, amyloid fibrils represent a protein conformational state that is even less accessible to common structure elucidation techniques. In the amyloid state, proteins with very different primary sequence and native structure form elongated fibrillar aggregates up to hundreds of nanometers in length (Fig. $1.1 \mathrm{~b}$ ). The protein backbone assumes a $\beta$-sheet conformation and runs perpendicular to the fibril long axis, forming backbone hydrogen bonds to the neighboring strand ('cross- $\beta$ ' arrangement) [42]. Amyloid aggregates occur in many devastating diseases, such as Alzheimer's and Parkinson's disease or Chorea Huntington. In prion diseases such as Creutzfeld-Jacob's disease or BSE, the alternative, pathological fold of the protein involved is even infectious [43, 44, 45]. Based on the variability of protein sequences involved in amyloid formation, it has been suggested 
that the amyloid conformation may be a general type of fold accessible to all proteins [46]. However, amyloid fibrils are too large for solution-state NMR investigations, and despite their high degree of long-range order, they do not yield X-ray diffraction patterns allowing for atomic structure determination. Only in some favorable cases, crystals could be grown from small peptides that also form amyloid fibrils [47, 48].

Solid-state NMR (ssNMR) represents a spectroscopic technique ideally suited for structural investigations on both membrane proteins and amyloid fibrils. No long-range order or fast molecular tumbling is required as for X-ray or solution NMR studies, and protein samples can be investigated in their native state, i.e. as full-size amyloid fibrils or, in case of membrane proteins, in a lipid bilayer environment. Other challenges arise, however. For example, strong anisotropic interactions are not averaged out as in the presence of fast overall molecular motion, and thus have to be removed by specific techniques in order to attain high-resolution spectra; isotope labelling is usually a necessity; and while system size, long-range order, or molecular motion do not limit ssNMR investigations in principle, spectral resolution and sensitivity may do so. Such challenges nonwithstanding, rapid progress has been made in this field in recent years. Near-complete resonance assignments and $3 \mathrm{D}$ structures have been reported for small and medium-sized globular model proteins $[49,50,51,52,53]$. While site-specifically labeled membrane proteins have been studied by ssNMR for a long time $[54,55]$, and structures of oriented samples of transmembrane peptides have been solved $[56,57]$, recently, also ssNMR structural studies on large, uniformly isotope-labeled membrane proteins were reported [58, 59, 60, 61, 62]. Finally, in the field of amyloid fibril research, ssNMR is the leading technique for structural investigations, providing constraints on fibril secondary structure and fold as well as structural models [20, 63, 64, 65], and first 3D structures have been published [66, 67].

In this context, the goal of this thesis is to contribute to the advancement of biomolecular solid-state NMR in three of the outlined fields of research: methods development on model systems, membrane proteins, and amyloid fibrils. The following chapters begin with an introduction to the theoretical principles of NMR spectroscopy in general (Chapter 2) and solid-state NMR in particular (Chapter 3). An overview of experimental methods used in the context of this thesis is given in Chapter 4. Chapter 5 then develops doublequantum (2Q) ssNMR spectroscopy as a novel method to measure protein dynamics in the solid state. As a model system, the small molecule L-tyrosine-ethylester (TEE) is used, a system for which previous studies have already shown the presence of different degrees of molecular motion $[68,69]$. Application of this method to the protein ubiquitin 
is then described in Chapter 6. Results are compared with other studies on the dynamics of this protein in different preparations and combined with a 3D structure calculation. In particular, the issue of the dependence of NMR chemical shift and local dynamics on sample preparation is addressed [70].

Chapters 7-9 then deal with the ssNMR investigation of structure and function of the chimeric potassium ion channel KcsA-Kv1.3, a tetramer of 160 amino acid residues per monomer $[62,71]$. As a basis for further investigations, Chapter 7 first describes ssNMR resonance assignments for a majority of residues of this large membrane protein, as well as its secondary structure in lipid bilayers. Based on these results, Chapter 8 discusses structural changes in KcsA-Kv1.3 upon its transition to an open-inactivated state at low pH. Data obtained in a functional lipid bilayer environment and in close reference to electrophysiological experiments provide a structural basis for the observed functional changes. Chapter 9 then investigates the dependence of the processes of opening and inactivation in this channel on the concentration of potassium. This dependence has so far not been studied on a structural level, and ssNMR data thus provide novel insight into the regulation of ion channels by the permeant ion itself.

Finally, Chapter 10 turns to the study of fibrillar protein aggregates by ssNMR. Polyglutamine peptides of different length are investigated as model systems for amyloidforming proteins that contain polyglutamine expansions, such as the huntingtin protein involved in Huntington's disease [72]. A range of different ssNMR methods is applied, leading to a model for the fold of polyglutamine peptides within their fibrillar aggregates. 


\section{Chapter 2}

\section{Elementary theory of NMR spectroscopy}

\subsection{Summary}

Nuclear magnetic resonance (NMR) spectroscopy makes use of interactions of nuclear spins with an external magnetic field and with each other to probe molecular structure and dynamics. This chapter introduces fundamental concepts of NMR spectroscopy and their quantum mechanical description. NMR interactions that are of interest in the context of this thesis are discussed.

\subsection{Nuclear magnetic resonance}

Spectroscopy deals with the interaction of matter with electromagnetic radiation to probe physical properties of the system under study. Nuclear magnetic resonance (NMR) spectroscopy makes use of an effect first described in 1946 by Bloch, Purcell and their coworkers $[73,74]$ (Nobel Prize in physics 1952). For atomic nuclei with nonzero spin angular momentum, the eigenstates of the spin angular momentum operator have different energies in the presence of an external magnetic field $B_{0}$ (Zeeman splitting). Transitions between these states can be induced by applying an additional oscillating magnetic field, orthogonal to the static field, whose frequency $\omega_{0}$ corresponds to the energy difference between eigenstates (Fig. 2.1):

$$
\omega_{0}=\frac{\Delta E}{\hbar}=-\gamma B_{0}
$$

This equation constitutes the fundamental resonance condition of NMR spectroscopy. $\gamma$ is the gyromagnetic ratio of the nucleus in question, and $\omega_{0}$ its Larmor frequency (in angular frequency units). For typical magnetic field strengths in NMR magnets (several Tesla, 
achieved with superconducting coils), $\omega_{0} / 2 \pi$ assumes values in the megahertz $(\mathrm{MHz})$, i.e. radiofrequency (r.f.) range.

The exact resonance frequency of any individual nucleus at field strength $B_{0}$ depends not only on $\gamma$, but also on its chemical environment $[75,76]$, chiefly because motions of electrons induced by the external magnetic field generate local secondary magnetic fields [77]. These variations of the resonance frequency, called chemical shifts, form the basis

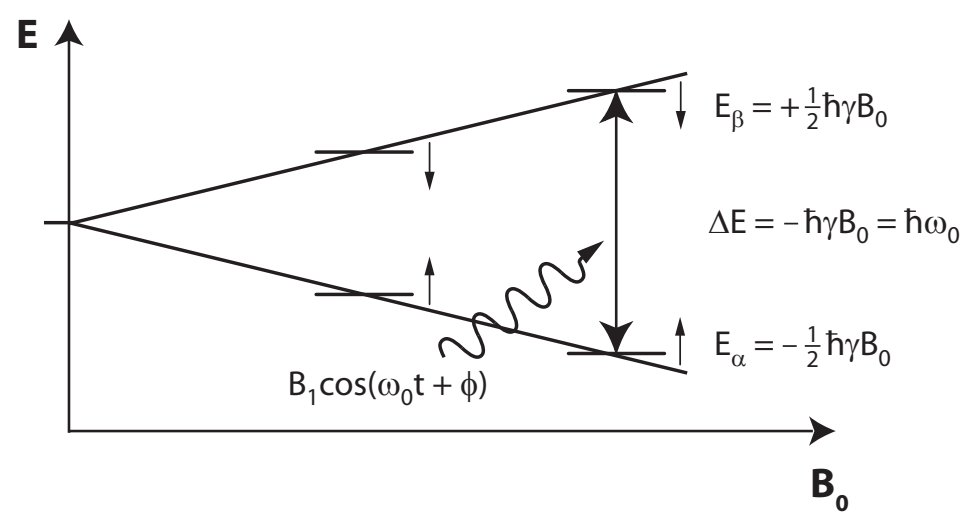

Figure 2.1: Illustration of the Zeeman splitting of spin states for a spin- $\frac{1}{2}$ nucleus. Energies $(E)$ are shown in dependence of magnetic field strength $\left(B_{0}\right)$. The oscillating line indicates a resonant radiofrequency field.

for obtaining structural information about a molecule from NMR. Chemical shifts usually are on the order of $10^{6}$ times smaller than the Larmor frequency and are thus commonly measured in ppm (parts per million) as offsets from the resonance frequency of a reference compound.

The measurement of NMR resonance signals was greatly facilitated by the introduction of Fourier-transform NMR spectroscopy by Ernst (Nobel Prize in chemistry 1991) and Anderson [78]. Here, short r.f. pulses, covering a range of frequencies around $\omega_{0}$, are used to excite resonant transitions in nuclei with different chemical shifts. Relaxation of the system back to equilibrium is then observable as a precession of the bulk magnetic moment around the direction of the $B_{0}$ field, which can be detected as induced current in a receiver coil. Fourier transformation of this free induction decay (FID) time-domain signal $s(t)$ then yields a spectrum that reveals the resonance frequencies of the nuclei contributing to the signal (Fig. 2.2 a):

$$
S(\omega) \propto \int_{0}^{\infty} s(t) e^{-i \omega t} d t
$$


a

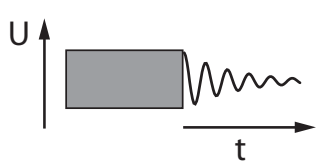

b

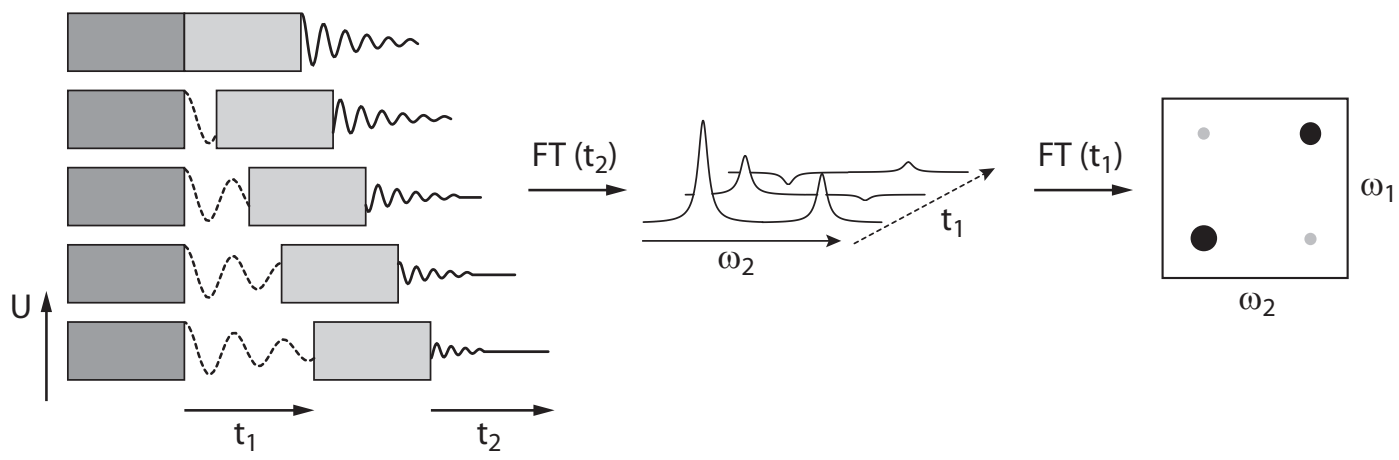

Figure 2.2: Fourier-transform NMR. Radiofrequency pulses or pulse sequences are indicated as shaded rectangles. $U$ : applied or detected voltage. (a) One-dimensional frequency $(\omega)$ spectrum with peak intensities $I$ obtained by Fourier transformation of a free induction decay (FID, oscillating line). (b) Two-dimensional spectrum obtained by double Fourier transformation of an FID with two time dependencies. 
Adding another period of free precession to the experiment and recording the final FID for different durations of this "indirect evolution" period yields a signal $s\left(t_{1}, t_{2}\right)$ with two time dependencies that can be Fourier transformed twice:

$$
S(\omega 1, \omega 2) \propto \int_{0}^{\infty} \int_{0}^{\infty} s\left(t_{1}, t_{2}\right) e^{-i \omega_{1} t_{1}} e^{-i \omega_{2} t_{2}} d t_{1} d t_{2}
$$

This leads to a two-dimensional spectrum in which interactions between different nuclei that take place between the indirect evolution and signal acquisition periods are directly revealed as off-diagonal "crosspeaks" [77, 79] (Fig. 2.2 b). Extension to more dimensions is also possible.

Important nuclei in biomolecular NMR spectroscopy are ${ }^{1} \mathrm{H},{ }^{13} \mathrm{C},{ }^{15} \mathrm{~N}$, and also ${ }^{31} \mathrm{P}$. All of these are spin- $\frac{1}{2}$ nuclei, i.e. they have two spin eigenstates and, correspondingly, two

Zeeman energy levels, $m=+\frac{1}{2}$ ( $\alpha$ state) and $m=-\frac{1}{2}$ ( $\beta$ state). The NMR effect depends on the presence of a population difference between the two states. Under equilibrium conditions, this is brought about by a Boltzmann distribution of the spins, yielding a relative population $N_{m} / N$ of state $m$ as:

$$
\begin{aligned}
\frac{N_{m}}{N} & =\exp \left(\frac{-E_{m}}{k_{B} T}\right) / \sum_{m^{\prime}=-1 / 2}^{+1 / 2} \exp \left(\frac{-E_{m^{\prime}}}{k_{B} T}\right) \\
& \approx \frac{1}{2}\left(1+\frac{m \hbar \gamma B_{0}}{k_{B} T}\right)
\end{aligned}
$$

The population difference is small, on the order of 1 in $10^{5}$ spins for ${ }^{1} \mathrm{H}$ nuclei at room temperature in a static magnetic field of $11.7 \mathrm{~T}$ (corresponding to a ${ }^{1} \mathrm{H}$ resonance frequency of $500 \mathrm{MHz}$ ) [77]. Thus, NMR is a comparatively insensitive spectroscopic technique, and many efforts have been taken to improve its sensitivity. As can be seen from Equation 2.4 , this can be achieved, e.g., by increasing magnetic field strength or measuring at lower temperatures, since in both cases, the population difference between the Zeeman levels is increased.

\subsection{Quantum-mechanical background}

\subsubsection{Density matrix formalism}

An appropriate description of NMR that also includes interactions between spins can only be obtained at the quantum-mechanical level. Since the spin degrees of freedom primarily determine the outcome of an NMR experiment, usually only spin wavefunctions 
are considered explicitly in the quantum-mechanical description. Other degrees of freedom contribute, e.g., to spin relaxation effects and are collectively termed the lattice into which the spins are embedded. This makes a statistical approach necessary since no unique wavefunction can be set up for all spins in a sample (i.e. it is in a mixed state) [77].

A suitable approach for the description of such a system is the density matrix formalism [80]. A system (with an orthonormal basis set $|n\rangle$ ) that consists of $k$ subensembles, each of which is described by a wavefunction $\left|\psi^{(k)}\right\rangle=\sum_{n} c_{n}^{(k)}|n\rangle$ and which contributes to the total system with a probability $p^{(k)}$, can be described by a density matrix $\hat{\rho}$ :

$$
\hat{\rho}=\sum_{k} p^{(k)}\left|\psi^{(k)}\right\rangle\left\langle\psi^{(k)}\right|
$$

The elements of $\hat{\rho}$ are ensemble averages of the products of the coefficients $c_{n}$, i.e. $\hat{\rho}_{n m}=$ $\overline{c_{n} c_{m}^{*}}=\sum_{k} p^{(k)} c_{n}^{(k)} c_{m}^{(k) *}$. The expectation value of a property $A$, represented by a Hermitian operator $\hat{A}$, is then calculated as $\overline{\langle A\rangle}=\operatorname{Tr}(\hat{\rho} \hat{A})[77]$.

\subsubsection{Time evolution}

Time evolution of the density matrix under a Hamiltonian $\hat{H}$ is described by rewriting the time-dependent Schrödinger equation in the density matrix formalism, yielding the Liouville-von Neumann equation:

$$
\frac{d}{d t} \hat{\rho}(t)=-\frac{i}{\hbar}[\hat{H}(t), \hat{\rho}(t)]
$$

Its solution is straightforward if $\hat{H}$ is time-independent:

$$
\hat{\rho}(t)=\exp \left(-\frac{i}{\hbar} \hat{H} t\right) \hat{\rho}(0) \exp \left(\frac{i}{\hbar} \hat{H} t\right)
$$

with $\exp \left(-\frac{i}{\hbar} \hat{H} t\right)$ the propagator for the system [77]. Approaches to deal with timedependent Hamiltonians are average Hamiltonian theory [81, 82] (AHT, see Section 3.6), Floquet theory [83] or numerical integration using a piecewise constant Hamiltonian.

\subsubsection{Form of the NMR density matrix and Hamiltonian}

The static magnetic field $B_{0}$ is usually chosen to be along the $z$ direction. The Hamiltonian governing interaction of the spin system with it, the so-called Zeeman Hamiltonian, has the form

$$
\hat{H}=-\boldsymbol{\mu} \cdot \boldsymbol{B}_{0}=-\gamma \hat{\boldsymbol{I}} \cdot B_{0} \boldsymbol{e}_{z}=\omega_{0} \hat{I}_{z}
$$


In the high-temperature approximation $\left(E_{n} \ll k_{B} T\right.$ for the energy of state $n$ ), the equilibrium density operator can also be cast in terms of the Zeeman Hamiltonian:

$$
\hat{\rho}_{0}=-\hat{H} /\left(N k_{B} T\right)=-\sum_{i=1}^{N} \frac{\omega_{0 i}}{N k_{B} T} \hat{I}_{i z}
$$

where the sum goes over all spins in the system and $\hat{I}_{z}=\sum_{i=1}^{N} \hat{I}_{i z}$. NMR experiments are commonly described in terms of a product operator formalism in which both the relevant Hamiltonians and the density matrix are written in terms of the spin angular momentum operators for the spin system considered. Time evolution of the density matrix can then be described as rotations in product operator space [77].

\subsubsection{Rotating frame transformation}

The oscillating r.f. field always introduces a time dependence to the system Hamiltonian in NMR experiments. It can be removed from $\hat{H}$ by transforming to a frame of reference rotating with the r.f. frequency. The transformation is represented by a unitary operator $\hat{U}$ which transforms the density matrix $\hat{\rho}$ as $\hat{\rho}^{\prime}=\hat{U} \hat{\rho} \hat{U}^{-1}$. The form of the Liouville-von Neumann equation is preserved in the rotating frame if $\hat{H}$ is transformed as

$$
\hat{H}^{\prime}=\hat{U} \hat{H} \hat{U}^{-1}-i \hat{U} \frac{d \hat{U}^{-1}}{d t}
$$

For transforming to a frame rotating at the r.f. frequency, $\hat{U}$ is chosen as $\hat{U}=\exp \left(i \omega_{\mathrm{rf}} \hat{I}_{z} t\right)$. This effectively eliminates the Zeeman interaction term which contains no structural information, leaving $\hat{H}^{\prime}=\left(\omega_{0}-\omega_{\mathrm{rf}}\right) \hat{I}_{z}=\Omega \hat{I}_{z}$, where $\Omega$ is the chemical shift [77]. NMR experiments are usually described in the rotating frame. If pulse sequences introduce additional time dependencies, further transformations can become necessary to describe the experiment in a 'toggling' or 'interaction' frame [84, 85].

\subsection{NMR interactions}

In the rotating frame, the relevant interactions contributing to the NMR Hamiltonian of a system of $\mathrm{N}$ spin- $\frac{1}{2}$ particles can be written as

$$
\hat{H}=\sum_{i=1}^{N}\left(\hat{H}_{\mathrm{CS}}^{(i)}+\hat{H}_{\mathrm{RF}}^{(i)}\right)+\sum_{i<j}^{N}\left(\hat{H}_{\mathrm{D}}^{(i j)}+\hat{H}_{\mathrm{J}}^{(i j)}\right)
$$

where the contributions are from chemical shielding (CS), radiofrequency fields (RF), dipolar coupling (D) and through-bond $\mathrm{J}$ coupling (J) interactions. Other interactions 
such as the quadrupolar coupling for nuclei with spin $>\frac{1}{2}$ or hyperfine interactions, as well as relaxation effects $[77,85,86]$ will not be considered further in the scope of this thesis.

According to perturbation theory, in the presence of a strong interaction $\hat{H}_{0}$ a weak interaction $\hat{H}_{1}$ can be reduced to those components that commute with $\hat{H}_{0}$. Since, at high magnetic fields, the Zeeman interaction dominates all others, only terms commuting with the Zeeman Hamiltonian (Equation 2.8) are usually considered for the treatment of NMR interactions (secular or high-field approximation) [87].

The parts of the Hamiltonian which are of interest here can generally be written in the form $\hat{H}=\hat{\boldsymbol{I}}_{i} \mathbf{A} \hat{\boldsymbol{I}}_{j}$, where $\hat{\boldsymbol{I}}_{i}, \hat{\boldsymbol{I}}_{j}$ are spin operators or, in case of chemical shielding and r.f. interactions, magnetic field vectors, and $\mathbf{A}$ is a second-rank Cartesian tensor. They are briefly introduced in the following.

\subsubsection{Chemical shielding}

Chemical shielding due to the electronic environment of a nucleus is the source of the chemical shift. It is described by a shielding tensor $\sigma$ :

$$
\hat{H}_{\mathrm{CS}}=\gamma \hat{\boldsymbol{I}} \boldsymbol{\sigma} \boldsymbol{B}_{0}
$$

It can be decomposed into an antisymmetric and a symmetric part, of which only the symmetric part contributes to the NMR Hamiltonian in the high-field approximation. Its isotropic average $\sigma_{\text {iso }}$, i.e. the average of its diagonal elements in the principal axis system (PAS) $\sigma_{\text {iso }}=\frac{1}{3} \sum_{i} \sigma_{i i}^{\mathrm{PAS}}$, is the isotropic chemical shift which is seen in NMR spectra under conditions of fast molecular tumbling (i.e. in solution). The orientation dependence of the anisotropic part leads to a broadening and characteristic lineshape for randomly oriented static samples (powder pattern) [84].

\subsubsection{Interaction with r.f. fields}

Interactions of the spin system with an r.f. field $\boldsymbol{B}_{1}$ are described in analogy to the Zeeman interaction, however $\boldsymbol{B}_{1} \ll \boldsymbol{B}_{0} . \boldsymbol{B}_{1}$ is usually linearly polarized with phase $\phi$ and orthogonal to $\boldsymbol{B}_{0}$. In the frame rotating about $z$ with $\omega_{\text {rf }}$, continuous r.f. irradiation at frequency $\omega_{\mathrm{rf}}$ is time-independent and described as

$$
\hat{H}_{\mathrm{rf}}=-\gamma \hat{\boldsymbol{I}} \cdot \boldsymbol{B}_{1}=\omega_{1}\left(\hat{I}_{x} \cos \phi+\hat{I}_{y} \sin \phi\right)
$$

with $\omega_{1}=-\gamma\left|\boldsymbol{B}_{1}\right|[77]$. 


\subsubsection{Dipolar coupling}

The magnetic dipole coupling between two spins arises from the magnetic moment of each individual nuclear spin which influences other spins in a distance- and orientationdependent manner. It is described by a dipolar tensor $\mathbf{D}$ which is traceless and symmetric. In the principal axis system of the dipolar interaction (with the internuclear vector along the $z$ direction), it can be written as

$$
\begin{aligned}
\hat{H}_{\mathrm{D}} & =\hat{\boldsymbol{I}}_{i} \mathbf{D}_{i j} \hat{\boldsymbol{I}}_{j}=d_{i j} \cdot \hat{\boldsymbol{I}}_{i}\left(\begin{array}{ccc}
1 & 0 & 0 \\
0 & 1 & 0 \\
0 & 0 & -2
\end{array}\right) \hat{\boldsymbol{I}}_{j}= \\
& =d_{i j} \cdot\left(\hat{\boldsymbol{I}}_{i} \hat{\boldsymbol{I}}_{j}-3 \hat{I}_{i z} \hat{I}_{j z}\right)
\end{aligned}
$$

with $d_{i j}=\frac{\mu_{0}}{4 \pi} \hbar \frac{\gamma_{i} \gamma_{j}}{r_{i j}^{3}}$ the dipolar coupling constant (in units of $\mathrm{rad} \cdot \mathrm{s}^{-1}$ ) for two nuclei $i, j$ with gyromagnetic ratios $\gamma_{i}, \gamma_{j}$ and an internuclear distance $r_{i j}$. Expansion in polar coordinates leads to the following expression for the secular terms of the homonuclear dipolar coupling:

$$
\hat{H}_{\mathrm{D}}=-d_{i j} \cdot \frac{3 \cos ^{2} \theta-1}{2}\left(2 \hat{I}_{1 z} \hat{I}_{2 z}-\frac{1}{2}\left(\hat{I}_{1}^{+} \hat{I}_{2}^{-}+\hat{I}_{1}^{-} \hat{I}_{2}^{+}\right)\right)
$$

with $\theta$ the angle between $\boldsymbol{r}_{i j}$ and $\boldsymbol{B}_{0}$. In the case of a heteronuclear spin pair, the latter term becomes nonsecular and can be neglected.

While the dipolar coupling, as an anisotropic interaction with no isotropic contribution, is averaged to zero by molecular tumbling in isotropic solution, it is a dominant interaction in solid samples. However, molecular motion may scale it also in the solid phase, making it a possible probe for dynamic processes. By virtue of its dependence on $\boldsymbol{r}_{i j}$, the dipolar coupling can be used for distance measurements [84].

\subsubsection{J coupling}

The $\mathrm{J}$ coupling between two nuclei is an indirect spin-spin interaction mediated by the electrons of the chemical bond. Its anisotropic component can usually be neglected, leaving only an isotropic value, the scalar coupling:

$$
\hat{H}_{J}=2 \pi J \hat{\boldsymbol{I}}_{i} \hat{\boldsymbol{I}}_{j}
$$

As an isotropic quantity, the scalar coupling also remains present under conditions of fast molecular tumbling and can be used to mediate polarization transfer both in solids and in solution $[77,88]$. 


\section{Chapter 3}

\section{Solid-state NMR}

\subsection{Summary}

Solid-state NMR (ssNMR) differs from NMR in solution by the absence of fast molecular tumbling, and consequently, anisotropic interactions are present. This chapter briefly describes the most important tools for the theoretical description of NMR experiments in the presence of such interactions and the fundamental approaches for selectively reducing or enhancing their influence on ssNMR experiments. A more detailed introduction to experimental ssNMR methods is then the subject of Chapter 4.

\subsection{Anisotropic interactions}

The chief difference between NMR in solution and in the solid state is the absence of fast overall molecular reorientation in solids. This entails that anisotropic interactions such as chemical shift anisotropy and dipolar coupling remain present and are not averaged out. They can thus directly be used for molecular structure determination, e.g. the dipolar coupling for distance measurements and chemical shift anisotropy for determining the relative orientation of functional groups [84]. However, the orientation dependence of anisotropic interactions leads to different resonance frequencies in identical nuclei for different molecular orientations. Severe line broadening is consequently observed (Fig. 3.1), precluding high-resolution spectroscopy in larger molecular systems. Thus, measures have to be taken to remove these interactions for recording high-resolution solid-state NMR (ssNMR) spectra (Section 3.4), while preserving or reintroducing them for obtaining information on molecular structure (Section 3.5). 


\subsection{Tensors and rotations}

For describing ssNMR experiments, rotations between different axis frames have to be taken into account (usually the principal axis system (PAS) of the interaction, the molecular frame (MOL), the rotor fixed frame for magic-angle-spinning experiments (ROT, see Section 3.4), and the laboratory frame (LAB)). This is facilitated by expression of the Hamiltonian in terms of irreducible spherical tensors [89] since these exhibit advantageous transformation properties under rotations.

For all relevant ssNMR interactions, the Hamiltonian can be separated into two parts containing either space $(\hat{\boldsymbol{A}})$ or the spin $(\hat{\boldsymbol{T}})$ coordinates only, both of which can be expressed as irreducible spherical tensors of rank 2 (or less). For an interaction $\Lambda$ (such as chemical shielding or dipolar coupling) that can be described by spherical tensors of rank $l(l \leq 2)$, the Hamiltonian can thus be written as

$$
\hat{H}^{\Lambda}=\sum_{l=0}^{2} \hat{\boldsymbol{A}}_{l}^{\Lambda} \cdot \hat{\boldsymbol{T}}_{l}^{\Lambda}=\sum_{l=0}^{2} \sum_{m=-l}^{l}(-1)^{m} \hat{A}_{l, m}^{\Lambda} \hat{T}_{l,-m}^{\Lambda}
$$

according to tensor multiplication rules [89]. For example, following the rules of expressing Cartesian tensors in a spherical tensor basis $[84,89]$, the only nonvanishing component of the $\hat{\boldsymbol{A}}^{D}$ tensor for the space components of the dipolar coupling between two spins $i, j$ in its principal axis frame is $\hat{A}_{20}^{D}=-\sqrt{6} d_{i j}=-\sqrt{6} \frac{\mu_{0}}{4 \pi} \hbar \frac{\gamma_{i} \gamma_{j}}{r_{i j}^{3}}$. Thus, the only component of the corresponding spin tensor $\hat{\boldsymbol{T}}^{D}$ that needs to be considered in the PAS is $\hat{\boldsymbol{T}}_{20}^{D}=$ $\sqrt{\frac{1}{6}}\left(3 \hat{I}_{i z} \hat{I}_{j z}-\hat{\boldsymbol{I}}_{\boldsymbol{i}} \hat{\boldsymbol{I}}_{\boldsymbol{j}}\right)$, yielding the same result for $\hat{H}^{D}$ as in Equation 2.14 .

Transformations to different axis frames are then described by rotations $R$ around Euler angles $(\alpha, \beta, \gamma)$ and applied to these tensors using Wigner rotation matrices [84, 89]:

$$
\begin{aligned}
\hat{\boldsymbol{A}}_{l}^{\prime} & =R(\alpha, \beta, \gamma) \hat{\boldsymbol{A}}_{l} R^{-1}(\alpha, \beta, \gamma) \\
\hat{A}_{l, m^{\prime}}^{\prime} & =\sum_{m=-l}^{l} \hat{A}_{l, m} D_{m, m^{\prime}}^{l}(\alpha, \beta, \gamma)
\end{aligned}
$$

where $D_{m, m^{\prime}}^{l}(\alpha, \beta, \gamma)=e^{-i m \alpha} d_{m, m^{\prime}}^{l}(\beta) e^{-i m^{\prime} \gamma}$ and $d_{m, m^{\prime}}^{l}(\beta)$ is a reduced Wigner element [89]. This procedure can be used analogously for spin tensors, e.g. for transforming to an interaction frame of an r.f. pulse sequence. In the high-field approximation, only laboratory-frame spin tensor components with $m=0\left(\hat{T}_{0,0}, \hat{T}_{1,0}, \hat{T}_{2,0}\right)$ need to be considered since only these commute with the Zeeman Hamiltonian. Additionally, antisymmetric contributions $(l=1)$ can usually be neglected in first-order average Hamiltonian theory 
which is commonly used for describing ssNMR experiments (see Section 3.6) [84]. The laboratory frame Hamiltonian of an interaction $\Lambda$ is thus usually sufficiently described as

$$
\hat{H}^{\Lambda, \text { lab }}=\hat{A}_{0,0}^{\Lambda, \text { lab }} \hat{T}_{0,0}^{\Lambda, \text { lab }}+\hat{A}_{2,0}^{\Lambda, \text { lab }} \hat{T}_{2,0}^{\Lambda, \text { lab }}
$$

\subsection{Magic angle spinning}

An important step towards high-resolution ssNMR spectroscopy was the finding that line broadening due to orientation-dependent anisotropic interactions can be removed or reduced by fast rotation of the sample around an axis inclined at the "magic angle" $\theta_{m}=\arccos \frac{1}{\sqrt{3}} \approx 54.74^{\circ}$, i.e. the angle of the cubic diagonal, with respect to the $\boldsymbol{B}_{0}$ field [90, 91] (Fig. 3.1 a). This "magic angle spinning" (MAS) is realized by packing the sample

a

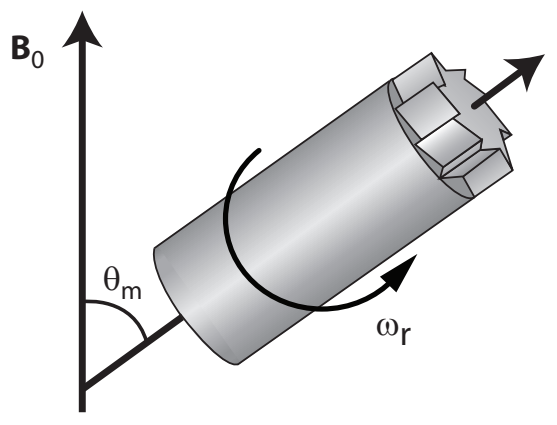

b

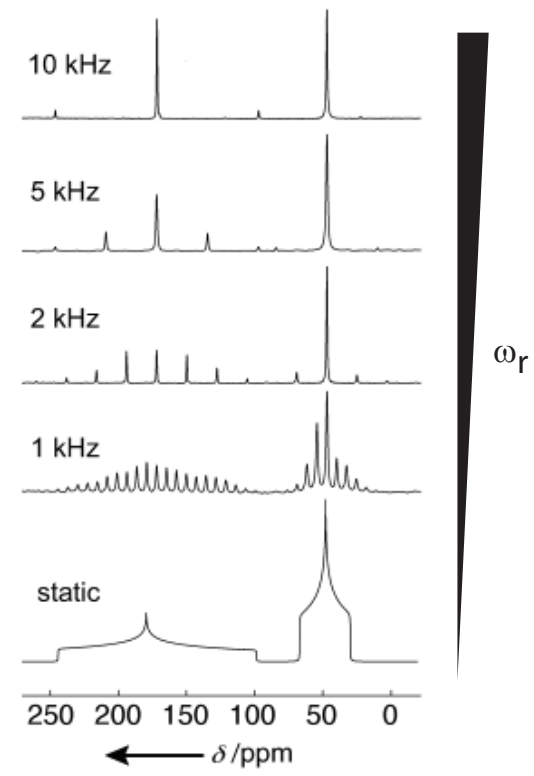

Figure 3.1: Magic angle spinning. (a) Illustration of an MAS rotor spinning at the magic angle with respect to $B_{0}$. Adapted from ref. [92]. (b) A broad static powder pattern (bottom) is resolved into a center band and many sidebands by slow MAS, while for fast MAS, only a sharp resonance line at the isotropic chemical shift remains. Figure obtained from ref. [93].

in a cylindrical rotor which is lifted and spun by gas streams inside a stator. The exact tilt angle of the stator in the NMR probehead can be adjusted to match the magic angle. MAS is routinely employed for most ssNMR spectroscopic investigations on biomolecules.

The effect of MAS can be explained by considering the laboratory frame component $\hat{A}_{2,0}^{l a b}$ of the rank 2 space tensor of the interaction in question. (As just discussed in Section 
3.3 , the only other relevant component for the laboratory frame Hamiltonian is $\hat{A}_{0,0}^{l a b}$, which is isotropic and unaffected by rotations.) For a sample rotated at $\theta_{m}$ with a frequency $\omega_{r}, \hat{A}_{2,0}^{l a b}$ is obtained by a transformation of $\hat{\boldsymbol{A}}_{2}^{\text {rot }}$ (in the rotor frame) about Euler angles $(\alpha, \beta, \gamma)=\left(\omega_{r} t, \theta_{m}, 0\right)$. The rotor-frame component $\hat{A}_{2,0}^{r o t}$ is transformed by the reduced Wigner element $d_{0,0}^{2}(\beta)=\frac{1}{2}\left(3 \cos ^{2} \beta-1\right)$, which is zero for $\beta=\theta_{m}$. The other rotor-frame components attain a time-dependence, such that $\hat{A}_{2,0}^{\text {lab }}$ is of the form

$$
\hat{A}_{2,0}^{l a b}=\sum_{m=-2,-1,1,2} \hat{A}_{2, m}^{r o t} d_{m, 0}^{2}\left(\theta_{m}\right) e^{-i m \omega_{r} t}
$$

Consequently, for sufficiently fast sample rotation (significantly faster than the anisotropy of the interaction in angular frequency units), anisotropic contributions are efficiently averaged out, and only the isotropic part remains (Fig. $3.1 \mathrm{~b}$ ). In case of the chemical shift interaction, a single resonance line is obtained in the spectrum at the position of the isotropic chemical shift, as in solution-state NMR. However, for slower spinning, spinning sidebands occur apart from the resonance line at the isotropic shift, spaced at multiples of $\omega_{r}$ from it [84]. Moreover, spinning speeds most commonly attainable $\left(\omega_{r} / 2 \pi\right.$ up to about $30 \mathrm{kHz})$ are insufficient to average out especially the homonuclear $\left({ }^{1} \mathrm{H},{ }^{1} \mathrm{H}\right)$ dipolar couplings, but also sizable heteronuclear $\left({ }^{1} \mathrm{H},{ }^{13} \mathrm{C}\right)$ and $\left({ }^{1} \mathrm{H},{ }^{15} \mathrm{~N}\right)$ dipolar couplings remain. Thus, additional measures have to be taken to achieve high-resolution conditions, such as r.f. decoupling (Section 3.5). Recent technological advances allow for MAS speeds up to $70 \mathrm{kHz}$, significantly improving the removal of unwanted interactions by MAS alone.

\subsection{Decoupling and recoupling}

To efficiently remove heteronuclear dipolar couplings of carbon and nitrogen nuclei to protons, r.f. irradiation can be used. Such r.f. decoupling is usually mandatory during signal acquisition periods if high-resolution ${ }^{13} \mathrm{C}$ or ${ }^{15} \mathrm{~N}$ ssNMR spectra are to be obtained. In the most simple implementation, a strong continuous-wave (CW) r.f. field is applied to the protons, causing fast ${ }^{1} \mathrm{H}$ spin flips and averaging out their coupling to other nuclei. More sophisticated multiple-pulse schemes such as TPPM [94] or SPINAL [95] provide improved decoupling efficiency. Homonuclear $\left({ }^{1} \mathrm{H},{ }^{1} \mathrm{H}\right)$ dipolar couplings can be attenuated, e.g., by Lee-Goldburg-type pulse sequences $[96,97]$. Here, off-resonance proton r.f. irradiation is employed such that an effective ${ }^{1} \mathrm{H}$ field is obtained which is inclined at the magic angle with respect to $\boldsymbol{B}_{0}$ and around which the proton spins nutate. More complex 
pulse sequences with quasi-continuous phase modulation of the r.f. irradiation have been developed in recent years [98].

On the other hand, interactions that have to be averaged out or attenuated to obtain high-resolution spectra contain useful information on molecular structure, such as internuclear distance (dipolar coupling) or orientation of functional groups (chemical shift anisotropy), as mentioned. The selective re-introduction or recoupling of such interactions under MAS conditions is thus desirable in parts of ssNMR pulse sequences that correlate different nuclei with each other. It can be accomplished by r.f. irradiation specifically tailored to reintroduce the interaction of interest. A large variety of ssNMR pulse se-
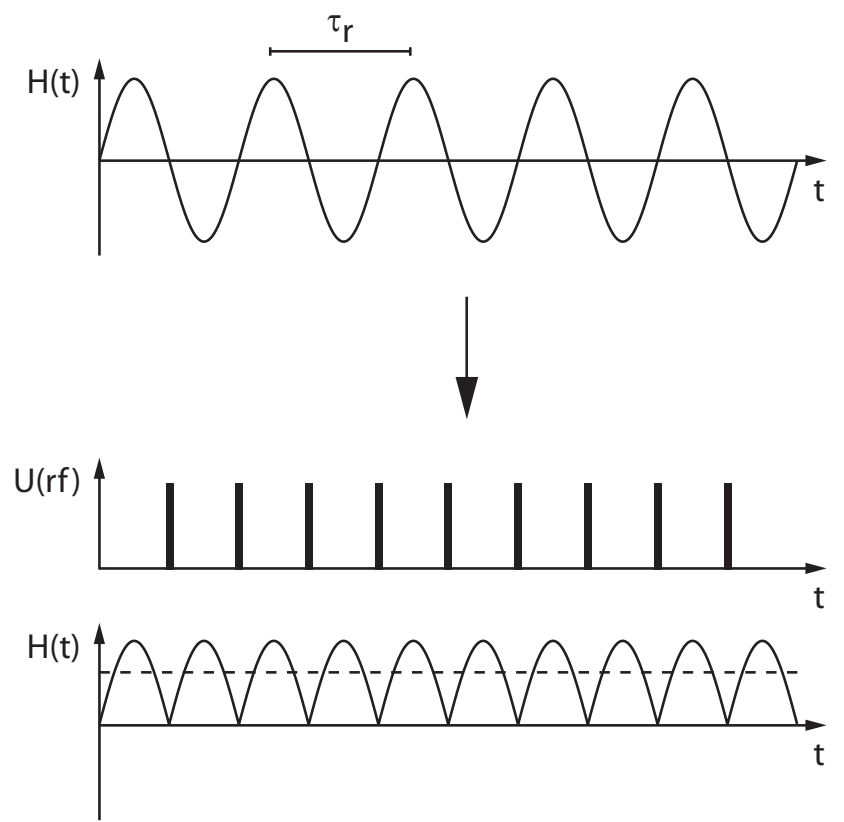

Figure 3.2: Illustration of the principle of recoupling. A Hamiltonian $H(t)$ that is time-dependent due to MAS is averaged out over one period of sample rotation $\left(\tau_{r}\right)$. Rotor-synchronized r.f. pulses can interfere with this averaging, and the Hamiltonian is no longer averaged to zero (indicated by a dashed line).

quences exist for this purpose, depending on the kind of correlation that is to be obtained $[99,100]$. A simple illustration of the principle of recoupling is shown in Figure 3.2. It involves r.f. irradiation that interferes with the averaging of the interaction of interest by MAS, leading to a nonzero average Hamiltonian over a period of sample rotation. For $90^{\circ}$ pulses, the pulse scheme shown in Figure 3.2 corresponds to the DRAMA (dipolar recovery at the magic angle) sequence for recoupling of the dipolar interaction (which, 
however, also reintroduces chemical shift anisotropy) [84, 101]. Examples of recoupling pulse sequences used in the context of this work are briefly discussed in Chapter 4 .

\subsection{Average Hamiltonian theory}

As mentioned in Section 2.3.2, integration of the Liouville-von Neumann equation is straightforward if the Hamiltonian $\hat{H}$ acting on the system is time-independent. This is often not the case in NMR, e.g. during the application of pulse sequences. However, in cases where $\hat{H}$ is periodic in time (e.g. over one or several MAS rotor periods), an average Hamiltonian can be found that correctly describes the effective evolution of the system over one period of the time dependence $[81,82,87,102]$. A time-dependent propagator of the form

$$
\hat{U}\left(t_{c}\right)=e^{-i \hat{H}_{n} t_{n} / \hbar} \cdots e^{-i \hat{H}_{1} t_{1} / \hbar} \cdot e^{-i \hat{H}_{0} t_{0} / \hbar}=\hat{T} \cdot \exp \left(-\frac{i}{\hbar} \sum_{k}^{n} \hat{H}_{k} t_{k}\right),
$$

with $t_{c}=\sum_{k} t_{k}$ and $\hat{T}$ the Dyson time ordering operator, is then described by a propagator using an average Hamiltonian $\overline{\hat{H}}$ :

$$
\hat{U}\left(t_{c}\right)=\exp \left(-\frac{i}{\hbar} \overline{\hat{H}} t_{c}\right)
$$

$\overline{\hat{H}}$ is obtained from a Magnus expansion [87] as

$$
\begin{aligned}
\overline{\hat{H}} & =\overline{\hat{H}^{(0)}}+\overline{\hat{H}^{(1)}}+\ldots \\
& =\frac{1}{t_{c}} \int_{0}^{t_{c}} d t_{1} \hat{H}\left(t_{1}\right)-\frac{i}{2 \hbar t_{c}} \int_{0}^{t_{c}} d t_{2} \int_{0}^{t_{2}} d t_{1}\left[\hat{H}\left(t_{1}\right), \hat{H}\left(t_{2}\right)\right]+\ldots
\end{aligned}
$$

Note that $\overline{\hat{H}^{(0)}}=\frac{1}{t_{c}} \int_{0}^{t_{c}} d t_{1} \hat{H}\left(t_{1}\right)$ is commonly called first-order average Hamiltonian. This term is in many cases a sufficient description of a time-dependent Hamiltonian acting during an NMR experiment; more specifically, it is a good approximation if the different Hamiltonians $\hat{H}_{0}, \hat{H}_{1}, \ldots \hat{H}_{n}$ that act during one period commute [84].

Average Hamiltonian theory is useful in many cases for describing ssNMR pulse sequences, e.g. symmetry-based sequences discussed in Section 4.3.3. More complex Hamiltonians with, e.g., two different time dependencies that cannot be treated independently require the use of Floquet theory for an adequate description [83]. 


\section{Chapter 4}

\section{Experimental techniques}

\subsection{Summary}

This chapter gives an overview over experimental methods important for investigations of protein structure and dynamics by solid-state NMR. Isotope labeling and pulse sequence schemes used in the context of this thesis are discussed. Since computational methods are often indispensable for the analysis of NMR data and are employed in essentially all experimental chapters of this thesis, they are also introduced here.

\subsection{Isotope labeling}

Since proton nuclei exhibit strong homonuclear dipolar couplings and small chemical shift dispersion, ${ }^{1} \mathrm{H}$ evolution and detection periods are normally of limited use in ssNMR spectroscopy of larger biomolecules. Therefore, ${ }^{13} \mathrm{C}$ and ${ }^{15} \mathrm{~N}$ spectral dimensions are routinely employed; however, the low natural abundance of these nuclei $\left(1.11 \%\right.$ for ${ }^{13} \mathrm{C}, 0.37 \%$ for ${ }^{15} \mathrm{~N}$ ) makes isotopic enrichment a necessity, especially for multidimensional correlation spectroscopy (see Section 2.2).

Specific labeling of selected ${ }^{13} \mathrm{C}$ or ${ }^{15} \mathrm{~N}$ nuclei by incorporation of chemically synthesized amino acids or functional groups has long been used in ssNMR, e.g. to measure protonation states of individual residues or specific internuclear distances [55, 99, 103, 104]. Such specific isotope labels or uniformly $\left[{ }^{13} \mathrm{C},{ }^{15} \mathrm{~N}\right]$-labeled amino acid residues can be incorporated into shorter peptides by solid-phase synthesis, as done for a polyglutamine peptide in Chapter 10 of this thesis. If recombinant expression, usually in bacteria, is employed, amino acid specific or forward labeling can be used for this purpose, where the amino acid type(s) with the labeling scheme of interest are added to the natural-abundance bacterial 
growth medium. However, this approach is only specific for amino acid types at termini of biosynthetic pathways; otherwise, metabolic interconversion and thus cross-labeling can occur [105]. Possible cross-labeling via transaminase activity also has to be taken into account.

Synthesis or purchase of (selectively) labeled amino acids is, however, expensive. Moreover, to measure the large amount of distance restraints required for protein structure determination by NMR (see Sections 4.3.4 and 4.4.3), many such samples of any individual protein would be required. Thus, recent years have seen a considerable increase in ssNMR studies on uniformly $\left[{ }^{13} \mathrm{C},{ }^{15} \mathrm{~N}\right]$-labeled proteins. Such uniform isotope labeling can be achieved by growing bacteria on minimal medium containing ${ }^{13} \mathrm{C}_{6}$-glucose and ${ }^{15} \mathrm{NH}_{4} \mathrm{Cl}$ as the only sources of carbon and nitrogen.

To again reduce spectral crowding and the density of the coupled spin network with respect to a uniformly labeled sample, without resorting to many site-specifically labeled samples, various advanced labeling schemes can be employed. One approach achieves ${ }^{13} \mathrm{C}$ spin dilution by using different specifically labeled precursors, such as $(1,3)-{ }^{13} \mathrm{C}$ - or $2-{ }^{13} \mathrm{C}$-glycerol [106]. Most amino acids are incompletely isotope-labeled by this method, such that two different samples have to be synthesized to cover all carbon resonances in a protein. This approach reduces spectral overlap and removes many strong one-bond dipolar $\left({ }^{13} \mathrm{C},{ }^{13} \mathrm{C}\right)$ couplings, facilitating ssNMR structural studies [49, 107, 108, 109]. On the other hand, similar to amino acid specific labeling, reverse labeling can be employed, where certain amino acid types are supplemented in natural abundance to growth medium containing ${ }^{13} \mathrm{C}_{6}$-glucose and ${ }^{15} \mathrm{NH}_{4} \mathrm{Cl}[20,59,110]$. This approach is used in Chapter 7 to reduce assignment ambiguities in spectra of an ion channel membrane protein.

\subsection{Spectroscopic techniques}

\subsubsection{Cross-polarization}

Cross-polarization (CP) is an essential technique in solid-state NMR especially to establish polarization transfer from high- $\gamma$ to low- $\gamma$ nuclei, such as from protons to carbons or nitrogens, to increase signal-to-noise $[111,112]$. As such, it is a common initial step in many ssNMR experiments. $\mathrm{CP}$ is established by irradiating both spin types, here denoted $I$ and $S$, with transversal spin-lock r.f. fields of strengths $\omega_{1 I}$ and $\omega_{1 S}$ in angular frequency units (Fig. 4.1). Under MAS conditions, nonzero heteronuclear dipolar coupling 

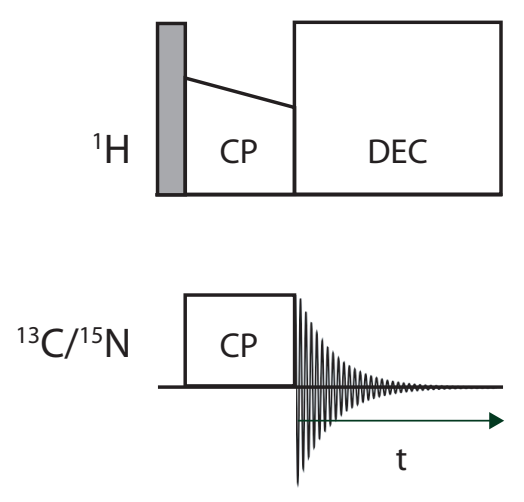

Figure 4.1: Cross-polarization (CP) pulse sequence. DEC indicates r.f. decoupling of protons from ${ }^{13} \mathrm{C}$ or ${ }^{15} \mathrm{~N}$ spins. The shaded rectangle represents a $90^{\circ}$ pulse (also in following figures).

terms corresponding to flip-flop transitions are obtained in the average Hamiltonian if the Hartmann-Hahn condition is fulfilled:

$$
\omega_{1 I}-\omega_{1 S}= \pm \omega_{r}, \pm 2 \omega_{r}
$$

with $\omega_{r}$ the MAS spinning frequency. For transfer from $I$ to $S$ spins, a polarization enhancement proportional to $\gamma_{I} / \gamma_{S}$ is found that also depends on the number of $I$ spins [112]. This makes CP more efficient than direct excitation of low- $\gamma S$ spins in the presence of dipolar coupled high- $\gamma I$ spins. The strength of one of the two spin-lock fields may be ramped through the Hartmann-Hahn condition to reduce dependence on an exact field match (as indicated in Fig. 4.1).

Since it relies on dipolar couplings, CP is effective only for more rigid spins, making it useful also for spectral editing if only rigid parts of the molecular system under study are to be probed. Complementary sequences exist for highly mobile regions of solid samples (based on INEPT-type transfers [113]) and for regions exhibiting intermediate dynamics [20]. CP can also be employed for polarization transfer between low- $\gamma$ nuclei (see Section 4.3.2).

\subsubsection{Correlations for sequential assignment}

As in NMR spectroscopy in general, resonance assignment of signals observed in NMR spectra to individual nuclei in the sample is a crucial problem also in protein ssNMR spectroscopy. Here, the assignment of resonances to amino acid types can in general easily be accomplished based on characteristic ${ }^{15} \mathrm{~N}$ and ${ }^{13} \mathrm{C}$ chemical shifts in different residue 
types (see, e.g., average values in the Biological Magnetic Resonance Data Bank (BMRB), http://www.bmrb.wisc.edu/). However, the position of individual residues in the protein sequence is usually not clear by default if several residues of the same type occur. Thus, a sequential resonance assignment has to be performed by correlating neighboring amino acid residues in the protein sequence [114]. By consecutively identifying neighbors of amino acid residues in interresidue correlation spectra, combinatorial ambiguity can be reduced until unambiguous assignment is achieved. For example, a specific triplett of amino acid

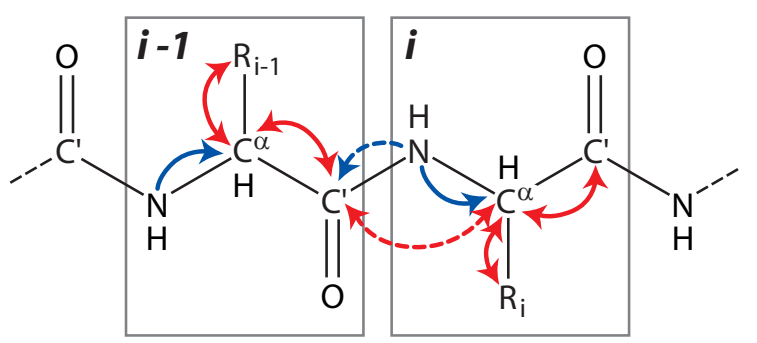

Figure 4.2: Schematic of the protein backbone. Two amino acid residues $i$ and $i-1$ with sidechains symbolized by $R_{i}$ and $R_{i-1}$ are shown. Possible (ss)NMR correlations for sequential resonance assignment are indicated by arrows. Red, $\left({ }^{13} \mathrm{C},{ }^{13} \mathrm{C}\right)$ correlations; blue, $\left({ }^{15} \mathrm{~N},{ }^{13} \mathrm{C}\right)$ correlations; solid lines denote intra-, dashed lines interresidue transfer. See text for experimental details.

residues identified as occurring sequentially may exist only once in the sequence of the protein under study; thus, these residues can then be considered as sequentially assigned. Both for intra- and interresidue correlation spectra, homo- and heteronuclear correlation sequences can be employed (Fig. 4.2).

Homonuclear ${ }^{13} \mathrm{C}$ correlations between spins within individual amino acid residues are obtained in a straightforward manner by longitudinal ${ }^{13} \mathrm{C}$ mixing sequences, where ${ }^{13} \mathrm{C}$ spin magnetization is aligned along the $z$ axis by a $90^{\circ}$ pulse after initial crosspolarization. In the context of this thesis, proton-driven spin diffusion (PDSD) is most frequently used [115, 116, 117, 118] (Fig. 4.3). Polarization transfer can be enhanced by additional $\left({ }^{1} \mathrm{H},{ }^{13} \mathrm{C}\right)$ recoupling using a ${ }^{1} \mathrm{H}$ r.f. field matched to the MAS frequency (dipolarassisted rotational resonance, DARR [119]). Since the strongest couplings chiefly determine dipolar-mediated polarization transfer [100, 120, 121], transfer to directly bonded nuclei dominates in uniformly ${ }^{13} \mathrm{C}$-labeled molecules. For mixing times up to few tens of milliseconds in case of spin diffusion (shorter for DARR), mostly intraresidue ${ }^{13} \mathrm{C}$ cor- 


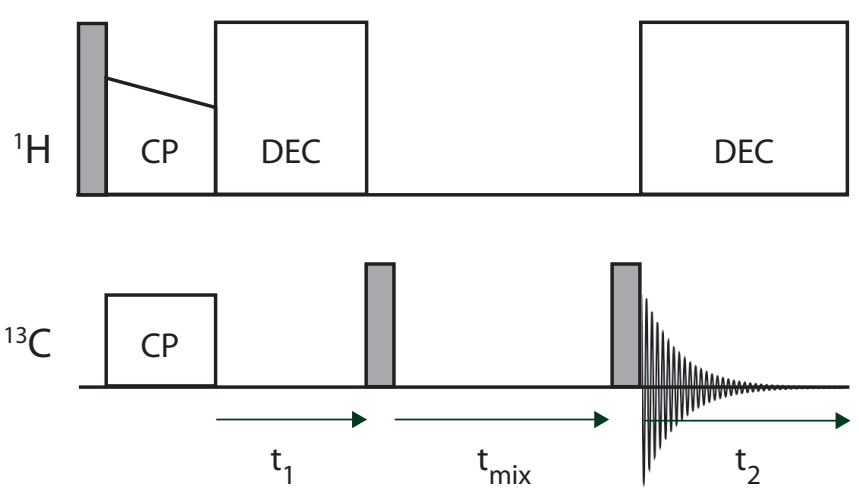

Figure 4.3: Pulse sequence for a two-dimensional proton-driven $\left({ }^{13} \mathrm{C},{ }^{13} \mathrm{C}\right)$ spin diffusion (PDSD) correlation spectrum. $t_{\text {mix }}$ : spin diffusion mixing time.

relations are thus obtained, since neighboring amino acid residues are separated by ${ }^{15} \mathrm{~N}$ spins.

Heteronuclear intraresidue correlations can be obtained using $\left({ }^{15} \mathrm{~N},{ }^{13} \mathrm{C}\right)$ cross polarization sequences. Since they are usually preceded by an initial $\left({ }^{1} \mathrm{H},{ }^{15} \mathrm{~N}\right) \mathrm{CP}$, they are often termed double CP sequences [122] (Fig. 4.4). Specific intraresidue transfer from backbone amide ${ }^{15} \mathrm{~N}$ to backbone ${ }^{13} \mathrm{C}^{\alpha}$ nuclei in amino acids can be obtained by achieving the Hartmann-Hahn match between ${ }^{15} \mathrm{~N}$ and ${ }^{13} \mathrm{C}$ irradiation with weak r.f. fields whose carrier frequency is centered on the resonance frequencies of interest (SPECIFIC CP $[123,124]$ ). Conversely, interresidue transfer from the backbone amide ${ }^{15} \mathrm{~N}$ of residue $i$ to the carbonyl ${ }^{13} \mathrm{C}$ ' of residue $i-1$ can be established analogously by centering weak ${ }^{13} \mathrm{C}$ r.f. irradiation in the spectral region of the carbonyl spins, which is well separated from that of the $\mathrm{C}^{\alpha}$ spins $(\sim 165-185 \mathrm{ppm}$ versus $\sim 40-70 \mathrm{ppm})$. Further intraresidue $\left({ }^{13} \mathrm{C},{ }^{13} \mathrm{C}\right)$ correlations to aid in identification of amino acid spin systems can in both cases be obtained by subsequent PDSD or DARR $\left({ }^{13} \mathrm{C},{ }^{13} \mathrm{C}\right)$ mixing. Both types of $\left({ }^{15} \mathrm{~N},{ }^{13} \mathrm{C}\right)$ correlation experiments yield essential information for sequential resonance assignment in ssNMR spectroscopy.

In addition, homonuclear sequential $\left({ }^{13} \mathrm{C},{ }^{13} \mathrm{C}\right)$ correlations can be obtained in the context of a PDSD experiment with intermediate $(\sim 50-150 \mathrm{~ms})$ mixing times if the weak coupling condition is established [125] (PDSD-WC) by setting the MAS frequency close to the $n=2$ rotational resonance condition for $\mathrm{C}^{\prime}$ and $\mathrm{C}^{\alpha}$ spins. This facilitates sequential $\left({ }^{13} \mathrm{C},{ }^{13} \mathrm{C}\right)$ polarization transfer across the peptide bond. Sequential correlations can thus be obtained without introducing a $\left({ }^{15} \mathrm{~N},{ }^{13} \mathrm{C}\right)$ transfer step, which usually reduces sensitiv- 


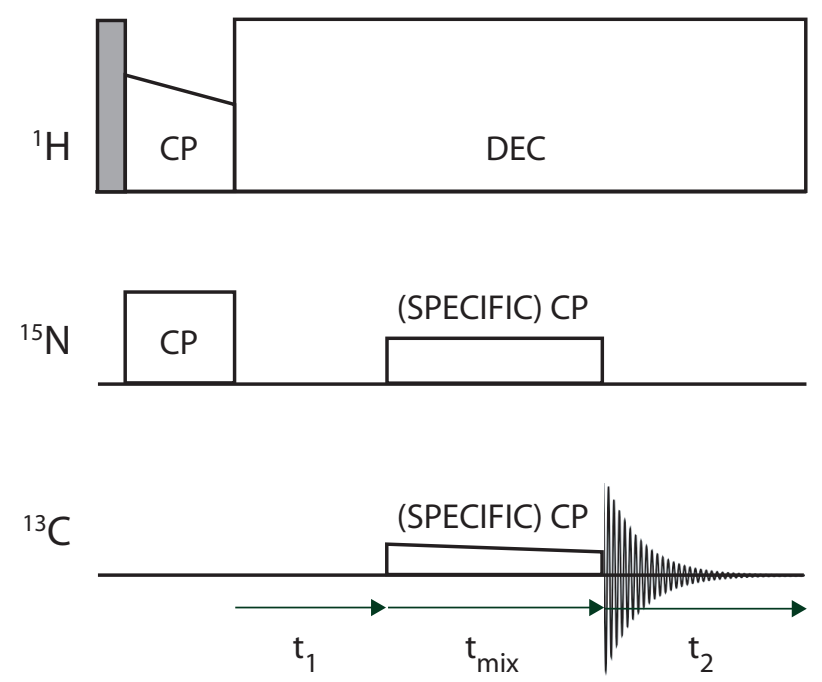

Figure 4.4: Pulse sequence of a two-dimensional $\left({ }^{15} \mathrm{~N},{ }^{13} \mathrm{C}\right)$ correlation spectrum employing $\mathrm{CP}$ or SPECIFIC CP $[123,124]$ for $\left({ }^{15} \mathrm{~N},{ }^{13} \mathrm{C}\right)$ mixing.

ity compared to $\left({ }^{13} \mathrm{C},{ }^{13} \mathrm{C}\right)$ spectroscopy. Additionally, in solid-state NMR, ${ }^{13} \mathrm{C}$ dimensions normally exhibit much better resolution and shift dispersion than ${ }^{15} \mathrm{~N}$ dimensions.

\subsubsection{Double-quantum recoupling}

Double-quantum (2Q) coherences between pairs of spin- $\frac{1}{2}$ nuclei are associated with transitions between their $|\alpha \alpha\rangle$ and $|\beta \beta\rangle$ states. During free precession under a Zeeman Hamiltonian, $2 \mathrm{Q}$ coherences evolve at a frequency corresponding to the sum of the chemical shifts of the two nuclei involved [77, 84]. 2Q coherences are not observable directly, but can be used, e.g., for frequency-labeling during evolution periods of multidimensional NMR experiments. This way, two-dimensional correlation spectra with an indirect $2 \mathrm{Q}$ dimension ((2Q,1Q) correlation spectra) can be obtained (Fig. 4.5 a; see Chapters 5 and 6). Only homonuclear $\left({ }^{13} \mathrm{C},{ }^{13} \mathrm{C}\right) 2 \mathrm{Q}$ coherences are considered in the context of this work.

In ssNMR, 2Q coherences can in principle be excited by any dipolar recoupling sequence (see Section 3.5 and refs. [99, 126]), possibly optimized for conditions without proton decoupling $[127,128,129]$. 2Q coherence must be reconverted to observable singlequantum coherence (corresponding to transversal magnetization) for acquisition. Selection for signals from nuclei that were involved in $2 \mathrm{Q}$ coherences during the pulse sequence is then accomplished by phase cycling of the r.f. pulses employed [130]. The efficiency of 2Q coherence excitation depends on the strength of the dipolar coupling involved. 2Q 
spectroscopy thus offers an opportunity to measure dipolar coupling strength and attenuation, e.g. due to mobility (see Chapter 5). Additionally, due to this dependence of $2 \mathrm{Q}$ coherences on strong dipolar couplings, $(2 \mathrm{Q}, 1 \mathrm{Q})$ correlation spectra usually only contain nearest-neighbor correlations, which can be of considerable help in resonance assignment of uniformly labeled biomolecules [100].

For efficient excitation of $2 \mathrm{Q}$ coherences (and other purposes), ssNMR pulse sequences have been developed that allow for selective recoupling of specific interactions based on symmetries of space and spin parts in the Hamiltonian. In these symmetry-based recoupling sequences, sets of r.f. pulses are employed which cover the duration of a defined integer number of MAS sample rotation periods and which are subsequently repeated with additional phase shifts [126, 131] (Fig. 4.5 b). One class of such sequences important for work discussed in this thesis is constituted by the $\mathrm{C} N_{n}^{\nu}$ sequences. In the terminology of Levitt and coworkers [126], this refers to sequences based on a simple or composite $360^{\circ}$ pulse, which constitutes the basic $\mathrm{C}$ block. $N$ such $\mathrm{C}$ r.f. blocks of duration $\tau_{C}$ each are applied successively, covering the duration of $n$ sample rotations. This naturally implies that the r.f. field strength has to be adjusted to the chosen MAS frequency. Additionally, the phase of the r.f. irradiation in each $\mathrm{C}$ block is shifted by $2 \pi \nu / N$ with respect to the preceding block. For the interaction-frame Hamiltonian $\hat{H}_{\operatorname{lm} \lambda \mu}^{\Lambda}$ of an interaction $\Lambda$ with space and spin ranks $l$ and $\lambda$, respectively, this leads to the following periodic symmetry:

$$
\hat{H}_{l m \lambda \mu}^{\Lambda}\left(t+q \tau_{C}\right)=\hat{H}_{l m \lambda \mu}^{\Lambda}(t) \cdot \exp \left(i \frac{2 \pi q}{N}(m n-\mu \nu)\right)
$$

with $q$ an integer. As a consequence, if $m n-\mu \nu \neq N \cdot$ integer, the first-order average Hamiltonian for this interaction is zero due to the variation of the phase factor in Equation 4.2. Otherwise, the interaction can be recoupled. This selection rule allows for the reintroduction of only specific components of the Hamiltonian [132, 133]. For example, the $\mathrm{C}_{2}^{1}$ sequence [134] suppresses all chemical shift anisotropy terms $(l=2, \lambda=1)$, while recoupling only $2 \mathrm{Q}$ dipolar coupling components $(l=2, \lambda=2)$ with $(m, \mu)=( \pm 1, \pm 2)$. The SPC5 sequence used in Chapters 5 and 6 is of the same type, exhibiting $\mathrm{C} 5{ }_{2}^{1}$ symmetry, but making use of an additional r.f. supercycle to suppress chemical shift anisotropy terms [121] (see Fig. 4.5 b).

\subsubsection{Through-space correlations}

To obtain three-dimensional (3D) molecular structures from NMR data, a large number of internuclear through-space distances has to be measured or estimated and used as 
a

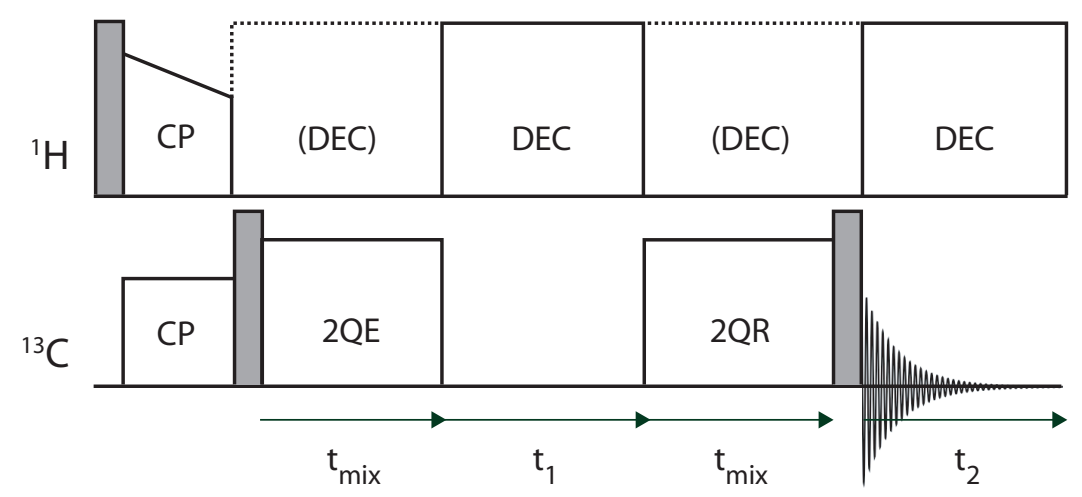

b

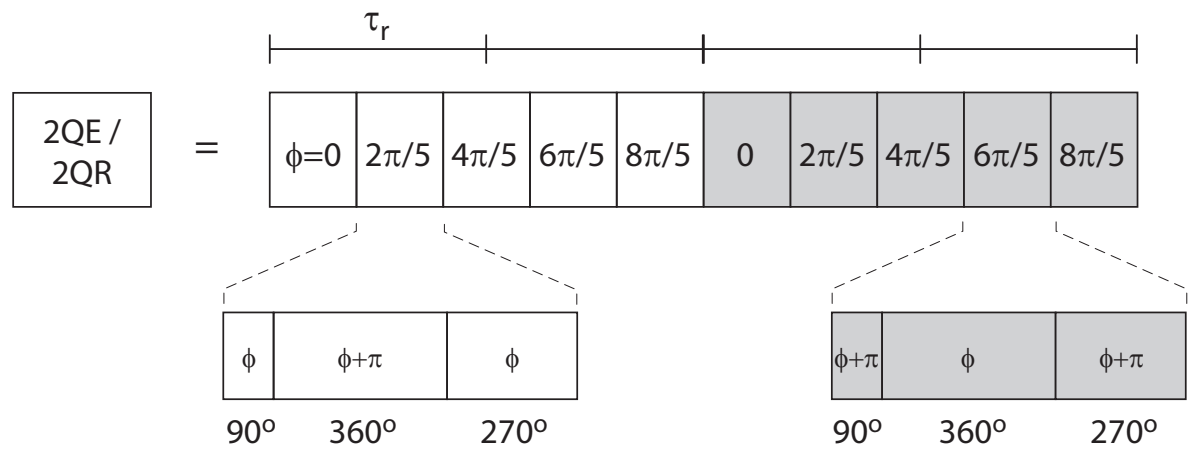

Figure 4.5: (a) Schematic pulse sequence of a $2 D(2 Q, 1 Q)$ correlation spectrum. $2 Q E$ / $2 Q R$ : double quantum excitation / reconversion. Dashed lines in boxes denoting proton decoupling during $2 \mathrm{QE}$ and $2 \mathrm{QR}$ indicate that no proton decoupling may be required depending on the recoupling pulse sequence used $[127,128,129]$. (b) Illustration of the composition of a symmetry-based recoupling sequence (SPC5 [121] in this case). Both 2QE and 2QR consist of composite $\left(90^{\circ}\right.$ $\left.360^{\circ}-270^{\circ}\right) 360^{\circ}$ pulses (C blocks) which are repeated with shifts in their phases $\phi$. Five such $C$ blocks cover two rotor periods $\left(\tau_{r}\right)$. In case of SPC5, every second set of five $\mathrm{C}$ blocks is repeated with an additional $\pi$ phase shift, indicated by shaded boxes. Mixing times can also be shorter than a full r.f. cycle (see Chapter 5). 
restraints in a structure calculation (see Section 4.4.3). Especially distances between nuclei which are distant in terms of the covalent structure of the molecule under study are important for defining its 3D fold. In solution-state NMR, NOESY experiments based on $\left({ }^{1} \mathrm{H},{ }^{1} \mathrm{H}\right)$ cross-relaxation are commonly used for this task [77, 135]. In the solid state, ${ }^{1} \mathrm{H}$ spectral dimensions usually do not offer enough resolution for identification and assignment of a large number of crosspeaks. $\left({ }^{1} \mathrm{H},{ }^{1} \mathrm{H}\right)$ mixing schemes with indirect detection on ${ }^{15} \mathrm{~N}$ or ${ }^{13} \mathrm{C}$ nuclei directly attached to protons (N/CHHC sequences $\left.[136,137]\right)$ are useful here (Fig. 4.6). Usually, longitudinal $\left({ }^{1} \mathrm{H},{ }^{1} \mathrm{H}\right)$ spin diffusion mixing is employed, leading to transfer rates depending on the inverse $6^{\text {th }}$ power of the $\left({ }^{1} \mathrm{H},{ }^{1} \mathrm{H}\right)$ distance in the initial rate regime [137]. The two $\mathrm{CPs}$ that bracket $\left({ }^{1} \mathrm{H},{ }^{1} \mathrm{H}\right)$ mixing are usually chosen to be short $\left(\sim 80-150 \mu\right.$ s for $\left({ }^{1} \mathrm{H},{ }^{13} \mathrm{C}\right), \sim 150-300 \mu \mathrm{s}$ for $\left({ }^{1} \mathrm{H},{ }^{15} \mathrm{~N}\right)$ transfer $)$ to ensure transfer between directly bonded nuclei only. Compared to selective recoupling of individual spin pairs that
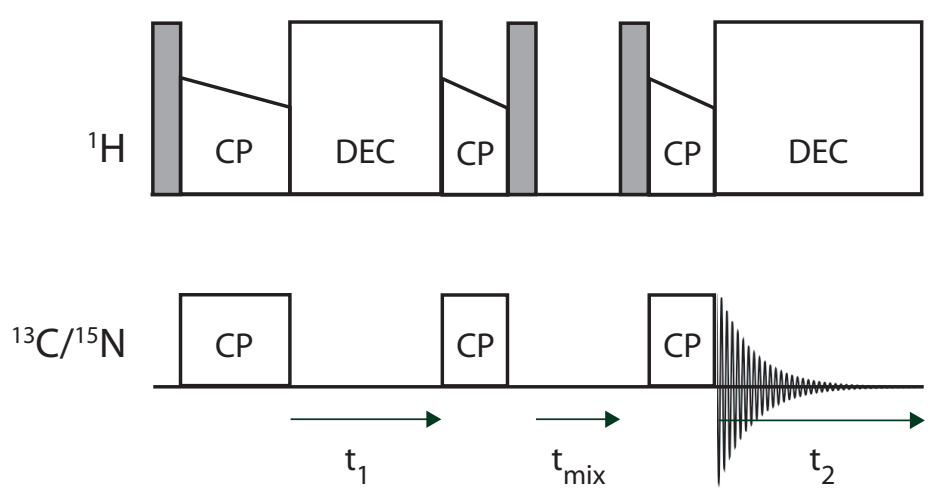

Figure 4.6: $\mathrm{N} / \mathrm{CHHC}$ pulse sequence. For $\mathrm{NHHC}$, the first two $\mathrm{CPs}$ are $\left({ }^{1} \mathrm{H},{ }^{15} \mathrm{~N}\right) \mathrm{CPs}$, and the last one is a $\left({ }^{1} \mathrm{H},{ }^{13} \mathrm{C}\right) \mathrm{CP}$. Detection is always on ${ }^{13} \mathrm{C}$ nuclei.

has long been used in ssNMR (see, e.g., refs. [138, 139, 140]; for review, see refs. [99, 100]), this approach has the considerable advantage that a large number of restraints can be obtained from a single or few experiments on a uniformly isotope-labeled sample [137]. $\mathrm{N} / \mathrm{CHHC}$ spectra are used for obtaining structural information in several parts of this thesis (see Chapters 6, 7, and 10).

On the other hand, also simple PDSD $\left({ }^{13} \mathrm{C},{ }^{13} \mathrm{C}\right)$ mixing schemes with longer mixing times (300 ms and above) have been successfully used to extract long-range distances $[49,52,53,108]$ (see also Section 10.4.5). To reduce nearest-neighbor transfer and spectral crowding, reduced ${ }^{13} \mathrm{C}$ labeling schemes can be employed [49] (see Section 4.2). Nevertheless, for shorter internuclear distances, $\left({ }^{1} \mathrm{H},{ }^{1} \mathrm{H}\right)$ contacts contain more structurally im- 
portant long-range information than $\left({ }^{13} \mathrm{C},{ }^{13} \mathrm{C}\right)$ contacts $[53,141]$. Other pulse sequences designed for measuring many long-range correlations at once, even in the presence of strong short-range interactions, have recently been described [129, 142, 143], offering increased opportunities to obtain large sets of independent through-space correlation data for structure determination by ssNMR.

\subsubsection{Water-edited experiments}

Water-edited ssNMR experiments adapt the Goldman-Shen pulse scheme [144] to first selectively excite water ${ }^{1} \mathrm{H}$ resonances and then transfer magnetization from water to the protein under study (Fig. 4.7) [3, 59, 145, 146]. Selective water ${ }^{1} \mathrm{H}$ excitation is accomplished by a weak Gaussian $\pi / 2$ pulse whose carrier frequency is centered at the resonance frequency of water protons. An additional dephasing period (of duration $2 \cdot \tau$, with a refocussing $\pi$ pulse in the middle) further selects for mobile proton resonances, since the more rigid ones dephase due to stronger dipolar couplings during this period. Longitudinal mixing (of duration $t_{m}$ ) then allows for polarization transfer between water and protein protons by way of chemical exchange, spin diffusion, and/or NOE effects [3, 147, 148]. For short $t_{m}$ times (few milliseconds), ${ }^{13} \mathrm{C}$ signals detected after a subsequent $\mathrm{CP}$

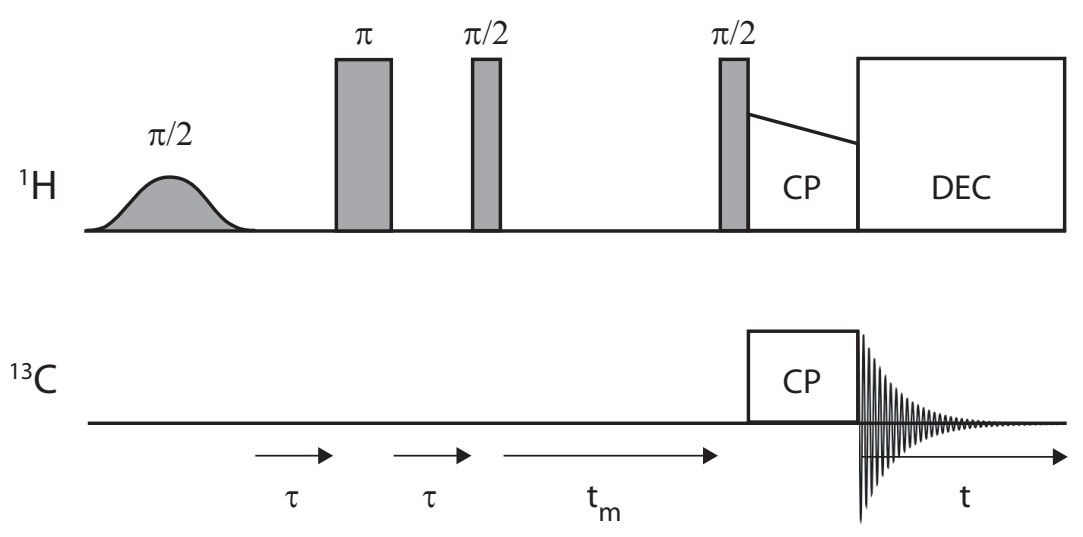

Figure 4.7: Pulse sequence of a one-dimensional water-edited $\left({ }^{1} \mathrm{H},{ }^{13} \mathrm{C}\right) \mathrm{CP}$ experiment.

originate from spins close to the water-protein interface. For longer $t_{m}$ times, saturation of the CP signal is observed. Buildup curves can be obtained by integrating the CP signal from a series of such experiments with different $t_{m}$. As detailed in ref. [3], the time $t_{m}^{s}$ 
at which maximum $\mathrm{CP}$ signal would be reached in the absence of saturation conditions is related to the ratio of protein volume $V$ to water-accessible surface $S$ as

$$
\frac{V}{S}=\sqrt{\frac{D_{e f f} t_{m}^{s}}{\pi}}
$$

with $D_{\text {eff }}$ the effective diffusion coefficient describing the magnetization transfer [3]. $t_{m}^{s}$ is obtained from a linear fit to the initial rate of a water-edited CP buildup curve. This type of experiment is used to probe the volume-to-surface ratio of proteins in Chapters 9 and 10.

\subsection{Computational Methods}

\subsubsection{Spin system simulations}

In many cases, quantum mechanical simulations are useful or essential to deduce structural and dynamical information about the spin system of interest from experimental NMR data. Simulations can be fit to experimental data to extract parameters such as motional rate and amplitude, chemical shift tensor orientation, and internuclear distance. For example, selective dipolar recoupling methods for distance measurements that yield dephasing or buildup curves, such as REDOR or rotational resonance, are commonly analyzed in this manner [138, 139, 140]. For spin systems with known geometry, such as directly bonded $\left({ }^{1} \mathrm{H},{ }^{13} \mathrm{C}\right)$ or $\left({ }^{13} \mathrm{C},{ }^{13} \mathrm{C}\right)$ pairs, fits of simulations to dipolar powder patterns or crosspeak buildup curves allow for estimates of local molecular mobility, which scales down dipolar couplings $[68,69,149]$. Such an approach is developed and applied in Chapters 5 and 6 of this thesis.

Apart from custom-written code, numerical simulation packages or libraries exist that can be used for ssNMR spin system simulations, most notably GAMMA [150], SIMPSON [151] and SPINEVOLUTION [152]. GAMMA, for example, which is used in Chapters 5 and 6 of this thesis, allows for direct specification of spin and space tensors defining the system of interest, along with Hamiltonians and propagators. Apart from the nuclear spins of interest and their interactions, simulation of MAS solid-state NMR experiments requires consideration of powder averaging and sample spinning. In addition, for simulations of multispin systems (as in the context of uniformly isotope-labeled molecules), tests may be required to investigate how many coupled spins have to be considered in addition to the spin system of interest per se (see Chapter 5). 


\subsubsection{Chemical shift and secondary structure prediction}

The nuclear chemical shift is a sensitive indicator of local geometry in proteins, in solution as well as in the solid state, with backbone ${ }^{15} \mathrm{~N}$ and ${ }^{13} \mathrm{C}$ nuclei being most informative for secondary structure [153]. However, the chemical shift it is affected by many other factors as well, such as hydrogen bonding, ring current effects, and sidechain orientation [154]. The relationship between local structure and chemical shift is therefore complex, and algorithms have been devised to estimate shift from structure and vice versa.

Since protein $\mathrm{C}^{\alpha}$ and $\mathrm{C}^{\beta}$ chemical shifts $\left(\delta_{C^{\alpha}}, \delta_{C^{\beta}}\right)$ are most affected by backbone $\phi$ and $\psi$ dihedral angles $[153,155,156,157]$, assignment of these resonances already provides an indication of local secondary structure. A secondary chemical shift $\Delta \delta$ is commonly defined [153], making use of tabulated reference values for average or random-coil $\mathrm{C}^{\alpha}$ and $\mathrm{C}^{\beta}$ chemical shifts of amino acid residues:

$$
\Delta \delta=\left(\delta_{C^{\alpha}}-\delta_{C^{\alpha}}(\mathrm{ref})\right)-\left(\delta_{C^{\beta}}-\delta_{C^{\beta}}(\mathrm{ref})\right)
$$

where values for $\delta_{C^{\alpha}}(\mathrm{ref}), \delta_{C^{\beta}}$ (ref) can be found, e.g., in the Biological Magnetic Resonance Data Bank (BMRB, http://www.bmrb.wisc.edu/) or in ref. [158]. Positive values of $\Delta \delta$ indicate $\alpha$-helical secondary structure, negative values $\beta$-sheet conformations. More detailed estimates of backbone dihedral angles can be obtained using computer programs that search databases of proteins with known structure and chemical shift assignments for residues whose shifts match those in the query, such as TALOS [159] and PREDITOR [160]. Such dihedral angle predictions can be used as restraints in a molecular structure calculation (Section 4.4.3).

Conversely, chemical shifts can be estimated based on protein primary, secondary, and/or tertiary structure. While full ab initio quantum-chemical calculations (such as in ref. [161]) as employed for this purpose in smaller molecular systems are still computationally too expensive for full-sized proteins, several fast approaches exist using precalculated quantum-chemical and empirical parameters as well as, possibly, reference databases [154, 162, 163, 164]. Such algorithms can be useful for purposes of NMR spectral assignment, e.g. by providing estimates for chemical shifts of the protein under study from a related protein for which a structure is available. If several models of the local or global structure of a protein exist, their chemical shifts can be predicted and compared with experimental spectra to extract the most likely conformation [165]. A similar approach is used in Chapter 8 to obtain estimates for the conformation of residues in functionally important regions of the potassium channel KcsA-Kv1.3 under different conditions. 
Chemical shift predictions have also been used iteratively for structure calculation and refinement $[166,167]$, and first successes in structure calculations based on chemical shifts as the only experimental source of information have been reported [168, 169].

\subsubsection{Structure calculation}

To obtain the structure of a protein from NMR data, many individual restraints on its conformation, such as distances between pairs of nuclei and dihedral bond angles, have to be derived from experiments. Computer algorithms are then employed for the complex task of subjecting the covalent protein structure to these restraints and modifying its conformation until as many of the restraints as possible are satisfied. This is most commonly done by molecular dynamics-based simulated annealing protocols [170]. Here, experimental restraints together with restraints enforcing covalent structure (bond lengths and angles, improper dihedrals) and van der Waals repulsion are formulated in terms of a potential energy function $V(\boldsymbol{r})$. Classical molecular dynamics are then performed for all atoms of the protein of interest subject to $V(\boldsymbol{r})$, i.e. Newton's equations of motion are integrated numerically for each particle $i$ :

$$
m_{i} \frac{d^{2}}{d t^{2}} \boldsymbol{r}_{i}=-\nabla_{i} V(\boldsymbol{r})
$$

Initially, high velocities are assigned to all particles (corresponding to a high temperature $T)$ to allow the system to sample the energy landscape. Energy is then gradually removed from the system via coupling to a "heat bath" [171] which appears as additional term $+\beta\left(\frac{T_{0}}{T}-1\right) \boldsymbol{v}_{i}$ on the right side of Equation 4.5. $\boldsymbol{v}_{i}$ is the velocity of particle $i$, and $T_{0}$ is the temperature of the heat bath which is reduced step by step in the course of the simulation. Ideally, the system thus settles into a global energy minimum. This process is referred to as simulated annealing [172]. Common software packages to perform simulated annealing structure calculations based on NMR data include Xplor-NIH [173, 174], CNS [175], and DYANA [176]. CNS is used for structure calculation (Chapter 6), local refinement (Chapter 8), and model building (Chapter 10) in the context of this thesis.

Instead of using Cartesian coordinates for all atoms, simulated annealing for protein structure determination may be conducted in torsion angle space, since the covalent structure is known and does not change. This significantly reduces the degrees of freedom of the system and, consequently, computation time [177]. Experimental restraints are commonly realized in a square-well form where no contribution to the energy $V(\boldsymbol{r})$ is obtained if the restrained quantity (e.g. an internuclear distance) is within the limits of a specified cutoff 
of its target value. Outside of these limits, a quadratically increasing energy contribution, i.e. a penalty, results.

Usually, simulated annealing is repeated on the order of 100-500 times with different initial velocities for a given protein structure to ensure convergence. A set of about 10-30 resultant structures with lowest overall energy, resulting from the lowest number of restraint violations, is commonly chosen as the final structural ensemble. Good convergence of a protein structure calculation is characterized chiefly by a small number of restraint violations, a low root-mean-square deviation (RMSD) of the positions of corresponding atoms in the final ensemble, and a distribution of dihedral angles mostly in most favored and allowed regions of the Ramachandran plot [170, 178]. Software exists to automatically perform such structure checks, such as WHATIF [179] and PROCHECKNMR [180]. Further structure refinements may be conducted, e.g. by short full molecular dynamics simulations in explicit water [170, 181].

A major problem in structure determination from NMR data is the assignment of crosspeaks in through-space correlation spectra (Section 4.3.4). Based on chemical shift alone, any given crosspeak can usually correspond to correlations between several different pairs of nuclei, resulting in a level of ambiguity that quickly becomes intractable with increasing molecular size. If crosspeaks can be found that can be assigned unambiguously, they may constrain the structural ensemble enough to disambiguate further crosspeaks, leading to iterative assignment of through-space spectra and finally a large enough set of restraints to calculate a well converged structural ensemble [51]. Similar principles of concurrent assignment and structure calculation are employed by automated algorithms designed for this task $[182,183,184]$. One of these is used for a structure calculation in Chapter 6 of this thesis. 


\section{Part II}

Molecular mobility 



\section{Chapter 5}

\section{Molecular motion detected by double-quantum $\left({ }^{13} \mathrm{C},{ }^{13} \mathrm{C}\right)$ solid-state NMR}

\subsection{Summary}

Double-quantum $(2 \mathrm{Q})\left({ }^{13} \mathrm{C},{ }^{13} \mathrm{C}\right)$ solid-state NMR is introduced as a method to probe molecular mobility in a site-resolved manner. Experimental buildups of magnetization under $2 \mathrm{Q}$ recoupling were recorded on the model system L-tyrosine-ethylester and fitted to quantum-mechanical simulations to extract dipolar order parameters. In agreement with previously determined $\left({ }^{1} \mathrm{H},{ }^{13} \mathrm{C}\right)$ order parameters, results reveal correlated motion of the ester tail. It is shown that already a small number of $2 \mathrm{D}$ correlation experiments is sufficient to probe molecular dynamics using $2 \mathrm{Q}\left({ }^{13} \mathrm{C},{ }^{13} \mathrm{C}\right)$ correlation spectroscopy.

\subsection{Introduction}

In contrast to X-ray crystallography, NMR yields not only structural information with atomic resolution, but can also precisely measure site-specific molecular mobility. In solution-state NMR, dynamic parameters are routinely measured using relaxation methods for motions up to the overall rotational correlation time of the molecule (in the picoto nanosecond regime), while slow dynamics on timescales in the order of $100 \mu$ s and slower can be probed using relaxation dispersion or exchange spectroscopy (for a review, see ref. [185]). While anisotropic interactions such as dipolar couplings are normally averaged out in solution by fast molecular tumbling, the gap between measuring dynamics on nanosecond and millisecond timescales in solution-state NMR has in recent years been closed by employing residual dipolar couplings measured in alignment media [186, 187]. 
On the other hand, solid-state NMR has long been used to study molecular motion in applications ranging from small molecules and polymers to biomolecules [188, 189, 190, 191, 192]. In the solid state, the analysis of intrinsic molecular mobility can be considerably simplified since no overall tumbling of the molecule has to be taken into account [193]. In addition to relaxation-based analyses [85, 192, 194], the effect of molecular motion upon anistropic parameters such as the quadrupolar coupling or the onebond $\left({ }^{1} \mathrm{H},{ }^{13} \mathrm{C} /{ }^{15} \mathrm{~N}\right)$ dipolar coupling has been used to probe dynamics in the solid state [85, 189, 190, 191]. Molecular motion reduces measured values of such couplings compared to the static case. This can be used to deduce order parameters that describe the extent of the motion, possibly within the framework of specific motional models. When applied to larger biomolecules, such experiments may require the use of three-dimensional correlation spectroscopy under magic angle spinning (MAS) conditions $[149,195,196]$. As in solution state, dipolar couplings probe motions up to the millisecond time scale [186, 187].

Molecular motion also impacts homonuclear dipolar couplings, as has been demonstrated using $\left({ }^{1} \mathrm{H},{ }^{1} \mathrm{H}\right)$ correlation spectroscopy to measure motion and the supramolecular organization of polymers [197, 198, 199]. Due to the limited spectral resolution, such methods are, however, of limited use for larger biomolecules which, on the other hand, can often be readily prepared in isotope-labeled form. Thus, in the following chapter, the use of $\left({ }^{13} \mathrm{C},{ }^{13} \mathrm{C}\right)$ double-quantum $(2 \mathrm{Q})$ spectroscopy to detect molecular motion in fully isotope-labeled biomolecules is investigated. This approach offers the opportunity

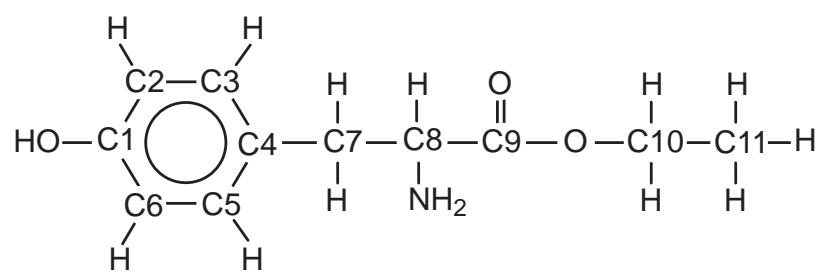

Figure 5.1: Schematic representation of L-tyrosine-ethylester (TEE) according to refs. [68, 69].

to detect modes of motion complementary to those covered by measuring $\left({ }^{1} \mathrm{H},{ }^{13} \mathrm{C} /{ }^{15} \mathrm{~N}\right)$ couplings. The method is laid out here using a small model system, L-tyrosine-ethylester (TEE) (Fig. 5.1), whose structure and dynamics have already been investigated using solid-state NMR [68, 69, 200, 201]. It is also examined whether a single data set or a small number of $2 \mathrm{D}(2 \mathrm{Q}, 1 \mathrm{Q})$ correlation experiments may be sufficient to identify regions 
of enhanced molecular mobility. Application of the method to studying dynamics in the protein ubiquitin is then described in Chapter 6.

\subsection{Experimental methods}

Experiments were conducted on the same sample of L-tyrosine-ethylester (TEE) (Fig. 5.1) as used in ref. [68]. It contained $4.5 \mathrm{mg}$ uniformly ${ }^{13} \mathrm{C}$-labeled TEE diluted in $45 \mathrm{mg}$ TEE with ${ }^{13} \mathrm{C}$ in natural abundance.

Spectra were recorded at a sample temperature of about $-2^{\circ} \mathrm{C}$ using a triple-resonance $\left({ }^{1} \mathrm{H},{ }^{13} \mathrm{C},{ }^{15} \mathrm{~N}\right)$ MAS probehead on a $400 \mathrm{MHz}$ wide-bore magnet (Bruker Biospin, Karlsruhe, Germany). MAS speed was set to $7.8 \mathrm{kHz}$. The general pulse scheme for recording (2Q,1Q) correlation spectra was discussed in Section 4.3 .3 (see Fig. 4.5 a). In the current application, the symmetry-based SPC5 recoupling scheme previously introduced by Griffin and coworkers was employed [121] (see Section 4.3.3 and Fig. 4.5 b). The effect of molecular motion was investigated by recording the buildup of $2 \mathrm{Q}$ coherence under this recoupling sequence. To do so, a series of $2 \mathrm{D}(2 \mathrm{Q}, 1 \mathrm{Q})$ correlation spectra was recorded, increasing 2Q excitation and reconversion times (i.e., $t_{\text {mix }}$ ) in synchrony from 154 to $1436 \mu$ s each. For proton decoupling during ${ }^{13} \mathrm{C}$ recoupling and $\left(t_{1}, t_{2}\right)$ evolution and detection periods, SPINAL64 decoupling [95] using a radiofrequency (r.f.) amplitude of $85 \mathrm{kHz}$ was employed. The carrier frequency of ${ }^{13} \mathrm{C}$ r.f. irradiation was set to $40 \mathrm{ppm}$.

SsNMR spectra were imported in MATLAB (The MathWorks, Natick, MA, USA) using the MatNMR add-on package [202], and crosspeak intensities were integrated using custom-written scripts. Spectral noise was determined from integrals in noise regions of the same size as crosspeak integrals.

\subsection{Simulations}

\subsubsection{Theory}

In order to relate experimental data to local mobility, polarization transfer dynamics must be examined in detail for a dipolar coupled ${ }^{13} \mathrm{C}$ spin network. In the absence of molecular motion, analytical functions have been given (see, e.g., refs. [100, 121]) for three-spin systems that take into account the details of the dipolar recoupling sequence under MAS conditions and the relative orientation of the dipolar vectors in the molecular frame. Motion further modulates the orientation-dependent rank 2 components of the 
anisotropic dipolar interaction [203] and can be represented by a phenomenological order parameter $S_{i j}\left(0 \leq S_{i j} \leq 1\right)$ that leads to a scaling of the effective dipolar coupling $d_{i j}^{\text {eff }}$ between two ${ }^{13} \mathrm{C}$ spins $i, j$ :

$$
d_{i j}^{\mathrm{eff}}=S_{i j} \cdot d_{i j}=S_{i j} \cdot \frac{\mu_{0}}{8 \pi^{2}} \frac{\hbar \gamma_{C} \gamma_{C}}{r_{i j}^{3}}
$$

(in units of $\mathrm{Hz}$ ). A smaller $S_{i j}$ value thus corresponds to greater mobility.

Here, dipolar coupled ${ }^{13} \mathrm{C}$ spin networks were numerically simulated on the quantummechanical (QM) level using the programm package GAMMA [150]. This allowed to also accommodate larger spin systems and effects such as chemical shift offsets important in realistic biomolecular systems. The $\mathrm{C}++$ simulation code is given in Appendix Section A.1. In the simulations, spin systems were defined via chemical shift tensors of all spins involved, as well as dipolar tensors determining spin system geometry and dipolar couplings between any two spins. Time evolution of the spin system was calculated by numerical integration of the Liouville-von Neumann equation assuming a piecewise constant Hamiltonian, considering the spin system parameters as well as powder averaging, MAS sample rotation, and r.f. irradiation corresponding to the SPC5 pulse sequence (see, e.g., refs. [100, 140, 204]. Time resolution of the simulations was $0.09 \mu \mathrm{s}$, and powder integration over 276 orientations was performed [205]. To simulate signal intensities corresponding to crosspeaks in a 2D SPC5 (2Q,1Q) correlation spectrum, all spins were set to be in the $\hat{I}_{z}$ state at the beginning of the simulation, and after evolution under the system Hamiltonian $H$ for a time $\tau$ corresponding to the duration of the 2Q excitation period (2QE), 2Q coherence between the two spins of interest $i, j$ was projected out from the density matrix using the projection superoperator $\hat{F}_{i j}^{2 Q}[121]$ :

$$
\hat{F}_{i j}^{2 Q}=\left|\hat{I}_{+}^{i} \hat{I}_{+}^{j}\right\rangle\left\langle\hat{I}_{+}^{i} \hat{I}_{+}^{j}|+| \hat{I}_{-}^{i} \hat{I}_{-}^{j}\right\rangle\left\langle\hat{I}_{-}^{i} \hat{I}_{-}^{j}\right|
$$

$\hat{I}_{z}$ magnetization on spins $i$ and $j$ was then detected after another period $\tau$ of evolution under the system Hamiltonian corresponding to double quantum reconversion (2QR). The intensity $p_{i}^{i j}$ of the crosspeak $\mathrm{CiC} j-\mathrm{C} i$ at the shift of spin $i$ in the 1Q dimension and at the sum of the shifts of spins $i$ and $j$ in the 2Q dimension, after 2QE and 2QR periods of length $\tau$ each, was thus given by [121]

$$
p_{i}^{i j}(\tau)=\operatorname{Tr}\left[e^{-i \tilde{H} \tau} \hat{F}_{i j}^{2 Q}\left\{e^{-i \tilde{H} \tau} \hat{I}_{z} e^{i \tilde{H} \tau}\right\} e^{i \tilde{H} \tau} \hat{I}_{z}^{i}\right]
$$

with $\tilde{H}$ denoting the average Hamiltonian active over the period $\tau[81,100]$. 


\subsubsection{Tests}

Simulations were first tested using rigid (i.e., $S_{i j}=1$ ) spin pairs and spin triplets. For example, a linear 3-spin system as shown in Figure 3 in ref. [121] was simulated. All chemical shift and anisotropy values were set to zero. Dipolar coupling constants were $d_{i j}=500 \mathrm{~Hz}$ between the directly bonded nuclei and $d_{i j}=0$ otherwise, and Euler angles for transforming dipolar tensors from the principal axis to the molecular frame were $(\alpha, \beta, \gamma)=$ $\left(0^{\circ}, 90^{\circ}, 0^{\circ}\right)$, corresponding to a linear arrangement along the $x$ axis of the molecular frame. Results were identical to those reported in ref. [121] (see Appendix Fig. A.1).

Simulations revealed that, to adequately capture the fastest observed experimental buildup characteristics, the ${ }^{13} \mathrm{C}$ spins directly bonded to the two ${ }^{13} \mathrm{C}$ spins contributing to the $2 \mathrm{Q}$ coherence of interest had to be included in the simulations, leading to 4 -spin simulations in most cases. The fact that larger spin systems yield faster buildups can be appreciated from the analytical expressions for $p_{i}^{i j}$ under SPC5 recoupling in a 3 -spin system given in ref. [121], whose leading terms are of the functional form $\cos ^{2}(r t) \sin ^{2}(r t)$, as also discussed in ref. [100], with $r$ formed from the three dipolar couplings as $r=$ $\sqrt{d_{i j}^{2}+d_{j k}^{2}+d_{i k}^{2}}$. A larger contribution from the third spin $k$ thus increases the oscillation frequency $r$ of crosspeak intensity, leading to a faster initial buildup [100].

Motion was subsequently introduced using uniform order parameters $S_{i j}=S$ for all couplings of 2-, 3- or 4-spin systems, yielding effective dipolar coupling constants $d_{i j}^{\text {eff }}$ as described in Equation 5.1. In Figure 5.2, theoretical results are shown for two- (a, c) and four-spin (b, d) systems. Here, the evolution of $\hat{I}_{z}$ magnetization on spin 1 (a) and spin 2 (b) after $t=2 t_{\text {mix }}$, with $t_{\text {mix }}=2 \mathrm{QE}=2 \mathrm{QR}$, was computed for $2 \mathrm{Q}$ coherence between the $(1,2)$ spin pair (a) and the (2,3) spin pair (b) as described above (Equation 5.3). Again, isotropic and anisotropic chemical shift values were set to zero. Geometry of the 4-spin system used was planar and extended with tetrahedral bond angles, as depicted in Figure 5.2. This led to Euler angles $(\alpha, \beta, \gamma)$ of $\left(0^{\circ}, 90^{\circ}, 0^{\circ}\right),\left(68^{\circ}, 90^{\circ}, 0^{\circ}\right)$, and $\left(0^{\circ}, 90^{\circ}, 0^{\circ}\right)$ for the PAS $\rightarrow$ MOL transformation of the dipolar coupling tensors corresponding to the $(1,2)$, $(2,3)$, and $(3,4)$ spin pairs, respectively.

For both the 2- and the 4-spin case, introduction of molecular motion reduces the 2Q coherence buildup rate. For identical $S$ values, the effective frequency of $2 \mathrm{Q}$ signal oscillation is enhanced in the 4-spin compared to the 2-spin system, in agreement with analytical predictions discussed above. Comparison to the rigid case $(S=1$, Fig. 5.2 c, d) reveals that the strongest deviations in signal intensity for different order parameters are seen in the initial rate regime corresponding to mixing times of about $0.5 \mathrm{~ms}$ ( 2 spin case) 

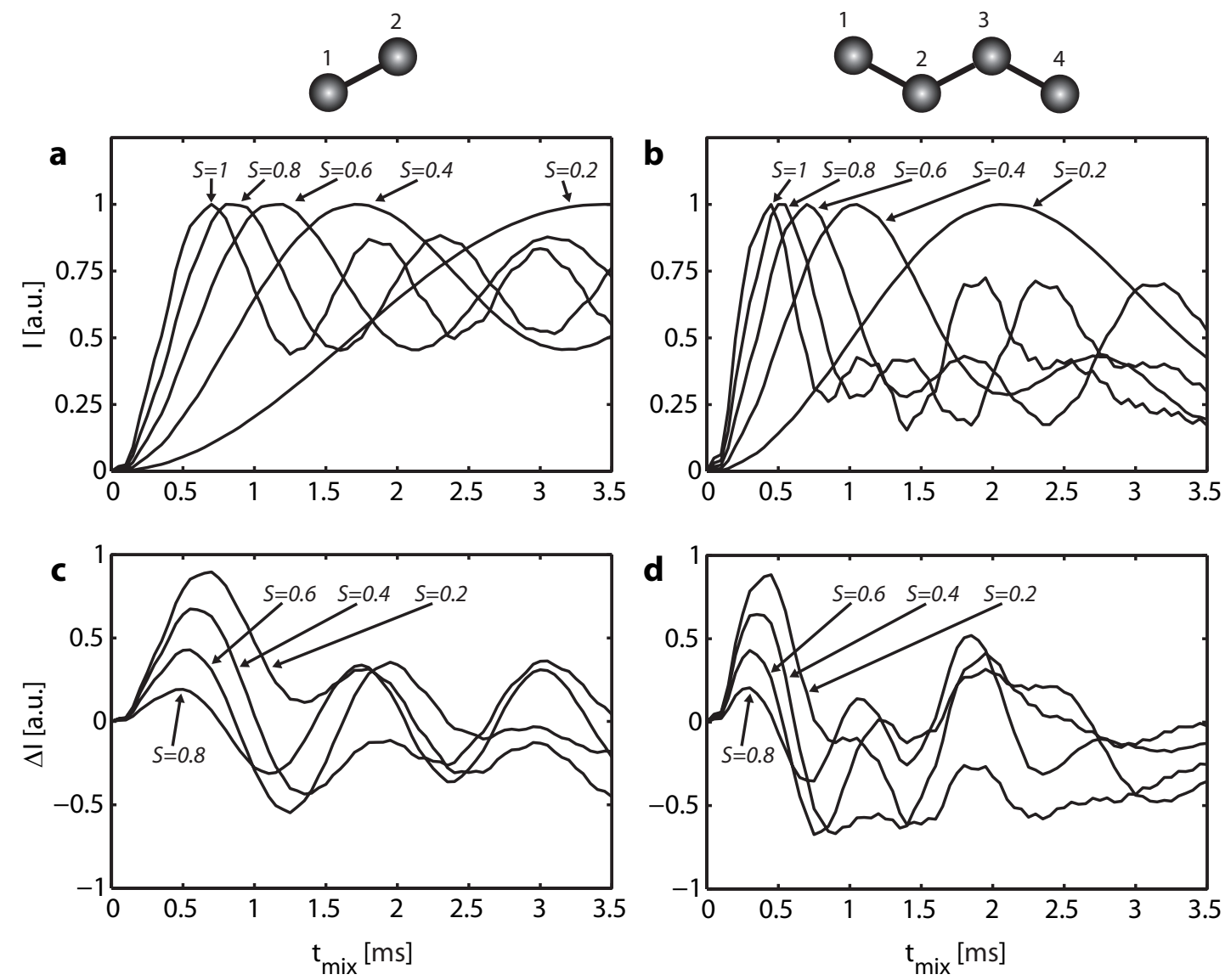

Figure 5.2: (a, b) Theoretical signal evolution for spin 1 (2-spin system, a) and spin 2 (4-spin system, b) in a $2 \mathrm{D}(2 \mathrm{Q}, 1 \mathrm{Q})$ correlation experiment for variable order parameters $S$. Intensities shown are calculated for double-quantum coherences between spins 1 and 2 (a) and between spins 2 and 3 (b), respectively, and are normalized to the maximum. 2QE and 2QR times of length $t_{\text {mix }}$ each were simulated for the SPC5 sequence [121]. (c, d) Differences in normalized signal intensities as shown in (a, b) between the rigid case $(S=1)$ and indicated $S$ parameters for spin 1 (2-spin system, c) and spin 2 (4-spin system, d). In (b) and (d), all one- and multi-bond dipolar $\left({ }^{13} \mathrm{C},{ }^{13} \mathrm{C}\right)$ couplings were scaled by the same order parameter. 
and $0.3 \mathrm{~ms}$ (4 spin case). Experimental variations for longer mixing times are in general smaller than expected from theory due to relaxation processes.
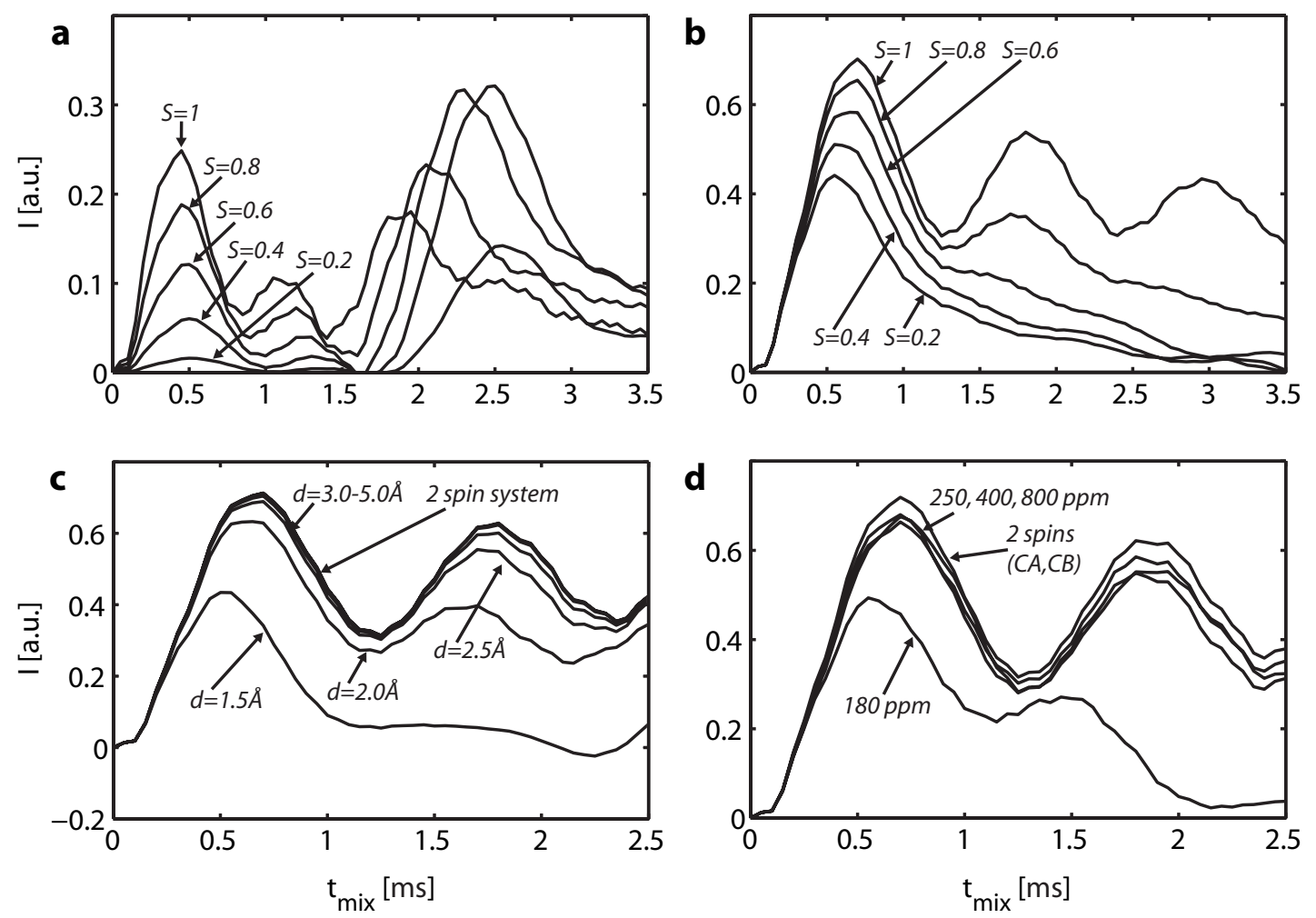

Figure 5.3: (a, b) Theoretical signal evolution in a 2D (2Q,1Q) correlation experiment for a 4-spin system in which only the order parameter $S$ of the C2-C3 bond and of all two- and three-bond couplings including this bond are varied as indicated in the panels. (a) shows the intensity of the C2C3-C2 peak, (b) that of the $\mathrm{C} 1 \mathrm{C} 2-\mathrm{C} 1$ peak. (c) Simulated $(2 \mathrm{Q}, 1 \mathrm{Q})$ signal evolution of the C1C2-C1 peak in a linear 3-spin system (C1-C2 distance 1.5 $\AA$ ) for different C2-C3 distances as indicated in the panel. Buildup of a pure 2-spin system is given as a reference. (d) Theoretical $(2 \mathrm{Q}, 1 \mathrm{Q})$ evolution of the CACB-CA signal for a $\left(\mathrm{C}^{\prime}, \mathrm{C}^{\alpha}, \mathrm{C}^{\beta}\right) 3$-spin system $\left(\mathrm{C}^{\alpha} 55 \mathrm{ppm}, \mathrm{C}^{\beta} 30 \mathrm{ppm}\right)$ with different chemical shifts of the $C^{\prime}$ spin as indicated in the panel. Evolution of the CACB-CA peak intensity in a pure $\left(C^{\alpha}, C^{\beta}\right)$ 2-spin system is shown for comparison.

Additional model calculations using 3- or 4-spin geometries were conducted to investigate the influence of different order parameters in different bonds, spin system geometry, chemical shift offset, chemical shift anisotropy, and the use of all or only one-bond dipolar couplings. Figure 5.3 shows (unnormalized) signal intensities for $\mathrm{C} 2 \mathrm{C} 3-\mathrm{C} 2$ (a) and $\mathrm{C} 1 \mathrm{C} 2-$ C1 (b) correlations in a planar extended 4-spin system for different order parameters $S_{i j}$ affecting only the $\mathrm{C} 2-\mathrm{C} 3$ bond and all two- and three-bond couplings across that bond 
(i.e. also $\mathrm{C} 1-\mathrm{C} 3, \mathrm{C} 1-\mathrm{C} 4$, and $\mathrm{C} 2-\mathrm{C} 4$ ). It can be seen that, in this context, the effect of varying these $S_{i j}$ values on the time course of the buildups up to the first maximum is much smaller than for the case of uniform scaling of all $S_{i j}$ and actually slightly reversed for the rigid spin pair (C1,C2) (Fig. 5.3 b). Rather, the 2Q correlation involving the weaker dipolar coupling is reduced in intensity, and magnetization distribution is determined more by the stronger couplings. In principle, such situations could be detected experimentally by simultaneously considering crosspeak intensity buildups for all coupled nuclei in a spin system, e.g. one amino acid residue of a protein. However, such an approach would lead to a considerable increase in the number of free parameters to be determined. Moreover, it is much more practical experimentally to consider normalized intensity buildup curves and extract information from their temporal characteristics.

Therefore, to determine order parameters for two ${ }^{13} \mathrm{C}$ spins within a coupled 2- to 4 -spin system, uniform scaling of all $S_{i j}$ values in these spin systems was assumed in this study. This leads to an effective order parameter for the two spins of interest as it would be in a uniformly mobile spin system. Notably, this does not assume that neighboring $\left({ }^{13} \mathrm{C},{ }^{13} \mathrm{C}\right)$ pairs in a chain of spins in fact have to exhibit identical order parameters, and different $S_{i j}$ values can be determined in such a context by separately fitting the corresponding crosspeak buildups (see Chapter 6). It can be seen from Figure 5.3 a, b, however, that this approach can lead to errors for $S_{i j}$ values of mobile spin pairs if large differences between $S_{i j}$ values in neighboring $\left({ }^{13} \mathrm{C},{ }^{13} \mathrm{C}\right)$ pairs occur, since variations in only one $S_{i j}$ parameter of a coupled spin system do not affect buildup kinetics much. Such situations should, however, be detectable by low crosspeak intensities for the more mobile spin pair (Fig. 5.3 a).

Figure 5.3 c shows that, in a linear 3-spin system, increasing the distance of one spin to the other two quickly leads to buildup characteristics approaching those of a 2-spin system, in agreement with expectations that the strongest couplings should chiefly govern the time evolution of the spin system $[100,206]$. A similar phenomenon is observed for increasing the chemical shift offset of the C' spin in the context of a 3-spin system corresponding to the $\mathrm{C}^{\prime}, \mathrm{C}^{\alpha}$, and $\mathrm{C}^{\beta}$ nuclei of an amino acid (Fig. $5.3 \mathrm{~d}$ ). However, this figure also shows that a C' nucleus with a chemical shift around $180 \mathrm{ppm}$ influences buildup kinetics of the neighboring nuclei and has to be taken into account in simulations, even if r.f. irradiation is centered in the aliphatic part of the spectrum. Under such conditions, usually no crosspeaks involving $C^{\prime}$ nuclei are detected in $2 \mathrm{D}(2 \mathrm{Q}, 1 \mathrm{Q})$ correlation experiments on larger biomolecules. This difference may in part be related to the fact that, experimentally, 
the initial condition $\hat{I}_{z}$ is not fulfilled for spins with large resonance frequency offsets from the r.f. carrier.

Further simulations show that, at least in the initial rate regime around the first maximum of the $2 \mathrm{Q}$ buildup curves, the effects of the conformation of a 4-spin system (extended as in Fig. 5.2 or bent) and of chemical shift anisotropy (assuming realistic values from the literature) can be neglected (Appendix Figs. A.2 and A.3). In particular, leaving out all but the one-bond couplings is found to have a negligible effect on SPC5 initial rate buildup characteristics (Appendix Fig. A.4), again in agreement with the expectation that the strongest couplings should dominate $2 \mathrm{Q}$ recoupling in a uniformly ${ }^{13} \mathrm{C}$-labeled molecule [100].

\subsubsection{Data fitting}

For simulations of TEE spin systems, four spins were considered for the aromatic ring moiety $(\mathrm{C} 1-\mathrm{C} 6)$ and for the aliphatic part of tyrosine $(\mathrm{C} 7-\mathrm{C} 9)$. 2-spin simulations were, on the other hand, sufficient for the ethyl ester tail $(\mathrm{C} 10-\mathrm{C} 11)$ which is separated from other carbons by an ester bond. A one-bond distance of $1.52 \AA$ was assumed for aliphatic carbons (corresponding to a dipolar coupling constant $d_{i j}$, as defined in Equation 5.1 , of $2160 \mathrm{~Hz}$ in the static case) and $1.4 \AA$ for the aromatic ring. Order parameters $S$ were determined for experimental spin systems by fitting corresponding simulations calculated for different uniform $S$ values to experimental data. A least-squares fit was determined using custom scripts in MATLAB. For $t_{\text {mix }}>1.2 \mathrm{~ms}$, simulations for more rigid $S$ values show again an increase in signal intensity, which was not observed in experimental data, most likely due to relaxation. Consequently, for fitting, only data points for $t_{\text {mix }}$ below 1.2 ms were considered. The error of $S$ was determined as the largest difference to another $S$ value that gave a fit to experimental data with a root-mean-square deviation (RMSD) differing by no more than $30 \%$ from that of the best fit. This usually yielded errors of \pm 0.05 . For this reason, simulations were run only for $S$ values in steps of 0.05 . Larger errors were found for aromatic TEE nuclei for which experimental data were more strongly affected by noise, presumably due to the larger chemical shift offset of these nuclei from the carrier frequency.

Since two nuclei are involved in a $2 \mathrm{Q}$ coherence, their individual crosspeak buildups should yield identical $S$ values. Within the experimental error of 0.05 , this was always the case. For differing $S$ values of the two nuclei in a $2 \mathrm{Q}$ coherence, the average is given. To compare $\left({ }^{13} \mathrm{C},{ }^{13} \mathrm{C}\right)$ order parameters thus determined (further designated as $S_{\mathrm{CC}}$ ) to 
$\left({ }^{1} \mathrm{H},{ }^{13} \mathrm{C}\right)$ order parameters $\left(S_{\mathrm{HC}}\right)$ measured previously [68], also the average of $S_{\mathrm{HC}}$ values for the two ${ }^{13} \mathrm{C}$ nuclei involved in a $2 \mathrm{Q}$ coherence was taken.

\subsection{Results and Discussion}

To test the validity of the approach of extracting dynamic information from $2 \mathrm{Q}$ coherence buildups, (2Q,1Q) correlation experiments were conducted for a series of mixing times on TEE using the SPC5 pulse sequence. In Figure 5.4, 2D ssNMR spectra are given for mixing times of 564 and $923 \mu \mathrm{s}$. For simplicity, only the spin pairs $(\mathrm{C} 7, \mathrm{C} 8),(\mathrm{C} 8, \mathrm{C} 9)$, and $(\mathrm{C} 10, \mathrm{C} 11)$ are shown. Relative intensities indicate that the $(\mathrm{C} 10, \mathrm{C} 11) 2 \mathrm{Q}$ correlation is more dominant in the latter experiment.
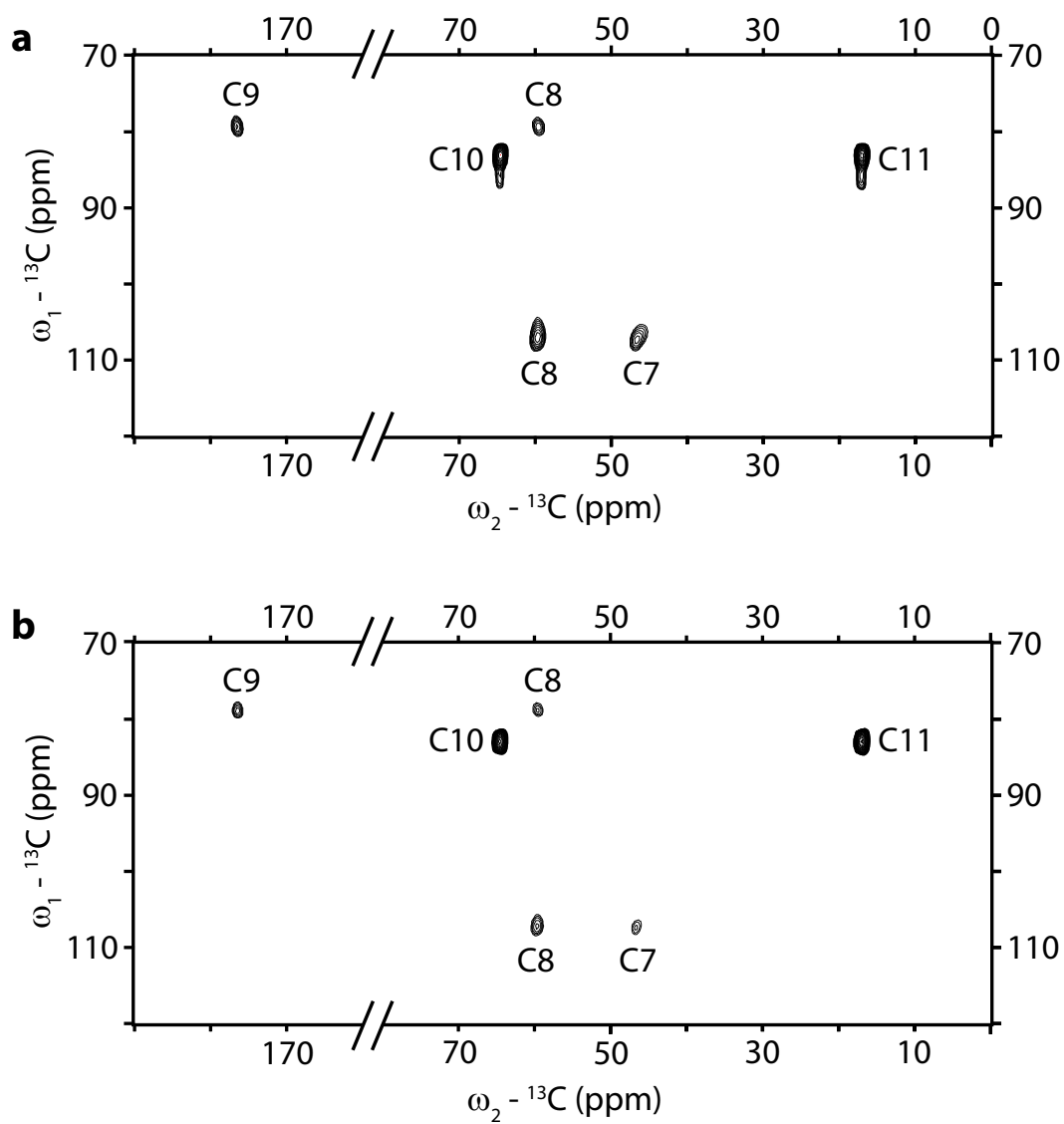

Figure 5.4: Cutouts of $2 \mathrm{D}(2 \mathrm{Q}, 1 \mathrm{Q})$ correlation experiments on TEE for $\mathrm{t}_{\mathrm{mix}}=564 \mu \mathrm{s}$ (a) and 923 $\mu$ s (b). 
Experimental 2Q crosspeak intensity buildup data together with best fits from simulations are shown in Figure 5.5. Simulations depicted in Figure 5.5 take chemical shifts of the nuclei involved into account, whereas for simulations in Figures 5.2 and 5.3, all chemical shifts and the r.f. carrier frequency were set to zero. This explains the different appearance of the simulated buildups in these figures. Notably, slightly negative crosspeak intensities were found at very short mixing times for aromatic nuclei, which exhibit large offsets of their resonance frequencies from the r.f. carrier. These negative intensities are reproduced in the simulations.

Within experimental accuracy, all spin pairs except for the $(\mathrm{C} 10, \mathrm{C} 11)$ moiety are described by $S_{\mathrm{CC}}$ order parameters close to 1 , in agreement with previous ssNMR studies of $\left({ }^{1} \mathrm{H},{ }^{13} \mathrm{C}\right)$ one-bond couplings [68]. The $(\mathrm{C} 8, \mathrm{C} 9)$ spin pair, which includes the $\mathrm{C} 9$ carbonyl, was excluded from the analysis since a wide range of different $S_{\mathrm{CC}}$ values leads to very similar buildup time courses for this spin pair. This effect can be traced to the large chemical shift offset of $\mathrm{C} 9$, reducing its impact on the $(\mathrm{C} 4, \mathrm{C} 7, \mathrm{C} 8, \mathrm{C} 9)$ spin system for lower order parameters, not unlike the situation with only one mobile $\left({ }^{13} \mathrm{C},{ }^{13} \mathrm{C}\right)$ bond discussed in Section 5.4.2.

The $(\mathrm{C} 10, \mathrm{C} 11)$ spin pair is best described by an order parameter of 0.80 . This value is larger than the average value $(0.62)$ of the $S_{\mathrm{HC}}$ order parameters determined for $\mathrm{C} 10$ and C11 [68]. This can be expected since $S_{\mathrm{HC}}$ should be sensitive also to rotations around the $\mathrm{C} 10-\mathrm{C} 11$ bond, which do not affect the effective $\left({ }^{13} \mathrm{C},{ }^{13} \mathrm{C}\right)$ coupling between these two nuclei. $S_{\mathrm{CC}}$ and $S_{\mathrm{HC}}$ thus probe different modes of motion. Conversely, the fact that $S_{\mathrm{CC}}$ is significantly lower than 1 for $(\mathrm{C} 10, \mathrm{C} 11)$ indicates that the ester tail exhibits modes of motion in addition to such bond rotation, e.g. a nutation as suggested in ref. [69]. On the other hand, the $S_{\mathrm{CC}}$ value of 0.80 is also larger than expected from the scaling of the (C10,C11) dipolar coupling determined by 2Q-filtered rotational resonance experiments and zero-quantum RIL [207] transfer [69, 208]. In these experiments, an apparent dipolar coupling of about $1400 \mathrm{~Hz}$ was found, which would amount to an $S_{\mathrm{CC}}$ value of 0.65 for the $\left({ }^{13} \mathrm{C},{ }^{13} \mathrm{C}\right)$ bond length of $1.52 \AA$ assumed here. This difference may be due to additional modulating effects on the experimentally detected dipolar coupling by the specific recoupling sequences used, which exhibit different effective dipolar coupling elements [121, 207]. Sample preparation may also play a role (see Chapter 6). Nevertheless, data shown here confirm previous results that polycrystalline TEE is rigid at the temperatures investigated, except for the ethyl ester tail. It should also be noted that the offset of 0.18 between $S_{\mathrm{HC}}$ and $S_{\mathrm{CC}}$ order parameters measured on the same TEE sample for the $(\mathrm{C} 10, \mathrm{C} 11)$ spin 
$(\mathrm{C} 2, \mathrm{C} 3)$
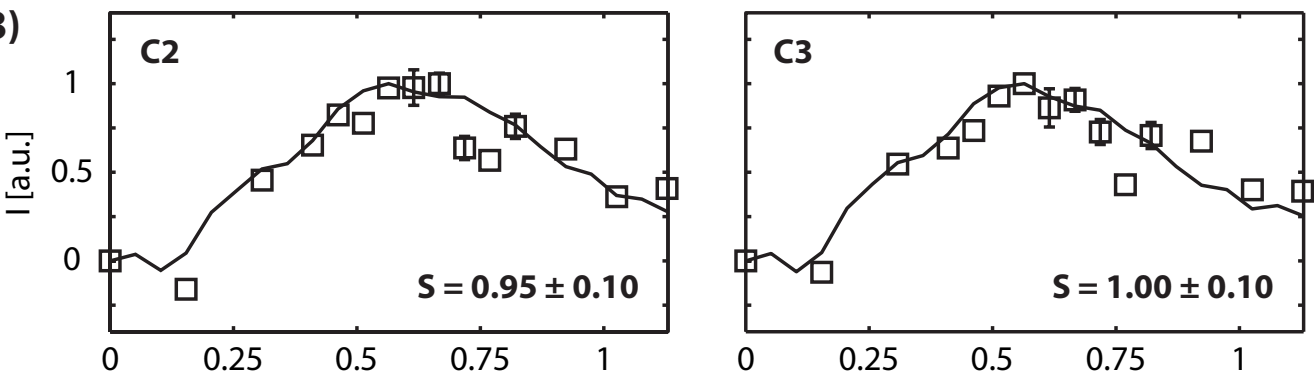

$(\mathrm{C} 5, \mathrm{C6})$
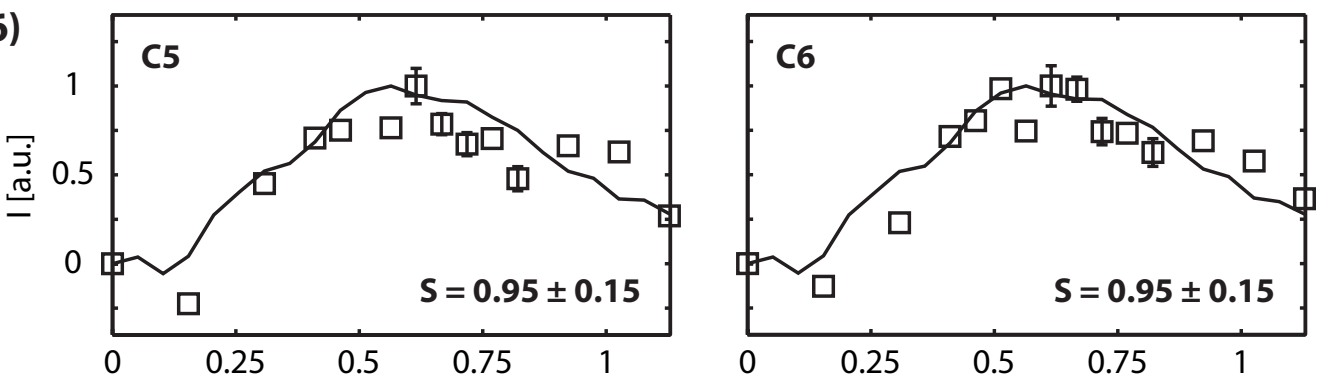

$(C 7, C 8)$
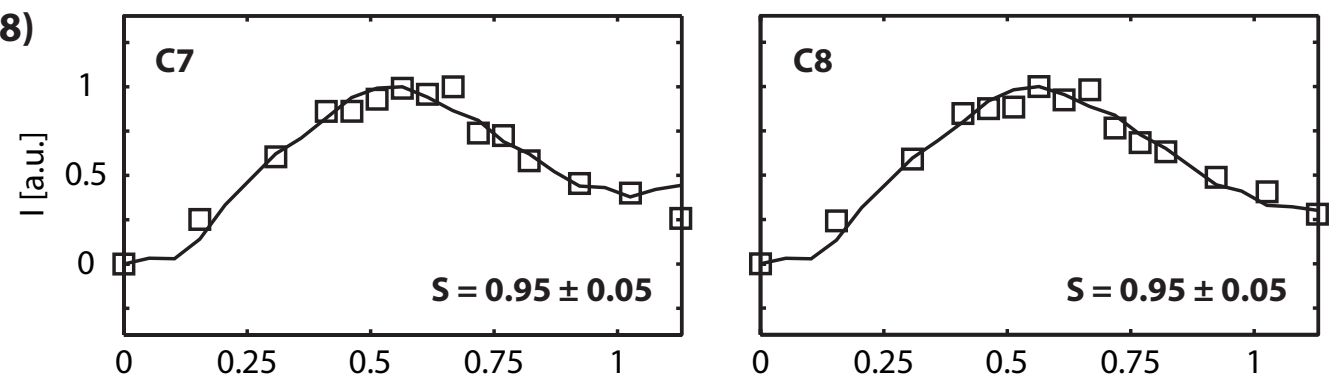

$(\mathrm{C} 10, \mathrm{C11})$
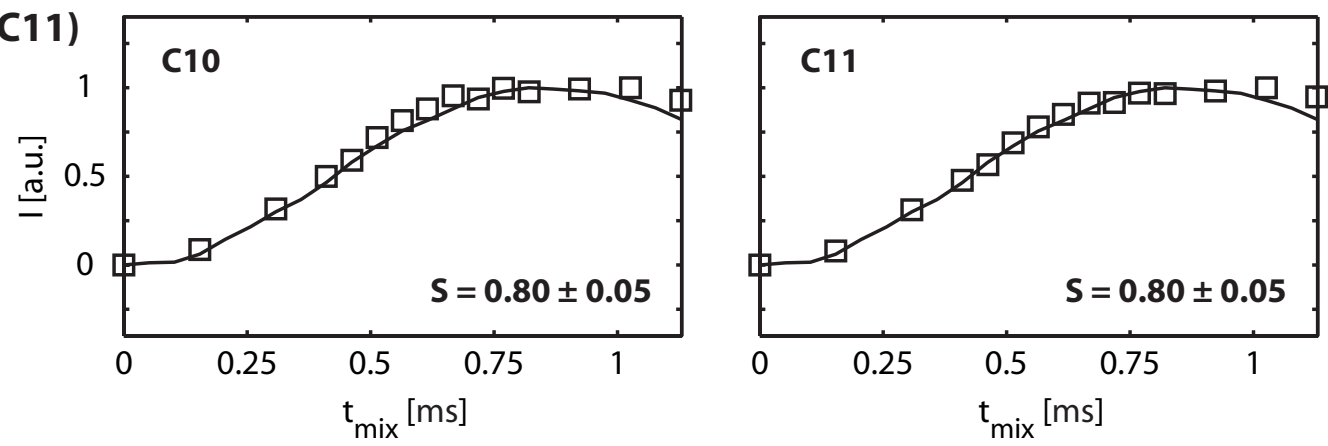

Figure 5.5: Experimental $2 Q$ crosspeak signal evolution (symbols) and best-fitting numerical simulations (lines) for the TEE ${ }^{13} \mathrm{C}$ spin pairs indicated in the panels. Order parameters $S$ of bestfitting simulations are given. Error bars determined from spectral noise are shown where they exceed the size of the data points. 
pair is in excellent agreement with the average difference of $S_{\mathrm{HC}}$ and $S_{\mathrm{CC}}$ values found for amino acid residues in a protein in Chapter 6.

Figure 5.6 summarizes $\left({ }^{1} \mathrm{H},{ }^{13} \mathrm{C}\right)$ and $\left({ }^{13} \mathrm{C},{ }^{13} \mathrm{C}\right)$ order parameters as determined for TEE by ssNMR. Comparison with spectra shown in Figure 5.4 reveals that a simple intensity analysis of two $2 \mathrm{D}(2 \mathrm{Q}, 1 \mathrm{Q})$ correlation experiments already provides insight into local molecular mobility, since the $(2 \mathrm{Q}, 1 \mathrm{Q})$ crosspeaks of mobile moieties reach maximum intensity only for longer mixing times. This is clearly visible in Figure $5.4 \mathrm{a}$ and b from

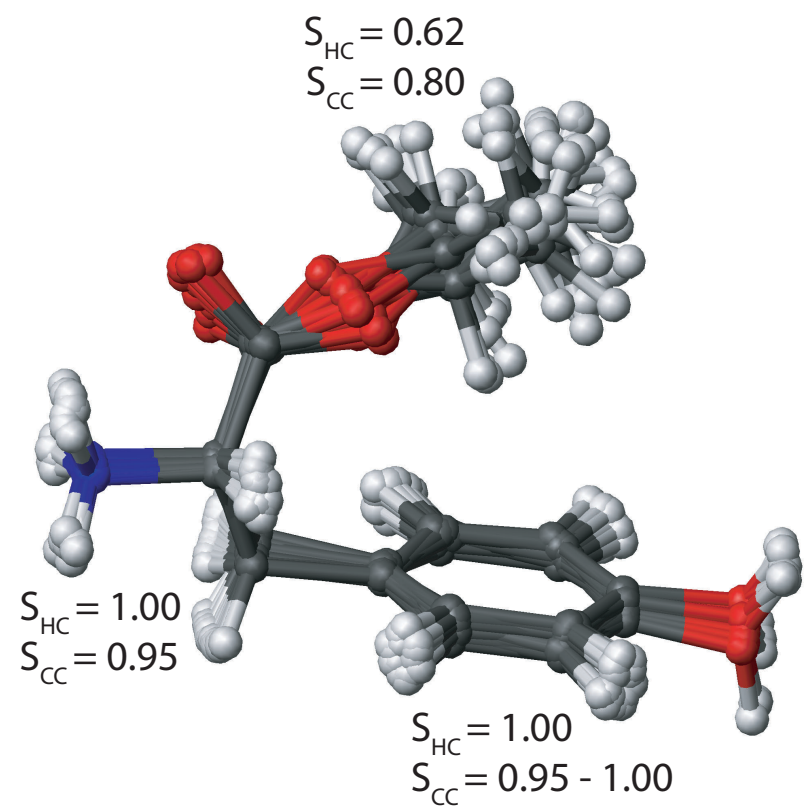

Figure 5.6: Comparison of dipolar $\left({ }^{1} \mathrm{H},{ }^{13} \mathrm{C}\right)$ and $\mathrm{DQ}\left({ }^{13} \mathrm{C},{ }^{13} \mathrm{C}\right)$ order parameters for the $(\mathrm{C} 2, \mathrm{C} 3)$, $(\mathrm{C} 5, \mathrm{C} 6),(\mathrm{C} 7, \mathrm{C} 8)$, and $(\mathrm{C} 10, \mathrm{C} 11)$ spin pairs of TEE. In both cases, values obtained from individual crosspeak buildups were averaged between the two nuclei involved. $\left({ }^{1} \mathrm{H},{ }^{13} \mathrm{C}\right)$ order parameters and the molecular model of polycrystalline TEE are taken from ref. [68].

the relative intensities of $(\mathrm{C} 7, \mathrm{C} 8)$ and $(\mathrm{C} 10, \mathrm{C} 11)$ correlations. However, intensities in individual spectra should be interpreted cautiously, as also evident from Figure 5.4. Absolute intensities of $(\mathrm{C} 10, \mathrm{C} 11)$ correlations are larger than those of $(\mathrm{C} 7, \mathrm{C} 8)$ correlations already at the shorter mixing time (Fig. 5.4 a); nevertheless, analysis of buildup kinetics clearly reveals that the $(\mathrm{C} 7, \mathrm{C} 8)$ correlation is characterized by a significantly more rigid order parameter. 


\subsection{Conclusions}

It could be shown that $\left({ }^{13} \mathrm{C},{ }^{13} \mathrm{C}\right)$ double-quantum spectroscopy can provide a sensitive means to probe molecular mobility up to the ms timescale. In the initial rate regime, the signal amplitude of $(2 \mathrm{Q}, 1 \mathrm{Q})$ correlation experiments is strongly modulated by molecular motion. Simulations and data fitting procedures were developed to analyze intensity buildup data quantitatively. In polypeptides, this readily allows for the study of differential motion in a residue-specific manner. Data obtained on the test system TEE are in good agreement with previous ssNMR order parameter measurements, showing a rigid molecule except for the ethyl ester tail, which exhibits correlated motion.

Protein dynamics play an important role in molecular recognition and complex formation [209]. Especially when the recording of entire three-dimensional data sets is prohibited by spectroscopic sensitivity, the approach outlined here may provide a useful means to identify molecular regions of enhanced mobility in a small number of $2 \mathrm{D}$ experiments. In the following chapter, this will be examined on the 76 -residue protein ubiquitin in a microcrystalline state. 


\section{Chapter 6}

\section{Structural and dynamical investigation of microcrystalline ubiquitin}

\subsection{Summary}

Microcrystalline preparations have successfully been used for structural investigations of proteins by solid-state NMR. However, local structure and dynamics in protein microcrystals may differ with respect to solution phase or single crystal preparations. Previously, chemical shift differences were reported for ubiquitin in different microcrystalline states and in solution [70]. This chapter describes that, additionally, several through-space correlations expected based on the crystal structure are absent in spectra of microcrystalline ubiquitin precipitated from poly(ethylene glycol) ("Ubi-P"). To investigate whether these phenomena are related to mobility, the approach to detect molecular motion developed in Chapter 5 was applied to Ubi-P. Results reveal the presence of dynamics on millisecond or faster time scales and differences in local mobility compared to methylpentanediolprecipitated ubiquitin ("Ubi-M"). Many absent CHHC correlations and large preparationdependent chemical shift changes occur in residues with elevated local mobility or mobility differences between the solid-state preparations. Structure calculation using an automated algorithm with the ubiquitin crystal structure as a reference indicates that loop residues with different local conformation may account for several of the remaining shift changes and absent CHHC correlations. Thus, results presented here confirm earlier hypotheses about the role of dynamics in the origin of preparation-dependent chemical shift differences. $^{1}$

\footnotetext{
${ }^{1}$ This chapter is related to the following manuscript in preparation for publication: Robert Schneider et al.: Probing molecular motion by double-quantum $\left({ }^{13} \mathrm{C},{ }^{13} \mathrm{C}\right)$ solid-state NMR spectroscopy: Application to ubiquitin.
} 


\subsection{Introduction}

Recent advances in instrumentation, methodology, and sample preparation have yielded considerable progress in the investigation of biomolecules by solid-state NMR [210, 211], leading to the first structures of uniformly $\left[{ }^{13} \mathrm{C},{ }^{15} \mathrm{~N}\right]$-labeled proteins determined by MAS ssNMR [49, 51, 67, 108]. First successful applications of partly and fully automated procedures for simultaneous assignment of through-space correlation spectra and structure calculation have been reported [5, 52, 53]. While ssNMR has also long been used to probe dynamics of solid biomolecules, in crystalline phase as well as in lipid membranes [190, 191, 212], only in recent years have studies of dynamics in uniformly labeled proteins by ssNMR become possible [113, 149, 194]. Microcrystalline protein preparations, shown to yield high quality ssNMR spectra while largely preserving native protein structure, have been used in many of these studies [213]. However, details of the preparation of protein microcrystals have been shown to influence NMR spectral parameters such as chemical shift $[70,213,214]$. In particular, considerable chemical shift variations between different microcrystalline preparations and with respect to solution-state NMR assignments have been reported for protein regions known to exhibit increased dynamics in solution [70, 215]. Thus, detailed knowledge of changes in protein conformation and dynamics depending on microcrystalline sample preparation would be highly desirable.

The 76-residue protein ubiquitin fulfils a wide variety of cellular functions, among the best known of which is the targeting of proteins for proteasomal degradation $[216,217]$. It has long been a classic model protein for studies of protein structure and dynamics by solution- and solid-state NMR [70, 108, 149, 218, 219, 220], and a high-resolution crystal structure is available [221] (PDB ID 1UBQ). Preparation-dependent chemical shift variations in putatively mobile protein regions as mentioned above have been described in this protein [70]. Thus, in work described in this chapter, structure and dynamics of ubiquitin in one specific microcrystalline preparation (poly(ethylene glycol) (PEG) precipitation, "Ubi-P") were investigated in detail and compared with data obtained using methylpentanediol (MPD) precipitation ("Ubi-M") [108, 149]). The method of detecting molecular mobility by double-quantum $(2 \mathrm{Q})\left({ }^{13} \mathrm{C},{ }^{13} \mathrm{C}\right)$ correlation spectroscopy as developed in Chapter 5 was applied, yielding $S_{\mathrm{CC}}$ order parameters for 40 residues. Ubiquitin CHHC through-space correlation spectra $[136,137]$ were analyzed in terms of their structural information content and used for an automated structure calculation procedure [5, 184]. Results show the sensitivity of $2 \mathrm{Q}$ ssNMR spectroscopy for residue-specific molecular mo- 
tion in proteins up to the millisecond ( $\mathrm{ms}$ ) time scale and relate preparation-dependent chemical shift changes to local differences in dynamics and conformation.

\subsection{Materials and Methods}

\subsubsection{Sample preparation}

Uniformly $\left[{ }^{13} \mathrm{C},{ }^{15} \mathrm{~N}\right.$-labeled, PEG-precipitated microcrystalline ubiquitin (see Appendix Table B.1) was prepared in the group of Stefan Becker as described in ref. [70]. The sample used here was identical to the one used in that study.

\subsubsection{Solid-state NMR}

NMR experiments were conducted using $4 \mathrm{~mm}$ triple-resonance $\left({ }^{1} \mathrm{H},{ }^{13} \mathrm{C},{ }^{15} \mathrm{~N}\right)$ probeheads at static magnetic fields of 14.1 and $18.8 \mathrm{~T}$ corresponding to 600 and $800 \mathrm{MHz}$ proton resonance frequencies (Bruker Biospin, Karlsruhe, Germany). CHHC [136, 137] spectra were recorded at $14.1 \mathrm{~T}$ using initial $\left({ }^{1} \mathrm{H},{ }^{13} \mathrm{C}\right)$ cross-polarization $(\mathrm{CP})$ times of $1 \mathrm{~ms}$, short CPs $(80 \mu \mathrm{s})$ bracketing $\left({ }^{1} \mathrm{H},{ }^{1} \mathrm{H}\right)$ mixing, and $\left({ }^{1} \mathrm{H},{ }^{1} \mathrm{H}\right)$ mixing times of $62.5,125,180$, and $250 \mu \mathrm{s}$, with 170 acquisition data points in the indirect and 1024 in the direct dimension. MAS frequency was set to $11 \mathrm{kHz}$, and effective sample temperature was about $+5^{\circ} \mathrm{C}$. ${ }^{1} \mathrm{H}$ field strengths were $83 \mathrm{kHz}$ for $90^{\circ}$ pulses and $72 \mathrm{kHz}$ for SPINAL64 [95] decoupling. $(2 \mathrm{Q}, 1 \mathrm{Q})$ correlation spectra were recorded at a static field of $18.8 \mathrm{~T}$ and $8 \mathrm{kHz}$ MAS frequency. The effective sample temperature (calibrated using nickelocene [222]) was $+17^{\circ} \mathrm{C}$, as in experiments for determination of $\left({ }^{1} \mathrm{H},{ }^{13} \mathrm{C}\right)$ order parameters on Ubi-M [149]. The SPC5 pulse sequence [121] (Section 4.3.3) was used for generation of 2Q coherence, with $2 \mathrm{Q}$ excitation and reconversion times each of $250,500,750$, or $1000 \mu \mathrm{s}$. Initial $\left({ }^{1} \mathrm{H},{ }^{13} \mathrm{C}\right)$ CPs for these experiments was set to $700 \mu \mathrm{s} .{ }^{1} \mathrm{H}$ field strengths of $83 \mathrm{kHz}$ were used for $90^{\circ}$ pulses and SPINAL64 decoupling, except during $2 \mathrm{Q}$ excitation and reconversion, where decoupling field strength was set to $90 \mathrm{kHz}$. (2Q,1Q) spectra were recorded with 240 and 1280 acquisition data points in indirect and direct dimensions, respectively. All spectra were processed in XWinNMR or Topspin (Bruker Biospin, Karlsruhe, Germany) using $1024 \times 1024$ processing data points and analyzed in Sparky [223]. 


\subsection{3 $\mathrm{CHHC}$ and chemical shift analysis}

For analysis of CHHC spectra, the software MOLMOL [224] was used to add protons to the ubiquitin crystal structure (PDB ID 1UBQ) and to list all $\left({ }^{1} \mathrm{H},{ }^{1} \mathrm{H}\right)$ pairs spaced no more than $2.7 \AA$ or $3.5 \AA$ apart (for analysis of CHHC spectra with 62.5 or $180 \mu \mathrm{s}$ mixing time, respectively). Crosspeak predictions were then obtained from assignments for ${ }^{13} \mathrm{C}$ nuclei bound to these protons, using Ubi-P assignments published in ref. [70]. The resultant correlations were considered as expected correlations, and percentages for present or absent crosspeaks assume these sets as $100 \%$ values. Only correlations involving ${ }^{13} \mathrm{C}$ nuclei which were both assigned in Ubi-P could thus be probed. A crosspeak was considered present in the spectrum if spectral intensity was found within $0.2 \mathrm{ppm}$ around its predicted position on at least one side of the diagonal, using a lower contour threshold of 2.3 times the spectral noise level as determined by Sparky.

Values of chemical shift changes between Ubi-P and Ubi-M were determined previously in ref. [70] and kindly provided by Karsten Seidel, using his assignments on Ubi-P [70] and Ubi-M assignments from ref. [225]. Summed chemical shift differences of C', $\mathrm{C}^{\alpha}$, and $\mathrm{C}^{\beta}$ nuclei were used.

\subsubsection{Analysis of dynamics}

Buildups of crosspeak intensities in $(2 \mathrm{Q}, 1 \mathrm{Q})$ correlation spectra were analyzed as described in Chapter 5, using previously determined Ubi-P resonance assignments [70]. Spectra were imported in MATLAB (The MathWorks, Natick, MA, USA) using the MatNMR addon package [202], and crosspeak intensities were integrated using custom-written scripts. Spectral noise was determined from integrals in noise regions of the same size as crosspeak integrals. Spin systems were simulated using the GAMMA [150] code described in Chapter 5 , considering the two ${ }^{13} \mathrm{C}$ spins involved in the formation of a $2 \mathrm{Q}$ state and any ${ }^{13} \mathrm{C}$ spins directly bonded to these. Details of spin system geometries used are given in Appendix Section B.3. For each spin system, simulations were conducted using dipolar $\left({ }^{13} \mathrm{C},{ }^{13} \mathrm{C}\right)$ couplings scaled by order parameters $S_{\mathrm{CC}}$ between 0.3 (3- and 4-spin simulations) or 0.5 (5-spin simulations) and 1 in steps of 0.05 . Chemical shifts were taken from the Biological Magnetic Resonance Data Bank (BMRB, http://www.bmrb.wisc.edu).

Least-squares fits of simulations to experimental data were determined by custom MATLAB scripts. Order parameters given are $S_{\mathrm{CC}}$ values used in the simulation yielding the best fit to experimental data, averaged over the two nuclei involved in the $2 \mathrm{Q}$ coherence 
if the two values differed. Errors of order parameters were determined as follows. On the one hand, simulations were fit to experimental data with noise (as determined from the spectra) added to or subtracted from each data point, considering all possible combinations (16 for 4 data points). On the other hand, the largest difference to another $S_{\mathrm{CC}}$ value was determined that gave a fit to experimental data with a root-mean-square deviation (RMSD) differing by no more than $30 \%$ from that of the best fit. Finally, the difference (if any) between the two $S_{\mathrm{CC}}$ values fitting best to the two crosspeak buildups of one $2 \mathrm{Q}$ coherence was considered. The largest of these three values was taken as the error of the respective $S_{\mathrm{CC}}$ value. It was usually found to be \pm 0.05 , comparing well to errors of $S_{\mathrm{HC}}$ order parameters in ref. [149]. This justified running spin system simulations only in $S_{\mathrm{CC}}$ steps of 0.05. Resultant $S_{\mathrm{CC}}$ values are thus always multiples of 0.05. Exceptions are $2 \mathrm{Q}$ coherences for which best-fitting simulations for the two corresponding crosspeak buildups yielded slightly different $S_{\mathrm{CC}}$ values; for those, the average is given.

$\left(\mathrm{C}^{\alpha}, \mathrm{C}^{\beta}\right) S_{\mathrm{CC}}$ order parameters could be obtained for $40 \mathrm{Ubi}-\mathrm{P}$ residues; $\left(\mathrm{C}^{\beta}, \mathrm{C}^{\gamma}\right)$, $\left(\mathrm{C}^{\beta}, \mathrm{C}^{\gamma 1}\right)$, or $\left(\mathrm{C}^{\beta}, \mathrm{C}^{\gamma 2}\right) S_{\mathrm{CC}}$ values were determined for 23 residues (Table B.3). $\left(\mathrm{C}^{\alpha}, \mathrm{C}^{\beta}\right)$ $S_{\mathrm{CC}}$ order parameters for three residues (Leu56, Leu67, Leu69) whose signals partly overlap with close sequence neighbors (Leu56 with Asp58; Leu67 with Leu69) were also included in the dataset (open circles in Fig. 6.4 a). Residues without $S_{\mathrm{CC}}$ order parameters are either not assigned in Ubi-P, exhibit overlapped resonances in (2Q,1Q) spectra, or are glycine residues lacking sidechain carbons for excitation of $\left({ }^{13} \mathrm{C},{ }^{13} \mathrm{C}\right) 2 \mathrm{Q}$ coherence.

Solution-state NMR order parameters based on backbone $\left({ }^{1} \mathrm{H},{ }^{15} \mathrm{~N}\right)$ residual dipolar couplings $\left(S_{\text {rdc }}^{2}\right)$ as given in refs. $[209,226]$ were kindly provided by Nils Lakomek. SsNMR $\left({ }^{1} \mathrm{H},{ }^{13} \mathrm{C}\right)$ order parameters measured on Ubi-M $\left(S_{\mathrm{HC}}\right)$ were taken from ref. [149]. For comparison with $S_{\mathrm{CC}}$ values, $S_{\mathrm{HC}}$ values were averaged over the corresponding two ${ }^{13} \mathrm{C}$ nuclei if both values were available. A parameter $\Delta S_{\text {rel,norm was defined as a normalized }}$ measure of the relative difference in $\left(\mathrm{C}^{\alpha}, \mathrm{C}^{\beta}\right) S_{\mathrm{HC}}$ and $S_{\mathrm{CC}}$ order parameters. For a residue $i$,

$$
\Delta S_{\text {rel, norm }}(i)=\frac{\left|\Delta S_{\text {rel }}(i)\right|}{\max _{j}\left(\left|\Delta S_{\text {rel }}(j)\right|\right)}
$$

where

$$
\Delta S_{\mathrm{rel}}(i)=\left(S_{\mathrm{CC}}(i)-S_{\mathrm{HC}}(i)\right)-\frac{1}{n} \sum_{j}\left(S_{\mathrm{CC}}(j)-S_{\mathrm{HC}}(j)\right)
$$

with $j$ in Equations 6.1 and 6.2 running over all $n$ residues for which both order parameters were available. $\Delta S_{\text {rel,norm }}$ is thus independent of an overall offset between $S_{\mathrm{HC}}$ and $S_{\mathrm{CC}}$ values. 


\subsubsection{Structure calculation}

For structure calculation, crosspeaks were picked manually in the four Ubi-P CHHC spectra using Sparky [223], with minimum contour levels set at 3.5 times the spectral noise level. Folded-in spinning sideband peaks and diagonal peaks were removed. The PASD algorithm for automated assignment of through-space correlations and structure calculation [184] implemented in Xplor-NIH [173, 174] was employed as previously published [5]. Tolerances for matching chemical shift assignments from ref. [70] to CHHC crosspeaks were set to $0.4 \mathrm{ppm}$, and upper limits for $\left({ }^{1} \mathrm{H},{ }^{1} \mathrm{H}\right)$ distance restraints associated with a given CHHC crosspeak assignment were set to $4.0,5.0,6.0$, or $6.5 \AA$ for peaks from spectra with $62.5,125,180$, or $250 \mu$ s mixing time, respectively, with a common lower distance cutoff of $1.8 \AA$. Torsion angle restraints that were previously derived from Ubi-P assignments using TALOS [159] as well as from NN and NHHC spectra of Ubi-P, as described in ref. [70], were kindly provided by Karsten Seidel. They were enforced using square-well potentials with no energy contribution for deviations within the predicted RMSD.

While three successive passes of PASD structure calculations in torsion angle space were carried out as published [5, 184], final likelihoods of crosspeak assignments were determined with the ubiquitin crystal structure (PDB 1UBQ) as a reference, effectively using PASD to assign the CHHC spectra with the crystal structure as a search model, similar as in ref. [5]. The distance violation cutoff for crosspeak assignments was set to $0.5 \AA$, i.e. a crosspeak assignment was discarded if the associated $\left({ }^{1} \mathrm{H},{ }^{1} \mathrm{H}\right)$ distance in the crystal structure exceeded the upper distance limit set for the corresponding CHHC spectrum by more than $0.5 \AA$. This approach thus always yields structures that are close to the reference (i.e. crystal) structure in three-dimensional space and can only detect smaller deviations from it. The validity of this approach follows from the findings described in Section 6.4.1 and ref. [70] that the Ubi-P structure must be closely related to the crystal structure. 368 unambiguous and 842 ambiguous distance restraints were obtained in this manner (counting also duplicates found in more than one spectrum). The unambiguous restraints involved 208 unique $\left({ }^{13} \mathrm{C},{ }^{13} \mathrm{C}\right)$ pairs; ambiguous restraints comprised 1186 unique $\left({ }^{13} \mathrm{C},{ }^{13} \mathrm{C}\right)$ pairs (Table B.4).

Together with the 92 dihedral angle restraints from ref. [70], these distance restraints were used for simulated annealing in CNS 1.1 [175]. Ambiguous restraints were accounted for by $R^{-6}$ sum averaging (CNS mode "sum") over all possible contacts. The simulated annealing protocol used the PARALLHDG5.3 parameter file and started from the crystal structure of ubiquitin (PDB 1UBQ). Three stages were used: 1) high-temperature anneal- 
ing in torsion angle space in 1000 time steps of 0.015 ps at $1000 \mathrm{~K}, 2)$ very slow cooling in torsion angle space in 10000 steps of 0.015 ps, reducing the temperature from $1000 \mathrm{~K}$ to zero in steps of $50 \mathrm{~K}$, and 3) a final conjugate gradient minimization in 20 cycles of 100 steps each. Force constants were 300,300 , and $150 \mathrm{kcal} \mathrm{mol}^{-1} \AA^{-2}$ for distance restraints and 100, 200, and $400 \mathrm{kcal} \mathrm{mol}^{-1} \mathrm{rad}^{-2}$ for dihedral angle restraints in the three stages, respectively. 100 structures were calculated from different initial velocities, of which the 10 structures with lowest overall energy were selected and aligned along the backbone atoms of residues Met1 to Val70 using MOLMOL [224].

\subsection{Results}

\subsubsection{Analysis of $\mathrm{CHHC}$ spectra}

Earlier investigations have already shown that the $\left({ }^{1} \mathrm{H},{ }^{1} \mathrm{H}\right)$ distances corresponding to crosspeaks visible in N/CHHC spectra correlate well with the $\left({ }^{1} \mathrm{H},{ }^{1} \mathrm{H}\right)$ mixing times used, at least in the initial rate regime up to few hundreds of microseconds [137, 227]. In a first step, a similar analysis was conducted here on Ubi-P. As a reference structure for the analysis of CHHC crosspeaks, the ubiquitin crystal structure was used (PDB ID 1UBQ) [221] which previous ssNMR studies have indicated to be closely similar to the structure(s) present in different solid-phase preparations [52, 70, 108]. Crosspeak predictions were obtained using previously determined Ubi-P resonance assignments covering $86 \%$ of the sequence [70]. A large majority of the crosspeaks predicted from the crystal structure was found to be present in $\mathrm{CHHC}$ spectra recorded at $600 \mathrm{MHz}{ }^{1} \mathrm{H}$ resonance frequency (see Section 6.3.3), and a good correlation with mixing time was observed. For unique correlations involving only protons attached to $\mathrm{C}^{\alpha}$ or $\mathrm{C}^{\beta}$ nuclei, $86 \%$ (49 of 57) of all contacts predicted for $\left({ }^{1} \mathrm{H},{ }^{1} \mathrm{H}\right)$ distances up to $2.7 \AA$ are present in a CHHC spectrum with $62.5 \mu$ s mixing time, while $93 \%$ (84 of 90) of all such contacts up to a $\left({ }^{1} \mathrm{H},{ }^{1} \mathrm{H}\right)$ distance of $3.5 \AA$ are found in a CHHC spectrum with $180 \mu$ s mixing time. This result confirms that valuable distance information can be obtained already from individual N/CHHC spectra and that the structure of Ubi-P must be closely related to the ubiquitin crystal structure.

Extending this analysis to all correlations with associated $\left({ }^{1} \mathrm{H},{ }^{1} \mathrm{H}\right)$ distances below 3.5 $\AA$ for which assignments are available shows that 186 of 196 (95\%) predicted correlations are found in the $180 \mu \mathrm{s}$ CHHC spectrum if only nonmethyl carbons are considered. However, only 177 (70\%) of 254 correlations involving at least one methyl group are represented in the spectrum (see Appendix Table B.2). This is in line with earlier results showing that, 
a

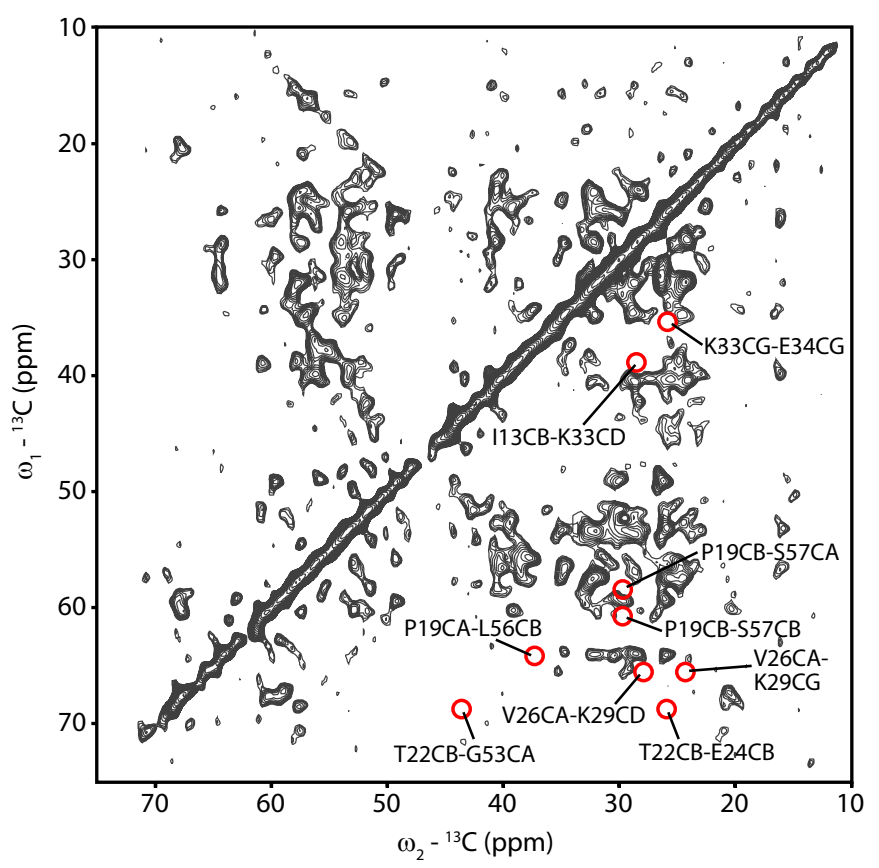

b

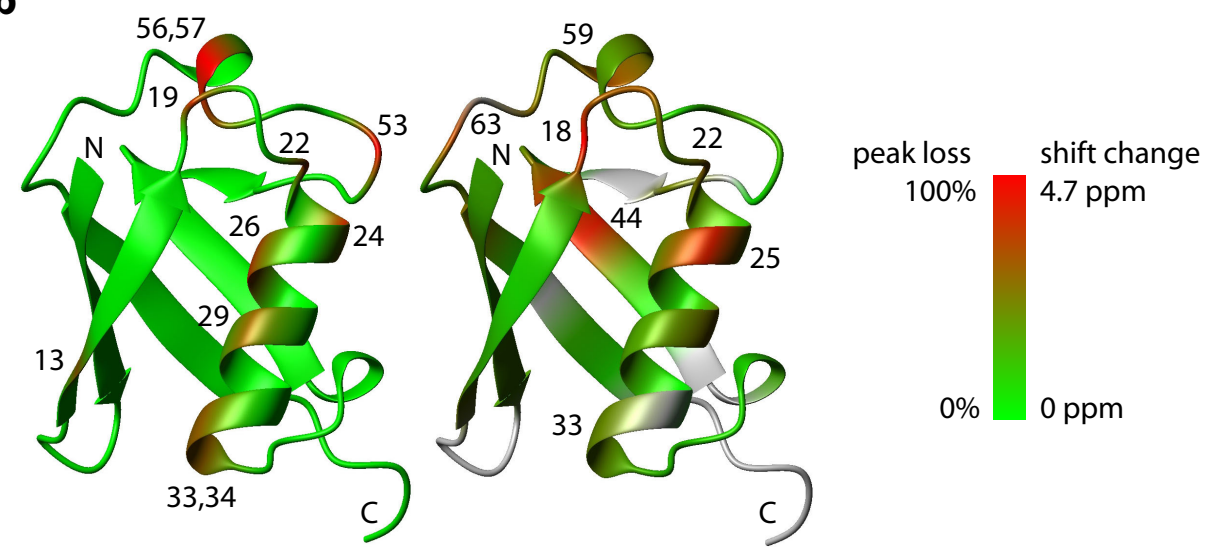

Figure 6.1: (a) CHHC spectrum of Ubi-P recorded at $14.1 \mathrm{~T}$ and $11 \mathrm{kHz}$ MAS with $180 \mu \mathrm{S}$ $\left({ }^{1} \mathrm{H},{ }^{1} \mathrm{H}\right)$ mixing time. Red circles indicate locations of absent interresidue correlations for nonmethyl carbons that were expected at this mixing time (i.e. associated $\left({ }^{1} \mathrm{H},{ }^{1} \mathrm{H}\right.$ ) distances in the crystal structure are $3.5 \AA$ or less). (b) Color code on the ubiquitin crystal structure (PDB ID 1UBQ) indicates the percentage of expected but absent non-methyl CHHC correlations per residue (left) and summed $\mathrm{C}^{\prime}, \mathrm{C}^{\alpha}$, and $\mathrm{C}^{\beta}$ chemical shift differences between Ubi-P and Ubi-M as described in ref. [70] (right). All residues with absent non-methyl $\mathrm{CHHC}$ correlations (left) and selected residues with large preparation-dependent chemical shift variations (right) are labeled, as well as the $\mathrm{N}$ - and $\mathrm{C}$-terminus of the molecule. 
due to the slower buildup of cross-polarization (CP) intensity in methyl groups exhibiting fast methyl rotation [68], methyl signals are less well represented in CHHC spectra with short CPs bracketing the $\left({ }^{1} \mathrm{H},{ }^{1} \mathrm{H}\right)$ mixing time $(80 \mu \mathrm{s}$ in this case) $[137,227]$. While methyl rotation also attenuates the $\left({ }^{1} \mathrm{H},{ }^{1} \mathrm{H}\right)$ coupling, increasing the $\mathrm{CP}$ time before and after $\left({ }^{1} \mathrm{H},{ }^{1} \mathrm{H}\right)$ mixing to, e.g., $125 \mu$ s already considerably increases the number of signals observed in the methyl region of the spectrum (data not shown).

Figure 6.1 a shows a CHHC spectrum recorded on Ubi-P with $180 \mu$ s mixing time and short $(80 \mu \mathrm{s})$ bracketing CPs. Red circles indicate locations of interresidue correlations between nonmethyl carbons expected at this mixing time (associated $\left({ }^{1} \mathrm{H},{ }^{1} \mathrm{H}\right)$ distance in the crystal structure below $3.5 \AA$ ), but absent from the spectrum on both sides of the diagonal. Mapping the residues involved onto the ubiquitin sequence (Fig. 6.1 b) reveals that they occur in the second $\beta$-sheet, the $\alpha$-helix, and in loop regions around residues 20 and 55. A comparison with an earlier analysis of residues exhibiting sizable preparationdependent chemical shift differences between Ubi-P and Ubi-M (Fig. 6.1 b, see ref. [70]) shows that overlapping regions of the protein are involved. Previously, it was suggested that such chemical shift changes between different solid-phase preparations are most likely to occur in protein regions exhibiting an enhanced degree of molecular mobility [70]. Thus, mobility may also be involved in the attenuation of signals in CHHC spectra.

\subsubsection{Residue-specific dynamics in ubiquitin}

To investigate a possible relation between absent CHHC correlations and molecular mobility in more detail, an analysis of dynamics in Ubi-P using $\left({ }^{13} \mathrm{C},{ }^{13} \mathrm{C}\right)$ double-quantum $(2 \mathrm{Q})$ ssNMR spectroscopy as developed in Chapter 5 was conducted. A set of dipolar $\left({ }^{1} \mathrm{H},{ }^{13} \mathrm{C}\right)$ order parameters had already been measured for Ubi-M [149], allowing for a comparison not only of chemical shift, but also of dynamical parameters between the two preparations.

In Figure 6.2, a $(2 \mathrm{Q}, 1 \mathrm{Q})$ correlation spectrum of Ubi-P recorded at $800 \mathrm{MHz}{ }^{1} \mathrm{H}$ resonance frequency with $250 \mu \mathrm{s}$ SPC5 mixing [121] is depicted. Already at this rather short mixing time, several ${ }^{13} \mathrm{C}$ resonances appear in the spectrum that exhibit considerable dynamics according to $\left({ }^{1} \mathrm{H},{ }^{13} \mathrm{C}\right)$ order parameters $\left(S_{\mathrm{HC}}\right)$ measured on Ubi-M [149]. Red circles mark selected pairs of nuclei for which at least one $S_{\mathrm{HC}}$ value is below 0.5 . Blue circles indicate resonances absent from the $S_{\mathrm{HC}}$ dataset in ref. [149], which has been suggested to be due to even larger mobility of these residues in Ubi-M. While, as discussed in Chapter 5 , the intensity of resonances in individual (2Q,1Q) spectra does not necessarily 
correlate with their mobility, these data suggest that different degrees of dynamics in individual residues of Ubi-P and Ubi-M may be present.

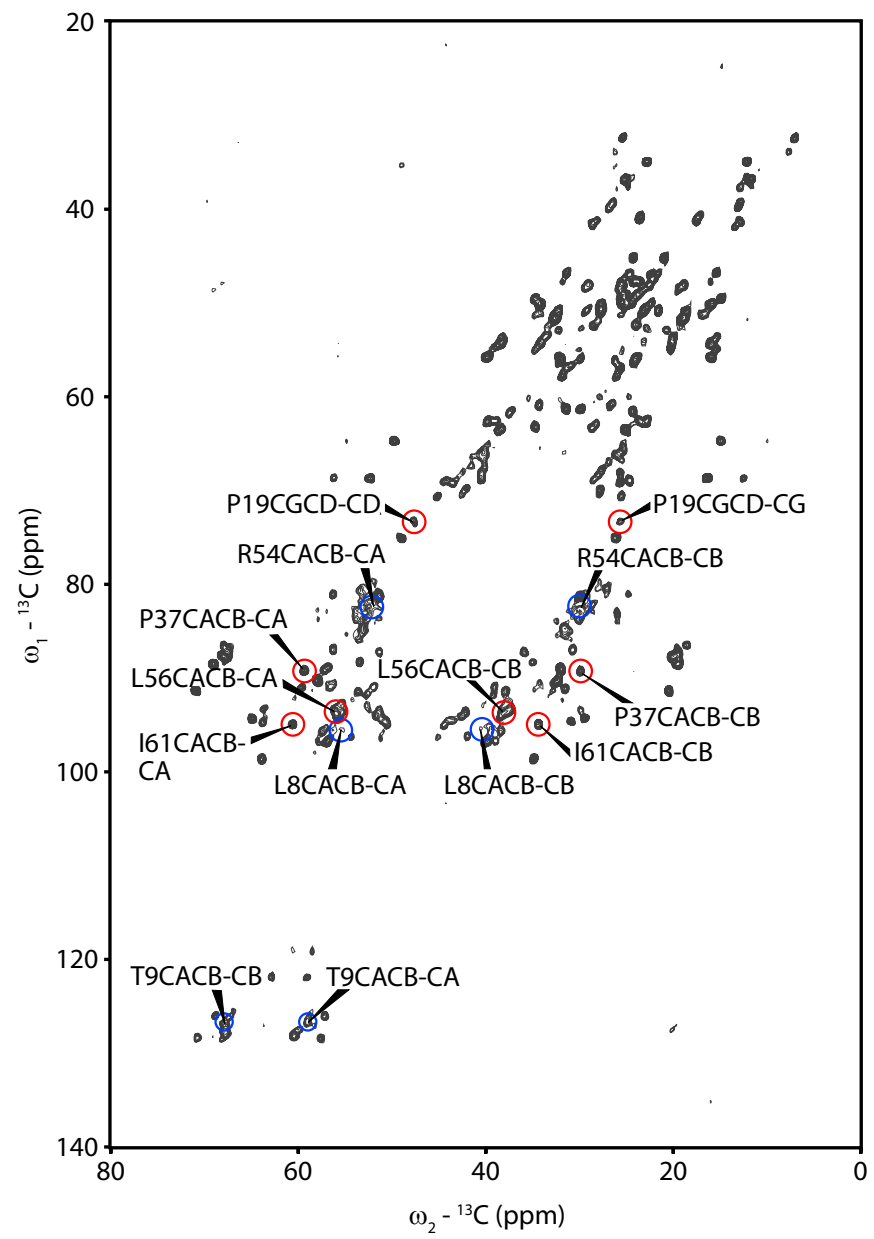

Figure 6.2: $(2 \mathrm{Q}, 1 \mathrm{Q})$ correlation spectrum of $\mathrm{Ubi}-\mathrm{P}$ recorded at $800 \mathrm{MHz}{ }^{1} \mathrm{H}$ resonance frequency with a $2 \mathrm{Q}$ excitation time of $250 \mu \mathrm{s}$. Labeled and circled in red are selected resonances clearly present already at this short mixing time, even though they exhibit low order parameters in Ubi-M [149]. Resonances circled in blue are absent from the Ubi-M dataset, possibly due to even larger degrees of motion, but present in this spectrum, despite lower signal intensity.

Thus, a comprehensive analysis of $2 \mathrm{Q}$ buildup data on Ubi-P was carried out. Experimental and best-fitting simulated intensity buildups for three resonance pairs also depicted in Figure 6.2 are shown in Figure 6.3. $2 \mathrm{Q}\left({ }^{13} \mathrm{C},{ }^{13} \mathrm{C}\right)$ order parameters $\left(S_{\mathrm{CC}}\right)$ for the $\left(\mathrm{C}^{\alpha}, \mathrm{C}^{\beta}\right)$ pairs in Thr9 and Pro37 indicate that these residues are indeed more rigid in Ubi-P than in Ubi-M. While Arg54 exhibits increased dynamics in Ubi-P compared to 
these residues, it is still observable in (2Q,1Q) spectra, also indicating a larger degree of rigidity compared to Ubi-M. For comparison, Figure 6.3 also shows buildup data and $S_{\mathrm{CC}}$ values for (overlapped) signals of lysine sidechain $\left(\mathrm{C}^{\delta}, \mathrm{C}^{\varepsilon}\right)$ pairs. In agreement with the expectation to find larger degrees of motion at the termini of long amino acid sidechains [149], these are the most dynamic sites observed in this dataset, illustrating the dispersion of $S_{\mathrm{CC}}$ order parameters in Ubi-P. Notably, similar buildup time courses can lead to differing order parameters for different spin systems (compare, e.g., Thr9 and Pro37 in Fig. 6.3) due to chemical shift offset effects. The $\mathrm{C}^{\beta}$ chemical shift is much closer to the r.f. carrier frequency for Pro than for Thr; thus, a faster $2 \mathrm{Q}$ buildup for the same $S_{\mathrm{CC}}$ value can be expected for the Pro $\left(\mathrm{C}^{\alpha}, \mathrm{C}^{\beta}\right)$ spin pair.
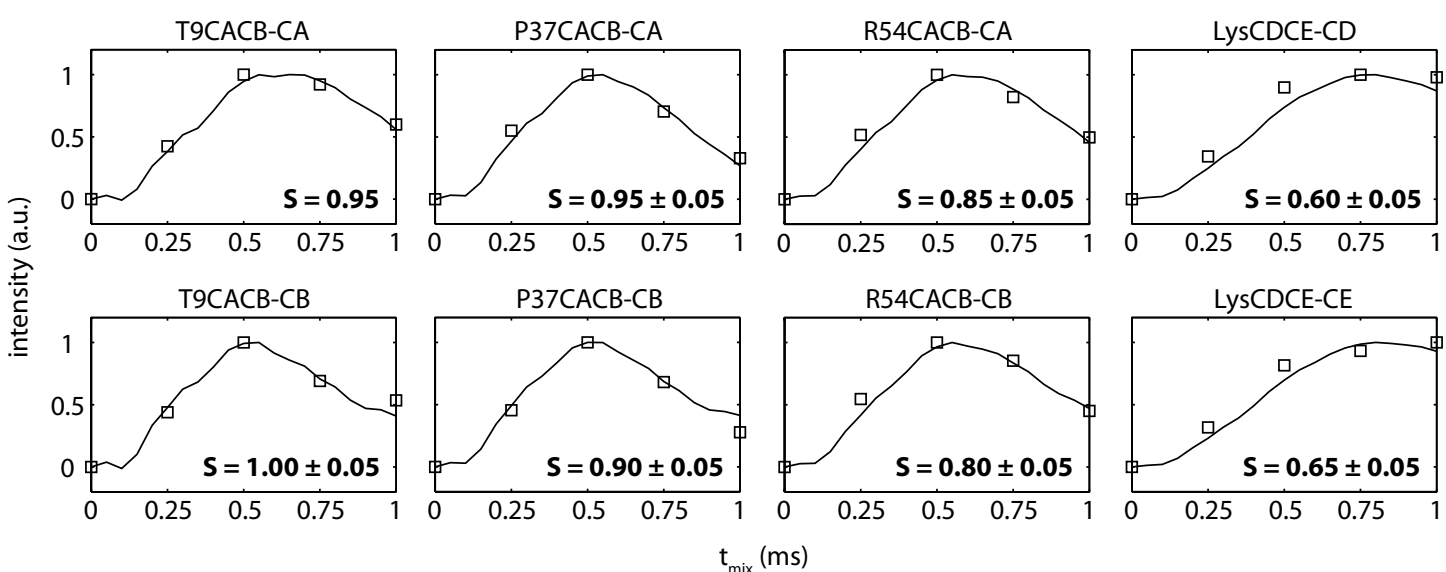

Figure 6.3: Experimental $2 \mathrm{Q}$ crosspeak buildups (squares) and best-fitting simulations (lines) for the $\left(\mathrm{C}^{\alpha}, \mathrm{C}^{\beta}\right)$ nuclei pairs in Thr9, Pro37 and Arg54. Also shown are data and fits for overlapped signals of lysine $\left(\mathrm{C}^{\delta}, \mathrm{C}^{\varepsilon}\right)$ nuclei pairs to illustrate the largest degrees of motion observed in the data. $S_{\mathrm{CC}}$ order parameters of best-fitting simulations are given with error values. For the Thr9 CACB-CA correlation, the error is smaller than 0.05 . Error bars of experimental data, calculated based on spectral noise, are smaller than the data points.

The complete set of $S_{\mathrm{CC}}$ order parameters for $\left(\mathrm{C}^{\alpha}, \mathrm{C}^{\beta}\right)$ pairs obtained on Ubi-P is shown in Figure 6.4 a and Appendix Table B.3. $\left(\mathrm{C}^{\alpha}, \mathrm{C}^{\beta}\right) S_{\mathrm{CC}}$ order parameters could be determined for 40 residues, i.e. $53 \%$ of the ubiquitin sequence. In the most mobile $20 \%$ of these residues, the $\left(\mathrm{C}^{\alpha}, \mathrm{C}^{\beta}\right) S_{\mathrm{CC}}$ value is below 0.85 (indicated by a solid line in Figure 6.4 a). These residues occur at the N-terminus, in second and third $\beta$-strand, and in loop regions. This shows that, also in the PEG-precipitated state, parts of the ubiquitin 
sequence exhibit considerable dynamics on the ms or faster time scales, comparable to the ester tail in L-tyrosine-ethylester (TEE, see Chapter 5).

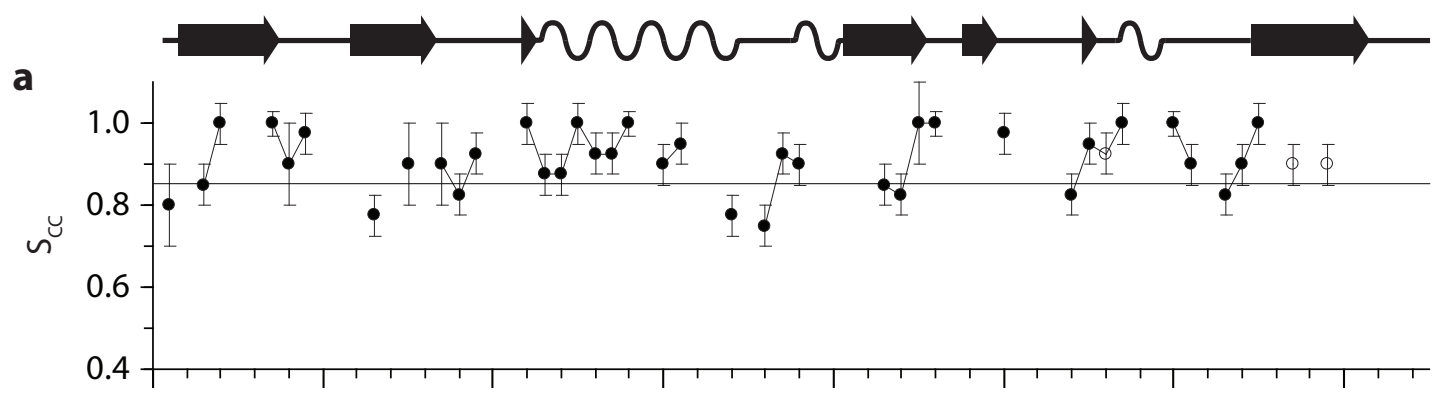

b
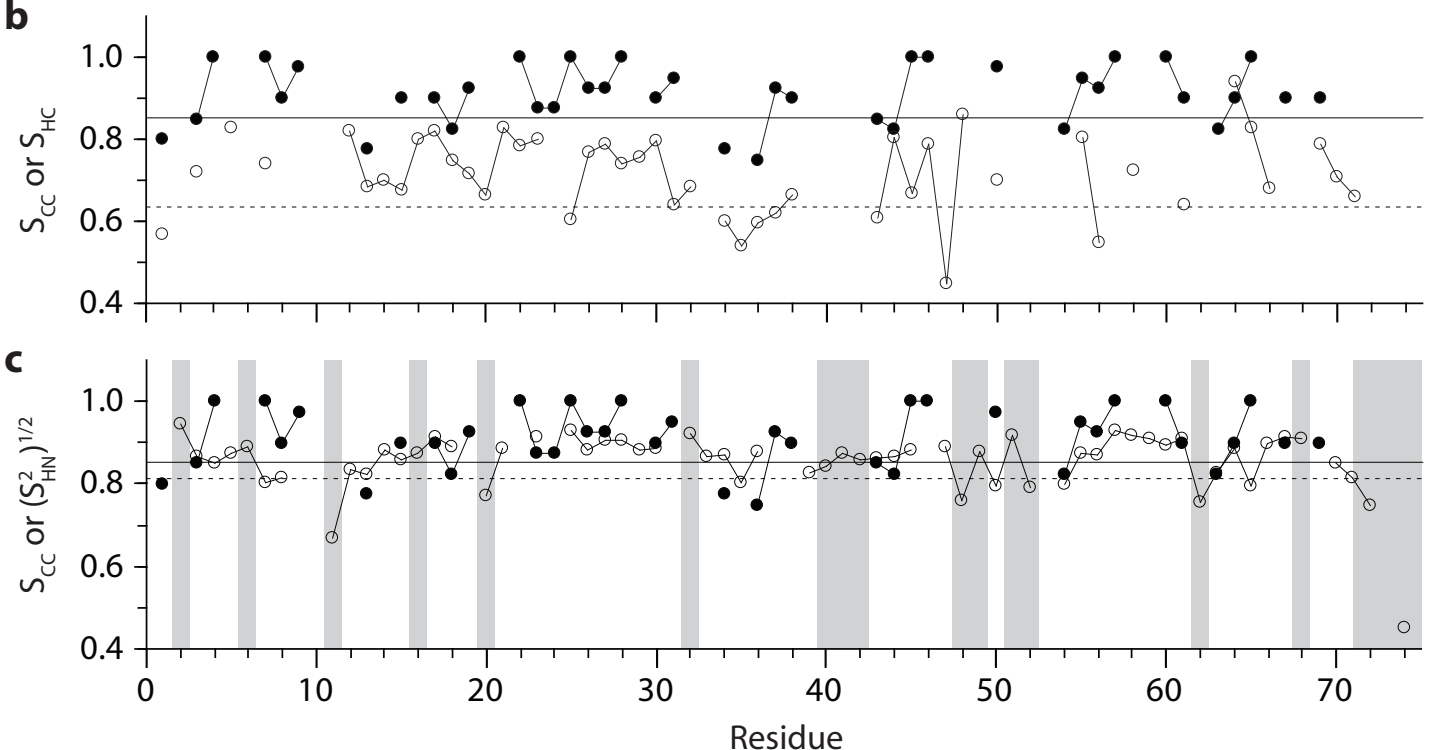

Figure 6.4: (a) Plot of Ubi-P $\left(\mathrm{C}^{\alpha}, \mathrm{C}^{\beta}\right) S_{\mathrm{CC}}$ order parameters. Open symbols indicate values obtained from signals overlapping with close sequence neighbors. Solid line indicates an $S_{\mathrm{CC}}$ value of 0.85 below which the most mobile $20 \%$ of determined $S_{\mathrm{CC}}$ values are found. Secondary structure of ubiquitin as found in the crystal structure (PDB 1UBQ) is sketched at the top. (b) Comparison of Ubi-P $\left(\mathrm{C}^{\alpha}, \mathrm{C}^{\beta}\right) S_{\mathrm{CC}}$ (filled symbols) and Ubi-M $S_{\mathrm{HC}}$ order parameters [149] (open symbols). $S_{\mathrm{HC}}$ values plotted are averaged over $\mathrm{C}^{\alpha}$ and $\mathrm{C}^{\beta}$ nuclei if both values are available. Solid line as in (a); dotted line denotes the upper limit $\left(S_{\mathrm{HC}}=0.63\right)$ for the most mobile $20 \%$ of residues detected in Ubi-M. (c) Comparison of Ubi-P (C $\left.\mathrm{C}^{\alpha}, \mathrm{C}^{\beta}\right) S_{\mathrm{CC}}$ (filled symbols) and RDCbased backbone $\left(S_{\text {rdc }}^{2}\right)^{1 / 2}$ order parameters from solution-state NMR [226] (open symbols). Solid line as in (a); dotted line $\left(\left(S_{\text {rdc }}^{2}\right)^{1 / 2}=0.81\right)$ corresponds to the upper limit for the most mobile $20 \%$ of residues measured in ref. [226]. Grey bars indicate residues for which $S_{\mathrm{CC}}$ order parameters could not be determined due to weak signal intensity in $(2 \mathrm{Q}, 1 \mathrm{Q})$ spectra (Ser20) or due to missing assignments (all other shaded residues). 
Figure 6.4 b compares Ubi-P $S_{\mathrm{CC}}$ order parameters with $S_{\mathrm{HC}}$ values measured on UbiM [149]. Notably, the same effective sample temperatures was used here as in ref. [149]. Both $S_{\mathrm{CC}}$ and $S_{\mathrm{HC}}$ values are averaged over $\mathrm{C}^{\alpha}$ and $\mathrm{C}^{\beta}$ nuclei to facilitate comparison. The difference between average $S_{\mathrm{HC}}$ and $S_{\mathrm{CC}}$ order parameters thus obtained (0.91 and 0.72 , respectively) is 0.19 , and the difference between the thresholds for the most mobile $20 \%$ of residues in each dataset ( 0.85 and 0.63 for $S_{\mathrm{CC}}$ and $S_{\mathrm{HC}}$, respectively) is 0.22 . This is well in line with results obtained on TEE (see Chapter 5), where the difference between $S_{\mathrm{HC}}$ and $S_{\mathrm{CC}}$ values for sites which are not fully rigid, i.e. the ester tail, amounts to 0.18.

Apart from the overall offset, clear local differences in relative mobility between Ubi-P and Ubi-M datasets are apparent. Especially residues in the loop between the first two $\beta$ sheets (Leu8, Thr9), in the $\alpha$-helix (Asn25), and near the 3-10 helix (Leu56) are more rigid in Ubi-P. Notably, loop residues Leu8 and Thr9 are absent from the Ubi-M dataset, which has been suggested to be caused by considerable local dynamics in these residues [149]. Interestingly, residues near the short 3-10 helix (consisting of residues Ser57 - Tyr59), where several absent CHHC correlations and larger preparation-dependent chemical shift changes are found (Fig. $6.1 \mathrm{~b}$ ), are nearly rigid in Ubi-P as seen by their $S_{\mathrm{CC}}$ values. Conversely, e.g. residues Ile13, Glu18, and Ile44 exhibit larger relative mobility in Ubi-P than in Ubi-M. Different patterns of $S_{\mathrm{CC}}$ and $S_{\mathrm{HC}}$ order parameters in consecutive residues are also apparent around residues 44, 56, and 64.On the other hand, some common regions of elevated dynamics can be found, most notably at the N-terminus of the molecule and at the end of the $\alpha$-helix (residues Glu34 - Ile36). Also, as discussed above, while residue Arg54 is rigid enough in Ubi-P to be detected by $(2 \mathrm{Q}, 1 \mathrm{Q})$ correlation spectroscopy, it belongs to the most mobile $20 \%$ of residues in the Ubi-P dataset, consistent with the hypothesis that it is dynamic in Ubi-M [149].

Residual dipolar couplings (RDCs) measured in alignment media in solution-state NMR probe similar time scales of dynamics as the $S_{\mathrm{CC}}$ and $S_{\mathrm{HC}}$ order parameters discussed so far $[186,187]$. While experimental and analytical methods are different, as well as the nuclei probed (backbone amide proton and nitrogen), a recent set of RDC-based $\left({ }^{1} \mathrm{H},{ }^{15} \mathrm{~N}\right)$ order parameters for solubilized ubiquitin (further denoted as $S_{\mathrm{rdc}}^{2}$ ) [209, 226] can be compared with data presented here in terms of the distribution of more mobile and more rigid residues along the sequence. Figure $6.4 \mathrm{c}$ plots Ubi-P $S_{\mathrm{CC}}$ values together with $S_{\text {rdc }}^{2}$ data. Square roots of $S_{\text {rdc }}^{2}$ values are shown for better comparison. Relative to the respective average values, mobility in the $\mathrm{N}$-terminal region up to the $\alpha$-helix appears more similar between Ubi-M and ubiquitin in solution, with elevated mobility found in 
residues of the first loop. Ser20, however, is more dynamic in solution (see below). On the other hand, as in Ubi-P, residues in the $\alpha$-helix (Ile23 - Lys33) as well as in the region of the 3-10 helix (Thr55 - Ile61) do not seem to exhibit large degrees of dynamics in solution, while some highly dynamic residues are found in these regions in Ubi-M. Notably, a quite similar pattern of $S_{\mathrm{rdc}}^{2}$ and $S_{\mathrm{CC}}$ order parameters is observed in the region between Arg54 and Leu67. Lys48 shows elevated mobility in solution, while its $S_{\mathrm{HC}}$ order parameter is one of the largest in the entire Ubi-M dataset. However, a "hotspot" of increased dynamics at or around Gly35 at the end of the $\alpha$-helix appears conserved in all preparations.

Another interesting observation in solution-state $S_{\text {rdc }}^{2}$ data is that, for several residues that could not be assigned in Ubi-P (Lys11, Lys48, Asp52, Gln62) or for which no $S_{\mathrm{CC}}$ order parameter could be determined due to lack of intensity in $(2 \mathrm{Q}, 1 \mathrm{Q})$ correlation spectra (Ser20; indicated by grey bars in Fig. 6.4 c), very low $S_{\text {rdc }}^{2}$ order parameters are found. While resonance overlap may be involved in missing assignments, this suggests that, in some of these residues, high levels of mobility may still be present after PEG precipitation.

Determination of $S_{\mathrm{CC}}$ order parameters was more difficult in sidechain regions due to more extensive resonance overlap and partly weaker signals, leading to less optimal fits. 23 order parameters were nevertheless obtained for $\left(\mathrm{C}^{\beta}, \mathrm{C}^{\gamma}\right),\left(\mathrm{C}^{\beta}, \mathrm{C}^{\gamma 1}\right)$, and $\left(\mathrm{C}^{\beta}, \mathrm{C}^{\gamma 2}\right)$ resonance pairs (see Appendix Fig. B.1 and Appendix Table B.3). In agreement with the expectation to find more mobility in sites more distal from the protein backbone, the average $S_{\mathrm{CC}}$ value is lower for $\left(\mathrm{C}^{\beta}, \mathrm{C}^{\gamma}\right)$ than for $\left(\mathrm{C}^{\alpha}, \mathrm{C}^{\beta}\right)$ (0.84 versus 0.91 , respectively). The offset from the average Ubi-M $S_{\mathrm{HC}}$ value for these sites $(0.62)$ is 0.22 and thus comparable to results for $\mathrm{C}^{\alpha}$ and $\mathrm{C}^{\beta}$ nuclei. Interestingly, a better correlation of $S_{\mathrm{CC}}$ and $S_{\mathrm{HC}}$ order parameters for $\left(\mathrm{C}^{\beta}, \mathrm{C}^{\gamma}\right)$ resonance pairs is found than for $\left(\mathrm{C}^{\alpha}, \mathrm{C}^{\beta}\right)$ (correlation coefficients 0.65 versus 0.27 , respectively).

\subsubsection{Correlation of mobility with shift changes and peak loss}

With order parameters for a majority of Ubi-P residues at hand, it became possible to investigate the involvement of mobility in absent CHHC correlations and preparationdependent chemical shift changes discussed in Section 6.4.1. Figure 6.5 a plots the percentage of expected CHHC correlations per residue (corresponding to $\left({ }^{1} \mathrm{H},{ }^{1} \mathrm{H}\right)$ distances up to $3.5 \AA$ in the crystal structure, excluding methyl groups) which are absent from the CHHC spectrum with $180 \mu$ s mixing time. In several of these residues, elevated levels of backbone or sidechain mobility are found (denoted by red bars, see also Appendix Table B.2). For Gly53, involved in an absent CHHC correlation with Thr22, no $\left(\mathrm{C}^{\alpha}, \mathrm{C}^{\beta}\right) S_{\mathrm{CC}}$ 

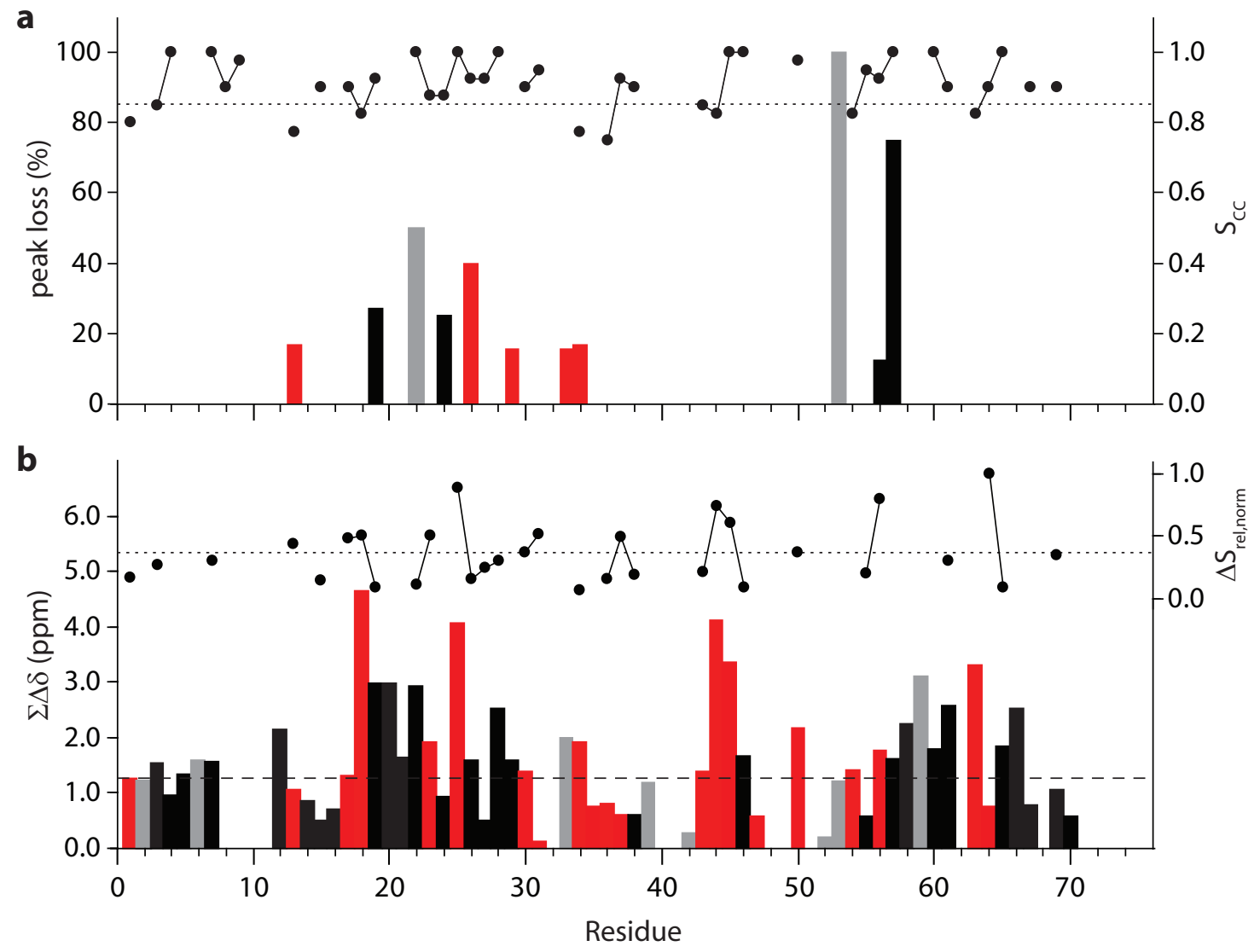

Figure 6.5: (a) Per-residue percentage of expected correlations $\left(\left({ }^{1} \mathrm{H},{ }^{1} \mathrm{H}\right)\right.$ distances up to $3.5 \AA$, only non-methyl ${ }^{13} \mathrm{C}$ nuclei) which are absent from the Ubi-P CHHC spectrum with $180 \mu$ s mixing time. Red bars denote residues where backbone or sidechain nuclei with elevated dynamics are involved in absent $\mathrm{CHHC}$ correlations. Grey bars denote absent correlations involving residue Gly53 for which no $\left(\mathrm{C}^{\alpha}, \mathrm{C}^{\beta}\right) S_{\mathrm{CC}}$ order parameter can be determined. Other absent correlations are shown as black bars. $\left(\mathrm{C}^{\alpha}, \mathrm{C}^{\beta}\right) S_{\mathrm{CC}}$ order parameters are given as a reference (filled circles, right axis), along with a dotted line indicating $S_{\mathrm{CC}}=0.85$. (b) Sum of C', $\mathrm{C}^{\alpha}$, and $\mathrm{C}^{\beta}$ chemical shift differences between Ubi-P and Ubi-M as determined in ref. [70]. Red bars indicate residues with $\left(\mathrm{C}^{\alpha}, \mathrm{C}^{\beta}\right) S_{\mathrm{CC}}$ or $S_{\mathrm{HC}}$ order parameters below 0.85 or 0.63 , respectively, or with an order parameter difference $\Delta S_{\text {rel,norm }}$ at or above the average (Section 6.3.4). Grey bars denote residues for which neither $S_{\mathrm{CC}}$ nor $S_{\mathrm{HC}}\left(\mathrm{C}^{\alpha}, \mathrm{C}^{\beta}\right)$ order parameters could be determined. Available data for residues with black bars do not indicate elevated mobility or large mobility differences. Dashed line indicates the average Ubi-P - Ubi-M summed chemical shift difference of $1.25 \mathrm{ppm} . \Delta S_{\text {rel,norm }}$ values (filled circles, right axis) and their average of 0.36 (dotted line) are given as a reference. 
order parameter can be determined; mobility in this region may nevertheless play a role in the absence of this crosspeak, considering the lack of assignments for residues Glu51 Asp52 from dipolar spectra and the low $S_{\mathrm{CC}}$ order parameter of residue Arg54.

Figure 6.5 b shows sums of $\mathrm{C}^{\prime}, \mathrm{C}^{\alpha}$, and $\mathrm{C}^{\beta}$ chemical shift differences between Ubi-P and Ubi-M as determined in ref. [70], together with a measure of relative difference of $S_{\mathrm{CC}}$ and $S_{\mathrm{HC}}$ order parameters independent of their overall offset $\left(\Delta S_{\text {rel,norm }}\right.$, see Section 6.3.4). While obviously no 1:1 correlation is present, especially the largest chemical shift changes clearly occur in the most mobile residues according to $S_{\mathrm{CC}}$ or $S_{\mathrm{HC}}$ order parameters, or in residues for which the largest relative mobility differences between the two datasets are detected, such as Asn25 or Phe45 (red bars in Fig. 6.5 b). Mobility may also be involved in other residues with larger shift changes for which $S_{\mathrm{CC}}, S_{\mathrm{HC}}$, or both order parameters could not be determined, such as Ser20 (see above).

\subsubsection{Structure calculation of Ubi-P}

In several residues, preparation-dependent chemical shift changes do not seem to be related to local mobility or mobility differences between Ubi-P and Ubi-M, most notably in residues Pro19 - Thr22 and Asp58 - Ile61 (with the possible exception of Ser20). To investigate whether structural changes play a role instead, a structure calculation of Ubi-P based on the CHHC spectra discussed in Section 6.4.1 was carried out. Four spectra with different $\left({ }^{1} \mathrm{H},{ }^{1} \mathrm{H}\right)$ mixing times up to $250 \mu$ s were used as input for the automated assignment and structure calculation algorithm PASD implemented in Xplor-NIH $[173,174,184]$. Similar to the approach described in ref. [5], the ubiquitin crystal structure (1UBQ) was used as a search model for CHHC crosspeak assignment. Due to the use of several spectra and looser distance bounds for crosspeak assignment, some restraints were also found that were classified as absent in the analysis in Section 6.4.1. Restraints obtained in this manner were used for a final simulated annealing step in CNS [175]. The resultant 10 lowest energy structures (Fig. 6.6 a) are well converged, with residues 1-70 exhibiting a backbone root-mean-square deviation (RMSD) to the mean of $0.49 \AA$ and of $1.79 \AA$ to the ubiquitin crystal structure (Table B.4).

While the structural ensemble is thus well defined, small but distinct changes are seen with respect to the crystal structure (Fig. 6.6 b and Appendix Fig. B.2). Deviations in residues for which no assignments and thus no restraints are available cannot be interpreted in terms of structural differences of Ubi-P compared to the crystal structure. However, other regions for which restraints are available and that are rigid as judged by $S_{\mathrm{CC}}$ order 
parameters indeed appear to assume slightly different conformations in Ubi-P than in single crystals. This is the case for the first loop around residue Leu8 and the 3-10 helix around Asp58. Thus, the preparation-dependent chemical shift changes and absent CHHC correlations occurring in the latter region appear to be related to a local conformational change.
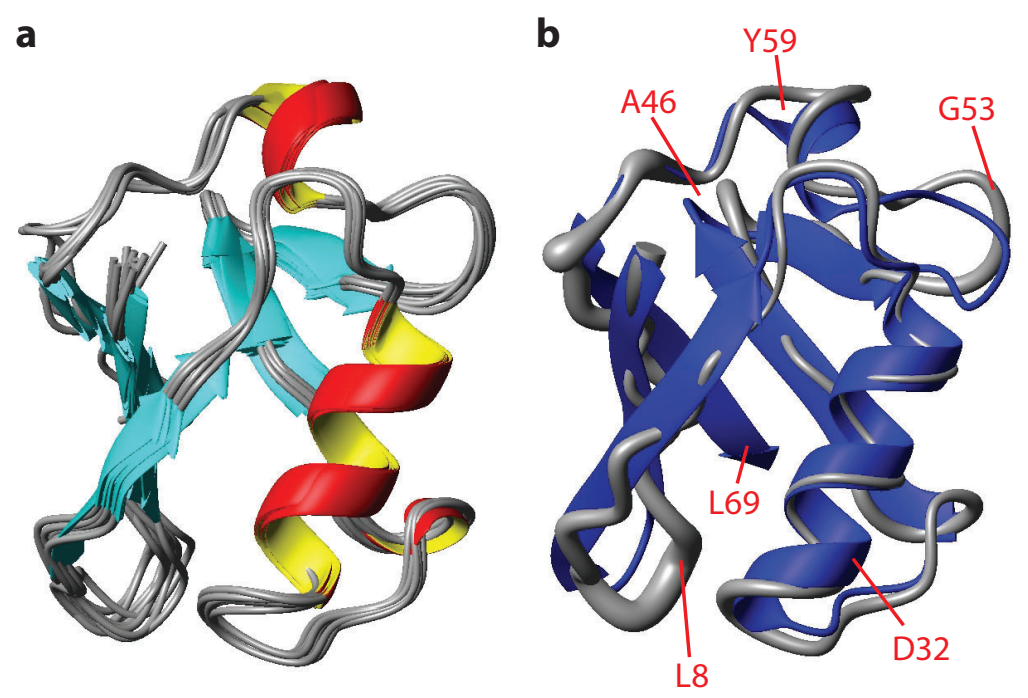

Figure 6.6: (a) Ensemble of 10 structures with lowest overall energy calculated from $4 \mathrm{CHHC}$ spectra of Ubi-P, using PASD [184] for crosspeak assignment and simulated annealing in CNS [175]. Structures were aligned along the backbone atoms of residues 1-70 using MOLMOL [224]. Only these residues are shown. (b) Sausage plot of residues 1-70 of the ensemble shown in (a) (grey) aligned with the ubiquitin crystal structure (PDB 1UBQ, blue). Residues in regions with largest local RMSD deviations between the two structures are labeled in red.

While analysis of structure and dynamics around residue Leu50 is difficult due to lack of assignments, available evidence suggests that both structural alterations and dynamic processes may play a role here. Flanking residues for which order parameters and / or distance restraints could be obtained exhibit increased dynamics (Arg54) and structural alterations (Ala46, Gly53, Arg54). Elevated dynamics are found in this region also in other ubiquitin preparations $[149,226]$. Moreover, the largest deviation of a backbone dihedral angle in Ubi-P with respect to the crystal structure is found in residue Arg54 [70], suggesting that the deviation of the calculated Ubi-P structural ensemble from the crystal structure around residue Gly53 indeed reflects a structural alteration of ubiquitin in PEG microcrystals. Conformational changes and increased dynamics in this region 
likely also affect residues Pro19 - Thr22 in the loop between second $\beta$-strand and $\alpha$-helix, which is close in space and partly hydrogen-bonded to residues Arg54 - Tyr59 in the ubiquitin crystal structure [228]. This likely explains the preparation-dependent chemical shift changes and absent CHHC correlations occurring in residues Pro19 - Thr22.

\subsection{Discussion}

In this chapter, it was shown that $2 \mathrm{Q}\left({ }^{13} \mathrm{C},{ }^{13} \mathrm{C}\right)$ ssNMR spectroscopy represents a viable approach to detect residue-specific dynamics on time scales up to milliseconds in larger biomolecules. As in solution and in MPD microcrystals, ubiquitin precipitated from PEG evidently exhibits sizable mobility on these time scales. Common regions of elevated dynamics can be found in all preparations, while the exact distribution of mobile and rigid residues appears to be distinctly preparation-dependent. In particular, several large preparation-dependent chemical shift differences as well as absent through-space correlations that would be expected based on the crystal structure occur in residues exhibiting increased molecular mobility or considerable differences in mobility between solid-phase preparations. Structure calculation indicates that in other residues, usually in exposed loop regions, shift changes and absent $\mathrm{CHHC}$ correlations are related to local structural alterations, while the overall ubiquitin structure is very well preserved. Data presented here thus confirm earlier hypotheses about the involvement of molecular mobility in the occurrence of chemical shift differences between different protein preparations [70]. However, as apparent from Figure $6.5 \mathrm{~b}$, mobility does not necessarily entail that a residue will exhibit large preparation-dependent chemical shift changes. Also, Figure 6.5 a shows that not in all of the more mobile residues, absent CHHC correlations are detected. This, however, may partly be due to the limits of the approach of identifying absent CHHC correlations. Especially in crowded spectral regions, absent through-space correlations may go unnoticed if they overlap with other, still present correlations. The above argumentation is only based on isolated, clearly identifiable absent crosspeaks (Fig. 6.1 a) and can thus be considered a conservative estimate.

Notably, $S_{\mathrm{CC}}$ order parameters determined here do not cover all motional time scales, and other dynamic processes may be present in microcrystalline ubiquitin. For example, for several residues in ubiquitin in solution, slow exchange processes on a $\mu$ s to ms time scale have been reported, most frequently for residues Ile23 - Asn25 and Glu51 - Gly53 [218, 228, 229], but also residues Thr9, Glu18, Ile30, Lys33, Ile36, Leu43, Phe45, Thr55 
- Asp58 and Val70 have been implicated in slow dynamic processes [228]. However, for many of these residues, available $S_{\mathrm{CC}}$ order parameters do not point to elevated mobility, with the exception of Glu18, Ile36, Leu43 and possibly Glu51 - Gly53. Slow exchange in the other residues, if present in Ubi-P, thus appears to occur on time scales slower than detectable by $2 \mathrm{Q}\left({ }^{13} \mathrm{C},{ }^{13} \mathrm{C}\right)$ spectroscopy or affect only a small proportion of these residues during a given time. The latter hypothesis is supported by solution-state NMR relaxation experiments indicating that, for several of these residues, the minor conformational state of the slow exchange process is populated only to an extent of about $2 \%$ [228], which is likely beyond the sensitivity limit of the experiments described here. These data suggest that some preparation-dependent shift changes may be related to dynamic processes not detected by $2 \mathrm{Q}$ spectroscopy. On the other hand, some interesting parallels between solidstate $S_{\mathrm{CC}}$ and solution-state $S_{\text {rdc }}^{2}$ values were found. For example, in both datasets, the backbone of the $\alpha$-helix and the 3-10 helix around residue Ser57 appear rather rigid, while several residues with low $S_{\text {rdc }}^{2}$ order parameters could not be assigned in the solid state or lack intensity in $(2 \mathrm{Q}, 1 \mathrm{Q})$ spectra. This suggests that, in these latter residues, large motional amplitudes may be preserved in PEG microcrystals.

Considering residues $1-70$, average $\left(\mathrm{C}^{\alpha}, \mathrm{C}^{\beta}\right) S_{\mathrm{CC}}$ and solution-state $\left(S_{\mathrm{rdc}}^{2}\right)^{1 / 2}$ order parameters of 0.91 and 0.86 , respectively, appear to indicate comparable overall backbone dynamics. The average Ubi-M $S_{\mathrm{HC}}$ value in these residues, averaged over $\mathrm{C}^{\alpha}$ and $\mathrm{C}^{\beta}$ nuclei, is 0.72 and thus considerably lower. As mentioned, the offset of average $S_{\mathrm{HC}}$ and $S_{\mathrm{CC}}$ values in ubiquitin is similar as found for the ester tail in TEE (Chapter 5). There, the lower $S_{\mathrm{HC}}$ value may be explained by rotation around the $\mathrm{C}-\mathrm{C}$ bond, which should attenuate the $\left({ }^{1} \mathrm{H},{ }^{13} \mathrm{C}\right)$ couplings, while leaving the $\left({ }^{13} \mathrm{C},{ }^{13} \mathrm{C}\right)$ coupling unaffected. The intepretation of the overall offset between average $\left(\mathrm{C}^{\alpha}, \mathrm{C}^{\beta}\right) S_{\mathrm{CC}}$ and $S_{\mathrm{HC}}$ order parameters, which is smaller but still significant even if only $\mathrm{C}^{\alpha} S_{\mathrm{HC}}$ values are considered (0.78 vs. 0.91, $p<0.001$, one-sided $t$-test), is less straightforward in the context of amino acid residues in a protein. One possible motional model to explain this difference would be a nutation of the $\mathrm{H}^{\alpha}-\mathrm{C}^{\alpha}$ bond vector around its main direction, i.e. "diffusion in a cone" similar as postulated for the TEE ester tail $[69,185] . \quad\left(\mathrm{C}^{\alpha}, \mathrm{C}^{\beta}\right)$ order parameters larger than corresponding $\left(\mathrm{H}^{\alpha}, \mathrm{C}^{\alpha}\right)$ values would then indicate that such motion is less pronounced in the $\mathrm{C}^{\alpha}-\mathrm{C}^{\beta}$ bond, e.g. due to steric restraints imposed by the other atoms bound to the $\mathrm{C}^{\beta}$. On the other hand, overall backbone dynamics may simply be higher in Ubi-M than in Ubi-P. However, apart from the nuclei involved, also the recoupling sequences and associated effective dipolar coupling elements used for determination of $S_{\mathrm{CC}}$ and 
$S_{\mathrm{HC}}$ values are different. Absolute $S_{\mathrm{CC}}$ and $S_{\mathrm{HC}}$ order parameter values should thus be compared with caution. This is of course also the case for comparisons with solution-state data. This chapter consequently focussed on relative mobility differences in individual protein regions, with respect to the average order parameters found in each dataset.

Recent studies have suggested that motions on time scales detected by measurements of dipolar couplings and exchange effects in solution occur in ubiquitin residues involved in protein-protein interactions [209, 228, 229]. This correlation is most pronounced for the exposed loop in residues Thr7 - Lys11 and the ubiquitin "interaction surface" between residues Gln40 and Leu50, although not for all residues a 1:1 correlation is seen. In particular, some residues crucial for protein-protein interactions are rather rigid in solution (Ile44, His68) [209, 230]. As far as assignments and $S_{\mathrm{CC}}$ values are available, the first loop is quite rigid in Ubi-P, however; on the other hand, enhanced dynamics can be detected in Ubi-P residues between Gln40 and Leu50 (Leu43, Ile44) or may be present in others that could not be assigned or analyzed in $(2 \mathrm{Q}, 1 \mathrm{Q})$ correlation spectra. Notably, key residues known to be involved in interactions with ubiquitin-binding domains (Ile44), E1 enzymes (Ile44, Arg54), and in polyubiquitination (Lys63) appear among the most dynamic 20\% of residues for which $S_{\mathrm{CC}}$ values could be obtained. This agrees with recent findings that free ubiquitin dynamically samples the conformations it assumes in complex with different interacting proteins, i.e. that conformational selection appears to govern interactions of ubiquitin with other proteins [209].

In solution, a correlation of local mobility in ubiquitin with exposure of amino acid sidechains to the solvent has been observed [231]. However, in Ubi-P, no correlation of $S_{\mathrm{CC}}$ order parameters and fractional accessible surface area of individual residues could be found (see Appendix Fig. B.3). This may reflect the fact that in microcrystals, in contrast to the solution state, the surface of the protein faces not only solvent, but also precipitant and neighboring molecules. Such contacts appear to be important for protein structure and dynamics in microcrystals. Similar to protein-protein interactions in ubiquitin complexes, intermolecular crystal contacts in the ubiquitin crystal structure also tend to cluster in regions around residues Thr9 and Lys48, although the region between residues 40 and 50 appears to be a less pronounced interaction surface than in complex structures $[209,221]$ (see Appendix Fig. B.4). Additionally, large numbers of crystal contacts are seen for residues Pro19, Ser20, Gly35, Arg54, Lys63, and the C-terminus. In or near all these residues, elevated dynamics and / or chemical shift changes and absent CHHC correlations 
are detected in Ubi-P, strongly suggesting effects of crystal packing and intermolecular contacts on protein dynamics and conformation also in PEG microcrystals.

The structure calculation approach used in this chapter employed the automated assignment and structure calculation algorithm PASD. This algorithm attempts to overcome the problem of crosspeak assignment in through-space correlation spectra (Section 4.4.3) by initially using all possible assignments as restraints for simulated annealing. The agreement of any given restraint with the emerging structural ensemble is analyzed repeatedly during the calculation, leading to its probabilistic inactivation (or reactivation). This approach relies on the basic assumption that the set of correct restraints is self-consistent and drives the structure calculation in a common direction [184], ideally leading to iterative assignment of through-space spectra and a large enough set of restraints to calculate a well converged structural ensemble. However, automated de novo structure calculation from solid-state NMR data on uniformly $\left[{ }^{13} \mathrm{C},{ }^{15} \mathrm{~N}\right]$-labeled molecules is currently still challenging, largely due to the high degrees of assignment ambiguities in through-space correlation spectra with typical ssNMR linewidths. If convergence cannot be obtained, a reference structure may be used to assign through-space correlations and collect restraints for a structure calculation if it can be assumed that the molecule under study has a similar fold as the reference structure. This approach was used for the ubiquitin structure calculation described in this chapter.

While useful for the detection of smaller structural alterations, using a reference structure does not yield a de novo structure. Success of automated de novo structure calculations will likely depend on several factors, such as the availability of enough non-redundant through-space data and the ability of the algorithm used to handle highly ambiguous data. In contrast to the solution state, where it is usually attempted to find one unambiguous assignment for a crosspeak [184], more assignment possibilities may have to be kept for solid-state data, reflecting the higher probability that a particular crosspeak may actually arise from more than one correlation. Thus, a probabilistic algorithm like PASD represents a promising approach, since it never discards a possible assignment completely [184]. First successes of automated ssNMR-based de novo structure calculations have already been reported $[52,53]$. Certainly, more advances in this field can be expected in the near future.

The approach outlined in this chapter should be useful for other studies of proteins by ssNMR as well, in particular for systems such as membrane proteins and protein fibrils which naturally occur in a non-solubilized state. In these systems, ssNMR can study 
structure and dynamics in their native state, such that additional influences from sample preparation as seen in this study are minimized. SsNMR investigations on a membrane protein and fibrillar peptide aggregates will be the focus of the remaining chapters of this thesis. 


\section{Part III}

The potassium channel KcsA-Kv1.3 



\section{Chapter 7}

\section{Assignment and structural analysis of the chimeric potassium channel KcsA-Kv1.3 in lipid bilayers}

\subsection{Summary}

This chapter describes the solid-state NMR investigation of structure and dynamics of the chimeric potassium channel KcsA-Kv1.3 in its resting state at pH 7.4 in lipid bilayers. Sequential resonance assignments for $59 \%$ of its $4 \times 160$ residues were obtained using a combination of $\left({ }^{15} \mathrm{~N},{ }^{13} \mathrm{C}\right)$ and $\left({ }^{13} \mathrm{C},{ }^{13} \mathrm{C}\right)$ correlation experiments conducted on fully and reverse-labeled as well as C-terminally truncated samples. Comparison of ssNMR data with those from X-ray crystallography and solution-state NMR in micelles on the parent KcsA $\mathrm{K}^{+}$channel reveal differences in length of $\alpha$-helical regions within the sequence. The results discussed here offer insight into the mechanism of ion channel selectivity and underline the important role of the lipid environment for membrane protein structure and function. They also form the basis for the investigation of gating and inactivation in KcsA-Kv1.3 in the following chapters. ${ }^{1}$

\subsection{Introduction}

The tetrameric bacterial potassium channel KcsA [232] is the first ion channel for which an atomic structure was determined [10]. It exhibits a high degree of homology to eukaryotic potassium channels such as Shaker and shares their core functional properties of an

\footnotetext{
${ }^{1}$ This chapter is related to the following publication: Robert Schneider, Christian Ader, Adam Lange, Karin Giller, Sönke Hornig, Olaf Pongs, Stefan Becker, and Marc Baldus: Solid-state NMR spectroscopy applied to a chimeric potassium channel in lipid bilayers. Journal of the American Chemical Society 130, 7427-7435, 2008. [2]
} 
exquisite selectivity for potassium over the smaller sodium ions (by at least two orders of magnitude [233]) and, at the same time, high throughput rates of up to $10^{8} \mathrm{~K}^{+}$ions per second [10]. Selectivity for potassium ions is conveyed by the selectivity filter region of the channel, whose conserved signature sequence Thr-Val-Gly-Tyr-Gly creates coordination spheres for dehydrated $\mathrm{K}^{+}$ions by means of backbone carbonyl moieties from all four subunits pointing towards the central ion conduction pathway [24]. In vitro, KcsA can be gated, i.e. its ion conduction pathway opened and closed, by a change in $\mathrm{pH}$ [234], and it exhibits an inactivation process comparable to C-type inactivation of voltage-gated channels $[38,39,235]$. These features, together with its relative ease of expression in bacteria and remarkable stability [236], have made it a widely used model system for structure and function of potassium channels in general.

As with other membrane proteins, however, the structure of KcsA in protein crystals or in detergent micelles may be affected by packing effects, antibody binding, the absence of a natural lipid environment or micelle curvature [33, 34, 35, 36]. Solid-state NMR offers the unique possibility to study ion channels in a native-like lipid environment which can also be used for parallel functional investigations by electrophysiology. This approach appears well suited to further understanding of several topics in ion channel research that are still a matter of discussion. Only models are available, for example, for structure and function of the intracellular N- and C-termini of KcsA [237]. The exact mechanism of potassium selectivity and, associated with it, the degree of dynamics exhibited by the selectivity filter, have been debated $[238,239]$. While other potassium channels were crystallized with their pore in an open conformation [40,41], the structure of open KcsA is still elusive, as well as its conformation in the inactivated state.

To address these questions, the potassium ion channel KcsA-Kv1.3 was investigated in lipid bilayers using solid-state NMR. The amino acid sequence of this tetramer of 4 $\times 160$ residues is identical to that of KcsA except for 11 amino acids between residues 52 and 64 in the extracellular turret loop and pore helix regions of the channel. They were replaced by the corresponding residues 368 to 380 from the human voltage-gated $\mathrm{K}^{+}$channel Kv1.3, which make KcsA-Kv1.3 a high-affinity receptor for scorpion toxins, similar to Kv1.3 itself [71, 240]. Otherwise, its function is closely related to that of KcsA [62] (see also Chapter 8). Previously, the binding of kaliotoxin (KTX) to KcsA-Kv1.3 was studied by ssNMR in lipid bilayers, and resonance assignments for 19 residues in the turret and selectivity filter regions of the channel were reported [62, 241]. This chapter reports on dipolar-based two- and three-dimensional ssNMR correlation experiments conducted 
on fully and reverse labeled as well as C-terminally truncated KcsA-Kv1.3 versions to obtain resonance assignments throughout the full-length channel and to investigate its structure and dynamics in lipid bilayers. Results are compared to those obtained on KcsA crystals [24] and in NMR studies of KcsA constructs in micelles [28, 242]. This study underlines the potential of ssNMR to investigate larger, membrane embedded proteins in a functional bilayer environment and reveals structural differences between a protein studied in a crystal lattice, in detergent micelles, and in the lipid bilayer.

\subsection{Materials and Methods}

\subsubsection{Expression and purification of $\left[{ }^{13} \mathrm{C},{ }^{15} \mathrm{~N}\right]$-labeled KcsA-Kv1.3 constructs}

Experiments were carried out using a $\left[{ }^{13} \mathrm{C},{ }^{15} \mathrm{~N}\right]$-uniformly labeled (in the following [U]KcsA-Kv1.3) and a reverse-labeled [20, 110] (containing leucine, valine, tryptophane, and tyrosine in natural abundance, $[\mathrm{R}] \mathrm{KcsA}-\mathrm{Kv} 1.3)$ version of the full length channel. In addition, a sample truncated after residue 125 by chymotrypsin digestion was used to assist in identification of C-terminal residues (denoted by [T]KcsA-Kv1.3). Expression, purification and reconstitution in asolectin liposomes were done by Karin Giller and Stefan Becker as described previously [62, 71]. The final KcsA-Kv1.3 buffer solution contained $50 \mathrm{mM}$ sodium phosphate, $\mathrm{pH} 7.4,50 \mathrm{mM} \mathrm{NaCl}$, and $4 \mathrm{mM} n$-decyl- $\beta$-D-maltopyranoside (DM). A 100:1 asolectin/KcsA-Kv1.3 molar ratio was used for the lipid bilayer reconstitution.

\subsubsection{Solid-state NMR experiments and analysis}

All NMR experiments were conducted using $4 \mathrm{~mm}$ triple-resonance $\left({ }^{1} \mathrm{H},{ }^{13} \mathrm{C},{ }^{15} \mathrm{~N}\right)$ probeheads at static magnetic fields of 14.1 and $18.8 \mathrm{~T}$ corresponding to 600 and $800 \mathrm{MHz}$ proton resonance frequencies (Bruker Biospin, Karlsruhe, Germany). Previous experiments have shown that ${ }^{1} \mathrm{H}$ evolution and detection periods provide limited resolution, even if homonuclear proton-proton decoupling sequences are applied [241]. Hence, $\left({ }^{13} \mathrm{C},{ }^{13} \mathrm{C}\right)$ and $\left({ }^{15} \mathrm{~N},{ }^{13} \mathrm{C}\right)$ two- and three-dimensional correlation experiments (see, e.g., ref. [100]) were performed on reverse-labeled and fully labeled samples at varying temperatures, corresponding to the liquid crystalline (sample temperature +2 to $+12^{\circ} \mathrm{C}$ ) and gel phase $(-5$ to $-20^{\circ} \mathrm{C}$ ) states of the lipid bilayer. $\left({ }^{13} \mathrm{C},{ }^{13} \mathrm{C}\right)$ mixing was accomplished by proton-driven spin diffusion under weak coupling conditions (PDSD-WC) at MAS speeds close to the rotational resonance condition for $\mathrm{C}^{\alpha}$ and $\mathrm{C}^{\prime}$ resonances $[125] .\left({ }^{15} \mathrm{~N},{ }^{13} \mathrm{C}\right)$ transfers typically involved SPECIFIC-CP $[123,124]$ transfer units of 1.5 to $4.5 \mathrm{~ms}$, followed by homonuclear 
$\left({ }^{13} \mathrm{C},{ }^{13} \mathrm{C}\right)$ DARR [119] mixing of up to $25 \mathrm{~ms}$. CHHC experiments [136] were recorded with 250 and $500 \mu$ s longitudinal proton-proton mixing times. Short CPs before and after $\left({ }^{1} \mathrm{H},{ }^{1} \mathrm{H}\right)$ mixing were set to $80 \mu \mathrm{s}$. 2D spectra were typically recorded using 1280 to 1536 acquisition data points in the direct dimension and 384, 45, or 170 acquisition data points in the indirect dimension for $\left({ }^{13} \mathrm{C},{ }^{13} \mathrm{C}\right),\left({ }^{15} \mathrm{~N},{ }^{13} \mathrm{C}\right)$, or CHHC spectra, respectively, and processed using $1024 \times 1024$ data points. Three-dimensional NCOCA data were recorded with $3 \mathrm{~ms}$ evolution times in indirect ${ }^{15} \mathrm{~N}$ and ${ }^{13} \mathrm{C}$ dimensions, using 21,19 , and 1024 acquisition data points in F1, F2, and F3 dimensions, respectively, and processed using $512 \times 256 \times 1024$ data points. Spectra were processed in XWinNMR or Topspin (Bruker Biospin, Karlsruhe, Germany) and analyzed with Sparky [223].

\subsubsection{Generation of the KcsA-Kv1.3 structural model}

A structural model for the chimeric channel was generated by Christian Ader and Robert Schneider. The KcsA-Kv1.3 protein sequence, the crystal structure of residues 22-124 of KcsA [24] (PDB ID 1K4C), and a structural model of full-length KcsA based on EPR spectroscopy [237] (PDB ID 1F6G) were used as input for homology modelling using MODELLER [243]. To conform to ssNMR data showing longer TM1 (TM = transmembrane) and C-terminal helices, the resultant model was subjected to a restrained simulated annealing protocol in CNS [175] in which all atoms were kept fixed except those in residues 20-24, 51-61, and 125-130, where helical structure in residues 22-24, 51-53, and 129 was enforced by applying $\alpha$-helical dihedral angle restraints. Dihedral angles for residues $56-$ 61 were kept close to their crystal structure values (see Appendix Section C.4 for further details). For visualization, the PyMOL software was used [244].

\subsection{Results and Discussion}

\subsubsection{D and 3D ssNMR correlation experiments on KcsA-Kv1.3}

In Figure 7.1, two-dimensional proton-driven $\left({ }^{13} \mathrm{C},{ }^{13} \mathrm{C}\right)$ spin diffusion (a) and NCACB (b) spectra of full-length [U]KcsA-Kv1.3 are shown. In addition, an NCOCA spectrum is given in Appendix Figure C.1. In line with earlier studies [62, 241], spectral resolution is given by ${ }^{13} \mathrm{C}$ and ${ }^{15} \mathrm{~N}$ line widths of $0.7 \mathrm{ppm}$ and $1.5 \mathrm{ppm}$, respectively, and compares favorably to ssNMR data obtained on microcrystals of globular proteins. While spectral resolution is high, resonance overlap due to the largely $\alpha$-helical nature of the protein and high levels of occurrence of particular residue types (especially Ala, Arg, Leu, Val) complicate the 

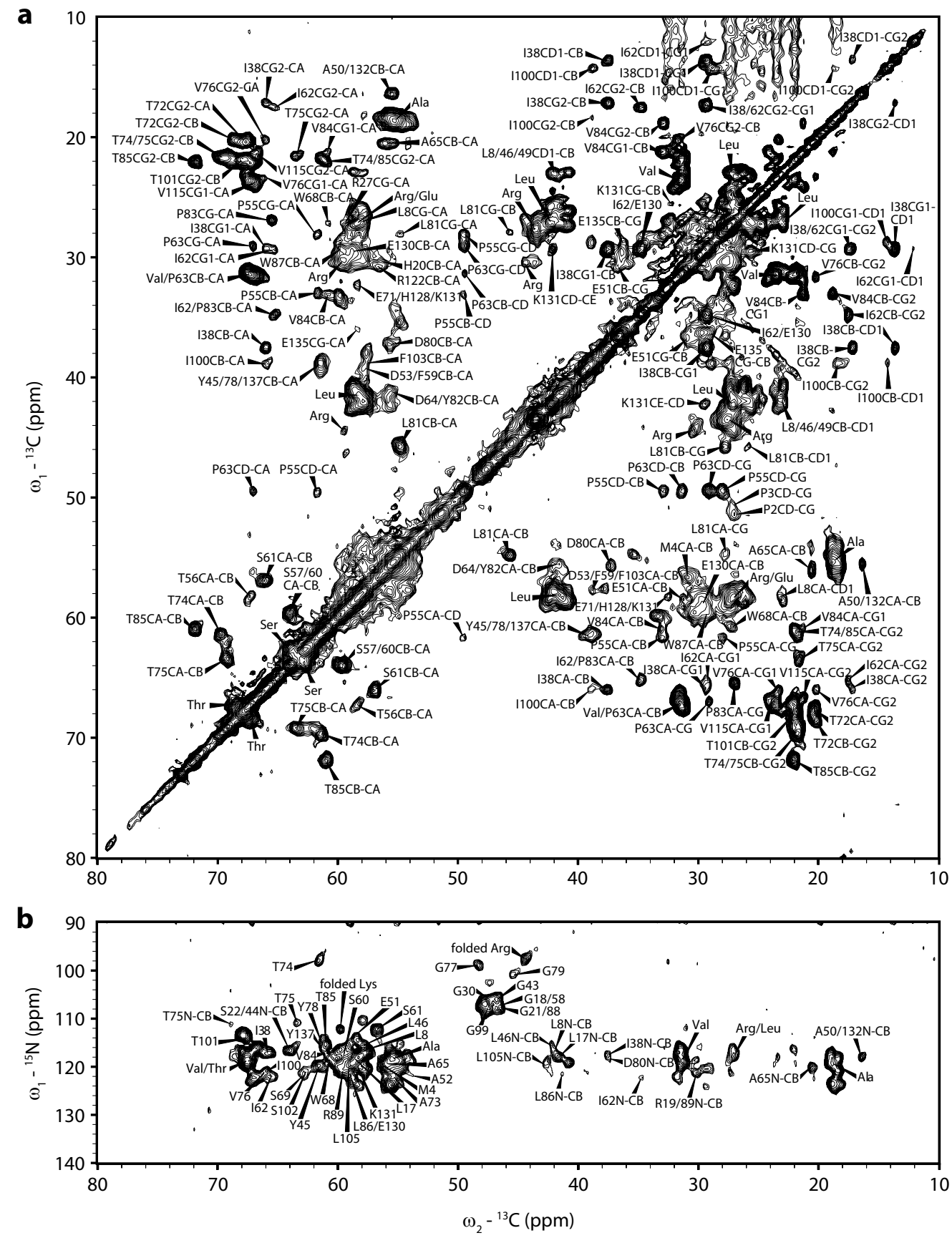

Figure 7.1: 2D ssNMR correlation spectra on [U]KcsA-Kv1.3 in liposomes recorded at $800 \mathrm{MHz}$ ${ }^{1} \mathrm{H}$ Larmor frequency, $12.5 \mathrm{kHz}$ MAS and with lipid bilayers in the liquid crystalline state. (a) Proton-driven $\left({ }^{13} \mathrm{C},{ }^{13} \mathrm{C}\right)$ spin diffusion spectrum (mixing time $20 \mathrm{~ms}$ ). (b) NCACB correlation spectrum using SPECIFIC-CP (3 ms) for the $\left({ }^{15} \mathrm{~N},{ }^{13} \mathrm{C}\right)$ transfer step and subsequent DARR $\left({ }^{13} \mathrm{C},{ }^{13} \mathrm{C}\right)$ mixing ( $8 \mathrm{~ms}$ ). Assigned residues as well as regions of significant overlap for certain residue types are indicated in both spectra. In the NCACB spectrum, peaks marked only with residue type and number represent $\left({ }^{15} \mathrm{~N},{ }^{13} \mathrm{C}^{\alpha}\right)$ correlations. 
assignment process. For this reason, data from the fully isotope-labeled channel were complemented with data obtained on a sample ([R]KcsA-Kv1.3) reverse labeled for some of the most abundant and spectrally overlapped amino acid types (Leu, Val) and for two of four aromatic residue types (Trp, Tyr) accounting for, in total, $29 \%$ of the entire amino acid sequence. This strategy facilitates assignment in the aromatic sidechain regions

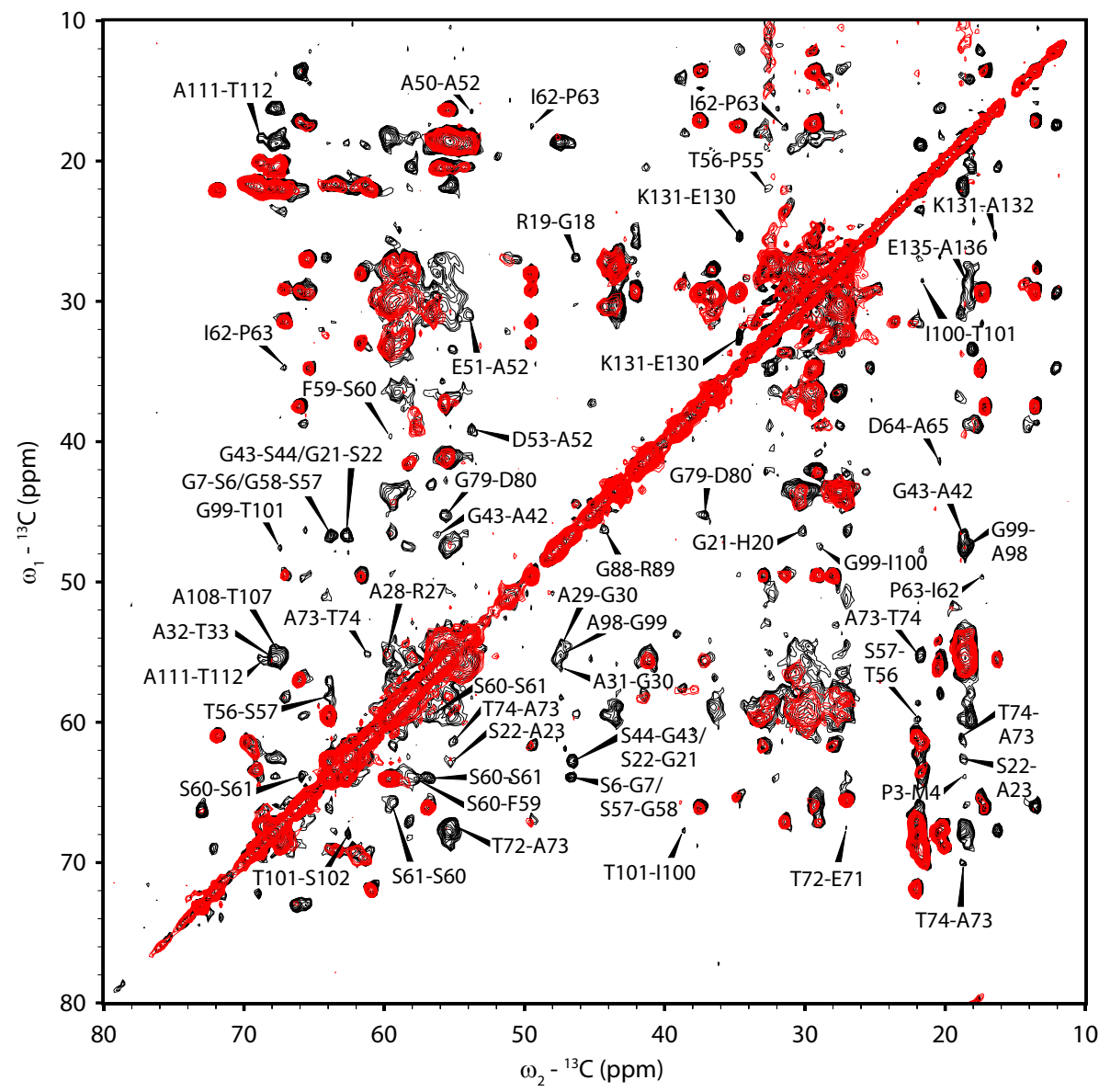

Figure 7.2: Overlay of proton-driven $\left({ }^{13} \mathrm{C},{ }^{13} \mathrm{C}\right)$ spin diffusion spectra of $[R] \mathrm{KcsA}-\mathrm{Kv} 1.3$ recorded with $20 \mathrm{~ms}$ (red) and $150 \mathrm{~ms}$ spin diffusion mixing time (black) under weak coupling conditions [125] ( $800 \mathrm{MHz}, 12.5 \mathrm{kHz}$ MAS, lipid bilayer in the liquid crystalline phase). Assigned sequential correlations are indicated.

$[20,110]$ and significantly improves spectral resolution as shown in Figure 7.2, where a 2D PDSD experiment conducted on [R]KcsA-Kv1.3 under weak coupling conditions [125] reveals a variety of sequential $\left({ }^{13} \mathrm{C},{ }^{13} \mathrm{C}\right)$ connectivities (see also Appendix Figure C.2). Additionally, spectra from a C-terminally truncated sample ([T]KcsA-Kv1.3) were used 
to assist in identification of signals from residues 126-160 in spectra of full-length samples, and a 3D NCOCA spectrum was recorded to resolve sequential $\left({ }^{15} \mathrm{~N},{ }^{13} \mathrm{C}\right)$ correlation information in an additional dimension. Data from $\left({ }^{13} \mathrm{C},{ }^{13} \mathrm{C}\right)$ correlation experiments were combined with $\left({ }^{15} \mathrm{~N},{ }^{13} \mathrm{C}\right)$-type dipolar transfer experiments [100] to derive sequential resonance assignments classified into three levels of reliability (see Appendix Section C.3).

Figures 7.3 and 7.4 show examples of the sequential assignment process. Combining $\left({ }^{15} \mathrm{~N},{ }^{13} \mathrm{C}^{\alpha}\right)$ correlation information obtained from $2 \mathrm{D}$ experiments as shown in Figure $7.1 \mathrm{~b}$ with results of a 3D NCOCA experiment (Fig. 7.3) readily leads to assignments for residues Ala73 to Asp80 that contain the selectivity filter region of KcsA-Kv1.3. In addition, a

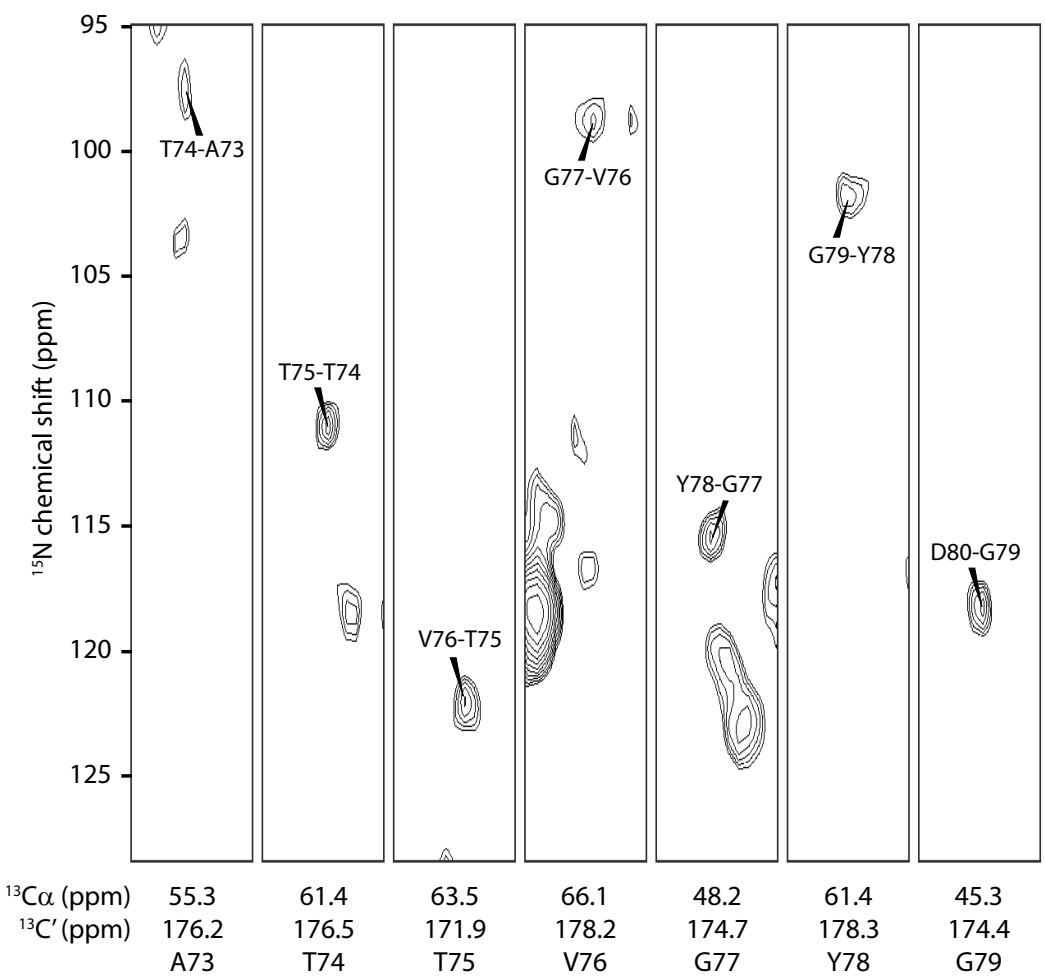

Figure 7.3: Strip plot from a 3D NCOCA experiment recorded on [T]KcsA-Kv1.3 (800 MHz, 12.5 $\mathrm{kHz}$ MAS, lipid bilayer in the gel phase) showing $\left({ }^{15} \mathrm{~N}_{\mathrm{i}},{ }^{13} \mathrm{C}_{\mathrm{i}-1}\right)$ correlations for residues in the selectivity filter region of KcsA-Kv1.3. Corresponding intraresidue $\left({ }^{15} \mathrm{~N}_{\mathrm{i}},{ }^{13} \mathrm{C}_{\mathrm{i}}\right)$ correlations were obtained using 2D NCACB experiments.

large number of resonances could be identified by combining $\left({ }^{15} \mathrm{~N},{ }^{13} \mathrm{C}\right)$ correlation experiments with sequential $\left({ }^{13} \mathrm{C},{ }^{13} \mathrm{C}\right)$ correlations from spectra obtained on $[\mathrm{R}] \mathrm{KcsA}-\mathrm{Kv} 1.3$. An example from the region around Gly99, which is assumed to contain the "gating hinge" 


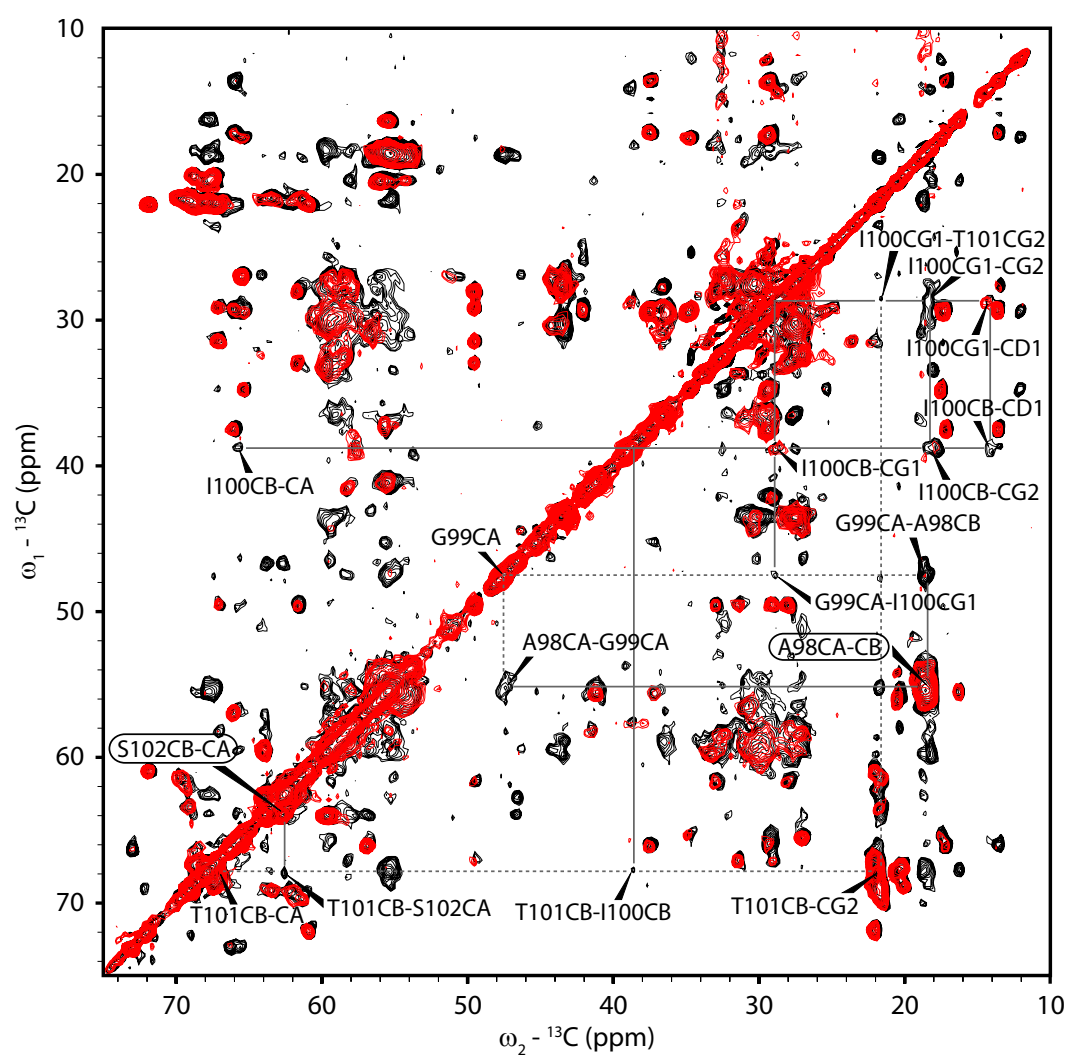

Figure 7.4: Sample sequential walk using only intraresidue and sequential $\left({ }^{13} \mathrm{C},{ }^{13} \mathrm{C}\right)$ correlations. Spectra of [R]KcsA-Kv1.3 are shown under experimental conditions as in Figure 7.2, i.e., recorded with mixing times of $20 \mathrm{~ms}$ (red) and $150 \mathrm{~ms}$ (black). Resonances of individual residues along the sequential stretch are connected by solid or dotted lines. A sequential walk along the same residues including $\left({ }^{15} \mathrm{~N},{ }^{13} \mathrm{C}\right)$ intraresidue and sequential information is shown in Appendix Figure C.3. 
for $\mathrm{pH}$-dependent opening of the channel, is shown in Figure 7.4 and Appendix Figure C.3.

Overall, sequential assignments could be obtained for $94(59 \%)$ of all 160 residues of KcsA-Kv1.3. 73 of these fall within those parts of the protein seen in KcsA crystals [24] (PDB ID 1K4C, residues 22-124), while 21 additional residues from the intracellular $\mathrm{N}$ - and C-termini that were not resolved or not included in the crystal structure were identified. Assigned residues are indicated in Figure 7.1 and listed in Appendix Table C.2.

\subsubsection{Structural analysis}

Using the well-known correlation between secondary chemical shifts and secondary structure $[153,155,245]$, helical and nonhelical stretches of the protein in lipid bilayers can be identified, and their distribution can be compared with those in crystalline and micelle preparations. Figure 7.5 shows residue-specific ssNMR secondary chemical shifts as bars,

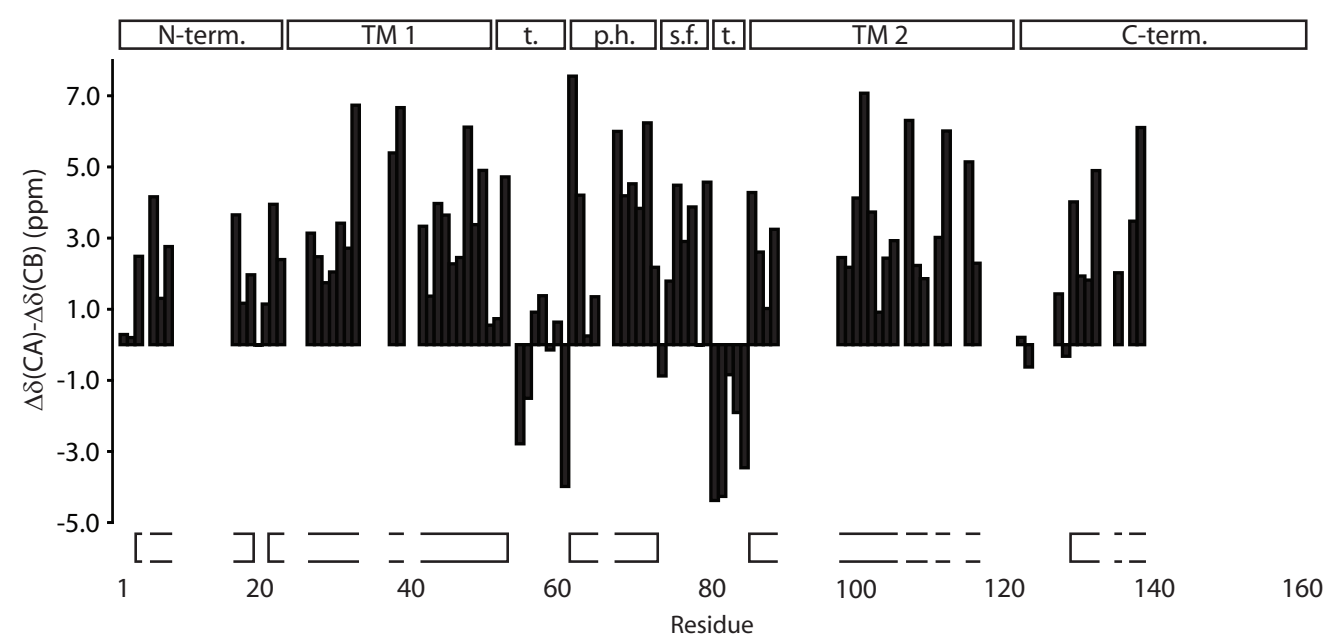

Figure 7.5: Secondary chemical shifts $\left(\Delta \delta\left(\mathrm{C}^{\alpha}\right)-\Delta \delta\left(\mathrm{C}^{\beta}\right)\right)$ of KcsA-Kv1.3 with reference to average values taken from the Biological Magnetic Resonance Data Bank (BMRB; http://www.bmrb.wisc.edu). Positive values indicate helical structure. Secondary structure elements of the crystal structure are given as horizontal bars at the top (TM: transmembrane helix; t.: turret loop; p.h.: pore helix; s.f.: selectivity filter), as well as $\mathrm{N}$ - and $\mathrm{C}$-terminal regions not covered by the crystal structure. Horizontal bars at the bottom show helical segments as seen by ssNMR.

where positive values indicate helical secondary structure. Secondary structure elements 
of the crystal structure are provided as reference at the top, while helical regions as apparent from ssNMR data are shown as horizontal bars at the bottom. In the transmembrane and extracellular parts of KcsA-Kv1.3, $\alpha$-helical structure is found in the regions between residues 22-53, 62-73 and from residue 86 to 116 or further, but not beyond residue 121 . While continuity of these helical segments cannot be inferred in regions that could not be assigned, they correspond very closely to helical segments found in the crystal structure (transmembrane helices TM1 and TM2 and the pore helix). Some differences are apparent for TM1. SsNMR data show strong indications for helical structure already at residue 22, while in the crystal structure, the TM1 helix begins only at residue 24, indicating a TM1 helix extending slightly longer at its N-terminus in lipid bilayers if continuity is assumed. The last residue exhibiting a strong $\alpha$-helical secondary chemical shift is Asp53, pointing towards an extension of the TM1 helix by two residues (compared to the crystal structure) also at its C-terminus. The extracellular end of the TM1 helix and the turret loop connecting TM1 and pore helix contain the Kv1.3 mutations in KcsA-Kv1.3 and constitute the binding interface of the antibody used for KcsA crystal preparation [24], such that structural differences in this region with respect to the crystal structure can be expected (see below and Appendix Figure C.4).

Further support for a slightly extended TM1 helix in liposomes comes from CHHC experiments [136] that probe through-space proton-proton contacts in an indirect manner. Three well resolved crosspeaks from CHHC spectra recorded with $\left({ }^{1} \mathrm{H},{ }^{1} \mathrm{H}\right)$ mixing times of 250 and $500 \mu$ s and the CHHC spectrum with $500 \mu$ s proton mixing are shown in Figure 7.6. Correlations shown in (b) and (c) are consistent with contacts between residues in the turret loops of KcsA-Kv1.3. Corresponding proton-proton contacts in the crystal structure of KcsA (1K4C, with L59 instead of F59) are shorter than $3.5 \AA$ and, in the absence of significant molecular motion (see below), would be expected to appear for significantly shorter $\left({ }^{1} \mathrm{H},{ }^{1} \mathrm{H}\right)$ mixing times (around 200-250 $\mu \mathrm{s}$ [70, 137]). However, these crosspeaks are only seen for the longer $\left({ }^{1} \mathrm{H},{ }^{1} \mathrm{H}\right)$ mixing time of $500 \mu \mathrm{s}$. This result is in line with the assumption of a structural change in the turret region due to the presence of a longer TM1 helix. Furthermore, in the CHHC spectrum for $250 \mu$ s proton mixing, a crosspeak is found that is consistent with an $(i, i+3)$ contact typical for $\alpha$-helical structure between residues Ala50 and Asp53 (Fig. 7.6). Taken together, chemical shift and proton-proton distance data give strong indications for a TM1 helix slightly longer than in the KcsA crystal structure. 


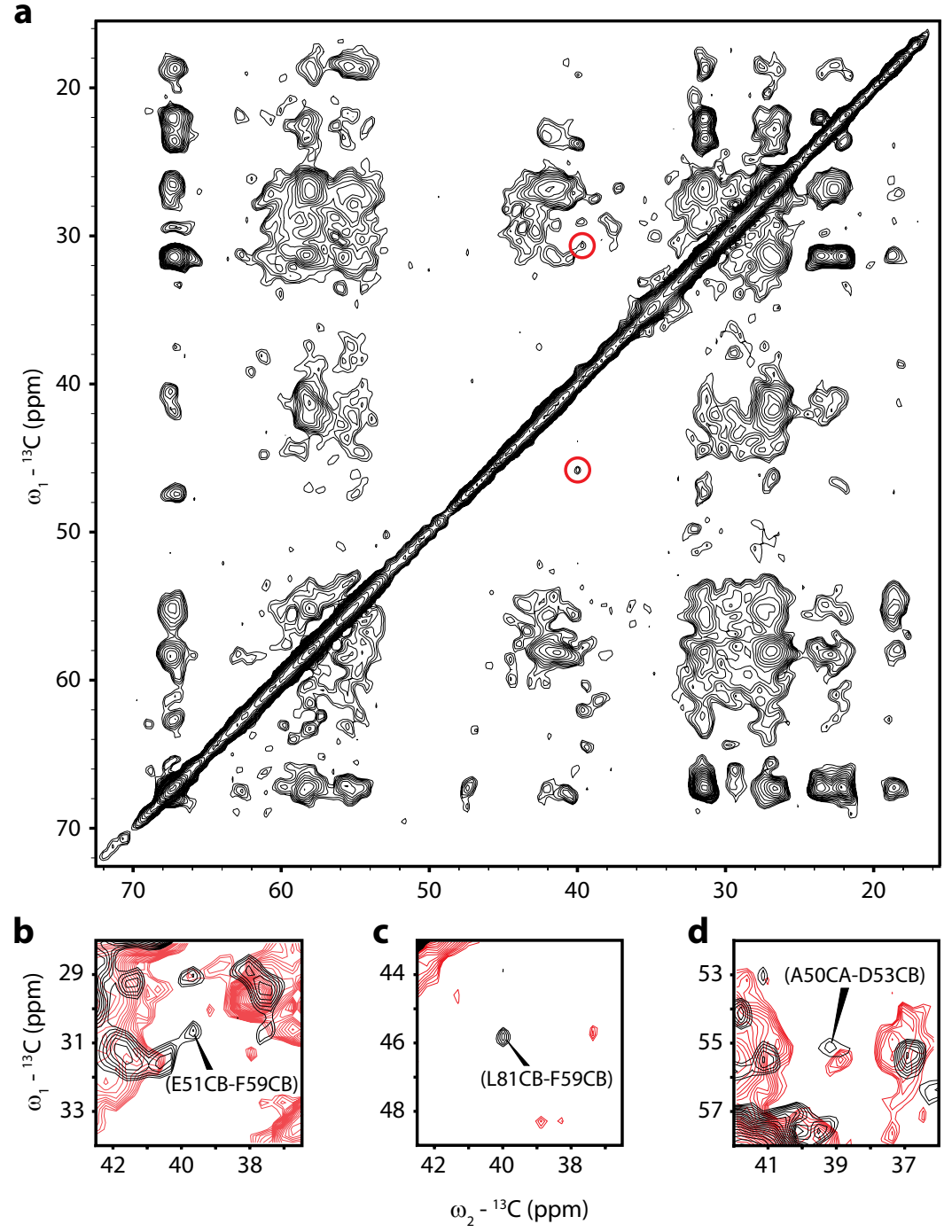

Figure 7.6: (a) CHHC spectrum of [U]KcsA-Kv1.3, recorded with a $\left({ }^{1} \mathrm{H},{ }^{1} \mathrm{H}\right)$ mixing time of 500 $\mu \mathrm{s}(600 \mathrm{MHz}, 9.375 \mathrm{kHz}$ MAS, lipid bilayer in the gel phase). Peaks enlarged in (b) and (c) are circled. (b-d) Spectral regions of the [U]KcsA-Kv1.3 CHHC spectrum shown in (a) (black, b and c) and of a $\mathrm{CHHC}$ spectrum with a $\left({ }^{1} \mathrm{H},{ }^{1} \mathrm{H}\right)$ mixing time of $250 \mu \mathrm{s}$ (black, d), superimposed onto a $\left({ }^{13} \mathrm{C},{ }^{13} \mathrm{C}\right)$ spin diffusion spectrum (red) with a mixing time of $150 \mathrm{~ms}(800 \mathrm{MHz}, 12.5 \mathrm{kHz}$ MAS, lipid bilayer in the gel phase). Crosspeaks consistent with contacts within and between turret loops and with an (i, i+3) $\alpha$-helical contact are shown in (b), (c), and (d), respectively. Labels in parentheses indicate that these peaks are not unambiguously assigned. 
In addition to structural alterations in the turret region, chemical shift data also clearly point to the presence of helical structure in the N-terminus, as seen from helical secondary chemical shifts observed for residues 4, 6-8, and 17-19, as well as in the C-terminus, beginning with residue 129. In line with EPR and solution-state NMR data, these results suggest that the N-terminus of KcsA-Kv1.3 contains an amphipathic $\alpha$-helix that might serve as membrane anchor. Similarly, the KcsA-Kv1.3 C-terminus appears to exhibit helical structure, possibly in the form of a helix bundle [28, 237, 242].

To delineate the differences between previous solution-state NMR data on KcsA constructs and this ssNMR analysis in further detail, a comparative analysis in reference to solution-state NMR chemical shifts measured for KcsA constructs in foscholine [242] and SDS [28] micelles was conducted. In addition, a structural model of full-length KcsA-

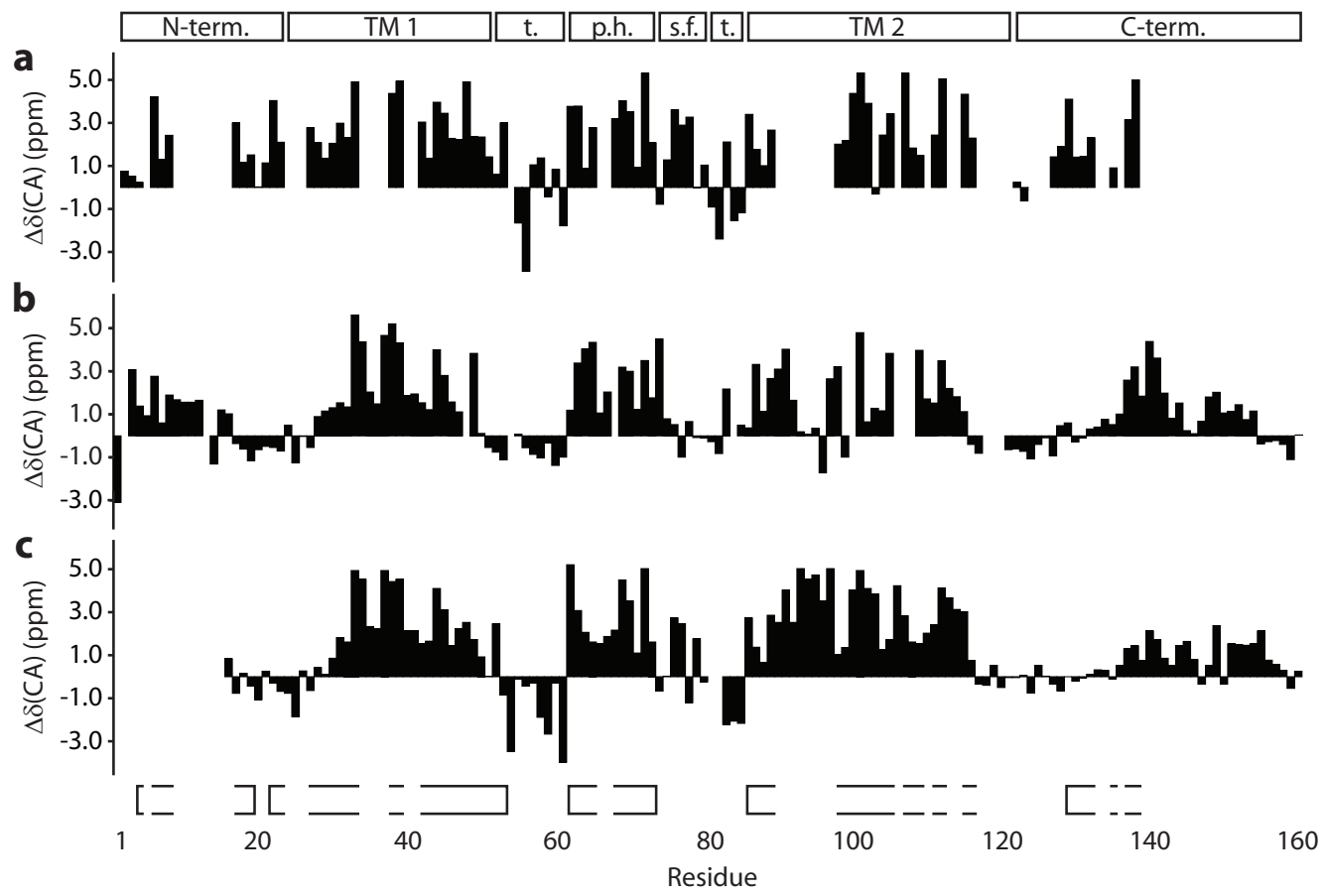

Figure 7.7: Comparison of $\mathrm{C}^{\alpha}$ secondary chemical shifts. (a) KcsA-Kv1.3 in liposomes as seen by solid-state NMR. (b) Full-length KcsA studied by solution-state NMR in foscholine micelles [242] (c) KcsA(16-160) measured by solution-state NMR in SDS micelles [28]. Positive values indicate helical structure. Secondary structure elements are given as in Figure 7.5.

Kv1.3 was created based on the crystal structure of KcsA [24] (PDB ID 1K4C), an EPR model [237] of the intracellular N- and C-termini (PDB ID 1F6G) and ssNMR data discussed here (see also Appendix Fig. C.5). Results are shown in Figure 7.7 as bar plots 
of $\mathrm{C}^{\alpha}$ secondary chemical shifts for the different datasets and in Figure $7.8 \mathrm{~b}$ and $\mathrm{c}$ as a color code of shift differences mapped onto the structural model of KcsA-Kv1.3. Residues colored in red exhibit $\mathrm{C}^{\alpha}$ chemical shift changes between solution- and solid-state NMR data larger than $2 \mathrm{ppm}$.

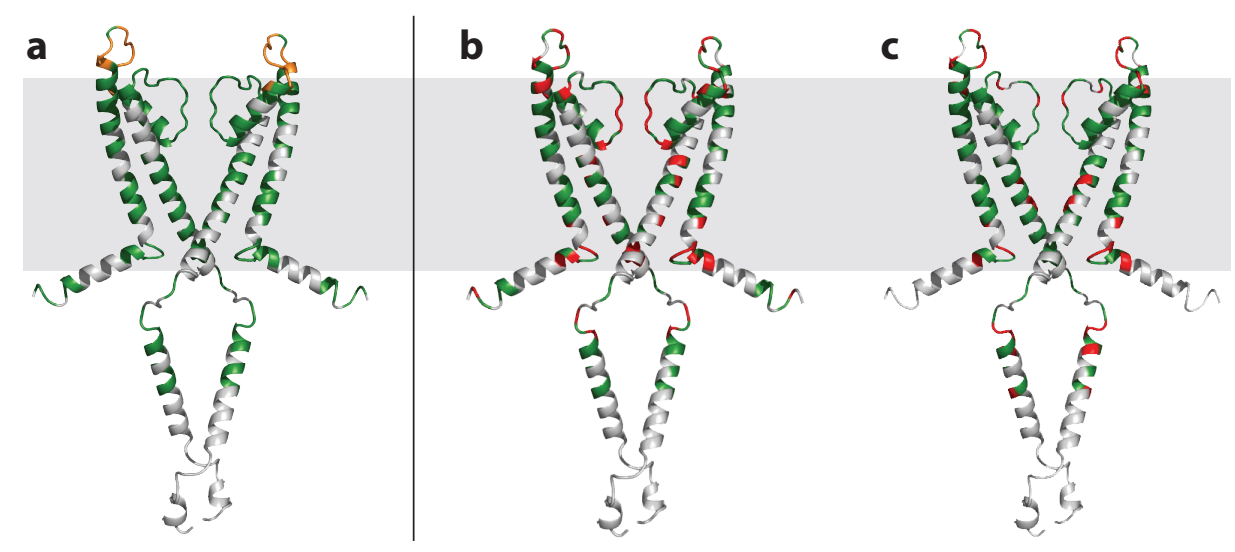

Figure 7.8: (a) Structural model of KcsA-Kv1.3 in lipid bilayers. Two of the four subunits have been removed for visual clarity. The grey rectangle reflects the approximate membrane environment. The color code indicates residues assigned by ssNMR. Among native KcsA residues, assigned ones are indicated in green, unassigned residues in grey. Residues mutated to Kv1.3 are colored orange, all of which except for Asp54 were assigned in lipid bilayers. (b, c) Differences in $\mathbf{C}^{\alpha}$ secondary chemical shift between ssNMR assignments on KcsA-Kv1.3 and solution-state NMR values for KcsA in foscholine micelles [242] (b) and SDS micelles [28] (c), color coded on the structural model of KcsA-Kv1.3. The color code shows residues with an agreement of $\Delta \delta\left(\mathrm{C}^{\alpha}\right)$ within $\pm 2 \mathrm{ppm}$ in green, deviations greater than $2 \mathrm{ppm}$ in red, and unassigned residues in grey.

Figure 7.8 a additionally shows which residues have been assigned and where the Kv1.3 mutations are located in KcsA-Kv1.3. Mutations are confined to the turret region where structural changes observed in reference to the crystal structure may, at least in part, be related to changes in protein sequence. On the other hand, the protein sequence and functional data for KcsA-Kv1.3 strongly suggest that the channel structure is otherwise closely related to KcsA. A test of self consistency comparing ssNMR secondary chemical shifts $\left(\Delta \delta\left(\mathrm{C}^{\alpha}\right)\right)$ to values predicted for the structural model depicted in Figure 7.8 using SHIFTX [163] showed very good agreement between ssNMR assignments and predictions within the accuracy limits of the program (Appendix Fig. C.5). Larger deviations are mostly confined to loop regions, suggesting that the structural model indeed captures the central structural features of KcsA-Kv1.3 in lipid bilayers. 
Compared to solution-state NMR shifts, differences are seen for the N-terminus of TM1 and the intracellular C-terminal helix. In lipid bilayers, considering those residues for which ssNMR assignments are available, helical structure in the C-terminus appears to be more pronounced and begins earlier in the sequence (residue 129), especially when compared to data measured in SDS micelles, where helical character becomes apparent at residue 142. This observation might be relevant for the tetrameric assembly of the channel, since the C-terminus has been shown to enhance thermal stability of the KcsA tetramer at neutral $\mathrm{pH}$ values [246]. On the other hand, ssNMR data indicate structural differences in the TM1 helix, where clear helical secondary chemical shifts are observed for all assigned residues between Ser22 and Gly30, while the TM1 helix begins only at residue 30 in micelles. This difference is likely of functional relevance, since residue His 25 in this amino acid stretch has been shown to be crucial for the opening of the intracellular activation gate of KcsA occurring at low pH values [247]. While His25 could not be assigned in this study, it is surrounded by helical residues, unlike in micellar preparations, indicating a different, likely helical, arrangement compared with that indicated by solutionstate NMR data. Interestingly, ssNMR $\mathrm{C}^{\alpha}$ secondary chemical shifts measured for two regions controlling ion conduction, i.e., the selectivity filter and the TM2 gating region, fit better to predictions using the KcsA crystal structure than to solution-state NMR values found for KcsA in SDS and foscholine micelles (Fig. 7.8 and Appendix Fig. C.6). In the selectivity filter, this difference may be related to different levels of potassium occupancy (R. Riek, personal communication). When comparing the total number of large chemical shift deviations $(>2 \mathrm{ppm})$ between the solid- and solution-state NMR datasets in transmembrane regions and the selectivity filter, SDS micelle data seem to fit somewhat better to solid-state NMR assignments (see Appendix Fig. C.6). This might be surprising since SDS has been shown to destabilize the tetramer at low $\mathrm{pH}$ [248] and was used in conjunction with an elevated temperature of $50^{\circ} \mathrm{C}$. However, it is known that, for proper function, KcsA requires negatively charged lipids, as they are present in the asolectin liposome preparation used here [249]. Negatively charged detergents such as SDS might hence offer advantages compared to zwitterionic lipids like foscholine to study functional aspects of the KcsA channel.

A recent report described ssNMR experiments on the full-length KcsA channel precipitated from detergent micelles and assignments for residues Val76-Asp80 and Pro83-Leu90 [250]. Compared to the results for KcsA-Kv1.3 in liposomes presented here, aliphatic carbon shifts in the selectivity filter are similar. Significant differences between the two 
datasets exist for backbone nitrogen and carbonyl shifts in the selectivity filter, as well as for most residues in the second turret loop and at the beginning of the TM2 helix (residues 83-89; see Appendix Table C.4). Especially the latter differences may be attributable to the details of sample preparation and to differences in the amino acid sequence (the KcsA-Kv1.3 construct used in this study carries the mutation L90C in this region). On the other hand, the similarity of aliphatic carbon shifts in the selectivity filter between the two datasets suggests a similar conformation in liposomal and precipitated preparations. As can also be seen from the good agreement of KcsA-Kv1.3 assignments with the KcsA crystal structure (see Appendix Figs. C.5 b, C.6, and ref. [62]), this conformation most likely corresponds to the conductive state of the selectivity filter as seen in high concentrations of potassium.

\subsubsection{Molecular mobility in KcsA-Kv1.3}

All spectra of KcsA-Kv1.3 discussed so far were based on dipolar magnetization transfer, which is attenuated or even averaged out in the presence of significant molecular motion. SsNMR experiments on other membrane proteins have shown that loop regions can exhibit fast dynamics. Such protein segments are absent from dipolar transfer-based spectra but can be detected using scalar (through-bond) transfer sequences [59, 113]. The presence or absence of significant levels of molecular mobility is of particular interest in the selectivity filter region. It has been a matter of debate whether ion selectivity in KcsA is a result of a relatively rigid selectivity filter that selects for potassium ions based on their size using a well-defined protein structure [239] or whether the selectivity filter is actually a highly mobile region exhibiting "liquid-like" dynamics, with selectivity determined by the electrostatic repulsion between the ligands coordinating the ions, in this case the selectivity filter carbonyls [238].

In KcsA-Kv1.3, experiments based on scalar transfer led to signal sets dominated by lipid resonances (data not shown), arguing against the presence of protein segments that exhibit fast dynamics as seen, e.g., in sensory rhodopsin II [59]. On the contrary, signals from all residues comprising the selectivity filter (residues 75-79) and adjacent residues could clearly be identified in dipolar spectra, as well as almost all resonances in the turret loops (residues 54-61 and 80-85, Fig. 7.9). For turret loop residues, this indicates that these residues can only exhibit limited molecular motion, even when not locked by antibody binding as in the crystal structure (see Appendix Fig. C.4). This observation is consistent with solution-state NMR relaxation studies on KcsA in SDS micelles [251] where it was 


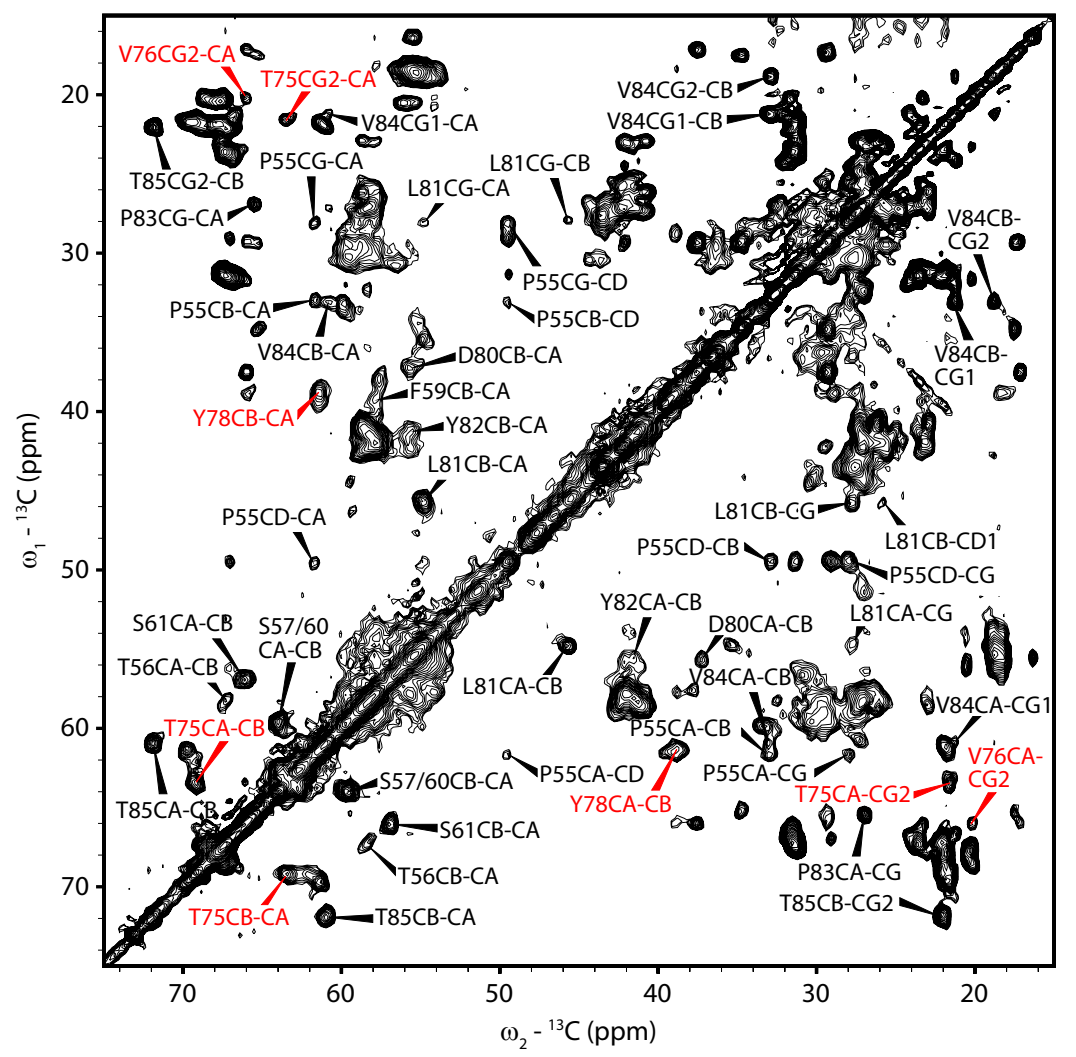

Figure 7.9: Proton-driven $\left({ }^{13} \mathrm{C},{ }^{13} \mathrm{C}\right)$ spin diffusion spectrum of [U]KcsA-Kv1.3 (800 MHz, $20 \mathrm{~ms}$ spin diffusion mixing time, $12.5 \mathrm{kHz}$ MAS, lipid bilayer in the liquid crystalline phase). Assignments indicate correlations arising from residues in the selectivity filter (red) and turret loops (black). Correlations for Gly77 and Gly79 in the selectivity filter are seen in the carbonyl region and in $\left({ }^{15} \mathrm{~N},{ }^{13} \mathrm{C}\right)$ correlation spectra (not shown). 
hypothesized that intersubunit contacts between turret regions could rigidify the structure of the turret loops. That study also reported the absence of large-amplitude motions on the pico- to nanosecond timescale in the selectivity filter region, in agreement with results presented here. While further ssNMR experiments are required to exclude small amplitude molecular motions in the selectivity filter of KcsA-Kv1.3, these data strongly argue against "liquid-like" fluctuations in its constituent residues and support views in which a well-defined protein structure plays a crucial role in ion selectivity.

\subsection{Conclusions}

In this chapter, it was shown that solid-state NMR experiments can be used to study structure and dynamics of a $4 \times 160$ residue chimeric potassium channel in lipid bilayers. Compared to previous structural work on the closely related KcsA channel, ssNMR data on KcsA-Kv1.3 indicate that transmembrane helix 1 is longer at both ends, especially at its N-terminus, when compared to the length apparent from NMR data in micelles. Consistent with results from an EPR study, clear evidence is found for the formation of an N-terminal $\alpha$-helix. Although ssNMR resonance assignment in the C-terminus is still incomplete, available data strongly suggest helical structure in the C-terminus as well (starting from residue 129), consistent with an EPR structural model proposing a four-helix bundle in this region. The clear presence of resonances from turret loops and selectivity filter in dipolar spectra suggests reduced mobility in these regions, speaking in favor of a mechanism for ion selectivity based on a defined protein structure.

Although 11 mutations are present in the construct used here, structural and functional evidence suggests that KcsA-Kv1.3 and the KcsA channel are structurally closely related (see Chapter 8). The observed differences are hence in line with increasing evidence that protein structure and function can be influenced by the surrounding membrane environment. Structural differences between bilayer preparations and micellar systems may, in part, reflect the fact that micelles have stronger curvature and lack the asymmetry and the closely packed headgroups of a bilayer [30]. In line with previous evidence [249], this work also points to the potential influence of lipid charges for structure and function of membrane proteins.

In this study, assignments for $59 \%$ of the residues of the entire protein and $71 \%$ of its putative transmembrane and extracellular regions were reported. Advanced labeling schemes, possibly involving cell-free based expression systems [252], are likely to improve 
the prospects of complete ssNMR assignments in larger membrane proteins in lipid bilayers. In the current context, an $\alpha$-helical protein was investigated. Similar studies may be important for $\beta$-barrel proteins that are under investigation by solution-state (see, e.g., refs. [25, 253]) and solid-state NMR [109, 254]. Such studies not only provide the basis for ssNMR-based structural studies in membrane proteins of unknown structure but also underline the importance of ssNMR for understanding functional aspects of membraneembedded proteins even if structural data from X-ray crystallography or solution-state NMR are available. 


\section{Chapter 8}

\section{Gating and inactivation in KcsA-Kv1.3}

\subsection{Summary}

Gating the ion-permeation pathway in $\mathrm{K}^{+}$channels requires conformational changes in activation and inactivation gates. This chapter describes structural alterations associated with $\mathrm{pH}$-dependent gating of the KcsA-Kv1.3 $\mathrm{K}^{+}$channel which were investigated using solid-state NMR spectroscopy in direct reference to electrophysiological and pharmacological experiments. Transition of the KcsA-Kv1.3 $\mathrm{K}^{+}$channel from a closed state at $\mathrm{pH}$ 7.5 to an inactivated state at $\mathrm{pH} 4.0$ revealed distinct structural changes within the pore domain, correlated with activation gate opening and inactivation gate closing. The open activation gate of KcsA-Kv1.3 is characterized by bent inner transmembrane helices as seen in crystal structures of other opened potassium channels. In the inactivated state of the channel, the selectivity filter adopts a nonconductive structure that can also be induced by binding of a pore-blocking tetraphenylporphyrin derivative. The results establish a structural link between inactivation and block of a $\mathrm{K}^{+}$channel in a membrane setting. ${ }^{1}$

\subsection{Introduction}

Gating of $\mathrm{K}^{+}$and other ion channels is an important mechanism to regulate the selective conduction of ions down their electrochemical gradient across the plasma membrane [37].

\footnotetext{
${ }^{1}$ This chapter is related to the following publication: Christian Ader*, Robert Schneider*, Sönke Hornig, Phanindra Velisetty, Erica M. Wilson, Adam Lange, Karin Giller, Iris Ohmert, Marie-France MartinEauclaire, Dirk Trauner, Stefan Becker, Olaf Pongs, and Marc Baldus: A structural link between inactivation and block of a $\mathrm{K}^{+}$channel. Nature Structural and Molecular Biology 15, 605-612, 2008. (*equally contributing authors) [1]
} 
In this process, the ion channel opens and closes for a defined duration of time in response to a specific external stimulus such as a change in the membrane electric field and/or in ligand concentration. Channel gating involves conformational changes at an activation gate in the permeation pathway $[39,40]$. Frequently, prolonged stimulation shuts the permeation pathway and renders the ion channel refractory to further activation. This process is called inactivation. Different inactivation mechanisms have been discovered involving distinct ion channel domains [39]. For example, N-type inactivation of voltage-gated delayed-rectifier type $\mathrm{K}^{+}(\mathrm{Kv})$ channels derives its name from an N-terminal inactivation domain that inserts into and blocks the channel pore after activation gate opening, while particular C-terminal domains are associated with so-called C-type or slow inactivation $[38,39]$.

C-type inactivation seems to be an effective mechanism for controlling Kv channel activity to regulate action-potential firing frequencies in excitable cells [255]. It is suggested that C-type inactivation of Shaker Kv channels is correlated with a nonconducting structure of the pore, reflecting a low occupancy of $\mathrm{K}^{+}$binding sites in the selectivity filter [39]. In this state, $\mathrm{K}^{+}$channels can conduct $\mathrm{Na}^{+}$and $\mathrm{Li}^{+}[256,257,258]$. Inactivation of the $\mathrm{KcsA} \mathrm{K}^{+}$channel shares many properties with C-type inactivation. Factors that influence $\mathrm{KcsA} \mathrm{K}^{+}$channel inactivation have been investigated in great detail, such as intracellular $\mathrm{pH}$ and transmembrane voltage $[259,260]$. The results supplemented by molecular dynamics simulations have provided detailed models on the regulation of $\mathrm{KcsA} \mathrm{K}^{+}$channel inactivation [235, 261, 262, 263].

Knowledge of the protein structural rearrangements that occur with activation and inactivation gating of the $\mathrm{K}^{+}$channel is of central importance for understanding the molecular mechanisms underlying $\mathrm{K}^{+}$channel gating. The available data suggest that two separate gates control activation and, respectively, C-type inactivation gating of the KcsA K ${ }^{+}$channel [264]. The activation gate seems to be associated with structures in the intracellular half of the channel, such as the gating hinge and inner helix bundle $[21,24,40,41]$. The inactivation gate has been localized to the selectivity filter in the extracellular half of the channel. Recent mutational analysis of inactivation gating in the KcsA $\mathrm{K}^{+}$channel has shown that stability of the closed inactivation gate is determined by a hydrogen bond network on the back side of the selectivity filter [235, 263]. The inactivation mechanism of the $\mathrm{KcsA} \mathrm{K}^{+}$channel, however, has been inferred from noninactivating mutants, because the structure of an inactivated $\mathrm{KcsA} \mathrm{K}^{+}$channel is still 
elusive; this highlights the need for direct structural approaches to this important $\mathrm{K}^{+}$ channel gating process.

Based on resonance assignments and structural information discussed in Chapter 7, this chapter describes the application of solid-state NMR spectroscopy to study activation and inactivation gating of functional KcsA-Kv1.3 $\mathrm{K}^{+}$channels in lipid bilayers in close reference to electrophysiological recordings. Functional data show that KcsA-Kv1.3, as its parent KcsA channel, gates open and subsequently inactivates under the influence of acidic pH. SsNMR spectra show that this transition from a closed state at $\mathrm{pH} 7.5$ to an inactivated state at $\mathrm{pH} 4.0$ induces distinct structural changes within the channel pore which can be correlated with an opened activation and a closed inactivation gate. Chemical shift analysis reveals a bend in the inner transmembrane helices at acidic $\mathrm{pH}$, opening the ion conduction pathway, and a nonconductive structure of the selectivity filter, comparable to its collapsed conformation seen in KcsA crystals grown in low potassium concentrations. This conformation of the selectivity filter can also be induced by binding a tetraphenylporphyrin derivative ("porphyrin"), which competes with scorpion toxins for binding to the Kv1.3 channel pore [265]. Inactivation and porphyrin block appear to induce similar conformations in the selectivity filter by depleting it of potassium ions.

\subsection{Materials and Methods}

\subsubsection{Sample preparation}

KcsA-Kv1.3 expression, purification and reconstitution into asolectin liposomes were carried out by Karin Giller and Stefan Becker as described [1, 62] (see also Chapter 7). Samples at $\mathrm{pH} 4.0$ were prepared by washing proteoliposomes with $100 \mathrm{mM}$ citrate buffer yielding the same ionic strength as the phosphate buffer used for $\mathrm{pH} 7.5$ samples. Porphyrinbound samples were prepared by adding porphyrin \#3 (ref. [265], with $\mathrm{R}=\mathrm{HN}-\left(\mathrm{CH}_{2}\right)_{2^{-}}$ $\mathrm{NH}_{2}$, in the following denoted porphyrin, see Appendix Fig. D.8 a) in two-fold molar excess to KcsA-Kv1.3 reconstituted in asolectin liposomes at pH 7.5. All samples were washed thoroughly with the respective buffer before ultracentrifugation and packing into standard $4 \mathrm{~mm}$ MAS rotors.

\subsubsection{Solid-state NMR}

NMR experiments were conducted using $4 \mathrm{~mm}$ triple-resonance $\left({ }^{1} \mathrm{H},{ }^{13} \mathrm{C},{ }^{15} \mathrm{~N}\right)$ probeheads at static magnetic fields of $14.1 \mathrm{~T}$ and $18.8 \mathrm{~T}$, corresponding to $600 \mathrm{MHz}$ and $800 \mathrm{MHz}$ pro- 
ton resonance frequencies (Bruker Biospin, Karlsruhe, Germany). $\left({ }^{13} \mathrm{C},{ }^{13} \mathrm{C}\right)$ correlations were obtained using proton-driven spin diffusion under weak coupling conditions (PDSDWC) [125], whereas $\left({ }^{15} \mathrm{~N},{ }^{13} \mathrm{C}\right)$ spectra were recorded using SPECIFIC-CP [123], with and without subsequent DARR [119] mixing for obtaining sidechain ${ }^{13} \mathrm{C}$ resonances. Throughspace constraints for the selectivity filter at $\mathrm{pH} 7.5$ were obtained via $\mathrm{NHHC}$ and $\mathrm{CHHC}$ [62] experiments using $100 \mu \mathrm{s}$ and $50 \mu \mathrm{s}\left({ }^{1} \mathrm{H},{ }^{1} \mathrm{H}\right)$ mixing times, respectively. MAS speeds used were $9.375 \mathrm{kHz}$ and $12.5 \mathrm{kHz}$ at $14.1 \mathrm{~T}$ and $18.8 \mathrm{~T}$ static magnetic field strengths, respectively, at effective sample temperatures (calibrated using nickelocene [222]) of approximately $+10^{\circ} \mathrm{C}$ (liquid crystalline bilayer state) or $-10^{\circ} \mathrm{C}$ (gel phase bilayer state). The typical proton field strength for $90^{\circ}$ pulses and SPINAL64 (ref. [95]) decoupling was $83 \mathrm{kHz}$.

\subsubsection{Structural analysis}

To analyze structural changes of the KcsA-Kv1.3 backbone upon pH change to $\mathrm{pH} 4.0$ and porphyrin binding, a grid search for dihedral angles accounting best for observed chemical shift changes was performed by Christian Ader. Backbone $(\phi, \psi)$ angles of the residues in question were systematically varied across the relevant regions of Ramachandran space in PDB models of KcsA-Kv1.3, and estimates for their chemical shifts were calculated using the SHIFTX software [163]. For every residue in the selectivity filter and the TM2 gate, $\phi$ and $\psi$ angles were altered independently in steps of $5^{\circ}$. Simulated secondary chemical shifts were calculated from SHIFTX results as defined previously [153]. For the selectivity filter and the TM2 gate of KcsA-Kv1.3 at pH 4.0, dihedral angle pairs were selected for which simulated secondary chemical shifts were closest to experimentally determined values and which required the smallest angular change compared to their values at $\mathrm{pH} 7.5$ (assumed to be identical to their values in the KcsA crystal structure at high potassium concentrations (PDB 1K4C), see below).

Owing to the large conformational space available to glycine residues, this chemical shift analysis did not yield an unambiguous result for the dihedral angles of Gly77 and Gly79 at pH 4.0. For this reason, a set of 500 structures of the KcsA-Kv1.3 monomer was calculated using restrained simulated annealing in CNS [175]. Gly77 and Gly79 were allowed to adjust freely, whereas dihedral angles of residues Thr75, Val76, and Tyr78 were restricted to a small region in the Ramachandran plot close to the values found previously in the secondary chemical shift analysis. All other atoms were kept fixed except residues Thr74 and Asp80 to allow for a smooth transition between flexible and rigid protein 
segments. Resultant structural models were selected on the basis of their overall energy as determined by CNS and a SHIFTX prediction of Gly77 and Gly79 ${ }^{\alpha}$ chemical shifts close to experimental values (deviations up to $1.5 \mathrm{ppm}$ accepted). The PyMOL [244] software was used to introduce obtained dihedral angles into the structural model of KcsA-Kv1.3 developed in Chapter 7 and for visualization.

\subsubsection{Electrophysiology}

Patch-clamp and lipid bilayer electrophysiological measurements on KcsA-Kv1.3 as well as porphyrin binding studies were done by Sönke Hornig, Phanindra Velisetty, and Olaf Pongs as described in ref. [1] (see Figure 8.1 legend for details).

\subsection{Results}

\subsubsection{Functional and structural investigation of KcsA-Kv1.3 at acidic pH}

Solution-state NMR studies using detergent micelles were previously conducted to probe conformational changes related to toxin binding and gating on the prototypical KcsA $\mathrm{K}^{+}$ channel [28, 242, 247, 248, 266]. With assignments and structural information discussed in Chapter 7, an investigation of conformational changes associated with gating and ligand binding of KcsA-Kv1.3 embedded in liposomes by solid-state NMR became possible.

Gating properties of KcsA-Kv1.3 were first investigated functionally by Olaf Pongs and coworkers. Inside-out patch-clamp experiments on KcsA-Kv1.3 in proteoliposomes showed that the KcsA-Kv1.3 channel is closed at pH 7.5 and activated by an increase in $\mathrm{H}^{+}$ concentration on the intracellular side, as occurs in the parent KcsA channel [234, 235, 267] (Fig. 8.1 a). Also, KcsA-Kv1.3 rapidly enters into an inactivated state during a prolonged pH stimulus. Current amplitudes of activated KcsA-Kv1.3 $\mathrm{K}^{+}$channels decay to about $8 \%$ of the initial amplitude within $1 \mathrm{~s}$ (Fig. 8.1 a). After reconstitution into lipid bilayers and with $\mathrm{pH} 4.0$ on the intracellular side, KcsA-Kv1.3 steady-state activity (Fig. $8.1 \mathrm{~b}$ ) similar to that reported for the KcsA $\mathrm{K}^{+}$channel was observed [234, 267]. The KcsAKv1.3 channel opens only briefly with a low open probability of about $6 \%$. In agreement with the previous observation that KTX occludes the KcsA-Kv1.3 pore [62], KcsA-Kv1.3 $\mathrm{K}^{+}$channel activity is completely inhibited by adding KTX to the extracellular side of the lipid bilayer (Fig. 8.1 b). Thus, both lipid bilayer and inside-out patch-clamp data show that, at $\mathrm{pH} 4.0$, the $\mathrm{KcsA}-\mathrm{Kv} 1.3 \mathrm{~K}^{+}$channel resides in proteoliposomes mostly in an inactivated state. 
a

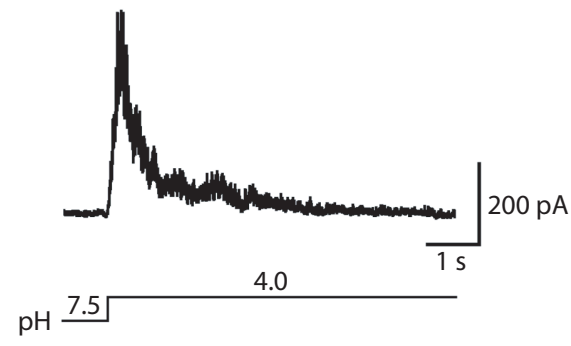

b

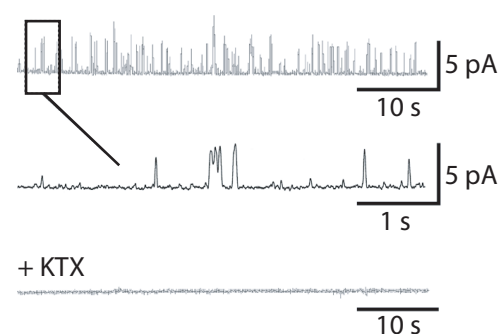

Figure 8.1: Inactivation and KTX binding properties of the KcsA-Kv1.3 channel. (a) KcsA-Kv1.3 current recorded from inside-out patches of proteoliposomes after jumping the $\mathrm{pH}$ from 7.5 to 4.0 at $+100 \mathrm{mV}$ in asymmetrical $\mathrm{K}^{+}$solutions (intracellular side $200 \mathrm{mM} \mathrm{KCl}$, extracellular side $8 \mathrm{mM}$ $\mathrm{KCl}$ ). (b) Representative current traces from lipid bilayers containing KcsA-Kv1.3 channels shown in a slow timescale (above) and as a higher-resolution detail (middle). Traces were obtained at $\mathrm{pH} 4.0$ on the intracellular side of the lipid bilayer and $+100 \mathrm{mV}$ in symmetrical $20 \mathrm{mM} \mathrm{KCl}$ solutions. Below, after addition of $100 \mathrm{nM} \mathrm{KTX}$ to the extracellular side of the lipd bilayer, KcsAKv1.3 channel activity was completely inhibited.

Next, structural changes in KcsA-Kv1.3 associated with a change in pH from 7.5 to 4.0 were monitored by ssNMR (Appendix Figs. D.1, D.2). Detailed ssNMR signal sets are shown in Figure 8.2 a, documenting distinct spectral changes for residues in the selectivity filter, TM2 helix and turret regions (see Appendix Table D.1 for assignments at pH 4.0). The bar diagram in Figure 8.2 b shows the complete set of differences in chemical shift for $\mathrm{C}^{\alpha}$ resonances at $\mathrm{pH} 4.0$ and 7.5. Alterations in side chain resonances are shown in Appendix Figure D.3. Substantial chemical shift changes were observed for the backbone of KcsA-Kv1.3 residues within the pore helix (Glu71, Thr72 and Ala73), selectivity filter (Thr74, Thr75, Val76, Gly77, Tyr78, Gly79 and Asp80), inner TM2 helix (Ala98, Gly99, Ile100, Thr101 and Gly116) and cytoplasmic C-terminal helix (Lys131). The magnitude of these backbone shift changes is $>0.6 \mathrm{ppm}$, that is, large enough to be correlated with substantial structural alteration (see, for example, ref. [153]). By contrast, chemical shift differences are negligible for residues of the TM1 helix and turret, and for TM2 residues facing the extracellular side of the lipid bilayer. Notably, attenuation of signals from some well-resolved residues in the turret and C-terminus was observed (Fig. 8.2 b, arrows). In particular, signals for residues Ala50, Glu51, Asp64, Tyr82, Ala132 and Glu135 could not be detected at $\mathrm{pH}$ 4.0. Taken together, among ssNMR assignments covering $71 \%$ of the KcsA-Kv1.3 pore domain, only a subset of residues shows sizable pH-dependent 
a
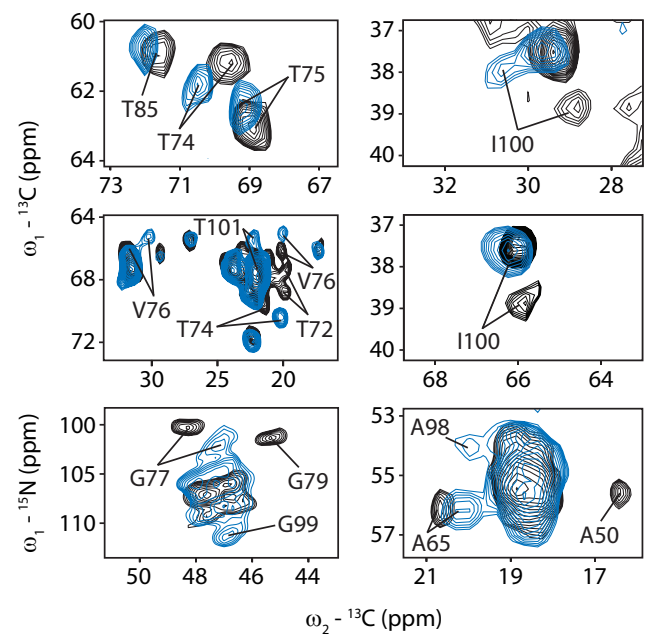

b

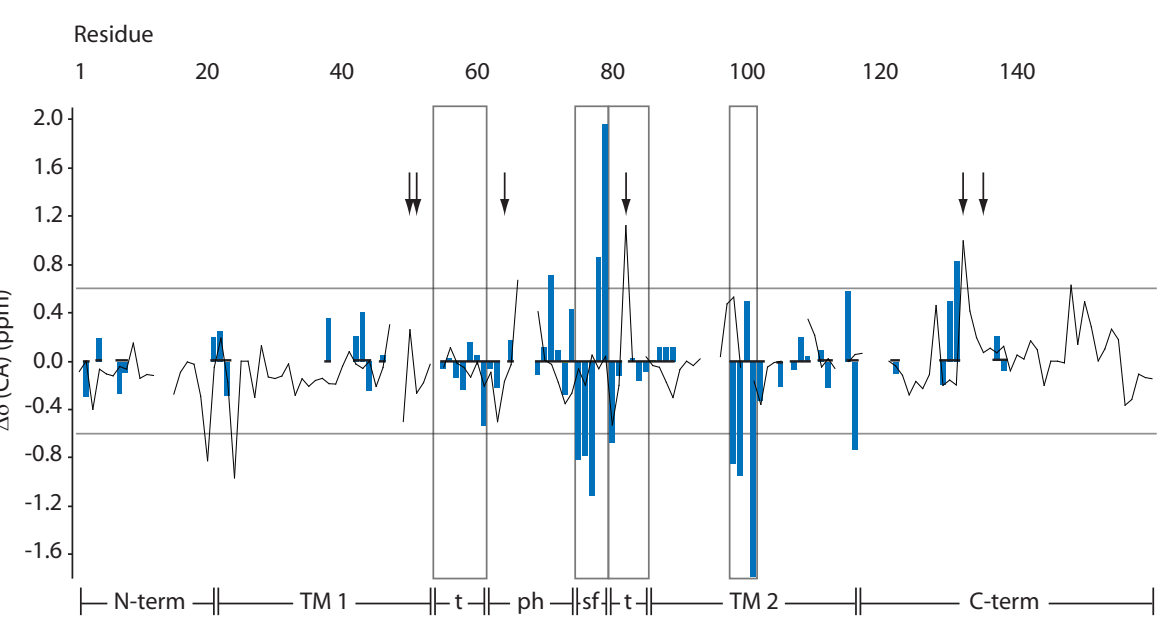

Figure 8.2: Comparison of ssNMR data for KcsA-Kv1.3 at pH 7.5 and $\mathrm{pH}$ 4.0. (a) Sections of homonuclear and heteronuclear ssNMR correlation spectra at pH 7.5 (black) and pH 4.0 (blue). Resonances assigned to residues within the selectivity filter, pore helix, TM2 helix and turret regions are labeled. (b) Summary of $\mathrm{C}^{\alpha}$ chemical shift changes observed for KcsA-Kv1.3 at pH 4.0 (blue bars) compared to $\mathrm{pH} 7.5$. Horizontal lines at $\pm 0.6 \mathrm{ppm}$ indicate the threshold chosen for substantial chemical shift changes. Lines below (N-term, $\mathrm{N}$-terminus; TM1, transmembrane helix 1; t, extracellular turret; ph, pore helix; sf, selectivity filter; TM2, transmembrane helix 2; C-term, C-terminus) schematically show secondary structure along the sequence as seen in ssNMR. Rectangular frames highlight the selectivity filter, TM2 gating hinge and turrets. Arrows point to residues not detectable at $\mathrm{pH}$ 4.0. For comparison, chemical shift changes between $\mathrm{pH} 7.0$ and $\mathrm{pH} 4.0$ described for KcsA in foscholine micelles [242] are shown as a solid black line. 
chemical shift changes. This implies that $\mathrm{pH}$-dependent gating of the KcsA-Kv1.3 channel is associated with defined local conformational changes, which occur within functionally important regions of the $\mathrm{K}^{+}$channel, such as the helix bundle crossing, gating hinge, and selectivity filter regions.

\subsubsection{Structural analysis}

To derive high-resolution structural information from ssNMR data, chemical shift data as presented here have to be complemented with through-space distance information. Indeed, an investigation of proton-proton distances using NHHC and CHHC [62] ssNMR experiments on KcsA-Kv1.3 at pH 7.5 (Appendix Fig. D.4) yielded distance constraints for the KcsA-Kv1.3 selectivity filter in good agreement with the conductive conformation of the selectivity filter in KcsA crystal structures [24, 40]. However, while NHHC and CHHC spectral data obtained for KcsA-Kv1.3 at pH 4.0 may be correlated with structural rearrangements involving lower selectivity filter and gating hinge, corresponding crosscorrelations in the spectra are ambiguous. Moreover, positional changes in (unprotonated) backbone carbonyls of selectivity filter residues are undetectable in CHHC and NHHC experiments [136]. Thus, no attempt was made here to derive high-resolution structural information. However, the distinct relationship between protein backbone structure and ssNMR $\mathrm{C}^{\alpha}$ and $\mathrm{C}^{\beta}$ chemical shift was used to evaluate structural changes associated with $\mathrm{pH}$-dependent gating. For this purpose, surfaces representing simulated secondary chemical shifts depending on the dihedral angles $\phi$ and $\psi$ for selectivity filter (Fig. 8.3 a) and gating hinge residues (Fig. 8.3 b) were computed using SHIFTX [163] (see Section 8.3.3). Simulated values were then compared to experimentally determined secondary chemical shifts [153]. Experimental values determined for KcsA-Kv1.3 at pH 7.5 (black) and $\mathrm{pH} 4.0$ (blue) are given for selectivity filter and gating hinge residues in Figure 8.3 c and Figure 8.3 d, respectively. As discussed in Section 7.4.2 (see Appendix Fig. C.6), secondary chemical shifts measured for KcsA-Kv1.3 at pH 7.5 show excellent agreement with their values predicted on the basis of the crystal structure of the closed KcsA K ${ }^{+}$ channel with a conductive selectivity filter [24] (PDB 1K4C). This is in line with earlier observations [62] and corroborates the findings from N/CHHC spectra discussed above, underlining the validity of using secondary chemical shifts for structural analysis.

With this approach, dihedral angle pairs for residues Thr75, Val76, and Tyr78 were obtained that show optimal agreement between simulated secondary chemical shifts and experimental ssNMR data at pH 4.0 (Fig. 8.3 a, red squares). Using these dihedral an- 
a
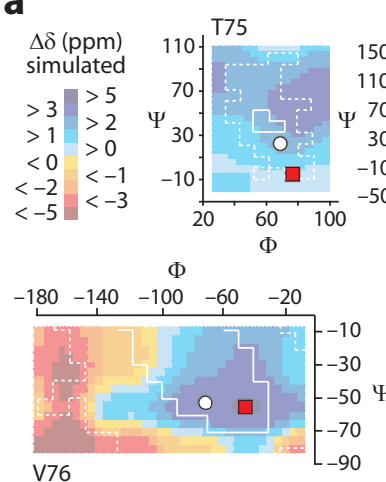

b
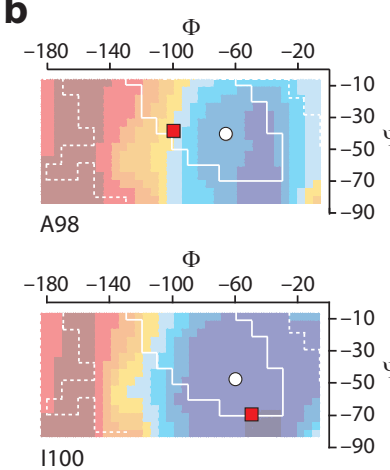
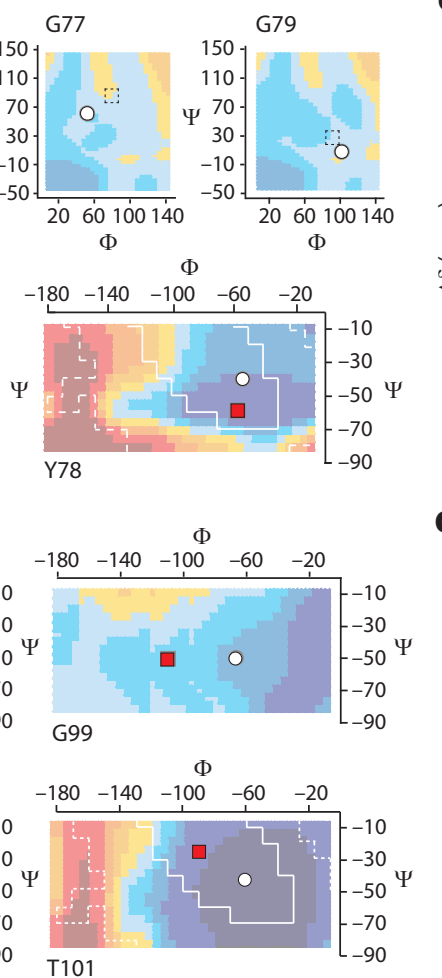

C

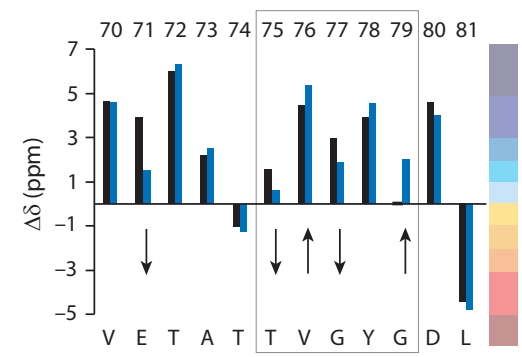

d

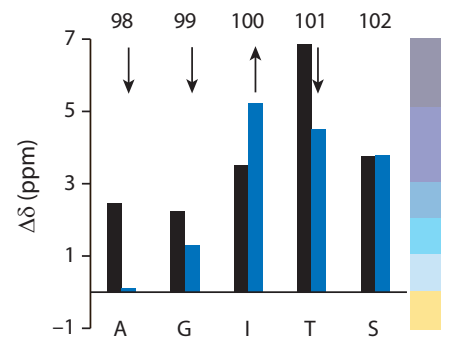

Figure 8.3: Analysis of changes in KcsA-Kv1.3 backbone conformation associated with $\mathrm{pH}$ dependent gating. (a, b) Surfaces representing SHIFTX-calculated secondary chemical shifts depending on dihedral angles $\phi$ and $\psi$ for residues composing the selectivity filter (a) and the TM2 gating hinge (b) are shown as a color code. White circles indicate dihedral angles for the crystal structure of KcsA (PDB 1K4C). Red or broken squares indicate dihedral angle pairs for which the best agreement between SHIFTX-calculated secondary chemical shifts and their experimental values at $\mathrm{pH} 4.0$ is found (for details, see text). Core (white, solid line) and allowed (white, dashed line) regions of Ramachandran space are given according to ref. [178]. (c, d) Experimental secondary chemical shifts (in ppm) for KcsA-Kv1.3 at pH 7.5 (black) and pH 4.0 (blue) are given for residues Val70 to Leu81 (c) and residues Ala98 to Ser102 (d). The black frame in (c) indicates the selectivity filter. Arrows show directions of changes in secondary chemical shift from $\mathrm{pH} 7.5$ to $\mathrm{pH}$ 4.0. For direct comparison to panels (a) and (b), the color gradient on the right illustrates the same color code for secondary chemical shifts as used for plotting SHIFTX-calculated secondary chemical shift surfaces in (a) and (b). 
gles as restraints for energy minimization of the selectivity filter in a CNS [175] simulated annealing protocol, dihedral angle values were also obtained for Gly77 and Gly79. The respective simulated secondary chemical shifts for these glycine residues are in close agreement with experimental data (Fig. 8.3 a, broken squares). A comparison of simulated and experimental secondary chemical shifts shows that backbone torsion angles are altered for selectivity filter residues Thr75 to Gly79 by a few tens of degrees (Appendix Table D.2), indicating that the KcsA-Kv1.3 selectivity filter has adopted a different conformation at $\mathrm{pH} 4.0$.

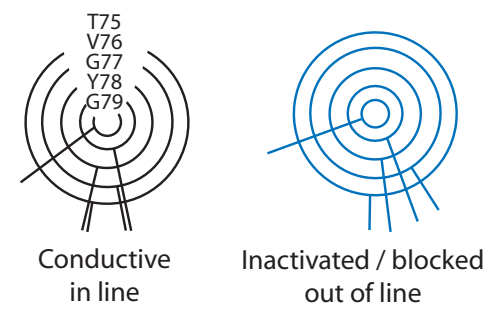

Figure 8.4: Symbolic illustration of $\mathrm{pH}$-dependent positional changes in backbone carbonyls of selectivity filter residues, shown as a Newman-like projection highlighting C'-O bonds. See also Appendix Figure D.6.

A hallmark of the conductive conformation of the $\mathrm{K}^{+}$channel selectivity filter (PDB $1 \mathrm{~K} 4 \mathrm{C}$ ) is a regular, in-line alignment of backbone carbonyls (Appendix Fig. D.6 a). Observed chemical shift changes for residues lining the selectivity filter of inactivated KcsAKv1.3 should thus reflect movement of backbone carbonyls away from their in-line arrangement, as shown in Figure 8.4. KcsA crystal structures in different ionic solutions have shown that the selectivity filter can potentially exist in two distinct, well-defined conformations, presumably corresponding to a conductive and a nonconductive conformation [24, 239]. In the nonconductive conformation, the backbone carbonyl group of Val76 has rotated away from the center of the pore, and the $\mathrm{C}^{\alpha}$ of Gly 77 faces toward the pore. As a result, the filter collapses shut [24]. This conformational change closely resembles that derived for the KcsA-Kv1.3 selectivity filter at $\mathrm{pH} 4.0$ (Appendix Figs. D.5-D.6). Conversely, secondary chemical shifts predicted by SHIFTX for the nonconductive KcsA conformation $[24,239,268]$ agree well with those measured for KcsA-Kv1.3 at pH 4.0 (Appendix Fig. D.7). Thus, a principal conclusion from combining electrophysiological and structural data is that inactivation of the KcsA-Kv1.3 $\mathrm{K}^{+}$channel can be associated with 
a change in selectivity filter conformation that resembles the switch to the nonconductive conformation of the KcsA $\mathrm{K}^{+}$channel.

For the gating hinge region in TM2, an analogous analysis indicated changes in dihedral $(\phi, \psi)$ angles by $20^{\circ}$ on average for four consecutive residues (Ala98, Gly99, Ile100 and Thr101) (Fig. 8.3 b and Appendix Table D.2). Dihedral angles obtained for the backbone of the gating hinge correspond to a bend in the inner TM2 helix in good agreement with structures of $\mathrm{K}^{+}$channels crystallized in an open conformation [40, 41, 269]. This bending is likely coupled to a "swinging out" of the lower part of the TM2 helix. Such a movement would open the inner TM2 helix bundle, which is associated with opening the activation gate at the cytoplasmic entrance to the pore of the channel [40]. While this constitutes a large overall structural rearrangement, local helical secondary structure should largely be preserved [40]. In agreement with this picture, generally, no large $\mathrm{C}^{\alpha}$ and $\mathrm{C}^{\beta}$ chemical shift changes were observed for residues in the lower TM2 helix. However, some substantial chemical shift changes do occur for residues in the C-terminal part of the TM2 helix (Gly116) and early in the C-terminal helix (Lys131) (Fig. 8.2 b), consistent with a change in chemical environment of residues in regions that may be affected by changes in intersubunit distances upon activation gating.

\subsubsection{Comparison to porphyrin binding}

Notably, as discussed in the publication related to this chapter [1], results by Christian Ader reveal that the selectivity filter exhibits similar chemical shift changes as seen in the free channel at pH 4.0 upon binding a water-soluble tetraphenylporphyrin derivative (Section 8.3, Fig. D.8 a) to KcsA-Kv1.3 at pH 7.5. No chemical shift changes are observed in regions outside of the selectivity filter, however (Appendix Figs. D.9, D.10). The porphyrin ligand binds to the $\mathrm{Kv} 1.3 \mathrm{~K}^{+}$channel with nanomolar affinity and inhibits its activity. In competition binding experiments [265], it was shown that porphyrin displaces a high-affinity pore blocker such as ${ }^{125}$ I-KTX (ref. [62]). The competition binding curve for KcsA-Kv1.3 is well described assuming a 1:1 stoichiometry and a direct displacement of ${ }^{125}$ I-KTX by porphyrin [1] (Appendix Fig. D.8 b). Further ssNMR experiments probing the ${ }^{1} \mathrm{H}$ environment of the channel-bound porphyrin, as well as a molecular dynamics-based in silico ligand-protein docking and electrophysiological tests of the voltage sensitivity of porphyrin binding, all point to the same binding mode of the porphyrin to KcsA-Kv1.3. One porphyrin molecule appears to bind the channel by inserting one of its four arms into 
the selectivity filter, where the protonated amine favorably interacts with $\mathrm{K}^{+}$binding site S3 [1] (Appendix Fig. D.11).

\subsection{Discussion}

Understanding structural alterations that accompany gating of ion channels is a key issue in ion channel physiology and biophysics. Here, the conformational changes that occur during gating of a functional $\mathrm{K}^{+}$channel were studied at the atomic level using ssNMR spectroscopy of KcsA-Kv1.3 channel preparations in liposomes. It could be shown that the KcsA-Kv1.3 channel is closed at pH 7.5, and that it is opened by an increase in intracellular ${ }^{1} \mathrm{H}$ concentration, as seen for the parent KcsA channel. The activated KcsA-Kv1.3 $\mathrm{K}^{+}$ channel rapidly enters an inactivated state during a persistent ${ }^{1} \mathrm{H}$ stimulus. Thus, the KcsA-Kv1.3 $\mathrm{K}^{+}$channel predominantly resides under steady-state conditions at $\mathrm{pH} 7.5$ in a closed state and at pH 4.0 in an inactivated state. SsNMR experiments conducted on the two channel states show distinct conformational differences in channel domains that are of prime importance for $\mathrm{K}^{+}$channel gating, such as the selectivity filter, gating hinge, and inner helix bundle. These differences are likely to reflect conformational changes associated with a transition from a closed to an inactivated state of the KcsA-Kv1.3 $\mathrm{K}^{+}$ channel.

Previous work established that conformational transitions occurring in the selectivity filter are related to $\mathrm{K}^{+}$channel inactivation [39]. The selectivity filter has the potential to adopt two distinct, well-defined structures corresponding to a conductive and a nonconductive conformation, respectively. The two conformations are coupled to $\mathrm{K}^{+}$ion occupancy in the filter, particularly at $\mathrm{K}^{+}$-selective sites 2 and 3 . At low $\mathrm{K}^{+}$concentration, the selectivity filter attains a nonconductive ("collapsed") conformation [24, 239, 268]. A nonconductive, potassium-depleted conformation of the selectivity filter has been implicated in C-type inactivation $[24,39,239,270]$. In particular, the collapsed conformation is also assumed upon binding of intracellular tetrabutylammonium (TBA) to KcsA, which is known to accelerate C-type inactivation [268]. A similar conformation in the selectivity filter is seen in KcsA-Kv1.3 preparations at pH 4.0 (Appendix Figs. D.5-D.7). Additional structural alterations were detected in the pore helix on the back side of the filter involving interactions between Glu71 and Asp80. This is in excellent agreement with mutational and biophysical studies, which indicate a hydrogen bond network behind the selectivity filter that promotes the inactivated state of the $\operatorname{KcsA~K}{ }^{+}$channel $[4,235,262,263,264]$. 
The strength of the interaction between Glu71 and Asp80, which are connected within this network by a water-mediated carboxyl-carboxylate interaction, has a key role in inactivation gating [4, 263]. Sizable chemical shift changes were observed for residues Glu71 and Asp80 associated with $\mathrm{pH}$-induced gating of the KcsA-Kv1.3 $\mathrm{K}^{+}$channel. In particular, the ${ }^{13} \mathrm{C}$ resonance of the sidechain carboxyl group of Glu71 experiences a change to a smaller isotropic chemical shift, indicative of increased protonation as expected for strengthened hydrogen-bonding at pH 4.0 [271, 272].

After a $\mathrm{pH}$ change from 7 to 4 , a recent NMR study using $\mathrm{KcsA}^{+}$channel preparations in foscholine micelles [242] reported only subtle structural changes regarding the hydrogen bond network between Tyr78, Gly79, Glu71 and Asp80 (see Fig. 8.2 b). The differences between solution and ssNMR data may not be surprising, considering that membrane proteins require a special membrane setting to function. Possibly, the conformation of KcsA in micelles is intrinsically unstable at pH 4.0 (as observed for SDS micelles at $\mathrm{pH} 4.2$, see ref. [248]) and fluctuates between a permeating and nonpermeating conformation. By contrast, the KcsA-Kv1.3 $\mathrm{K}^{+}$channel attains in liposomes a more stable, nonconducting conformation, in which the inner helix shows an opened activation gate and the selectivity filter has collapsed shut (Fig. 8.5 a, b).

By inserting one of its arms into the selectivity filter during binding, porphyrin appears to induce the same nonconductive selectivity filter conformation in KcsA-Kv1.3 at pH 7.5 as seen at pH 4.0 in the free channel (ref. [1] and Appendix Fig. D.11). The underlying common principle seems to be that both channel block by porphyrin and inactivation generate a low $\mathrm{K}^{+}$occupancy in the selectivity filter. Consistent with a large body of functional $\mathrm{K}^{+}$channel data [39, 235, 263], data presented here imply that inactivation gate closure can be associated with a switch from a conductive to a nonconductive conformation in the selectivity filter. It is proposed that this event is accompanied by distinct alterations in backbone structure such that the carbonyl oxygen atoms are moved out of line, disrupting $\mathrm{K}^{+}$ion binding and conduction. The structural changes inferred from ssNMR analysis, however, are smaller than those predicted from molecular dynamics simulations [261] or those seen in a crystal structure of a non-inactivating KcsA mutant [235].

In comparison to the porphyrin-bound state of KcsA-Kv1.3, ssNMR data obtained at $\mathrm{pH} 4.0$ indicate unique $\mathrm{pH}$-induced structural alterations in the KcsA-Kv1.3 pore domain. These include sidechain chemical shift changes for residues in the lower part of the selectivity filter and the pore helix, as well as additional conformational changes in backbone 
a

Kv1.2 pore domain
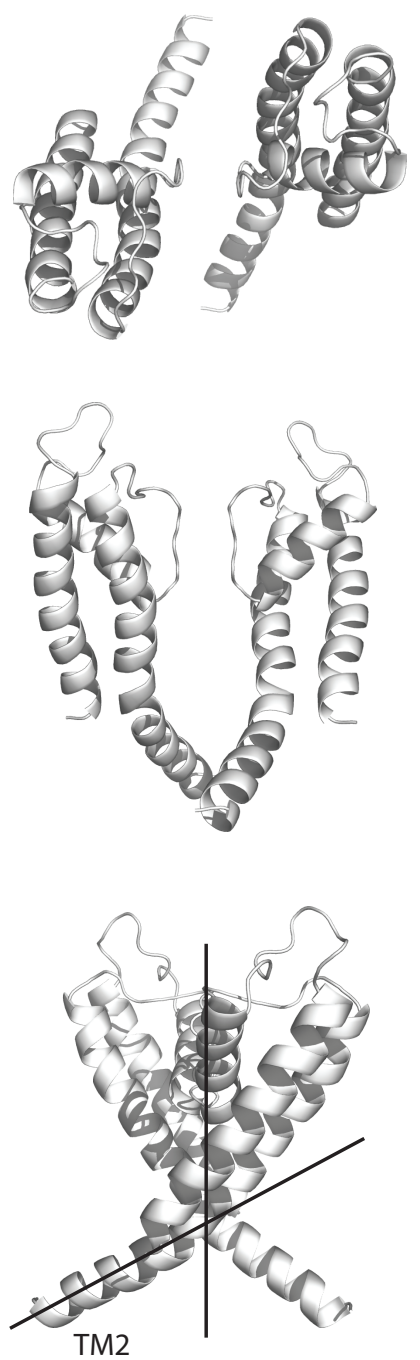

b
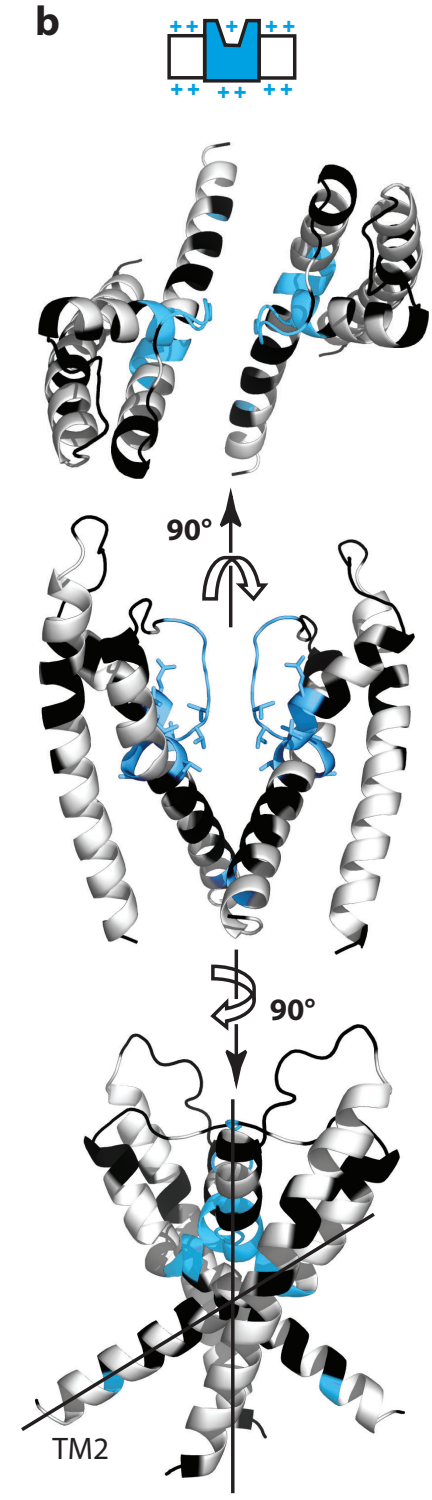

C
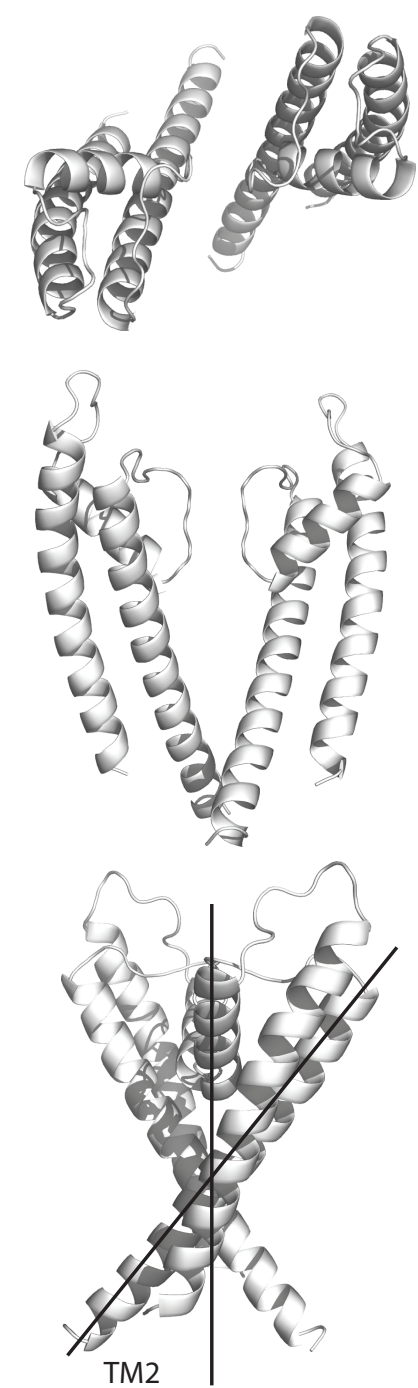

Figure 8.5: Structural model of $\mathrm{KcsA}-\mathrm{Kv} 1.3$ at $\mathrm{pH}$ 4.0. For comparison, the crystal structure of the pore domain of Kv1.2 (ref. [41]; PDB 2A79) is shown in (a). The KcsA-Kv1.3 structural model from Chapter 7 is depicted in (b), with dihedral angles in selectivity filter and TM2 gate as obtained by chemical shift analysis and restrained simulated annealing (see Section 8.3.3). Residues assigned both at $\mathrm{pH} 4.0$ and 7.5 are colored blue if chemical shift changes above 0.6 ppm were observed between the two states and black otherwise; unassigned residues are grey. Sidechains are indicated for residues with considerable sidechain chemical shift changes. (c) shows the pore domain of the KcsA-Kv1.3 structural model at $\mathrm{pH}$ 7.5. Lines in the bottom row represent the four-fold symmetry axis of the channel and the helical TM2 axis, highlighting the structural reorientation of the TM2 helix associated with a bend in the TM2 gating hinge. 
and sidechains of residues within the intracellular half of the KcsA-Kv1.3 $\mathrm{K}^{+}$channel. Here, structural data are consistent with those derived from crystal structures of opened $\mathrm{K}^{+}$channel conformations [21, 40,41] (Fig. 8.5 a, b). In particular, secondary chemical shift analysis indicates bending of the inner TM2 helix at the highly conserved gating hinge around residue Gly99. Bending at a gating hinge of the inner TM2 helix has been attributed a key role in opening the activation gate associated with the inner helix bundle [40]. SsNMR data can thus resolve a debate about the exact nature of the conformational change occurring during KcsA activation gate opening. While EPR spectroscopy data have suggested a rotation and a relatively small tilt of the entire TM2 helices [273], site-directed mass-tagging has indicated larger rearrangements limited to the lower pore region below the TM2 gating hinge, as observed in crystal structures of open potassium channels $[40,274]$. KcsA-Kv1.3 data presented here thus clearly support the latter view.

Direction and magnitude of the bend in the KcsA-Kv1.3 TM2 helix at pH 4.0 are consistent with the idea that the inner helix bundle of the KcsA-Kv1.3 $\mathrm{K}^{+}$channel adopts an opened conformation at $\mathrm{pH}$ 4.0. The implication is then that, at $\mathrm{pH} 4.0$, the KcsAKv1.3 channel shows an opened activation gate and a collapsed selectivity filter, in good agreement with a recent proposal for the conformation of the inactivated $\mathrm{KcsA} \mathrm{K}^{+}$channel characterized by an opened activation and a closed inactivation gate [263]. The observed changes in sidechain chemical shifts for residues in gating hinge, pore helix and lower selectivity filter regions suggest that steric interaction between the two gates is possible (see also Chapter 9).

Notably, in the ssNMR experiments shown in this chapter, the final reconstitution buffer for KcsA-Kv1.3 contained no added potassium. Different $\mathrm{K}^{+}$concentrations may, however, affect the KcsA-Kv1.3 conformation. This will be the focus of Chapter 9 . 


\section{Chapter 9}

\section{Effects of potassium on activation and inactivation gating in KcsA-Kv1.3}

\subsection{Summary}

Ion concentration and effector molecules widely control the activity of ion channels by modulating the opening and closing of the channel pores. This chapter describes solidstate NMR and electrophysiological experiments on KcsA-Kv1.3 that were conducted to delineate the influence of potassium, $\mathrm{pH}$ and channel blockers on its activation and inactivation gates in a lipid bilayer environment. Data presented here show that activation gate opening at acidic $\mathrm{pH}$ is correlated with protonation of residues in the lower TM2 gate. Potassium strongly reduces the steady-state open probability of the activation gate as seen from ssNMR chemical shift data and experiments probing the water-accessible surface of KcsA-Kv1.3. Furthermore, toxin (KTX) binding to the external mouth of the KcsA-Kv1.3 channel inhibits gate opening at $\mathrm{pH} 4.0$ even in the absence of external potassium. These results reveal that potassium as well as the toxin influence the open probability of the TM2 gate via the selectivity filter. Thus, the two gates are coupled, and effects of the permeant ion on the inactivation gate modulate activation gating, suggesting a mechanism for coordinated opening and closing of activation and inactivation gates. ${ }^{1}$

\footnotetext{
${ }^{1}$ This chapter is related to the following publication: Christian Ader*, Robert Schneider*, Sönke Hornig, Phanindra Velisetty, Vitya Vardanyan, Karin Giller, Iris Ohmert, Stefan Becker, Olaf Pongs, and Marc Baldus: Coupling of activation and inactivation gate in a $\mathrm{K}^{+}$channel: potassium and ligand sensitivity. EMBO Journal, accepted for publication, 2009. (*equally contributing authors)
} 


\subsection{Introduction}

As described in Chapter 8, the KcsA-Kv1.3 $\mathrm{K}^{+}$channel, like other $\mathrm{K}^{+}$channels, contains two gates controlling ion flow, an activation (lower) and an inactivation (upper) gate $[39,235,275]$. These gates are associated with the inner transmembrane helix (TM2) bundle and the selectivity filter, respectively. Both gates may occur in opened or closed states. Hence, KcsA-Kv1.3 channel gating can be depicted with a simplified gating cycle describing equilibria between four composite gating states, namely closed state C, opened state $\mathrm{O}$, and inactivated states I and I* (Fig. 9.1) [275]. Within this framework, different KcsA-Kv1.3 channel conformations can operationally be correlated with distinct gating states. For example, as shown in Chapter 8 , inactivated states are characterized by a collapsed selectivity filter, which may occur together with either an opened (I) or a closed lower activation gate $\left(\mathrm{I}^{*}\right)$.

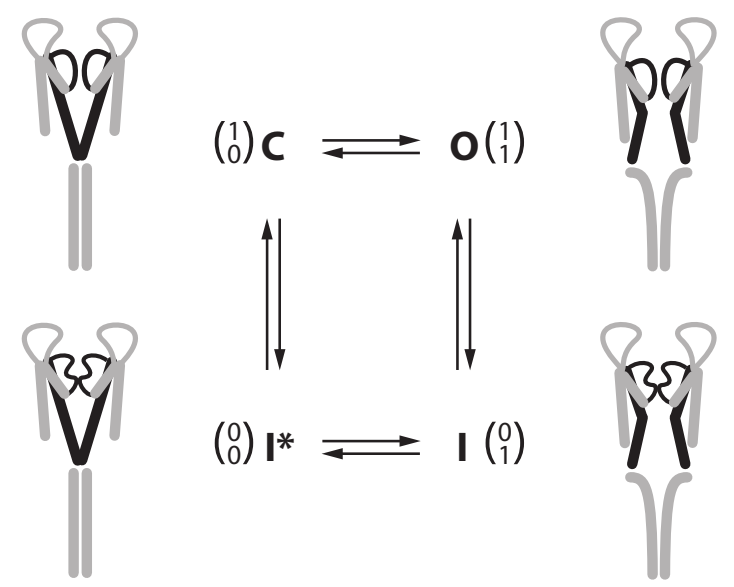

Figure 9.1: A four-state minimal gating model with an upper (inactivation) and a lower (activation) gate in the conduction pathway of the KcsA-Kv1.3 channel. Gate positions ( 0 for closed and 1 for opened) are diagrammed in one-column matrices with upper gate in upper and lower gate in lower row. $\mathrm{C}$ - closed channel in resting state; $\mathrm{O}$ - opened channel; I and $\mathrm{I}^{*}$ - inactivated states. Pictograms of two KcsA-Kv1.3 subunits (compare Fig. 7.8) sketch corresponding conformational states of selectivity filter and TM2 helix (black). TM1 and pore helices, turret loop and C-terminus are also indicated (grey). The $\mathrm{N}$-terminus is omitted for clarity.

In Chapters 7 and 8, structural information on two states within this gating cycle was obtained, namely states $\mathrm{C}$ and I. Starting from state $\mathrm{C}$ at neutral $\mathrm{pH}$, it was shown that acidic $\mathrm{pH}$ elicits opening of the activation gate by bending the TM2 helices, followed by a conformational change in the selectivity filter which closes the inactivation gate, leading to 
state I (Chapter 8). It is likely that coupling between the two gates regulates channel open probability and sequentially coordinates activation and inactivation gating. In the family of voltage-gated $\mathrm{K}^{+}(\mathrm{Kv})$ channels, opening and closing of activation and inactivation gates were shown to be coupled [275, 276, 277, 278]. However, it is not well understood how sequential activity of the two gates in the $\mathrm{K}^{+}$channel pore domain is coordinated.

Extensive electrophysiological studies on Shaker channels indicated that inactivation and possibly also activation gating are $\mathrm{K}^{+}$-sensitive $[38,39,276]$. Thus, in the following, combined functional and ssNMR-structural investigations on $\mathrm{pH}$ - and $\mathrm{K}^{+}$-sensitive gating transitions in KcsA-Kv1.3 are reported. Using the exquisite sensitivity of ssNMR data to changes in both local and global structure as well as protonation state, the influence of $\mathrm{pH}$ and $\left[\mathrm{K}^{+}\right]$on activation and inactivation gates is delineated. In order to further study coordinated structural changes at the two gates, the inactivation gate was trapped in two different functional states with external pore blockers to the $\mathrm{K}^{+}$channel, kaliotoxin (KTX) and porphyrin (see Chapter 8 and refs. [1, 62]). Structural and functional results show that both $\mathrm{pH}$ and potassium strongly influence the open probability of the activation gate. The effect of potassium is mediated by distinct binding sites in the selectivity filter, which shows that the inactivation gate can influence activation gating. These findings suggest a general self-regulatory mechanism for controlling sequential gating activity in $\mathrm{K}^{+}$channels, with the permeant ion regulating the onset of ion channel activation as well as inactivation.

\subsection{Materials and Methods}

\subsubsection{Sample preparation}

KcsA-Kv1.3 expression, purification and reconstitution into asolectin liposomes were done by Karin Giller and Stefan Becker as described [1, 62] (see also Chapter 7). Reconstitution was performed at a 100:1 asolectin / KcsA-Kv1.3 molar ratio in either $50 \mathrm{mM}$ sodium phosphate, $\mathrm{pH} 7.4$, with $50 \mathrm{mM} \mathrm{NaCl}$ or $\mathrm{KCl}$, or in $10 \mathrm{mM}$ MOPS (3-(Nmorpholino)propanesulfonic acid), pH 7.4, with $150 \mathrm{mM} \mathrm{KCl}$. KTX and porphyrin were added in twofold molar excess as described (see Chapter 8 and refs. [1, 62]). pH and potassium titrations were performed by washing the proteoliposomal pellet three times with $1 \mathrm{ml}$ of the desired buffer, followed by $30 \mathrm{~min}$ ultracentrifugation at $45000 \mathrm{rpm}$ and $+4^{\circ} \mathrm{C}$. Buffers used for $\mathrm{pH} 4.0$ were $100 \mathrm{mM}$ citric acid / sodium citrate, $50 \mathrm{mM}$ citric acid / sodium citrate with up to $50 \mathrm{mM} \mathrm{KCl}$, or $10 \mathrm{mM}$ MOPS with $150 \mathrm{mM} \mathrm{KCl}$. 


\subsubsection{Solid-state NMR}

All NMR experiments were conducted using $4 \mathrm{~mm}$ triple-resonance $\left({ }^{1} \mathrm{H},{ }^{13} \mathrm{C},{ }^{15} \mathrm{~N}\right)$ probeheads at static magnetic fields of 9.4, 14.1, and $18.8 \mathrm{~T}$ corresponding to 400,600, and 800 $\mathrm{MHz}$ proton resonance frequencies (Bruker Biospin, Karlsruhe, Germany). Assignments obtained previously (Chapter 7 ) were extended and verified by $2 \mathrm{D}\left({ }^{13} \mathrm{C},{ }^{13} \mathrm{C}\right)$ correlation experiments using proton-driven spin diffusion under weak coupling conditions (PDSD-WC) [125]. Water-edited and ligand binding ssNMR experiments were performed by Christian Ader. Water-edited experiments were conducted and analyzed as described in ref. [3], using a $3 \mathrm{~ms}$ Gaussian $\pi / 2$ pulse, a T2 filter containing two delays $(\tau)$ of $1 \mathrm{~ms}$, and a cross-polarization contact time of $700 \mu \mathrm{s}$. MAS speeds used were $6.5,9.375$, and $12.5 \mathrm{kHz}$ at $9.4,14.1$, and $18.8 \mathrm{~T}$ static magnetic field strengths, respectively, at an effective sample temperature of approximately $+7^{\circ} \mathrm{C}$ (calibrated using nickelocene [222]). Integration of spectral cross peaks was performed using the software Topspin 2.1 (Bruker Biospin, Karlsruhe, Germany). Error estimates for spectral integrals were obtained from integrals in noise regions with sizes equal to those of the signal integration regions.

\subsubsection{Electrophysiology}

Electrophysiological measurements on KcsA-Kv1.3 in proteoliposomes were conducted and analyzed by Sönke Hornig, Phanindra Velisetty, Vitya Vardanyan, and Olaf Pongs as described in Chapter 8 and ref. [1]. However, in patch-clamp recordings, $\mathrm{K}^{+}$concentrations in solutions corresponding to intracellular $\left(\left[\mathrm{K}^{+}\right]_{\text {in }}\right)$ and extracellular $\left(\left[\mathrm{K}^{+}\right]_{\text {out }}\right)$ solutions were varied from 0 to $150 \mathrm{mM}$ while keeping the total monovalent cation concentration constant at $150 \mathrm{mM}$ with sodium (see Fig. 9.6 legend for details).

\subsection{Results}

\subsubsection{Potassium sensitivity of KcsA-Kv1.3 conformation}

In Chapters 7 and 8 it was shown that, in proteoliposomes at pH 7.5, the KcsA-Kv1.3 channel adopts a structure resembling the crystal structure of detergent-solubilized KcsA obtained at high $\mathrm{K}^{+}$concentrations [24] (PDB 1K4C). The structure, designated as closedconductive conformation, represents a KcsA-Kv1.3 channel with a closed activation gate and a conductive filter conformation, corresponding functionally to a closed $\mathrm{K}^{+}$channel at rest. To investigate the $\mathrm{K}^{+}$-sensitivity of the closed-conductive conformation of 
KcsA-Kv1.3 in asolectin liposomes, ssNMR spectra at $\mathrm{pH} 7.5$ were recorded with $\mathrm{K}^{+}$concentrations varying from 0 to $150 \mathrm{mM}$ (see Fig. 9.2 and Appendix Fig. E.1). SsNMR

a no $K^{+}$
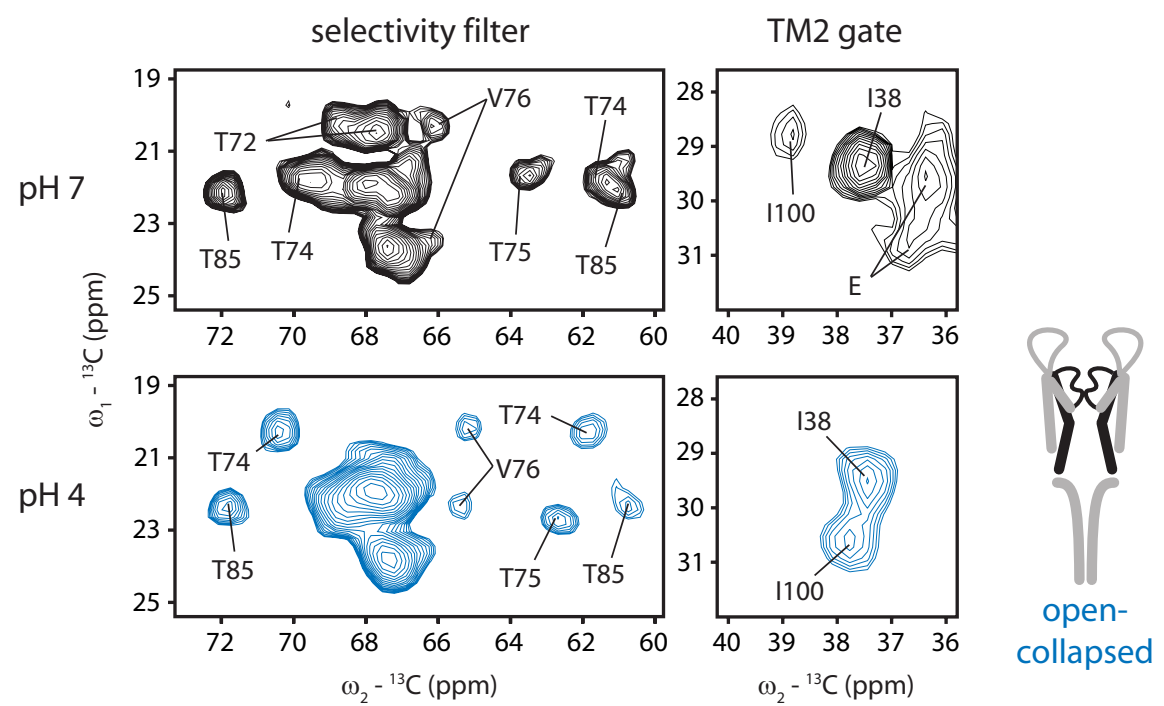

b

$$
50 \mathrm{mM} \mathrm{K}^{+}
$$
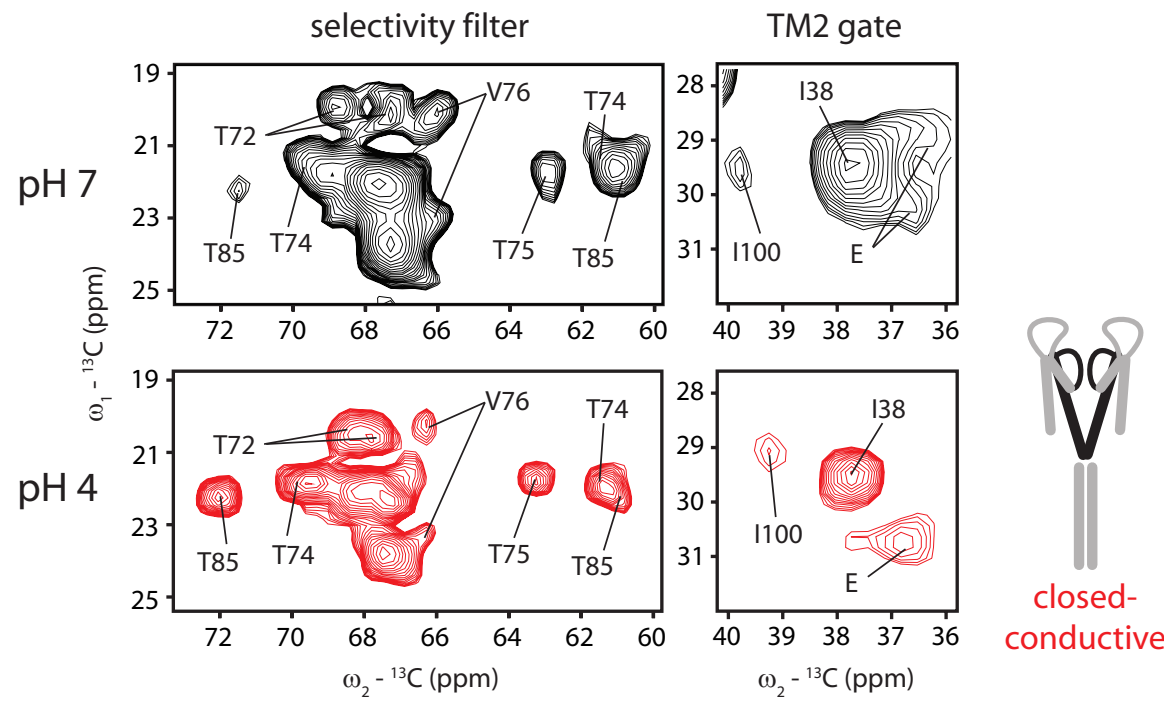

conductive

Figure 9.2: The open-collapsed conformation of KcsA-Kv1.3 is induced stably at $\mathrm{pH} 4.0$ in the absence of potassium (a), but not in the presence of millimolar concentrations of potassium (b) as seen from chemical shift analysis.

results show a closed-conductive $\mathrm{K}^{+}$channel conformation irrespective of the $\mathrm{K}^{+}$concentration in the buffer. For example, virtually identical ssNMR spectra were obtained 
for KcsA-Kv1.3 proteoliposomes reconstituted in no- $\mathrm{K}^{+}$and high- $\mathrm{K}^{+}$buffers (Fig. 9.2 a, b, black spectra). This was unexpected, since previous KcsA crystallization experiments showed a collapsed conformation of the selectivity filter in low $\left[\mathrm{K}^{+}\right](5 \mathrm{mM})[24]$. A likely explanation is that, in the lipidic environment used, the conductive filter conformation is less sensitive to absence of $\mathrm{K}^{+}$in the buffer.

A change in $\mathrm{pH}$ from 7.5 to 4.0 activates the KcsA-Kv1.3 channel, and in the continued presence of protons the activated channel inactivates within seconds, reflecting a conformational transition from a conductive to a non-conductive state (see Chapter 8 and ref. [1]). Accordingly, ssNMR spectra were recorded with KcsA-Kv1.3 proteoliposome preparations at different $\mathrm{K}^{+}$concentrations (0 to $150 \mathrm{mM}$ ) after shifting the $\mathrm{pH}$ from 7.5 to 4.0. Overall ionic strength of the buffers was kept constant in all samples by replacing potassium with sodium. Spectra measured at $\mathrm{K}^{+}$concentrations $\geq 10 \mathrm{mM}$ display resonances essentially identical to those recorded at $\mathrm{pH} 7.5$, both in phosphate/citrate and MOPS buffer systems. This was also observed in C-terminally truncated KcsA-Kv1.3 comprising only residues 1125 (Fig. 9.2 b, red spectra, and Appendix Fig. E.1). Only at $10 \mathrm{mM}\left[\mathrm{K}^{+}\right]$, one additional peak corresponding to a Ile100 sidechain conformation as seen in the open-collapsed state begins to appear, while all other signals from gating hinge and selectivity filter still clearly correspond to the closed-conductive state (Appendix Fig. E.1). The data demonstrate that, in the presence of millimolar $\mathrm{K}^{+}$concentrations, the closed-conductive conformation representing a KcsA-Kv1.3 channel at rest is the prevailing conformation at pH 4.0 in asolectin liposomes. This observation is consistent with electrophysiological recordings showing a low open probability $\left(P_{o}=\sim 0.06\right)$ for the activated KcsA-Kv1.3 channel at steady-state (see Chapter 8). In contrast, as discussed in Chapter 8, spectra at pH 4.0 in low $\mathrm{K}^{+}$concentrations $(\leq 1 \mu \mathrm{M})$ are compatible with an open-collapsed conformation of the $\mathrm{K}^{+}$channel. Apparent chemical shift changes for pore-domain residues, e.g. Thr74, Val76, Ile100, indicate a pore structure in which the activation gate has opened and the selectivity filter has adopted a collapsed conformation (Fig. 9.2 a, blue spectra).

Activation-gate opening of $\mathrm{K}^{+}$channels is associated with an outward movement of the inner (TM2) helices around a gating hinge (Chapter 8). This relatively large conformational change consequently increases the water accessible surface of the channel, particularly of the inner half of the pore domain, and provides an independent means to study activation-gate opening of the KcsA-Kv1.3 channel at acidic pH. Consequently, the water-accessible channel surface was evaluated by measuring magnetization transfer from selectively excited water protons to channel protein spins [3, 59, 145] (see Section 
4.3.5). Magnetization build-up is well described with an initial-rate time constant $\sqrt{t_{m}^{s}}$ that is sensitive to changes in water-accessible channel surface [3]. Results obtained from

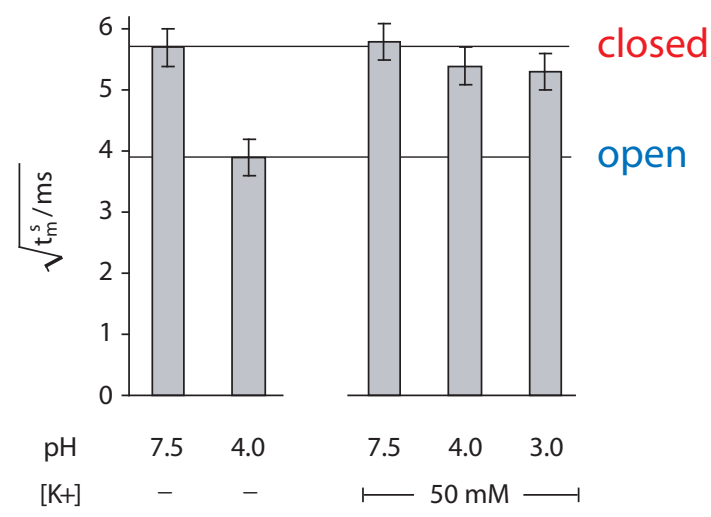

Figure 9.3: Water-edited ssNMR spectroscopy reveals water-protein magnetization transfer dynamics corresponding to a closed channel down to $\mathrm{pH} 3.0$ in the presence of $50 \mathrm{mM}$ potassium.

this type of experiment are bar graphed in Figure 9.3. The data show that the time constant $\sqrt{t_{m}^{s}}$ is unaffected by changing the $\mathrm{pH}$ from 7.5 to 4.0 in $50 \mathrm{mM} \mathrm{K}^{+}$(Fig. 9.3) $\left(\sqrt{t_{m}^{s}} \mathrm{pH} 7.5=5.8 \pm 0.4 \mathrm{~ms}^{1 / 2} ; \sqrt{t_{m}^{s}} \mathrm{pH} 4.0=5.4 \pm 0.4 \mathrm{~ms}^{1 / 2}\right)$, in agreement with the observation that a closed-conductive $\mathrm{K}^{+}$channel conformation largely prevails under these conditions. In contrast, in low $\mathrm{K}^{+}$concentrations $(\leq 1 \mu \mathrm{M})$, a shift to acidic $\mathrm{pH}$ markedly decreases $\sqrt{t_{m}^{s}}\left(\sqrt{t_{m}^{s}} \mathrm{pH} 4.0=3.9 \pm 0.4 \mathrm{~ms}^{1 / 2}\right)$ (Fig. 9.3). The observed change in $\sqrt{t_{m}^{s}}$ corresponds to an increase of the water-accessible protein surface of approximately $60 \%$. It signifies a marked conformational change of the channel and lends independent support to the observation that, in the absence of $\mathrm{K}^{+}$, acidic $\mathrm{pH}$ induces a conformational change characterized - in addition to a collapsed filter structure - by a stably opened activation gate. These results have two important implications. First, acidic $\mathrm{pH}$, which opens the KcsA-Kv1.3 channel, renders the selectivity filter vulnerable to inactivation. Second, the probability of activation gate opening at acidic $\mathrm{pH}$ is $\mathrm{K}^{+}$-sensitive.

\subsubsection{Correlation of glutamate protonation with activation gating}

Mutational studies on KcsA have suggested that activation gate opening at acidic $\mathrm{pH}$ is associated with protonation of glutamate side chains, notably Glu118 and Glu120 at the lower end of the inner TM2 helix [279]. The isotropic chemical shift of glutamate $\delta$ carbon atoms is correlated with protonation state $[271,272]$. Thus, the influence of $\mathrm{pH}$ on NMR resonance frequencies of KcsA-Kv1.3 glutamate residues was investigated to 
monitor activation gate opening. KcsA-Kv1.3 subunits contain nine glutamates each, four in the pore domain (residues 51, 71, 118 and 120) and five in the cytoplasmic C-terminus. Figure 9.4 a-d depicts sections of $\left({ }^{13} \mathrm{C},{ }^{13} \mathrm{C}\right)$ correlation spectra showing glutamate $\mathrm{C}^{\gamma}-\mathrm{C}^{\delta}$
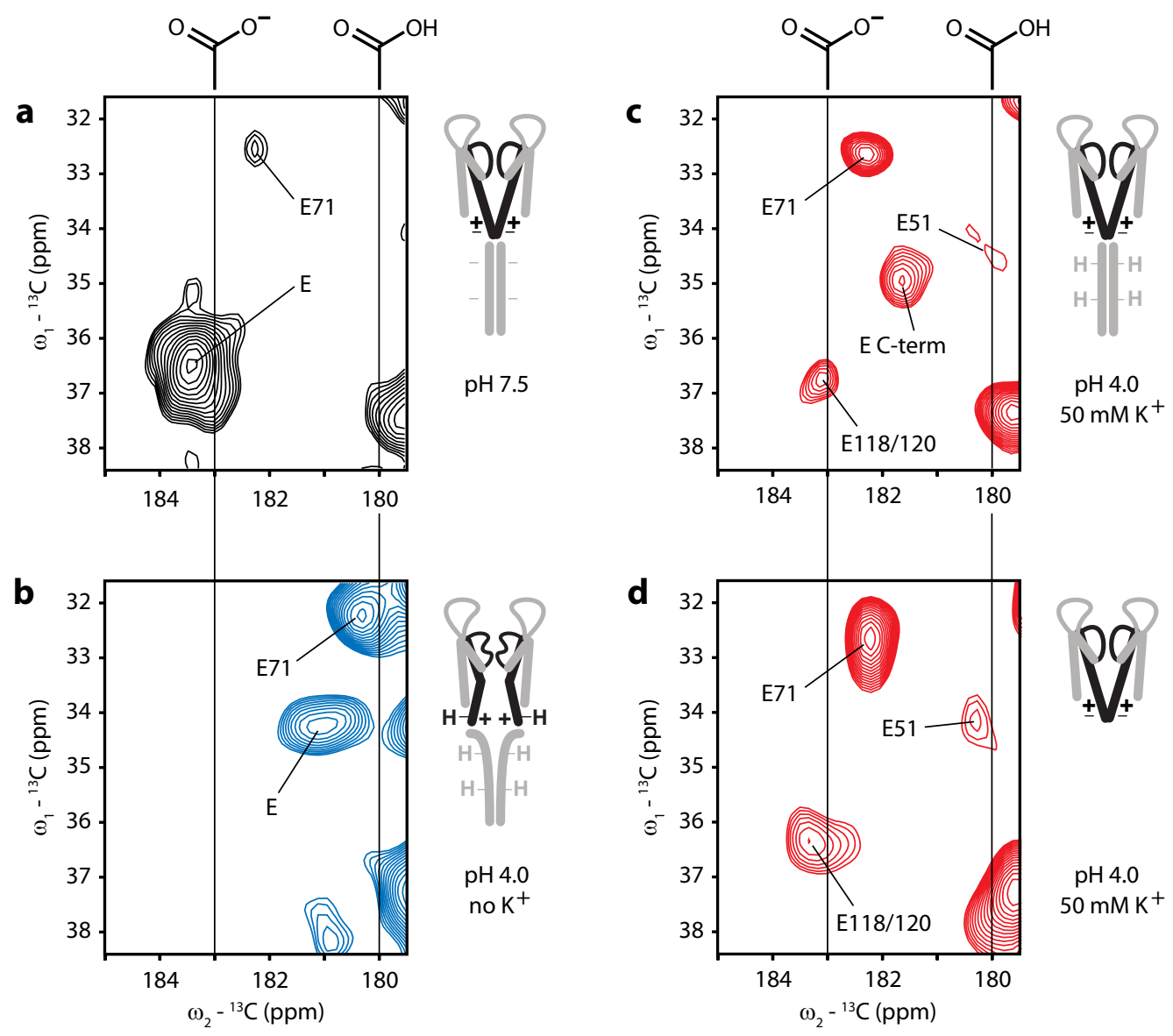

Figure 9.4: Protonation of glutamate sidechain carboxyls depends on $\mathrm{pH}$ and $\left[\mathrm{K}^{+}\right]$. (a-d) Regions from $\left({ }^{13} \mathrm{C},{ }^{13} \mathrm{C}\right)$ correlation spectra showing glutamate $\mathrm{C}^{\gamma}-\mathrm{C}^{\delta}$ crosspeaks for different $\mathrm{pH}$ and $\left[\mathrm{K}^{+}\right]$ conditions, as indicated below the pictograms. Literature values for protonated and deprotonated glutamate $\mathrm{C}^{\delta}$ chemical shifts are indicated by vertical lines in the spectra. Charge and protonation states in lower TM2 helix and C-terminus are shown schematically in the pictograms.

crosspeaks providing information about glutamate protonation states present at different $\mathrm{pH}$ and $\left[\mathrm{K}^{+}\right]$conditions. The chemical shift of $\mathrm{C}^{\delta}$ resonances suggests that, at $\mathrm{pH} 7.5$, all glutamates but Glu71 are in a deprotonated state and at $\mathrm{pH} 4.0$, in the absence of $\mathrm{K}^{+}$, in a more protonated state (Fig. 9.4 a, b). Notably, the magnitudes of chemical shift changes are significantly larger than in previous pH-dependent NMR studies of KcsA in solution 
$[242,247]$. The Glu71 $\mathrm{C}^{\delta}$ resonance at $\mathrm{pH} 7.5$ is consistent with a (partly) protonated glutamate side chain, in agreement with the finding that Glu71 and Asp80 form a watermediated hydrogen bond behind the selectivity filter in KcsA crystal structures [24, 263]. In contrast, in the presence of $>10 \mathrm{mM}$ potassium at $\mathrm{pH} 4.0$, two new glutamate $\mathrm{C}^{\gamma}-\mathrm{C}^{\delta}$ crosspeaks are observed in addition to those previously assigned to Glu51 and Glu71 (see Chapter 7) (Fig. 9.4 c). Comparison of ssNMR spectra of full-length and C-terminally truncated KcsA-Kv1.3 (Fig. 9.4 c, d) shows that removal of the C-terminus selectively eliminates one of these two glutamate $\mathrm{C}^{\gamma}-\mathrm{C}^{\delta}$ crosspeaks. This peak was therefore assigned to the five C-terminal glutamates and the remaining one to Glu118 and Glu120 (Fig. 9.4 $\mathrm{c}, \mathrm{d})$.

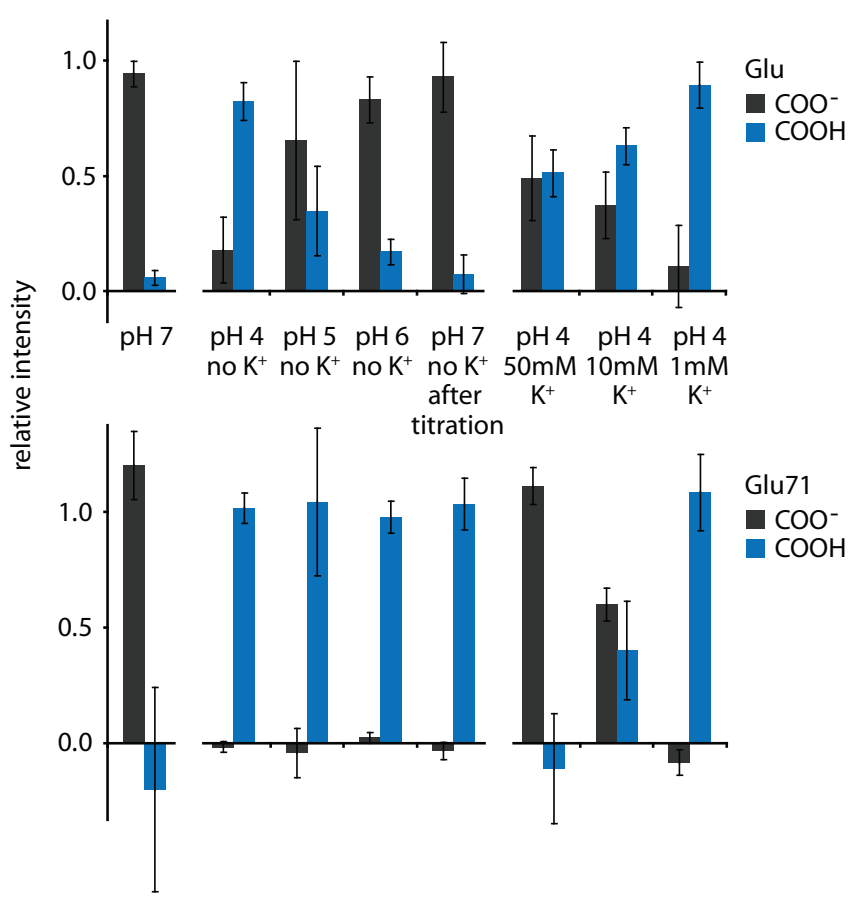

Figure 9.5: Relative integrals of spectral regions corresponding to deprotonated (black) and protonated (blue) glutamate $\mathrm{C}^{\gamma}-\mathrm{C}^{\delta}$ crosspeak regions for all glutamates except Glu71 (top panel) and Glu71 (bottom panel). For each condition, integrals of regions corresponding to protonated and deprotonated Glu or Glu71 sidechains were normalized to their sum, such that bars represent fractions of protonated and deprotonated Glu or Glu71 sidechain signal with respect to total Glu or Glu71 sidechain signal.

Following relative intensities of $\mathrm{C}^{\gamma}-\mathrm{C}^{\delta}$ crosspeaks corresponding to more protonated or to deprotonated glutamate side chains throughout titration experiments (Figs. 9.4 and 
9.5), the following observations can be made. First, Glu51, having an exposed localization in the extracellular turret region, and the C-terminal glutamates are protonated at $\mathrm{pH}$ 4.0, as expected for water-exposed glutamate sidechains. Sidechain protonation of these glutamates thus appears to be $\mathrm{K}^{+}$-insensitive. In contrast, $\mathrm{C}^{\delta}$ resonances of pore-domain glutamates 71,118 , and 120 are markedly $\mathrm{K}^{+}$-sensitive at acidic $\mathrm{pH}$. Their sidechain chemical shifts remain at values seen for neutral $\mathrm{pH}$ even at $\mathrm{pH} 4.0$ in the presence of $50 \mathrm{mM}$ potassium, whereas they shift to values corresponding to full protonation in the absence of potassium (Fig. 9.4 b-d). Notably, for Glu118 and Glu120 as well as the Cterminal glutamates, this behavior can also be observed in their $\mathrm{C}^{\beta}-\mathrm{C}^{\gamma}$ crosspeaks seen in Figure $9.2 \mathrm{a}, \mathrm{b}$ in the panels showing correlations from the TM2 gate. These data show that protonation is $\mathrm{K}^{+}$-sensitive for pore-domain glutamates that have important roles in activation (Glu118 and Glu120) [279] and inactivation (Glu71) gating of KcsA $[235,263]$. The observed $\mathrm{K}^{+}$-sensitivity correlates with the observation that an opencollapsed conformation of the KcsA-Kv1.3 channel predominates at low $\left[\mathrm{K}^{+}\right]$in acidic $\mathrm{pH}$. Taken together, the data imply that protonation of pore-domain glutamates is associated both with a stably opened activation gate and with collapse of the selectivity filter.

\subsubsection{Coupling of activation and inactivation gates}

Next, structural changes in the open-collapsed channel upon back-titration from $\mathrm{pH} 4.0$ to pH 7.5 in the absence of potassium were investigated (Fig. 9.5). SsNMR spectra (Appendix Fig. E.2) were obtained that reveal a channel conformation with a collapsed selectivity filter and a closed activation gate. Residues of gating hinge, lower pore helix, and turret regions had returned to their conformational states originally observed at neutral $\mathrm{pH}$. Thus, structural information was obtained about a closed-collapsed conformation (state $I^{*}$ in Fig. 9.1) in addition to a closed-conductive and an open-collapsed conformation of the channel. Interestingly, in this state, also sidechain resonances of the lower selectivity filter assume values as seen at $\mathrm{pH} 7.5$, while corresponding backbone resonances correspond to the collapsed state (Appendix Fig. E.3). The fact that these sidechain resonances are only seen to shift together with resonances originating from the TM2 gate suggests that they are part of an interaction surface coupling inactivation gate to activation gate. Importantly, the closed-conductive conformation of the $\mathrm{K}^{+}$channel at $\mathrm{pH} 7.5$ could be fully restored by adding $50 \mathrm{mM}$ potassium to the buffer (Appendix Fig. E.2).

Notably, the back-titration was conducted in the KcsA-Kv1.3 channel truncated Cterminally after residue 125 . Both activation and inactivation gating as well as their 
potassium dependence were thus found to be preserved with respect to the full-length channel. This entails that the C-terminus can play at most a modulatory role in activation and inactivation gating, in agreement with findings of earlier studies [237, 280].

\subsubsection{Modulation of gating by internal and external potassium}

Since it was unclear from which side of the lipid membrane (intra- or extracellular) $\mathrm{K}^{+}$ exerts its effects on KcsA-Kv1.3 conformation, effects of both internal $\left(\left[\mathrm{K}^{+}\right]_{\text {in }}\right)$ and external $\mathrm{K}^{+}\left(\left[\mathrm{K}^{+}\right]_{\text {out }}\right)$ on KcsA-Kv1.3 channel gating were investigated functionally by Olaf Pongs and coworkers (see Section 9.3.3). First, the influence of $\left[\mathrm{K}^{+}\right]_{\text {out }}$ on KcsA-Kv1.3 channel inactivation was studied. As described in Chapter 8, upon a pH-jump from 7.5 to 4.0 the KcsA-Kv1.3 channel activates rapidly and then inactivates within seconds to finally reach a steady-state current level $\left(I_{\mathrm{ss}}\right)$ (Fig. 9.6 a). It was found that KcsA-Kv1.3 steadystate current levels $\left(I_{\mathrm{ss}} / I_{\max }\right)$ remaining at the end of the test pulse are very sensitive to $\left[\mathrm{K}^{+}\right]_{\text {out }}($ Fig. $9.6 \mathrm{~b})$. A plot of $I_{\mathrm{sS}} / I_{\max }$ versus $\left[\mathrm{K}^{+}\right]_{\text {out }}$ reveals that $I_{\mathrm{ss}} / I_{\max }$ titrates with $\left[\mathrm{K}^{+}\right]_{\text {out }}$ (Fig. $9.6 \mathrm{~b}$ ), showing a $K_{D}$ of $0.9 \mathrm{mM}$ with a maximal $I_{\mathrm{ss}} / I_{\max }$ value of 0.44 at $150 \mathrm{mM}\left[\mathrm{K}^{+}\right]_{\text {out }}$ and a minimum $I_{\mathrm{sS}} / I_{\max }$ value of 0.02 at $0 \mathrm{mM}\left[\mathrm{K}^{+}\right]_{\text {out }}$. The $K_{D}$ value found for $\mathrm{K}^{+}$-sensitive $\mathrm{KcsA}-\mathrm{Kv} 1.3$ inactivation is conspicuously similar to the $K_{D}$ of $0.43 \mathrm{mM}$ determined in calorimetric studies for an external $\mathrm{K}^{+}$binding site of the closed KcsA channel [239]. Combination of electrophysiological and structural data indicates that $\mathrm{K}^{+}$-sensitive collapse of the filter structure corresponds to this $\mathrm{K}^{+}$-sensitive KcsA-Kv1.3 channel inactivation process associated with closure of the upper (inactivation) pore-gate. Occupancy of an external $\mathrm{K}^{+}$binding site with $\mathrm{K}^{+}$is thus essential for a stable conductive structure of the selectivity filter, which otherwise is prone to collapse [24].

Next, the influence of $\left[\mathrm{K}^{+}\right]_{\text {in }}$ on KcsA-Kv1.3 gating properties was investigated. Upon a pH jump from 7.5 to 4.0, hyperpolarizing test pulses induce rapid activation of $\mathrm{K}^{+}$inward current (Fig. 9.6 c). In agreement with previously reported outward-rectification properties of KcsA-mediated currents, inactivation of KcsA-Kv1.3 inward-current is essentially complete [267]. The most important observation was that $\left[\mathrm{K}^{+}\right]_{\text {in }}$ significantly accelerates KcsA-Kv1.3 inactivation with a minimal inactivation time constant $\left(\tau_{\text {inact }}=0.11 \pm 0.01 \mathrm{~s}\right.$, s.e.m., $n=7$ ) at $\left[\mathrm{K}^{+}\right]_{\text {in }} \geq 0.2 \mathrm{mM}$ (Fig. $9.6 \mathrm{c}, \mathrm{d}$ ). A plot of $\tau_{\text {inact }}$ versus $\left[\mathrm{K}^{+}\right]_{\text {in }}$ shows that $\tau_{\text {inact }}$ titrates with $\left[\mathrm{K}^{+}\right]_{\text {in }}$ (Fig. $9.6 \mathrm{~d}$ ), revealing an internal $\mathrm{K}^{+}$binding site with $K_{D}=6.5 \mu \mathrm{M}$. Combining this result with the observation that the lower gate can be trapped in an opened conformation in the absence of $\mathrm{K}^{+}\left(\left[\mathrm{K}^{+}\right] \leq 1 \mu \mathrm{M}\right)$ suggests that occupancy of an internal $\mathrm{K}^{+}$binding site regulates activation gate closure. 

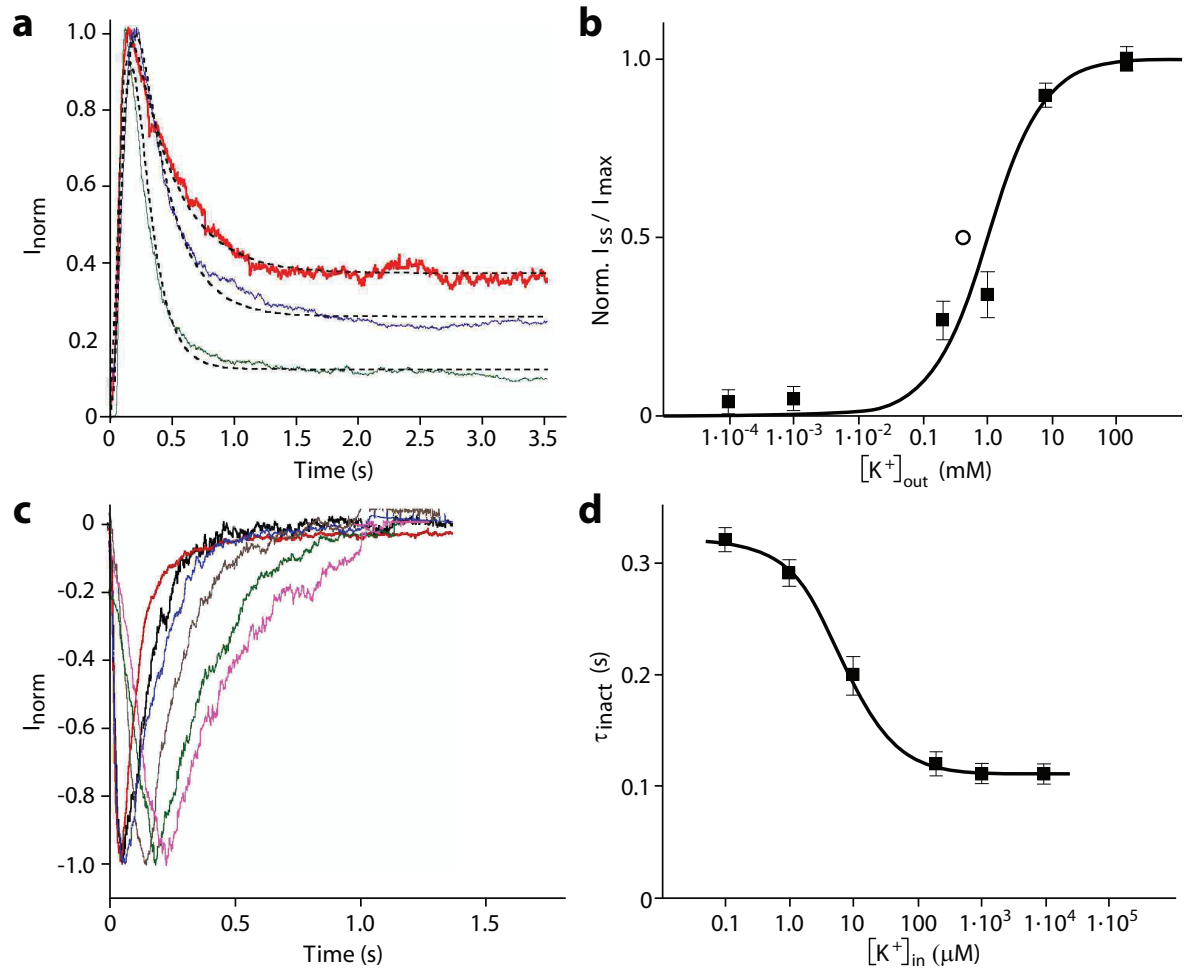

Figure 9.6: $\mathrm{K}^{+}$-sensitivity of KcsA-Kv1.3 channel gating. (a) Normalized KcsA-Kv1.3 outwardcurrents $\left(I_{\text {norm }}\right)$ recorded at $+100 \mathrm{mV}$ with different external $\mathrm{K}^{+}\left(\left[\mathrm{K}^{+}\right]_{\text {out }}\right)$ concentrations $(150 \mathrm{mM}$, red; $1 \mathrm{mM}$, blue; $1 \mu \mathrm{M}$, green). Inside (bath) solution always contained $150 \mathrm{mM} \mathrm{KCl}$. Dashed curves are fits to the data according to ref. [281]. (b) Normalized relative steady-state current amplitudes $\left(I_{\mathrm{ss}} / I_{\max }\right)$ plotted against $\left[\mathrm{K}^{+}\right]_{\text {out }}$. Solid line represents fitted curve to the dose-response data (filled squares) with $K_{D}$ for external $\mathrm{K}^{+}$binding of $0.9 \mathrm{mM}$. Error bars are s.e.m. $(\mathrm{n}=5-14)$. Open circle represents the $K_{D}$ for an extracellular $\mathrm{K}^{+}$binding site in $\mathrm{KcsA}$ according to ref. [239]. (c) Normalized KcsA-Kv1.3 inward currents $\left(I_{\text {norm }}\right)$ recorded at $-100 \mathrm{mV}$ with different internal $\mathrm{K}^{+}$ $\left(\left[\mathrm{K}^{+}\right]_{\text {in }}\right)$ solutions (0 mM, magenta; $1 \mu \mathrm{M}$, green; $10 \mu \mathrm{M}$, brown; $0.2 \mathrm{mM}$, black; $1 \mathrm{mM}$, blue; 150 $\mathrm{mM}$, red). Outside (pipette) solution contained $150 \mathrm{mM} \mathrm{KCl}$. (d) $\left[\mathrm{K}^{+}\right]_{\text {in }}$-sensitivity of inactivation rate $\left(\tau_{\text {inact }}\right)$. Error bars are s.e.m. $(\mathrm{n}=5-12)$. The $K_{D}$ value of $6.5 \mu \mathrm{M}$ was obtained by fitting a smooth curve to the dose-response data (filled squares) as described in ref. [276]. 
In order to probe the structural impact of the internal $\mathrm{K}^{+}$binding site found in electrophysiological experiments, ssNMR experiments were performed at a potassium concentration of $1 \mu \mathrm{M}$. If $\mathrm{pH}$ was lowered together with the addition of $1 \mu \mathrm{M}$ potassium, quantitative channel opening was observed in water-edited experiments (Fig. 9.7) [3]. However, if 1

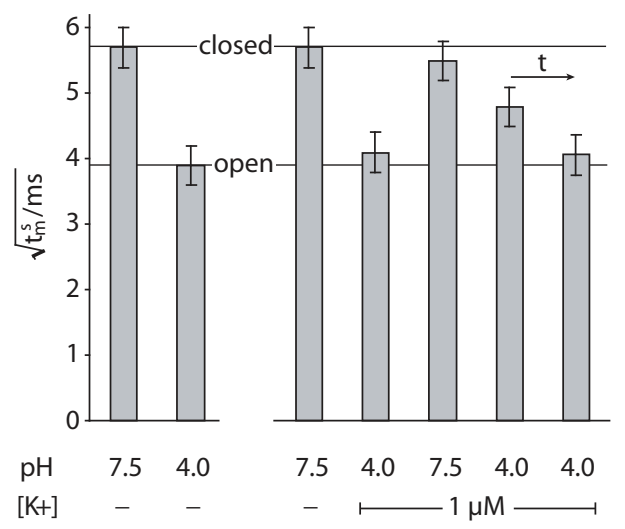

Figure 9.7: Water-edited ssNMR spectroscopy shows that a micromolar concentration of potassium drastically slows activation gate opening if it is present before a jump to $\mathrm{pH} 4.0$. The time $t$ indicates an incubation of the sample over several days at $280 \mathrm{~K}$.

$\mu \mathrm{M}$ potassium was present already at $\mathrm{pH} 7.5$, a jump to $\mathrm{pH} 4.0$ first elicited fractional channel opening $\left(\sqrt{t_{m}^{s}} \mathrm{pH} 4.0=4.5 \pm 0.4 \mathrm{~ms}^{1 / 2}\right)$ and quantitative channel opening only after prolonged incubation $\left(\sim 1\right.$ week; $\left.\sqrt{t_{m}^{s}} \mathrm{pH}_{4.0}=3.9 \pm 0.4 \mathrm{~ms}^{1 / 2}\right)$. Thus, kinetics of activation gate opening are markedly slowed at low $(1 \mu \mathrm{M})$ potassium concentrations. This suggests that potassium binding to the internal binding site with a $K_{D}$ of $6.5 \mu \mathrm{M}$ indeed affects activation gate opening in agreement with electrophysiological data. The fact that potassium has to be present before the $\mathrm{pH}$ jump in order to slow activation gate opening indicates that this potassium binding site changes upon activation gating. This makes the selectivity filter with the inactivation gate a prime candidate for locating this high-affinity potassium binding site.

\subsubsection{Ligand binding unmasks gate coupling}

Previous reports have already indicated that lower activation gate and upper inactivation gate are coupled in potassium channels. In N- and C-type inactivation, opening of the lower activation gate induces closure of the upper inactivation gate [38, 262]. On the other hand, upper gate closure can promote lower gate opening [275, 282]. Data discussed so far imply 
that the observed $\mathrm{K}^{+}$- and $\mathrm{pH}$-sensitivity of both upper and lower gate conformations has a profound influence on coupling activation gate opening and inactivation gate closure. In order to test a potential coupling between upper and lower gate conformations further, two ligands were employed which affect KcsA-Kv1.3 selectivity filter conformation in different ways. The first one was the tetraphenylporphyrin derivative (porphyrin) already used in Chapter 8 which binds to KcsA-Kv1.3 with nanomolar affinity [265]. As discussed in Chapter 8 and ref. [1], this ligand induces a collapsed, non-conductive conformation of the selectivity filter. Binding of the second ligand used, kaliotoxin (KTX), only affects upper selectivity filter residues [62] and stabilizes a conductive filter conformation [4]. This particular situation made it possible to study the effect of acidic $\mathrm{pH}$ on the lower activation gate in the context of an opened or a closed upper inactivation gate.

First, ssNMR spectra were recorded from the porphyrin-KcsA-Kv1.3 complex at pH 4.0 in the absence of $\mathrm{K}^{+}$. The spectra are similar to those obtained for the unliganded channel, i.e. they indicate an open-collapsed structure, exhibiting an opened lower activation gate and a closed upper inactivation gate (Fig. 9.8 a, b). In contrast, ssNMR spectra from the KTX-bound channel reveal that, in this state, the lower activation gate remains closed at pH 4.0 even if no potassium is added to the buffer solution (Fig. 9.8 c, d). $\mathrm{C}^{\delta}$ shifts of Glu118 and Glu120 remain at values corresponding to deprotonated carboxyl groups, while C-terminal glutamates exhibit resonances pointing to protonated carboxyl groups. The lower selectivity filter preserves its conductive conformation, and chemical shifts observed for residues 77-80 confirm that KTX remains bound to KcsA-Kv1.3 at pH 4.0 (Fig. E.4). The important implication of these results is that activation gate and inactivation gate are coupled. A closed upper gate promotes a stably opened lower activation gate, whereas an opened upper gate is linked with the more stable closed-conductive conformation of the channel.

\subsection{Discussion}

KcsA-Kv1.3 $\mathrm{K}^{+}$channel activation and inactivation is well described by a simple cyclic gating scheme (Fig. 9.1 and Fig. 9.9) which defines main channel states by different combinations of opened and closed activation and inactivation gates, respectively [275]. Here, direct structural information was obtained for three of the four states: closed-conductive (C), open-collapsed (I), and closed-collapsed (I*) in a membrane setting, with a focus on residues located in activation and inactivation gates (Fig. 9.10). 


$$
\text { no } K^{+}
$$
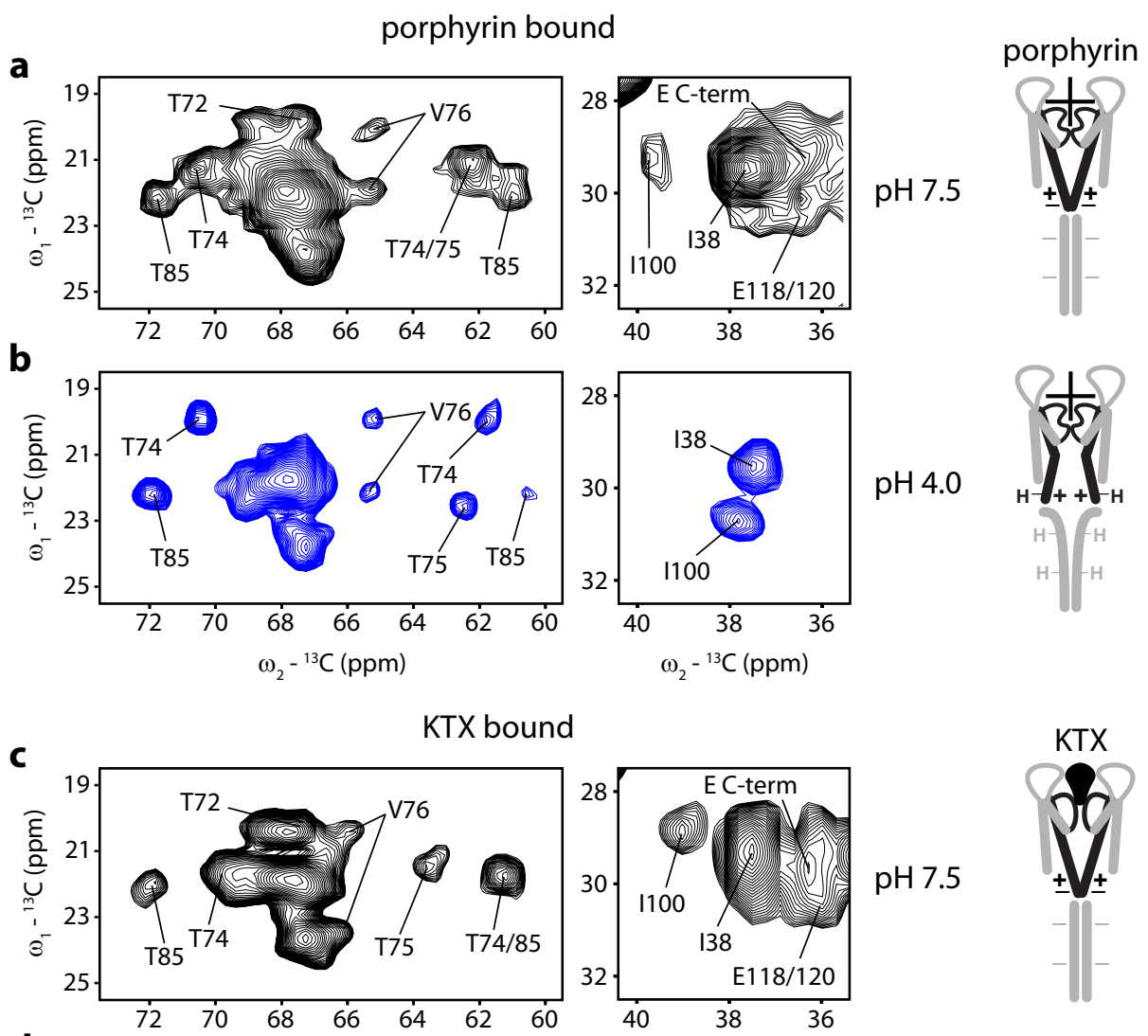

$\mathrm{pH} 7.5$
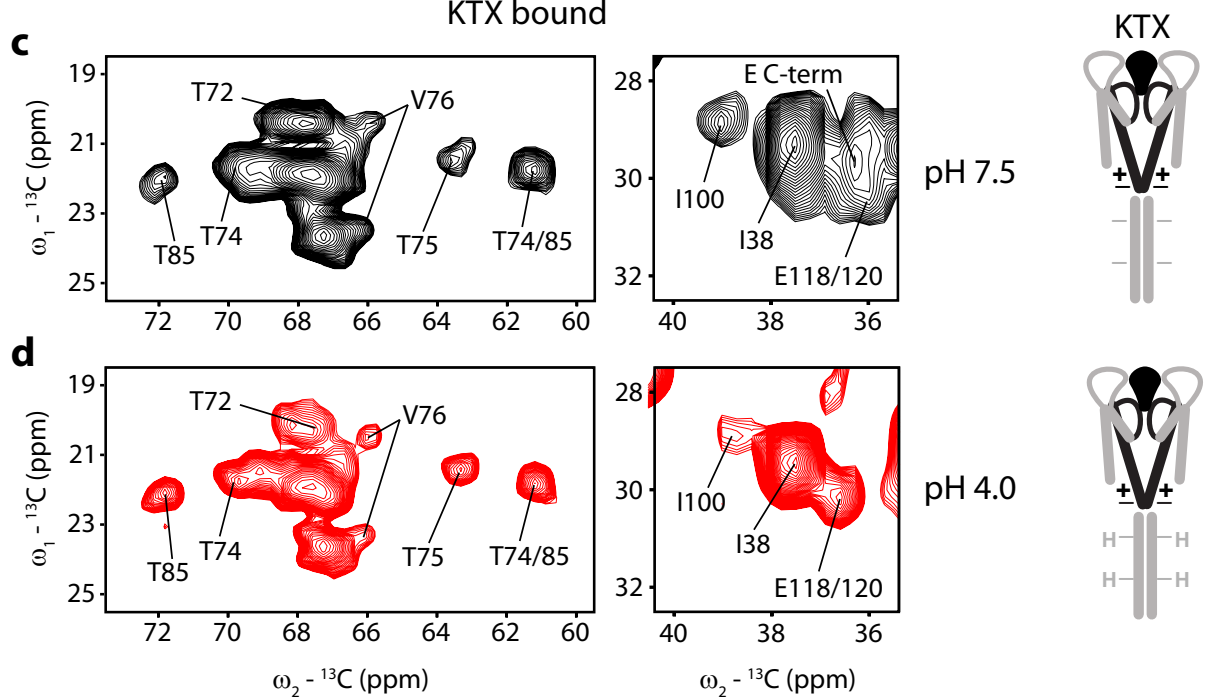

Figure 9.8: Spectral regions covering resonances of selectivity filter, gating hinge, and glutamate residues from turret, TM2 helix and C-terminus for KcsA-Kv1.3 bound to porphyrin (a, b) and $\mathrm{KTX}(\mathbf{c}, \mathbf{d})$ in the absence of potassium at pH $7.5(\mathrm{a}, \mathrm{c})$ and pH $4.0(\mathrm{~b}, \mathrm{~d})$. Cartoons on the right illustrate the state of KcsA-Kv1.3 under the respective conditions in terms of selectivity filter and TM2 gate conformations as well as glutamate protonation and charge distribution in the lower TM2 helix linked to activation gating. 
The most important observation was that open probabilities of both activation gate and inactivation gate are distinctly $\mathrm{K}^{+}$-sensitive. In agreement with previous electrophysiological studies on $\mathrm{K}^{+}$-sensitive gating of the KcsA channel [233, 283], the opened KcsA-Kv1.3 activation gate is more stable in low than in high $\mathrm{K}^{+}$concentrations. In contrast to those studies, however, in the work presented here the total monovalent cation strength was explicitly kept constant by replacing potassium with sodium. Thus, results in this chapter can be correlated to the influence of potassium per se. It could be shown that, under the conditions used in this study, the closed-conductive conformation of the KcsA-Kv1.3 channel is quantitatively converted to an open-collapsed conformation at pH 4.0 if the buffer solution contains no or only submicromolar potassium concentrations. The $\mathrm{K}^{+}$-sensitivity of the open-collapsed conformation is correlated with a $\mathrm{K}^{+}$-sensitive protonation of glutamate residues 71,118 , and 120 . These residues have an important influence on selectivity filter conformation (Glu71) [235, 263] and on pH-induced activation gate opening (Glu118 and 120), respectively [279]. Conversely, in high (mM) K ${ }^{+}$concentrations, as the most stable conformation in asolectin liposomes a closed-conductive state of the KcsA-Kv1.3 channel was observed at $\mathrm{pH} 7.5$ as well as at $\mathrm{pH} 4.0$.

Results shown here underline previous reports that potassium concentration and lipidic environment have an important influence on functional and thermodynamic properties of the $\mathrm{K}^{+}$channel pore domain $[249,284,285,286,287]$ (see also Chapters 7 and 8). The structural data agree well with electrophysiological results showing a low open probability of the channel at pH 4.0. They complement earlier studies on KcsA, as well as results for KcsA-Kv1.3 without added potassium discussed in Chapter 8, that trace low channel open probability at acidic $\mathrm{pH}$ to an open-inactivated state $[235,242,264,273,280]$. In light of the results presented in this chapter, it is crucial to consider the exact potassium concentrations and lipid environments used in such studies in order to discuss the individual structural findings in a common context. For example, the buffer used in EPRspectroscopic studies on KcsA, which have indicated an opened TM2 activation gate under steady-state conditions at $\mathrm{pH} 4.0$, appears to contain only low millimolar concentrations of potassium, which might explain the difference to results shown here for $\left[\mathrm{K}^{+}\right] \geq 10$ $\mathrm{mM}[235,236]$. It is also important to note that electrochemical gradients as present in electrophysiology have so far not been reproduced in structural studies. Nevertheless, the finding that the closed state of the KcsA-Kv1.3 activation gate is accessible at acidic pH is consistent with functional studies suggesting that recovery of $\mathrm{K}^{+}$channels from inac- 


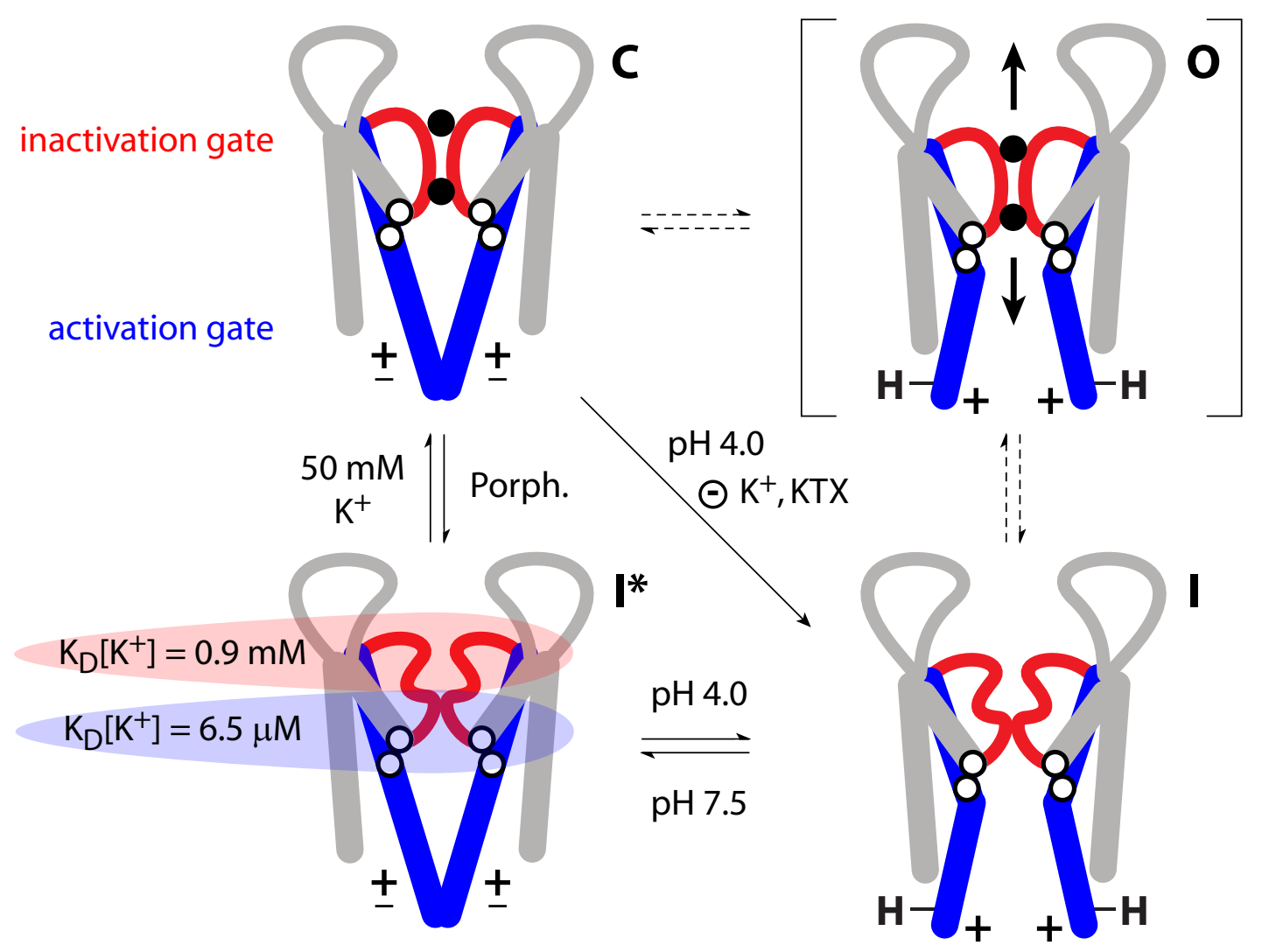

Figure 9.9: Gating scheme of KcsA-Kv1.3. N- and C-termini are omitted for clarity. Activation (blue) and inactivation (red) gates can both reside in an open and a closed conformation. Gating states are labeled as in Figure 9.1. Filled black circles sketch potassium ions, open black circles represent sidechains of Ile100 in the TM2 gate and of residues in the lower selectivity filter and pore helix (e.g. Thr74). H indicates protonation of Glu118 and Glu120 at the helix bundle crossing. Solid arrows are labeled with the conditions used to interconvert states for ssNMR-spectroscopic and electrophysiological investigations. The circled minus represents the antagonistic effect of $\mathrm{K}^{+}$and $\mathrm{KTX}$ on the channel open probability. Dashed arrows indicate interconversions not investigated on a structural level and brackets mark the open-conductive state of KcsA-Kv1.3 not yet accessible to structural characterization by ssNMR. The red and blue clouds point towards the different intra- and extra-vesicular potassium binding events related to the potassium dependence of channel activation and inactivation gating. 
tivation, required to explain steady-state current, usually occurs via closure of the TM2 gate, possibly also at acidic $\mathrm{pH}[259,260,275]$.

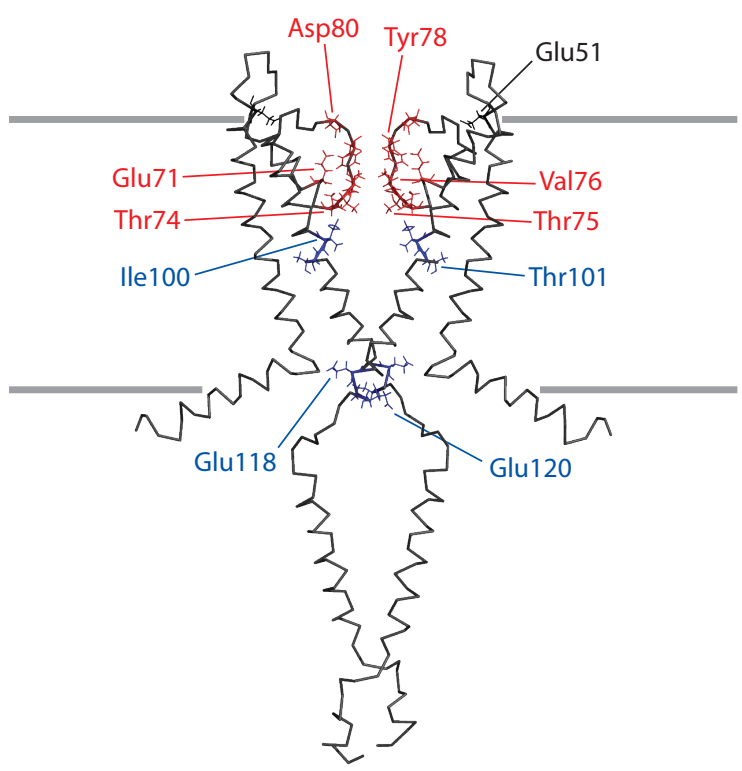

Figure 9.10: Structural model of KcsA-Kv1.3 from Chapter 7. Residues in activation and inactivation gates used as probes for channel conformation throughout the ssNMR experiments are indicated in blue and red, respectively. Only two channel subunits are shown for clarity. The approximate extent of the lipid bilayer is indicated by grey lines.

The influence of external $\mathrm{K}^{+}$on selectivity filter stability and $\mathrm{K}^{+}$channel gating, e.g. C-type inactivation of Shaker channels, has been extensively studied [259, 270, 288, 289, 290]. Data shown in this chapter reveal a higher KcsA-Kv1.3 steady-state current at high external $\mathrm{K}^{+}$concentration, a finding which agrees well with these earlier studies and is consistent with the idea that C-type inactivation is correlated with the collapse of a conductive selectivity filter structure (Chapter 8). A previous study found a $K_{D}$ of $0.43 \mathrm{mM}$ for $\mathrm{K}^{+}$binding to the selectivity filter of $\mathrm{KcsA}$ [239]. The $K_{D}$ value of $0.9 \mathrm{mM}$ found here for the sensitivity of KcsA-Kv1.3 inactivation to external $\mathrm{K}^{+}$as measured by steady-state current is conspicuously similar. However, a second, higher-affinity $\mathrm{K}^{+}$binding process was observed in electrophysiology by varying the internal $\mathrm{K}^{+}$concentration. A higher $\left[\mathrm{K}^{+}\right]_{\text {in }}$ accelerates KcsA-Kv1.3 channel inactivation kinetics with a $K_{D}$ of $6.5 \mu \mathrm{M}$. A comparable micromolar concentration of potassium was found to influence activation gating 
in water-edited ssNMR experiments. Micromolar $\mathrm{K}^{+}$binding constants have also been described in functional studies for block of $\mathrm{Na}^{+}$conduction through C-type inactivated $\mathrm{K}^{+}$channels [291, 292]. The implication of these data is that the occupancy of internal and external $\mathrm{K}^{+}$binding sites of the KcsA-Kv1.3 channel has distinct effects on gating transitions and channel open probability.

KTX binding was observed to stabilize a conductive selectivity filter conformation and to prevent activation gate opening at $\mathrm{pH} 4.0$ even in the absence of potassium. This provides strong evidence that stabilizing the conductive conformation of the selectivity filter is sufficient to lower the open probability of the activation gate and, consequently, that both gates are coupled. The synchrony observed in pH-dependent sidechain chemical shift changes in lower pore helix and lower selectivity filter on the one hand and TM2 gating hinge on the other hand also speaks in favour of coupling between lower and upper gates. This observation fits nicely with reports that the presence of ions in the selectivity filter of voltage-gated $\mathrm{K}^{+}$channels can accelerate activation gate closure [275, 282]. Thus, both $\mathrm{K}^{+}$binding sites observed in the electrophysiological experiments discussed here can be traced to the selectivity filter. One might then speculate that the two $K_{D}$ values measured correspond to two sequential binding events of $\mathrm{K}^{+}$ions to different binding sites within the filter $[239,270,292]$. The important implication of data presented in this chapter is thus that $\mathrm{K}^{+}$, the permeant ion itself, modulates activation as well as inactivation gating, providing a scenario for coordinated, consecutive gating transitions involving opening, inactivation, and closing. 


\section{Part IV}

Fibrillar aggregates 



\section{Chapter 10}

\section{Structural characterization of polyglutamine fibrils by solid-state NMR}

\subsection{Summary}

Protein aggregation via polyglutamine stretches occurs in a number of severe diseases such as Huntington's disease. This chapter describes the investigation of fibrillar aggregates formed by polyglutamine peptides below, at and above the toxicity limit of around 40 glutamine residues using solid-state NMR and electron microscopy. Experimental data for all constructs reveal a largely $\beta$-strand character and are consistent with a dry fibril core of at least $70-80 \AA$ diameter, arguing against a water-filled $\beta$-helix structure. $2 \mathrm{D}$ ssNMR dipolar correlation experiments point to tight interdigitation of hydrogen bonded glutamine sidechains from different sheets in agreement with 'polar zipper' models. Two populations of glutamine residues with distinct sets of chemical shifts are found, possibly related to distinct sidechain conformations or backbone dihedral angles mutually compensating for $\beta$-strand twist. While $Q_{15}$ peptides are present as single extended $\beta$-strands, data obtained for longer constructs are most compatible with a superpleated arrangement, in which individual molecules contribute $\beta$-strands to more than one sheet, and with an antiparallel assembly of strands within $\beta$-sheets. ${ }^{1}$

\subsection{Introduction}

A variety of diseases involving proteins with expanded glutamine repeat stretches are known, including Huntington's disease and various types of spinocerebellar ataxia [72,

\footnotetext{
${ }^{1}$ This chapter is related to the following manuscript in preparation for publication: Robert Schneider et al.: Structural characterization of polyglutamine fibrils by solid-state NMR spectroscopy.
} 
293, 294, 295]. While the proteins involved are usually unrelated except for the presence of polyglutamine regions, there is a common critical length of the glutamine stretch of $35-45$ residues above which disease occurs [293, 295]. Proteins with glutamine repeat stretches as well as polyglutamine peptides can form aggregates that bear the characteristic features of amyloid fibrils, i.e. linear and unbranched fibrils which exhibit a cross- $\beta$ Xray diffraction pattern, indicating $\beta$-strands running perpendicular to the fibril axis, and which can be stained by the dyes Congo red and thioflavin T [296]. Disease progression is correlated with the accumulation of neuronal intranuclear inclusions of such amyloid-like aggregates [293, 294, 297]. However, toxicity has recently been attributed to mono- or oligomeric polyglutamine species [298, 299]. Polyglutamine aggregation kinetics and age of onset of Huntington's disease have been shown to correlate with the glutamine repeat length [300].

In addition to the role of polyglutamine-containing proteins in human disease, regions rich in glutamine and asparagine have been found in a number of yeast prions, where they mediate aggregation to amyloid fibrils as a mechanism of epigenetic regulation and inheritance [301]. An example is the yeast translation termination factor Sup35. Evidence is also emerging for a role of glutamine- and asparagine-rich regions in protein-protein interactions [302]. However, while a large number of biochemical and structural as well as molecular dynamics studies have investigated aggregates of proteins and peptides with polyglutamine stretches, their structural arrangement is still a matter of debate. Crystal structures of shorter asparagine- and glutamine-rich peptides related to amyloid fibrils have been reported $[47,48]$, showing extended strands in a parallel cross- $\beta$ arrangement with densely packed sidechains. For longer peptides, various models have been proposed. Many of these involve extended $\beta$-strands of varying length, connected by short turns within the plane of a $\beta$-sheet or by longer $\beta$-bends between stacked sheets $[303,304,305$, 306]. The latter arrangement has also been postulated for glutamine- and asparagine-rich fragments of yeast prions $[307,308]$. In contrast, Perutz et al. have developed a waterfilled $\beta$-helix model believed to be in better agreement with some features of the fibril X-ray diffractogram [309]. However, it has been shown that a $\beta$-hairpin structure with $\beta$-sheets stacked tightly on top of each other may account for these features as well [310]. Additionally, molecular dynamics simulations have indicated that water-filled circular $\beta$ helix structures are unstable $[306,311]$ and suggested triangular $\beta$-helices instead, with inward-pointing sidechains forming a dry fibril core [311]. Some of these models also allow 
for parallel or anti-parallel arrangement of the strands within a $\beta$-sheet and between the sheets.

As a spectroscopic method that allows for the investigation of insoluble, noncrystalline materials, solid-state NMR has become the leading method for structure elucidation of amyloid fibrils (see, e.g., refs. [20, 63, 64, 65, 66, 67]). This chapter describes an ssNMR study of fibrillar aggregates formed by polyglutamine peptides of varying length, both below and above the disease threshold. Data obtained are consistent with a 'polar zipper' arrangement of sidechains and tight stacking of $\beta$-sheets composed of two distinct glutamine populations discernible by their chemical shift. As the overall fold of polyglutamine molecules within their fibrils, an antiparallel superpleated arrangement emerges in which a monomer folds in a plane orthogonal to the fibril axis and contributes strands to more than one $\beta$-sheet.

\subsection{Materials and Methods}

\subsubsection{Peptide synthesis, expression and fibrillization}

The peptide $\mathrm{D}_{2} \mathrm{Q}_{15} \mathrm{~K}_{2}$ with residues Asp2, Gln3, Gln6, Gln9, Gln10, Gln17, and Lys18 uniformly $\left[{ }^{13} \mathrm{C},{ }^{15} \mathrm{~N}\right.$-labeled was prepared by Volker Klaukien and Gerhard Wolf in Nterminally acetylated and C-terminally amidated form using manual solid-phase synthesis. Unlabeled and labeled Fmoc-protected amino acids were purchased from Merck Biosciences and Cambridge Isotope Laboratories, respectively. After cleavage from the resin, the crude peptide was precipitated with diethyl ether, washed and lyophilized. Subsequently, it was purified on a Jasco HPLC (high performance liquid chromatography) system and analyzed by electrospray ionization mass spectrometry (ESI-MS).

Expression and purification of uniformly $\left[{ }^{13} \mathrm{C},{ }^{15} \mathrm{~N}\right]$-labeled $\mathrm{GK}_{2} \mathrm{Q}_{38} \mathrm{~K}_{2}$ and $\mathrm{GK}_{2} \mathrm{Q}_{54} \mathrm{~K}_{2}$ constructs was performed by Miria Schumacher in the group of Martin Engelhard. The constructs were recombinantly expressed as GST (glutathione S-transferase) fusion proteins in E. coli and purified from the cytoplasmic fraction via a glutathione sepharose column. After cleavage of the GST tag by TEV protease, the water-insoluble $\mathrm{GK}_{2} \mathrm{Q}_{38} \mathrm{~K}_{2}$ and $\mathrm{GK}_{2} \mathrm{Q}_{54} \mathrm{~K}_{2}$ peptides were solubilized and purified using trifluoroacetic acid (TFA) and hexafluoroisopropanol (HFIP) as described in ref. [312] and stored in water with $0.1 \%$ TFA at $\mathrm{pH} 3$.

For fibrillization, either the lyophilized peptide $\left(\mathrm{D}_{2} \mathrm{Q}_{15} \mathrm{~K}_{2}\right)$ or the peptide in water with $0.1 \%$ TFA at $\mathrm{pH} 3\left(\mathrm{GK}_{2} \mathrm{Q}_{38} \mathrm{~K}_{2}, \mathrm{GK}_{2} \mathrm{Q}_{54} \mathrm{~K}_{2}\right)$ was dissolved in or transferred to TBS buffer 
(10 mM Tris/HCl, $150 \mathrm{mM} \mathrm{NaCl}$ ) at $\mathrm{pH} 7.4$ in a concentration of $100 \mu \mathrm{M}$ and stored at $37^{\circ} \mathrm{C}$ for four days. Alternatively, prolonged incubation (12 weeks) in water with $0.1 \%$ TFA was used $\left(\mathrm{GK}_{2} \mathrm{Q}_{54} \mathrm{~K}_{2}\right)$, which also leads to aggregation [312]. Then, aggregates were harvested by ultracentrifugation at 208,000 g for $1 \mathrm{~h}$.

\subsubsection{Solid-state NMR}

Experiments were conducted using $2.5 \mathrm{~mm}, 3.2 \mathrm{~mm}$, or $4 \mathrm{~mm}$ Bruker triple-resonance $\left({ }^{1} \mathrm{H}\right.$, ${ }^{13} \mathrm{C},{ }^{15} \mathrm{~N}$ ) magic-angle-spinning (MAS) probeheads at static magnetic fields of 9.4, 14.1, 18.8 or $20.0 \mathrm{~T}$ corresponding to proton resonance frequencies of $400,600,800$, or $850 \mathrm{MHz}$, respectively (Bruker Biospin, Karlsruhe, Germany). Effective sample temperatures were calibrated using nickelocene $[222]$ and varied from about $-10^{\circ} \mathrm{C}$ for $\left({ }^{15} \mathrm{~N},{ }^{13} \mathrm{C}\right)$ correlation spectra at high field to about $+7^{\circ} \mathrm{C}$ for $\left({ }^{13} \mathrm{C},{ }^{13} \mathrm{C}\right)$ correlation spectra. MAS frequencies used were $11 \mathrm{kHz}$ for $\left({ }^{15} \mathrm{~N},{ }^{13} \mathrm{C}\right)$ spectra and, for $\left({ }^{13} \mathrm{C},{ }^{13} \mathrm{C}\right)$ spectra, close to the rotational resonance condition for $\mathrm{C}^{\alpha}$ and $\mathrm{C}^{\prime}$ resonances to achieve weak coupling conditions [125] (11.2 or $12.5 \mathrm{kHz}$ at $800 \mathrm{MHz}$ proton resonance frequency). ${ }^{1} \mathrm{H}$ field strengths for $90^{\circ}$ pulses and SPINAL64 decoupling [95] were around $80 \mathrm{kHz}$. Initial $\left({ }^{1} \mathrm{H},{ }^{13} \mathrm{C}\right)$ or $\left({ }^{1} \mathrm{H},{ }^{15} \mathrm{~N}\right)$ cross-polarization (CP) times were set to $700 \mu \mathrm{s}$.

$\left({ }^{13} \mathrm{C},{ }^{13} \mathrm{C}\right)$ mixing was performed using proton-driven spin diffusion under weak coupling conditions (PDSD-WC) [125] with mixing times of 20, 150 or $500 \mathrm{~ms}$ to obtain intraresidue, sequential or long-range correlations, respectively. $\left({ }^{15} \mathrm{~N},{ }^{13} \mathrm{C}\right)$ spectra were recorded using SPECIFIC-CP $[123,124]$ as the $\left({ }^{15} \mathrm{~N},{ }^{13} \mathrm{C}\right)$ transfer step, using mixing times of 3 to $4.5 \mathrm{~ms}$, followed by homonuclear $\left({ }^{13} \mathrm{C},{ }^{13} \mathrm{C}\right)$ spin diffusion mixing of up to $50 \mathrm{~ms}$. N/CHHC $[136,137]$ spectra used short CPs bracketing the longitudinal $\left({ }^{1} \mathrm{H},{ }^{1} \mathrm{H}\right)$ mixing block (80 to $100 \mu \mathrm{s}$ for $\left({ }^{1} \mathrm{H},{ }^{13} \mathrm{C}\right.$ )-CPs, $150 \mu \mathrm{s}$ for $\left({ }^{1} \mathrm{H},{ }^{15} \mathrm{~N}\right)$-CPs) to limit transfer to directly bonded nuclei. $\left({ }^{1} \mathrm{H},{ }^{1} \mathrm{H}\right)$ mixing times in N/CHHC spectra were 100 or 250 $\mu$ s. Frequency-switched homonuclear Lee-Goldburg (FSLG) $[96,97,313]$ decoupling at a ${ }^{1} \mathrm{H}$ field strength of $83 \mathrm{kHz}$ was used for the indirect ${ }^{1} \mathrm{H}$ evolution of the $\left({ }^{1} \mathrm{H},{ }^{15} \mathrm{~N}\right) \mathrm{HET}$ COR correlation spectrum in Figure 10.2. The proton dimension of this spectrum was calibrated to match peak positions and shift dispersion of regular $\left({ }^{1} \mathrm{H},{ }^{15} \mathrm{~N}\right)$ and $\left({ }^{1} \mathrm{H},{ }^{13} \mathrm{C}\right)$ HETCOR spectra. Water-edited one-dimensional (1D) CP buildup experiments [3] were recorded on 400 or $850 \mathrm{MHz}$ instruments at 6.5 or $10 \mathrm{kHz}$ MAS and $+7^{\circ} \mathrm{C}$ effective sample temperature. A $3 \mathrm{~ms}$ Gaussian pulse and a $\mathrm{T}_{2}$ filter containing two delays of $1 \mathrm{~ms}$ each were used for selective water excitation before longitudinal $\left({ }^{1} \mathrm{H},{ }^{1} \mathrm{H}\right)$ mixing and $\left({ }^{1} \mathrm{H},{ }^{13} \mathrm{C}\right)$ CP transfer of $700 \mu \mathrm{s}$. 
Two-dimensional (2D) spectra were typically acquired using 1280 data points in the direct and 375,34 or 25 data points in the indirect dimension of $\left({ }^{13} \mathrm{C},{ }^{13} \mathrm{C}\right),\left({ }^{15} \mathrm{~N},{ }^{13} \mathrm{C}\right)$, or NHHC correlation spectra, respectively, for measurements at $800 \mathrm{MHz}$ proton resonance frequency. CHHC spectra in Figure 10.8 were recorded using 1024 and 60 data points in direct and indirect dimensions, respectively, at $600 \mathrm{MHz}$ proton resonance frequency. Spectra were processed in XWinNMR or Topspin (Bruker Biospin, Karlsruhe, Germany), using $1024 \times 1024$ processing data points, and analyzed with Sparky [223].

\subsubsection{Analysis of water-edited buildup experiments}

Evaluation of ssNMR water-edited buildups depicted in Figure 10.3 was done as described in ref. [3]. Magnetization build-up in water-edited experiments is described by an initialrate time constant $t_{m}^{s}$ which is related to the ratio of volume $V$ to water-accessible surface

$S$ as $\frac{V}{S}=\sqrt{\frac{D_{\text {eff }} t_{m}^{s}}{\pi}}$ (Section 4.3.5). For the analysis, an effective magnetization diffusion coefficient $D_{\text {eff }}$ of $0.2 \mathrm{~nm}^{2} / \mathrm{ms}$, shown to be appropriate for low-resolution structural models with a flat surface [3], was assumed. In an elongated fibril with length $l$ and a circular or quadratic cross-section of diameter or edge length $d, \frac{V}{S}$ equals $\frac{d}{4}$ if $l \gg d$.

\subsubsection{Generation of structural models}

The models of polyglutamine fibrils shown in Figures 10.6 to 10.9 were obtained from extended $\mathrm{D}_{2} \mathrm{Q}_{15} \mathrm{~K}_{2}, \mathrm{GK}_{2} \mathrm{Q}_{38} \mathrm{~K}_{2}$, or $\mathrm{GK}_{2} \mathrm{Q}_{54} \mathrm{~K}_{2}$ strands by a standard simulated annealing protocol in CNS [175], enforcing $\beta$-sheet-like dihedral angles $(\phi, \psi)=\left(-119^{\circ}, 114^{\circ}\right)$ with a tolerance of $\pm 30^{\circ}$ and planarity in one, two, or three 15-residue segments along the sequence, respectively, by way of dihedral and planarity restraints. Distances corresponding to hydrogen bonding between backbone and sidechain amide and carbonyl groups as well as a stacking of backbone $\mathrm{C}^{\alpha}$ atoms between sheets of $8.3 \AA$ were enforced by distance restraints between the corresponding atoms. Four repeat units along the fibril axis were generated for each model, each consisting of two $\mathrm{D}_{2} \mathrm{Q}_{15} \mathrm{~K}_{2}$ or one $\mathrm{GK}_{2} \mathrm{Q}_{38} \mathrm{~K}_{2}$ or $\mathrm{GK}_{2} \mathrm{Q}_{54} \mathrm{~K}_{2}$ molecule(s), respectively. For the analysis of $\chi_{1}$ sidechain dihedral angles, only the central $\beta$-strand of the inner two molecules of the $\mathrm{GK}_{2} \mathrm{Q}_{54} \mathrm{~K}_{2}$ model was considered to exclude edge effects. 


\subsection{Results}

\subsubsection{Electron microscopy}

Figure 10.1 shows electron micrographs of aggregates grown from the three peptides investigated (courtesy of Dietmar Riedel). All of them form fibrillar aggregates whose length and degree of order appear to increase with the length of the construct. In agreement with electron microscopy (EM) data from an earlier study [296], $\mathrm{D}_{2} \mathrm{Q}_{15} \mathrm{~K}_{2}$ does not form the highly ordered, long filaments that are characteristic for typical amyloid fibrils (Fig. 10.1 a). Rather, smaller fibrillar aggregates of around $70 \AA$ in diameter and few hundreds of angstroms in length are visible that cluster together laterally and longitudinally to form higher-order aggregates. $\mathrm{GK}_{2} \mathrm{Q}_{38} \mathrm{~K}_{2}$ aggregates (Fig. $10.1 \mathrm{~b}$ ) are in the order of $100-$ $200 \mathrm{~nm}$ in length, with a broader, ribbon-like appearance and diameters up to about 200 $\AA$, likely due to lateral clustering of individual protofibrillar aggregates. Minimal fibril diameters observed are around $70-80 \AA$ and are more easily found in earlier stages of fibrillization (Appendix Fig. F.1). $\mathrm{GK}_{2} \mathrm{Q}_{54} \mathrm{~K}_{2}$ forms fibers several hundred nanometers in length (Fig. 10.1 c) that are often wound around each other. In this construct, various fibril diameters up to about $160 \AA$ can be found, while the minimum diameter is again about $80 \AA$ A. Additionally, in several samples and constructs, some loose, unfibrillized protein is present.

The EM data agree well with earlier studies on length-dependent properties of polyglutamine aggregates, which indicated that only aggregates from peptides with a glutamine repeat length above the disease threshold around 40 residues exhibit all features of classical amyloid fibrils [296]. They also agree with observations that polyglutamine peptides of intermediate length (in the range of 20 - 40 repeats) tend to form broader, more ribbon-like aggregates than longer constructs, which appear to assemble in a more typical amyloid fibril-like fashion with diameters around $80 \AA$ [296]. Nevertheless, also the shorter peptides form fibrillar aggregates, which are known to exhibit a cross- $\beta$ X-ray diffraction pattern and bind thioflavin $\mathrm{T}$ similar to bona fide amyloid fibrils [296, 309, 310]. The fibril diameters indicate that the minimal fibrillar unit of polyglutamine aggregates is about $70-80$ $\AA$ wide. Thus, at least the longer constructs should be folded instead of fully extended. The distribution of fibril diameters in the samples investigated here indicates that there may be more than one overall fold accessible to polyglutamine monomers within fibrils, apart from different levels of lateral aggregation of protofilaments. 


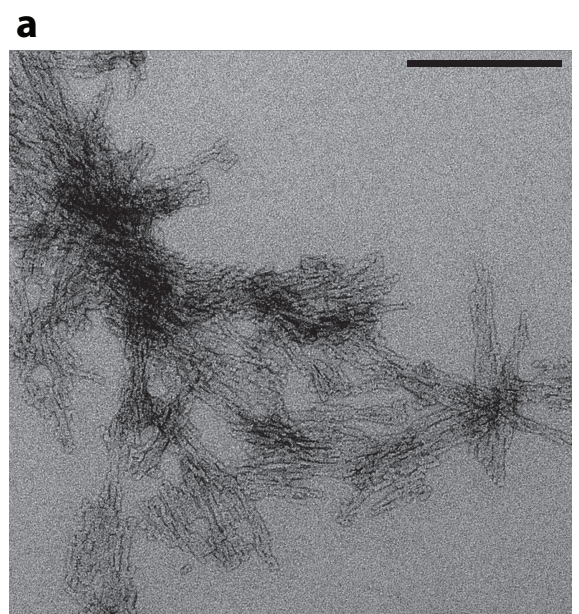

b
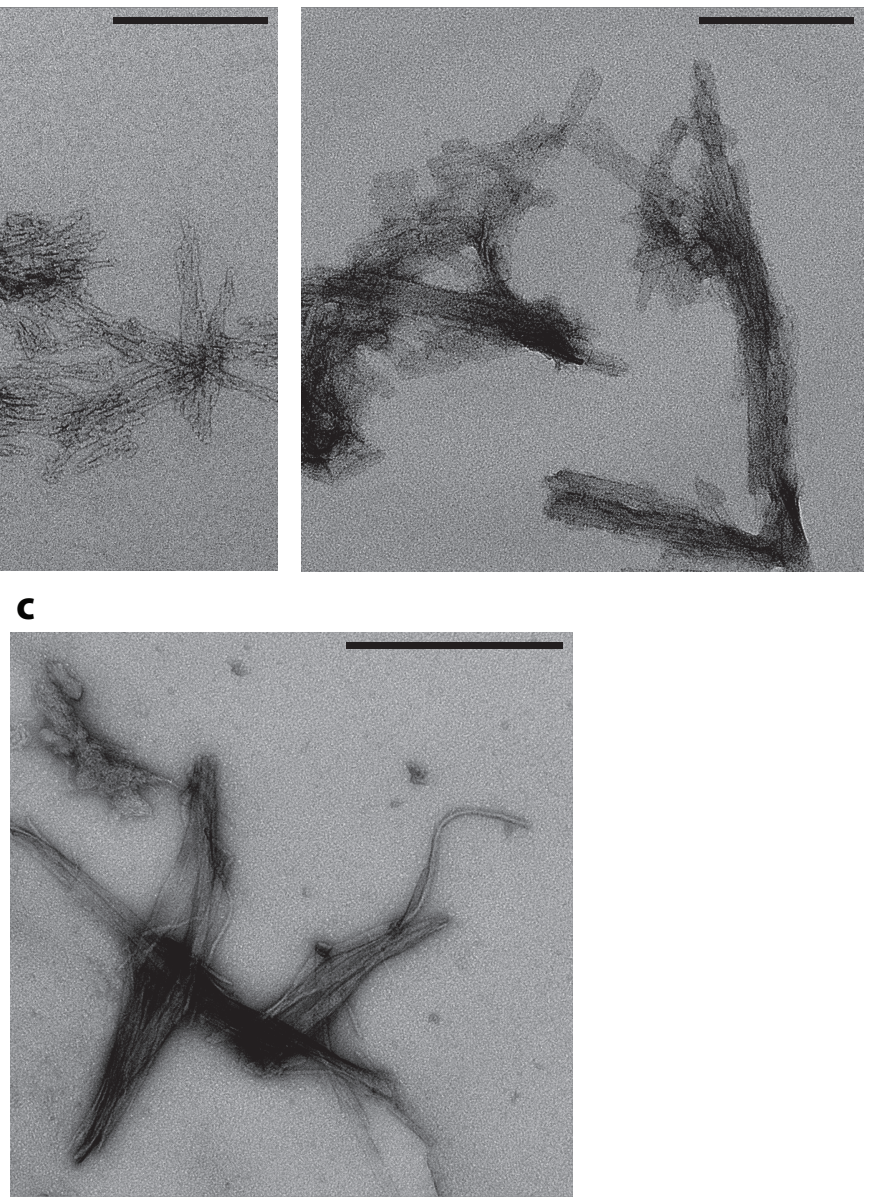

C

Figure 10.1: Electron micrographs of (a) $\mathrm{D}_{2} \mathrm{Q}_{15} \mathrm{~K}_{2}$, (b) $\mathrm{GK}_{2} \mathrm{Q}_{38} \mathrm{~K}_{2}$, and (c) $\mathrm{GK}_{2} \mathrm{Q}_{54} \mathrm{~K}_{2}$ fibrillar aggregates, courtesy of Dietmar Riedel. Scale bars are $200 \mathrm{~nm}$ in (a, b) and $500 \mathrm{~nm}$ in (c). 


\subsubsection{Overall fibril organization}

Initially, ssNMR experiments were conducted to investigate the overall arrangement of polyglutamine fibrils. In order to probe hydrogen bonding interactions, proton-nitrogen HETCOR correlation spectra on $\mathrm{D}_{2} \mathrm{Q}_{15} \mathrm{~K}_{2}$ fibrillar aggregates were recorded, using homonuclear frequency-switched Lee-Goldburg proton decoupling (FSLG) [96, 97, 313] (Fig. 10.2). Since it relies on strong dipolar couplings, this type of spectrum selects for rigid residues. The sizable downfield shift of backbone amide proton resonances, which appear

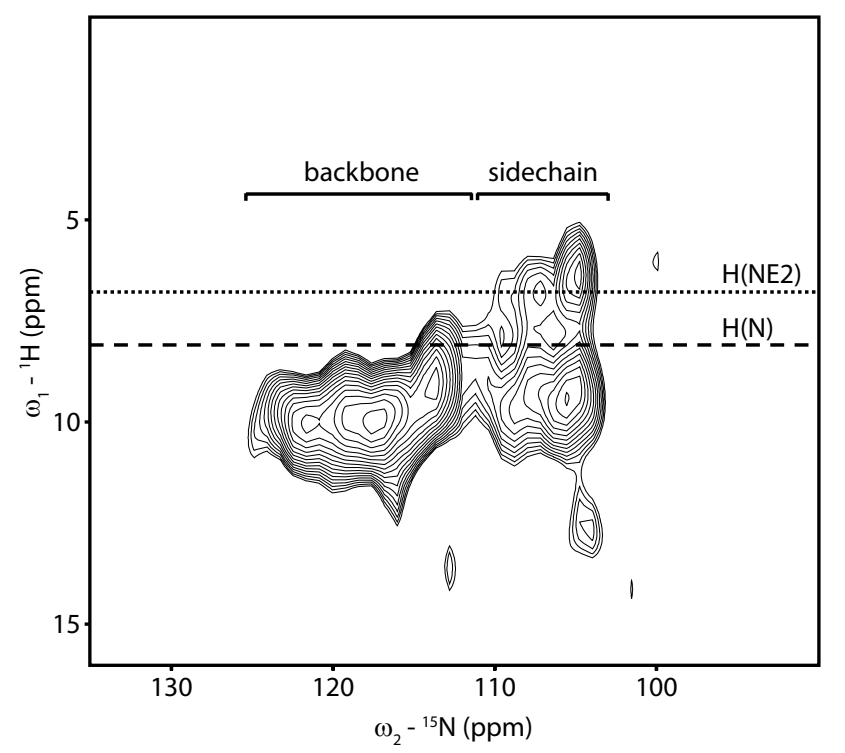

Figure 10.2: FSLG-HETCOR $\left({ }^{1} \mathrm{H},{ }^{15} \mathrm{~N}\right)$ correlation spectrum of $\mathrm{D}_{2} \mathrm{Q}_{15} \mathrm{~K}_{2}$. Chemical shift regions of glutamine backbone and sidechain nitrogen resonances are indicated. Dashed line shows the BMRB average value for the glutamine backbone amide proton chemical shift $(\mathrm{H}(\mathrm{N}), 8.2 \mathrm{ppm})$, dotted line that of the sidechain amide protons (H(NE2), $7.1 \mathrm{ppm})$. The spectrum was recorded by Henrik Müller.

around $10 \mathrm{ppm}$, indicates strong backbone hydrogen bonding of all rigid glutamine residues $[157,164,314]$, consistent with a $\beta$-sheet organization of the fibril core. Interestingly, such downfield shifts are also detected for a majority of glutamine sidechain amides, in line with a structural arrangement of polyglutamine molecules as postulated in steric or polar zipper models. According to these models, hydrogen bonds between glutamine sidechain amide and carbonyl groups along the sheet axis enforce an ordered arrangement of sidechains within a sheet, which enables tight interdigitation of sidechains from adjacent $\beta$-sheets $[303,310]$. 
In addition, water-edited ssNMR experiments were performed to probe the water accessibility of polyglutamine fibrils $[3,64,145]$ (see also Chapter 9 and Section 4.3.5). As described in Section 10.3.3, in the context of protein fibrils, these experiments give information about the fibril diameter. Data from water-edited buildup experiments on the three polyglutamine constructs are shown in Figure 10.3 and Appendix Table F.3. The analysis yields average fibril diameters of about $70 \AA$ for $\mathrm{D}_{2} \mathrm{Q}_{15} \mathrm{~K}_{2}, 110 \AA$ for $\mathrm{GK}_{2} \mathrm{Q}_{38} \mathrm{~K}_{2}$, and $85 \AA$ for $\mathrm{GK}_{2} \mathrm{Q}_{54} \mathrm{~K}_{2}$, respectively. These values correspond well to the diameters most commonly found in electron micrographs and accurately reflect the different width distributions and levels of lateral aggregation seen in EM. Importantly, these diameters were

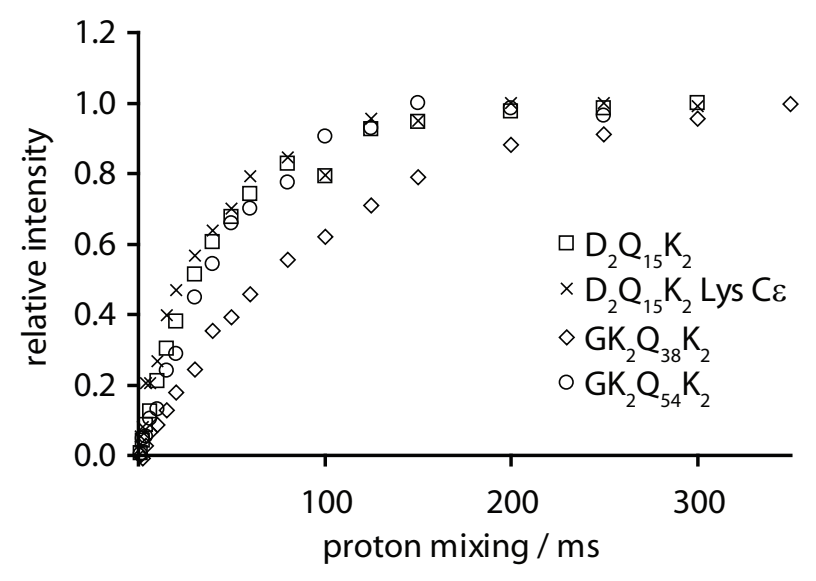

Figure 10.3: Buildups of water-edited cross-polarization intensity of the aliphatic spectral region $\left(15-65 \mathrm{ppm}\right.$ ) of the three polyglutamine constructs (open symbols) and of the resolved Lys $\mathrm{C}^{\varepsilon}$ peak of $D_{2} Q_{15} K_{2}$ (crosses).

calculated assuming compact, dry fibrils with no water inside. A water-filled structure of the same diameter would be characterized by a much larger surface and consequently a faster experimental magnetization buildup in water-edited experiments. In order to match experimental data, a water-filled structure would require a larger volume, which would be in conflict with fibril dimensions observed in EM. Thus, these data provide clear evidence that polyglutamine fibrils are not organized as water-filled nanotubes. Rather, they appear to have a dry, tightly packed fibrillar core as seen or postulated for other types of amyloid [20, 47, 63, 64, 67]. Figure 10.3 also shows the buildup of an isolated lysine sidechain $\mathrm{C}^{\varepsilon}$ peak of $\mathrm{D}_{2} \mathrm{Q}_{15} \mathrm{~K}_{2}$, originating from its only isotope-labeled lysine residue Lys18. The buildup of this peak is slightly faster than the average buildup of aliphatic resonances in $\mathrm{D}_{2} \mathrm{Q}_{15} \mathrm{~K}_{2}$. While this may partially be traced to chemical exchange processes of water 
protons with lysine sidechain amine protons [148], it nevertheless indicates that Lys18 is likely not buried within the $\mathrm{D}_{2} \mathrm{Q}_{15} \mathrm{~K}_{2}$ fibril, as suggested by some models [306], but rather exposed on its surface.

\subsubsection{Residue-specific ssNMR analysis}

Two-dimensional $\left({ }^{13} \mathrm{C},{ }^{13} \mathrm{C}\right)$ ssNMR spectra of $\mathrm{D}_{2} \mathrm{Q}_{15} \mathrm{~K}_{2}$, $\mathrm{GK}_{2} \mathrm{Q}_{38} \mathrm{~K}_{2}$, and $\mathrm{GK}_{2} \mathrm{Q}_{54} \mathrm{~K}_{2}$ fibrillar aggregates reveal striking similarities indicative of a common structural organization (Fig. 10.4). Most prominently, in all constructs and across different types of spectra selecting for more rigid parts of the sample, two major populations of glutamine residues are apparent whose resonance signals fall into distinct spectral regions (in the following denoted as populations 1 and 2). A third glutamine population is seen in $\left({ }^{13} \mathrm{C},{ }^{13} \mathrm{C}\right)$ correlation spectra with longer mixing times, likely due to enhanced mobility in these residues. A rather high spectral linewidth of around $2 \mathrm{ppm}$ in ${ }^{13} \mathrm{C}$ and $3 \mathrm{ppm}$ in ${ }^{15} \mathrm{~N}$ dimensions indicates some structural heterogeneity in all samples. Apparently, glutamine-rich sequences can assume slightly different molecular conformations within fibrillar aggregates, as also suggested by the distribution of fibril diameters observed in EM for $\mathrm{GK}_{2} \mathrm{Q}_{38} \mathrm{~K}_{2}$ and $\mathrm{GK}_{2} \mathrm{Q}_{54} \mathrm{~K}_{2}$ constructs. The observations of rather large spectral linewidths and of distinct sets of NMR signals from residues of the same type are consistent with recent ssNMR data on fibrils from asparagine- and glutamine-rich peptides and proteins $[307,315]$. Notably, crosspeaks corresponding to population 1 and 2 glutamines are also found in spectra of lyophilized polyglutamine samples recorded after addition of water or buffer solution, even though only oligomers can be detected in such samples by EM (Appendix Fig. F.2). This might indicate that the local organization leading to the occurrence of two distinct glutamine populations forms already in early stages of aggregation.

The two main populations of glutamine residues appear in ssNMR spectra with approximately equal intensities in all constructs, suggesting a distribution close to 1:1. Resonances of all nuclei of population 1 glutamines are consistently shifted to higher frequencies with respect to population 2 (Table 10.1). This effect has also been observed for a glutamine residue in fibrils formed by a asparagine- and glutamine-rich peptide from the yeast prion Sup35 [315]. Compared to average $\mathrm{C}^{\alpha}$ and $\mathrm{C}^{\beta}$ chemical shift values for glutamine, both populations exhibit a strongly negative secondary chemical shift of about -5 ppm (Table 10.1; see ref. [158] and http://www.bmrb.wisc.edu/). This clearly indicates that both groups of glutamine residues participate in $\beta$-sheet secondary structure [153] and 

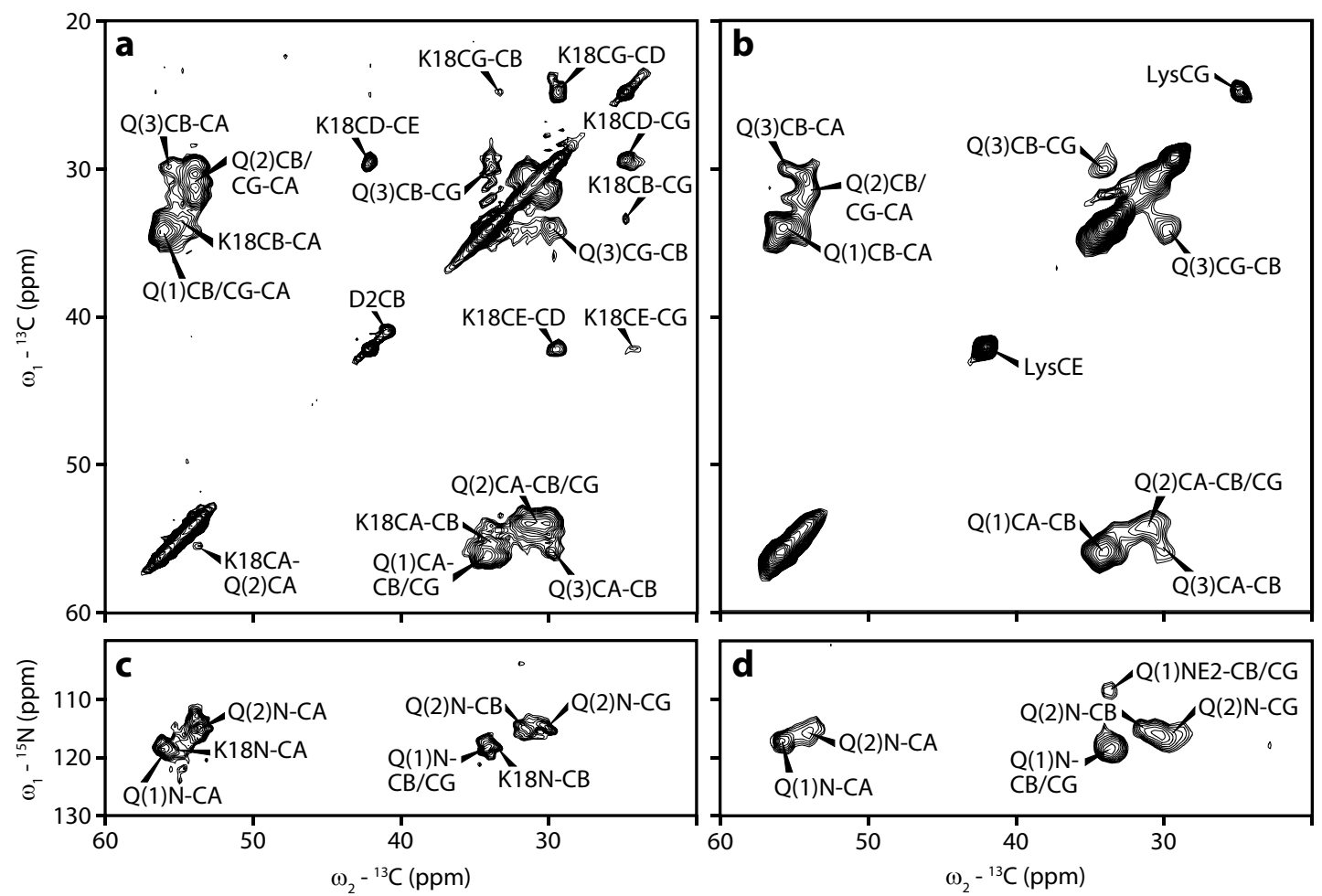

Figure 10.4: (a, b) $\left({ }^{13} \mathrm{C},{ }^{13} \mathrm{C}\right.$ ) spin diffusion correlation spectra (150 ms mixing time) and (c, d) intraresidue NCACB correlation spectra of $\mathrm{D}_{2} \mathrm{Q}_{15} \mathrm{~K}_{2}$ (a, c) and $\mathrm{GK}_{2} \mathrm{Q}_{54} \mathrm{~K}_{2}$ (b, d). Bracketed numbers in assignments refer to the different glutamine populations (see text). Corresponding carbonyl regions are depicted in Appendix Figure F.3.

\begin{tabular}{c|cccccccc}
\hline \hline $\begin{array}{c}\text { Gln population or } \\
\mathrm{D}_{2} \mathrm{Q}_{15} \mathrm{~K}_{2} \text { residue }\end{array}$ & $\mathrm{N}$ & $\mathrm{CO}$ & $\mathrm{CA}$ & $\mathrm{CB}$ & $\mathrm{CG}$ & $\mathrm{CD}$ & $\mathrm{CE}$ & $\mathrm{NE} 2$ \\
\hline 1 & 118.0 & 175.9 & 55.9 & 34.2 & 33.9 & 178.5 & 107.9 \\
2 & 115.4 & 174.0 & 54.0 & 31.6 & 29.8 & 177.4 & 104.4 \\
3 & & 174.0 & 55.7 & 29.9 & 33.9 & 180.2 & & \\
\hline $\mathrm{D} 2$ & & 177.6 & 54.6 & 40.9 & 180.0 & & & \\
$\mathrm{~K} 18$ & 118.6 & 174.8 & 55.1 & 33.5 & 24.7 & 29.5 & 42.1 & \\
\hline \hline
\end{tabular}

Table 10.1: List of average chemical shifts (in ppm) observed for the three glutamine populations in $\mathrm{D}_{2} \mathrm{Q}_{15} \mathrm{~K}_{2}, \mathrm{GK}_{2} \mathrm{Q}_{38} \mathrm{~K}_{2}$, and $\mathrm{GK}_{2} \mathrm{Q}_{54} \mathrm{~K}_{2}$ samples. Populations 1 and 2 constitute $\beta$-sheets, population 3 corresponds to residues in turn regions or unstructured molecules. For completeness, assignments for residues Asp2 and Lys18 of $D_{2} Q_{15} K_{2}$ are also given. 
that polyglutamine fibrillar aggregates are largely composed of $\beta$-sheets, consistent with their cross- $\beta$ X-ray diffraction pattern.

On the other hand, $\mathrm{C}^{\alpha}$ and $\mathrm{C}^{\beta}$ chemical shifts of glutamine population 3 visible in long mixing time $\left({ }^{13} \mathrm{C},{ }^{13} \mathrm{C}\right)$ spectra are close to random-coil values. These residues could constitute turns or unstructured regions within the fibrils, or they could occur in unstructured monomers with enhanced, but limited mobility. The appearance of loose protein in some electron micrographs is consistent with this assumption. However, the presence of highly mobile individual monomers as seen in fibril preparations of $\alpha$-synuclein [20] can be excluded for $\mathrm{D}_{2} \mathrm{Q}_{15} \mathrm{~K}_{2}$, where INEPT-based spectra probing molecules with solution statelike dynamics [113] only reveal minuscule lysine sidechain signals (Appendix Fig. F.4). In contrast, clear signal in INEPT spectra can be observed for $\mathrm{GK}_{2} \mathrm{Q}_{38} \mathrm{~K}_{2}$ and $\mathrm{GK}_{2} \mathrm{Q}_{54} \mathrm{~K}_{2}$. However, compared to cross-polarization $(\mathrm{CP})$ and direct excitation spectra, lysine signals are strongly enhanced with respect to glutamine signals, indicating that the INEPT signal predominantly arises from mobile termini of molecules that are otherwise rigid. These data suggest that, in the longer constructs, the lysine-containing $\mathrm{N}$ - and C-termini are not part of the fibril core, but protrude from it.

The presence of distinct glutamine populations with different chemical shifts raises the question whether these populations occur within a fibril monomer unit. Sequential NCOCA $\left(\mathrm{N}_{i}, \mathrm{C}_{i-1}\right)$ correlation spectra on the polyglutamine constructs exhibit spectral intensity predominantly at the same positions as seen in intraresidue $\operatorname{NCACB}\left(\mathrm{N}_{i}, \mathrm{C}_{i}\right)$ correlation spectra (Fig. 10.5 a-c), suggesting that glutamine residues of the two major $\beta$-sheet populations are mostly flanked by residues of the same population within the amino acid sequence. However, some peaks do occur that correspond to correlations between the two populations in the longer constructs. Sequential $\left({ }^{13} \mathrm{C},{ }^{13} \mathrm{C}\right)$ correlation spectroscopy [125] with inherently greater resolution and sensitivity confirms the existence of sequential correlations between the two main $\beta$-sheet populations 1 and 2 in $\mathrm{GK}_{2} \mathrm{Q}_{38} \mathrm{~K}_{2}$ (Fig. $10.5 \mathrm{~d}$ ) and $\mathrm{GK}_{2} \mathrm{Q}_{54} \mathrm{~K}_{2}$. This means that, at least in the longer polyglutamine constructs, glutamine residues of different chemical shift populations can occur within the same monomer. They appear to be clustered, however, in stretches of several residues of one population, as seen from sequential $\left(\mathrm{N}_{i}, \mathrm{C}_{i-1}\right)$ correlation spectra.

Spectral resolution, uniform isotope labeling, and the dominant presence of only one residue type preclude residue-specific sequential resonance assignments in the longer polyglutamine constructs. More insight into the distribution of glutamine residues with different shifts can be expected from the selectively labeled $\mathrm{D}_{2} \mathrm{Q}_{15} \mathrm{~K}_{2}$ construct (Section 10.3.1) 

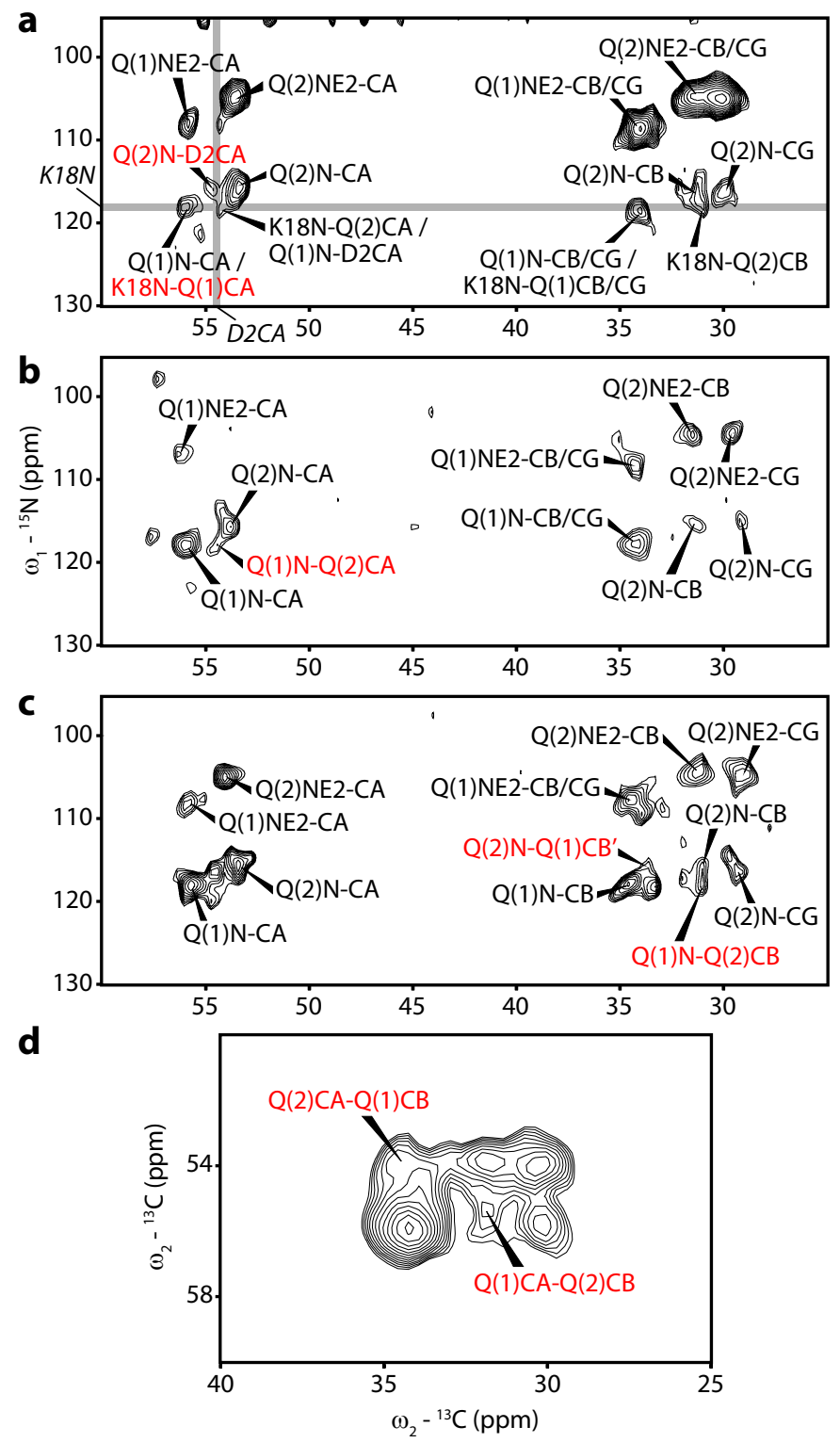

Figure 10.5: Interresidue correlation spectra. (a) - (c) Interresidue $\left(\mathrm{N}_{\mathrm{i}}, \mathrm{C}_{i-1}\right)$ NCOCA correlation spectra of (a) $D_{2} Q_{15} K_{2}$, (b) $G_{2} Q_{38} K_{2}$ (recorded by Henrike Heise), and (c) $G_{2}{ }_{2} Q_{54} K_{2}$. Grey lines in (a) indicate chemical shifts of Lys18 $\mathrm{N}$ and Asp2 $\mathrm{C}^{\alpha}$ important for assignment; red labels mark their most likely correlation partners. Red assignments in (b, c) indicate sequential correlations between different glutamine populations. (d) Section representing $C^{\alpha}-C^{\beta}$ correlations of a sequential $\left({ }^{13} \mathrm{C},{ }^{13} \mathrm{C}\right)$ spin diffusion spectrum of $\mathrm{GK}_{2} \mathrm{Q}_{38} \mathrm{~K}_{2}$ recorded under weak coupling conditions [125] with $150 \mathrm{~ms}$ mixing time. Sequential correlations between different glutamine populations are indicated. 
in which sequential correlations from only three residue pairs can be expected. The labeled residues Asp2 and Lys18 can be identified unambiguously in ssNMR spectra based on their chemical shifts, although Asp2 resonances are usually weak (Fig. 10.4 b). The main interresidue cross-correlations in an $\left(\mathrm{N}_{i}, \mathrm{C}_{i-1}\right)$ correlation spectrum of $\mathrm{D}_{2} \mathrm{Q}_{15} \mathrm{~K}_{2}$ (Fig. 10.5 a) then indicate that Gln17 belongs to glutamine population 1 and Gln3, Gln9 and Gln10 to population 2. However, shoulders of the main peaks within the observed range of Lys18 amide nitrogen and Asp2 $\mathrm{C}^{\alpha}$ chemical shifts (grey lines in Fig. 10.5 a) show that the respective other possibilities cannot be ruled out completely. In fact, the NCOCA spectrum in Figure 10.5 a could in principle even be explained by two groups of $\mathrm{D}_{2} \mathrm{Q}_{15} \mathrm{~K}_{2}$ molecules composed entirely of one population of glutamines, since the chemical shifts of the labeled residues cannot be correlated across gaps in the labeling pattern. These data indicate that glutamine populations 1 and 2 do not necessarily correspond to specific positions within the peptide sequence, although preferences at different residue positions seem to exist.

It is important to note that the interresidue correlation corresponding to neighboring glutamines of population 2 in the $\mathrm{D}_{2} \mathrm{Q}_{15} \mathrm{~K}_{2}$ NCOCA spectrum (labeled $\mathrm{Q}(2) \mathrm{N}-\mathrm{CA}$ in Fig. 10.5 a) is outside of the chemical shift ranges of both the Lys18 amide nitrogen and the Asp2 $\mathrm{C}^{\alpha}$ nucleus. Thus, to account for this peak, the Gln9-Gln10 residue pair has to be invoked. This means that, at least in a substantial part of the sample, two consecutive central glutamines exhibit chemical shifts corresponding to an extended $\beta$ sheet conformation (see also below), i.e. they cannot form a bend or turn.

\subsubsection{Structural significance of the different shift populations}

To investigate a possible correlation between chemical shift population and backbone conformation in the glutamine residues, an in silico grid search was conducted to identify glutamine backbone conformations with optimal agreement between SHIFTX-predicted [163] and experimental chemical shifts (Appendix Section F.1; compare Section 8.3.3). In addition, the Biological Magnetic Resonance Data Bank (BMRB) database was searched for glutamine residues with assignments corresponding to the more unusual population 1 values, for which no good fits could be obtained in the grid search (see Appendix Section F.1 for details). Glutamine backbone dihedral angles found in these analyses were nearly exclusively in most favored or allowed $\beta$-sheet regions of the Ramachandran plot for populations 1 and 2, while best fitting dihedral angles for population 3 glutamines corresponded to polyproline helix or $\alpha$-helix secondary structure (Appendix Fig. F.5). These findings 
thus confirm that both population 1 and 2 glutamines participate in $\beta$-sheet secondary structure, while population 3 residues appear to be in coil, turn or bend regions.

The dihedral angle distributions found for the two $\beta$-sheet populations exhibit considerable scatter and overlap; thus, assigning distinct preferred backbone dihedral angles to the two residue groups is difficult. However, average values of the two dihedral angle distributions are different (see Appendix Section F.1), which may partly account for the presence of two groups of $\beta$-sheet glutamine residues with distinct chemical shifts. The two glutamine populations may, for example, exhibit complementary dihedral angles that compensate for $\beta$-strand twist and allow for assembly of relatively flat strands and sheets, as required for elongated amyloid fibrils with no or only little fibril twist [316, 317].

Alternatively, the occurrence of two distinct sets of glutamine chemical shifts may be related to different sidechain rotameric states in the corresponding residues. It is well known that sidechain dihedral angles, especially $\chi_{1}$, can have a sizable impact on sidechain and backbone chemical shifts [154, 164, 318, 319] (see Appendix Fig. F.6). Sidechain conformations can be expected to depend on the exact arrangement of polyglutamine monomers in the fibrils. For example, in a polar zipper arrangement as postulated by Xray studies [303, 310], which so far appears consistent with ssNMR data, hydrogen bonds between sidechain amide and carboxyl groups along the fibril axis and tight stacking of $\beta$-sheets are likely to strongly constrain individual sidechain orientations.

A model exhibiting the core features of a polyglutamine polar zipper, assembled using simulated annealing in CNS [175], indeed shows a well ordered sidechain arrangement (Fig. 10.6 a). This is the case even if, for assembling the model, no further explicit constraints on sidechain atoms are imposed except that the polar sidechain termini of residues within one sheet should be hydrogen bonded along the fibril axis. $\chi_{1}$ angles in central $\beta$-strands of this model are broadly clustered around the gauche ${ }^{+}\left(-60^{\circ}\right)$, gauche $^{-}$ $\left(60^{\circ}\right)$, and trans $\left(180^{\circ}\right)$ orientations preferred in proteins $[164,320]$. Additionally, they alternate quite regularly between consecutive residues if all sidechain carbonyl groups are forced to point in the same direction (Fig. 10.6 b), while such alternation is much less pronounced if sidechain carbonyl groups of consecutive residues point in opposite directions (Fig. 10.6 c). In 60 modeled strands, the average absolute difference between consecutive residues is $91^{\circ}$ in the former case, and differences between consecutive $\chi_{1}$ angles above $60^{\circ}$ occur about seven times in a strand of 15 residues. In the latter case, the average absolute difference is reduced to $41^{\circ}$, and in a 15 -residue strand, on average only three consecutive $\chi_{1}$ angles differ by more than $60^{\circ}$. 

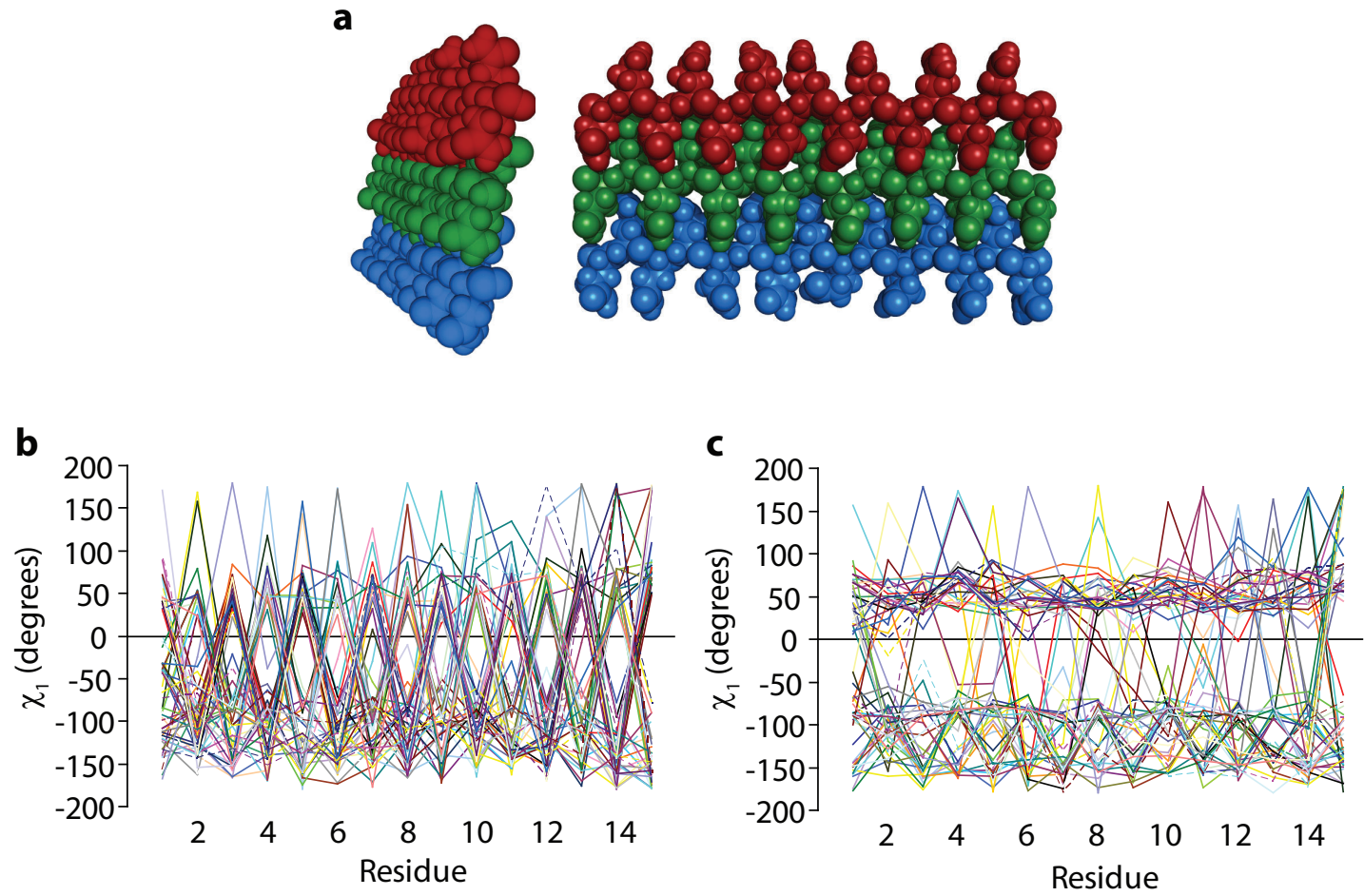

Figure 10.6: (a) Illustration of the repeat unit of a model of cross- $\beta$ polyglutamine fibrils obtained by enforcing tight sheet stacking and hydrogen bonding in both backbone and sidechain regions. View in the left panel is down the covalent chain axis, with the fibril axis running from left to right, while in the right panel, view is down the fibril axis into the paper plane. Backbone and sidechain hydrogen bonds are along the fibril axis (into the paper plane in the right panel). Three stacked polyglutamine $\beta$-strands are shown in different colors. (b) Pattern of $\chi_{1}$ dihedral angles observed in residues of the central $\beta$-strand of the model if all sidechain hydrogen bonds are oriented identically, i.e. all sidechain carbonyls are forced to point in the same direction along the fibril axis. (c) Same as (b), but with sidechain carbonyls of consecutive residues pointing in alternating directions. 
While these data were obtained on a model, they nevertheless indicate that, in a polar zipper arrangement, individual sidechains are indeed sterically strongly constrained. It also appears that, in such a structural context, the orientation of the sidechain carbonyl group can influence the $\chi_{1}$ angle, which is known to affect backbone chemical shifts. Certain sidechain hydrogen bonding patterns may then lead to a clustering of similar $\chi_{1}$ angles along the peptide sequence, consistent with the clustering observed in chemical shift populations.

\subsubsection{Through-space correlations}

In order to probe intra- and intermolecular through-space contacts, N/CHHC spectra [136, 137] and $\left({ }^{13} \mathrm{C},{ }^{13} \mathrm{C}\right)$ spin diffusion spectra with long mixing times were recorded (Figs. 10.7 and 10.8). For the selectively labeled $\mathrm{D}_{2} \mathrm{Q}_{15} \mathrm{~K}_{2}$ sample, an NHHC spectrum with a $\left({ }^{1} \mathrm{H},{ }^{1} \mathrm{H}\right)$ mixing time of $100 \mu \mathrm{s}$ is depicted in Figure 10.7 a (upper panel) in black, overlaid with the same spectrum recorded on a $\mathrm{D}_{2} \mathrm{Q}_{15} \mathrm{~K}_{2}$ sample diluted in unlabeled material before fibrillization (blue). Peaks observable only in the spectrum of the undiluted sample consequently correspond to intermolecular contacts. The variability of chemical shifts observed in polyglutamine fibrils, especially in ${ }^{15} \mathrm{~N}$ dimensions, complicates comparisons of $\left({ }^{15} \mathrm{~N},{ }^{13} \mathrm{C}\right)$ correlation spectra across different sample preparations. Nevertheless, while the relatively short $\left({ }^{1} \mathrm{H},{ }^{1} \mathrm{H}\right)$ mixing time of $100 \mu$ s leads to the dominant presence of intraresidue and sequential correlations [70], some peaks can be identified that are not present in NCACB or NCOCA correlation spectra or in the NHHC spectrum of diluted $\mathrm{D}_{2} \mathrm{Q}_{15} \mathrm{~K}_{2}$. Already at this mixing time, weak intermolecular correlations between glutamine sidechain amide nitrogens of one population and sidechain as well as backbone carbons of the respective other population become apparent. This points to a well-ordered, rather rigid arrangement of glutamine sidechains in the fibrillar aggregates. Moreover, the distance between a sidechain amide proton and a backbone $\mathrm{C}^{\alpha}$ proton in a neighboring strand within the same $\beta$-sheet should be on the order of $5-6 \AA$ (assuming extended sidechains and a standard interstrand separation of $4.8 \AA$ ) and would thus most likely be too long to be observed with the short $\left({ }^{1} \mathrm{H},{ }^{1} \mathrm{H}\right)$ mixing time employed here [137]. These correlations are thus best explained by a close proximity of the glutamine sidechain termini and the next $\beta$-sheet layer stacked above or below them. Together with sidechain hydrogen bonding observed in the $\left({ }^{1} \mathrm{H},{ }^{15} \mathrm{~N}\right)$ FSLG-HETCOR spectrum in Figure 10.2, these data provide support for the presence of tightly stacked $\beta$-sheets with interdigitated sidechains in polyglutamine aggregates, as proposed by polar zipper models [303, 310]. 


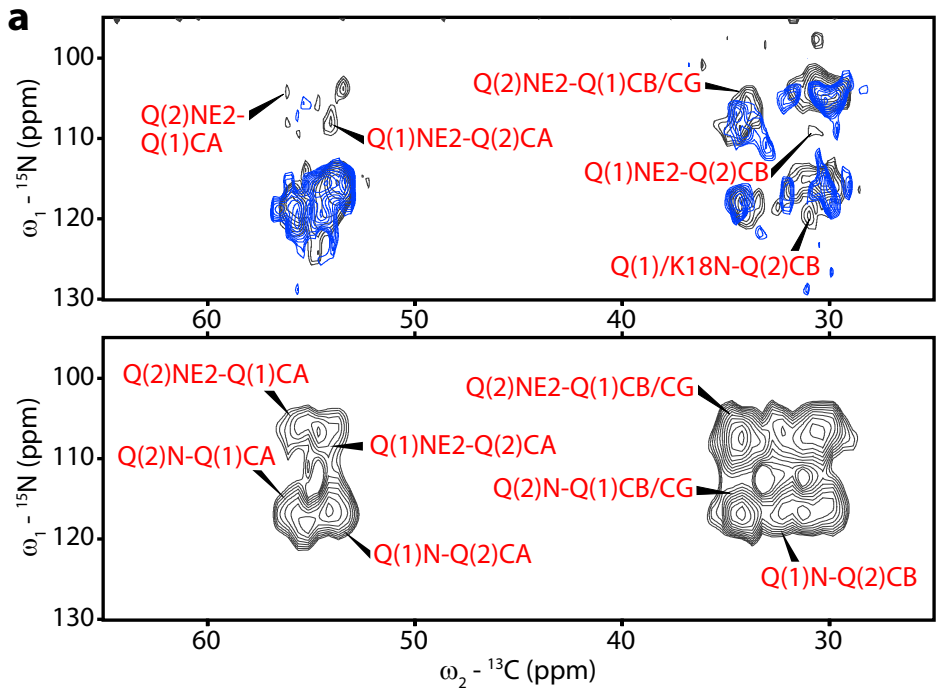

b

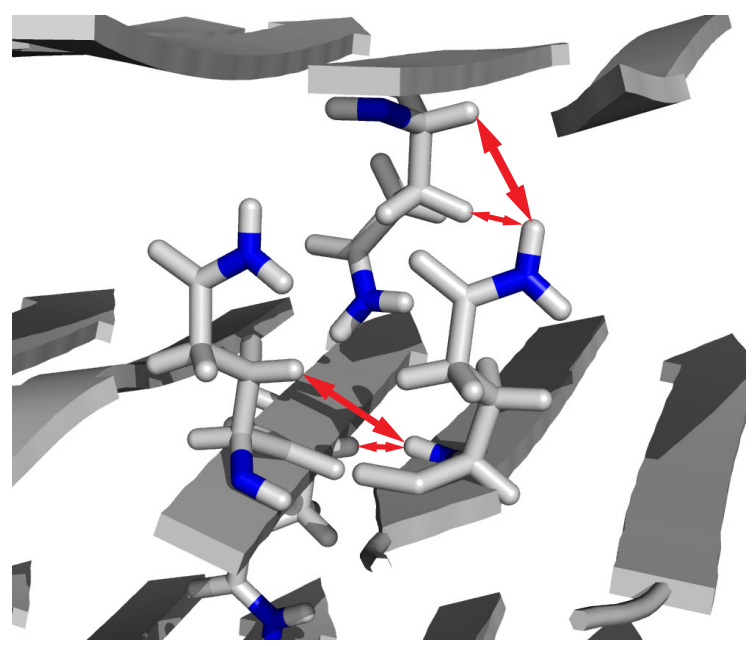

Figure 10.7: $\left({ }^{15} \mathrm{~N},{ }^{13} \mathrm{C}\right)$ through-space correlations. Crosspeaks between different glutamine populations are labeled in red. (a) Top: Overlay of NHHC spectra (100 $\mu$ s mixing time) of undiluted, selectively labeled $D_{2} Q_{15} K_{2}$ fibrils (black) and selectively labeled $D_{2} Q_{15} K_{2}$ diluted in 2.5 parts of unlabeled material before fibrillization (blue). Bottom: NHHC spectrum (250 $\mu$ s mixing) of uniformly $\left[{ }^{15} \mathrm{~N},{ }^{13} \mathrm{C}\right]$-labeled $\mathrm{GK}_{2} \mathrm{Q}_{54} \mathrm{~K}_{2}$ fibrils (recorded by Henrik Müller). (b) Scheme illustrating the contacts seen in $\mathrm{NHHC}$ spectra in a hypothetical arrangement of stacked antiparallel $\beta$-sheets. 
In addition, a peak in the backbone amide nitrogen region confirms intermolecular contacts within the $\beta$-sheet plane between population 1 and 2 glutamines or between Lys18 and a population 2 glutamine. Although, as discussed, the labeled glutamine residues in $\mathrm{D}_{2} \mathrm{Q}_{15} \mathrm{~K}_{2}$ cannot unambiguously be assigned to the two shift populations, the main interresidue NCOCA crosspeaks suggest that Gln3, Gln9, and Gln10 mainly belong to population 2 and Gln17 mainly to population 1. This intermolecular crosspeak thus gives an indication that $\mathrm{D}_{2} \mathrm{Q}_{15} \mathrm{~K}_{2}$ monomers should assemble into fibrils in an antiparallel fashion.

An NHHC spectrum with a longer mixing time of $250 \mu$ s, recorded on uniformly $\left[{ }^{15} \mathrm{~N},{ }^{13} \mathrm{C}\right]$-labeled $\mathrm{GK}_{2} \mathrm{Q}_{54} \mathrm{~K}_{2}$ fibrils, is depicted in the lower panel of Figure 10.7 a. Here, the through-space correlations between different glutamine populations are equally well represented as the correlations within individual residues or populations. Especially the sidechain-backbone correlations between the populations further confirm a tight stacking of $\beta$-sheets as assumed in polar zipper models. Figure $10.7 \mathrm{~b}$ illustrates the NHHC contacts seen in the spectra of Figure 10.7 a in a hypothetical arrangement of closely stacked antiparallel $\beta$-sheets.

Further constraints on the folding of polyglutamine monomers into fibrils were obtained from $\left({ }^{13} \mathrm{C},{ }^{13} \mathrm{C}\right) \mathrm{CHHC}$ and spin diffusion spectra recorded on $\mathrm{GK}_{2} \mathrm{Q}_{38} \mathrm{~K}_{2}$ and $\mathrm{GK}_{2} \mathrm{Q}_{54} \mathrm{~K}_{2}$ fibrils. In order to differentiate between intra- and intermolecular correlations, spectra of uniformly $\left[{ }^{15} \mathrm{~N},{ }^{13} \mathrm{C}\right]$-labeled fibrils were compared with spectra of fibrils prepared from a 1:1 mixture of either ${ }^{15} \mathrm{~N}$ - or ${ }^{13} \mathrm{C}$-labeled monomers. Figure 10.8 a shows a comparison of $\left({ }^{13} \mathrm{C},{ }^{13} \mathrm{C}\right)$ spin diffusion spectra recorded with a long mixing time of $500 \mathrm{~ms}$ on uniformly $\left[{ }^{15} \mathrm{~N},{ }^{13} \mathrm{C}\right]$-labeled (top) and mixed ${ }^{15} \mathrm{~N}$ - or ${ }^{13} \mathrm{C}$-labeled $\mathrm{GK}_{2} \mathrm{Q}_{38} \mathrm{~K}_{2}$ fibrils (bottom). Correlations between glutamine residues of populations 1 and 2 are strong in the uniformly labeled sample and attenuated with respect to the intra-population crosspeaks in the mixed labeled sample. This observation shows that the inter-population crosspeaks contain an intermolecular contribution.

In order to clearly differentiate between intermolecular contributions and magnetization transfer along the carbon-carbon bond network dominant in $\left({ }^{13} \mathrm{C},{ }^{13} \mathrm{C}\right)$ spin diffusion spectra $[136,137]$, CHHC spectra with $250 \mu$ s mixing time were recorded on the $\mathrm{GK}_{2} \mathrm{Q}_{38} \mathrm{~K}_{2}$ samples (Fig. 10.8 b). Whereas correlations between glutamine populations 1 and 2 are clearly visible in the uniformly labeled sample, they are virtually absent in the mixed labeled sample. This indicates that these crosspeaks originate from intermolecular contacts and is consistent with the previously discussed finding that most sequential contacts occur 

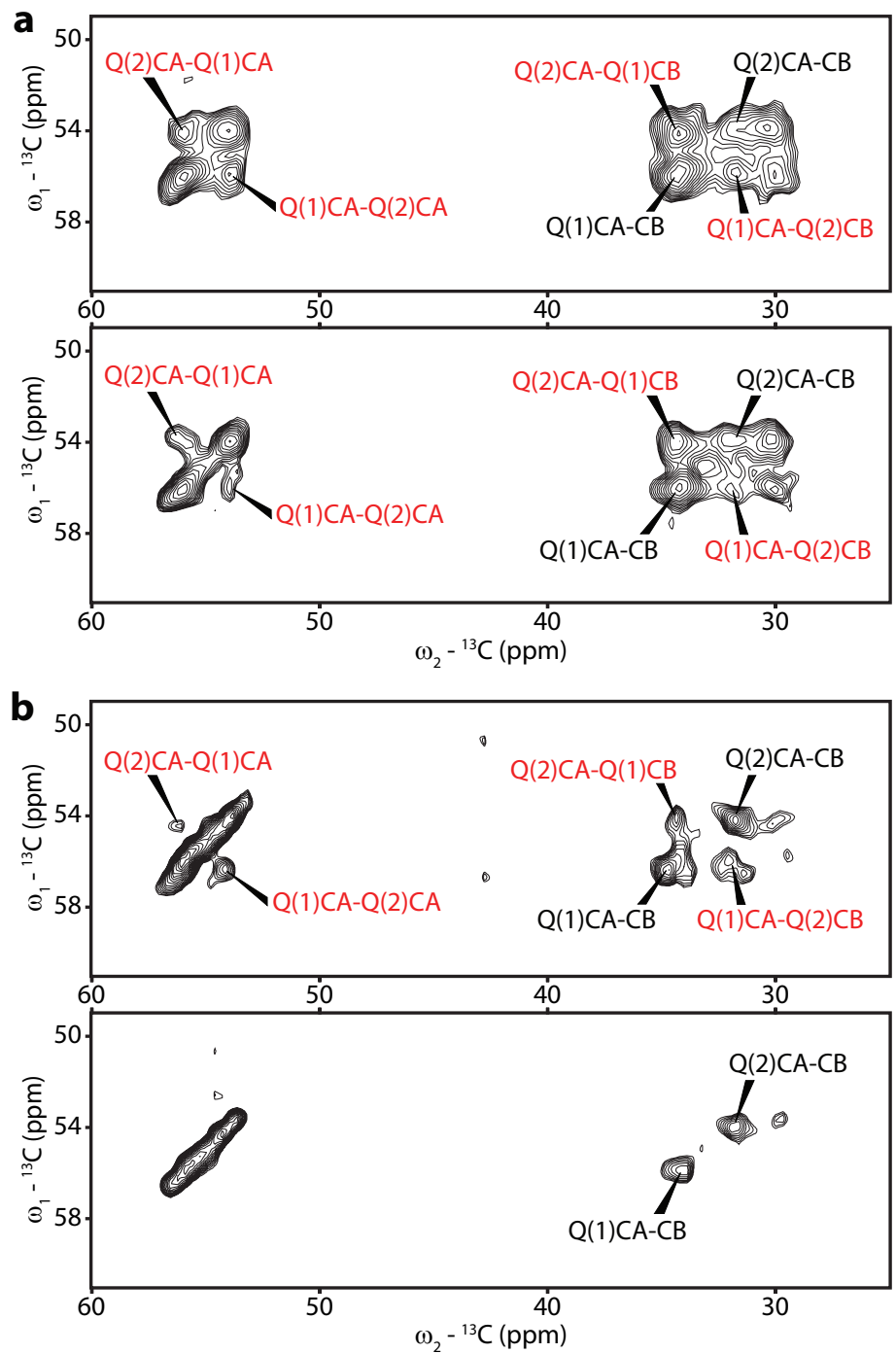

Figure 10.8: $\left({ }^{13} \mathrm{C},{ }^{13} \mathrm{C}\right)$ through-space correlations. Crosspeaks between different glutamine populations are labeled in red. (a) Cut-outs from $\left({ }^{13} \mathrm{C},{ }^{13} \mathrm{C}\right.$ ) spin diffusion spectra with $500 \mathrm{~ms}$ mixing time of uniformly $\left[{ }^{15} \mathrm{~N},{ }^{13} \mathrm{C}\right.$ ]-labeled $\mathrm{GK}_{2} \mathrm{Q}_{38} \mathrm{~K}_{2}$ fibrils (top) and of $\mathrm{GK}_{2} \mathrm{Q}_{38} \mathrm{~K}_{2}$ fibrils consisting of a $1: 1$ mixture of ${ }^{15} \mathrm{~N}$ - or ${ }^{13} \mathrm{C}$-labeled monomers (bottom). (b) Cut-outs from $\mathrm{CHHC}$ spectra (250 $\mu$ s mixing time) recorded on uniformly $\left[{ }^{15} \mathrm{~N},{ }^{13} \mathrm{C}\right]$-labeled $\mathrm{GK}_{2} \mathrm{Q}_{38} \mathrm{~K}_{2}$ fibrils (top) and on $\mathrm{GK}_{2} \mathrm{Q}_{38} \mathrm{~K}_{2}$ fibrils consisting of a $1: 1$ mixture of ${ }^{15} \mathrm{~N}$ - or ${ }^{13} \mathrm{C}$-labeled monomers (bottom). 
between glutamine residues of the same population. Since the distance between backbone atoms of residues in stacked sheets should not be smaller than about $8 \AA$ [310], it can be assumed that especially $\mathrm{C}^{\alpha}-\mathrm{C}^{\alpha}$ contacts between different populations occur chiefly between neighboring strands within the same $\beta$-sheet. The fact that they are not visible in the spectrum of the mixed labeled sample strongly suggests that $\mathrm{GK}_{2} \mathrm{Q}_{38} \mathrm{~K}_{2}$ does not fold back within one $\beta$-sheet as an antiparallel hairpin. Individual monomers rather appear to fold in a superpleated $\beta$-sandwich fashion [321, 322] where one molecule contributes strands to different $\beta$-sheets stacked on top of each other. In this arrangement, all contacts between strands within one sheet are intermolecular, consistent with CHHC data.

In addition, one of the $\mathrm{CHHC}$ crosspeaks between $\mathrm{C}^{\alpha}$ nuclei of different glutamine populations appears with an intensity comparable to intraresidue or intra-population crosspeaks in the spectrum of uniformly labeled $\mathrm{GK}_{2} \mathrm{Q}_{38} \mathrm{~K}_{2}$ (Fig. $10.8 \mathrm{~b}$, upper panel). This suggests that $\beta$-sheets in $\mathrm{GK}_{2} \mathrm{Q}_{38} \mathrm{~K}_{2}$ fibrils are arranged in an antiparallel fashion, since the distance between $\mathrm{H}^{\alpha}$ protons in neighboring strands is much shorter in antiparallel $\beta$-sheets (about $2.3 \AA$ ) than in parallel sheets (about $4.8 \AA$ ) [114]. Data on $\mathrm{GK}_{2} \mathrm{Q}_{54} \mathrm{~K}_{2}$ fibrils confirm these conclusions (Appendix Fig. F.7).

\subsection{Discussion}

The structural arrangement of polyglutamine fibrils was investigated using solid-state NMR and electron microscopy. Electron micrographs show that the length of the fibrillar aggregates increases with the length of the construct. Different fibril diameters likely reflect different levels of lateral aggregation of protofilaments as well as different $\beta$-strand lengths. Water-edited spectroscopy shows that polyglutamine fibrils possess a dry fibrillar core of $70-80 \AA$ diameter or larger, depending on the construct. Data presented here are thus incompatible with water-filled, $\beta$-helical nanotubes [309] in agreement with molecular dynamics studies suggesting their limited stability [306, 311]. Proton spectroscopy and through-space correlations suggest that glutamine sidechains in fibrillar aggregates are well ordered, largely hydrogen bonded, and tightly interdigitated as postulated by polar zipper models of polyglutamine amyloid fibrils [303, 310].

Local sequential and spatial organization appears to be similar in the different constructs. Despite rather large spectral linewidths, indicating structural variability, two distinct main populations of glutamine residues were identified in all samples which constitute the $\beta$-sheet fibrillar core and are discernible by chemical shift. They occur within 
individual monomers and are clustered in stretches of several residues. The findings of an increased spectral linewidth and of distinct chemical shift populations are in agreement with earlier studies on fibrils from asparagine- and glutamine-rich peptides [307, 315]. However, it could be shown that residue groups with different chemical shifts can occur within individual fibrils and monomers, while they have so far been assumed to constitute different fibrils [315].

Thorough analysis of conformation-dependent ssNMR chemical shifts reveals that residues of both major glutamine chemical shift populations are in $\beta$-sheet conformations. Their dihedral angle distributions appear to cover overlapping, but different regions of Ramachandran space. One explanation for the existence of two chemical shift populations may thus be that they represent groups of residues with different average backbone dihedral angles. It has been demonstrated by cryo-EM and Fourier-transform infrared spectroscopy (FTIR) that $\beta$-sheets in amyloid fibrils are more planar than in globular proteins, which allows for the assembly of long filaments with little or no filament twist $[316,317]$. A flat, planar $\beta$-sheet requires $\beta$-strands with only a small degree of strand twist along the polypeptide chain. Such flat $\beta$-strands have been found or postulated for amyloid fibrils also in X-ray or ssNMR studies [47, 63, 66, 316, 323]. There, they are assembled from residues with narrow dihedral angle distributions centered around the $\psi=-\phi$ diagonal of the Ramachandran plot. Alternatively, different residues or groups of residues may exhibit complementary backbone dihedral angles mutually compensating for $\beta$-strand twist, which could lead to distinct sets of chemical shifts in these residues.

On the other hand, sidechain dihedral angles, especially $\chi_{1}$, are known to influence sidechain and backbone chemical shifts to an extent comparable to the shift differences between the populations observed here $[154,164,318,319]$. Already in a simple model of polyglutamine fibrils, sidechain conformational freedom is restrained considerably if tightly stacked $\beta$-sheets and hydrogen bonds between polar sidechain groups are present, as postulated by polar zipper models $[303,310]$. In particular, the orientations of glutamine sidechain carbonyl and amide groups can apparently lead to quite regular patterns of sidechain $\chi_{1}$ dihedral angles in consecutive residues. This hypothesis thus offers an alternative explanation for the presence of populations of residues with distinct sets of chemical shifts. Notably, this explanation would well account for the experimental findings that the populations appear clustered in the sequence, that sidechain chemical shifts are distinct between the populations as well, and that only few such shift populations are found. Additional ssNMR experiments that probe backbone torsion angles using the evolution of 
multiple-quantum coherence or that correlate anisotropic chemical shielding interactions may help to distinguish between the effects of backbone and sidechain conformation.

For $\mathrm{D}_{2} \mathrm{Q}_{15} \mathrm{~K}_{2}$, the observed diameter of fibrillar aggregates of about $70 \AA$ corresponds nearly exactly to the length of a fully extended strand of this 19-residue peptide, assuming a standard $\beta$-sheet-like extension of around $3.5 \AA$ per residue. Earlier investigations have assumed the $\mathrm{D}_{2} \mathrm{Q}_{15} \mathrm{~K}_{2}$ peptide to fold back to form a hairpin-like structure in fibrillar aggregates [296, 304, 310]. However, no evidence of substructures or smaller protofilaments (e.g. via signs of fibril twist) was found in electron micrographs of the polyglutamine aggregates investigated here. In line with these findings, ssNMR data suggest that, at least in a considerable fraction of $\mathrm{D}_{2} \mathrm{Q}_{15} \mathrm{~K}_{2}$ molecules, two consecutive central glutamines must be in a standard extended $\beta$-sheet conformation. Finally, water-edited experiments indicate that the terminal lysine residues face the outside of the fibril. Taken together, data presented here strongly suggest that $\mathrm{D}_{2} \mathrm{Q}_{15} \mathrm{~K}_{2}$ is present in fibrillar aggregates as a single extended strand, indicating that $\beta$-strands in amyloid fibrils may be considerably longer than they are on average in globular proteins [324, 325].

With a similar $\beta$-strand length as in $\mathrm{D}_{2} \mathrm{Q}_{15} \mathrm{~K}_{2}, \mathrm{GK}_{2} \mathrm{Q}_{38} \mathrm{~K}_{2}$ would form two and $\mathrm{GK}_{2} \mathrm{Q}_{54} \mathrm{~K}_{2}$ three $\beta$-strands, connected by turns or bends, compatible with minimum fibril diameters observed in EM. However, the variations in fibril diameter suggest not only lateral aggregation of individual protofilaments, but also a polymorphic nature of the longer constructs in terms of $\beta$-strand length. This is also indicated by mutation studies investigating aggregation kinetics of glutamine homopolypeptides interspersed with turninducing proline-glycine pairs. Such peptides aggregate as rapidly as wild-type polyglutamine peptides of equal length, provided $\beta$-strands can be at least seven residues in length [326]. This agrees well with ssNMR data, since the finding that $\mathrm{D}_{2} \mathrm{Q}_{15} \mathrm{~K}_{2}$ does not form a turn or bend indicates that $\beta$-strands in polyglutamine fibrils should be at least seven or eight residues long. With such an organization of the peptide sequence, a peptide of around 40 residues in length would form four $\beta$-strands. These results thus underline the variable folding options inherently available in the context of a homopolypeptide, where similar interactions can in principle occur between any two residues, as opposed to regular proteins in which a specific amino acid sequence places more restraints on folding.

Neighboring strands within a $\beta$-sheet, even in the longer polyglutamine constructs, appear to belong to different monomers. This finding is best explained by a superpleated $\beta$-sandwich-like organization $[321,322]$ in which individual monomers are folded in a plane orthogonal to the fibril axis and contribute strands to more than one $\beta$-sheet. A similar 
type of fold has also been postulated for several other types of amyloid, such as fibrils formed from the prion domains of Ure2p [327], Sup35p [308], and the A $\beta$ peptide [63]. In the case of $\mathrm{A} \beta$, a staggering of strands from different monomers has been assumed [328], which would also be consistent with data discussed in this chapter. The aforementioned mutation study on polyglutamine peptides with interspersed proline-glycine residue pairs suggested the presence of hairpin-like turns, entailing monomers folded within the plane of one $\beta$-sheet. However, proline-glycine pairs could also be accommodated within $\beta$ bends connecting strands between different sheets [306, 326]. Moreover, if monomers were folded in an antiparallel hairpin-like fashion within the plane of the same $\beta$-sheet, short and long constructs would present similar aggregation surfaces in the direction of fibril growth, i.e. one $\beta$-strand. This would make the accelerated aggregation kinetics observed in longer constructs difficult to explain [296]. In a superpleated arrangement, an increase in monomer length directly leads to an increase in aggregation surface, offering a plausible explanation for the observed aggregation kinetics.

In most amyloid fibrils investigated to date, parallel $\beta$-sheets have been found or postulated. N/CHHC data from the polyglutamine constructs studied here, however, are more consistent with antiparallel sheets. Fibrils of shorter peptides have also been reported to adopt an antiparallel organization, depending on their sequence and factors such as charge and amphiphilicity [329, 330, 331, 332, 333]. Both parallel and antiparallel arrangements of uniform polyglutamine sequences allow for stabilizing sidechain hydrogen bonding interactions. Parallel sheets would, however, bring like charges at the peptide termini into close contact. Electrostatic interactions thus make an antiparallel arrangement of the constructs investigated here appear more favorable.

Apart from a superpleated $\beta$-sandwich structure, a triangular or similar $\beta$-helical arrangement with a tightly packed hydrophobic core might be envisioned for polyglutamine fibrils, as suggested by some molecular dynamics studies [311, 334]. Such structures have been found in a large number of proteins [335] and similarly in a recent solid-state NMR study of amyloid fibrils from the HET-s(218-289) prion [67]. The presence of random-coillike population 3 chemical shifts already in spectra of $\mathrm{D}_{2} \mathrm{Q}_{15} \mathrm{~K}_{2}$ appears to be consistent with a triangular arrangement of this molecule. However, these signals should probably rather be attributed to the loose, unstructured material visible in some electron micrographs, for the following reasons. Triangular fibrils would have a smaller diameter (no more than about $30 \AA$ ) than observed in electron micrographs (Fig. 10.1). Additionally, the sidechain-backbone contacts seen in NHHC spectra are best explained by a regular pat- 

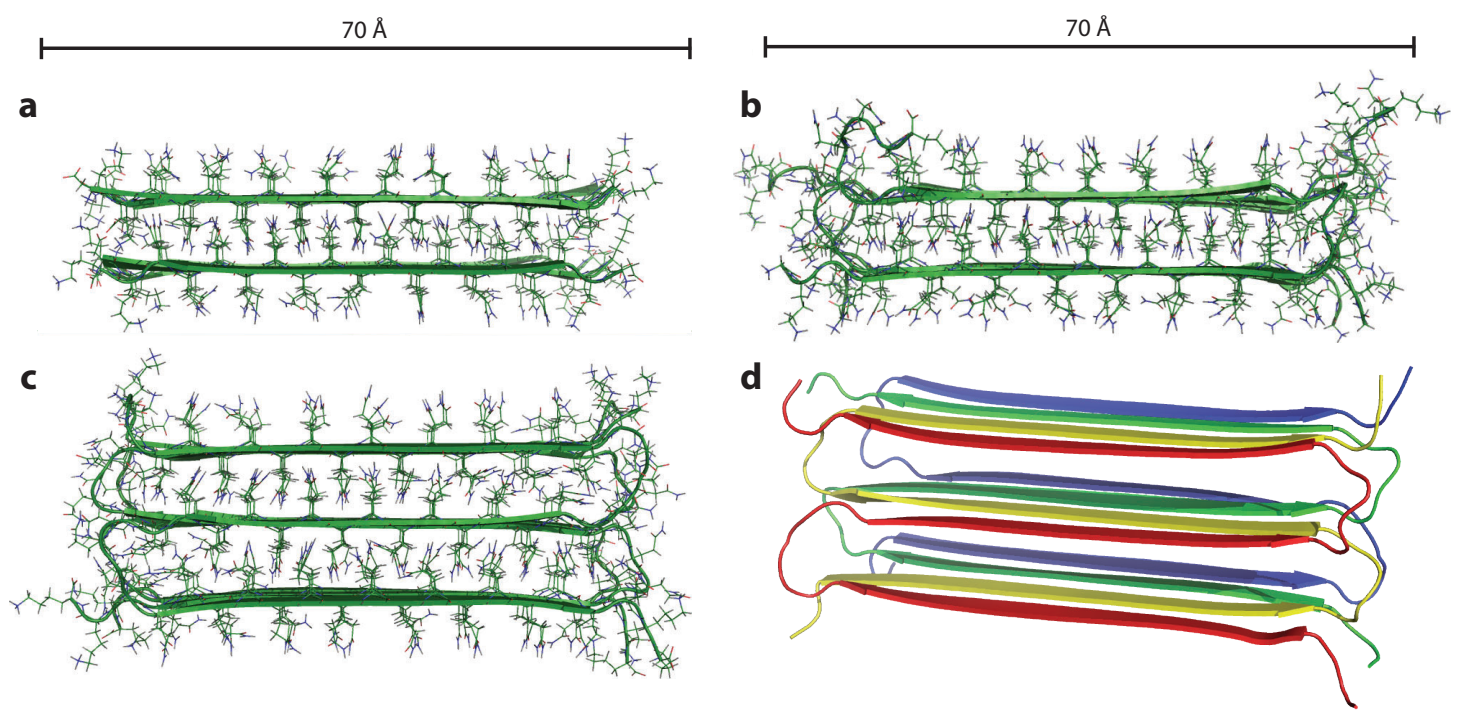

Figure 10.9: Models of polyglutamine fibrils as suggested by ssNMR data. (a - c) View down the fibril axis of (a) $D_{2} Q_{15} K_{2}$, (b) $G_{K} \mathrm{Q}_{38} \mathrm{~K}_{2}$, and (c) $\mathrm{GK}_{2} \mathrm{Q}_{54} \mathrm{~K}_{2}$ models, illustrating the regular arrangement and interdigitation of glutamine sidechains hydrogen-bonded along the fibril axis. (d) Illustration of the superpleated antiparallel cross- $\beta$ arrangement in a $\mathrm{GK}_{2} \mathrm{Q}_{54} \mathrm{~K}_{2}$ model, viewed down the fibril axis. Different monomers are shown in different colors. The fibril repeat unit consists of one $\mathrm{GK}_{2} \mathrm{Q}_{54} \mathrm{~K}_{2}$ molecule which forms three $\beta$-strands and contributes to three stacked $\beta$-sheets. 
tern of interdigitated sidechains from adjacent $\beta$-sheets, which is more difficult to achieve in a triangular arrangement if all sidechains have equal length, as in a homopolypeptide. A triangular $\beta$-helical structure would also not account for the $8.3 \AA$ reflection in fibril X-ray diffractograms [310]. Finally, ssNMR data indicate that even in the longer polyglutamine constructs, neighboring strands within a $\beta$-sheet originate from different monomers and are arranged in an antiparallel fashion, in contrast to $\beta$-helical models. Thus, the antiparallel superpleated $\beta$-sandwich structure with tightly stacked sheets and interdigitated sidechains can be considered the most likely arrangement of polyglutamine fibrils. This type of structure has also been shown to be stable in molecular dynamics simulations [305].

Models of polyglutamine protofilament units consistent with ssNMR data are shown in Figure 10.9. They exhibit a $\beta$-strand length of 15 residues, which leads to a fibril diameter in the strand direction of around $70 \AA$ including turns. Extended glutamine sidechains are hydrogen bonded along the fibril axis and tightly interdigitated between the sheets. Monomers of the longer constructs are folded back between different sheets stacked on top of each other in a superpleated arrangement, with an intersheet distance of around 8.3 A. Sheets are composed of antiparallel strands. Some residues at the N-and C-termini of the monomers protrude from the fibril core. To attain a dimension of 70 to $80 \AA$ not only in the strand direction, but also in the sheet stacking direction, three $\left(\mathrm{GK}_{2} \mathrm{Q}_{54} \mathrm{~K}_{2}\right)$ or four $\left(\mathrm{D}_{2} \mathrm{Q}_{15} \mathrm{~K}_{2}, \mathrm{GK}_{2} \mathrm{Q}_{38} \mathrm{~K}_{2}\right)$ such protofilaments would have to be stacked on top of each other. As already mentioned, no single uniform arrangement of monomers is probably present in fibrillar polyglutamine aggregates. However, the model accounts well for all experimental data and illustrates the most likely general arrangement of polyglutamine peptides in fibrillar aggregates.

The results discussed here provide no clear indication for a structural basis of the toxicity limit of polyglutamine stretches, known to be around 40 residues. SsNMR spectra indicate the local structural organization of fibrillar aggregates from constructs of different length to be highly similar, although EM shows increasing long-range order with increasing monomer length. This may be related to the presence of more $\beta$-strands per monomer in the longer constructs. Assuming a $\beta$-strand length of around 15 residues as apparent for $\mathrm{D}_{2} \mathrm{Q}_{15} \mathrm{~K}_{2}$, about 40 residues would be the minimum length for a single polyglutamine peptide to assume a conformation as postulated here for the aggregated state, i.e. two $\beta$-strands folded back on top of each other with interdigitating sidechains. However, in view of the rather 'soft' toxicity limit varying from 35 to 45 glutamine repeats, the 
conformational transition leading to toxicity may not be accessible only for glutamine repeats above that limit. Length-dependent differences in thermodynamics or kinetics of the folding reaction might be sufficient to explain the different degrees of toxicity.

While aggregation kinetics and, in case of Huntington's disease, age of onset have been shown to correlate with glutamine repeat length [300], the toxic polyglutamine species appear to be mono- or oligomeric rather than fibrillar [298, 299]. However, since monomers seem to become toxic only after a conformational transition to a $\beta$-sheet-rich state [298], and the rate-limiting step in polyglutamine aggregation has been traced to an unfavorable conformational transition within a single molecule [300, 336], it may be assumed that the toxic mono- and oligomeric species exhibit similar structural features as the monomers within the fibrils investigated in this study. This is also indicated by the finding mentioned above that the characteristic two $\beta$-sheet chemical shift populations appear already relatively early in the aggregation process, when only oligomers can be detected by EM. The observation of toxicity in polyglutamine monomers or small soluble aggregates certainly points to the need for more detailed structural investigation of these species. In addition, recent evidence suggests that, in vivo, flanking sequences and other residues in the host protein of the polyglutamine stretch as well as the cellular context can be crucial for toxicity and disease progression [337, 338, 339]. This means that a complete picture of polyglutamine aggregation and toxicity may actually not be obtained if only the polyglutamine stretches per se are considered. 


\section{Part V}

Summary 



\section{Chapter 11}

\section{Summary and conclusions}

This thesis described development and application of solid-state NMR methods for the structural and dynamical investigation of biomolecules. Systems investigated ranged from a small amino acid derivative to a large transmembrane protein in lipid bilayers. Work presented here provides novel insight into systems of considerable biological relevance and underlines the potential of solid-state NMR to study larger proteins also in the presence of uniform $\left[{ }^{13} \mathrm{C},{ }^{15} \mathrm{~N}\right]$ isotope labeling. In particular, the advantages of being able to observe insoluble, noncrystalline biomolecules in a native-like environment became apparent. Especially in such systems, solid-state NMR can link functional data with atomic-resolution structural information.

Chapter $\mathbf{5}$ - Mobility by $\mathbf{2 Q}$ ssNMR. Double-quantum ssNMR spectroscopy was introduced as a novel method to probe residue-specific local molecular mobility of the ${ }^{13} \mathrm{C}$ spin network. Spin system simulations were developed and successfully tested. Together with routines for fitting simulations to experimental data, a set of tools for quantitative analysis of $2 \mathrm{Q}$ buildup data in terms of mobility is now available. Results on the test system L-tyrosine-ethylester agree well with earlier data on local mobility in this molecule $[68,69]$, confirming the validity of the approach. A small set of $2 \mathrm{D}$ experiments was shown to already be sufficient for obtaining estimates of relative mobility.

Chapter 6 - Structure and dynamics of ubiquitin. A detailed analysis of molecular mobility was conducted on PEG-precipitated microcrystalline ubiquitin (Ubi-P), using 2Q $\left({ }^{13} \mathrm{C},{ }^{13} \mathrm{C}\right)$ ssNMR spectroscopy as developed in Chapter 5 . Site-specific mobility data could be obtained for 40 residues in the context of uniform $\left[{ }^{13} \mathrm{C},{ }^{15} \mathrm{~N}\right]$ labeling. Results show the presence of local backbone dynamics on the ms or faster time scales in Ubi-P. While 
common regions of elevated dynamics in different ubiquitin preparations could be found, sample preparation was seen to affect the distribution of mobile and rigid residues along the protein sequence. In addition to the analysis of dynamics, CHHC through-space spectra of Ubi-P were analyzed in terms of their information content and used for a structure calculation, employing an automated algorithm with the ubiquitin crystal structure as a reference. The Ubi-P structure thus obtained was found to be well defined and close to the crystal structure, with local differences in loop regions. With structural and dynamical data at hand, sizable preparation-dependent chemical shift changes reported earlier [70] as well as absent correlations in CHHC spectra could largely be traced to residues differing in mobility or conformation between ubiquitin preparations. These results, for the first time, offer a coherent picture of structure and dynamics in microcrystalline ubiquitin.

Chapter 7 - Assignment and secondary structure of KcsA-Kv1.3. Using uniformly and reverse labeled as well as C-terminally truncated samples and a set of 2D and $3 \mathrm{D}\left({ }^{13} \mathrm{C},{ }^{13} \mathrm{C}\right)$ and $\left({ }^{15} \mathrm{~N},{ }^{13} \mathrm{C}\right)$ correlation experiments, resonance assignments were obtained for $59 \%$ of the $4 \times 160$ residues of the potassium channel KcsA-Kv1.3 in lipid bilayers. Secondary structure was seen to be in close agreement with the crystal structure of the parent KcsA channel [24] in transmembrane and extracellular regions. The transmembrane helix 1 (TM1) was found to be slightly longer than in the crystal structure and several residues longer than in micellar preparations investigated by solution-state NMR [28, 242]. This result has potential implications for channel gating and illustrates the sensitivity of membrane protein structure and function for the surrounding membrane environment. In agreement with EPR and solution-state NMR, helical structure was also found in the intracellular N- and C-termini. Clear presence of turret loop and selectivity filter residues in dipolar correlation spectra argues against elevated mobility in these regions and suggests that ion size and defined protein structure play a crucial role in the mechanism of ion selectivity.

Chapter 8 - Gating and inactivation in KcsA-Kv1.3. Solid-state NMR was used to follow structural changes associated with gating of the KcsA-Kv1.3 $\mathrm{K}^{+}$channel in a lipid bilayer environment in reference to electrophysiological experiments. Acidic pH induced distinct local chemical shift changes in functionally important regions of the channel, while overall channel structure was well preserved. Data recorded at neutral $\mathrm{pH}$ confirm that KcsA-Kv1.3 is present in a closed-conductive conformation under these conditions 
[24], while analysis of chemical shifts at $\mathrm{pH} 4.0$ show a transition to an open-inactivated conformation. This state is characterized on the one hand by a bent inner transmembrane helix (TM2), i.e. an open activation gate, as seen in crystal structures of potassium channels in an open conformation [40,41]. On the other hand, the selectivity filter assumes a nonconductive conformation similar to its known collapsed structure [24, 239, 268]. This conformation could also be induced by binding a porphyrin ligand to the channel, with potassium depletion of the selectivity filter being the likely common mechanism at work. Results underline the importance of the lipid environment for membrane protein function and provide a structural explanation for functional data obtained in electrophysiological experiments.

Chapter 9 - Influence of potassium on KcsA-Kv1.3 gating. The influence of $\mathrm{pH}$ and potassium concentration on the KcsA-Kv1.3 $\mathrm{K}^{+}$channel was studied in lipid bilayers using solid-state NMR and electrophysiology. It was shown that activation gate opening at acidic $\mathrm{pH}$, as described in Chapter 8, is correlated with protonation of residues Glu118 and Glu120. These residues belong to a network of charged amino acids which was identified as the $\mathrm{pH}$ sensor of KcsA by mutational studies [279]. Chemical shift data and experiments probing the water-accessible surface of KcsA-Kv1.3 showed that potassium strongly reduces the steady-state open probability of the activation gate. Electrophysiology revealed distinct $\mathrm{K}^{+}$binding sites with micromolar and millimolar affinities that modulate KcsA-Kv1.3 gating. The high-affinity site seems to influence activation gating, while the low-affinity site most likely affects the interconversion between conductive and inactivated filter conformations. KTX binding to the channel was found to prevent gate opening at $\mathrm{pH} 4.0$ even in the absence of potassium. Together with titration experiments at a potassium concentration of $1 \mu \mathrm{M}$, these data strongly suggest that potassium influences the open probability of the TM2 gate via the selectivity filter and that both $\mathrm{K}^{+}$binding sites observed are located in this region of the channel. Data presented in this chapter establish that the inactivation gate can act upon activation gating, i.e. that both gates are coupled, and that the permeant ion itself modulates inactivation as well as activation gating, providing a scenario for the coordination of the different gating transitions in an ion channel.

Chapter 10 - Polyglutamine fibrils. Disease-relevant fibrillar aggregates formed by polyglutamine peptides of different length were investigated. Despite the presence of only 
a single dominant amino acid type and fibril polymorphism, useful constraints could be deduced on the structural organization of the fibrils using solid-state NMR and electron microscopy. Data presented in this chapter indicate that the polyglutamine fibrils investigated are antiparallel, superpleated cross- $\beta$ structures with $\beta$-sheets stacked tightly on top of each other. Experimental evidence supports a polar zipper arrangement with sidechains from adjacent sheets being closely interdigitated and participating in hydrogen bonding interactions. $\beta$-helical structures, especially if assumed to be water-filled, are disfavored. The $\beta$-sheet fibrillar core is formed by two populations of glutamine residues discernible by chemical shift, which is likely related to backbone dihedral angles compensating for $\beta$-strand twist or to distinct sidechain conformations enforced by tight sheet packing. These data should be useful for further studies investigating toxic mono- and oligomeric polyglutamine species and effects of inhibitors of fibril formation. 
Part VI

Appendix 



\title{
Appendix A
}

\section{Molecular motion detected by double-quantum $\left({ }^{13} \mathrm{C},{ }^{13} \mathrm{C}\right)$ solid-state NMR (Supplement)}

\author{
A.1 GAMMA $\mathrm{C}++$ code for simulation of $2 \mathrm{D} 2 \mathrm{Q}$ buildups \\ \# include "gamma.h"

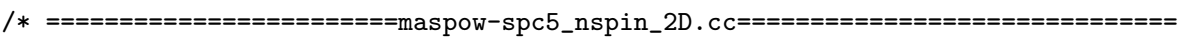 \\ * Program to simulate 2D DQ buildups under SPC5 sequence \\ * Arbitrary spin system setup possible \\ * Considers 4 reference systems: PAS, MOL, ROT, LAB. \\ * Calculation is based on piecewise time constant Hamiltonian integration \\ $*$ \\ * MAS and powder loop, basic Hamiltonian setup based on code by \\ * Ovidiu Andronesi, MPIBPC \\ * Spin system setup based on code by Lars Sonnenberg, MPIBPC \\ $*$
}

int main (int argc, char* argv[])

\{

coord $\mathrm{B}(0,0,1)$;

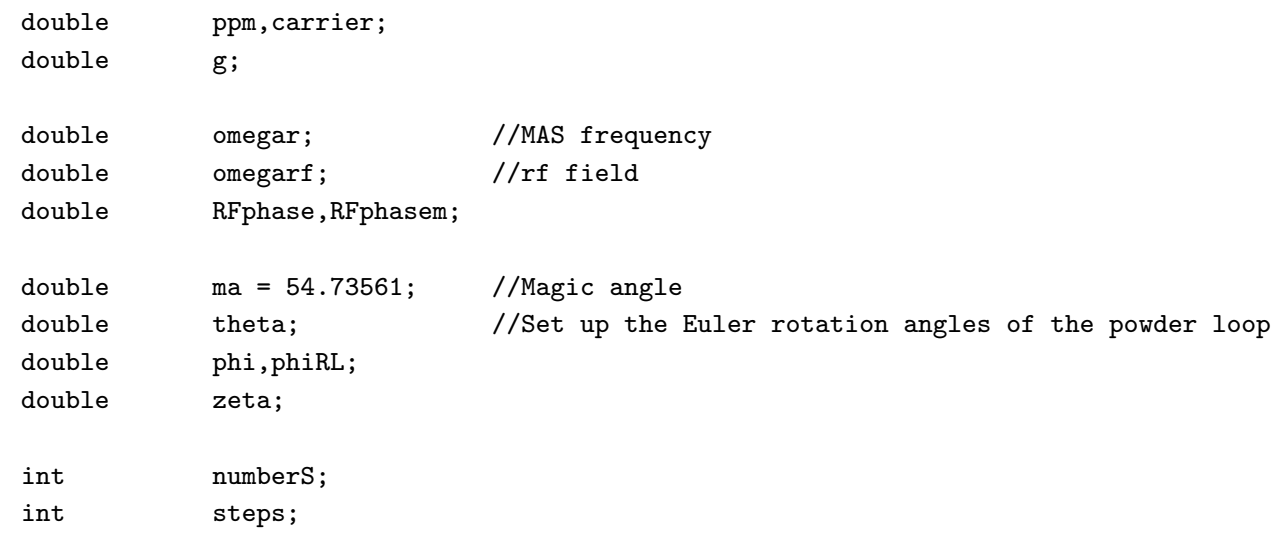




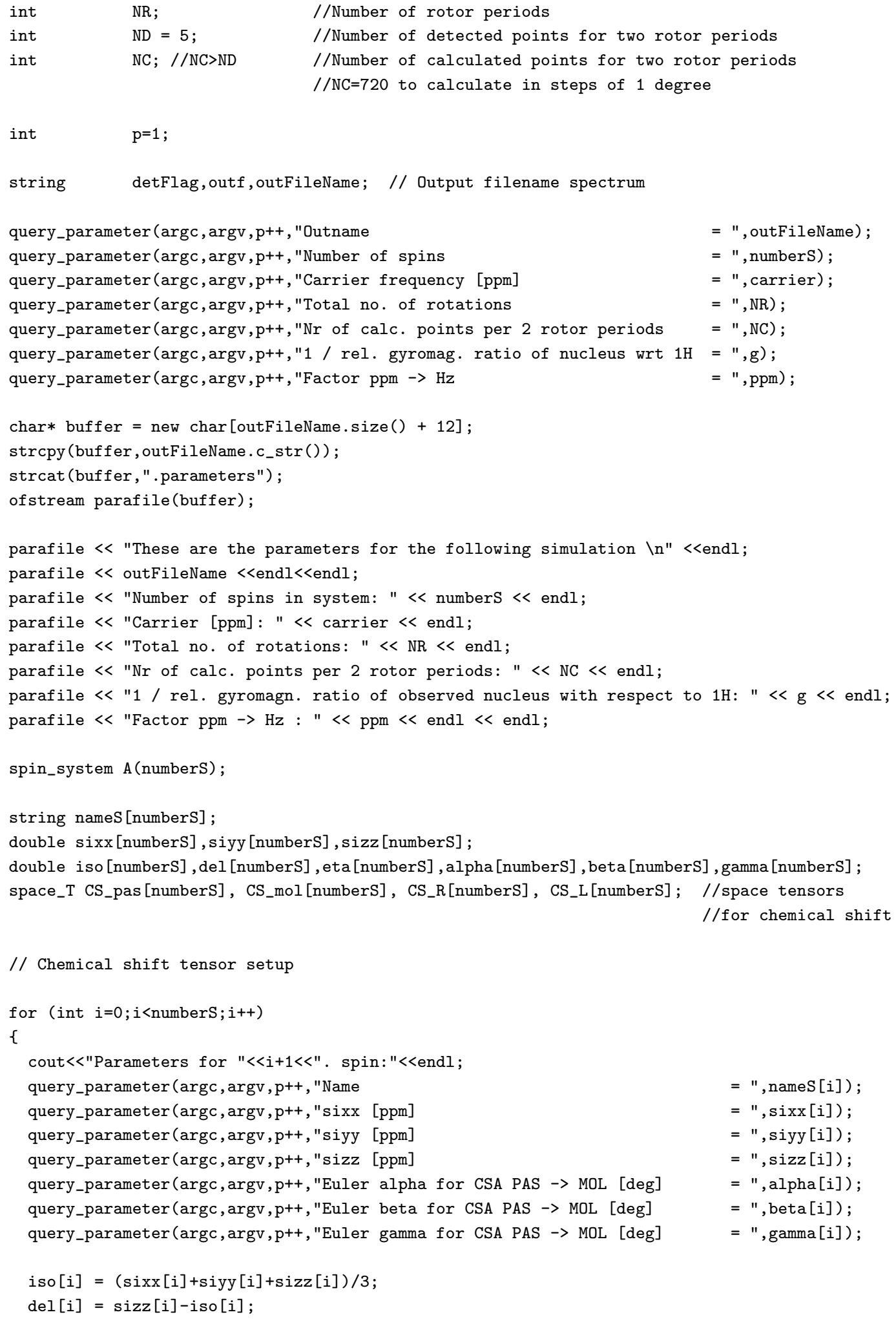




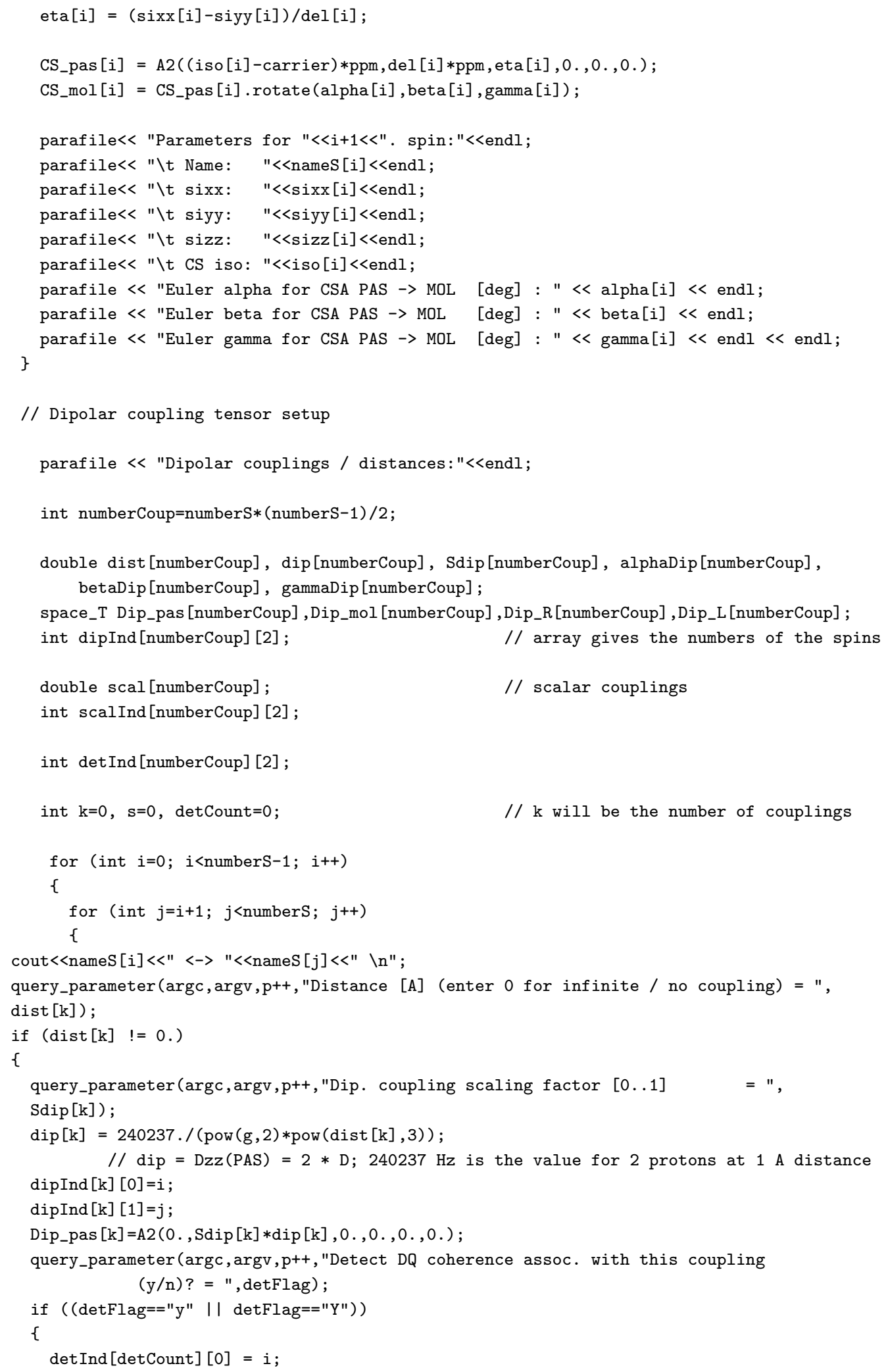




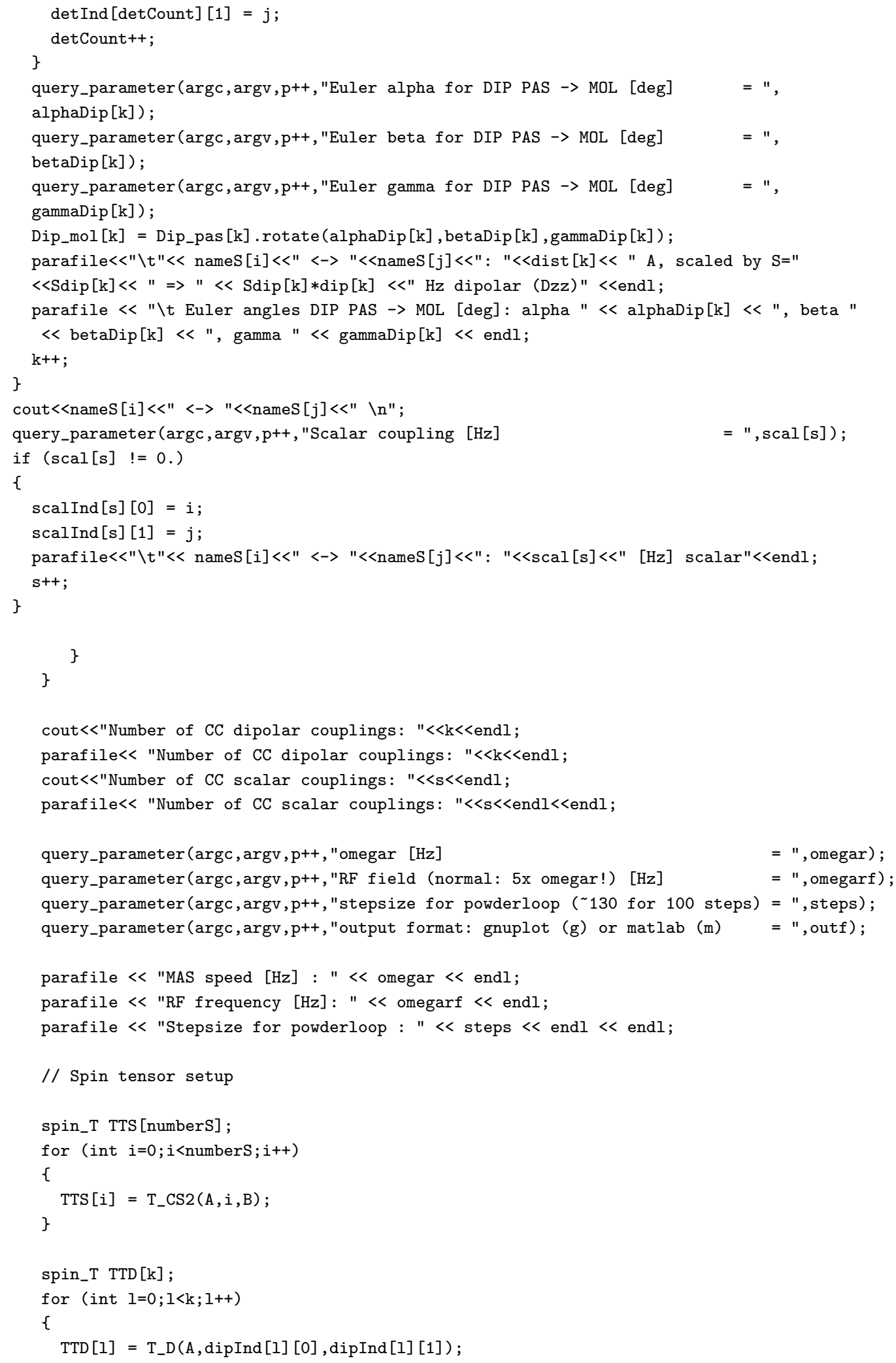


\}

// Detection setup

int $\mathrm{NP}=\operatorname{int}((\mathrm{NR} * \mathrm{ND}) / 2)+1$;

matrix $\operatorname{spect}(\operatorname{det} C o u n t * 2, N P), \operatorname{specsum}(\operatorname{det} \operatorname{Coun} t * 2, N P), \operatorname{data}(\operatorname{det} \operatorname{Count} * 2, N P)$;

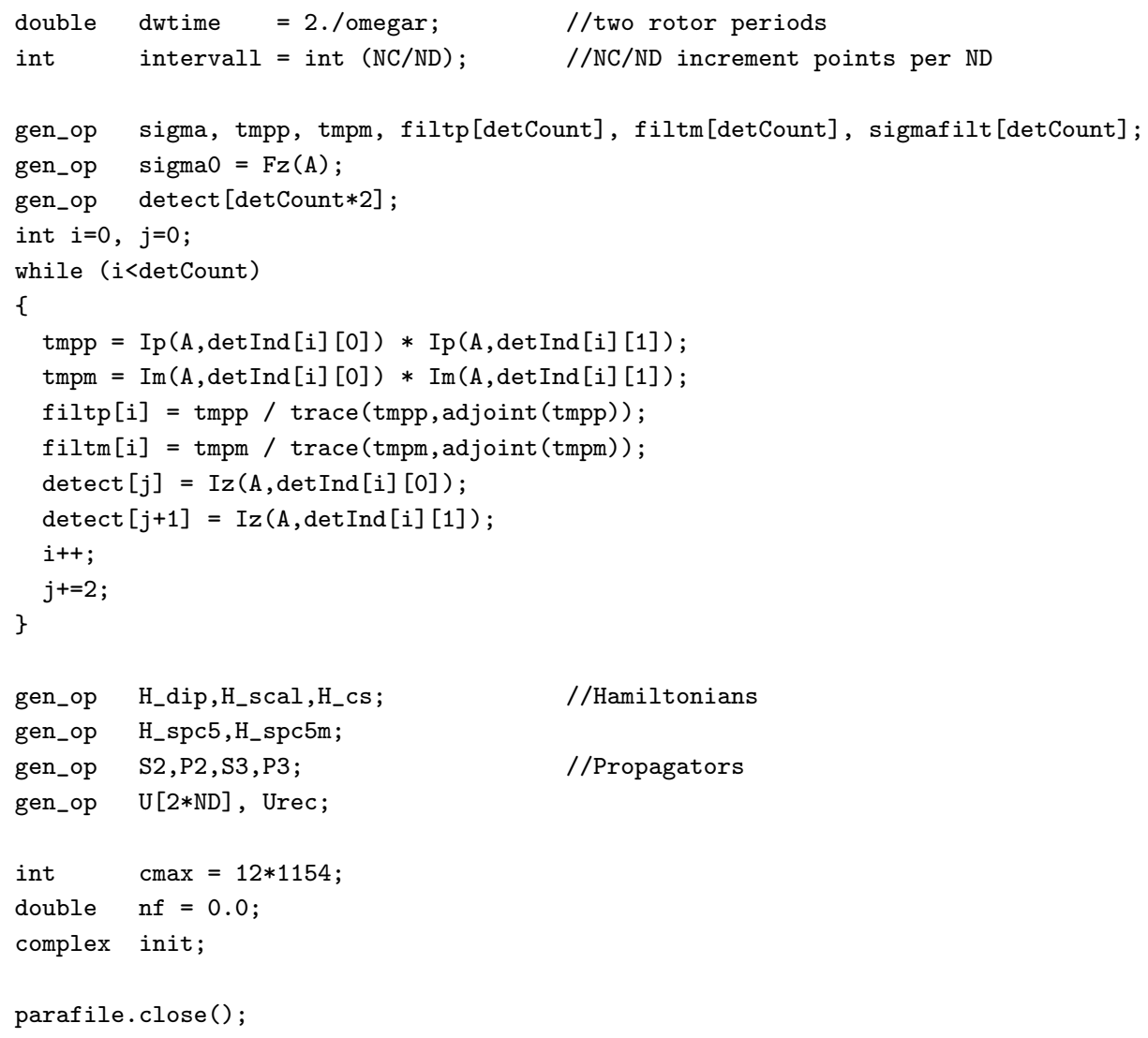




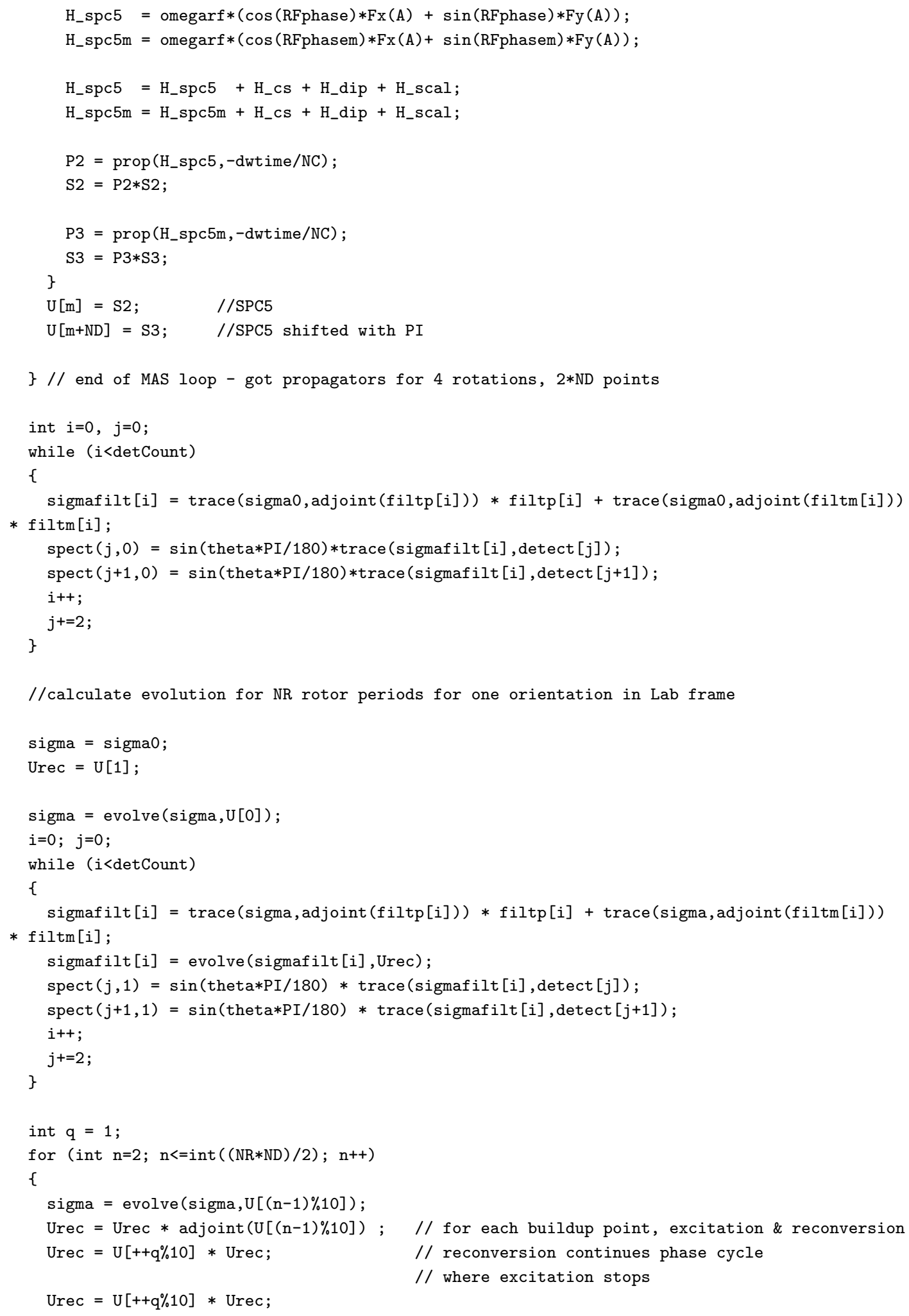




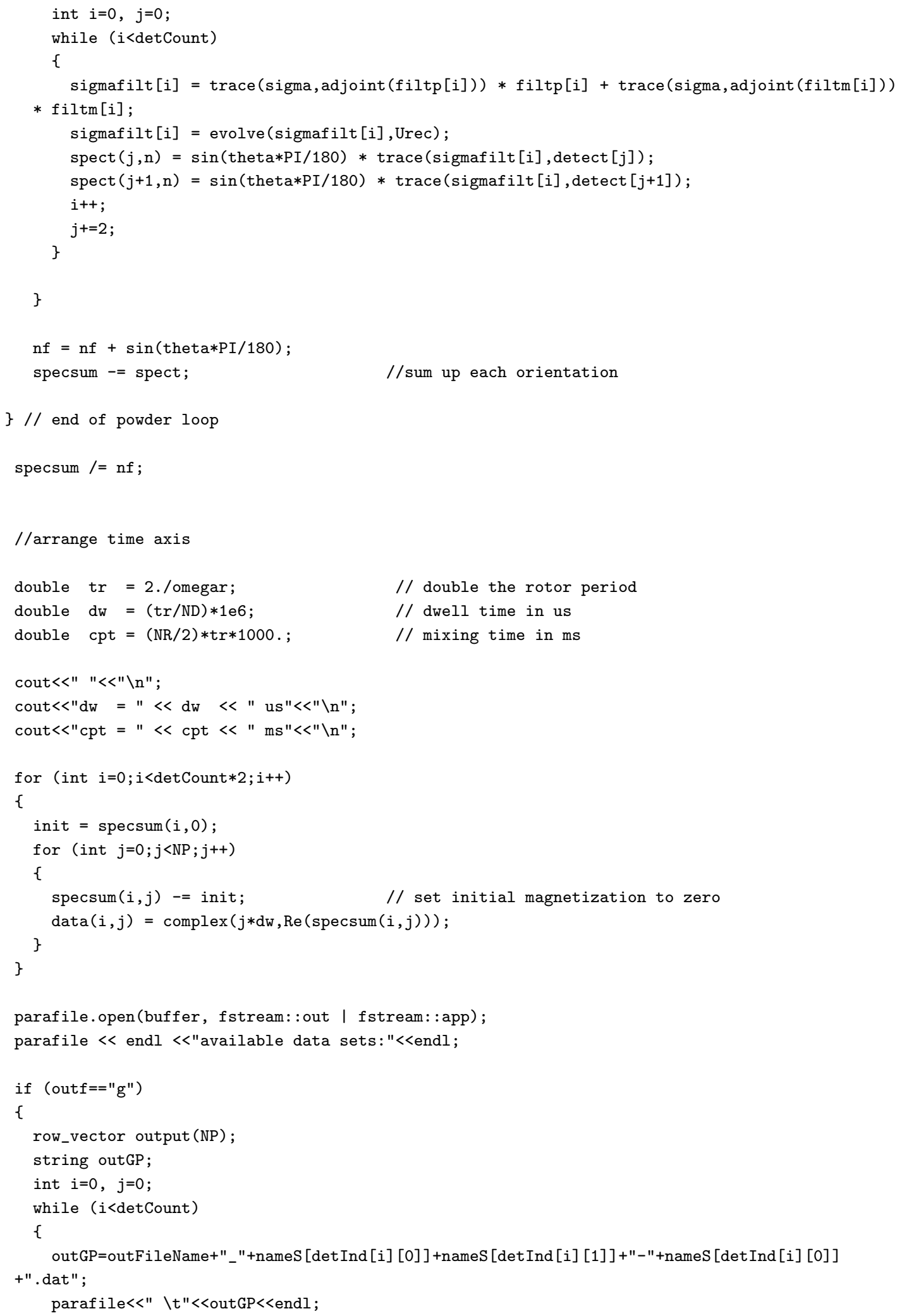




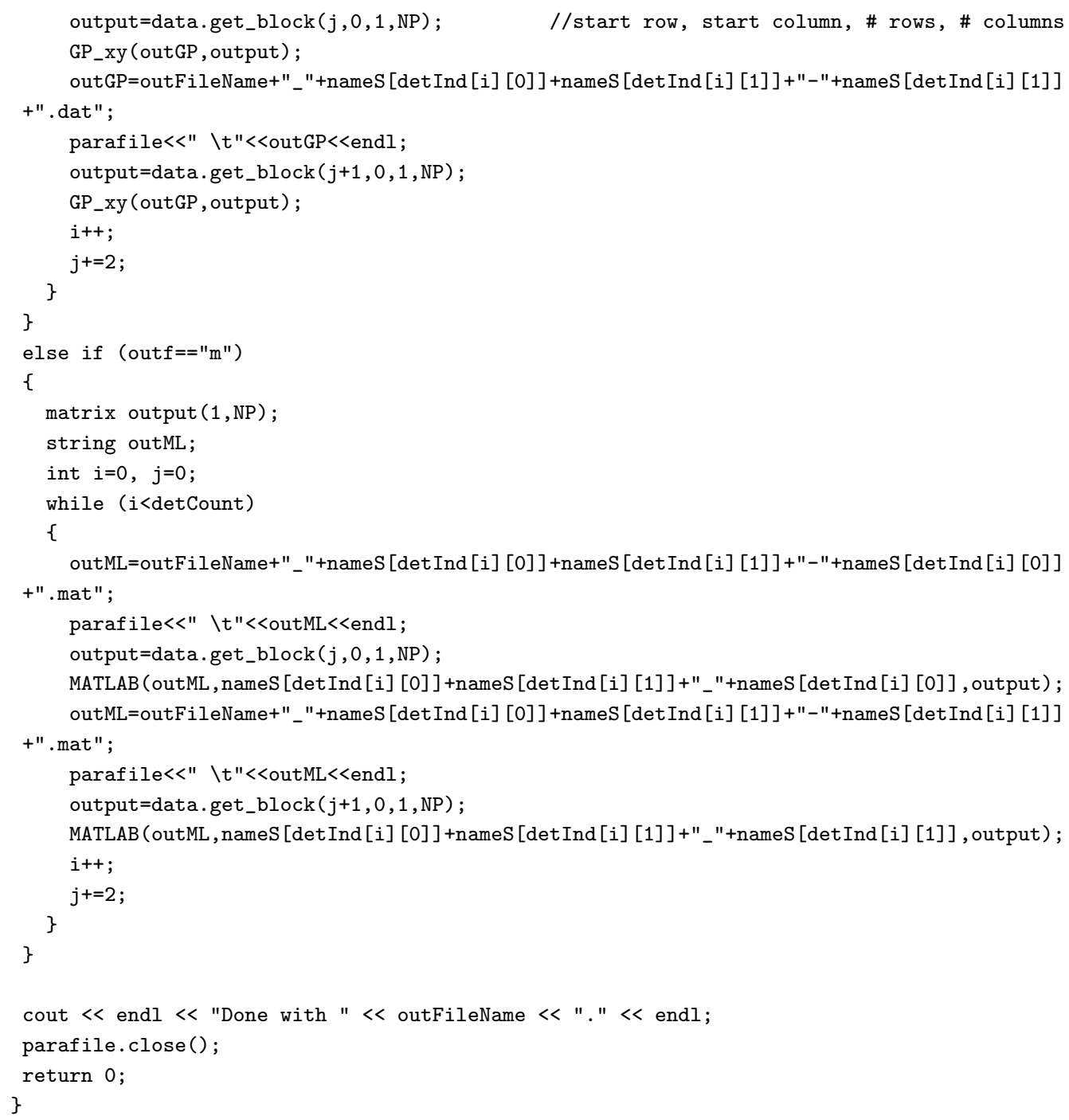




\section{A.2 Supplementary figures}

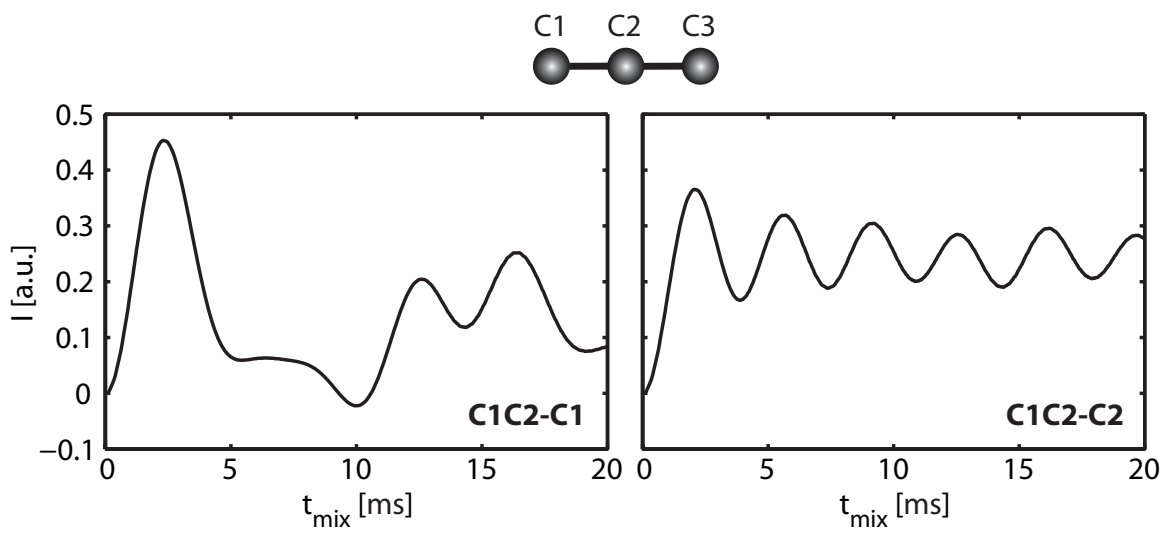

Figure A.1: Simulated intensity buildup of crosspeaks $\mathrm{C} 1 \mathrm{C} 2-\mathrm{C} 1$ (left) and $\mathrm{C} 1 \mathrm{C} 2-\mathrm{C} 2$ (right) in a $2 \mathrm{D}(2 \mathrm{Q}, 1 \mathrm{Q})$ correlation spectrum of a linear 3 -spin system as depicted above. Isotropic and anisotropic chemical shifts were set to zero, and dipolar couplings were $d_{i j}=500 \mathrm{~Hz}$ for $(i, j)=$ $(1,2)$ and $(2,3)$ and 0 otherwise. This corresponds to the setup used for the simulations depicted in Figure $3 \mathrm{~d}$, e in ref. [121]. Results shown here are identical to those in ref. [121]. MAS speed in the simulation was $8 \mathrm{kHz}$.

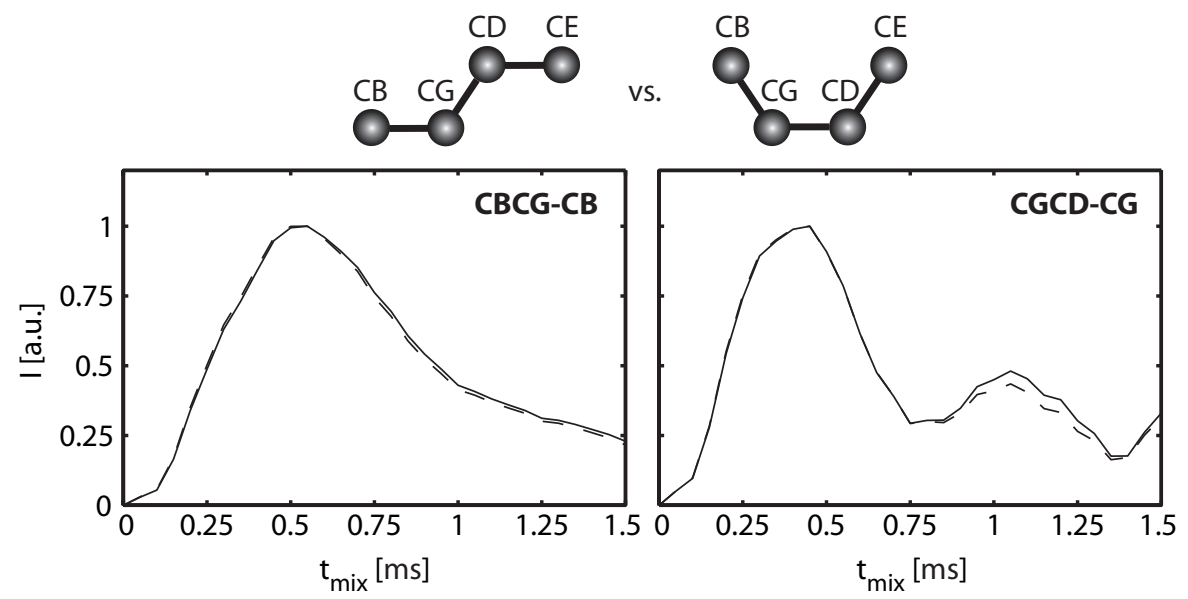

Figure A.2: Comparison of simulated $(2 \mathrm{Q}, 1 \mathrm{Q})$ crosspeak buildups for a lysine sidechain 4-spin system with extended geometry (solid line, left sketch) and with bent geometry (dashed line, right sketch). CBCG-CB (left) and CGCD-CG (right) crosspeak buildups are shown. Isotropic chemical shifts were set to $32.74 \mathrm{ppm}\left(\mathrm{C}^{\beta}\right), 24.89 \mathrm{ppm}\left(\mathrm{C}^{\gamma}\right), 28.89 \mathrm{ppm}\left(\mathrm{C}^{\delta}\right)$, and $41.85 \mathrm{ppm}$ $\left(\mathrm{C}^{\varepsilon}\right)$. Euler angles for the transformations of $\left(\mathrm{C}^{\beta}, \mathrm{C}^{\gamma}\right),\left(\mathrm{C}^{\gamma}, \mathrm{C}^{\delta}\right)$, and $\left(\mathrm{C}^{\delta}, \mathrm{C}^{\varepsilon}\right)$ dipolar tensors from principal axis to molecular frame were $(\alpha, \beta, \gamma)=\left(0^{\circ}, 90^{\circ}, 0^{\circ}\right),\left(68^{\circ}, 90^{\circ}, 0^{\circ}\right)$, and $\left(0^{\circ}, 90^{\circ}, 0^{\circ}\right)$ for extended geometry and $\left(0^{\circ}, 90^{\circ}, 0^{\circ}\right),\left(78^{\circ}, 90^{\circ}, 0^{\circ}\right)$, and $\left(156^{\circ}, 90^{\circ}, 0^{\circ}\right)$ for bent geometry. 

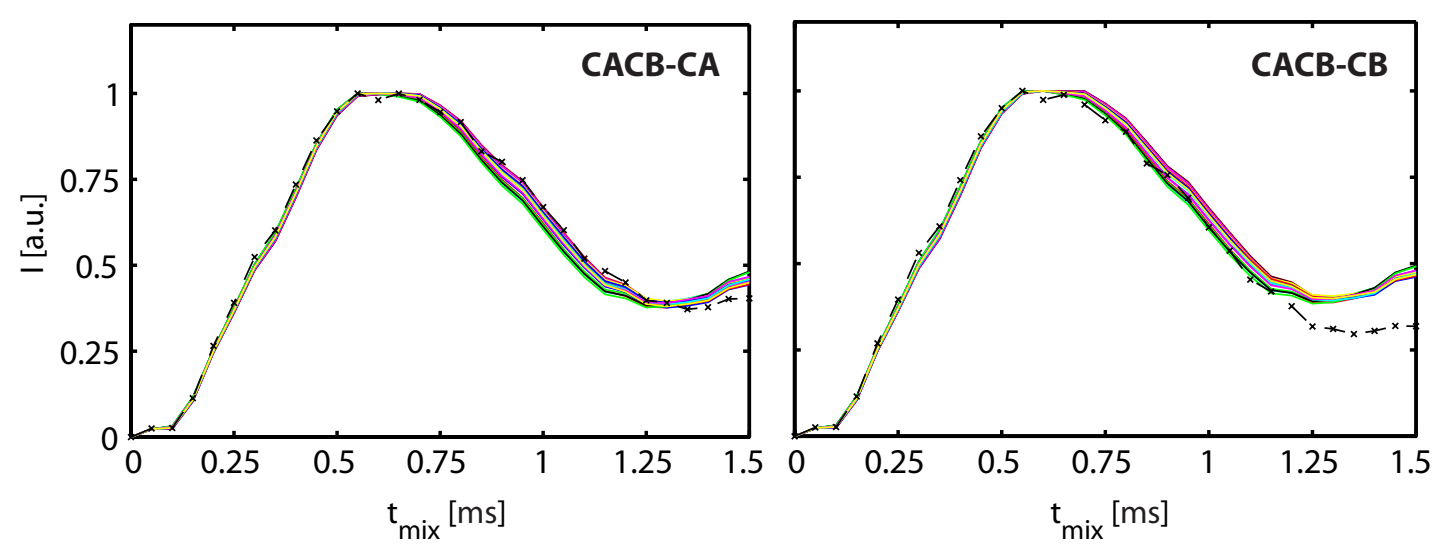

Figure A.3: Comparison of $(2 \mathrm{Q}, 1 \mathrm{Q})$ crosspeak buildup simulations for an aspartic acid / asparagine 4-spin system with and without chemical shift anisotropy. An extended geometry was again assumed, with Euler angles for the transformations of $\left(\mathbf{C}^{\prime}, \mathbf{C}^{\alpha}\right),\left(\mathbf{C}^{\alpha}, \mathbf{C}^{\beta}\right)$, and $\left(\mathbf{C}^{\beta}, \mathbf{C}^{\gamma}\right)$ dipolar tensors from principal axis to molecular frame chosen as $(\alpha, \beta, \gamma)=\left(0^{\circ}, 90^{\circ}, 0^{\circ}\right),\left(-68^{\circ}, 90^{\circ}, 0^{\circ}\right)$, and $\left(0^{\circ}, 90^{\circ}, 0^{\circ}\right)$. Buildups for CACB-CA (left) and CACB-CB (right) crosspeaks are shown. Dashed black lines with crosses represent buildups if all nuclei have isotropic chemical shift tensors ( $C^{\prime} 176$ ppm, $C^{\alpha} 52.7$ ppm, $C^{\beta} 37$ ppm, $C^{\gamma} 178.7$ ppm). Colored lines are buildups resulting from assuming anisotropic chemical shift tensors for all four nuclei. Principal axis values $\left(\sigma_{x x}, \sigma_{y y}, \sigma_{z z}\right)$ for CSA tensors were taken from refs. [340] and were set to (in ppm) $(253,178,97)$ for C', $(29,58,71)$ for $\mathbf{C}^{\alpha},(49,44,18)$ for $\mathbf{C}^{\beta}$, and $(255,181,100)$ for $\mathbf{C}^{\gamma}$. $\mathrm{C}^{\prime}$ and $\mathrm{C}^{\gamma}$ values were adapted from values for alanine in ref. [340] to match BMRB isotropic shifts for aspartic acid / asparagine. C', $C^{\alpha}$ and $C^{\gamma}$ tensor orientations were as described in refs. [161, 341]. That is, for the $\mathrm{C}^{\alpha}$ CSA tensor, the axis with the largest principal value formed an angle of $76^{\circ}$ with the $\mathrm{C}^{\alpha}-\mathrm{H}^{\alpha}$ bond, and the one with the intermediate principal value formed an angle of $60^{\circ}$ with the $\mathrm{C}^{\alpha}-\mathrm{N}$ bond. For $\mathrm{C}^{\prime}$ and $\mathrm{C}^{\gamma} \mathrm{CSA}$ tensors, the axis with the intermediate principal value was oriented parallel to the C'-O bond, and (for C') the axis with the smallest principal value was perpendicular to the peptide bond plane. This led to Euler angles $(\alpha, \beta, \gamma)$ for the PAS $\rightarrow$ MOL transformation of $\mathrm{C}^{\prime}, \mathrm{C}^{\alpha}$, and $\mathrm{C}^{\gamma} \mathrm{CSA}$ tensors of $\left(90^{\circ}, 77^{\circ},-60^{\circ}\right),\left(51^{\circ}, 67^{\circ}, 90^{\circ}\right)$, and $\left(90^{\circ}, 20^{\circ},-120^{\circ}\right)$, respectively. $\mathrm{C}^{\beta}$ CSA tensor orientation was varied around a sphere in 20 steps, leading to the different buildup curves. 


\section{CACB-CA}
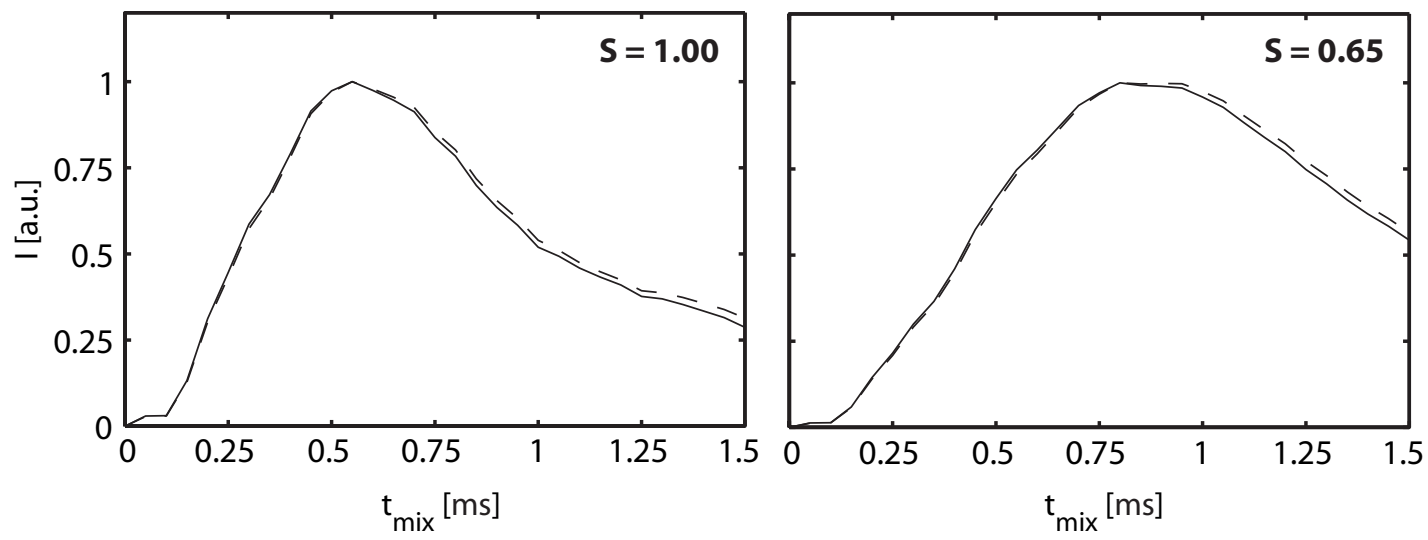

CBCG-CB
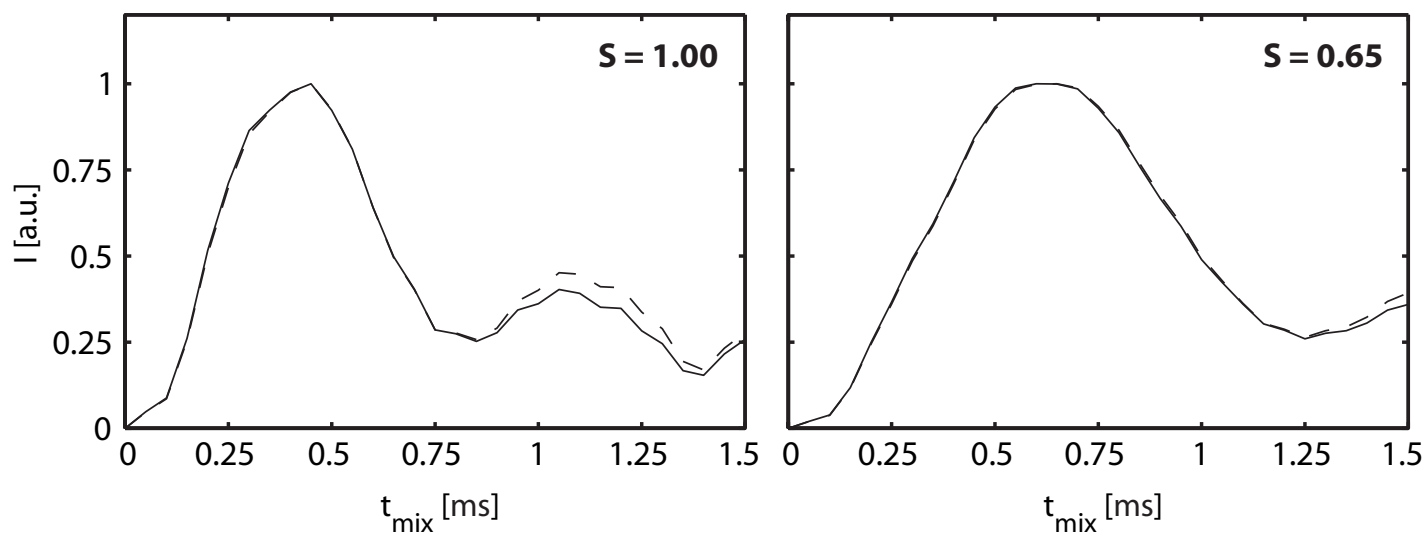

Figure A.4: Comparison of simulated $(2 \mathrm{Q}, 1 \mathrm{Q})$ crosspeak buildups in a lysine sidechain 4-spin system with extended geometry (see Fig. A.2) if all dipolar couplings are considered (solid lines) or only one-bond couplings (dashed line). Buildups shown are for CACB-CA (top) and CBCGCB (bottom) crosspeaks. $S_{\mathrm{CC}}$ order parameters of 1 (left) or 0.65 were assumed for scaling of all considered couplings. 


\section{Appendix B}

Structural and dynamical investigation of microcrystalline ubiquitin (Supplement)

\section{B.1 Primary sequence}




\section{B.2 $\mathrm{CHHC}$ analysis}

\begin{tabular}{|c|c|c|c|}
\hline \multicolumn{3}{|c|}{ Correlation } & Comment \\
\hline 13 ILE CB & - & 33 LYS CD & Ile13 $\left(\mathrm{C}^{\alpha}, \mathrm{C}^{\beta}\right) S_{\mathrm{CC}}<0.85 ;$ mobile Lys sidechain \\
\hline 19 PRO CA & - & $56 \mathrm{LEU} \mathrm{CB}$ & \\
\hline $19 \mathrm{PRO} \mathrm{CB}$ & - & 57 SER CB & \\
\hline $19 \mathrm{PRO} \mathrm{CB}$ & - & 57 SER CA & \\
\hline 22 THR CB & - & 24 GLU CB & \\
\hline 22 THR CB & - & 53 GLY CA & Gly53 possibly mobile \\
\hline 26 VAL CA & - & 29 LYS CG & mobile Lys sidechain \\
\hline 26 VAL CA & - & 29 LYS CD & mobile Lys sidechain \\
\hline 33 LYS CG & - & 34 GLU CG & $\begin{array}{l}\text { Glu34 }\left(\mathrm{C}^{\alpha}, \mathrm{C}^{\beta}\right) \quad S_{\mathrm{CC}}<0.85 ; \text { mobile Lys sidechain } \\
\left(\text { Lys33 }\left(\mathrm{C}^{\gamma}, \mathrm{C}^{\delta}\right) \text { correlation not found in }(2 \mathrm{Q}, 1 \mathrm{Q}) \text { spec- }\right. \\
\text { tra) }\end{array}$ \\
\hline 57 SER CA & - & 57 SER CB & \\
\hline
\end{tabular}

Table B.2: List of non-methyl $\left({ }^{13} \mathrm{C},{ }^{13} \mathrm{C}\right)$ correlations expected in the Ubi-P CHHC spectrum with $180 \mu \mathrm{s}$ mixing time (associated $\left({ }^{1} \mathrm{H},{ }^{1} \mathrm{H}\right.$ ) distance below $3.5 \AA$ ) but absent from the spectrum. Right column indicates for which of these mobile moieties are (possibly) involved (red or grey bars in Fig. 6.5 a).

\section{B.3 Spin system simulations}

In GAMMA simulations, the two spins contributing to the $2 \mathrm{Q}$ coherence of interest and any ${ }^{13} \mathrm{C}$ spins directly bonded to them were considered. This yielded 3 -spin simulations for Ala and Ser and 4-spin simulations for the other unbranched amino acids. For residues with longer sidechains (e.g. Lys), separate 4-spin simulations were conducted for sidechain 2Q coherence buildups (e.g. considering $\mathrm{C}^{\beta}, \mathrm{C}^{\gamma}, \mathrm{C}^{\delta}$, and $\mathrm{C}^{\varepsilon}$ for fitting an experimental $\left(\mathrm{C}^{\gamma}, \mathrm{C}^{\delta}\right)$ buildup). Extended geometry was assumed, with Euler angles $(\alpha, \beta, \gamma)$ for the PAS $\rightarrow$ MOL transformation set to $\left(0^{\circ}, 90^{\circ}, 0^{\circ}\right),\left(68^{\circ}, 90^{\circ}, 0^{\circ}\right)$, and $\left(0^{\circ}, 90^{\circ}, 0^{\circ}\right)$ for dipolar tensors connecting spins $(1,2),(2,3)$, and $(3,4)$ of a 4-spin system, respectively. All dipolar couplings were considered in setup of the system Hamiltonian for 3- and 4-spin systems. Bond lengths were set to $1.52 \AA$, and an additional scalar coupling of $40 \mathrm{~Hz}$ between directly bonded nuclei was considered. Chemical shifts for individual nuclei were taken from the BMRB database.

For amino acids with branched sidechains, i.e. Ile, Leu, and Val, 5-spin systems had to be considered. For example, for fitting Ile $\left(\mathrm{C}^{\beta}, \mathrm{C}^{\gamma 1}\right)$ or $\left(\mathrm{C}^{\beta}, \mathrm{C}^{\gamma 2}\right)$ buildups, a spin system consisting of $\mathrm{C}^{\alpha}, \mathrm{C}^{\beta}, \mathrm{C}^{\gamma 1}, \mathrm{C}^{\gamma 2}$, and $\mathrm{C}^{\delta 1}$ was simulated. Corresponding Euler angles $(\alpha, \beta, \gamma)$ for the PAS $\rightarrow$ MOL transformation of dipolar coupling tensors were as follows:

\begin{tabular}{c|rrr} 
Dipolar tensor & $\alpha$ & $\beta$ & $\gamma$ \\
\hline$\left(\mathrm{C}^{\alpha}, \mathrm{C}^{\beta}\right)$ & $0^{\circ}$ & $90^{\circ}$ & $0^{\circ}$ \\
$\left(\mathrm{C}^{\beta}, \mathrm{C}^{\gamma 1}\right)$ & $-68^{\circ}$ & $90^{\circ}$ & $0^{\circ}$ \\
$\left(\mathrm{C}^{\beta}, \mathrm{C}^{\gamma 2}\right)$ & $51^{\circ}$ & $37^{\circ}$ & $0^{\circ}$ \\
$\left(\mathrm{C}^{\gamma 1}, \mathrm{C}^{\delta 1}\right)$ & $0^{\circ}$ & $90^{\circ}$ & $0^{\circ}$
\end{tabular}


Only one-bond dipolar couplings were considered in 5-spin simulations for computational reasons (see Section 5.4.2 and Fig. A.4). As above, an additional scalar coupling contribution of $40 \mathrm{~Hz}$ between bonded nuclei was included in the calculations.

\section{B.4 Ubi-P order parameters}

Listed are all determined $S_{\mathrm{CC}}$ order parameters for $\left(\mathrm{C}^{\alpha}, \mathrm{C}^{\beta}\right)$ and $\left(\mathrm{C}^{\beta}, \mathrm{C}^{\gamma}\right)$ pairs. If the $S$ values of simulations fitting best to individual crosspeak buildups $\left(\mathrm{C}^{\alpha}\right.$ and $\mathrm{C}^{\beta}$ or $\mathrm{C}^{\beta}$ and $\mathrm{C}^{\gamma}$, respectively) are different, the average is given. Errors were determined as described in Section 6.3.4. For threonine residues, $\left(\mathrm{C}^{\beta}, \mathrm{C}^{\gamma}\right)$ corresponds to $\left(\mathrm{C}^{\beta}, \mathrm{C}^{\gamma 2}\right)$; for all residues with $\mathrm{C}^{\gamma 1}$ and $\mathrm{C}^{\gamma 2}$ nuclei, the $\left(\mathrm{C}^{\beta}, \mathrm{C}^{\gamma 1}\right) S_{\mathrm{CC}}$ value is given.

\begin{tabular}{c|cc|cc}
\hline Residue & $\left(\mathrm{C}^{\alpha}, \mathrm{C}^{\beta}\right) S_{\mathrm{CC}}$ & Error & $\left(\mathrm{C}^{\beta}, \mathrm{C}^{\gamma}\right) S_{\mathrm{CC}}$ & Error \\
\hline M1 & 0.80 & 0.1 & 0.80 & 0.05 \\
Q2 & - & - & - & - \\
I3 & 0.85 & 0.05 & 0.78 & 0.10 \\
F4 & 1.00 & 0.05 & - & - \\
V5 & - & - & 0.90 & 0.05 \\
K6 & - & - & - & - \\
T7 & 1.00 & $<0.05$ & 0.98 & 0.05 \\
L8 & 0.90 & 0.1 & - & - \\
T9 & 0.98 & 0.05 & - & - \\
G10 & - & - & - & - \\
K11 & - & - & - & - \\
T12 & - & - & - & - \\
I13 & 0.78 & 0.05 & - & - \\
T14 & - & - & - & - \\
L15 & 0.90 & 0.1 & 0.80 & 0.05 \\
E16 & - & - & - & - \\
V17 & 0.90 & 0.1 & 0.95 & 0.05 \\
E18 & 0.83 & 0.05 & - & - \\
P19 & 0.93 & 0.05 & 0.80 & 0.1 \\
S20 & - & - & - & - \\
D21 & - & - & - & - \\
T22 & 1.00 & 0.05 & 0.93 & 0.05 \\
I23 & 0.88 & 0.05 & - & - \\
E24 & 0.88 & 0.05 & - & - \\
N25 & 1.00 & 0.05 & - & - \\
V26 & 0.93 & 0.05 & 0.98 & 0.25 \\
K27 & 0.93 & 0.05 & 0.80 & 0.05 \\
A28 & 1.00 & $<0.05$ & - & - \\
K29 & - & - & 0.80 & 0.05 \\
I30 & 0.90 & 0.05 & 0.85 & 0.05 \\
Q31 & 0.95 & 0.05 & - & - \\
D32 & - & - & - & - \\
K33 & - & - & - & - \\
E34 & 0.78 & 0.05 & 0.95 & -10 \\
\hline & & & & \\
\hline
\end{tabular}




\begin{tabular}{|c|c|c|c|c|}
\hline Residue & $\left(\mathrm{C}^{\alpha}, \mathrm{C}^{\beta}\right) S_{\mathrm{CC}}$ & Error & $\left(\mathrm{C}^{\beta}, \mathrm{C}^{\gamma}\right) S_{\mathrm{CC}}$ & Error \\
\hline G35 & - & - & - & - \\
\hline I36 & 0.75 & 0.05 & - & 0.05 \\
\hline P37 & 0.93 & 0.05 & - & 0.05 \\
\hline P38 & 0.90 & 0.05 & - & 0.05 \\
\hline D39 & - & - & - & - \\
\hline Q40 & - & - & - & - \\
\hline Q41 & - & - & - & - \\
\hline $\mathrm{R} 42$ & - & - & - & - \\
\hline $\mathrm{L} 43$ & 0.85 & 0.05 & 0.78 & 0.05 \\
\hline I44 & 0.83 & 0.05 & - & - \\
\hline F 45 & 1.00 & 0.1 & - & - \\
\hline A 46 & 1.00 & $<0.05$ & - & - \\
\hline G47 & - & - & - & - \\
\hline K48 & - & - & - & - \\
\hline Q49 & - & - & - & - \\
\hline L50 & 0.98 & 0.05 & 0.83 & 0.05 \\
\hline E51 & - & - & - & - \\
\hline D52 & - & - & - & - \\
\hline G53 & - & - & - & - \\
\hline $\mathrm{R} 54$ & 0.83 & 0.05 & - & - \\
\hline $\mathrm{T} 55$ & 0.95 & 0.05 & 1.00 & $<0.05$ \\
\hline $\mathrm{L} 56$ & 0.93 & 0.05 & 0.83 & 0.1 \\
\hline $\mathrm{S} 57$ & 1.00 & 0.05 & - & - \\
\hline D58 & - & - & - & - \\
\hline Y59 & - & - & - & - \\
\hline N60 & 1.00 & $<0.05$ & - & - \\
\hline I61 & 0.90 & 0.05 & 0.85 & 0.1 \\
\hline Q62 & - & - & - & - \\
\hline K63 & 0.83 & 0.05 & 0.80 & 0.20 \\
\hline E64 & 0.90 & 0.05 & 0.85 & 0.05 \\
\hline S65 & 1.00 & 0.05 & - & - \\
\hline T66 & - & - & - & - \\
\hline L67 & 0.90 & 0.05 & 0.73 & 0.05 \\
\hline H68 & - & - & - & - \\
\hline L69 & 0.90 & 0.05 & 0.63 & 0.15 \\
\hline V70 & - & - & 0.85 & 0.05 \\
\hline L71 & - & - & - & - \\
\hline $\mathrm{R} 72$ & - & - & - & - \\
\hline L73 & - & - & - & - \\
\hline R74 & - & - & - & - \\
\hline G75 & - & - & - & - \\
\hline G76 & - & - & - & - \\
\hline
\end{tabular}

Table B.3: Backbone $\left(\mathrm{C}^{\alpha}, \mathrm{C}^{\beta}\right)$ and sidechain $\left(\mathrm{C}^{\beta}, \mathrm{C}^{\gamma}\right) S_{\mathrm{CC}}$ order parameters determined for Ubi-P. 


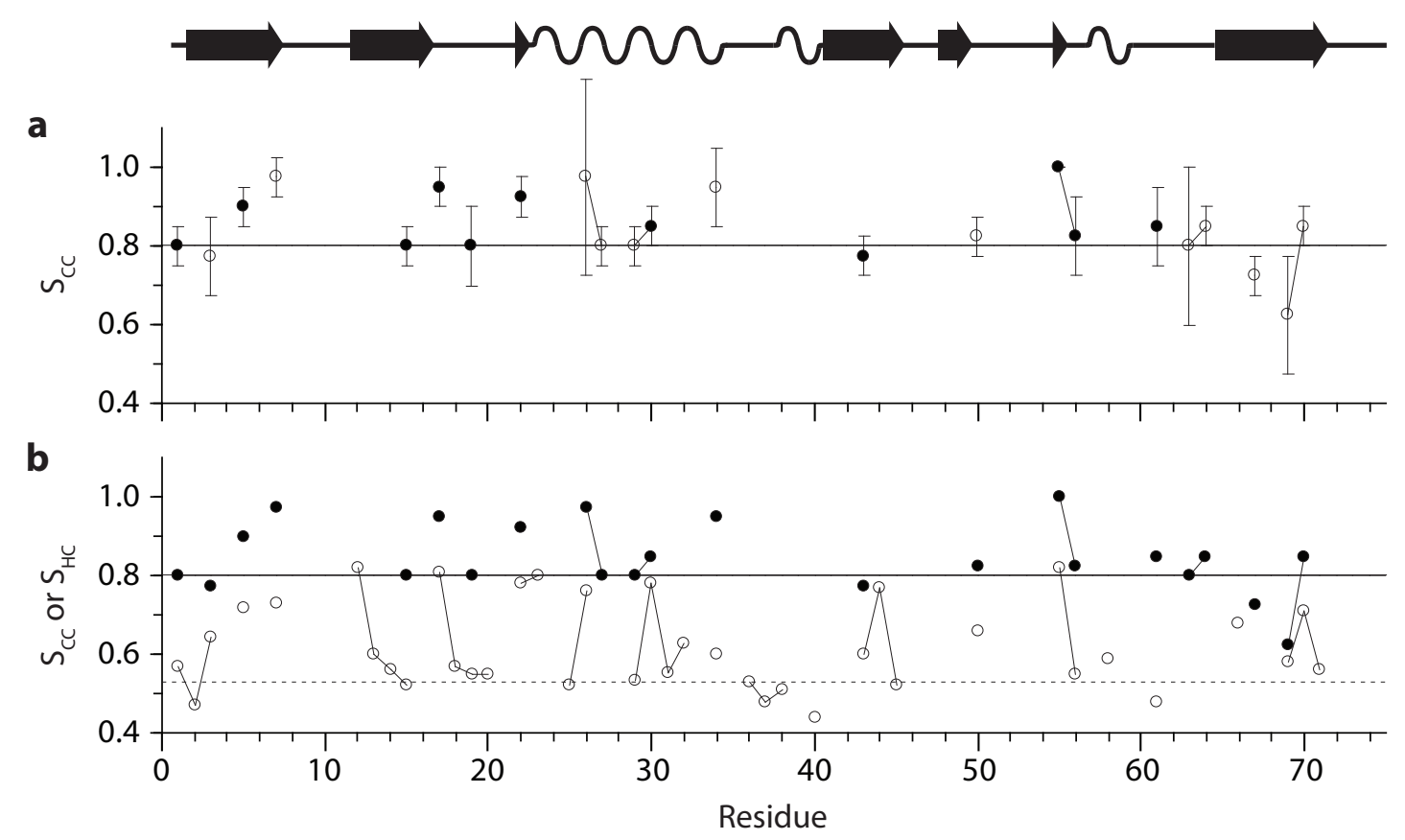

Figure B.1: (a) Plot of Ubi-P $\left(\mathrm{C}^{\beta}, \mathrm{C}^{\gamma}\right) S_{\mathrm{CC}}$ order parameters from Table B.3. Open symbols indicate values with fit RMSDs $>0.1$ or that were obtained from signals not fully resolved in spectra. Solid line indicates an $S_{\mathrm{CC}}$ value of 0.80 below which the most mobile $17 \%$ of determined $S_{\mathrm{CC}}$ values are found. Secondary structure of ubiquitin as found in the crystal structure (PDB 1UBQ) is sketched at the top. (b) Comparison of Ubi-P $\left(\mathrm{C}^{\beta}, \mathrm{C}^{\gamma}\right) S_{\mathrm{CC}}$ (filled symbols) and Ubi$\mathrm{M} S_{\mathrm{HC}}$ order parameters [149] (open symbols). $S_{\mathrm{HC}}$ values plotted are averaged over $\mathrm{C}^{\beta}$ and $\mathrm{C}^{\gamma}$ nuclei if both values are available. Solid line as in (a); dotted line denotes the upper limit $\left(S_{\mathrm{HC}}=0.53\right)$ for the most mobile $21 \%$ of residues detected in Ubi-M. 


\section{B.5 Structure calculation}

\begin{tabular}{|c|c|}
\hline \multicolumn{2}{|l|}{$\left({ }^{1} \mathbf{H},{ }^{1} \mathbf{H}\right)$ distance restraints } \\
\hline Total & 1210 \\
\hline Unambiguous restraints & 368 \\
\hline Unique $\left({ }^{13} \mathrm{C},{ }^{13} \mathrm{C}\right)$ pairs involved & 208 \\
\hline Intraresidue & 68 \\
\hline Sequential & 33 \\
\hline Medium-range & 37 \\
\hline Long-range & 70 \\
\hline Ambiguous restraints & 842 \\
\hline Unique $\left({ }^{13} \mathrm{C},{ }^{13} \mathrm{C}\right)$ pairs involved & 1186 \\
\hline Intraresidue & 170 \\
\hline Sequential & 174 \\
\hline Medium-range & 249 \\
\hline Long-range & 593 \\
\hline Distance violations $(>0.5 \AA)$ & 0 \\
\hline Dihedral angle restraints & 92 \\
\hline Dihedral violations $\left(>5^{\circ}\right)$ & 2 \\
\hline \multicolumn{2}{|l|}{ Energies (kcal/mol) } \\
\hline Total & $1098.6 \pm 4.2$ \\
\hline $\mathrm{CHHC}$ & $28.0 \pm 1.3$ \\
\hline Dihedral & $11.6 \pm 2.1$ \\
\hline \multicolumn{2}{|c|}{ Coordinate precision and accuracy (residues $1-70$ ) } \\
\hline \multicolumn{2}{|l|}{ Average RMSD to mean coordinates $(\AA)$} \\
\hline Backbone atoms & 0.49 \\
\hline Heavy atoms & 0.87 \\
\hline \multicolumn{2}{|l|}{ Average RMSD to 1 UBQ coordinates $(\AA)$} \\
\hline Backbone atoms & 1.79 \\
\hline Heavy atoms & 2.54 \\
\hline \multicolumn{2}{|c|}{ Ramachandran statistics (excluding Pro, Gly) } \\
\hline Residues in most favored regions (\%) & 86.7 \\
\hline Residues in allowed regions (\%) & 9.4 \\
\hline Residues in generously allowed regions (\%) & 2.6 \\
\hline Residues in disallowed regions (\%) & 1.4 \\
\hline
\end{tabular}

Table B.4: Structural statistics for the 10 calculated Ubi-P structures with lowest overall energy. The residue difference $i-j$ of two nuclei involved in medium-range distance restraints is $2 \leq$ $i-j \leq 4$ and $i-j \geq 5$ for long-range restraints. 

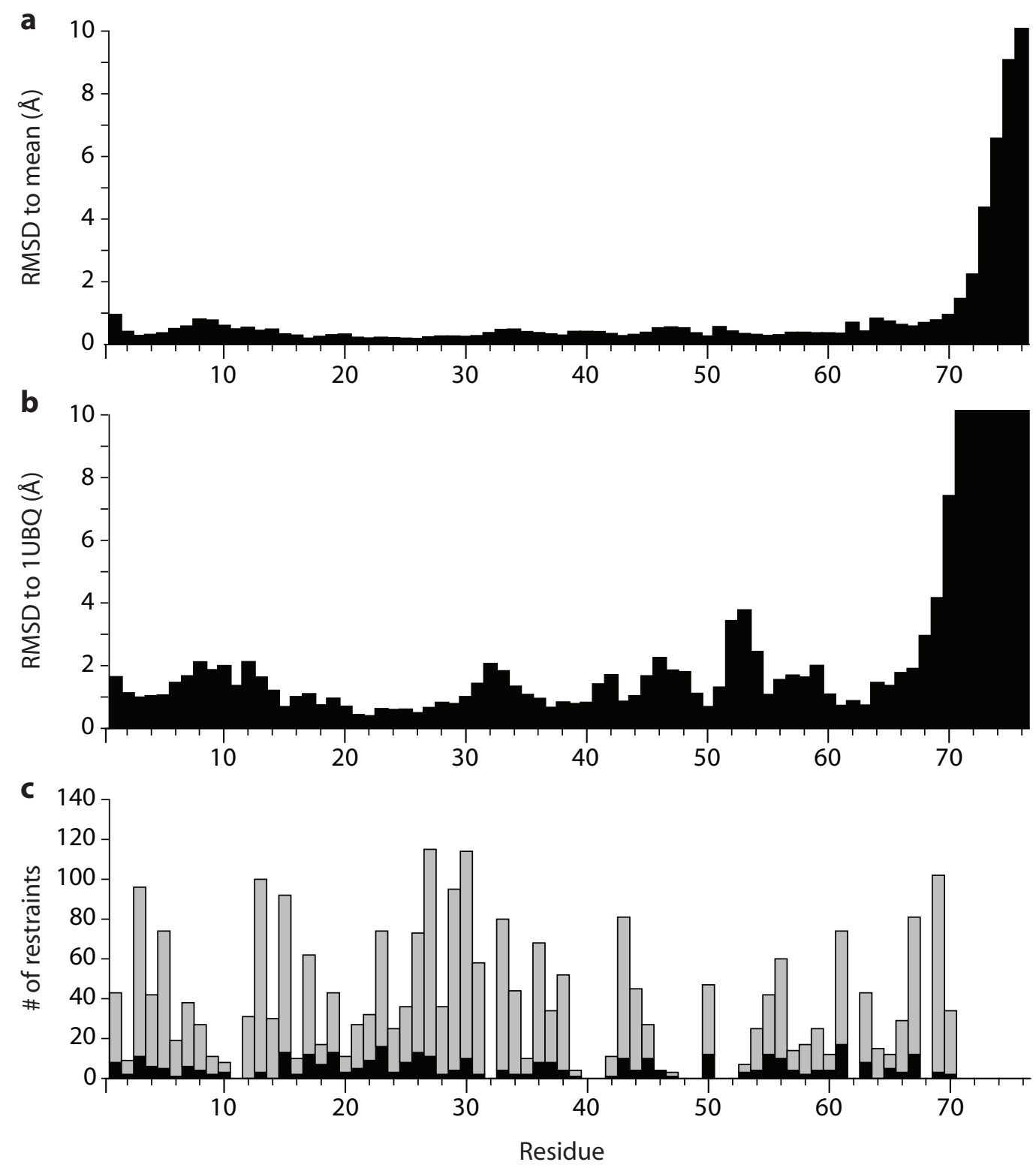

Figure B.2: (a) Average RMSD per residue of the 10 calculated Ubi-P structures with lowest overall energy to their mean coordinates. (b) Average RMSD per residue of the 10 lowest-energy calculated Ubi-P structures to the ubiquitin crystal structure (1UBQ). Bars for residues 71-76 for which no restraints are available are truncated at $10 \AA$. (c) Number of $\mathrm{CHHC}$ restraints per residue obtained by PASD, counting only restraints unique in terms of the ${ }^{13} \mathrm{C}$ nuclei involved (total 208 unambiguous, 1186 ambiguous). Interresidue restraints are counted in both residues involved. Black: unambiguous restraints; grey: ambiguous restraints. 


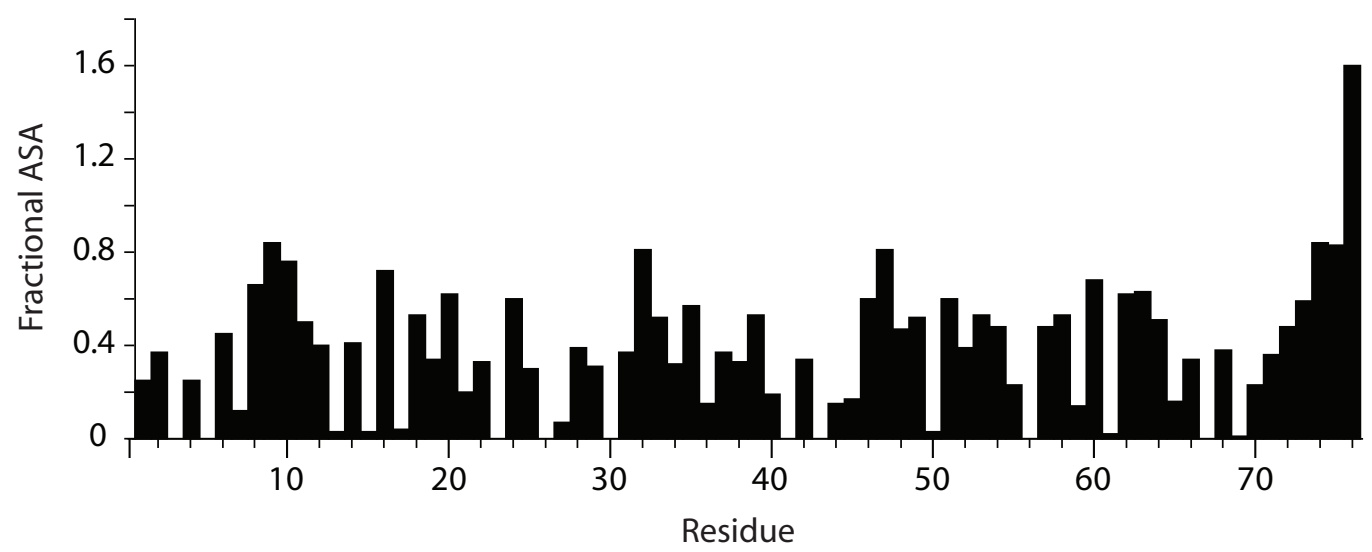

Figure B.3: Fractional accessible surface area (ASA) per residue as determined for the ubiquitin crystal structure (1UBQ) by the VADAR web server [342] used with standard settings. Fractional ASA is defined as the observed ASA for a residue (in $\AA^{2}$ ) divided by its ASA in an extended GlyXaa-Gly peptide. The Pearson correlation coefficient of fractional ASA with $\left(\mathrm{C}^{\alpha}, \mathrm{C}^{\beta}\right) S_{\mathrm{CC}}$ values along the ubiquitin sequence is 0.16 .

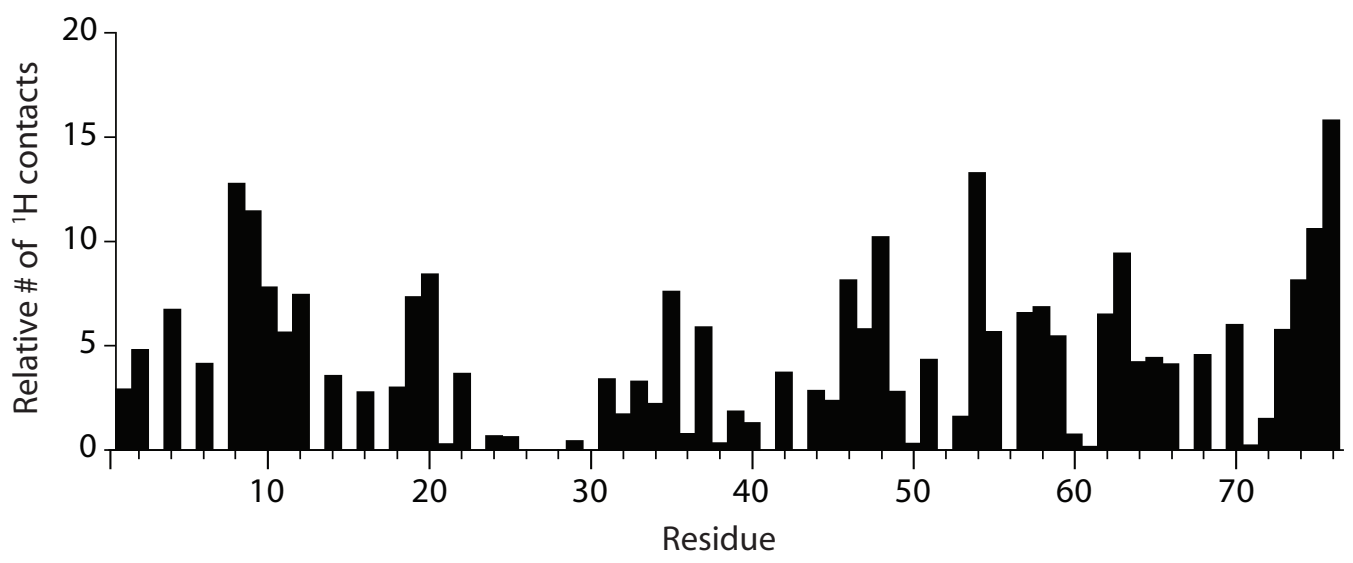

Figure B.4: Number of close crystal contacts per residue for the ubiquitin crystal structure (1UBQ). Addition of protons to $1 \mathrm{UBQ}$ and construction of the crystal unit cell with neighboring molecules were performed using PyMOL [244]. The graph shows the number of protons in a neighboring ubiquitin molecule that are within $6 \AA$ of any proton of a given ubiquitin residue, divided by the number of protons in that residue. 


\section{Appendix C}

\section{Assignment and structural analysis of the chimeric potassium channel KcsA-Kv1.3 in lipid bilayers (Supplement)}

C.1 Primary sequence

\begin{tabular}{|c|c|c|c|c|}
\hline & 10 & 20 & 30 & 40 \\
\hline $\mathrm{KcsA}$ & MPPMLSGLLA & RLVKLLLGRH & GSALHWRAAG & AATVLLVIVL \\
\hline \multirow[t]{2}{*}{$\mathrm{Kcs} A-\mathrm{Kv} 1.3$} & MPPMLSGLLA & RLVKLLLGRH & GSALHWRAAG & AATVLLVIVL \\
\hline & 50 & 60 & 70 & 80 \\
\hline $\mathrm{KcsA}$ & LAGSYLAVLA & ERGAPGAQLI & TYPRALWWSV & ETATTVGYGD \\
\hline \multirow[t]{2}{*}{$\mathrm{KcsA}-\mathrm{Kv} 1.3$} & LAGSYLAVLA & EADDPTSGFS & SIPDALWWSV & ETATTVGYGD \\
\hline & 90 & 100 & 110 & 120 \\
\hline $\mathrm{KcsA}$ & LYPVTLWGRL & VAVVVMVAGI & TSFGLVTAAL & ATWFVGREQE \\
\hline \multirow[t]{2}{*}{$\mathrm{KcsA}-\mathrm{Kv} 1.3$} & LYPVTLWGRC & VAVVVMVAGI & TSFGLVTAAL & ATWFVGREQE \\
\hline & 130 & 140 & 150 & 160 \\
\hline $\mathrm{KcsA}$ & RRGHFVRHSE & KAAEEAYTRT & TRALHERFDR & LERMLDDNRR \\
\hline KcsA-Kv1.3 & RRGHFVRHSE & KAAEEAYTRT & TRALHERFDR & LERMLDDNRR \\
\hline
\end{tabular}

Table C.1: Amino acid sequences of KcsA and KcsA-Kv1.3. In the KcsA-Kv1.3 sequence, mutations to Kv1.3 residues in the turret region as well as the L90C mutation increasing expression yield are underlined. 


\section{C.2 Supplementary figures}

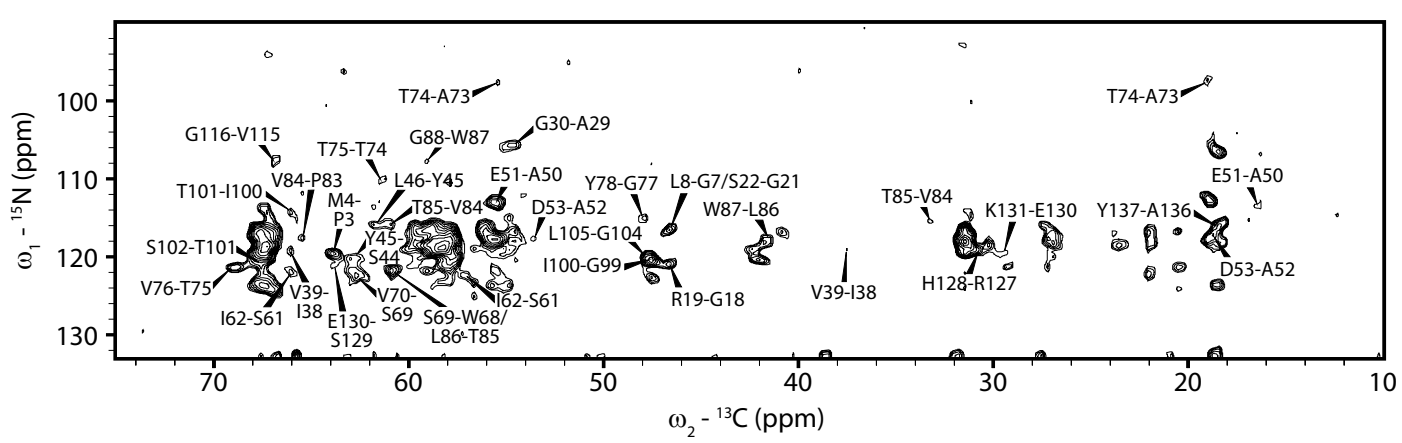

Figure C.1: 2D NCOCA $\left({ }^{15} \mathrm{~N},{ }^{13} \mathrm{C}\right)$ correlation spectrum of [U]KcsA-Kv1.3 in liposomes $(800 \mathrm{MHz}$ ${ }^{1} \mathrm{H}$ Larmor frequency, $12.5 \mathrm{kHz}$ MAS, lipid bilayer in liquid crystalline phase). SPECIFIC-CP $[123,124](1.5 \mathrm{~ms})$ was used for the $\left({ }^{15} \mathrm{~N},{ }^{13} \mathrm{C}\right)$ transfer step; subsequently, DARR mixing [119] (20 ms) was employed to obtain sidechain ${ }^{13} \mathrm{C}$ resonances. Assigned sequential correlations are indicated.

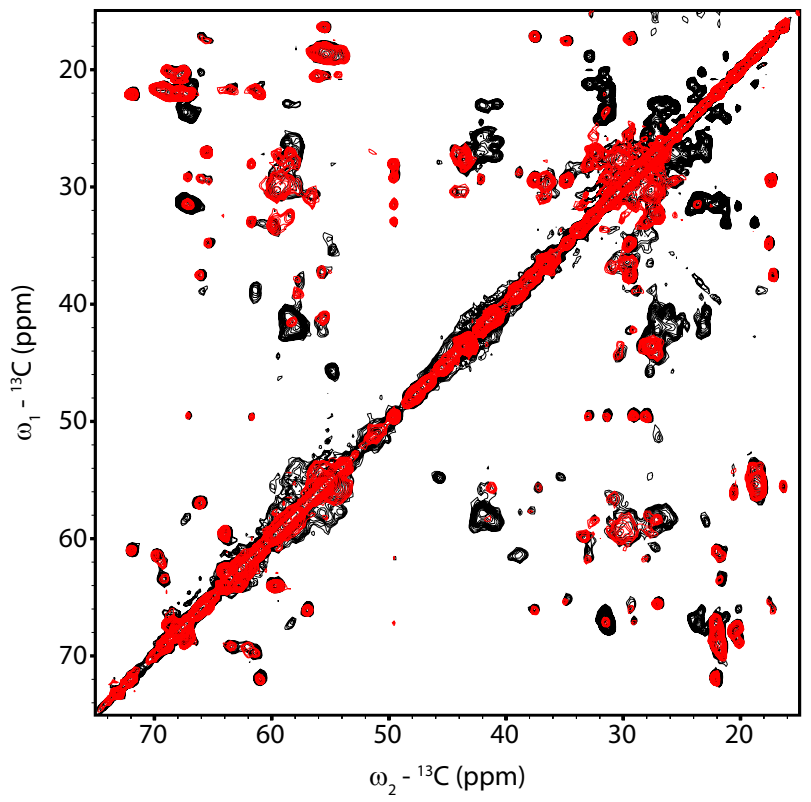

Figure C.2: Comparison of proton-driven $\left({ }^{13} \mathrm{C},{ }^{13} \mathrm{C}\right)$ spin diffusion (PDSD) spectra of [U]KcsAKv1.3 (black) and [R]KcsA-Kv1.3 (red). Both spectra were recorded with lipid bilayers in the liquid crystalline phase, using a spin diffusion mixing time of $20 \mathrm{~ms}$, at $800 \mathrm{MHz}{ }^{1} \mathrm{H}$ Larmor frequency and $12.5 \mathrm{kHz}$ MAS. 


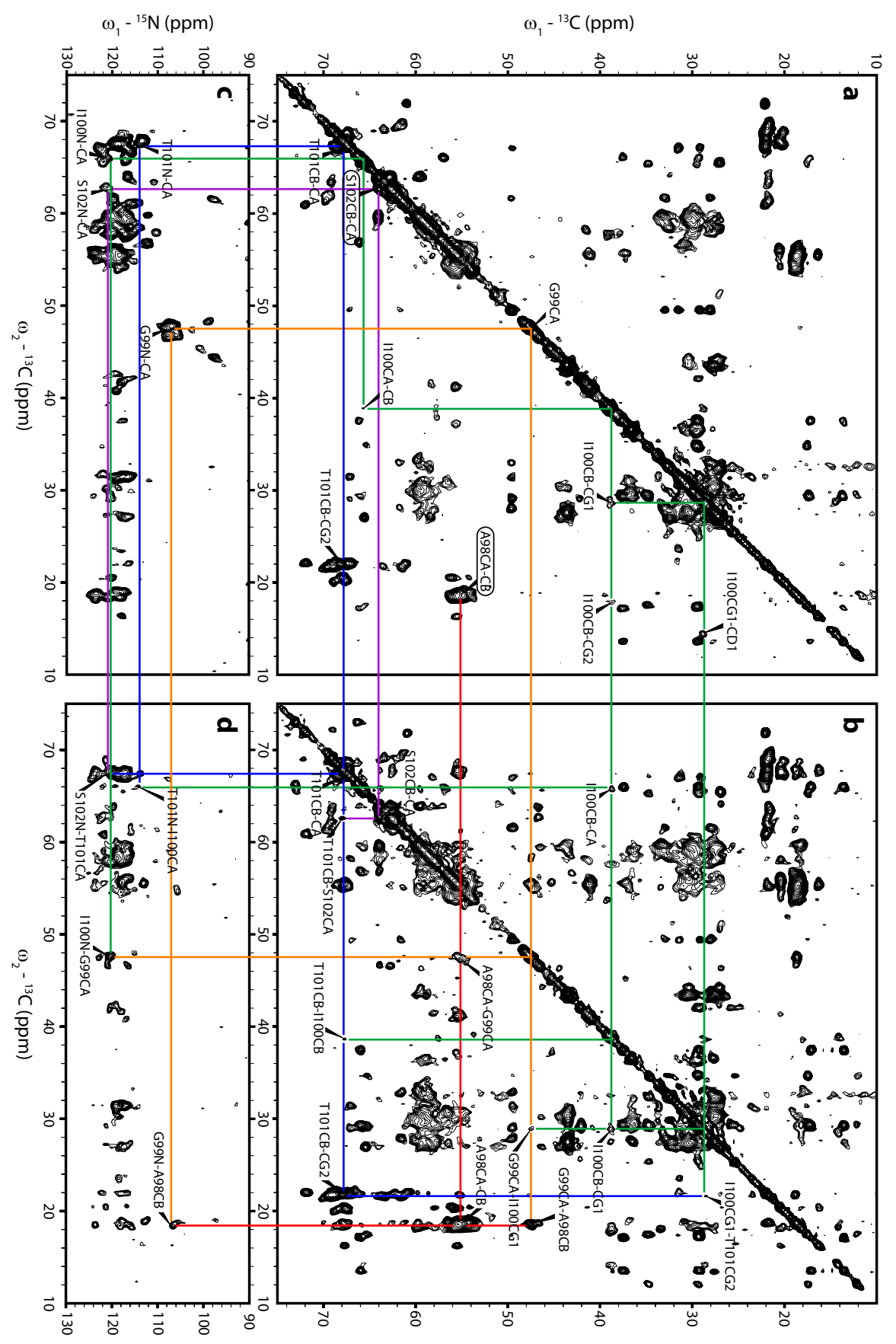

Figure C.3: Sequential walk through amino acid residues 98-102 in the putative gating region of KcsA-Kv1.3. Shown on top are PDSD-WC $[125]\left({ }^{13} \mathrm{C},{ }^{13} \mathrm{C}\right)$ correlation spectra of [R]KcsA-Kv1.3 recorded with $20 \mathrm{~ms}$ (a) and $150 \mathrm{~ms}$ (b) spin diffusion mixing times. At bottom, NCACB (c) and NCOCA (d) $\left({ }^{15} \mathrm{~N},{ }^{13} \mathrm{C}\right)$ correlation spectra of fully labeled KcsA-Kv1.3 are shown. All spectra were recorded at $800 \mathrm{MHz}{ }^{1} \mathrm{H}$ Larmor frequency, in the liquid crystalline phase of lipid bilayers and using $12.5 \mathrm{kHz}$ MAS. Note that (a) and (c) exhibit intraresidue correlations only, while (d) shows $\left({ }^{15} \mathrm{~N}_{i},{ }^{13} \mathrm{C}_{i-1}\right)$ sequential correlations and (b) contains intra- and interresidue $\left({ }^{13} \mathrm{C}_{i},{ }^{13} \mathrm{C}_{i \pm 1}\right)$ crosspeaks. Resonances of individual residues are connected by lines of a specific color. 


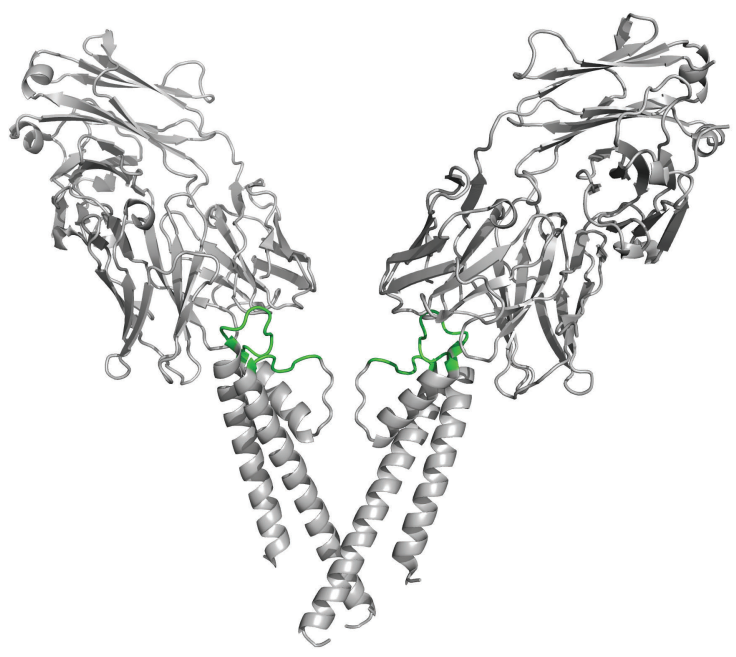

Figure C.4: Two of four subunits of the $\mathrm{KcsA} \mathrm{K} \mathrm{K}^{+}$channel with bound antibody as found in the crystal structure [24] (PDB ID 1K4C). Turret residues (52-61 and 80-85 in KcsA) are shown in green. Residues in the first turret loop (52-61) and at the extracellular ends of the TM1 and pore helices form the binding interface.

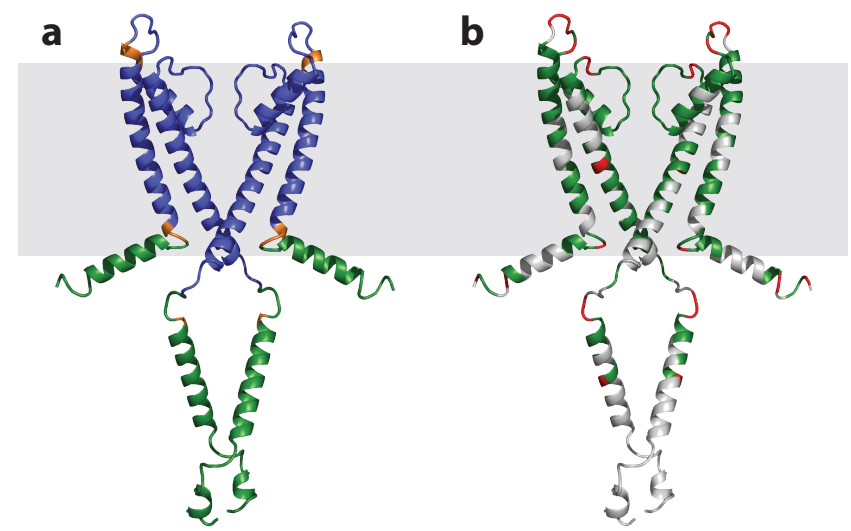

Figure C.5: Structural model of KcsA-Kv1.3. Approximate insertion into lipid bilayers is indicated by a grey rectangle. (a) Residues are colored blue where the model is based on the KcsA crystal structure [24], green for model parts derived from an EPR spectroscopy-based structural model [237], and orange where secondary structure was adapted according to ssNMR data. (b) Differences in $\mathrm{C}^{\alpha}$ secondary chemical shift between ssNMR assignments on KcsA-Kv1.3 and SHIFTX [163] predictions for the structural model of KcsA-Kv1.3. The color code on the model backbone shows residues with an agreement of $\Delta \delta\left(\mathrm{C}^{\alpha}\right)$ within $\pm 2 \mathrm{ppm}$ in green, deviations greater than 2 ppm in red, and unassigned residues in grey. 


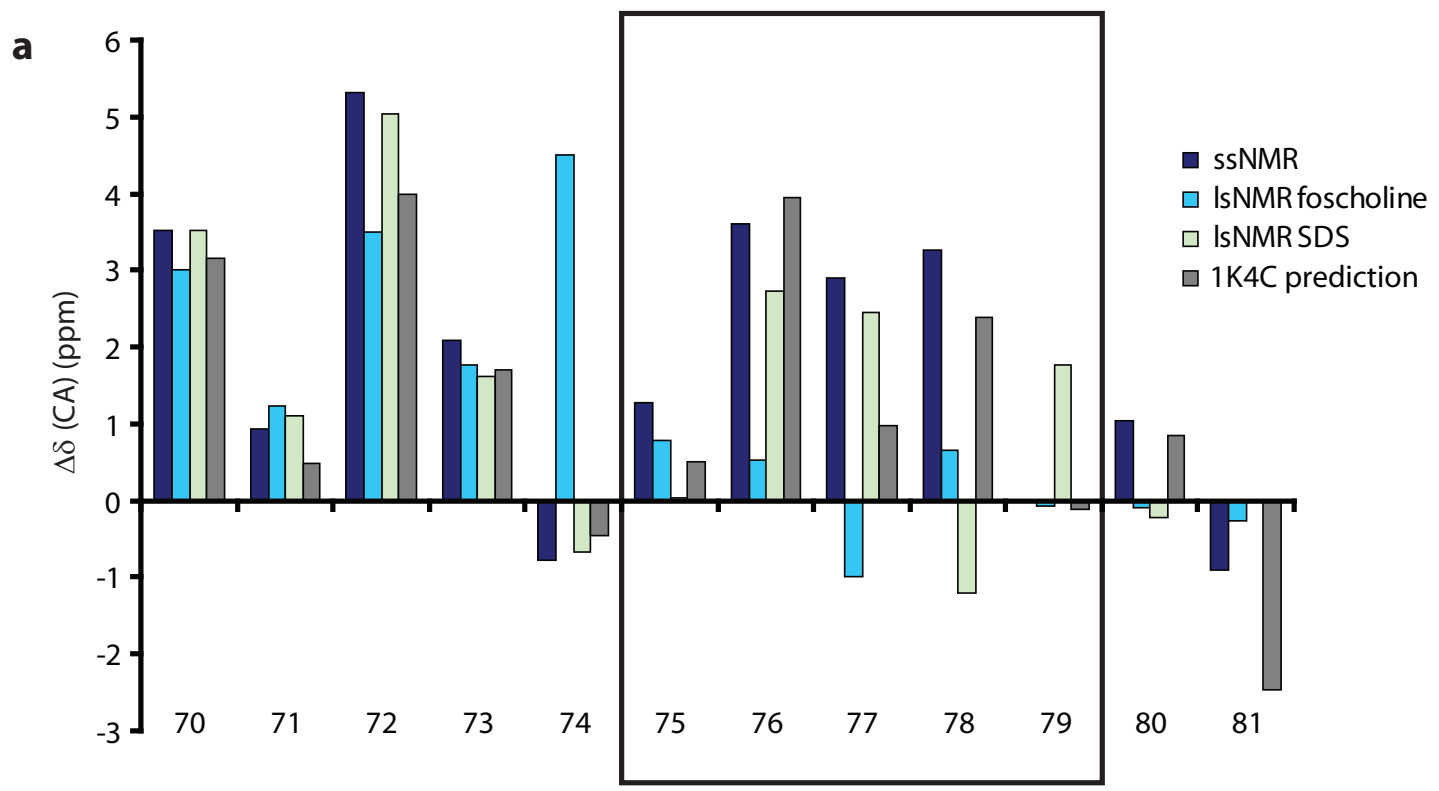

Residue

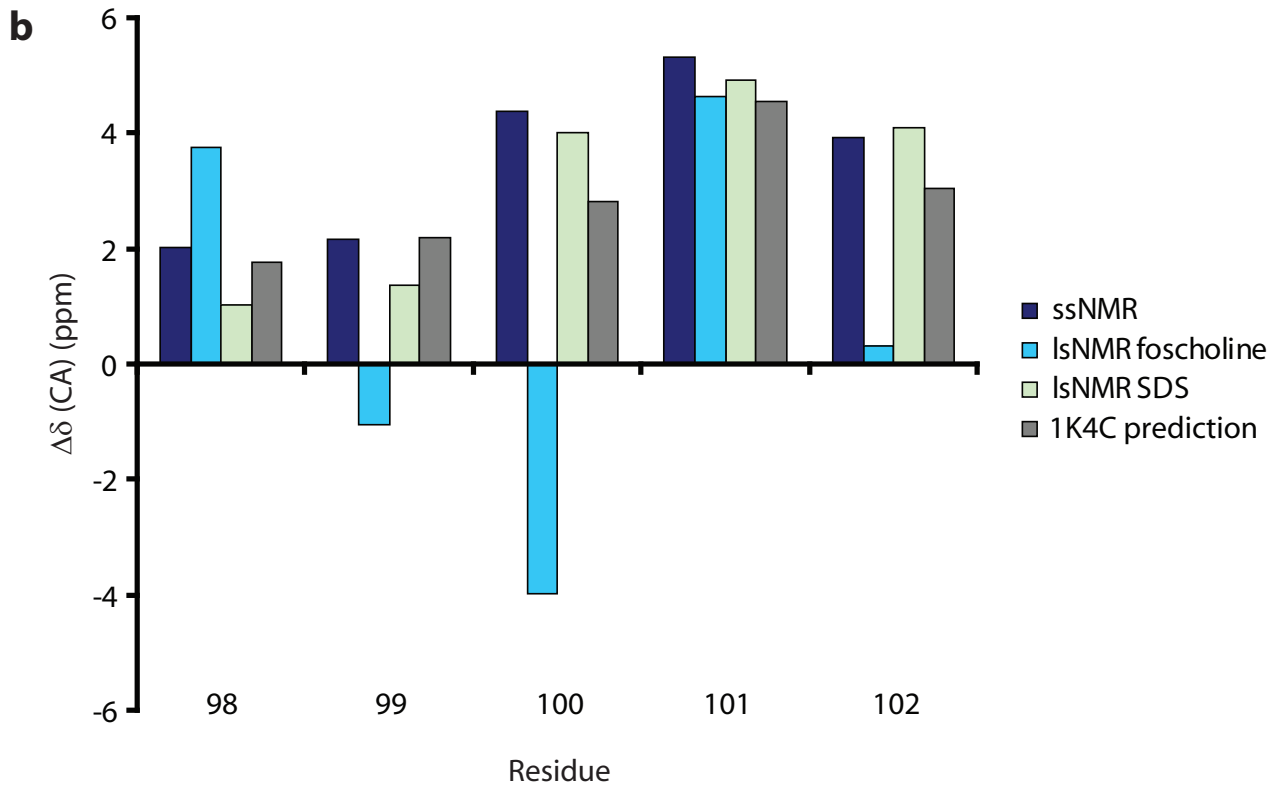

Figure C.6: Comparison of $\mathrm{C}^{\alpha}$ secondary chemical shifts in the selectivity filter region (a) and the putative TM2 gate (b) between solid-state NMR data measured on KcsA-Kv1.3 in liposomes ("ssNMR"), solution-state NMR data measured on KcsA in foscholine micelles [242] ("IsNMR foscholine") and SDS micelles [28] ("IsNMR SDS"), and SHIFTX [163] predictions for the KcsA crystal structure [24] ("1K4C prediction"). 


\section{C.3 Solid-state NMR resonance assignments}

${ }^{13} \mathrm{C}$ resonances were calibrated using adamantane as external reference. The upfield resonance of adamantane was set to $31.47 \mathrm{ppm}$ to allow for direct comparison with solutionstate NMR data referenced to DSS (2,2-dimethyl-2-silapentane-5-sulphonic acid). Accordingly, ${ }^{15} \mathrm{~N}$ data were calibrated using the tripeptide AGG as external reference [153].

Resonance assignments are self-consistent and based on sequential $\left({ }^{13} \mathrm{C},{ }^{13} \mathrm{C}\right)$ and / or $\left({ }^{15} \mathrm{~N},{ }^{13} \mathrm{C}\right)$ correlations. Similar to ref. [59], they were classified into three categories, based on amount and reliability of sequential information, as very good (1), good (2) and reasonable (3) (see column "class" of Table C.3). While usually several sequential correlations from $\left({ }^{13} \mathrm{C},{ }^{13} \mathrm{C}\right)$ and $\left({ }^{15} \mathrm{~N},{ }^{13} \mathrm{C}\right)$ spectra are available for category 1 assignments, category 3 assignments are generally characterized by one sequential correlation and crosspeaks with weaker intensity and/or in regions of strong spectral overlap.

Assignments obtained in the study described in Chapter 7 cover 94 of 160 KcsA-Kv1.3 residues (59\%). The following table also lists assignments for residues Glu118 and Glu120 obtained as described in Chapter 9, increasing the number of assigned residues to 96 (60\% of the KcsA-Kv1.3 sequence).

\begin{tabular}{|c|c|c|c|c|c|c|c|c|c|c|c|}
\hline Residue & Class & $\mathrm{N}$ & $\mathrm{CO}$ & CA & CB & CG(1) & CG2 & $\mathrm{CD}(1)$ & CD2 & $\mathrm{CE}(1)$ & $\mathrm{CZ}$ \\
\hline \multicolumn{12}{|l|}{ M1 } \\
\hline P2 & 2 & & & 64.1 & 32.3 & 27.0 & & 51.2 & & & \\
\hline P3 & 2 & & & 63.8 & 32.1 & 27.1 & & 50.6 & & & \\
\hline M4 & 2 & 119.5 & & 56.4 & 30.7 & & & & & 18.7 & \\
\hline \multicolumn{12}{|l|}{ L5 } \\
\hline S6 & 3 & & & 62.9 & 63.8 & & & & & & \\
\hline G7 & 3 & 109.3 & 175.3 & 46.6 & & & & & & & \\
\hline L8 & 3 & 116.7 & 177.5 & 58.1 & 41.9 & 27.1 & & 23.3 & & & \\
\hline \multicolumn{12}{|l|}{ L9 } \\
\hline \multicolumn{12}{|l|}{ A10 } \\
\hline \multicolumn{12}{|l|}{ R11 } \\
\hline \multicolumn{12}{|l|}{ L12 } \\
\hline \multicolumn{12}{|l|}{ V13 } \\
\hline \multicolumn{12}{|l|}{ K14 } \\
\hline \multicolumn{12}{|l|}{ L15 } \\
\hline \multicolumn{12}{|l|}{ L16 } \\
\hline L17 & 3 & 118.1 & & 58.7 & 41.6 & & & & & & \\
\hline G18 & 3 & 107.1 & 176.2 & 46.5 & & & & & & & \\
\hline R19 & 3 & 121.2 & 178.5 & 58.4 & 30.2 & 26.8 & & & & & \\
\hline $\mathrm{H} 20$ & 3 & & 178.2 & 56.5 & 30.3 & 132.1 & & & & 133.4 & \\
\hline G21 & 1 & 107.6 & 176.4 & 46.5 & & & & & & & \\
\hline $\mathrm{S} 22$ & 1 & 116.6 & 175.7 & 62.7 & 63.9 & & & & & & \\
\hline A23 & 1 & & & 55.3 & 18.7 & & & & & & \\
\hline \multicolumn{12}{|l|}{ L24 } \\
\hline H25 & & & & & & & & & & & \\
\hline
\end{tabular}




\begin{tabular}{|c|c|c|c|c|c|c|c|c|c|c|c|}
\hline Residue & Class & $\mathrm{N}$ & $\mathrm{CO}$ & $\mathrm{CA}$ & $\mathrm{CB}$ & CG(1) & CG2 & $\mathrm{CD}(1)$ & CD2 & $\mathrm{CE}(1)$ & $\mathrm{CZ}$ \\
\hline \multicolumn{12}{|l|}{ W26 } \\
\hline $\mathrm{R} 27$ & 3 & & & 59.6 & 30.3 & 27.3 & & 44.3 & & & \\
\hline A28 & 3 & & & 55.3 & 18.6 & & & & & & \\
\hline A29 & 3 & & & 54.5 & 18.6 & & & & & & \\
\hline G30 & 3 & 105.7 & & 47.4 & & & & & & & \\
\hline A31 & 3 & & & 56.2 & 18.5 & & & & & & \\
\hline A32 & 3 & & & 55.5 & 18.6 & & & & & & \\
\hline T33 & 3 & & & 67.1 & 67.8 & & & & & & \\
\hline \multicolumn{12}{|l|}{ V34 } \\
\hline \multicolumn{12}{|l|}{ L35 } \\
\hline \multicolumn{12}{|l|}{ L36 } \\
\hline \multicolumn{12}{|l|}{ V37 } \\
\hline I38 & 1 & 117.6 & 177.3 & 65.9 & 37.6 & 29.3 & 17.2 & 13.6 & & & \\
\hline V39 & 2 & 119.3 & & 67.4 & 30.9 & & & & & & \\
\hline \multicolumn{12}{|l|}{ L40 } \\
\hline \multicolumn{12}{|l|}{ L41 } \\
\hline A 42 & 1 & & 180.1 & 56.2 & 18.7 & & & & & & \\
\hline G43 & 1 & 105.8 & 175.1 & 46.7 & & & & & & & \\
\hline S44 & 1 & 116.5 & 174.5 & 62.7 & 63.8 & & & & & & \\
\hline Y45 & 1 & 119.9 & 178.1 & 61.6 & 39.0 & & & & & & \\
\hline L46 & 1 & 115.7 & & 58.0 & 42.3 & & & 23.4 & & & \\
\hline A47 & 3 & & & 55.4 & 18.7 & & & & & & \\
\hline V48 & 3 & & & 67.4 & 31.4 & 21.9 & & & & & \\
\hline L49 & 3 & & & 58.0 & 41.2 & & & & & & \\
\hline A50 & 1 & 118.1 & 177.5 & 55.5 & 16.4 & & & & & & \\
\hline E51 & 1 & 113.4 & 175.9 & 58.8 & 30.8 & 36.7 & & 183.2 & & & \\
\hline A52 & 1 & 121.8 & 178.4 & 53.8 & 18.8 & & & & & & \\
\hline D53 & 1 & 117.3 & 175.4 & 57.6 & 39.1 & 179.1 & & & & & \\
\hline \multicolumn{12}{|l|}{ D54 } \\
\hline $\mathrm{P} 55$ & 1 & 131.0 & 176.6 & 61.7 & 32.9 & 28.0 & & 49.5 & & & \\
\hline T56 & 1 & & & 58.3 & 67.2 & & 22.0 & & & & \\
\hline $\mathrm{S} 57$ & 1 & & 175.5 & 59.8 & 63.9 & & & & & & \\
\hline G58 & 1 & 107.2 & 175.4 & 46.7 & & & & & & & \\
\hline F59 & 1 & & 179.2 & 57.7 & 39.6 & 138.7 & & & & 130.2 & \\
\hline S60 & 1 & 117.9 & & 59.5 & 64.0 & & & & & & \\
\hline S61 & 1 & 112.2 & 173.8 & 56.9 & 66.0 & & & & & & \\
\hline I 62 & 1 & 122.1 & 175.4 & 65.3 & 34.8 & 29.4 & 17.5 & 12.1 & & & \\
\hline P63 & 1 & 134.6 & & 67.1 & 31.4 & 29.1 & & 49.5 & & & \\
\hline D64 & 2 & & 177.3 & 55.5 & 41.4 & 179.7 & & & & & \\
\hline A65 & 2 & 119.4 & & 56.0 & 20.4 & & & & & & \\
\hline \multicolumn{12}{|l|}{ L66 } \\
\hline \multicolumn{12}{|l|}{ W67 } \\
\hline W68 & 2 & 118.2 & & 60.8 & 27.2 & 113.4 & & & & & \\
\hline S69 & 2 & 121.7 & 175.7 & 62.7 & 63.6 & & & & & & \\
\hline V70 & 2 & 122.4 & & 66.0 & 31.7 & 20.2 & & & & & \\
\hline E71 & 1 & & & 58.3 & 27.1 & 32.5 & & 181.9 & & & \\
\hline T72 & 1 & & 176.1 & 67.5 & 68.7 & & 20.3 & & & & \\
\hline
\end{tabular}




\begin{tabular}{|c|c|c|c|c|c|c|c|c|c|c|c|}
\hline Residue & Class & $\mathrm{N}$ & $\mathrm{CO}$ & $\mathrm{CA}$ & CB & CG(1) & CG2 & $\mathrm{CD}(1)$ & CD2 & $\mathrm{CE}(1)$ & $\mathrm{CZ}$ \\
\hline A73 & 1 & 122.3 & 176.2 & 55.3 & 18.9 & & & & & & \\
\hline $\mathrm{T} 74$ & 1 & 96.2 & 176.5 & 61.4 & 69.7 & & 21.7 & & & & \\
\hline $\mathrm{T} 75$ & 1 & 110.7 & 171.9 & 63.5 & 69.1 & & 21.7 & & & & \\
\hline V76 & 1 & 121.5 & 178.2 & 66.1 & 31.8 & 23.4 & 20.2 & & & & \\
\hline G77 & 1 & 99.2 & 174.7 & 48.2 & & & & & & & \\
\hline Y78 & 1 & 115.2 & 178.3 & 61.4 & 38.6 & & & & & 118.0 & 157.9 \\
\hline G79 & 1 & 101.2 & 174.3 & 45.3 & & & & & & & \\
\hline D80 & 1 & 118.4 & & 55.7 & 37.3 & 179.4 & & & & & \\
\hline L81 & 3 & & 174.9 & 54.8 & 45.7 & 27.8 & & 25.8 & 24.6 & & \\
\hline Y82 & 3 & & & 55.7 & 41.1 & & & & & 115.5 & \\
\hline P83 & 2 & & & 65.4 & 34.7 & 26.9 & & 49.4 & & & \\
\hline V84 & 1 & 117.5 & & 60.9 & 33.0 & 21.3 & 18.8 & & & & \\
\hline T85 & 1 & 115.5 & 174.8 & 61.0 & 71.9 & & 22.1 & & & & \\
\hline L86 & 1 & 121.4 & & 59.1 & 41.4 & & & & & & \\
\hline W87 & 2 & 117.7 & 178.4 & 59.4 & 29.2 & & & & & & \\
\hline G88 & 2 & 107.9 & 176.3 & 46.4 & & & & & & & \\
\hline R89 & 2 & 120.5 & 178.6 & 59.5 & 30.1 & 27.0 & & 44.3 & & & \\
\hline \multicolumn{12}{|l|}{ C90 } \\
\hline \multicolumn{12}{|l|}{ V91 } \\
\hline \multicolumn{12}{|l|}{ A92 } \\
\hline \multicolumn{12}{|l|}{ V93 } \\
\hline \multicolumn{12}{|l|}{ V94 } \\
\hline \multicolumn{12}{|l|}{ V95 } \\
\hline \multicolumn{12}{|l|}{ M96 } \\
\hline \multicolumn{12}{|l|}{ V97 } \\
\hline A98 & 1 & & 178.4 & 55.2 & 18.5 & & & & & & \\
\hline G99 & 1 & 107.5 & 175.8 & 47.5 & & & & & & & \\
\hline I100 & 1 & 120.6 & 175.7 & 66.0 & 38.8 & 28.7 & 18.4 & 14.2 & & & \\
\hline T101 & 1 & 114.2 & 175.9 & 67.5 & 67.9 & & 21.7 & & & & \\
\hline S102 & 1 & 120.8 & 176.1 & 62.6 & 64.0 & & & & & & \\
\hline F103 & 2 & & & 57.9 & 38.7 & 139.8 & & 132.0 & & & 128.2 \\
\hline G104 & 2 & & 174.9 & 47.8 & & & & & & & \\
\hline L105 & 3 & 119.6 & & 59.1 & 42.8 & & & & & & \\
\hline \multicolumn{12}{|l|}{ V106 } \\
\hline T107 & 3 & & & 67.5 & 68.6 & & & & & & \\
\hline A108 & 3 & & & 55.0 & 18.6 & & & & & & \\
\hline A109 & 3 & & & 54.7 & 18.6 & & & & & & \\
\hline \multicolumn{12}{|l|}{ L110 } \\
\hline A111 & 3 & & & 55.6 & 18.4 & & & & & & \\
\hline $\mathrm{T} 112$ & 3 & & & 67.2 & 68.7 & & & & & & \\
\hline \multicolumn{12}{|l|}{ W113 } \\
\hline \multicolumn{12}{|l|}{ F114 } \\
\hline V115 & 1 & & 178.6 & 66.8 & 31.9 & 23.8 & 21.9 & & & & \\
\hline G116 & 1 & 107.8 & & 47.6 & & & & & & & \\
\hline \multicolumn{12}{|l|}{$\mathrm{R} 117$} \\
\hline E118 & 2 & & 178.4 & 58.2 & 30.6 & 36.7 & & 183.1 & & & \\
\hline Q119 & & & & & & & & & & & \\
\hline
\end{tabular}




\begin{tabular}{|c|c|c|c|c|c|c|c|c|c|c|c|}
\hline Residue & Class & $\mathrm{N}$ & $\mathrm{CO}$ & $\mathrm{CA}$ & CB & $\mathrm{CG}(1)$ & CG2 & $\mathrm{CD}(1)$ & CD2 & $\mathrm{CE}(1)$ & $\mathrm{CZ}$ \\
\hline E120 & 2 & & 178.4 & 58.2 & 30.6 & 36.7 & & 183.1 & & & \\
\hline \multicolumn{12}{|l|}{$\mathrm{R} 121$} \\
\hline R122 & 2 & & 178.2 & 57.1 & 30.7 & 27.2 & & 44.3 & & & \\
\hline G123 & 2 & 107.6 & & 44.7 & & & & & & & \\
\hline \multicolumn{12}{|l|}{ H124 } \\
\hline \multicolumn{12}{|l|}{ F125 } \\
\hline \multicolumn{12}{|l|}{ V126 } \\
\hline $\mathrm{R} 127$ & 2 & & 178.4 & 58.3 & 30.6 & 27.0 & & 44.4 & & & \\
\hline H128 & 3 & 119.3 & 175.7 & 58.4 & 32.4 & 131.8 & & & & & \\
\hline S129 & 2 & & & 62.8 & 63.9 & & & & & & \\
\hline E130 & 2 & 121.2 & 178.8 & 58.8 & 29.4 & 34.7 & & 183.0 & & & \\
\hline K131 & 1 & 119.6 & 178.4 & 58.4 & 32.4 & 25.5 & & 29.4 & & 42.1 & \\
\hline A132 & 1 & 117.8 & 177.5 & 55.5 & 16.4 & & & & & & \\
\hline \multicolumn{12}{|l|}{ A133 } \\
\hline \multicolumn{12}{|l|}{ E134 } \\
\hline E135 & 3 & & & 58.3 & 28.9 & 36.2 & & 183.2 & & & \\
\hline A136 & 1 & & & & 18.4 & & & & & & \\
\hline Y137 & 2 & 116.2 & & 61.2 & 38.9 & & & & & & \\
\hline $\mathrm{T} 138$ & 3 & & & 67.2 & 68.5 & & & & & & \\
\hline \multicolumn{12}{|l|}{ R139 } \\
\hline \multicolumn{12}{|l|}{$\mathrm{T} 140$} \\
\hline \multicolumn{12}{|l|}{$\mathrm{T} 141$} \\
\hline \multicolumn{12}{|l|}{$\mathrm{R} 142$} \\
\hline \multicolumn{12}{|l|}{ A143 } \\
\hline \multicolumn{12}{|l|}{ L144 } \\
\hline \multicolumn{12}{|l|}{ H145 } \\
\hline \multicolumn{12}{|l|}{ E146 } \\
\hline \multicolumn{12}{|l|}{$\mathrm{R} 147$} \\
\hline \multicolumn{12}{|l|}{ F148 } \\
\hline \multicolumn{12}{|l|}{ D149 } \\
\hline \multicolumn{12}{|l|}{$\mathrm{R} 150$} \\
\hline \multicolumn{12}{|l|}{ L151 } \\
\hline \multicolumn{12}{|l|}{ E152 } \\
\hline \multicolumn{12}{|l|}{$\mathrm{R} 153$} \\
\hline \multicolumn{12}{|l|}{ M154 } \\
\hline L155 & & & & & & & & & & & \\
\hline D156 & & & & & & & & & & & \\
\hline D157 & & & & & & & & & & & \\
\hline N158 & & & & & & & & & & & \\
\hline R159 & & & & & & & & & & & \\
\hline R160 & & & & & & & & & & & \\
\hline
\end{tabular}

Table C.2: Solid-state NMR resonance assignments for KcsA-Kv1.3 in liposomes. "Class" denotes classification of a residue's resonance assignments into three categories of reliability (see text). 


\begin{tabular}{|c|c|c|c|c|c|}
\hline Sample & $\begin{array}{l}\text { Spectrum } \\
\text { type }\end{array}$ & Mixing & $\begin{array}{l}\text { Lipid bilayer } \\
\text { state }\end{array}$ & MAS rate & Field \\
\hline \multirow[t]{5}{*}[\mathrm{R}]{$\mathrm{K} \operatorname{cs} \mathrm{A}-\mathrm{Kv} 1.3$} & $\mathrm{CC}$ & $\begin{array}{l}\text { PDSD 20,150, } \\
300 \mathrm{~ms}\end{array}$ & Liquid crystalline & $12.5 \mathrm{kHz}$ & $800 \mathrm{MHz}$ \\
\hline & $\mathrm{NCA}$ & SPECIFIC-CP & Gel phase & $8.5 \mathrm{kHz}$ & $600 \mathrm{MHz}$ \\
\hline & & $1.5 \mathrm{~ms}$ & & & \\
\hline & NCACB & $\begin{array}{l}\text { SPECIFIC-CP } 1.5 \\
\mathrm{~ms}, \text { PDSD } 50 \mathrm{~ms}\end{array}$ & Gel phase & $8.5 \mathrm{kHz}$ & $600 \mathrm{MHz}$ \\
\hline & NCOCA & $\begin{array}{l}\text { SPECIFIC-CP } 4 \mathrm{~ms}, \\
\text { PDSD } 50 \mathrm{~ms}\end{array}$ & Gel phase & $9.375 \mathrm{kHz}$ & $600 \mathrm{MHz}$ \\
\hline \multirow[t]{3}{*}{ [U]KcsA-Kv1.3 } & $\mathrm{CC}$ & PDSD 20, $150 \mathrm{~ms}$ & $\begin{array}{l}\text { Liquid crystalline } \\
\text { / gel phase }\end{array}$ & $12.5 \mathrm{kHz}$ & $800 \mathrm{MHz}$ \\
\hline & NCACB & $\begin{array}{c}\text { SPECIFIC-CP } 3 \mathrm{~ms}, \\
\text { DARR } 8 \mathrm{~ms}\end{array}$ & Liquid crystalline & $12.5 \mathrm{kHz}$ & $800 \mathrm{MHz}$ \\
\hline & $\mathrm{NCOCA}$ & $\begin{array}{l}\text { SPECIFIC-CP } 1.5 \\
\text { ms, DARR } 20 \mathrm{~ms}\end{array}$ & Liquid crystalline & $12.5 \mathrm{kHz}$ & $800 \mathrm{MHz}$ \\
\hline \multirow[t]{4}{*}[\mathrm{T}]{$\mathrm{KcsA}-\mathrm{Kv} 1.3$} & $\mathrm{CC}$ & PDSD 20, $150 \mathrm{~ms}$ & $\begin{array}{l}\text { Liquid crystalline } \\
\text { / gel phase }\end{array}$ & $12.5 \mathrm{kHz}$ & $800 \mathrm{MHz}$ \\
\hline & $\mathrm{NCA}$ & $\begin{array}{l}\text { SPECIFIC-CP } \\
4.5 \mathrm{~ms}\end{array}$ & Gel phase & $12.5 \mathrm{kHz}$ & $800 \mathrm{MHz}$ \\
\hline & NCACB & $\begin{array}{c}\text { SPECIFIC-CP } 2 \mathrm{~ms}, \\
\text { DARR } 15 \mathrm{~ms}\end{array}$ & Gel phase & $12.5 \mathrm{kHz}$ & $800 \mathrm{MHz}$ \\
\hline & $\begin{array}{l}\text { NCOCA } \\
2 \mathrm{D}, 3 \mathrm{D}\end{array}$ & $\begin{array}{l}\text { SPECIFIC-CP } 3.5 \\
\text { ms, DARR } 25 \mathrm{~ms}\end{array}$ & Gel phase & $12.5 \mathrm{kHz}$ & $800 \mathrm{MHz}$ \\
\hline
\end{tabular}

Table C.3: List of KcsA-Kv1.3 spectra used for the assignment. 


\begin{tabular}{cccccccc}
\hline Residue & $\mathrm{N}$ & $\mathrm{CO}$ & $\mathrm{CA}$ & $\mathrm{CB}$ & $\mathrm{CG}(1)$ & $\mathrm{CG} 2$ & $\mathrm{CD}$ \\
\hline V76 & -0.4 & -0.1 & 0.3 & 0.0 & 0.1 & -0.2 & \\
G77 & -2.6 & 0.0 & 0.3 & & & & \\
Y78 & -8.0 & -2.1 & 0.3 & & & & \\
G79 & -6.5 & -1.1 & -0.9 & & & & \\
D80 & 1.3 & & & & & & \\
P83 & & & -1.4 & 3.3 & -2.8 & & -1.0 \\
V84 & -2.2 & & 0.6 & 0.4 & 0.1 & 0.9 & \\
T85 & 6.7 & 1.2 & 2.9 & & & -0.1 & \\
L86 & -0.9 & & 0.5 & -0.8 & & & \\
W87 & -5.1 & -0.1 & -0.5 & -1.2 & & & \\
G88 & 0.0 & -0.2 & -1.1 & & & & \\
R89 & -4.0 & 0.2 & 4.6 & -3.4 & & & 0.6 \\
\hline
\end{tabular}

Table C.4: SsNMR chemical shift differences between KcsA-Kv1.3 in liposomes and KcsA precipitated from detergent micelles. The table shows the results of subtracting assignments reported in ref. [250] from assignments on KcsA-Kv1.3 discussed here, where values are available in both datasets. No further corrections or recalibrations were applied.

\section{C.4 Supplementary Methods}

The simulated annealing protocol used to refine the full-length KcsA-Kv1.3 structural model generated by MODELLER [243] consisted of the following stages: 1) high-temperature annealing in torsion angle space in 1000 time steps of 0.015 ps at $10000 \mathrm{~K}, 2$ ) slow cooling in torsion angle space in 2000 steps of 0.015 ps, reducing the temperature from $10000 \mathrm{~K}$ to zero in steps of $125 \mathrm{~K}, 3$ ) an additional cooling stage in Cartesian space in 3000 steps of $0.005 \mathrm{ps}$, cooling from $2000 \mathrm{~K}$ to zero in steps of $25 \mathrm{~K}$, and 4) a final conjugate gradient minimization in 10 cycles of 100 steps each. The force constant for dihedral angle restraints was always set to $200 \mathrm{kcal} \mathrm{mol}^{-1} \mathrm{rad}^{-2}$. All atoms were kept fixed except those in residues 20-24, 51-61, and 125-130. Helical structure was enforced in residues $22-24,51-53$, and 129 by applying $\alpha$-helical dihedral angle restraints, and dihedral angles for residues 56-61 were kept close to their crystal structure values. 20 structures were calculated and one of those with lowest overall energy selected for further analysis. 


\section{Appendix D}

Gating and inactivation in KcsA-Kv1.3 (Supplement)

D.1 Solid-state NMR resonance assignments at $\mathrm{pH} 4.0$

\begin{tabular}{|c|c|c|c|c|c|c|c|c|c|c|}
\hline Residue & $\mathrm{N}$ & $\mathrm{CO}$ & $\mathrm{CA}$ & $\mathrm{CB}$ & $\mathrm{CG}(1)$ & CG2 & $\mathrm{CD}(1)$ & CD2 & $\mathrm{CE}(1)$ & $\mathrm{CZ}$ \\
\hline \multicolumn{11}{|l|}{ M1 } \\
\hline P2 & & & 63.80 & 31.90 & 27.40 & & 50.70 & & & \\
\hline \multicolumn{11}{|l|}{ P3 } \\
\hline M4 & & & 56.60 & 31.00 & & & & & & \\
\hline \multicolumn{11}{|l|}{ L5 } \\
\hline \multicolumn{11}{|l|}{ S6 } \\
\hline G7 & & & 46.40 & & & & & & & \\
\hline L8 & & & 58.00 & 42.30 & & & 23.60 & & & \\
\hline \multicolumn{11}{|l|}{ L9 } \\
\hline \multicolumn{11}{|l|}{ A10 } \\
\hline \multicolumn{11}{|l|}{ R11 } \\
\hline \multicolumn{11}{|l|}{ L12 } \\
\hline \multicolumn{11}{|l|}{ V13 } \\
\hline \multicolumn{11}{|l|}{ K14 } \\
\hline \multicolumn{11}{|l|}{ L15 } \\
\hline \multicolumn{11}{|l|}{ L16 } \\
\hline \multicolumn{11}{|l|}{ L17 } \\
\hline \multicolumn{11}{|l|}{ G18 } \\
\hline \multicolumn{11}{|l|}{ R19 } \\
\hline $\mathrm{H} 20$ & & & & 30.37 & & & & & & \\
\hline G21 & & 176.60 & 46.66 & & & & & & & \\
\hline S22 & & & 63.00 & & & & & & & \\
\hline A 23 & & & 55.00 & 18.50 & & & & & & \\
\hline \multicolumn{11}{|l|}{ L24 } \\
\hline \multicolumn{11}{|l|}{$\mathrm{H} 25$} \\
\hline \multicolumn{11}{|l|}{ W26 } \\
\hline $\mathrm{R} 27$ & & & & 30.40 & & & 44.26 & & & \\
\hline \multicolumn{11}{|l|}{ A 28} \\
\hline \multicolumn{11}{|l|}{ A29 } \\
\hline G30 & & & & & & & & & & \\
\hline
\end{tabular}




\begin{tabular}{|c|c|c|c|c|c|c|c|c|c|c|}
\hline Residue & $\mathrm{N}$ & $\mathrm{CO}$ & $\mathrm{CA}$ & $\mathrm{CB}$ & CG(1) & CG2 & $\mathrm{CD}(1)$ & CD2 & $\mathrm{CE}(1)$ & $\mathrm{CZ}$ \\
\hline A31 & & & & & & & & & & \\
\hline A 32 & & & & & & & & & & \\
\hline T33 & & & & & & & & & & \\
\hline V34 & & & & & & & & & & \\
\hline L35 & & & & & & & & & & \\
\hline L36 & & & & & & & & & & \\
\hline V37 & & & & & & & & & & \\
\hline I38 & 118.16 & & 66.47 & 37.80 & 29.73 & 17.65 & 14.34 & & & \\
\hline V39 & & & & & & & & & & \\
\hline $\mathrm{L} 40$ & & & & & & & & & & \\
\hline L41 & & & & & & & & & & \\
\hline A 42 & & & 56.40 & 18.58 & & & & & & \\
\hline G43 & & & 47.10 & & & & & & & \\
\hline $\mathrm{S} 44$ & & & 62.40 & 63.60 & & & & & & \\
\hline Y45 & & & & & & & & & & \\
\hline $\mathrm{L} 46$ & & & 58.00 & 42.30 & & & 23.20 & & & \\
\hline A47 & & & & & & & & & & \\
\hline V48 & & & & & & & & & & \\
\hline L49 & & & & & & & & & & \\
\hline A50 & & & & & & & & & & \\
\hline E51 & & & & 31.17 & & & & & & \\
\hline A 52 & & & & 18.59 & & & & & & \\
\hline D53 & & & & & & & & & & \\
\hline D54 & & & & & & & & & & \\
\hline P55 & & & 61.67 & 32.72 & 27.99 & & 49.61 & & & \\
\hline T56 & & & 58.30 & 67.40 & & & & & & \\
\hline $\mathrm{S} 57$ & & & 59.62 & 63.80 & & & & & & \\
\hline G58 & & & 46.49 & & & & & & & \\
\hline F59 & & & 57.88 & 40.00 & & & & & & \\
\hline S60 & & & 59.60 & 63.50 & & & & & & \\
\hline S61 & & & 56.40 & 65.60 & & & & & & \\
\hline I62 & & & 65.58 & 35.10 & 29.46 & 17.79 & 12.40 & & & \\
\hline P63 & & & 66.82 & 31.48 & 29.33 & & 49.75 & & & \\
\hline D64 & & & & & & & & & & \\
\hline A65 & & & 56.18 & 20.07 & & & & & & \\
\hline L66 & & & & & & & & & & \\
\hline W67 & & & & & & & & & & \\
\hline W68 & & & & & & & & & & \\
\hline S69 & & & 62.60 & & & & & & & \\
\hline V70 & & & 66.10 & 31.60 & & & & & & \\
\hline E71 & & & 59.14 & 30.09 & 32.12 & & 180.35 & & & \\
\hline $\mathrm{T} 72$ & & & 67.45 & 68.62 & & 21.68 & & & & \\
\hline A73 & & & 55.01 & 18.22 & & & & & & \\
\hline $\mathrm{T} 74$ & 98.90 & 177.59 & 61.88 & 70.52 & & 20.23 & & & & \\
\hline $\mathrm{T} 75$ & 113.98 & 173.44 & 62.65 & 69.09 & & 22.67 & & & & \\
\hline V76 & 117.23 & & 65.19 & 30.25 & 22.21 & 20.01 & & & & \\
\hline G77 & 101.54 & & 47.29 & & & & & & & \\
\hline
\end{tabular}




\begin{tabular}{|c|c|c|c|c|c|c|c|c|c|c|}
\hline Residue & $\mathrm{N}$ & $\mathrm{CO}$ & $\mathrm{CA}$ & CB & $\mathrm{CG}(1)$ & CG2 & $\mathrm{CD}(1)$ & CD2 & $\mathrm{CE}(1)$ & $\mathrm{CZ}$ \\
\hline Y78 & & & 62.33 & 38.79 & & & & & & \\
\hline G79 & & & 47.35 & & & & & & & \\
\hline D80 & & & 55.09 & 37.31 & 179.70 & & & & & \\
\hline L81 & & & 54.65 & 46.13 & 27.28 & & & & & \\
\hline \multicolumn{11}{|l|}{ Y82 } \\
\hline P83 & & & 65.22 & 34.58 & 27.00 & & & & & \\
\hline V84 & & & 60.84 & 33.34 & & & & & & \\
\hline T85 & & & 60.90 & 72.00 & & & & & & \\
\hline L86 & & & 59.07 & 41.24 & & & & & & \\
\hline W87 & & & 59.47 & 29.24 & & & & & & \\
\hline G88 & & & 46.53 & & & & & & & \\
\hline R89 & & & 59.62 & 30.24 & & & 44.06 & & & \\
\hline \multicolumn{11}{|l|}{ C90 } \\
\hline \multicolumn{11}{|l|}{ V91 } \\
\hline \multicolumn{11}{|l|}{ A92 } \\
\hline \multicolumn{11}{|l|}{ V93 } \\
\hline \multicolumn{11}{|l|}{ V94 } \\
\hline \multicolumn{11}{|l|}{ V95 } \\
\hline \multicolumn{11}{|l|}{ M96 } \\
\hline \multicolumn{11}{|l|}{ V97 } \\
\hline A98 & & & 54.20 & 19.96 & & & & & & \\
\hline G99 & 111.53 & & 46.73 & & & & & & & \\
\hline I100 & 117.72 & 180.84 & 66.28 & 38.00 & 30.76 & 17.12 & 14.24 & & & \\
\hline $\mathrm{T} 101$ & 109.81 & 177.52 & 65.70 & 68.59 & & 22.48 & & & & \\
\hline S102 & & & 62.29 & 63.59 & & & & & & \\
\hline \multicolumn{11}{|l|}{ F103 } \\
\hline \multicolumn{11}{|l|}{ G104 } \\
\hline L105 & & & 58.94 & 42.73 & & & & & & \\
\hline \multicolumn{11}{|l|}{ V106 } \\
\hline T107 & & & 67.42 & 68.71 & & & & & & \\
\hline A108 & & & 55.16 & & & & & & & \\
\hline A109 & & & 54.74 & 18.53 & & & & & & \\
\hline \multicolumn{11}{|l|}{ L110 } \\
\hline A111 & & & 55.69 & & & & & & & \\
\hline $\mathrm{T} 112$ & & & 67.05 & 68.55 & & & & & & \\
\hline \multicolumn{11}{|l|}{ W113 } \\
\hline \multicolumn{11}{|l|}{ F114 } \\
\hline V115 & & & 67.37 & & & & & & & \\
\hline G116 & & & 46.89 & & & & & & & \\
\hline \multicolumn{11}{|l|}{$\mathrm{R} 117$} \\
\hline \multicolumn{11}{|l|}{ E118 } \\
\hline \multicolumn{11}{|l|}{ Q119 } \\
\hline \multicolumn{11}{|l|}{ E120 } \\
\hline \multicolumn{11}{|l|}{$\mathrm{R} 121$} \\
\hline $\mathrm{R} 122$ & & & 57.01 & 30.47 & & & & & & \\
\hline G123 & & & & & & & & & & \\
\hline H124 & & & & & & & & & & \\
\hline
\end{tabular}




\begin{tabular}{|c|c|c|c|c|c|c|c|c|c|c|}
\hline Residue & $\mathrm{N}$ & $\mathrm{CO}$ & $\mathrm{CA}$ & $\mathrm{CB}$ & CG(1) & CG2 & $\mathrm{CD}(1)$ & CD2 & $\mathrm{CE}(1)$ & $\mathrm{CZ}$ \\
\hline F125 & & & & & & & & & & \\
\hline V126 & & & & & & & & & & \\
\hline $\mathrm{R} 127$ & & & & & & & & & & \\
\hline H128 & & & & & & & & & & \\
\hline S129 & & & 62.64 & 63.84 & & & & & & \\
\hline E130 & & & 59.28 & 29.46 & 34.81 & & & & & \\
\hline K131 & & & 59.21 & 32.44 & 25.67 & & & & & \\
\hline A132 & & & & & & & & & & \\
\hline A133 & & & & & & & & & & \\
\hline E134 & & & & & & & & & & \\
\hline E135 & & & & & & & & & & \\
\hline A136 & & & & & & & & & & \\
\hline Y137 & & & 61.38 & 39.24 & & & & & & \\
\hline $\mathrm{T} 138$ & & & 67.11 & 68.75 & & & & & & \\
\hline R139 & & & & & & & & & & \\
\hline T140 & & & & & & & & & & \\
\hline T141 & & & & & & & & & & \\
\hline R142 & & & & & & & & & & \\
\hline A143 & & & & & & & & & & \\
\hline L144 & & & & & & & & & & \\
\hline H145 & & & & & & & & & & \\
\hline E146 & & & & & & & & & & \\
\hline $\mathrm{R} 147$ & & & & & & & & & & \\
\hline F148 & & & & & & & & & & \\
\hline D149 & & & & & & & & & & \\
\hline $\mathrm{R} 150$ & & & & & & & & & & \\
\hline L151 & & & & & & & & & & \\
\hline E152 & & & & & & & & & & \\
\hline $\mathrm{R} 153$ & & & & & & & & & & \\
\hline M154 & & & & & & & & & & \\
\hline L155 & & & & & & & & & & \\
\hline D156 & & & & & & & & & & \\
\hline D157 & & & & & & & & & & \\
\hline N158 & & & & & & & & & & \\
\hline R159 & & & & & & & & & & \\
\hline R160 & & & & & & & & & & \\
\hline
\end{tabular}

Table D.1: Solid-state NMR resonance assignments for KcsA-Kv1.3 in liposomes at pH 4.0. 


\section{D.2 Supplementary data}
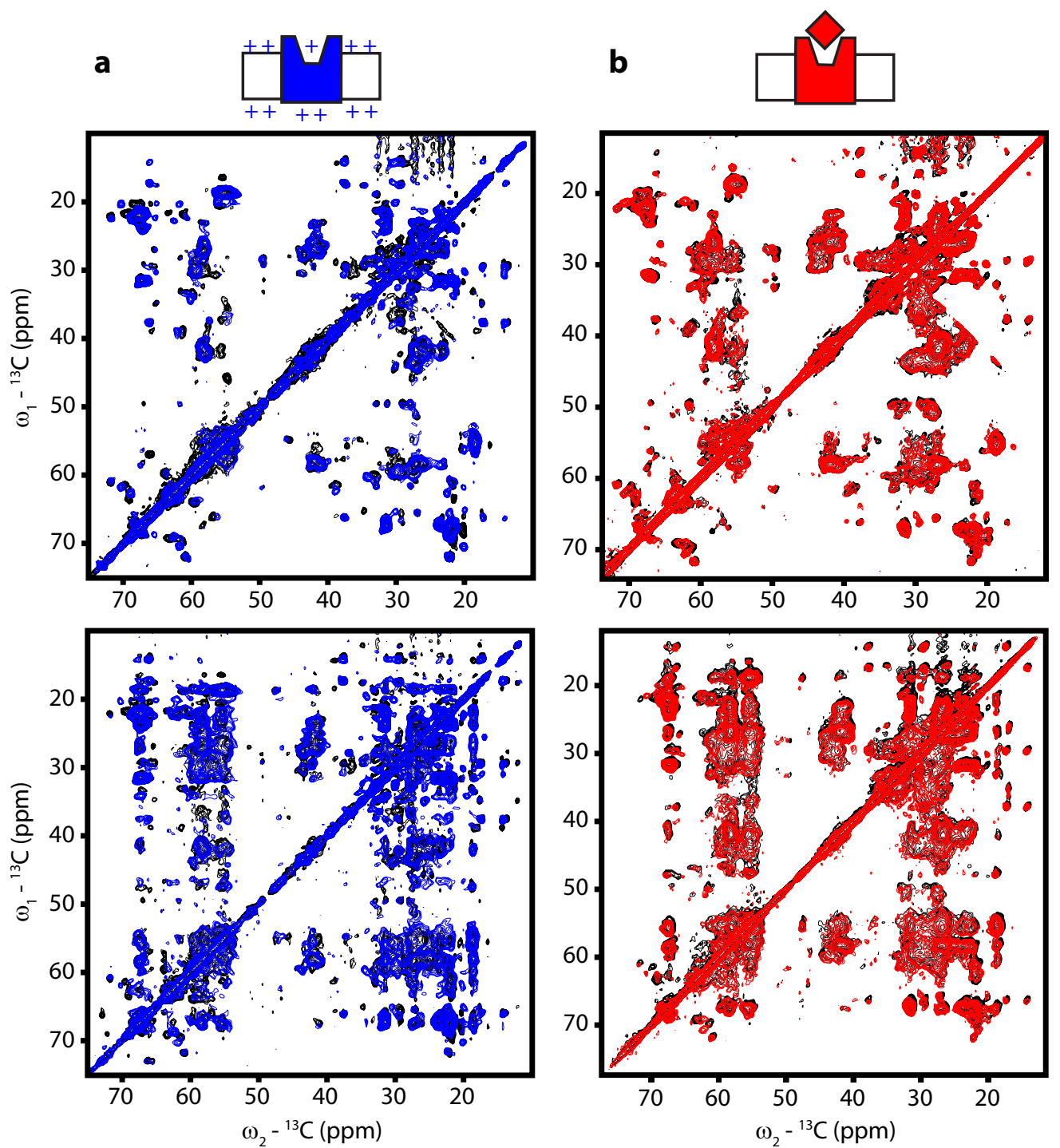

Figure D.1: $\left({ }^{13} \mathrm{C},{ }^{13} \mathrm{C}\right)$ ssNMR spectra of KcsA-Kv1.3 at pH 4.0 (blue, a) and complexed with porphyrin \#3 (red, b). In both cases, spectra are superimposed on corresponding spectra of free KcsA-Kv1.3 at pH 7.5 (black). Top row: PDSD-WC [125] intraresidue correlation spectra (20 ms mixing, $800 \mathrm{MHz}{ }^{1} \mathrm{H}$ Larmor frequency, sample temperature $\left.+10^{\circ} \mathrm{C}, 12.5 \mathrm{kHz} \mathrm{MAS}\right)$. Bottom row: as top row, but with $150 \mathrm{~ms}$ PDSD-WC mixing to obtain sequential correlations. 


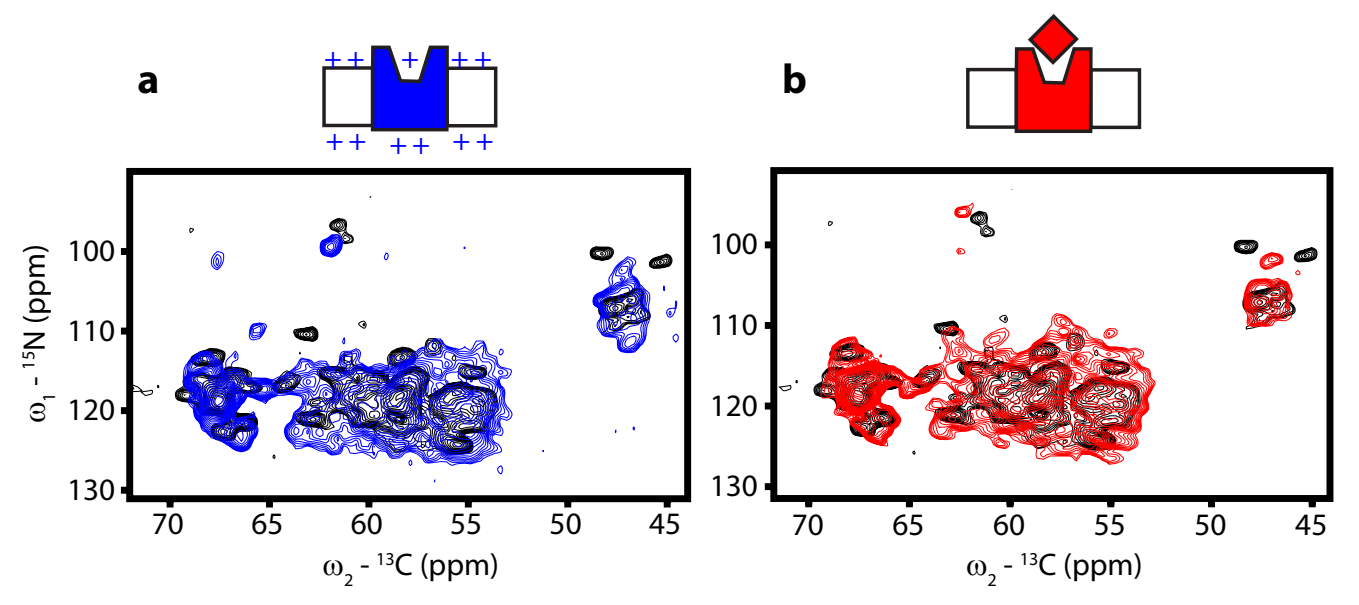

Figure D.2: N-CA ssNMR spectra of KcsA-Kv1.3 at pH 4.0 (blue, a) and complexed with porphyrin \#3 (red, b). In both cases, spectra are superimposed on corresponding spectra of free KcsAKv1.3 at pH 7.5 (black). SPECIFIC-CP $[123,124]$ was used for $\left({ }^{15} \mathrm{~N},{ }^{13} \mathrm{C}\right)$ polarization transfer. Spectra for free KcsA-Kv1.3 at pH 7.5 (black) and KcsA-Kv1.3 with bound porphyrin (red) were recorded at $600 \mathrm{MHz}{ }^{1} \mathrm{H}$ Larmor frequency, $-10^{\circ} \mathrm{C}$ sample temperature and $9.375 \mathrm{kHz}$ MAS, while the spectrum for KcsA-Kv1.3 at pH 4.0 (blue) was recorded at $800 \mathrm{MHz}{ }^{1} \mathrm{H}$ Larmor frequency, $-10^{\circ} \mathrm{C}$ sample temperature and $12.5 \mathrm{kHz}$ MAS. 
a
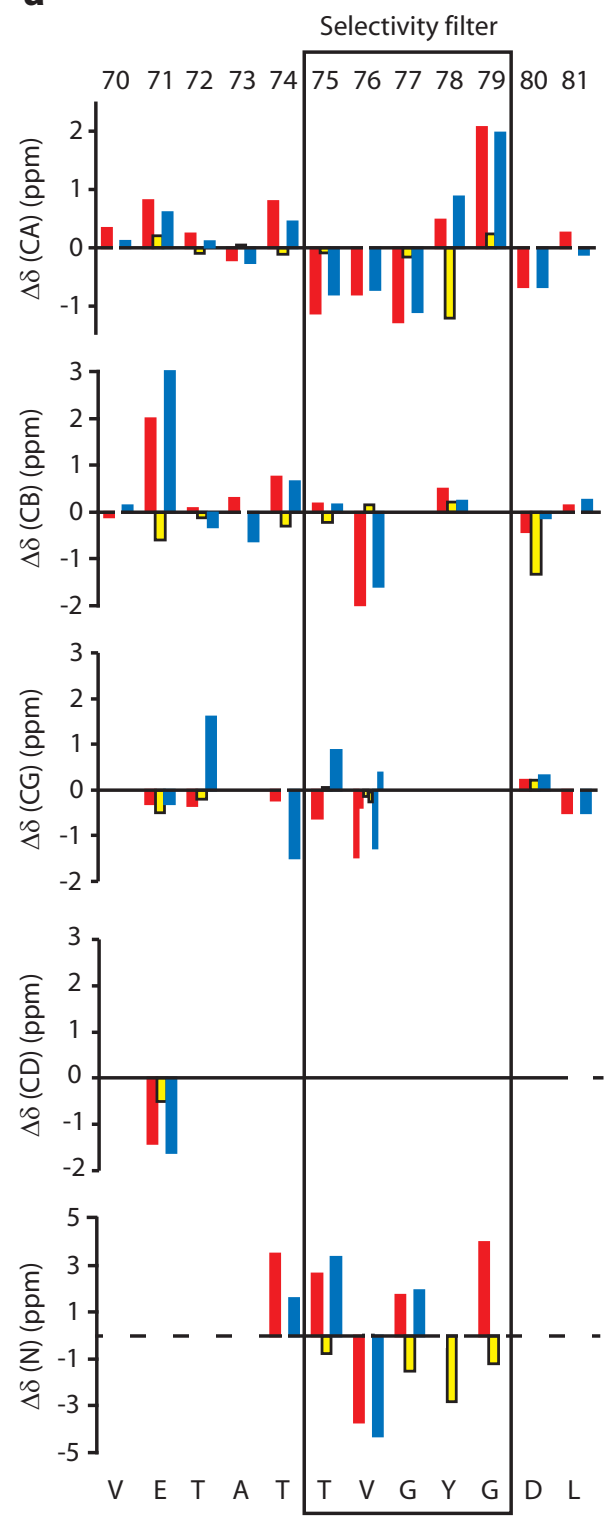

b
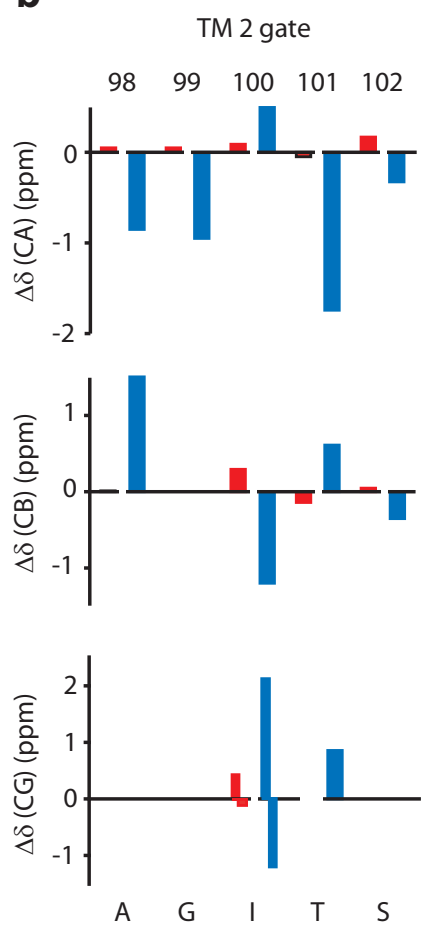

KcsA-Kv1.3 + porphyrin

$\square \mathrm{KcsA}-\mathrm{Kv} 1.3+\mathrm{KTX}$

KcsA-Kv1.3 at pH4.0

Figure D.3: Chemical shift changes observed for residues in the selectivity filter (a) and the TM 2 gate (b) under different experimental conditions. Shown are data recorded at pH 4.0 (blue), with bound porphyrin (red), and with bound KTX [62] (yellow) relative to free KcsA-Kv1.3 at pH 7.5. The selectivity filter is emphasized by a grey frame. Chemical shift changes for $\mathrm{C}^{\alpha}, \mathrm{C}^{\beta}, \mathrm{C}^{\gamma}$, and $\mathrm{C}^{\delta}{ }^{13} \mathrm{C}$ resonances and backbone amide ${ }^{15} \mathrm{~N}$ resonances $(\Delta \delta(\mathrm{CA}), \Delta \delta(\mathrm{CB}), \Delta \delta(\mathrm{CG}), \Delta \delta(\mathrm{CD})$, and $\Delta \delta(\mathrm{N}))$ are in ppm. 
a

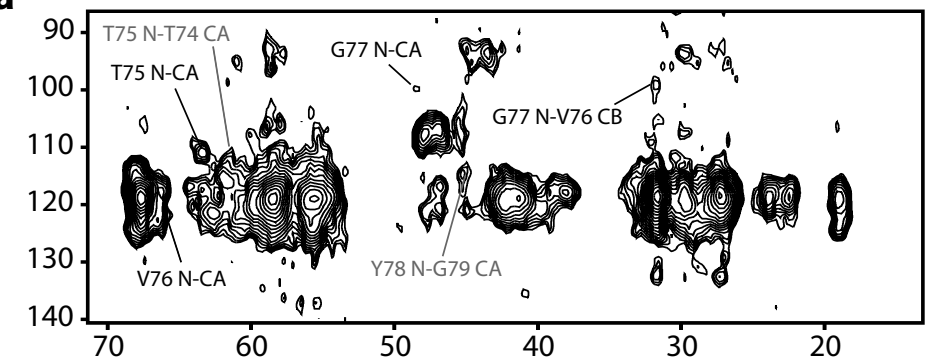

b

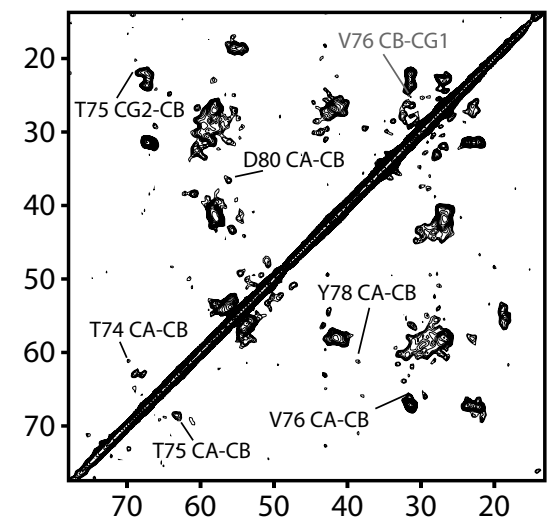

c

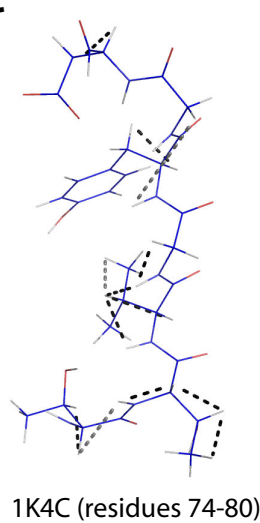

Figure D.4: (a) NHHC (100 $\mu$ s proton mixing) and (b) CHHC (50 $\mu$ s proton mixing) spectra [136] for the KcsA-Kv1.3 channel at pH 7.5. Signals corresponding to residues Thr74 to Asp80 are labeled (unambiguous: black, ambiguous: grey), and corresponding distances are indicated by dashed lines in the selectivity filter of the KcsA crystal structure (PDB ID 1K4C) (c). Measured distance constraints are compatible with a structure of the selectivity filter as observed in the crystal structure of conductive KcsA obtained at high potassium concentration [24]. 

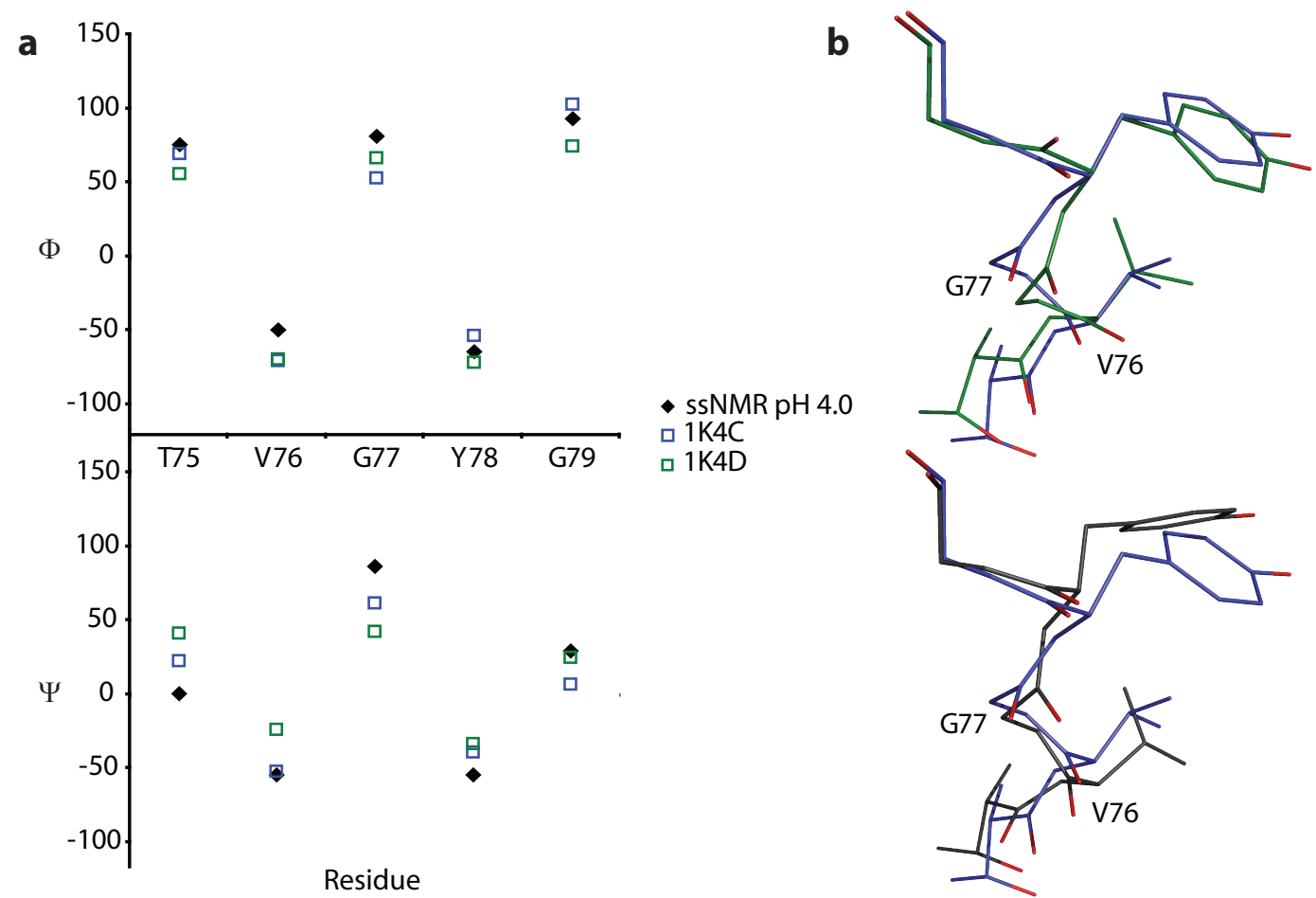

Figure D.5: Structural model of the selectivity filter of KcsA-Kv1.3 at pH 4.0 derived from ssNMR data. (a) Dihedral angles obtained for selectivity filter residues 75 to 79 from ssNMR data measured at $\mathrm{pH} 4.0$ by chemical shift analysis and simulated annealing as described in Section 8.3.3. Resulting angles are shown as black diamonds. Values for the conductive (PDB ID 1K4C) and collapsed (1K4D) KcsA crystal structures [24] are given for comparison. (b) Selectivity filter residues 75 to 79 seen from the center of the ion conduction pore. On top, the collapsed crystal structure (1K4D, green) has been superimposed on the conductive crystal structure ( $1 \mathrm{~K} 4 \mathrm{C}$, blue); at bottom, a structural model with the dihedral angles derived from ssNMR data at $\mathrm{pH} 4.0$ (dark grey) is seen together with $1 \mathrm{~K} 4 \mathrm{C}$ (blue). Alignment was done along the $\mathrm{C}^{\alpha}$ atoms of residues 75 to 79 . This figure indicates that neither $1 \mathrm{~K} 4 \mathrm{C}$ nor $1 \mathrm{~K} 4 \mathrm{D}$ fully explain ssNMR findings at $\mathrm{pH}$ 4.0. However, as discussed in Chapter 8, dihedral angles that fit best to experimental data lead to a structural model of the selectivity filter which is characterized by an out-of-line movement of backbone carbonyls. This arrangement is comparable to the conformation seen in collapsed filter structures. 


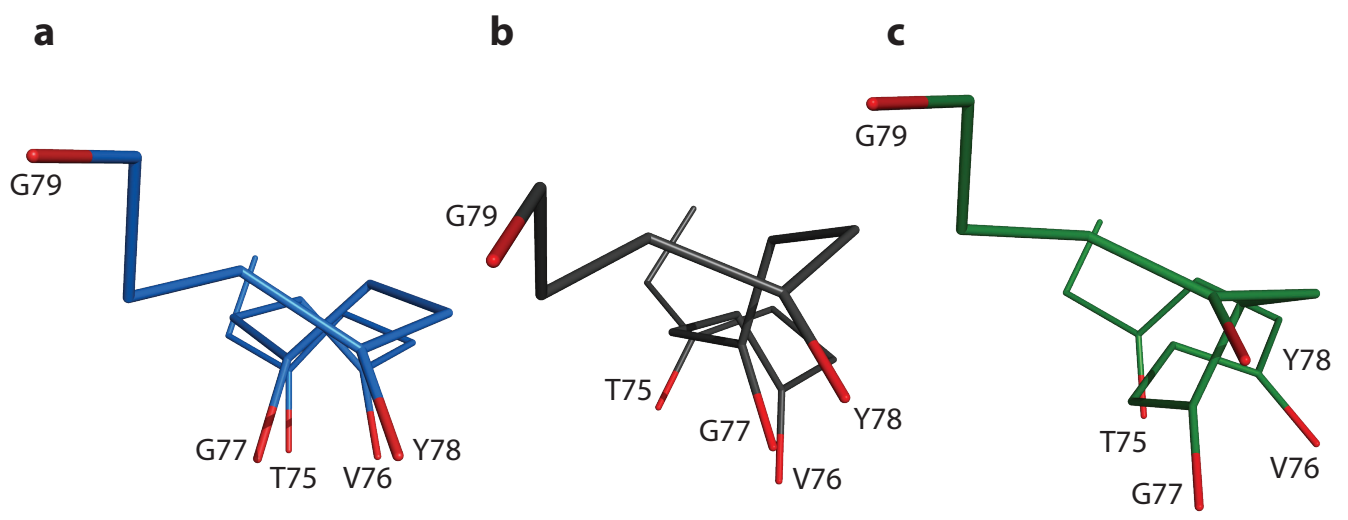

Figure D.6: View on the selectivity filter backbone of one channel subunit from the extracellular side, down the ion conduction pore. (a) shows the conductive conformation of the selectivity filter as seen in KcsA at high potassium concentrations [24] (PDB ID 1K4C), with an in-line arrangement of carbonyl moieties. (b) displays the model of the selectivity filter of KcsA-Kv1.3 at pH 4.0, with dihedral angles determined as described in Section 8.3.3 (Table D.2). (c) shows the collapsed $\mathrm{KcsA}$ selectivity filter conformation seen in low potassium concentrations [24] (1K4D). Both (b) and (c) exhibit a comparable out-of-line carbonyl arrangement, likely interfering with ion complexation and conduction.

\begin{tabular}{c|cc|cc|cc|cc|cc|} 
Residue & \multicolumn{2}{|c|}{ Thr75 } & \multicolumn{2}{|c|}{ Val76 } & \multicolumn{2}{|c|}{ Gly77 } & \multicolumn{2}{|c|}{ Tyr78 } & \multicolumn{2}{|c}{ Gly79 } \\
Angle & $\phi$ & $\psi$ & $\phi$ & $\psi$ & $\phi$ & $\psi$ & $\phi$ & $\psi$ & $\phi$ & $\psi$ \\
\hline 1K4C & 69 & 22 & -71 & -52 & 53 & 61 & -54 & -40 & 102 & 6 \\
1K4D & 55 & 41 & -70 & -25 & 66 & 42 & -72 & -34 & 74 & 24 \\
\hline pH4, ssNMR & 75 & 0 & -50 & -55 & 81 & 86 & -65 & -55 & 93 & 29 \\
Residue & \multicolumn{2}{|c|}{ Ala98 } & \multicolumn{2}{|c|}{ Gly99 } & Ile100 & \multicolumn{2}{|c}{ Thr101 } \\
Angle & $\phi$ & $\psi$ & $\phi$ & $\psi$ & $\phi$ & $\psi$ & $\phi$ & $\psi$ \\
\hline 1K4C & -66 & -42 & -66 & -50 & -59 & -48 & -60 & -43 \\
pH4, ssNMR & -100 & -40 & -110 & -50 & -50 & -70 & -90 & -25
\end{tabular}

Table D.2: Comparison of dihedral angles in the selectivity filter and the TM 2 gating hinge. The upper table lists dihedral angles (in degrees) of selectivity filter residues 75 to 79 of the conductive (PDB ID 1K4C) and collapsed (1K4D) KcsA crystal structures [24] and dihedral angles determined from ssNMR data on KcsA-Kv1.3 at pH 4.0 by chemical shift analysis and simulated annealing (Section 8.3.3). The lower table shows dihedral angles (in degrees) of residues 98 to 101 in the TM2 gating region of the closed KcsA crystal structure (PDB ID 1K4C) and as determined from ssNMR data for KcsA-Kv1.3 at pH 4.0. 


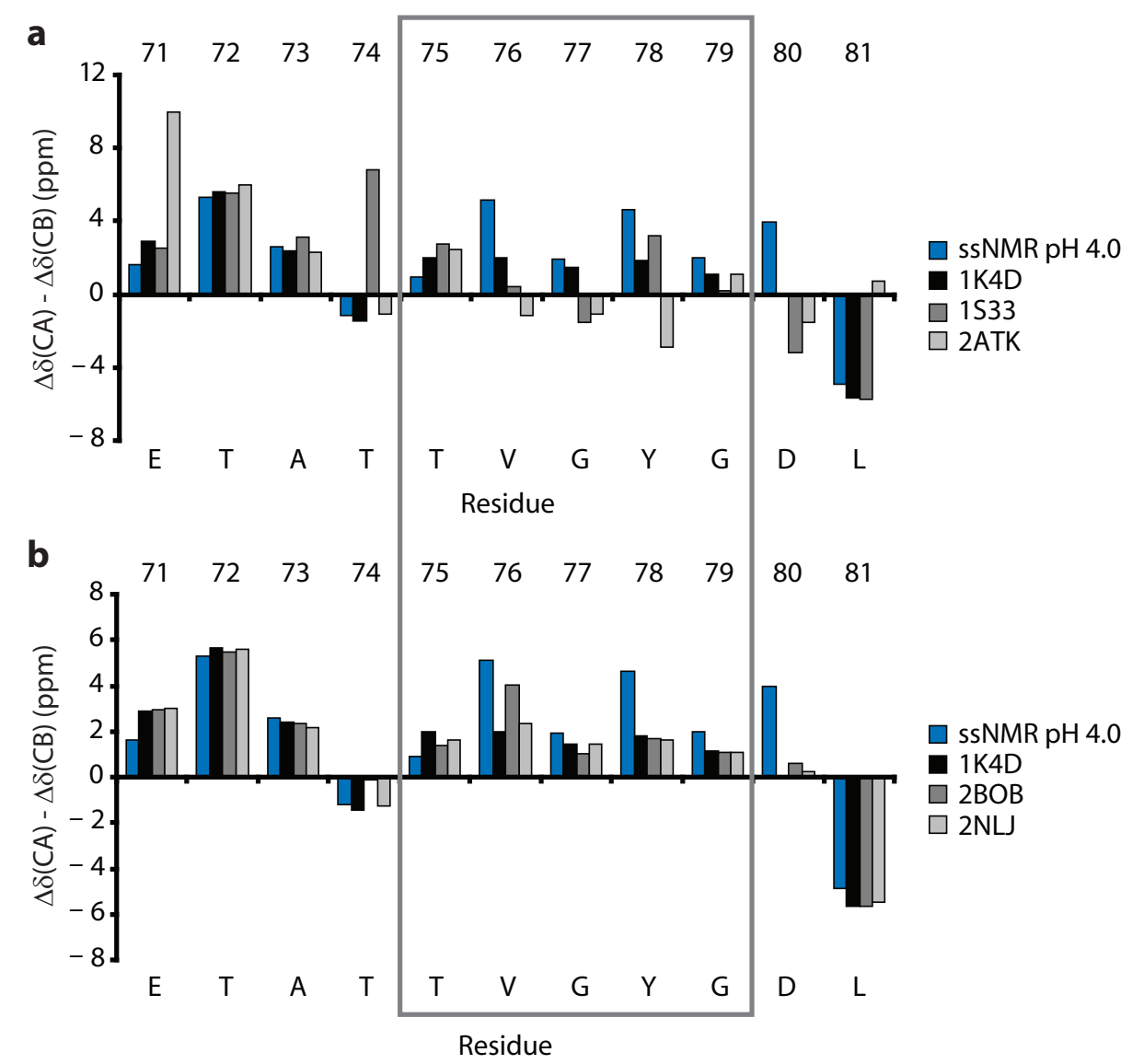

Figure D.7: Comparison of experimental secondary chemical shifts $(\Delta \delta(\mathrm{CA})-\Delta \delta(\mathrm{CB}))$ in the selectivity filter region at pH 4.0 (blue) with SHIFTX [163] predictions for secondary chemical shifts of various KcsA structures or models. Both plots show predictions for the crystal structure obtained with a low concentration of potassium [24] (black, PDB ID 1K4D). The selectivity filter is marked by a grey rectangle. (a) Comparison with secondary chemical shift predictions for a MD model of KcsA with Val76C' flipped [261] (dark grey, 1S33), and for the crystal structure of the E71A mutant with both Val76C' and Tyr78C' flipped [235] (light grey, 2ATK). (b) Comparison with secondary chemical shift predictions for crystal structures of KcsA crystallized with intracellular tetrabutylammonium (TBA) [268] (dark grey, 2BOB) and of the KcsA mutant M96V [239] (light grey, 2NLJ). Both of these structures display a high degree of similarity in the selectivity filter to the KcsA structure obtained in low potassium concentration (1K4D) in terms of dihedral angles and backbone RMSD (residues 74 to $80: 2 B O B$ vs. $1 \mathrm{~K} 4 \mathrm{D}: 0.16 \AA$, 2NLJ vs. $1 \mathrm{~K} 4 \mathrm{D}: 0.09 \AA$ ). 

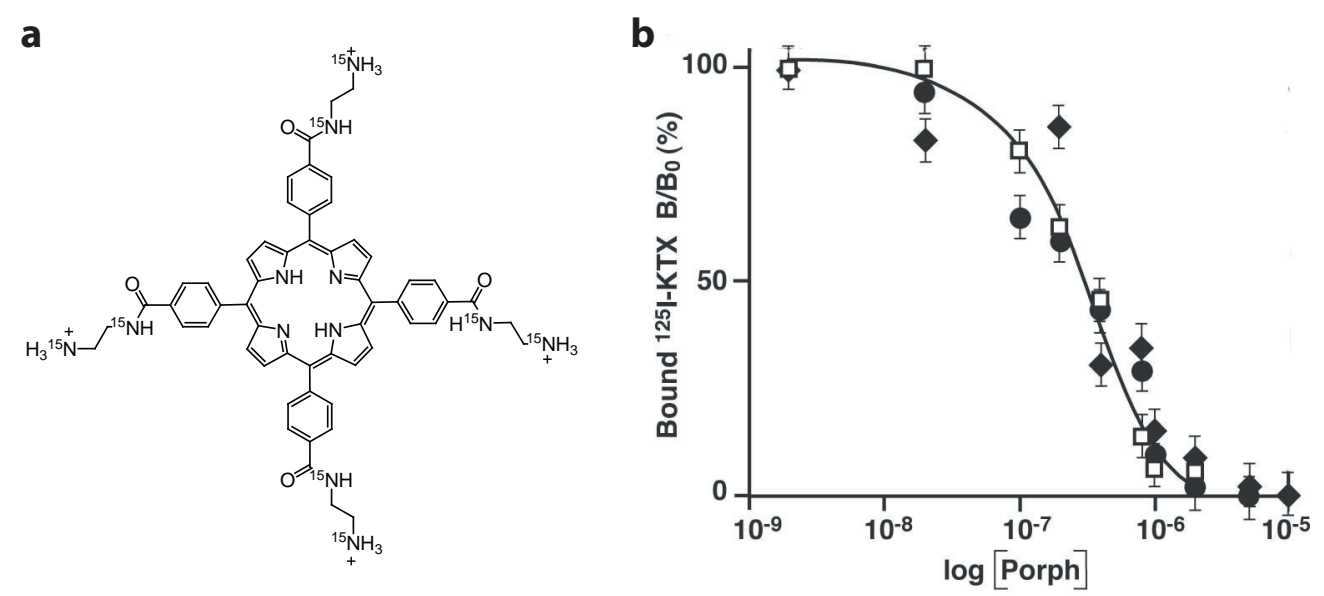

Figure D.8: (a) Structure of porphyrin \#3. (b) Binding of ${ }^{125} \mathrm{I}-\mathrm{KTX}$ to purified KcsA-Kv1.3 (open squares), KcsA-Kv1.3 G58A (filled circles) or KcsA-Kv1.3 G58V (filled diamonds) in the absence $\left(B_{0}\right)$ or presence $(B)$ of porphyrin \#3 (Porph) [265]. Error bars indicate s.e.m.

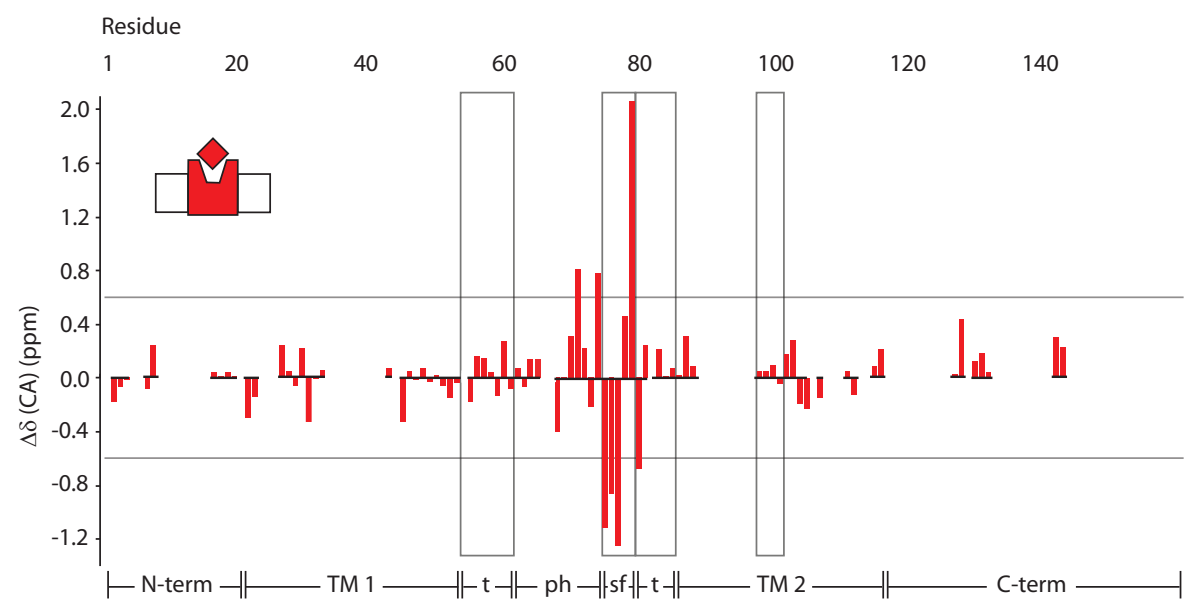

Figure D.9: Summary of $\mathrm{C}^{\alpha}$ chemical shift changes observed for KcsA-Kv1.3 with bound porphyrin compared to the free state at $\mathrm{pH} 7.5$. Horizontal lines at $\pm 0.6 \mathrm{ppm}$ indicate the threshold chosen for substantial chemical shift changes. Lines below ( $\mathrm{N}$-term, $\mathrm{N}$ terminus; TM1, transmembrane helix 1; t, extracellular turret; ph, pore helix; sf, selectivity filter; TM2, transmembrane helix 2; C-term, C terminus) schematically show secondary structure along the sequence as seen in ssNMR. Rectangular frames highlight the selectivity filter, TM2 gating hinge and turrets. 
a

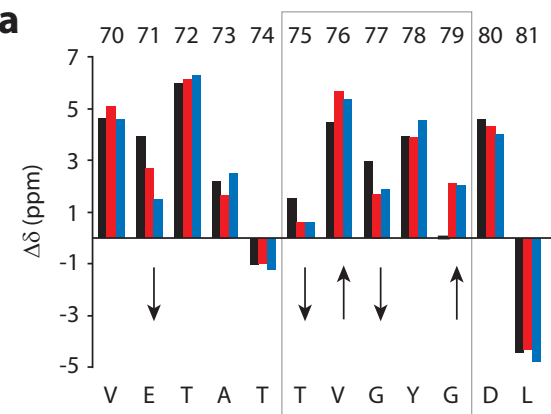

b

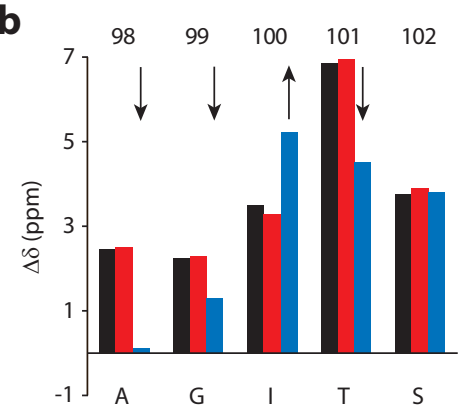

Figure D.10: Experimental secondary chemical shifts (in ppm) for KcsA-Kv1.3 at pH 7.5 (black), $\mathrm{pH} 4.0$ (blue), and in the porphyrin-bound state (red) are given for residues Val70 to Leu81 (a) and residues Ala98 to Ser102 (b). The black frame in (a) indicates the selectivity filter. Arrows show directions of changes in secondary chemical shift.

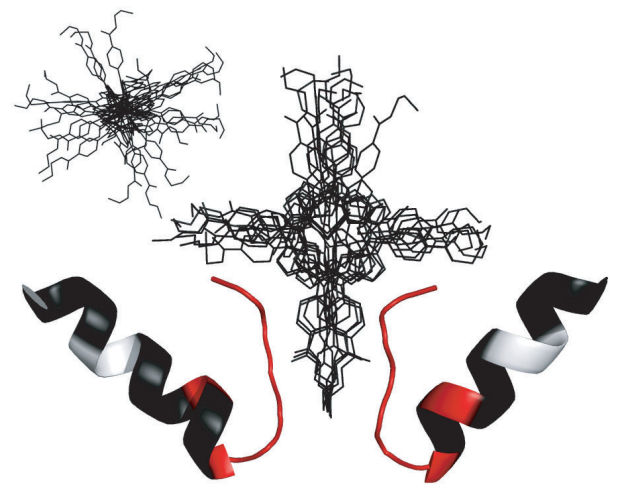

Figure D.11: Model of the KcsA-Kv1.3-porphyrin complex obtained by HADDOCK docking [343] as discussed in ref. [1]. An overlay of the ten lowest-energy structures is shown together with selectivity filter and pore helix region of two KcsA-Kv1.3 subunits. Residues for which substantial chemical shift changes upon binding were observed are colored red; black signifies no chemical shift changes above the threshold; and grey indicates unassigned residues. The inset on the upper left displays the ten bound porphyrin structures alone from a different viewpoint to illustrate the rotational freedom of porphyrin binding. 


\section{Appendix E}

Effects of potassium on activation and inactivation gating in KcsA-Kv1.3 (Supplement) 
Full-length KcsA-Kv1.3 (residues 1-160)

$\mathrm{pH} 7$

$150 \mathrm{mM} \mathrm{K}^{+}$

MOPS buffer

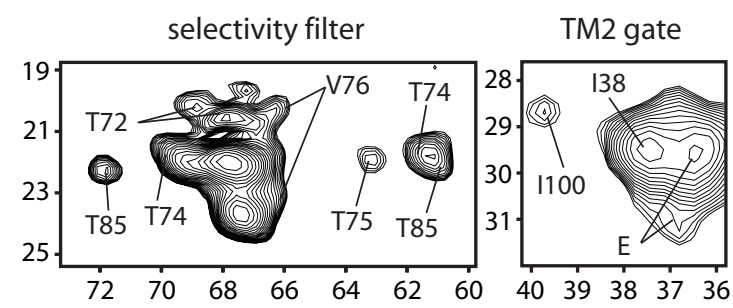

$\mathrm{pH} 4$

$150 \mathrm{mM} \mathrm{K}^{+}$

MOPS buffer
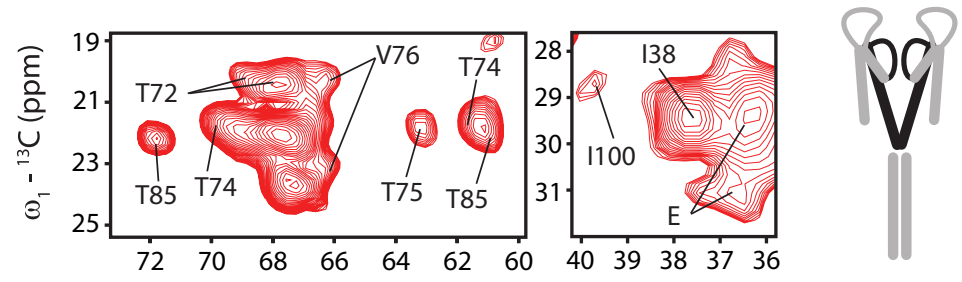

$\mathrm{pH} 4$

$10 \mathrm{mM} \mathrm{K}^{+}$

citrate buffer

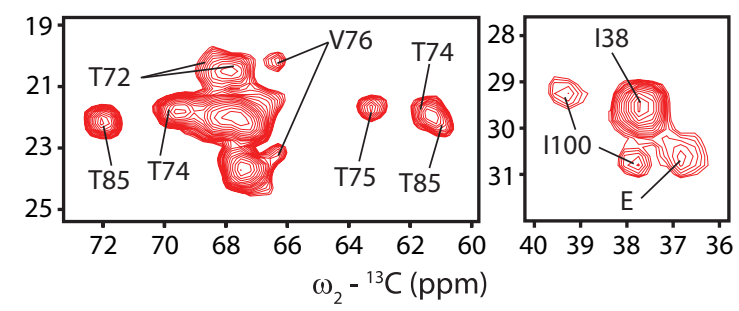

C-terminally truncated KcsA-Kv1.3 (residues 1-125)

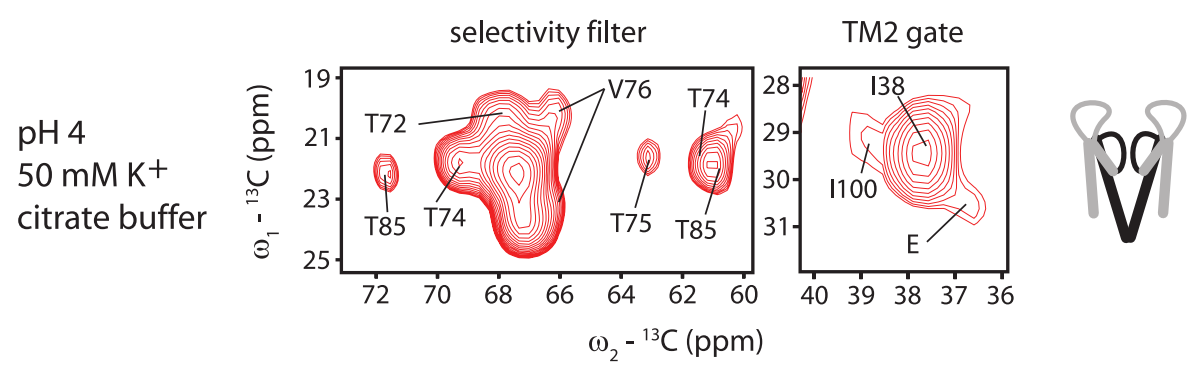

Figure E.1: Spectral cut-outs from selectivity filter and TM2 gating hinge regions of full length and C-terminally truncated KcsA-Kv1.3 in asolectin liposomes at different buffer, $\mathrm{pH}$ and $\left[\mathrm{K}^{+}\right]$conditions as indicated in labels on the left. In all conditions shown, $\mathrm{KcsA}-\mathrm{Kv} 1.3$ is found exclusively or predominantly in the closed-conductive conformation, as in phosphate buffer at pH 7.5 (Fig. 9.2 $a, b$ in Section 9.4.1, see cartoons on the right). 

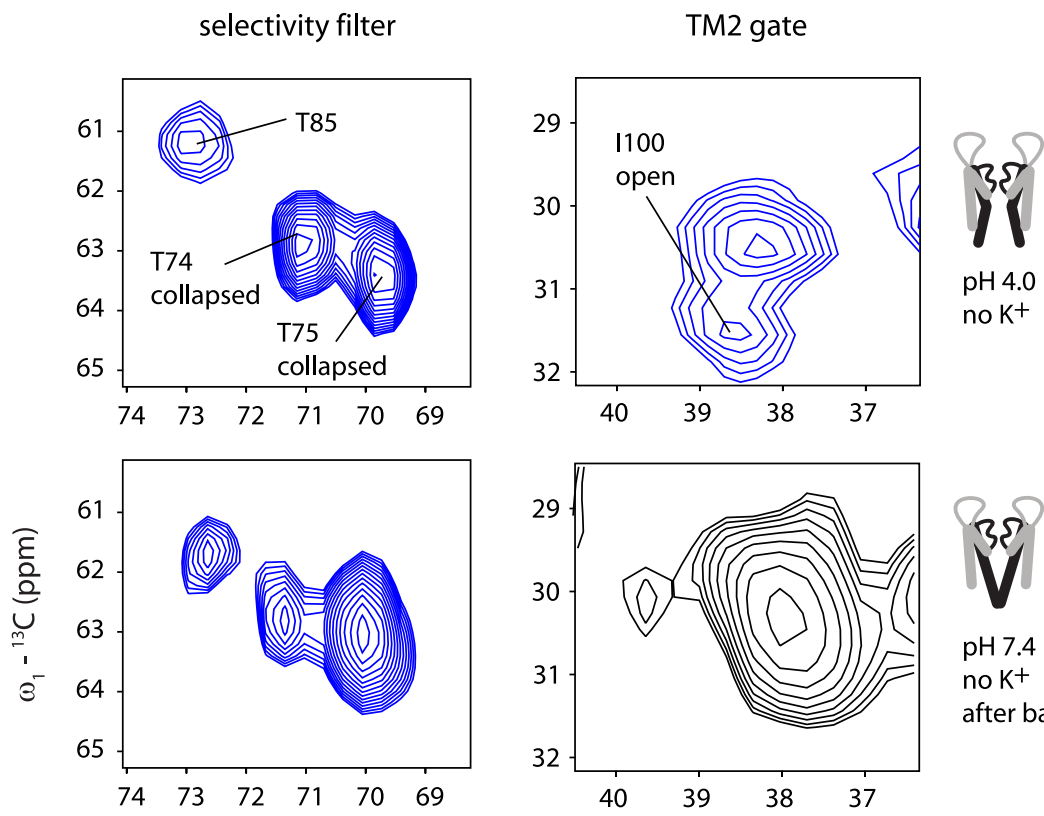

$\mathrm{pH} 7.4$

no $\mathrm{K}^{+}$

after backtitration
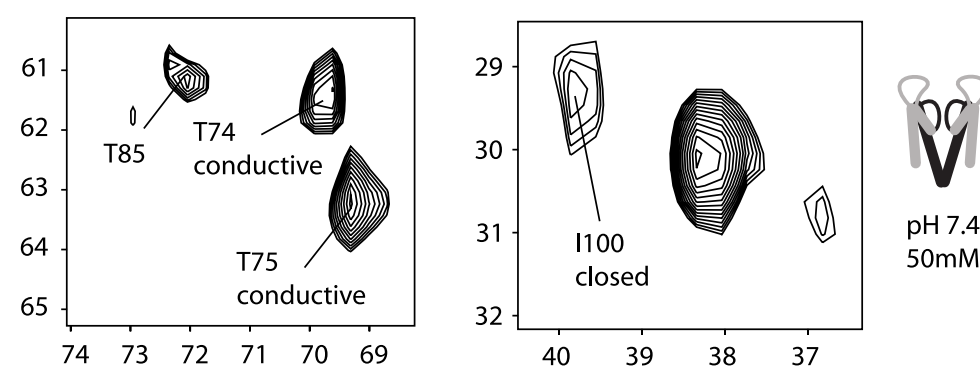

$50 \mathrm{mM} \mathrm{K}^{+}$

$\omega_{2}-{ }^{13} \mathrm{C}(\mathrm{ppm})$

Figure E.2: Spectral cut-outs from selectivity filter and TM2 gating hinge regions following the backtitration from $\mathrm{pH} 4.0$ (upper row) to $\mathrm{pH} 7.5$ (middle row) in the absence of potassium and after addition of $50 \mathrm{mM}$ potassium at pH 7.5 (bottom row). The KcsA-Kv1.3 conformations observed throughout these titrations are open-collapsed, closed-collapsed, and closed-conductive, respectively (see cartoons on the right). 


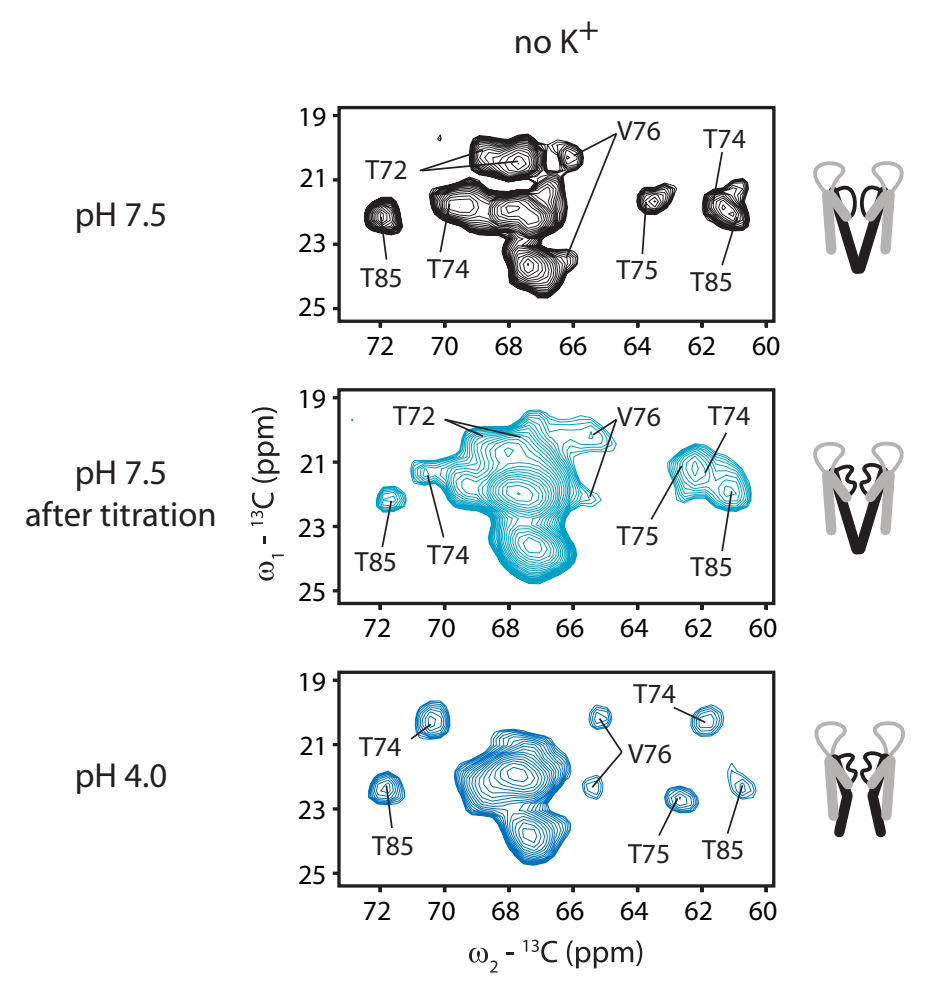

Figure E.3: Cut-out from $\left({ }^{13} \mathrm{C},{ }^{13} \mathrm{C}\right)$ spin diffusion spectrum illustrating the conformation of the selectivity filter of KcsA-Kv1.3 after back-titration from pH 4.0 to $\mathrm{pH} 7.5$ in the absence of potassium (center panel). Sidechain $\mathrm{C}^{\gamma 2}$ shifts of lower pore helix and lower selectivity filter threonines (Thr72, Thr74, Thr75) have shifted back to values seen at neutral pH before the titration, while $\mathrm{C}^{\alpha}$ and $\mathrm{C}^{\beta}$ shifts of Thr74 and Thr75 as well as those of the other selectivity filter residues still correspond to the collapsed conformation as seen at $\mathrm{pH} 4.0$ in the absence of potassium. For comparison, top and bottom panels show the same region in $\left({ }^{13} \mathrm{C},{ }^{13} \mathrm{C}\right)$ spectra of $\mathrm{KcsA}-\mathrm{Kv} 1.3$ at $\mathrm{pH} 7.5$ and $\mathrm{pH} 4.0$ without potassium, corresponding to the closed-conductive and open-collapsed conformations, respectively. Pictograms on the right illustrate the overall conformations of selectivity filter and TM2 helix in the respective states. 


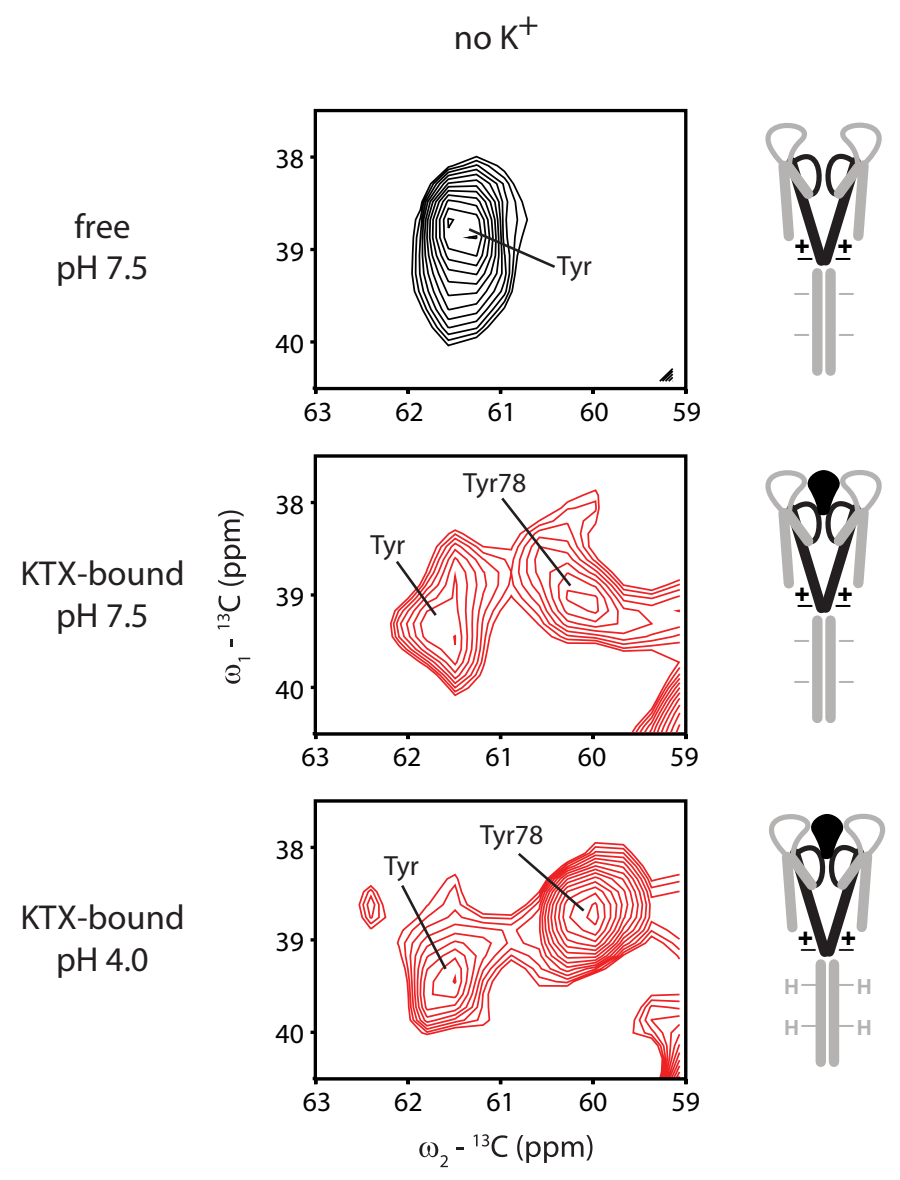

Figure E.4: Tyrosine $\mathrm{C}^{\beta}-\mathrm{C}^{\alpha}$ correlation region of $\left({ }^{13} \mathrm{C},{ }^{13} \mathrm{C}\right.$ ) spin diffusion spectra (20 ms mixing) recorded on KcsA-Kv1.3 with bound $\mathrm{KTX}$ at $\mathrm{pH} 7.5$ (center panel) and at $\mathrm{pH} 4.0$ (bottom panel), illustrating that KTX remained bound after the shift to $\mathrm{pH}$ 4.0. Top panel shows the same region in free KcsA-Kv1.3 at pH 7.5 for comparison. All samples were in buffers without added potassium. Pictograms illustrate KcsA-Kv1.3 conformation and KTX binding as well as charge distribution and protonation in the respective states. 


\section{Appendix F}

\section{Structural characterization of polyglutamine fibrils by solid-state NMR (Supplement)}

\section{F.1 Conformational analysis}

A grid search comparable to the one described in Chapter 8 was performed by independently varying backbone dihedral angles $\phi$ and $\psi$ of Gln10 in a PDB model of extended $\mathrm{D}_{2} \mathrm{Q}_{15} \mathrm{~K}_{2}$ from $-180^{\circ}$ to $180^{\circ}$ in steps of $5^{\circ}$ and predicting associated Gln10 chemical shifts using SHIFTX [163]. Those $(\phi, \psi)$ pairs within most favored or allowed regions of the Ramachandran plot [178] were selected for which the sum of the absolute differences of predicted and measured average $\mathrm{C}^{\alpha}$ and $\mathrm{C}^{\beta}$ chemical shifts did not exceed $1 \mathrm{ppm}$. Averaged experimental $\mathrm{C}^{\alpha}$ and $\mathrm{C}^{\beta}$ shifts used in this analysis were 56.0 and $34.1 \mathrm{ppm}$ for population 1, 54.2 and $31.7 \mathrm{ppm}$ for population 2 , and 55.8 and $29.8 \mathrm{ppm}$ for population 3 glutamines, respectively. No good fits to experimental values within most favored regions of the Ramachandran plot were obtained for the chemical shifts of glutamine population 1. For population 2, backbone dihedral angles nearly exclusively in the $\beta$-sheet most favored region of the Ramachandran plot were found, with best fitting $(\phi, \psi)$ values being $\left(-130^{\circ}, 145^{\circ}\right)$ (Fig. F.5 a). Dihedral angles found for population 3 chemical shifts were much more spread out in Ramachandran space, with closest matches corresponding to polyproline helix $\left(-80^{\circ}, 135^{\circ}\right)$ or $\alpha$-helix $\left(-120^{\circ}, 0^{\circ}\right)$ secondary structure, indicative of a coil, turn or bend organization for these glutamine residues (Fig. F.5 b).

To find estimates for population 1 dihedral angles, the Biological Magnetic Resonance Data Bank (BMRB, http://www.bmrb.wisc.edu/) was searched for proteins containing glutamine residues whose $\mathrm{C}^{\alpha}$ and $\mathrm{C}^{\beta}$ resonances were assigned to values corresponding to population 1 glutamines (56 and $34 \mathrm{ppm}$, respectively, with an allowed tolerance of $\pm 0.5 \mathrm{ppm}$ each) and for which structures in the Protein Data Bank (PDB, http://www.pdb.org/) were available. 17 such residues in 15 proteins were found (see Appendix Table F.2). One of these was discarded since, in the corresponding protein construct, it was a C-terminal residue without a $\psi$ dihedral angle (Gln75, PDB ID 2HDM); 
however, an alternative PDB for this protein was available in which the sequence extended longer C-terminally (1J9O). One residue could not be analyzed since the associated PDB structure contained only $\mathrm{C}^{\alpha}$ atoms (Gln81 of PDB ID 3B61). Backbone dihedral angles were calculated for the remaining residues using MOLMOL [224]. Another residue (Gln9 of PDB ID 1EIK) was discarded since its backbone dihedral angles in the PDB NMR ensemble were spread out over three quadrants of the Ramachandran plot. Of the remaining 15 residues in 13 proteins, the conformation in the mean or lowest energy structure was selected if such a structure was provided as a separate PDB file. Otherwise, a conformation in the center of the dihedral angle distribution of the NMR ensemble was selected. Figure F.5 c shows that nearly all resulting dihedral angle values are in most favored or allowed $\beta$-sheet regions of the Ramachandran plot. About half of the glutamines found with this approach are located near the ends of $\beta$-strands.

A trend is apparent for the distribution of population 1 dihedral angles to be centered more around the $\psi=-\phi$ diagonal than that of population 2 dihedral angles (Fig. F.5). This difference is more prominent if only those glutamine residues of the BMRB search are considered for which SHIFTX chemical shift predictions are close to their experimentally determined values (white diamonds in Fig. F.5 c). This approach should select for less unusual conformations and avoid potential assignment errors. The comparison suggests that the presence of two groups of $\beta$-sheet glutamine residues with distinct chemical shifts may partly be related to different average backbone dihedral angles in these residues.

\section{F.2 Supplementary figures}

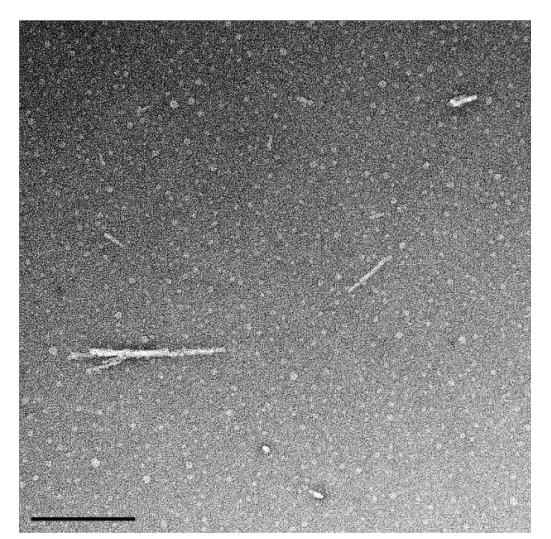

Figure F.1: EM image of $\mathrm{GK}_{2} \mathrm{Q}_{38} \mathrm{~K}_{2}$ kept in water with $0.1 \%$ TFA at pH 3 (courtesy of Dietmar Riedel). After about four weeks, some fibrillar aggregates can be found, with minimum diameters of $70 \AA$. Additionally, small spherical oligomers are visible. Scale bar is $200 \mathrm{~nm}$. 

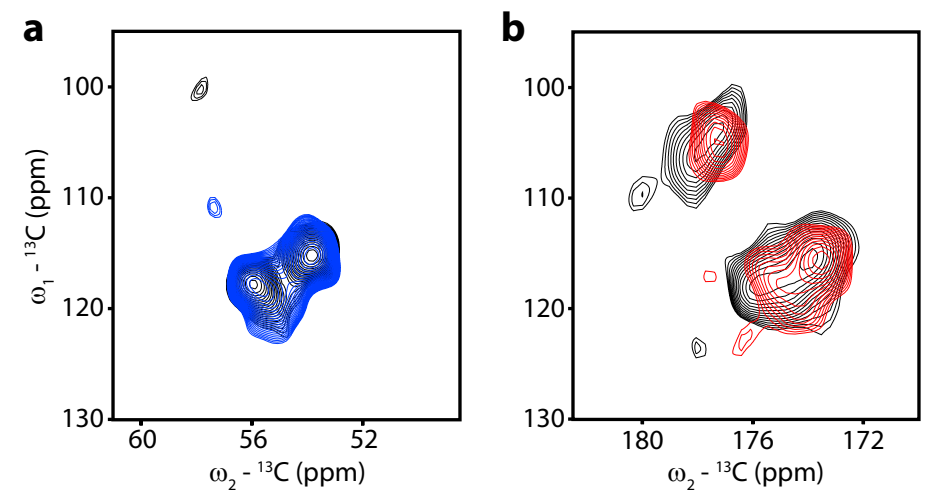

Figure F.2: (a) Comparison of NCA correlation spectra of $\mathrm{GK}_{2} \mathrm{Q}_{38} \mathrm{~K}_{2}$ fibrils (black) and $\mathrm{GK}_{2} \mathrm{Q}_{38} \mathrm{~K}_{2}$ raw material kept in $\mathrm{H}_{2} \mathrm{O}$ with $0.1 \%$ TFA after extraction (blue), corresponding to the sample state shown in Figure F.1. (b) Comparison of NCO correlation spectra of $\mathrm{GK}_{2} \mathrm{Q}_{38} \mathrm{~K}_{2}$ fibrils (black) and lyophilized $\mathrm{GK}_{2} \mathrm{Q}_{38} \mathrm{~K}_{2}$ after addition of about $2 \mu$ l of water (red). EM images of the latter sample showed only oligomers, no fibrils (data not shown). Spectra were recorded by Henrike Heise.

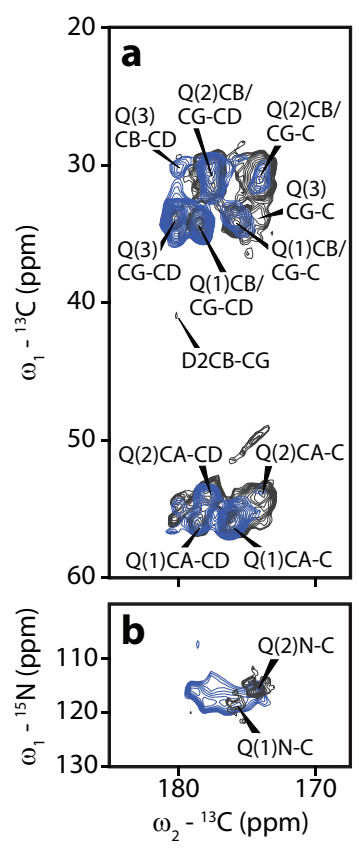

Figure F.3: Overlay of carbonyl regions of $\left({ }^{13} \mathrm{C},{ }^{13} \mathrm{C}\right.$ ) spin diffusion spectra (150 ms mixing time) (a) and NCACB spectra (b) of $\mathrm{D}_{2} \mathrm{Q}_{15} \mathrm{~K}_{2}$ (black) and $\mathrm{GK}_{2} \mathrm{Q}_{54} \mathrm{~K}_{2}$ (blue). Bracketed numbers in assignments refer to the different glutamine populations. Note the longer $\left({ }^{13} \mathrm{C},{ }^{13} \mathrm{C}\right)$ mixing time used in the $\mathrm{GK}_{2} \mathrm{Q}_{54} \mathrm{~K}_{2} \mathrm{NCACB}$ spectrum (blue, b) after the $\left({ }^{15} \mathrm{~N},{ }^{13} \mathrm{C}\right)$ transfer step, explaining the correlations of the backbone amide nitrogen with sidechain carbonyl resonances for $\mathrm{GK}_{2} \mathrm{Q}_{54} \mathrm{~K}_{2}$ in (b). 


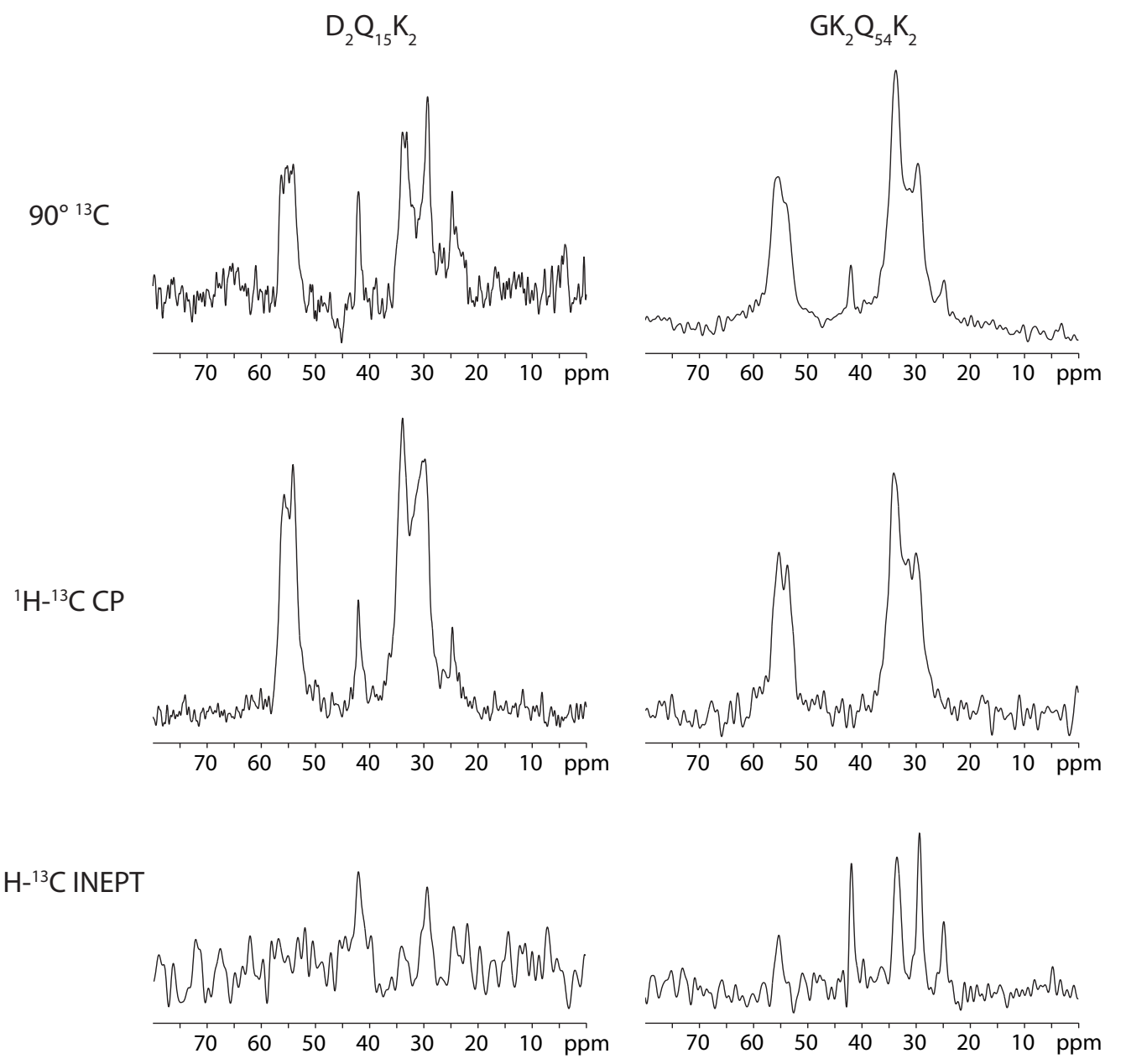

Figure F.4: Carbon 1D direct excitation, CP, and INEPT spectra for $D_{2} Q_{15} K_{2}$ and $G_{K_{2}} Q_{54} K_{2}$. Spectra of individual samples were processed identically and scaled to equal height of the peak at $42 \mathrm{ppm}$ (Lys $\mathrm{C}^{\varepsilon}$ in the case of $\mathrm{D}_{2} \mathrm{Q}_{15} \mathrm{~K}_{2}$ (left column) or to the peak at $30 \mathrm{ppm}$ ( $G \ln \mathrm{C}^{\beta}, \mathrm{C}^{\gamma}$, and Lys $\mathrm{C}^{\delta}$ ) for $\mathrm{GK}_{2} \mathrm{Q}_{54} \mathrm{~K}_{2}$ (right column). Spectra were recorded as follows: $\mathrm{D}_{2} \mathrm{Q}_{15} \mathrm{~K}_{2}$ : direct excitation: 3584 scans, $800 \mathrm{MHz}$; CP: 8 scans, $800 \mathrm{MHz}$; INEPT: 1536 scans, $400 \mathrm{MHz}$; $\mathrm{GK}_{2} \mathrm{Q}_{54} \mathrm{~K}_{2}$ : direct excitation: 256 scans; CP: 8 scans; INEPT: 1024 scans; all $\mathrm{GK}_{2} \mathrm{Q}_{54} \mathrm{~K}_{2}$ spectra recorded on a $400 \mathrm{MHz}$ magnet. While the three $\mathrm{GK}_{2} \mathrm{Q}_{54} \mathrm{~K}_{2}$ spectra were recorded on the exact same sample, the $D_{2} Q_{15} K_{2}$ spectra were recorded on different quantities of the same sample in different rotors, explaining the observed variations in signal to noise. 

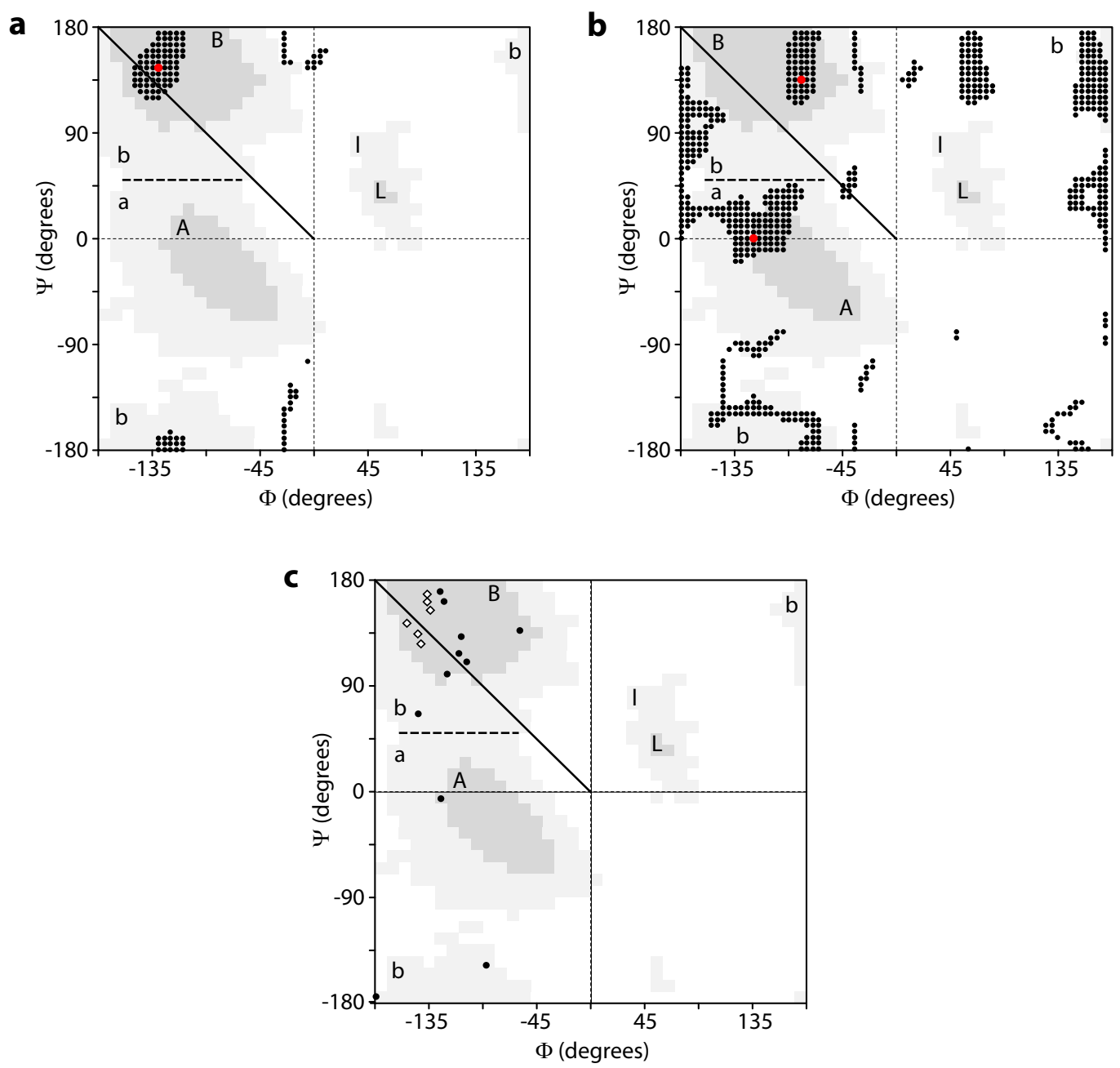

Figure F.5: Results from SHIFTX grid search (a, b) and BMRB search (c) for dihedral angles fitting best to chemical shifts of glutamine populations 2 (a), 3 (b) and 1 (c). Most favored (grey, capital letters) and allowed (light grey, small letters) regions of Ramachandran space are given [178]. Letters indicate regions corresponding to $\alpha$-helix $(\mathrm{A} / \mathrm{a}), \beta$-sheet $(\mathrm{B} / \mathrm{b})$, and left-handed helix $(\mathrm{L} / \mathrm{l})$, with a dashed line marking the border between $\alpha$-helix and $\beta$-sheet regions. A solid line indicates the $\psi=-\phi$ diagonal. In (a) and (b), glutamine conformations whose predicted $\mathrm{C}^{\alpha}$ and $\mathrm{C}^{\beta}$ chemical shifts are close to experimental values for populations 2 and 3 (i.e. sum of absolute shift differences for both nuclei does not exceed 1 ppm) are depicted as black circles on the Ramachandran plot. Red circles indicate dihedral angle values with best fitting chemical shift predictions within most favored or allowed regions. (c) shows dihedral angles of glutamines found in the BMRB to have been assigned close to population 1 chemical shifts as black circles and white diamonds, with the latter indicating those residues whose SHIFTX predictions are close to their experimental assignments (see Section F.1). 


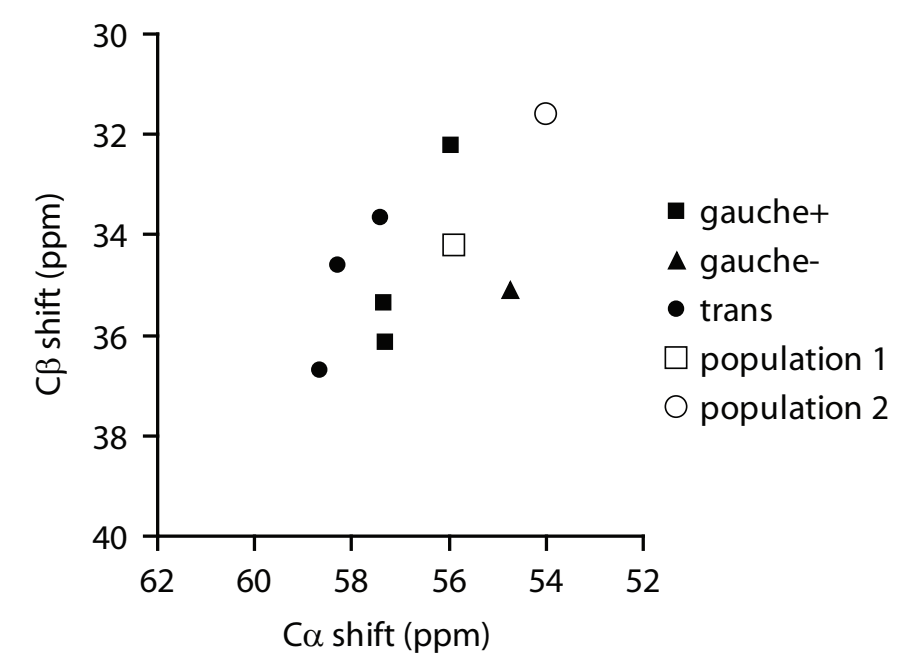

Figure F.6: $\mathbf{C}^{\alpha}$ and $\mathrm{C}^{\beta}$ chemical shifts of glutamine residues in $\beta$-sheet secondary structure with different sidechain dihedral angles, calculated at the density functional theory (DFT) level by Scheraga and coworkers [319] (filled symbols), compared with average values for glutamine populations 1 and 2 found in this study (open symbols; see Table 10.1). DFT chemical shift values in ref. [319] were calculated for the lowest-energy conformations of seven clustered families of sidechain conformations; they are grouped according to their $\chi_{1}$ rotameric state here. DFT-calculated chemical shifts were calibrated with respect to a scale on which tetramethylsilane (TMS) was set to $1.7 \mathrm{ppm}$, which corresponds to setting $0.5 \% 2,2-$ dimethyl-2-silapentane-5sulfonic acid (DSS) in $\mathrm{D}_{2} \mathrm{O}$ under MAS to $0.43 \mathrm{ppm}$ [344]. Experimental chemical shifts measured in this thesis were referenced to DSS set to $0 \mathrm{ppm}$ under these conditions, such that chemical shifts referenced as in ref. [319] should on average be $0.43 \mathrm{ppm}$ larger. There appears to be an additional offset of DFT values of unknown origin which is also reproduced in comparison with average experimental values found in proteins (see Table F.1). 


\begin{tabular}{ccccccc} 
Family number & CA shift $(\mathrm{ppm})$ & $\mathrm{CB}$ shift $(\mathrm{ppm})$ & $\chi_{1}\left(^{\circ}\right)$ & $\chi_{2}\left(^{\circ}\right)$ & $\chi_{3}\left(^{\circ}\right)$ & $\chi_{4}\left(^{\circ}\right)$ \\
\hline 1 & 57.32 & 36.16 & -64.4 & -176.32 & -101 & 179.5 \\
2 & 57.33 & 35.37 & -63.1 & -168.5 & 99.18 & -178.5 \\
3 & 55.94 & 32.23 & -61 & 178.24 & 36.3 & -1.2 \\
4 & 58.27 & 34.62 & -161.3 & 160.7 & 94.1 & 1.14 \\
5 & 58.66 & 36.70 & 161 & 159 & -100 & 179 \\
6 & 57.39 & 33.67 & -161 & 159.67 & -100.74 & -0.09 \\
7 & 54.72 & 35.11 & 67.1 & 179.34 & -98.6 & 0.49 \\
\hline Average & 57.09 & 34.84 & & & & \\
Exp. average & 54.33 & 31.92 & & & & \\
\hline Population 1 & 55.9 & 34.2 & & & &
\end{tabular}

Table F.1: Exact values of DFT-calculated $\mathrm{C}^{\alpha}$ and $\mathrm{C}^{\beta}$ chemical shifts shown in Figure F.6 and corresponding sidechain dihedral angles, as given in Table S1 of ref. [319]. Shown are also average values for these calculated $C^{\alpha}$ and $C^{\beta}$ chemical shifts as well as experimentally observed average values for glutamine residues in $\beta$-sheet secondary structure [158] and average population 1 and 2 chemical shifts as discussed in Chapter 10. Notably, experimental average values for $\beta$-sheet glutamines as found in ref. [158] correspond to population 2 chemical shifts, while average DFT values appear shifted about 3 ppm downfield.
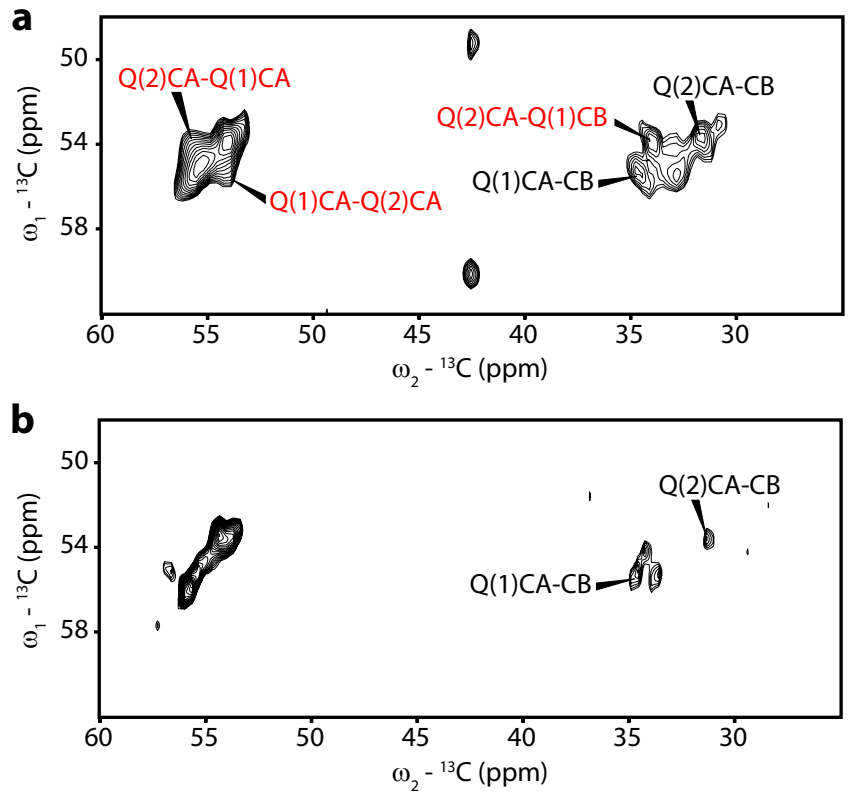

Figure F.7: Cut-outs from CHHC spectra (250 $\mu$ s mixing time) recorded on uniformly $\left[{ }^{15} \mathrm{~N},{ }^{13} \mathrm{C}\right]-$ labeled $\mathrm{GK}_{2} \mathrm{Q}_{54} \mathrm{~K}_{2}$ fibrils (a) and on $\mathrm{GK}_{2} \mathrm{Q}_{54} \mathrm{~K}_{2}$ fibrils consisting of a $1: 1$ mixture of ${ }^{15} \mathrm{~N}$ - or ${ }^{13} \mathrm{C}$ labeled monomers (b). Correlations between different glutamine populations are labeled in red. 


\begin{tabular}{|c|c|c|c|c|c|c|}
\hline BMRB ID & Residue & $\mathrm{C}^{\alpha}$ shift $(\mathrm{ppm})$ & $\mathrm{C}^{\beta}$ shift (ppm) & PDB ID & $\phi\left(^{\circ}\right)$ & $\psi\left({ }^{\circ}\right)$ \\
\hline 4712 & 55 & 55.78 & 34.05 & $1 \mathrm{FMM}$ & -88 & $\begin{array}{l}-148 \\
\end{array}$ \\
\hline 5267 & 160 & 56.02 & 33.65 & 2B59 & -143 & 126 \\
\hline 5327 & 54 & 55.52 & 34.17 & $1 \mathrm{~L} 8 \mathrm{C}$ & -125 & -6 \\
\hline 6298 & 23 & 55.5 & 34.37 & $1 \mathrm{TEY}$ & -104 & 111 \\
\hline 6299 & 12 & 55.57 & 34.02 & $1 \mathrm{U} 5 \mathrm{M}$ & -120 & 100 \\
\hline 6322 & $16(41)$ & 55.98 & 33.57 & $1 \mathrm{XFQ}$ & -145 & 135 \\
\hline 6405 & 89 & 55.71 & 34.47 & $1 \mathrm{WUZ}$ & -180 & -175 \\
\hline 6406 & 6 & 55.77 & 34.21 & 1B88 & -108 & 133 \\
\hline 6447 & 64 & 55.6 & 33.6 & $1 \mathrm{Y} 7 \mathrm{X}$ & -60 & 137 \\
\hline 10002 & $28(638)$ & 55.64 & 33.88 & 1VEX & -137 & 169 \\
\hline 4136 & 81 & 55.8 & 33.8 & $3 \mathrm{~B} 61$ & & \\
\hline 4678 & 9 & 55.66 & 33.73 & $1 \mathrm{EIK}$ & & \\
\hline 6100 & 8 & 56.4 & 34 & & & \\
\hline 6995 & 36 & 56.01 & 33.91 & & & \\
\hline 7205 & 5 & 56.48 & 33.58 & & & \\
\hline 4106 & 226 & 55.62 & 33.72 & $1 \mathrm{EZA}$ & -155 & 144 \\
\hline 4264 & 226 & 55.69 & 33.72 & 3EZA & -135 & 156 \\
\hline 5251 & 75 & 55.8 & 33.9 & $1 \mathrm{~J} 9 \mathrm{O} / 1 \mathrm{~J} 8 \mathrm{I}$ & -145 & 67 \\
\hline 15215 & 75 & 55.81 & 33.92 & $2 \mathrm{HDM}$ & & \\
\hline 15215 & 82 & 56.15 & 33.92 & $1 \mathrm{~J} 9 \mathrm{O} / 1 \mathrm{~J} 8 \mathrm{I}$ & -127 & 171 \\
\hline 15215 & 83 & 56.03 & 33.84 & $1 \mathrm{~J} 9 \mathrm{O} / 1 \mathrm{~J} 8 \mathrm{I}$ & -123 & 162 \\
\hline 5570 & 21 & 55.6 & 34.3 & 1Q27 & -111 & 118 \\
\hline 5570 & 21 & 55.6 & 34.3 & $2 \mathrm{O} 5 \mathrm{~F}$ & -137 & 162 \\
\hline
\end{tabular}

Table F.2: List of residues with corresponding BMRB and PDB (if available) entries found in the BMRB search (Section F.1) for glutamine residues with assigned chemical shifts close to population 1 values for $\mathrm{C}^{\alpha}$ and $\mathrm{C}^{\beta}$ nuclei (56 and $34 \mathrm{ppm}$, respectively, with an allowed tolerance of $\pm 0.5 \mathrm{ppm}$ ). Entries originating from the same protein (e.g. in different complexation states, with some mutations, or investigated by NMR and X-ray) are grouped in the lower part of the table. Residues for which no dihedral angles are listed were discarded as described in Section F.1, or no associated PDB file was found.

\begin{tabular}{c|cccc} 
Sample & $\begin{array}{c}\text { Initial rate } \\
\text { slope }(1 / \mathrm{ms})\end{array}$ & $t_{m}^{s}(\mathrm{~ms})$ & $V / S(\AA)$ & diameter $(\AA)$ \\
\hline $\mathrm{D}_{2} \mathrm{Q}_{15} \mathrm{~K}_{2}$ & 0.019 & 53 & 18 & 73 \\
$\mathrm{GK}_{2} \mathrm{Q}_{38} \mathrm{~K}_{2}$ & 0.0089 & 112 & 27 & 107 \\
$\mathrm{GK}_{2} \mathrm{Q}_{54} \mathrm{~K}_{2}$ & 0.014 & 71 & 21 & 85
\end{tabular}

Table F.3: Water-edited buildup data for the three polyglutamine constructs investigated. The initial rate slope was obtained from a linear fit to the data points of the buildup curve below $50 \%$ of its maximum value. For calculation of $t_{m}^{s}, V / S$, and the estimated diameter, see Section 10.3.3. 


\section{List of Symbols and Abbreviations}

$\begin{array}{ll}2 \mathrm{Q} & \text { Double quantum (coherence) } \\ 2 \mathrm{QE} / 2 \mathrm{QR} & \text { Double quantum coherence excitation / reconversion } \\ \hat{A} & \text { Spatial tensor } \\ \text { AHT } & \text { Average Hamiltonian theory } \\ \boldsymbol{B} & \text { Magnetic field (magnetic flux density) } \\ \boldsymbol{B}_{0} & \text { Static external magnetic field } \\ \boldsymbol{B}_{1} & \text { Magnetic field generated by r.f. irradiation } \\ \text { BMRB } & \text { Biological Magnetic Resonance Data Bank } \\ & \text { (http://www.bmrb.wisc.edu/) } \\ \text { CHHC } & \left.\text { (') }{ }^{13} \mathrm{C}\right) \text {-encoded }\left({ }^{1} \mathrm{H},{ }^{1} \mathrm{H}\right) \text { correlation experiment } \\ \text { CNS } & \text { Crystallography and NMR System }[175] \\ \text { CP } & \text { Cross-polarization } \\ \text { CSA } & \text { Chemical shielding anisotropy } \\ \text { CW } & \text { Continuous wave decoupling } \\ d_{i j} & \text { Dipolar coupling constant for two spins } i, j \\ \text { DARR } & \text { Dipolar-assisted rotational resonance } \\ \text { DEC } & \text { Decoupling } \\ \delta & \text { Chemical shift } \\ \Delta \delta & \text { Secondary chemical shift } \\ \text { DQ } & \text { Double quantum (coherence) } \\ \text { DRAMA } & \text { Dipolar recovery at the magic angle } \\ \text { DSS } & \text { sodium salt of } 2,2 \text {-dimethyl-2-silapentane-5-sulphonic acid } \\ E & \text { Energy } \\ \Delta E & \text { Energy difference } \\ E . c o l i & \text { Escherichia coli } \\ \text { EM } & \text { electron microscopy } \\ \text { FID } & \text { Free induction decay } \\ \text { FSLG } & \text { Frequency-switched Lee-Goldburg }\left({ }^{1} \mathrm{H},{ }^{1} \mathrm{H}\right) \text { decoupling } \\ \gamma & \text { Gyromagnetic ratio } \\ \hbar & \text { Planck's constant (divided by } 2 \pi) \\ & \end{array}$




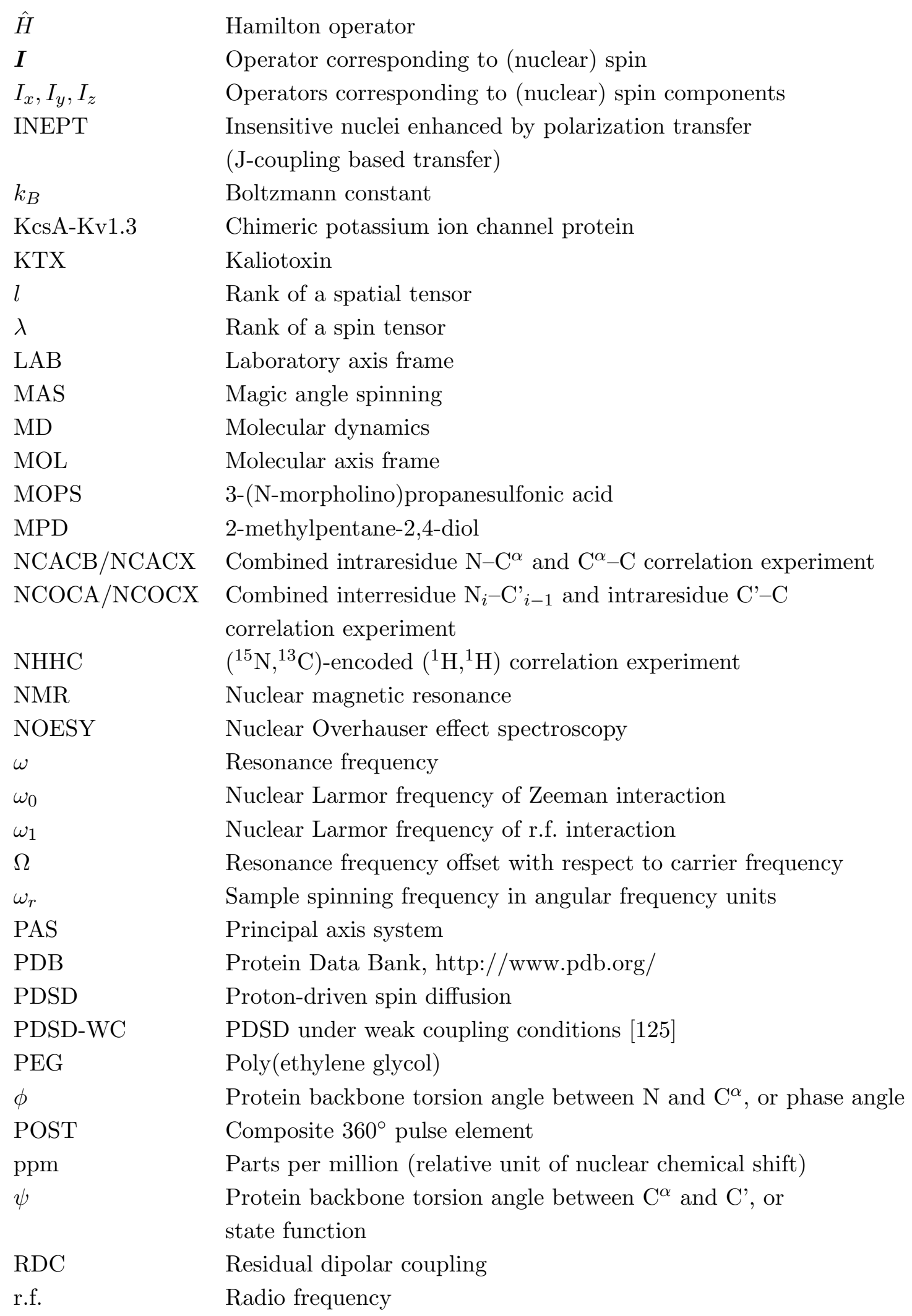




$\begin{array}{ll}\text { REDOR } & \text { Rotational echo double resonace } \\ \hat{\rho} & \text { Density operator } \\ \text { RMSD } & \text { Root-mean-square deviation } \\ \text { ROT } & \text { Rotor axis frame } \\ \text { s.e.m. } & \text { standard error of the mean } \\ S_{i j} & \text { Scaling factor (order parameter) of dipolar coupling between } \\ & \text { spins } i, j \\ \sigma & \text { Chemical shielding tensor } \\ \text { SPC5 } & \text { Supercycled POST-C5 } \\ \text { SPECIFIC-CP } & \text { Spectrally induced filtering in combination with cross-polarization } \\ \text { SPINAL } & \text { Small phase incremental alternation decoupling } \\ \text { SsNMR } & \text { solid-state nuclear magnetic resonance } \\ t & \text { Time } \\ \hat{T} & \text { Spin tensor } \\ \text { TEE } & \text { L-tyrosine-ethylester } \\ \theta_{m} & \text { Magic angle } \\ \text { TM1/2 } & \text { Transmembrane helix } 1 \text { or } 2 \text { of KcsA-Kv1.3 } \\ \text { TPPM } & \text { Two-pulse phase modulation decoupling } \\ \hat{U} & \text { Propagator } \\ \text { Ubi-M } & \text { Microcrystalline ubiquitin, precipitated using MPD } \\ \text { Ubi-P } & \text { Microcrystalline ubiquitin, precipitated using PEG }\end{array}$




\section{Bibliography}

[1] C. Ader, R. Schneider, S. Hornig, P. Velisetty, E. M. Wilson, A. Lange, K. Giller, I. Ohmert, M.-F. Martin-Eauclaire, D. Trauner, S. Becker, O. Pongs, and M. Baldus. A structural link between inactivation and block of a $\mathrm{K}^{+}$channel. Nat. Struct. Mol. Biol., 15(6):605-612, Jun 2008. DOI $10.1038 /$ nsmb.1430.

[2] R. Schneider, C. Ader, A. Lange, K. Giller, S. Hornig, O. Pongs, S. Becker, and M. Baldus. Solid-state NMR spectroscopy applied to a chimeric potassium channel in lipid bilayers. $J$. Am. Chem. Soc., 130(23):7427-7435, Jun 2008. DOI 10.1021/ja800190c.

[3] C. Ader, R. Schneider, K. Seidel, M. Etzkorn, S. Becker, and M. Baldus. Structural rearrangements of membrane proteins probed by water-edited solid-state NMR spectroscopy. $J$. Am. Chem. Soc., 131(1):170-176, Jan 2009. DOI 10.1021/ja806306e.

[4] U. Zachariae, R. Schneider, P. Velisetty, A. Lange, D. Seeliger, S. J. Wacker, Y. KarimiNejad, G. Vriend, S. Becker, O. Pongs, M. Baldus, and B. L. de Groot. The molecular mechanism of toxin-induced conformational changes in a potassium channel: relation to C-type inactivation. Structure, 16(5):747-754, May 2008. DOI 10.1016/j.str.2008.01.018.

[5] J. Korukottu, R. Schneider, V. Vijayan, A. Lange, O. Pongs, S. Becker, M. Baldus, and M. Zweckstetter. High-resolution 3D structure determination of kaliotoxin by solid-state NMR spectroscopy. PLoS ONE, 3(6):e2359, 2008. DOI 10.1371/journal.pone.0002359.

[6] C. Ader, R. Schneider, K. Seidel, M. Etzkorn, and M. Baldus. Magic-angle-spinning NMR spectroscopy applied to small molecules and peptides in lipid bilayers. Biochem. Soc. Trans., 35:991-995, 2007. DOI 10.1042/BST0350991.

[7] K. Seidel, M. Etzkorn, R. Schneider, C. Ader, and M. Baldus. Comparative analysis of NMR chemical shift predictions for proteins in the solid phase. Solid State NMR, 35(4):235-242, Jul 2009. DOI 10.1016/j.ssnmr.2008.12.008.

[8] F. Crick. What Mad Pursuit: A Personal View of Scientific Discovery. Basic Books, 1990.

[9] J. D. Watson and F. H. Crick. Molecular structure of nucleic acids; a structure for deoxyribose nucleic acid. Nature, 171(4356):737-738, Apr 1953. DOI 10.1038/171737a0.

[10] D. A. Doyle, J. Morais Cabral, R. A. Pfuetzner, A. Kuo, J. M. Gulbis, S. L. Cohen, B. T. Chait, and R. MacKinnon. The structure of the potassium channel: molecular basis of $\mathrm{K}^{+}$ conduction and selectivity. Science, 280(5360):69-77, Apr 1998. DOI 10.1126/science.280. 5360.69 . 
[11] H. M. Berman, J. Westbrook, Z. Feng, G. Gilliland, T. N. Bhat, H. Weissig, I. N. Shindyalov, and P. E. Bourne. The Protein Data Bank. Nucl. Acids Res., 28(1):235-242, 2000. DOI 10.1093/nar/28.1.235. URL http://www.pdb.org/.

[12] H. A. Scheraga, M. Khalili, and A. Liwo. Protein-folding dynamics: overview of molecular simulation techniques. Annu. Rev. Phys. Chem., 58:57-83, 2007. DOI 10.1146/annurev. physchem.58.032806.104614.

[13] Y. Zhang. Progress and challenges in protein structure prediction. Curr. Opin. Struct. Biol., 18(3):342-348, 2008. DOI 10.1016/j.sbi.2008.02.004.

[14] R. Das and D. Baker. Macromolecular modeling with Rosetta. Annu. Rev. Biochem., 77: 363-382, 2008. DOI 10.1146/annurev.biochem.77.062906.171838.

[15] J. Moult. A decade of CASP: progress, bottlenecks and prognosis in protein structure prediction. Curr. Opin. Struct. Biol., 15(3):285-289, 2005. DOI 10.1016/j.sbi.2005.05.011.

[16] L. E. Kay. Protein dynamics from NMR. Nat. Struct. Biol., 5 Suppl:513-517, Jul 1998. DOI $10.1038 / 755$.

[17] C. M. Fraser, J. D. Gocayne, O. White, et al. The minimal gene complement of mycoplasmagenitalium. Science, 270:397-403, 1995. DOI 10.1126/science.270.5235.397.

[18] B. Alberts, A. Johnson, J. Lewis, M. Raff, K. Roberts, and P. Walter. Molecular Biology of the Cell. Garland Science, 4th edition, 2002.

[19] S. H. White. The progress of membrane protein structure determination. Prot. Sci., 13(7): 1948-1949, Jul 2004. DOI 10.1110/ps.04712004.

[20] H. Heise, W. Hoyer, S. Becker, O. C. Andronesi, D. Riedel, and M. Baldus. Molecularlevel secondary structure, polymorphism, and dynamics of full-length alpha-synuclein fibrils studied by solid-state NMR. Proc. Natl. Ac. Sci. USA, 102(44):15871-15876, Nov 2005. DOI 10.1073/pnas.0506109102.

[21] S. B. Long, X. Tao, E. B. Campbell, and R. MacKinnon. Atomic structure of a voltagedependent $\mathrm{K}^{+}$channel in a lipid membrane-like environment. Nature, 450(7168):376-382, Nov 2007. DOI 10.1038/nature06265.

[22] V. Cherezov, D. M. Rosenbaum, M. A. Hanson, S. G. F. Rasmussen, F. S. Thian, T. S. Kobilka, H.-J. Choi, P. Kuhn, W. I. Weis, B. K. Kobilka, and R. C. Stevens. High-resolution crystal structure of an engineered human beta2-adrenergic G protein-coupled receptor. Science, 318(5854):1258-1265, Nov 2007. DOI 10.1126/science.1150577.

[23] S. G. F. Rasmussen, H.-J. Choi, D. M. Rosenbaum, T. S. Kobilka, F. S. Thian, P. C. Edwards, M. Burghammer, V. R. P. Ratnala, R. Sanishvili, R. F. Fischetti, G. F. X. Schertler, W. I. Weis, and B. K. Kobilka. Crystal structure of the human beta2 adrenergic G-protein-coupled receptor. Nature, 450(7168):383-387, Nov 2007. DOI 10.1038/nature06325. 
[24] Y. Zhou, J. H. Morais-Cabral, A. Kaufman, and R. MacKinnon. Chemistry of ion coordination and hydration revealed by a $\mathrm{K}^{+}$channel-Fab complex at $2.0 \AA$ resolution. Nature, 414 (6859):43-48, Nov 2001. DOI 10.1038/35102009.

[25] B. Liang and L. K. Tamm. Structure of outer membrane protein G by solution NMR spectroscopy. Proc. Natl. Ac. Sci. USA, 104(41):16140-16145, Oct 2007. DOI 10.1073/pnas. 0705466104 .

[26] S. Hiller, R. G. Garces, T. J. Malia, V. Y. Orekhov, M. Colombini, and G. Wagner. Solution structure of the integral human membrane protein VDAC-1 in detergent micelles. Science, 321(5893):1206-1210, Aug 2008. DOI 10.1126/science.1161302.

[27] M. Bayrhuber, T. Meins, M. Habeck, S. Becker, K. Giller, S. Villinger, C. Vonrhein, C. Griesinger, M. Zweckstetter, and K. Zeth. Structure of the human voltage-dependent anion channel. Proc. Natl. Ac. Sci. USA, 105(40):15370-15375, Oct 2008. DOI 10.1073/ pnas. 0808115105 .

[28] J. H. Chill, J. M. Louis, C. Miller, and A. Bax. NMR study of the tetrameric KcsA potassium channel in detergent micelles. Prot. Sci., 15(4):684-698, Apr 2006. DOI $10.1110 /$ ps.051954706.

[29] A. G. Lee. How lipids affect the activities of integral membrane proteins. Biochim. Biophys. Acta, 1666(1-2):62-87, Nov 2004. DOI 10.1016/j.bbamem.2004.05.012.

[30] E. E. Matthews, M. Zoonens, and D. M. Engelman. Dynamic helix interactions in transmembrane signaling. Cell, 127(3):447-450, Nov 2006. DOI 10.1016/j.cell.2006.10.016.

[31] J. A. Killian and T. K. M. Nyholm. Peptides in lipid bilayers: the power of simple models. Curr. Opin. Struct. Biol., 16(4):473-479, Aug 2006. DOI 10.1016/j.sbi.2006.06.007.

[32] J. P. Klare, E. Bordignon, M. Doebber, J. Fitter, J. Kriegsmann, I. Chizhov, H.-J. Steinhoff, and M. Engelhard. Effects of solubilization on the structure and function of the sensory rhodopsin II/transducer complex. J. Mol. Biol., 356(5):1207-1221, Mar 2006. DOI 10.1016/ j.jmb.2005.12.015.

[33] R. Moukhametzianov, J. P. Klare, R. Efremov, C. Baeken, A. Göppner, J. Labahn, M. Engelhard, G. Büldt, and V. I. Gordeliy. Development of the signal in sensory rhodopsin and its transfer to the cognate transducer. Nature, 440(7080):115-119, Mar 2006. DOI 10.1038/nature04520.

[34] G. F. X. Schertler. Structure of rhodopsin and the metarhodopsin I photointermediate. Curr. Opin. Struct. Biol., 15(4):408-415, Aug 2005. DOI 10.1016/j.sbi.2005.07.010.

[35] S. F. Poget and M. E. Girvin. Solution NMR of membrane proteins in bilayer mimics: small is beautiful, but sometimes bigger is better. Biochim. Biophys. Acta, 1768(12):3098-3106, Dec 2007. DOI 10.1016/j.bbamem.2007.09.006. 
[36] C. M. Franzin, X.-M. Gong, K. Thai, J. Yu, and F. M. Marassi. NMR of membrane proteins in micelles and bilayers: the FXYD family proteins. Methods, 41(4):398-408, Apr 2007. DOI 10.1016/j.ymeth.2006.08.011.

[37] B. Hille. Ionic Channels of Excitable Membranes. Sinauer Associates Inc., Sunderland, MA, 3rd edition, 2001.

[38] H. T. Kurata and D. Fedida. A structural interpretation of voltage-gated potassium channel inactivation. Prog. Biophys. Mol. Biol., 92(2):185-208, Oct 2006. DOI 10.1016/j.pbiomolbio. 2005.10.001.

[39] G. Yellen. The moving parts of voltage-gated ion channels. Q. Rev. Biophys., 31(3):239-295, Aug 1998. DOI 10.1017/S0033583598003448.

[40] Y. Jiang, A. Lee, J. Chen, M. Cadene, B. T. Chait, and R. MacKinnon. The open pore conformation of potassium channels. Nature, 417(6888):523-526, May 2002. DOI 10.1038/ 417523a.

[41] S. B. Long, E. B. Campbell, and R. Mackinnon. Crystal structure of a mammalian voltagedependent Shaker family K ${ }^{+}$channel. Science, 309(5736):897-903, Aug 2005. DOI 10.1126/ science.1116269.

[42] F. Chiti and C. M. Dobson. Protein misfolding, functional amyloid, and human disease. Annu. Rev. Biochem., 75:333-366, 2006. DOI 10.1146/annurev.biochem.75.101304.123901.

[43] C. Soto. Unfolding the role of protein misfolding in neurodegenerative diseases. Nat. Rev. Neurosci., 4(1):49-60, Jan 2003. DOI 10.1038/nrn1007.

[44] S. B. Prusiner. Molecular biology of prion diseases. Science, 252(5012):1515-1522, Jun 1991. DOI $10.1126 /$ science.1675487.

[45] S. B. Prusiner. Novel proteinaceous infectious particles cause scrapie. Science, 216(4542): 136-144, Apr 1982. DOI 10.1126/science.6801762.

[46] M. Fändrich and C. M. Dobson. The behaviour of polyamino acids reveals an inverse side chain effect in amyloid structure formation. EMBO J., 21(21):5682-5690, Nov 2002. DOI $10.1093 / \mathrm{emboj} / \mathrm{cdf573}$.

[47] R. Nelson, M. R. Sawaya, M. Balbirnie, A. Ø. Madsen, C. Riekel, R. Grothe, and D. Eisenberg. Structure of the cross-[beta] spine of amyloid-like fibrils. Nature, 435(7043):773-778, 2005. DOI 10.1038/nature03680.

[48] M. R. Sawaya, S. Sambashivan, R. Nelson, M. I. Ivanova, S. A. Sievers, M. I. Apostol, M. J. Thompson, M. Balbirnie, J. J. W. Wiltzius, H. T. McFarlane, A. Ø. Madsen, C. Riekel, and D. Eisenberg. Atomic structures of amyloid cross-beta spines reveal varied steric zippers. Nature, 447(7143):453-457, May 2007. DOI 10.1038/nature05695.

[49] F. Castellani, B. van Rossum, A. Diehl, M. Schubert, K. Rehbein, and H. Oschkinat. Structure of a protein determined by solid-state magic-angle-spinning NMR spectroscopy. Nature, 420(6911):98-102, Nov 2002. DOI 10.1038/nature01070. 
[50] D. H. Zhou, J. J. Shea, A. J. Nieuwkoop, W. T. Franks, B. J. Wylie, C. Mullen, D. Sandoz, and C. M. Rienstra. Solid-state protein-structure determination with proton-detected tripleresonance 3D magic-angle-spinning NMR spectroscopy. Angew. Chem. Int. Ed. Engl., 46 (44):8380-8383, 2007. DOI 10.1002/anie.200702905.

[51] A. Lange, S. Becker, K. Seidel, K. Giller, O. Pongs, and M. Baldus. A concept for rapid protein-structure determination by solid-state NMR spectroscopy. Angew. Chem. Int. Ed. Engl., 44(14):2089-2092, Mar 2005. DOI 10.1002/anie.200462516.

[52] T. Manolikas, T. Herrmann, and B. H. Meier. Protein structure determination from 13C spin-diffusion solid-state NMR spectroscopy. J. Am. Chem. Soc., 130(12):3959-3966, Mar 2008. DOI $10.1021 / \mathrm{ja} 078039 \mathrm{~s}$.

[53] A. Loquet, B. Bardiaux, C. Gardiennet, C. Blanchet, M. Baldus, M. Nilges, T. Malliavin, and A. Böckmann. 3D structure determination of the Crh protein from highly ambiguous solid-state NMR restraints. J. Am. Chem. Soc., 130(11):3579-3589, Mar 2008. DOI 10.1021/ ja078014t.

[54] F. Creuzet, A. McDermott, R. Gebhard, K. Vanderhoef, M. B. Spijkerassink, J. Herzfeld, J. Lugtenburg, M. H. Levitt, and R. G. Griffin. Determination of membrane protein structure by rotational resonance NMR - bacteriorhodopsin. Science, 251(4995):783-786, Feb 1991.

[55] L. K. Thompson, A. E. McDermott, J. Raap, C. M. van der Wielen, J. Lugtenburg, J. Herzfeld, and R. G. Griffin. Rotational resonance NMR study of the active site structure in bacteriorhodopsin: conformation of the Schiff base linkage. Biochemistry, 31(34): 7931-7938, Sep 1992. DOI 10.1021/bi00149a026.

[56] T. A. Cross and S. J. Opella. Solid-state NMR structural studies of peptides and proteins in membranes. Curr. Op. Struct. Biol., 4(4):574-581, Aug 1994. DOI 10.1073/pnas.112677599.

[57] S. H. Park, A. A. Mrse, A. A. Nevzorov, M. F. Mesleh, M. Oblatt-Montal, M. Montal, and S. J. Opella. Three-dimensional structure of the channel-forming trans-membrane domain of virus protein "u" (Vpu) from HIV-1. J. Mol. Biol., 333(2):409-424, Oct 2003. DOI 10.1016/j.jmb.2003.08.048.

[58] T. A. Egorova-Zachernyuk, J. Hollander, N. Fraser, P. Gast, A. J. Hoff, R. Cogdell, H. J. de Groot, and M. Baldus. Heteronuclear 2D-correlations in a uniformly $\left[{ }^{13} \mathrm{C},{ }^{15} \mathrm{~N}\right]$ labeled membrane-protein complex at ultra-high magnetic fields. J. Biomol. NMR, 19(3):243-253, Mar 2001. DOI 10.1023/A:1011235417465.

[59] M. Etzkorn, S. Martell, O. C. Andronesi, K. Seidel, M. Engelhard, and M. Baldus. Secondary structure, dynamics, and topology of a seven-helix receptor in native membranes, studied by solid-state NMR spectroscopy. Angew. Chem. Int. Ed. Engl., 46(3):459-462, 2007. DOI 10.1002/anie.200602139.

[60] Y. Li, D. A. Berthold, R. B. Gennis, and C. M. Rienstra. Chemical shift assignment of the transmembrane helices of DsbB, a 20-kDa integral membrane enzyme, by 3D magic-angle spinning NMR spectroscopy. Prot. Sci., 17(2):199-204, Feb 2008. DOI 10.1110/ps.073225008. 
[61] K. Seidel, O. C. Andronesi, J. Krebs, C. Griesinger, H. S. Young, S. Becker, and M. Baldus. Structural characterization of $\mathrm{Ca}^{2+}$-ATPase-bound phospholamban in lipid bilayers by solidstate nuclear magnetic resonance (NMR) spectroscopy. Biochemistry, 47(15):4369-4376, Apr 2008. DOI 10.1021/bi7024194.

[62] A. Lange, K. Giller, S. Hornig, M.-F. Martin-Eauclaire, O. Pongs, S. Becker, and M. Baldus. Toxin-induced conformational changes in a potassium channel revealed by solid-state NMR. Nature, 440(7086):959-962, Apr 2006. DOI 10.1038/nature04649.

[63] A. T. Petkova, Y. Ishii, J. J. Balbach, O. N. Antzutkin, R. D. Leapman, F. Delaglio, and R. Tycko. A structural model for Alzheimer's beta-amyloid fibrils based on experimental constraints from solid state NMR. Proc. Natl. Ac. Sci. USA, 99(26):16742-16747, Dec 2002. DOI 10.1073/pnas.262663499.

[64] O. C. Andronesi, M. von Bergen, J. Biernat, K. Seidel, C. Griesinger, E. Mandelkow, and M. Baldus. Characterization of Alzheimer's-like paired helical filaments from the core domain of tau protein using solid-state NMR spectroscopy. J. Am. Chem. Soc., 130(18):5922-5928, May 2008. DOI 10.1021/ja7100517.

[65] R. Tycko. Molecular structure of amyloid fibrils: insights from solid-state NMR. Q. Rev. Biophys., 39(1):1-55, Feb 2006. DOI 10.1017/S0033583506004173.

[66] C. P. Jaroniec, C. E. MacPhee, V. S. Bajaj, M. T. McMahon, C. M. Dobson, and R. G. Griffin. High-resolution molecular structure of a peptide in an amyloid fibril determined by magic angle spinning NMR spectroscopy. Proc. Natl. Ac. Sci. USA, 101(3):711-716, Jan 2004. DOI 10.1073/pnas.0304849101.

[67] C. Wasmer, A. Lange, H. van Melckebeke, A. B. Siemer, R. Riek, and B. H. Meier. Amyloid fibrils of the HET-s(218-289) prion form a beta solenoid with a triangular hydrophobic core. Science, 319(5869):1523-1526, Mar 2008. DOI 10.1126/science.1151839.

[68] K. Seidel, M. Etzkorn, L. Sonnenberg, C. Griesinger, A. Sebald, and M. Baldus. Studying molecular 3D structure and dynamics by high-resolution solid-state NMR: Application to L-tyrosine-ethylester. J. Phys. Chem. A, 109(11):2436-2442, Mar 2005. DOI $10.1021 / \mathrm{jp} 045605 \mathrm{~m}$.

[69] X. Helluy and A. Sebald. Structure and dynamic properties of solid L-tyrosine-ethylester as seen by C-13 MAS NMR. J. Phys. Chem. B, 107(14):3290-3296, Apr 2003. DOI 10.1021/ jp0277210.

[70] K. Seidel, M. Etzkorn, H. Heise, S. Becker, and M. Baldus. High-resolution solid-state NMR studies on uniformly $\left[{ }^{13} \mathrm{C},{ }^{15} \mathrm{~N}\right]$-labeled ubiquitin. ChemBioChem, 6(9):1638-1647, Sep 2005. DOI 10.1002/cbic.200500085.

[71] C. Legros, V. Pollmann, H. G. Knaus, A. M. Farrell, H. Darbon, P. E. Bougis, M. F. Martin-Eauclaire, and O. Pongs. Generating a high affinity scorpion toxin receptor in KcsAKv1.3 chimeric potassium channels. J. Biol. Chem., 275(22):16918-16924, Jun 2000. DOI 10.1074/jbc.275.22.16918. 
[72] G. Bates. Huntingtin aggregation and toxicity in Huntington's disease. Lancet, 361(9369): 1642-1644, May 2003. DOI 10.1016/S0140-6736(03)13304-1.

[73] F. Bloch, W. W. Hansen, and M. Packard. Nuclear induction. Phys. Rev., 69(3-4):127, Feb 1946. DOI 10.1103/PhysRev.69.127.

[74] E. M. Purcell, H. C. Torrey, and R. V. Pound. Resonance absorption by nuclear magnetic moments in a solid. Phys. Rev., 69(1-2):37-38, Jan 1946. DOI 10.1103/PhysRev.69.37.

[75] W. C. Dickinson. Dependence of the $F^{19}$ nuclear resonance position on chemical compound. Phys. Rev., 77(5):736-737, Mar 1950. DOI 10.1103/PhysRev.77.736.2.

[76] W. G. Proctor and F. C. Yu. On the magnetic moments of $\mathrm{Mn}^{55}, \mathrm{Co}^{59}, \mathrm{Cl}^{37}, \mathrm{~N}^{15}$, and $\mathrm{N}^{14}$. Phys. Rev., 77(5):716-717, Mar 1950. DOI 10.1103/PhysRev.77.716.

[77] J. Cavanagh, W. J. Fairbrother, A. G. Palmer, M. Rance, and N. J. Skelton. Protein NMR Spectroscopy. Academic Press, 2nd edition, 2006.

[78] R. R. Ernst and W. A. Anderson. Application of Fourier transform spectroscopy to magnetic resonance. Rev. Sci. Instr., 37:93-102, 1966. DOI 10.1063/1.1719961.

[79] J. Jeener. Pulse pair techniques in high resolution NMR. In Ampere International Summer School II, Basko Polje, Yugoslavia, volume Pulsed Magnetic and Optical Resonance, 1971.

[80] J. J. Sakurai. Modern Quantum Mechanics. Addison-Wesley, revised edition, 1994.

[81] U. Haeberlen and J. S. Waugh. Coherent averaging effects in magnetic resonance. Phys. Rev., 175:453-467, 1968. DOI 10.1103/PhysRev.175.453.

[82] J. S. Waugh. Average Hamiltonian theory. In D. M. Grant and R. K. Harris, editors, Encyclopedia of Nuclear Magnetic Resonance. John Wiley \& Sons, 2007. DOI 10.1002/ 9780470034590.emrstm0020.

[83] T. O. Levante, M. Baldus, B. H. Meier, and R. R. Ernst. Formalized quantum-mechanical Floquet theory and its application to sample spinning in nuclear magnetic resonance. Mol. Phys., 86(5):1195-1212, Dec 1995.

[84] M. J. Duer. Introduction to solid-state NMR spectroscopy. Blackwell Publishing, 2004.

[85] K. Schmidt-Rohr and H. W. Spiess. Multidimensional Solid-State NMR and Polymers. Academic Press, London, 1994.

[86] C. P. Slichter. Principles of Magnetic Resonance. Springer, 3rd edition, 1989.

[87] R. R. Ernst, G. Bodenhausen, and A. Wokaun. Principles of Nuclear Magnetic Resonance in One and Two Dimensions. Oxford University Press, 1st edition, 1987.

[88] M. Baldus and B. H. Meier. Total correlation spectroscopy in the solid state. The use of scalar couplings to determine the through-bond connectivity. J. Magn. Reson. A, 121(1): 65-69, Jul 1996. DOI 10.1006/jmra.1996.0137. 
[89] D. M. Brink and G. R. Satchler. Angular momentum. Oxford University Press, 1961.

[90] E. R. Andrew, A. Bradbury, and R. G. Eades. Removal of dipolar broadening of nuclear magnetic resonance spectra of solids by specimen rotation. Nature, 183:1802-1803, 1959. DOI 10.1038/1831802a0.

[91] E. R. Andrew, A. Bradbury, and R. G. Eades. Nuclear magnetic resonance spectra from a crystal rotated at high speed. Nature, 182:1659, 1958. DOI 10.1038/1821659a0.

[92] L. Sonnenberg. Abstandsmessungen an uniform isotopenmarkierten Biomolekülen mit Festkörper-NMR. Master's thesis, Universiät Göttingen, 2003.

[93] D. D. Laws, H. M. L. Bitter, and A. Jerschow. Solid-state NMR spectroscopic methods in chemistry. Angew. Chem. Int. Ed. Engl., 41:3096-3129, 2002. DOI 10.1002/ 1521-3773(20020902)41:17〈3096::AID-ANIE3096〉3.0.CO;2-X.

[94] A. E. Bennett, C. M. Rienstra, M. Auger, K. V. Lakshmi, and R. G. Griffin. Heteronuclear decoupling in rotating solids. J. Chem. Phys., 103(16):6951-6958, Oct 1995. DOI 10.1063/ 1.470372 .

[95] B. M. Fung, A. K. Khitrin, and K. Ermolaev. An improved broadband decoupling sequence for liquid crystals and solids. J. Magn. Reson., 142(1):97-101, Jan 2000. DOI 10.1006/jmre. 1999.1896 .

[96] M. Lee and W. I. Goldburg. Nuclear-magnetic-resonance line narrowing by a rotating rf field. Phys. Rev., 140(4A):1261, 1965. DOI 10.1103/PhysRev.140.A1261.

[97] B. J. van Rossum, H. Forster, and H. J. M. deGroot. High-field and high-speed CP-MAS C-13 NMR heteronuclear dipolar-correlation spectroscopy of solids with frequency-switched Lee-Goldburg homonuclear decoupling. J. Magn. Reson., 124(2):516-519, 1997. DOI 10. 1006/jmre.1996.1089.

[98] D. Sakellariou, A. Lesage, P. Hodgkinson, and L. Emsley. Homonuclear dipolar decoupling in solid-state NMR using continuous phase modulation. Chem. Phys. Lett., 319:253, 2000. DOI 10.1016/S0009-2614(00)00127-5.

[99] R. G. Griffin. Dipolar recoupling in MAS spectra of biological solids. Nat. Struct. Biol., 5: 508-512, Jul 1998. DOI 10.1038/749.

[100] M. Baldus. Correlation experiments for assignment and structure elucidation of immobilized polypeptides under magic angle spinning. Progr. NMR Spectr., 41(1-2):1-47, Sep 2002. DOI 10.1016/S0079-6565(02)00007-9.

[101] R. Tycko and G. Dabbagh. Measurement of nuclear magnetic dipole-dipole couplings in magic angle spinning NMR. Chem. Phys. Lett., 173(5-6):461-465, Oct 1990. DOI 10.1016/ 0009-2614(90)87235-J.

[102] U. Haeberlen. High resolution NMR of solids. Adv. Magn. Reson.: Supplement 1, 1976. Academic Press, San Diego. 
[103] J. Herzfeld, S. K. Das Gupta, M. R. Farrar, G. S. Harbison, A. E. McDermott, S. L. Pelletier, D. P. Raleigh, S. O. Smith, C. Winkel, and J. Lugtenburg. Solid-state 13C NMR study of tyrosine protonation in dark-adapted bacteriorhodopsin. Biochemistry, 29(23):5567-5574, Jun 1990. DOI 10.1021/bi00475a022.

[104] L. M. McDowell and J. Schaefer. High-resolution NMR of biological solids. Curr. Opin. Struct. Biol., 6(5):624-629, Oct 1996. DOI 10.1016/S0959-440X(96)80028-5.

[105] L. Lian and D. A. Middleton. Labelling approaches for protein structural studies by solutionstate and solid-state NMR. Progr. NMR Spectr., 39(3):171-190, Oct 2001. DOI 10.1016/ S0079-6565(01)00034-6.

[106] D. M. LeMaster and D. M. Kushlan. Dynamical mapping of E. coli thioredoxin via C-13 NMR relaxation analysis. J. Am. Chem. Soc., 118(39):9255-9264, Oct 1996. DOI 10.1021/ ja960877r.

[107] A. J. van Gammeren, F. B. Hulsbergen, J. G. Hollander, and H. J. M. de Groot. Biosynthetic site-specific C-13 labeling of the light-harvesting 2 protein complex: A model for solid state NMR structure determination of transmembrane proteins. J. Biomol. NMR, 30(3):267-274, Nov 2004. DOI 10.1007/s10858-004-3736-7.

[108] S. G. Zech, A. J. Wand, and A. E. McDermott. Protein structure determination by highresolution solid-state NMR spectroscopy: application to microcrystalline ubiquitin. J. Am. Chem. Soc., 127(24):8618-8626, Jun 2005. DOI 10.1021/ja0503128.

[109] M. Hiller, L. Krabben, K. R. Vinothkumar, F. Castellani, B. J. van Rossum, W. Kuhlbrandt, and H. Oschkinat. Solid-state magic-angle spinning NMR of outer-membrane protein G from Escherichia coli. ChemBioChem, 6(9):1679-1684, Sep 2005. DOI 10.1002/cbic.200500132.

[110] G. W. Vuister, S. J. Kim, C. Wu, and A. Bax. 2D and 3D NMR study of phenylalanine residues in proteins by reverse isotopic labeling. J. Am. Chem. Soc., 116(20):9206-9210, Oct 1994. DOI 10.1021/ja00099a041.

[111] S. R. Hartmann and E. L. Hahn. Nuclear double resonance in the rotating frame. Phys. Rev., 128(5):2042-2053, 1962. DOI 10.1103/PhysRev.128.2042.

[112] A. Pines, M. G. Gibby, and J. S. Waugh. Proton-enhanced NMR of dilute spins in solids. J. Chem. Phys., 59(2):569-590, 1973. DOI 10.1063/1.1680061.

[113] O. C. Andronesi, S. Becker, K. Seidel, H. Heise, H. S. Young, and M. Baldus. Determination of membrane protein structure and dynamics by magic-angle-spinning solid-state NMR spectroscopy. J. Am. Chem. Soc., 127(37):12965-12974, Sep 2005. DOI 10.1021/ja0530164.

[114] K. Wüthrich. NMR of Proteins and Nucleic Acids. Wiley Interscience, 1986. ISBN 9780471828938 .

[115] N. Bloembergen. On the interaction of nuclear spins in a crystalline lattice. Physica, 15 (3-4):386-426, 1949. DOI 10.1016/0031-8914(49)90114-7. 
[116] A. Kubo and C. A. McDowell. Spectral spin diffusion in polycrystalline solids under magicangle spinning. J. Chem. Soc. Faraday Trans. I, 84:3713-3730, 1988.

[117] D. Suter and R. R. Ernst. Spin diffusion in resolved solid-state NMR spectra. Phys. Rev. B, 32(9):5608-5627, 1985. DOI 10.1103/PhysRevB.32.5608.

[118] D. Suter and R. R. Ernst. Spectral spin diffusion in the presence of an extraneous dipolar reservoir. Phys. Rev. B, 25(9):6038-6041, 1982. DOI 10.1103/PhysRevB.25.6038.

[119] K. Takegoshi, S. Nakamura, and T. Terao. ${ }^{13} \mathrm{C}-{ }^{1} \mathrm{H}$ dipolar-assisted rotational resonance in magic-angle spinning NMR. Chem. Phys. Lett., 344(5-6):631-637, Aug 2001. DOI 10.1016/ S0009-2614(01)00791-6.

[120] M. Hohwy, C. M. Rienstra, and R. G. Griffin. Band-selective homonuclear dipolar recoupling in rotating solids. J. Chem. Phys., 117(10):4973-4987, September 2002. DOI 10.1063/1. 1488136.

[121] M. Hohwy, C. M. Rienstra, C. P. Jaroniec, and R. G. Griffin. Fivefold symmetric homonuclear dipolar recoupling in rotating solids: Application to double quantum spectroscopy. J. Chem. Phys., 110(16):7983-7992, Apr 1999. DOI 10.1063/1.478702.

[122] J. Schaefer, R. A. McKay, and E. O. Stejskal. Double-cross-polarization NMR of solids. J. Magn. Reson., 34(2):443-447, 1979.

[123] M. Baldus, A. T. Petkova, J. Herzfeld, and R. G. Griffin. Cross polarization in the tilted frame: assignment and spectral simplification in heteronuclear spin systems. Mol. Phys., 95 (6):1197-1207, Dec 1998. DOI 10.1080/002689798166215.

[124] A. T. Petkova, M. Baldus, M. Belenky, M. Hong, R. G. Griffin, and J. Herzfeld. Backbone and side chain assignment strategies for multiply labeled membrane peptides and proteins in the solid state. J. Magn. Reson., 160(1):1-12, Jan 2003. DOI 10.1016/S1090-7807(02)00137-4.

[125] K. Seidel, A. Lange, S. Becker, C. E. Hughes, H. Heise, and M. Baldus. Protein solid-state NMR resonance assignments from $\left({ }^{13} \mathrm{C},{ }^{13} \mathrm{C}\right)$ correlation spectroscopy. Phys. Chem. Chem. Phys., 6(22):5090-5093, 2004. DOI 10.1039/b411689e.

[126] M. H. Levitt. Symmetry-based pulse sequences in magic-angle spinning solid-state NMR. In D. M. Grant and R. K. Harris, editors, Encyclopedia of Nuclear Magnetic Resonance: Supplementary Volume, pages 165-196. John Wiley \& Sons, 2002.

[127] C. E. Hughes, S. Luca, and M. Baldus. Radio-frequency driven polarization transfer without heteronuclear decoupling in rotating solids. Chem. Phys. Lett., 385(5-6):435-440, Feb 2004.

[128] I. Marin-Montesinos, D. H. Brouwer, G. Antonioli, W. C. Lai, A. Brinkmann, and M. H. Levitt. Heteronuclear decoupling interference during symmetry-based homonuclear recoupling in solid-state NMR. J. Magn. Reson., 177(2):307-317, Dec 2005. DOI 10.1016/j.jmr. 2005.07.020. 
[129] G. De Paëpe, M. J. Bayro, J. Lewandowski, and R. G. Griffin. Broadband homonuclear correlation spectroscopy at high magnetic fields and MAS frequencies. J. Am. Chem. Soc., 128(6):1776-1777, Feb 2006. DOI 10.1021/ja0550430.

[130] G. Bodenhausen, H. Kogler, and R. R. Ernst. Selection of coherence-transfer pathways in NMR pulse experiments. J. Magn. Reson., 58(3):370-388, 1984. DOI 10.1016/0022-2364(84) 90142-2.

[131] M. H. Levitt. Symmetry in the design of NMR multiple-pulse sequences. J. Chem. Phys., 128(5):052205, Feb 2008. DOI 10.1063/1.2831927.

[132] A. Brinkmann, M. Eden, and M. H. Levitt. Synchronous helical pulse sequences in magicangle spinning nuclear magnetic resonance: Double quantum recoupling of multiple-spin systems. J. Chem. Phys., 112(19):8539-8554, May 2000.

[133] M. Eden and M. H. Levitt. Pulse sequence symmetries in the nuclear magnetic resonance of spinning solids: Application to heteronuclear decoupling. J. Chem. Phys., 111(4):1511-1519, 1999. DOI $10.1063 / 1.479410$.

[134] Y. K. Lee, N. D. Kurur, M. Helmle, O. G. Johannessen, N. C. Nielsen, and M. H. Levitt. Efficient dipolar recoupling in the NMR of rotating solids. A sevenfold symmetric radiofrequency pulse sequence. Chem. Phys. Lett., 242(3):304-309, 1995. DOI 10.1016/0009-2614(95)00741-L.

[135] D. Neuhaus and N. Williamson. The nuclear Overhauser effect in structural and conformational analysis. VCH Publishers, New York, 1989.

[136] A. Lange, S. Luca, and M. Baldus. Structural constraints from proton-mediated rare-spin correlation spectroscopy in rotating solids. J. Am. Chem. Soc., 124(33):9704-9705, Aug 2002. DOI $10.1021 / \mathrm{ja} 026691 \mathrm{~b}$.

[137] A. Lange, K. Seidel, L. Verdier, S. Luca, and M. Baldus. Analysis of proton-proton transfer dynamics in rotating solids and their use for 3D structure determination. J. Am. Chem. Soc., 125(41):12640-12648, Oct 2003. DOI 10.1021/ja034555g.

[138] D. P. Raleigh, M. H. Levitt, and R. G. Griffin. Rotational resonance in solid-state NMR. Chem. Phys. Lett., 146(1-2):71-76, Apr 1988. DOI 10.1016/0009-2614(88)85051-6.

[139] T. Gullion and J. Schaefer. Rotational-echo double-resonance NMR. J. Magn. Reson., 81 (1):196-200, Jan 1989. DOI 10.1016/0022-2364(89)90280-1.

[140] L. Sonnenberg, S. Luca, and M. Baldus. Multiple-spin analysis of chemical-shift-selective (13C, 13C) transfer in uniformly labeled biomolecules. J. Magn. Reson., 166(1):100-110, Jan 2004. DOI 10.1016/j.jmr.2003.10.014.

[141] M. Baldus. ICMRBS founder's medal 2006: biological solid-state NMR, methods and applications. J. Biomol. NMR, 39(1):73-86, Sep 2007. DOI 10.1007/s10858-007-9177-3. 
[142] J. R. Lewandowski, G. De Paëpe, and R. G. Griffin. Proton assisted insensitive nuclei cross polarization. J. Am. Chem. Soc., 129(4):728-729, Jan 2007. DOI 10.1021/ja0650394.

[143] G. De Paëpe, J. R. Lewandowski, and R. G. Griffin. Spin dynamics in the modulation frame: application to homonuclear recoupling in magic angle spinning solid-state NMR. J. Chem. Phys., 128(12):124503, Mar 2008. DOI 10.1063/1.2834732.

[144] M. Goldman and L. Shen. Spin-spin relaxation in LaF3. Phys. Rev., 144(1):321-\&, 1966. DOI 10.1103/PhysRev.144.321.

[145] K. K. Kumashiro, K. Schmidt-Rohr, O. J. Murphy, K. L. Ouellette, W. A. Cramer, and L. K. Thompson. A novel tool for probing membrane protein structure: Solid-state NMR with proton spin diffusion and X-nucleus detection. J. Am. Chem. Soc., 120(20):5043-5051, May 1998. DOI 10.1021/ja972655e.

[146] G. J. Gallagher, M. Hong, and L. K. Thompson. Solid-state NMR spin diffusion for measurement of membrane-bound peptide structure: gramicidin A. Biochemistry, 43(24):7899-7906, Jun 2004. DOI 10.1021/bi0356101.

[147] A. Lesage, L. Emsley, F. Penin, and A. Böckmann. Investigation of dipolar-mediated waterprotein interactions in microcrystalline Crh by solid-state NMR spectroscopy. J. Am. Chem. Soc., 128(25):8246-8255, Jun 2006. DOI 10.1021/ja060866q.

[148] A. Lesage, C. Gardiennet, A. Loquet, R. Verel, G. Pintacuda, L. Emsley, B. H. Meier, and A. Böckmann. Polarization transfer over the water-protein interface in solids. Angew. Chem. Int. Ed. Engl., 47(31):5851-5854, 2008. DOI 10.1002/anie.200801110.

[149] J. L. Lorieau and A. E. McDermott. Conformational flexibility of a microcrystalline globular protein: order parameters by solid-state NMR spectroscopy. J. Am. Chem. Soc., 128(35): 11505-11512, Sep 2006. DOI 10.1021/ja062443u.

[150] S. A. Smith, T. O. Levante, B. H. Meier, and R. R. Ernst. Computer simulations in magnetic resonance. An object-oriented programming approach. J. Magn. Reson. A, 106(1):75-105, Jan 1994. DOI 10.1006/jmra.1994.1008.

[151] M. Bak, J. T. Rasmussen, and N. C. Nielsen. SIMPSON: A general simulation program for solid-state NMR spectroscopy. J. Magn. Reson., 147:296-330, 2000. DOI 10.1006/jmre.2000. 2179 .

[152] M. Veshtort and R. G. Griffin. SPINEVOLUTION: a powerful tool for the simulation of solid and liquid state NMR experiments. J. Magn. Reson., 178(2):248-282, Feb 2006. DOI 10.1016/j.jmr.2005.07.018.

[153] S. Luca, D. V. Filippov, J. H. van Boom, H. Oschkinat, H. J. M. de Groot, and M. Baldus. Secondary chemical shifts in immobilized peptides and proteins: A qualitative basis for structure refinement under Magic Angle Spinning. J. Biomol. NMR, 20(4):325-331, Aug 2001. DOI 10.1023/A:1011278317489. 
[154] X.-P. Xu and D. A. Case. Probing multiple effects on 15N, 13C alpha, 13C beta, and 13C' chemical shifts in peptides using density functional theory. Biopolymers, 65(6):408-423, Dec 2002. DOI 10.1002/bip.10276.

[155] H. Saito. Conformation-dependent C-13 chemical-shifts - a new means of conformational characterization as obtained by high-resolution solid-state C-13 NMR. Magn. Res. Chem., 24(10):835-852, Oct 1986. DOI 10.1002/mrc.1260241002.

[156] S. Spera and A. Bax. Empirical correlation between protein backbone conformation and C-alpha and C-beta C-13 nuclear magnetic resonance chemical shifts. J. Am. Chem. Soc., 113(14):5490-5492, Jul 1991. DOI 10.1021/ja00014a071.

[157] D. S. Wishart, B. D. Sykes, and F. M. Richards. Relationship between nuclear magnetic resonance chemical shift and protein secondary structure. J. Mol. Biol., 222(2):311-333, Nov 1991. DOI 10.1016/0022-2836(91)90214-Q.

[158] Y. J. Wang and O. Jardetzky. Probability-based protein secondary structure identification using combined NMR chemical-shift data. Prot. Sci., 11(4):852-861, Apr 2002. DOI 10. $1110 /$ ps.3180102.

[159] G. Cornilescu, F. Delaglio, and A. Bax. Protein backbone angle restraints from searching a database for chemical shift and sequence homology. J. Biomol. NMR, 13(3):289-302, Mar 1999. DOI 10.1023/A:1008392405740.

[160] M. V. Berjanskii, S. Neal, and D. S. Wishart. PREDITOR: a web server for predicting protein torsion angle restraints. Nucl. Acids Res., 34(suppl 2):W63-69, July 1, 20062006. DOI 10.1093/nar/gkl341.

[161] H. H. Sun, L. K. Sanders, and E. Oldfield. Carbon-13 NMR shielding in the twenty common amino acids: Comparisons with experimental results in proteins. J. Am. Chem. Soc., 124 (19):5486-5495, May 2002. DOI 10.1021/ja011863a.

[162] J. Meiler. PROSHIFT: protein chemical shift prediction using artificial neural networks. $J$. Biomol. NMR, 26(1):25-37, May 2003. DOI 10.1023/A:1023060720156.

[163] S. Neal, A. M. Nip, H. Y. Zhang, and D. S. Wishart. Rapid and accurate calculation of protein H-1, C-13 and N-15 chemical shifts. J. Biomol. NMR, 26(3):215-240, Jul 2003. DOI 10.1023/A:1023812930288.

[164] Y. Shen and A. Bax. Protein backbone chemical shifts predicted from searching a database for torsion angle and sequence homology. J. Biomol. NMR, 38(4):289-302, Aug 2007. DOI $10.1007 / \mathrm{s} 10858-007-9166-6$.

[165] M. Etzkorn, H. Kneuper, P. Dünnwald, V. Vijayan, J. Krämer, C. Griesinger, S. Becker, G. Unden, and M. Baldus. Plasticity of the PAS domain and a potential role for signal transduction in the histidine kinase DcuS. Nat. Struct. Mol. Biol., 15(10):1031-1039, Oct 2008. DOI 10.1038/nsmb.1493. 
[166] H. Heise, S. Luca, B. L. de Groot, H. Grubmüller, and M. Baldus. Probing conformational disorder in neurotensin by two-dimensional solid-state NMR and comparison to molecular dynamics simulations. Biophys. J., 89(3):2113-2120, Sep 2005. DOI 10.1529/biophysj.105. 059964 .

[167] Y. Matsuki, H. Akutsu, and T. Fujiwara. Spectral fitting for signal assignment and structural analysis of uniformly 13C-labeled solid proteins by simulated annealing based on chemical shifts and spin dynamics. J. Biomol. NMR, 38(4):325-339, Aug 2007. DOI 10.1007/s10858-007-9170-x.

[168] A. Cavalli, X. Salvatella, C. M. Dobson, and M. Vendruscolo. Protein structure determination from NMR chemical shifts. Proc. Natl. Ac. Sci. USA, 104(23):9615-9620, Jun 2007. DOI 10.1073/pnas.0610313104.

[169] Y. Shen, O. Lange, F. Delaglio, P. Rossi, J. M. Aramini, G. Liu, A. Eletsky, Y. Wu, K. K. Singarapu, A. Lemak, A. Ignatchenko, C. H. Arrowsmith, T. Szyperski, G. T. Montelione, D. Baker, and A. Bax. Consistent blind protein structure generation from NMR chemical shift data. Proc. Natl. Ac. Sci. USA, 105(12):4685-4690, Mar 2008. DOI 10.1073/pnas. 0800256105 .

[170] P. Güntert. Structure calculation of biological macromolecules from NMR data. Q. Rev. Biophys., 31:145-237, 1998. DOI 10.1017/S0033583598003436.

[171] H. J. C. Berendsen, J. P. M. Postma, W. F. van Gunsteren, A. Dinola, and J. R. Haak. Molecular dynamics with coupling to an external bath. J. Chem. Phys., 81:3684-3690, 1984. DOI 10.1063/1.448118.

[172] S. Kirkpatrick, C. D. Gelatt, and M. P. Vecchi. Optimization by simulated annealing. Science, 220:671-680, 1983. DOI 10.1126/science.220.4598.671.

[173] C. D. Schwieters, J. J. Kuszewski, and G. M. Clore. Using Xplor-NIH for NMR molecular structure determination. Progr. NMR Spectr., 48(1):47-62, 2006. DOI 10.1016/j.pnmrs.2005. 10.001 .

[174] C. D. Schwieters, J. J. Kuszewski, N. Tjandra, and G. M. Clore. The Xplor-NIH NMR molecular structure determination package. J. Magn. Reson., 160(1):65-73, Jan 2003. DOI 10.1016/S1090-7807(02)00014-9.

[175] A. T. Brunger, P. D. Adams, G. M. Clore, W. L. DeLano, P. Gros, R. W. GrosseKunstleve, J. S. Jiang, J. Kuszewski, M. Nilges, N. S. Pannu, R. J. Read, L. M. Rice, T. Simonson, and G. L. Warren. Crystallography \& NMR system: A new software suite for macromolecular structure determination. Acta Cryst. D, 54:905-921, Sep 1998. DOI $10.1107 /$ S0907444998003254.

[176] P. Güntert, C. Mumenthaler, and K. Wüthrich. Torsion angle dynamics for NMR structure calculation with the new program DYANA. J. Mol. Biol., 273:283-298, 1997. DOI 10.1006/ jmbi.1997.1284. 
[177] E. G. Stein, L. M. Rice, and A. T. Brunger. Torsion-angle molecular dynamics as a new efficient tool for NMR structure calculation. J. Magn. Reson., 124:154-164, 1997. DOI 10.1006/jmre.1996.1027.

[178] A. L. Morris, M. W. MacArthur, E. G. Hutchinson, and J. M. Thornton. Stereochemical quality of protein structure coordinates. Proteins, 12(4):345-364, Apr 1992. DOI 1002/prot. 340120407.

[179] G. Vriend. WHAT IF: A molecular modeling and drug design program. J. Mol. Graph., 8: 52-56, 1990. DOI 10.1016/0263-7855(90)80070-V.

[180] R. A. Laskowski, J. A. Rullmannn, M. W. MacArthur, R. Kaptein, and J. M. Thornton. AQUA and PROCHECK-NMR: programs for checking the quality of protein structures solved by NMR. J. Biomol. NMR, 8(4):477-486, Dec 1996. DOI 10.1007/BF00228148.

[181] J. P. Linge, M. A. Williams, C. Spronk, A. M. J. J. Bonvin, and M. Nilges. Refinement of protein structures in explicit solvent. Prot. Struct. Funct. Gen., 50(3):496-506, Feb 2003. DOI 10.1002/prot.10299.

[182] J. P. Linge, M. Habeck, W. Rieping, and M. Nilges. ARIA: automated NOE assignment and NMR structure calculation. Bioinformatics, 19(2):315-316, Jan 2003.

[183] T. Herrmann, P. Güntert, and K. Wüthrich. Protein NMR structure determination with automated NOE assignment using the new software CANDID and the torsion angle dynamics algorithm DYANA. J. Mol. Biol., 319(1):209-227, May 2002. DOI 10.1016/S0022-2836(02) 00241-3.

[184] J. Kuszewski, C. D. Schwieters, D. S. Garrett, R. A. Byrd, N. Tjandra, and G. M. Clore. Completely automated, highly error-tolerant macromolecular structure determination from multidimensional nuclear Overhauser enhancement spectra and chemical shift assignments. J. Am. Chem. Soc., 126(20):6258-6273, May 2004. DOI 10.1021/ja049786h.

[185] A. G. Palmer. NMR characterization of the dynamics of biomacromolecules. Chem. Rev., 104(8):3623-3640, Aug 2004. DOI 10.1021/cr030413t.

[186] M. Blackledge. Recent progress in the study of biomolecular structure and dynamics in solution from residual dipolar couplings. Progr. NMR Spectr., 46(1):23-61, 2005. DOI 10.1016/j.pnmrs.2004.11.002.

[187] J. R. Tolman and K. Ruan. NMR residual dipolar couplings as probes of biomolecular dynamics. Chem. Rev., 106(5):1720-1736, May 2006. DOI 10.1021/cr040429z.

[188] H. W. Spiess. Molecular motion studied by NMR powder spectra. 1. Lineshape calculation for axially-symmetric shielding tensors. Chem. Phys., 6(2):217-225, 1974. DOI 10.1016/ 0301-0104(74)85062-7.

[189] R. G. Griffin. Solid state nuclear magnetic resonance of lipid bilayers. Meth. Enzymol., 72: 108-174, 1981. DOI 10.1016/S0076-6879(81)72010-X. 
[190] D. A. Torchia. Solid-state NMR studies of protein internal dynamics. Annu. Rev. Biophys. Bioeng., 13:125-144, 1984. DOI 10.1146/annurev.bb.13.060184.001013.

[191] S. J. Opella. Protein dynamics by solid state nuclear magnetic resonance. Meth. Enzymol., 131:327-361, 1986. DOI 10.1016/0076-6879(86)31048-6.

[192] A. G. Palmer, J. Williams, and A. E. McDermott. Nuclear magnetic resonance studies of biopolymer dynamics. J. Phys. Chem., 100(31):13293-13310, Aug 1996. DOI 10.1021/ jp9606117.

[193] N. Giraud, A. Böckmann, A. Lesage, F. Penin, M. Blackledge, and L. Emsley. Site-specific backbone dynamics from a crystalline protein by solid-state NMR spectroscopy. J. Am. Chem. Soc., 126(37):11422-11423, Sep 2004. DOI 10.1021/ja046578g.

[194] N. Giraud, M. Blackledge, M. Goldman, A. Böckmann, A. Lesage, F. Penin, and L. Emsley. Quantitative analysis of backbone dynamics in a crystalline protein from nitrogen15 spin-lattice relaxation. J. Am. Chem. Soc., 127(51):18190-18201, Dec 2005. DOI $10.1021 / \mathrm{ja} 055182 \mathrm{~h}$.

[195] W. T. Franks, D. H. Zhou, B. J. Wylie, B. G. Money, D. T. Graesser, H. L. Frericks, G. Sahota, and C. M. Rienstra. Magic-angle spinning solid-state NMR spectroscopy of the beta 1 immunoglobulin binding domain of protein G (GB1): N-15 and C-13 chemical shift assignments and conformational analysis. J. Am. Chem. Soc., 127(35):12291-12305, Sep 2005. DOI 10.1021/ja044497e.

[196] M. Hologne, K. Faelber, A. Diehl, and B. Reif. Characterization of dynamics of perdeuterated proteins by MAS solid-state NMR. J. Am. Chem. Soc., 127(32):11208-11209, Aug 2005. DOI 10.1021/ja0518301.

[197] R. Graf, D. E. Demco, S. Hafner, and H. W. Spiess. Selective residual dipolar couplings in cross-linked elastomers by $1 \mathrm{H}$ double-quantum NMR spectroscopy. Solid State NMR, 12 (2-3):139-152, Sep 1998. DOI 10.1016/S0926-2040(98)00058-7.

[198] L. Gasper, D. E. Demco, and B. Blümich. Proton residual dipolar couplings by NMR magnetization exchange in cross-linked elastomers: determination and imaging. Solid State NMR, 14(2):105-116, Jul 1999. DOI 10.1016/S0926-2040(99)00012-0.

[199] I. Schnell and H. W. Spiess. High-resolution H-1 NMR spectroscopy in the solid state: Very fast sample rotation and multiple-quantum coherences. J. Magn. Reson., 151(2):153-227, Aug 2001. DOI 10.1006/jmre.2001.2336.

[200] D. P. Raleigh, F. Creuzet, S. K. D. Gupta, M. H. Levitt, and R. G. Griffin. Measurement of internuclear distances in polycrystalline solids: Rotationally enhanced transfer of nuclear spin magnetization. J. Am. Chem. Soc., 111(12):4502-4503, Jun 1989. DOI 10.1021/ja00194a057.

[201] L. Frydman, S. Vallabhaneni, Y. K. Lee, and L. Emsley. Solid-state dynamic processes in complex systems analyzed by two-dimensional isotropic-anisotropic correlation nuclear magnetic resonance. J. Chem. Phys., 101(1):111-117, 1994. DOI 10.1063/1.468185. 
[202] J. D. van Beek. matNMR: a flexible toolbox for processing, analyzing and visualizing magnetic resonance data in Matlab. J. Magn. Reson., 187(1):19-26, Jul 2007. DOI 10.1016/j.jmr.2007.03.017. URL http://matnmr. sourceforge.net/.

[203] D. A. Torchia and A. Szabo. The information-content of powder lineshapes in the fast motion limit. J. Magn. Reson., 64(1):135-141, 1985.

[204] O. C. Andronesi, J. R. Pfeifer, L. Al-Momani, S. Ozdirekcan, D. T. S. Rijkers, B. Angerstein, S. Luca, U. Koert, J. A. Killian, and M. Baldus. Probing membrane protein orientation and structure using fast magic-angle-spinning solid-state NMR. J. Biomol. NMR, 30(3):253-265, Nov 2004. DOI 10.1007/s10858-004-3452-3.

[205] V. B. Cheng, H. H. Suzukawa, and M. Wolfsberg. Investigations of a nonrandom numerical method for multidimensional integration. J. Chem. Phys., 59(8):3992-3999, 1973. DOI $10.1063 / 1.1680590$.

[206] B. H. Meier. Polarization transfer and spin diffusion in solid-state NMR. In W. S. Warren, editor, Advances in Magnetic and Optical Resonance, volume 18, pages 1-116. Academic Press, New York, 1994.

[207] M. Baldus and B. H. Meier. Broadband polarization transfer under magic-angle spinning: Application to total through-space-correlation NMR spectroscopy. J. Magn. Reson., 128(2): 172-193, Oct 1997. DOI 10.1006/jmre.1997.1219.

[208] S. Dusold and A. Sebald. Double-quantum filtration under rotational-resonance conditions: numerical simulations and experimental results. J. Magn. Reson., 145(2):340-356, Aug 2000. DOI 10.1006/jmre.2000.2118.

[209] O. F. Lange, N.-A. Lakomek, C. Farès, G. F. Schröder, K. F. A. Walter, S. Becker, J. Meiler, H. Grubmüller, C. Griesinger, and B. L. de Groot. Recognition dynamics up to microseconds revealed from an RDC-derived ubiquitin ensemble in solution. Science, 320(5882):1471-1475, Jun 2008. DOI 10.1126/science.1157092.

[210] M. Baldus. Magnetic resonance in the solid state: applications to protein folding, amyloid fibrils and membrane proteins. Eur. Biophys. J., 36(Suppl. 1):37-48, May 2007. DOI 10. 1007/s00249-007-0174-y.

[211] A. E. McDermott. Structural and dynamic studies of proteins by solid-state NMR spectroscopy: rapid movement forward. Curr. Opin. Struct. Biol., 14(5):554-561, Oct 2004. DOI 10.1016/j.sbi.2004.09.007.

[212] H. B. R. Cole and D. A. Torchia. An NMR study of the backbone dynamics of staphylococcal nuclease in the crystalline state. Chem. Phys., 158(2-3):271-281, Dec 1991. DOI 10.1016/ 0301-0104(91)87071-3.

[213] R. W. Martin and K. W. Zilm. Preparation of protein nanocrystals and their characterization by solid state NMR. J. Magn. Reson., 165(1):162-174, Nov 2003. 
[214] A. Böckmann, A. Lange, A. Galinier, S. Luca, N. Giraud, M. Juy, H. Heise, R. Montserret, F. Penin, and M. Baldus. Solid state NMR sequential resonance assignments and conformational analysis of the $2 \times 10.4 \mathrm{kDa}$ dimeric form of the Bacillus subtilis protein Crh. $J$. Biomol. NMR, 27(4):323-339, Dec 2003. DOI 10.1023/A:1025820611009.

[215] P. Robustelli, A. Cavalli, and M. Vendruscolo. Determination of protein structures in the solid state from NMR chemical shifts. Structure, 16(12):1764-1769, Dec 2008. DOI 10.1016/ j.str.2008.10.016.

[216] A. Hershko and A. Ciechanover. The ubiquitin system. Annu. Rev. Biochem., 67:425-479, 1998. DOI 10.1146/annurev.biochem.67.1.425.

[217] C. M. Pickart and M. J. Eddins. Ubiquitin: structures, functions, mechanisms. Biochim. Biophys. Acta, 1695(1-3):55-72, Nov 2004. DOI 10.1016/j.bbamcr.2004.09.019.

[218] N. Tjandra, S. E. Feller, R. W. Pastor, and A. Bax. Rotational diffusion anisotropy of human ubiquitin from N-15 NMR relaxation. J. Am. Chem. Soc., 117(50):12562-12566, Dec 1995. DOI $10.1021 / \mathrm{ja} 00155 \mathrm{a} 020$.

[219] G. Cornilescu, J. L. Marquardt, M. Ottiger, and A. Bax. Validation of protein structure from anisotropic carbonyl chemical shifts in a dilute liquid crystalline phase. J. Am. Chem. Soc., 120(27):6836-6837, Jul 1998. DOI 10.1021/ja9812610.

[220] W. Peti, J. Meiler, R. Bruschweiler, and C. Griesinger. Model-free analysis of protein backbone motion from residual dipolar couplings. J. Am. Chem. Soc., 124(20):5822-5833, May 2002. DOI $10.1021 /$ ja011883c.

[221] S. Vijay-Kumar, C. E. Bugg, and W. J. Cook. Structure of ubiquitin refined at $1.8 \AA$ resolution. J. Mol. Biol., 194(3):531-544, Apr 1987. DOI 10.1016/0022-2836(87)90679-6.

[222] H. Heise, F. H. Köhler, and X. Xie. Solid-state NMR spectroscopy of paramagnetic metallocenes. J. Magn. Reson., 150(2):198-206, Jun 2001. DOI 10.1006/jmre.2001.2343.

[223] T. D. Goddard and D. G. Kneller. SPARKY 3. URL http://www.cgl.ucsf .edu/home/ sparky/. University of California, San Francisco.

[224] R. Koradi, M. Billeter, and K. Wüthrich. MOLMOL: A program for display and analysis of macromolecular structures. J. Mol. Graph., 14(1):51, Feb 1996. DOI 10.1016/0263-7855(96) 00009-4.

[225] T. I. Igumenova, A. E. McDermott, K. W. Zilm, R. W. Martin, E. K. Paulson, and A. J. Wand. Assignments of carbon NMR resonances for microcrystalline ubiquitin. J. Am. Chem. Soc., 126(21):6720-6727, Jun 2004. DOI 10.1021/ja030547o.

[226] N.-A. Lakomek, K. F. A. Walter, C. Farès, O. F. Lange, B. L. de Groot, H. Grubmüller, R. Brüschweiler, A. Munk, S. Becker, J. Meiler, and C. Griesinger. Self-consistent residual dipolar coupling based model-free analysis for the robust determination of nanosecond to microsecond protein dynamics. J. Biomol. NMR, 41(3):139-155, Jul 2008. DOI 10.1007/ s10858-008-9244-4. 
[227] C. Gardiennet, A. Loquet, M. Etzkorn, H. Heise, M. Baldus, and A. Böckmann. Structural constraints for the Crh protein from solid-state NMR experiments. J. Biomol. NMR, 40(4): 239-250, Apr 2008. DOI 10.1007/s10858-008-9229-3.

[228] F. Massi, M. J. Grey, and A. G. Palmer. Microsecond timescale backbone conformational dynamics in ubiquitin studied with NMR R-1p relaxation experiments. Prot. Sci., 14(3): 735-742, 2005. DOI 10.1110/ps.041139505.

[229] A. Majumdar and R. Ghose. Probing slow backbone dynamics in proteins using TROSYbased experiments to detect cross-correlated time-modulation of isotropic chemical shifts. $J$. Biomol. NMR, 28(3):213-227, 2004. DOI 10.1023/B:JNMR.0000013705.98136.99.

[230] L. Hicke, H. L. Schubert, and C. P. Hill. Ubiquitin-binding domains. Nat. Rev. Mol. Cell Biol., 6(8):610-621, Aug 2005. DOI 10.1038/nrm1701.

[231] N.-A. Lakomek, C. Farès, S. Becker, T. Carlomagno, J. Meiler, and C. Griesinger. Side-chain orientation and hydrogen-bonding imprint supra-tau(c) motion on the protein backbone of ubiquitin. Angew. Chem. Int. Ed. Engl., 44(47):7776-7778, Dec 2005. DOI 10.1002/anie. 200502573.

[232] H. Schrempf, O. Schmidt, R. Kümmerlen, S. Hinnah, D. Müller, M. Betzler, T. Steinkamp, and R. Wagner. A prokaryotic potassium ion channel with two predicted transmembrane segments from Streptomyces lividans. EMBO J., 14(21):5170-5178, Nov 1995.

[233] M. LeMasurier, L. Heginbotham, and C. Miller. KcsA: it's a potassium channel. J. Gen. Physiol., 118(3):303-314, Sep 2001. DOI 10.1085/jgp.118.3.303.

[234] L. G. Cuello, J. G. Romero, D. M. Cortes, and E. Perozo. pH-dependent gating in the Streptomyces lividans $\mathrm{K}^{+}$channel. Biochemistry, 37(10):3229-3236, Mar 1998. DOI 10. 1021/bi972997x.

[235] J. F. Cordero-Morales, L. G. Cuello, Y. Zhao, V. Jogini, D. M. Cortes, B. Roux, and E. Perozo. Molecular determinants of gating at the potassium-channel selectivity filter. Nat. Struct. Mol. Biol., 13(4):311-318, Apr 2006. DOI 10.1038/nsmb1069.

[236] D. M. Cortes and E. Perozo. Structural dynamics of the Streptomyces lividans $\mathrm{K}^{+}$channel (SKC1): oligomeric stoichiometry and stability. Biochemistry, 36(33):10343-10352, Aug 1997. DOI 10.1021/bi971018y.

[237] D. M. Cortes, L. G. Cuello, and E. Perozo. Molecular architecture of full-length KcsA: role of cytoplasmic domains in ion permeation and activation gating. J. Gen. Physiol., 117(2): 165-180, Feb 2001. DOI 10.1085/jgp.117.2.165.

[238] S. Y. Noskov, S. Bernèche, and B. Roux. Control of ion selectivity in potassium channels by electrostatic and dynamic properties of carbonyl ligands. Nature, 431(7010):830-834, Oct 2004. DOI 10.1038/nature02943. 
[239] S. W. Lockless, M. Zhou, and R. MacKinnon. Structural and thermodynamic properties of selective ion binding in a $\mathrm{K}^{+}$channel. PLoS Biol., 5(5):e121, May 2007. DOI 10.1371/ journal.pbio.0050121.

[240] C. Legros, C. Schulze, M. L. Garcia, P. E. Bougis, M.-F. Martin-Eauclaire, and O. Pongs. Engineering-specific pharmacological binding sites for peptidyl inhibitors of potassium channels into KcsA. Biochemistry, 41(51):15369-15375, Dec 2002. DOI 10.1021/bi026264a.

[241] A. Lange, K. Giller, O. Pongs, S. Becker, and M. Baldus. Two-dimensional solid-state NMR applied to a chimeric potassium channel. J. Recept. Signal Transduct. Res., 26(5-6):379-393, 2006. DOI 10.1080/10799890600932188.

[242] K. A. Baker, C. Tzitzilonis, W. Kwiatkowski, S. Choe, and R. Riek. Conformational dynamics of the KcsA potassium channel governs gating properties. Nat. Struct. Mol. Biol., 14(11): 1089-1095, Nov 2007. DOI 10.1038/nsmb1311.

[243] A. Sali and T. L. Blundell. Comparative protein modelling by satisfaction of spatial restraints. J. Mol. Biol., 234(3):779-815, Dec 1993. DOI 10.1006/jmbi.1993.1626.

[244] W. L. DeLano. The PyMOL Molecular Graphics System. URL http://www.pymol.org. DeLano Scientific, Palo Alto, CA, USA, 2002.

[245] D. S. Wishart and B. D. Sykes. Chemical-shifts as a tool for structure determination. Nucl. Magn. Reson. C, 239:363-392, 1994.

[246] V. P. T. Pau, Y. Zhu, Z. Yuchi, Q. Q. Hoang, and D. S. C. Yang. Characterization of the C-terminal domain of a potassium channel from Streptomyces lividans (KcsA). J. Biol. Chem., 282(40):29163-29169, Oct 2007. DOI 10.1074/jbc.M703277200.

[247] K. Takeuchi, H. Takahashi, S. Kawano, and I. Shimada. Identification and characterization of the slowly exchanging $\mathrm{pH}$-dependent conformational rearrangement in KcsA. J. Biol. Chem., 282(20):15179-15186, May 2007. DOI 10.1074/jbc.M608264200.

[248] J. H. Chill, J. M. Louis, F. Delaglio, and A. Bax. Local and global structure of the monomeric subunit of the potassium channel KcsA probed by NMR. Biochim. Biophys. Acta, 1768(12): 3260-3270, Dec 2007. DOI 10.1016/j.bbamem.2007.08.006.

[249] F. I. Valiyaveetil, Y. Zhou, and R. MacKinnon. Lipids in the structure, folding, and function of the KcsA K ${ }^{+}$channel. Biochemistry, 41(35):10771-10777, Sep 2002. DOI 10.1021/bi026215y.

[250] K. Varga, L. Tian, and A. E. McDermott. Solid-state NMR study and assignments of the KcsA potassium ion channel of S. lividans. Biochim. Biophys. Acta, 1774(12):1604-1613, Dec 2007.

[251] J. H. Chill, J. M. Louis, J. L. Baber, and A. Bax. Measurement of ${ }^{15} \mathrm{~N}$ relaxation in the detergent-solubilized tetrameric KcsA potassium channel. J. Biomol. NMR, 36(2):123-136, Oct 2006. DOI 10.1007/s10858-006-9071-4. 
[252] N. Trbovic, C. Klammt, A. Koglin, F. Löhr, F. Bernhard, and V. Dötsch. Efficient strategy for the rapid backbone assignment of membrane proteins. J. Am. Chem. Soc., 127(39): 13504-13505, Oct 2005. DOI 10.1021/ja0540270.

[253] L. K. Tamm and B. Y. Liang. NMR of membrane proteins in solution. Progr. NMR Spectr., 48(4):201-210, 2006.

[254] R. Mahalakshmi, C. M. Franzin, J. Choi, and F. M. Marassi. NMR structural studies of the bacterial outer membrane protein OmpX in oriented lipid bilayer membranes. Biochim. Biophys. Acta, 1768(12):3216-3224, Dec 2007. DOI 10.1016/j.bbamem.2007.08.008.

[255] B. P. Bean. The action potential in mammalian central neurons. Nat. Rev. Neurosci., 8(6): 451-465, Jun 2007. DOI 10.1038/nrn2148.

[256] J. G. Starkus, L. Kuschel, M. D. Rayner, and S. H. Heinemann. Ion conduction through C-type inactivated shaker channels. J. Gen. Physiol., 110(5):539-550, Nov 1997. DOI 10. 1085/jgp.110.5.539.

[257] J. G. Starkus, L. Kuschel, M. D. Rayner, and S. H. Heinemann. Macroscopic $\mathrm{Na}^{+}$currents in the "nonconducting" Shaker potassium channel mutant W434F. J. Gen. Physiol., 112(1): 85-93, Jul 1998. DOI 10.1085/jgp.112.1.85.

[258] F. I. Valiyaveetil, M. Leonetti, T. W. Muir, and R. MacKinnon. Ion selectivity in a semisynthetic $\mathrm{K}^{+}$channel locked in the conductive conformation. Science, 314(5801):1004-1007, Nov 2006. DOI 10.1126/science.1133415.

[259] S. Chakrapani, J. F. Cordero-Morales, and E. Perozo. A quantitative description of KcsA gating I: macroscopic currents. J. Gen. Physiol., 130(5):465-478, Nov 2007. DOI 10.1085/ jgp.200709843.

[260] S. Chakrapani, J. F. Cordero-Morales, and E. Perozo. A quantitative description of KcsA gating II: single-channel currents. J. Gen. Physiol., 130(5):479-496, Nov 2007. DOI 10.1085/ jgp.200709844.

[261] S. Bernèche and B. Roux. A gate in the selectivity filter of potassium channels. Structure, 13(4):591-600, Apr 2005. DOI 10.1016/j.str.2004.12.019.

[262] J. F. Cordero-Morales, L. G. Cuello, and E. Perozo. Voltage-dependent gating at the KcsA selectivity filter. Nat. Struct. Mol. Biol., 13(4):319-322, Apr 2006. DOI 10.1038/nsmb1070.

[263] J. F. Cordero-Morales, V. Jogini, A. Lewis, V. Vásquez, D. M. Cortes, B. Roux, and E. Perozo. Molecular driving forces determining potassium channel slow inactivation. Nat. Struct. Mol. Biol., 14(11):1062-1069, Nov 2007. DOI 10.1038/nsmb1309.

[264] R. Blunck, J. F. Cordero-Morales, L. G. Cuello, E. Perozo, and F. Bezanilla. Detection of the opening of the bundle crossing in KcsA with fluorescence lifetime spectroscopy reveals the existence of two gates for ion conduction. J. Gen. Physiol., 128(5):569-581, Nov 2006. DOI 10.1085/jgp.200609638. 
[265] S. N. Gradl, J. P. Felix, E. Y. Isacoff, M. L. Garcia, and D. Trauner. Protein surface recognition by rational design: nanomolar ligands for potassium channels. J. Am. Chem. Soc., 125(42):12668-12669, Oct 2003. DOI 10.1021/ja036155z.

[266] L. Yu, C. Sun, D. Song, J. Shen, N. Xu, A. Gunasekera, P. J. Hajduk, and E. T. Olejniczak. Nuclear magnetic resonance structural studies of a potassium channel-charybdotoxin complex. Biochemistry, 44(48):15834-15841, Dec 2005. DOI 10.1021/bi051656d.

[267] L. Heginbotham, M. LeMasurier, L. Kolmakova-Partensky, and C. Miller. Single Streptomyces lividans $\mathrm{K}^{+}$channels: functional asymmetries and sidedness of proton activation. $J$. Gen. Physiol., 114(4):551-560, Oct 1999. DOI 10.1085/jgp.114.4.551.

[268] M. J. Lenaeus, M. Vamvouka, P. J. Focia, and A. Gross. Structural basis of TEA blockade in a model potassium channel. Nat. Struct. Mol. Biol., 12(5):454-459, May 2005. DOI $10.1038 /$ nsmb929.

[269] Y. Jiang, A. Lee, J. Chen, V. Ruta, M. Cadene, B. T. Chait, and R. MacKinnon. X-ray structure of a voltage-dependent $\mathrm{K}^{+}$channel. Nature, 423(6935):33-41, May 2003. DOI 10.1038/nature01580.

[270] L. Kiss and S. J. Korn. Modulation of C-type inactivation by $\mathrm{K}^{+}$at the potassium channel selectivity filter. Biophys. J., 74(4):1840-1849, Apr 1998.

[271] P. Keim, R. A. Vigna, J. S. Morrow, R. C. Marshall, and F. R. Gurd. Carbon 13 nuclear magnetic resonance of pentapeptides of glycine containing central residues of serine, threonine, aspartic and glutamic acids, asparagine, and glutamine. J. Biol. Chem., 248(22): 7811-7818, Nov 1973.

[272] Z. T. Gu and A. McDermott. Chemical shielding anisotropy of protonated and deprotonated carboxylates in amino-acids. J. Am. Chem. Soc., 115(10):4282-4285, 1993. DOI 10.1021/ ja00063a051.

[273] Y. S. Liu, P. Sompornpisut, and E. Perozo. Structure of the KcsA channel intracellular gate in the open state. Nat. Struct. Biol., 8(10):883-887, Oct 2001. DOI 10.1038/nsb1001-883.

[274] B. L. Kelly and A. Gross. Potassium channel gating observed with site-directed mass tagging. Nat. Struct. Biol., 10(4):280-284, 2003. DOI 10.1038/nsb908.

[275] G. Panyi and C. Deutsch. Cross talk between activation and slow inactivation gates of Shaker potassium channels. J. Gen. Physiol., 128(5):547-559, Nov 2006. DOI 10.1085/jgp. 200609644 .

[276] T. Baukrowitz and G. Yellen. Modulation of $\mathrm{K}^{+}$current by frequency and external $\left[\mathrm{K}^{+}\right]$: a tale of two inactivation mechanisms. Neuron, 15(4):951-960, Oct 1995. DOI 10.1016/ 0896-6273(95)90185-X.

[277] T. Baukrowitz and G. Yellen. Use-dependent blockers and exit rate of the last ion from the multi-ion pore of a $\mathrm{K}^{+}$channel. Science, 271(5249):653-656, Feb 1996. DOI 10.1126/science. 271.5249 .653 . 
[278] G. Panyi and C. Deutsch. Probing the cavity of the slow inactivated conformation of Shaker potassium channels. J. Gen. Physiol., 129(5):403-418, May 2007. DOI 10.1085/ jgp.200709758.

[279] A. N. Thompson, D. J. Posson, P. V. Parsa, and C. M. Nimigean. Molecular mechanism of pH sensing in KcsA potassium channels. Proc. Natl. Ac. Sci. USA, 105(19):6900-6905, May 2008. DOI 10.1073/pnas.0800873105.

[280] E. Perozo, D. M. Cortes, and L. G. Cuello. Structural rearrangements underlying K ${ }^{+}$-channel activation gating. Science, 285(5424):73-78, Jul 1999. DOI 10.1126/science.285.5424.73.

[281] J. Roeper, C. Lorra, and O. Pongs. Frequency-dependent inactivation of mammalian A-type $\mathrm{K}^{+}$channel Kv1.4 regulated by $\mathrm{Ca}^{2+} /$ calmodulin-dependent protein kinase. J. Neurosci., 17(10):3379-3391, May 1997.

[282] R. S. Hurst, M. J. Roux, L. Toro, and E. Stefani. External barium influences the gating charge movement of Shaker potassium channels. Biophys. J., 72(1):77-84, Jan 1997.

[283] E. Zakharian and R. N. Reusch. Streptomyces lividans potassium channel KcsA is regulated by the potassium electrochemical gradient. Biochem. Biophys. Res. Commun., 316(2):429436, Apr 2004. DOI 10.1016/j.bbrc.2004.02.069.

[284] M. L. Renart, F. N. Barrera, M. L. Molina, J. A. Encinar, J. A. Poveda, A. M. Fernández, J. Gómez, and J. M. González-Ros. Effects of conducting and blocking ions on the structure and stability of the potassium channel KcsA. J. Biol. Chem., 281(40):29905-29915, Oct 2006. DOI 10.1074/jbc.M602636200.

[285] M. N. Krishnan, J.-P. Bingham, S. H. Lee, P. Trombley, and E. Moczydlowski. Functional role and affinity of inorganic cations in stabilizing the tetrameric structure of the KcsA K ${ }^{+}$ channel. J. Gen. Physiol., 126(3):271-283, Sep 2005. DOI 10.1085/jgp.200509323.

[286] F. N. Barrera, M. L. Renart, J. A. Poveda, B. de Kruijff, J. A. Killian, and J. M. GonzálezRos. Protein self-assembly and lipid binding in the folding of the potassium channel KcsA. Biochemistry, 47(7):2123-2133, Feb 2008. DOI 10.1021/bi700778c.

[287] P. Marius, M. Zagnoni, M. E. Sandison, J. M. East, H. Morgan, and A. G. Lee. Binding of anionic lipids to at least three nonannular sites on the potassium channel KcsA is required for channel opening. Biophys. J., 94(5):1689-1698, Mar 2008. DOI 10.1529/biophysj.107.117507.

[288] T. W. Claydon, M. Vaid, S. Rezazadeh, D. C. H. Kwan, S. J. Kehl, and D. Fedida. A direct demonstration of closed-state inactivation of $\mathrm{K}^{+}$channels at low pH. J. Gen. Physiol., 129 (5):437-455, May 2007. DOI 10.1085/jgp.200709774.

[289] S. D. Demo and G. Yellen. The inactivation gate of the Shaker $\mathrm{K}^{+}$channel behaves like an open-channel blocker. Neuron, 7(5):743-753, Nov 1991. DOI 10.1016/0896-6273(91)90277-7.

[290] W. N. Zagotta and R. W. Aldrich. Voltage-dependent gating of Shaker A-type potassium channels in Drosophila muscle. J. Gen. Physiol., 95(1):29-60, Jan 1990. DOI 10.1085/jgp. 95.1.29. 
[291] E. M. Ogielska and R. W. Aldrich. Functional consequences of a decreased potassium affinity in a potassium channel pore. Ion interactions and C-type inactivation. J. Gen. Physiol., 113 (2):347-358, Feb 1999. DOI 10.1085/jgp.113.2.347.

[292] L. Kiss, D. Immke, J. LoTurco, and S. J. Korn. The interaction of $\mathrm{Na}^{+}$and $\mathrm{K}^{+}$in voltagegated potassium channels. Evidence for cation binding sites of different affinity. J. Gen. Physiol., 111(2):195-206, Feb 1998. DOI 10.1085/jgp.111.2.195.

[293] H. T. Orr. Beyond the Qs in the polyglutamine diseases. Genes Dev., 15(8):925-932, Apr 2001. DOI 10.1101/gad. 888401.

[294] E. E. Wanker. Protein aggregation and pathogenesis of Huntington's disease: mechanisms and correlations. Biol. Chem., 381(9-10):937-942, 2000. DOI 10.1515/BC.2000.114.

[295] J. F. Gusella and M. E. MacDonald. Molecular genetics: unmasking polyglutamine triggers in neurodegenerative disease. Nat. Rev. Neurosci., 1(2):109-115, Nov 2000. DOI 10.1038/ $\operatorname{nrd} 1077$.

[296] S. Chen, V. Berthelier, J. B. Hamilton, B. O'Nuallain, and R. Wetzel. Amyloid-like features of polyglutamine aggregates and their assembly kinetics. Biochemistry, 41(23):7391-7399, Jun 2002. DOI 10.1021/bi011772q.

[297] W. Yang, J. R. Dunlap, R. B. Andrews, and R. Wetzel. Aggregated polyglutamine peptides delivered to nuclei are toxic to mammalian cells. Hum. Mol. Genet., 11(23):2905-2917, Nov 2002. DOI 10.1093/hmg/11.23.2905.

[298] Y. Nagai, T. Inui, H. A. Popiel, N. Fujikake, K. Hasegawa, Y. Urade, Y. Goto, H. Naiki, and T. Toda. A toxic monomeric conformer of the polyglutamine protein. Nat. Struct. Mol. Biol., 14(4):332-340, Apr 2007. DOI 10.1038/nsmb1215.

[299] T. Takahashi, S. Kikuchi, S. Katada, Y. Nagai, M. Nishizawa, and O. Onodera. Soluble polyglutamine oligomers formed prior to inclusion body formation are cytotoxic. Hum. Mol. Genet., 17(3):345-356, Feb 2008. DOI 10.1093/hmg/ddm311.

[300] S. Chen, F. A. Ferrone, and R. Wetzel. Huntington's disease age-of-onset linked to polyglutamine aggregation nucleation. Proc. Natl. Ac. Sci. USA, 99(18):11884-11889, Sep 2002. DOI 10.1073/pnas.182276099.

[301] J. Shorter and S. Lindquist. Prions as adaptive conduits of memory and inheritance. Nat. Rev. Genet., 6(6):435-450, Jun 2005. DOI 10.1038/nrg1616.

[302] M. D. Michelitsch and J. S. Weissman. A census of glutamine/asparagine-rich regions: implications for their conserved function and the prediction of novel prions. Proc. Natl. Ac. Sci. USA, 97(22):11910-11915, Oct 2000. DOI 10.1073/pnas.97.22.11910.

[303] M. F. Perutz. Glutamine repeats as polar zippers: their role in inherited neurodegenerative disease. Mol. Med., 1(7):718-721, Nov 1995. 
[304] D. Sharma, L. M. Shinchuk, H. Inouye, R. Wetzel, and D. A. Kirschner. Polyglutamine homopolymers having 8-45 residues form slablike beta-crystallite assemblies. Proteins, 61 (2):398-411, Nov 2005. DOI 10.1002/prot.20602.

[305] L. Esposito, A. Paladino, C. Pedone, and L. Vitagliano. Insights into structure, stability, and toxicity of monomeric and aggregated polyglutamine models from molecular dynamics simulations. Biophys. J., 94(10):4031-4040, May 2008. DOI 10.1529/biophysj.107.118935.

[306] D. Zanuy, K. Gunasekaran, A. M. Lesk, and R. Nussinov. Computational study of the fibril organization of polyglutamine repeats reveals a common motif identified in beta-helices. $J$. Mol. Biol., 358(1):330-345, Apr 2006. DOI 10.1016/j.jmb.2006.01.070.

[307] U. Baxa, R. B. Wickner, A. C. Steven, D. E. Anderson, L. N. Marekov, W.-M. Yau, and R. Tycko. Characterization of beta-sheet structure in Ure2p1-89 yeast prion fibrils by solidstate nuclear magnetic resonance. Biochemistry, 46(45):13149-13162, Nov 2007. DOI 10. 1021/bi700826b.

[308] F. Shewmaker, R. B. Wickner, and R. Tycko. Amyloid of the prion domain of Sup35p has an in-register parallel beta-sheet structure. Proc. Natl. Ac. Sci. USA, 103(52):19754-19759, Dec 2006. DOI 10.1073/pnas.0609638103.

[309] M. F. Perutz, J. T. Finch, J. Berriman, and A. Lesk. Amyloid fibers are water-filled nanotubes. Proc. Natl. Ac. Sci. USA, 99(8):5591-5595, Apr 2002. DOI 10.1073/pnas.042681399.

[310] P. Sikorski and E. Atkins. New model for crystalline polyglutamine assemblies and their connection with amyloid fibrils. Biomacromolecules, 6(1):425-432, 2005. DOI 10.1021/ bm0494388.

[311] M. Stork, A. Giese, H. A. Kretzschmar, and P. Tavan. Molecular dynamics simulations indicate a possible role of parallel beta-helices in seeded aggregation of poly-Gln. Biophys. J., 88(4):2442-2451, Apr 2005. DOI 10.1529/biophysj.104.052415.

[312] S. Chen and R. Wetzel. Solubilization and disaggregation of polyglutamine peptides. Prot. Sci., 10(4):887-891, Apr 2001. DOI 10.1110/ps.42301.

[313] M. H. Levitt, A. C. Kolbert, A. Bielecki, and D. J. Ruben. High-resolution 1H NMR in solids with frequency-switched multiple-pulse sequences. Solid State NMR, 2(4):151-163, Sep 1993. DOI 10.1016/0926-2040(93)90021-E.

[314] S. Moon and D. A. Case. A new model for chemical shifts of amide hydrogens in proteins. J. Biomol. NMR, 38(2):139-150, Jun 2007. DOI 10.1007/s10858-007-9156-8.

[315] P. C. A. van der Wel, J. R. Lewandowski, and R. G. Griffin. Solid-state NMR study of amyloid nanocrystals and fibrils formed by the peptide GNNQQNY from yeast prion protein Sup35p. J. Am. Chem. Soc., 129(16):5117-5130, Apr 2007. DOI 10.1021/ja068633m.

[316] G. Zandomeneghi, M. R. H. Krebs, M. G. McCammon, and M. Fändrich. FTIR reveals structural differences between native beta-sheet proteins and amyloid fibrils. Prot. Sci., 13 (12):3314-3321, Dec 2004. DOI 10.1110/ps.041024904. 
[317] J. L. Jiménez, E. J. Nettleton, M. Bouchard, C. V. Robinson, C. M. Dobson, and H. R. Saibil. The protofilament structure of insulin amyloid fibrils. Proc. Natl. Ac. Sci. USA, 99 (14):9196-9201, Jul 2002. DOI 10.1073/pnas.142459399.

[318] X. P. Xu and D. A. Case. Automated prediction of 15N, 13Calpha, 13Cbeta and 13C' chemical shifts in proteins using a density functional database. J. Biomol. NMR, 21(4): 321-333, Dec 2001. DOI 10.1023/A:1013324104681.

[319] M. E. Villegas, J. A. Vila, and H. A. Scheraga. Effects of side-chain orientation on the 13C chemical shifts of antiparallel beta-sheet model peptides. J. Biomol. NMR, 37(2):137-146, Feb 2007. DOI 10.1007/s10858-006-9118-6.

[320] M. J. McGregor, S. A. Islam, and M. J. Sternberg. Analysis of the relationship between side-chain conformation and secondary structure in globular proteins. J. Mol. Biol., 198(2): 295-310, Nov 1987. DOI 10.1016/0022-2836(87)90314-7.

[321] A. V. Kajava, U. Baxa, R. B. Wickner, and A. C. Steven. A model for Ure2p prion filaments and other amyloids: the parallel superpleated beta-structure. Proc. Natl. Ac. Sci. USA, 101 (21):7885-7890, May 2004. DOI 10.1073/pnas.0402427101.

[322] H. Heise. Solid-state NMR spectroscopy of amyloid proteins. ChemBioChem, 9(2):179-189, Jan 2008. DOI 10.1002/cbic.200700630.

[323] C. P. Jaroniec, C. E. MacPhee, N. S. Astrof, C. M. Dobson, and R. G. Griffin. Molecular conformation of a peptide fragment of transthyretin in an amyloid fibril. Proc. Natl. Ac. Sci. USA, 99(26):16748-16753, Dec 2002. DOI 10.1073/pnas.252625999.

[324] W. Kabsch and C. Sander. Dictionary of protein secondary structure: pattern recognition of hydrogen-bonded and geometrical features. Biopolymers, 22(12):2577-2637, Dec 1983. DOI 10.1002/bip.360221211.

[325] K. A. Dill. Dominant forces in protein folding. Biochemistry, 29(31):7133-7155, Aug 1990. DOI 10.1021/bi00483a001.

[326] A. K. Thakur and R. Wetzel. Mutational analysis of the structural organization of polyglutamine aggregates. Proc. Natl. Ac. Sci. USA, 99(26):17014-17019, Dec 2002. DOI $10.1073 /$ pnas.252523899.

[327] J. C. C. Chan, N. A. Oyler, W.-M. Yau, and R. Tycko. Parallel beta-sheets and polar zippers in amyloid fibrils formed by residues 10-39 of the yeast prion protein Ure2p. Biochemistry, 44(31):10669-10680, Aug 2005. DOI 10.1021/bi050724t.

[328] A. T. Petkova, W.-M. Yau, and R. Tycko. Experimental constraints on quaternary structure in Alzheimer's beta-amyloid fibrils. Biochemistry, 45(2):498-512, Jan 2006. DOI 10.1021/ bi051952q.

[329] P. T. Lansbury, P. R. Costa, J. M. Griffiths, E. J. Simon, M. Auger, K. J. Halverson, D. A. Kocisko, Z. S. Hendsch, T. T. Ashburn, R. G. S. Spencer, B. Tidor, and R. G. Griffin. Structural model for the beta-amyloid fibril based on interstrand alignment of an antiparallel 
sheet comprising a C-terminal peptide. Nat. Struct. Biol., 2(11):990-998, Nov 1995. DOI 10.1038/nsb1195-990.

[330] A. T. Petkova, G. Buntkowsky, F. Dyda, R. D. Leapman, W.-M. Yau, and R. Tycko. Solid state NMR reveals a $\mathrm{pH}$-dependent antiparallel beta-sheet registry in fibrils formed by a betaamyloid peptide. J. Mol. Biol., 335(1):247-260, Jan 2004. DOI 10.1016/j.jmb.2003.10.044.

[331] Z. Bu, Y. Shi, D. J. E. Callaway, and R. Tycko. Molecular alignment within beta-sheets in Abeta(14-23) fibrils: solid-state NMR experiments and theoretical predictions. Biophys. J., 92(2):594-602, Jan 2007. DOI 10.1529/biophysj.106.091017.

[332] P. Sikorski, E. D. T. Atkins, and L. C. Serpell. Structure and texture of fibrous crystals formed by Alzheimer's Abeta(11-25) peptide fragment. Structure, 11(8):915-926, Aug 2003. DOI 10.1016/S0969-2126(03)00149-7.

[333] D. J. Gordon, J. J. Balbach, R. Tycko, and S. C. Meredith. Increasing the amphiphilicity of an amyloidogenic peptide changes the beta-sheet structure in the fibrils from antiparallel to parallel. Biophys. J., 86(1 Pt 1):428-434, Jan 2004.

[334] S. D. Khare, F. Ding, K. N. Gwanmesia, and N. V. Dokholyan. Molecular origin of polyglutamine aggregation in neurodegenerative diseases. PLoS Comp. Biol., 1(3):230-235, Aug 2005. DOI 10.1371/journal.pcbi.0010030.

[335] J. Jenkins and R. Pickersgill. The architecture of parallel beta-helices and related folds. Prog. Biophys. Mol. Biol., 77(2):111-175, Oct 2001. DOI 10.1016/S0079-6107(01)00013-X.

[336] A. M. Bhattacharyya, A. K. Thakur, and R. Wetzel. Polyglutamine aggregation nucleation: thermodynamics of a highly unfavorable protein folding reaction. Proc. Natl. Ac. Sci. USA, 102(43):15400-15405, Oct 2005. DOI 10.1073/pnas.0501651102.

[337] R. Truant, R. S. Atwal, C. Desmond, L. Munsie, and T. Tran. Huntington's disease: revisiting the aggregation hypothesis in polyglutamine neurodegenerative diseases. FEBS J., 275(17): 4252-4262, Sep 2008. DOI 10.1111/j.1742-4658.2008.06561.x.

[338] B. Dehay and A. Bertolotti. Critical role of the proline-rich region in Huntingtin for aggregation and cytotoxicity in yeast. J. Biol. Chem., 281(47):35608-35615, Nov 2006. DOI 10.1074/jbc.M605558200.

[339] A. Bhattacharyya, A. K. Thakur, V. M. Chellgren, G. Thiagarajan, A. D. Williams, B. W. Chellgren, T. P. Creamer, and R. Wetzel. Oligoproline effects on polyglutamine conformation and aggregation. J. Mol. Biol., 355(3):524-535, Jan 2006. DOI 10.1016/j.jmb.2005.10.053.

[340] Y. F. Wei, D. K. Lee, and A. Ramamoorthy. Solid-state ${ }^{13}$ C NMR chemical shift anisotropy tensors of polypeptides. J. Am. Chem. Soc., 123(25):6118-6126, Jun 2001. DOI 10.1021/ ja010145l.

[341] X. L. Yao and M. Hong. Determination of C alpha chemical shift tensor orientation in peptides by dipolar-modulated chemical shift recoupling NMR spectroscopy. J. Am. Chem. Soc., 124(11):2730-2738, 2002. DOI 10.1021/ja017137p. 
[342] L. Willard, A. Ranjan, H. Zhang, H. Monzavi, R. F. Boyko, B. D. Sykes, and D. S. Wishart. VADAR: a web server for quantitative evaluation of protein structure quality. Nucl. Acids Res., 31(13):3316-3319, Jul 2003. DOI 10.1093/nar/gkg565.

[343] C. Dominguez, R. Boelens, and A. M. J. J. Bonvin. HADDOCK: A protein-protein docking approach based on biochemical or biophysical information. J. Am. Chem. Soc., 125(7): 1731-1737, 2003. DOI 10.1021/ja026939x. URL http://www.nmr.chem.uu.nl/haddock/.

[344] C. R. Morcombe and K. W. Zilm. Chemical shift referencing in MAS solid state NMR. J. Magn. Reson., 162(2):479-486, Jun 2003. 


\section{Lebenslauf}

Robert Schneider

Zimmermannstr. 64, 37075 Göttingen

Geboren am 21. September 1974 in München. Deutsche Staatsangehörigkeit.

\section{Werdegang}

Seit 2005

2003-2004

1996-2002

$1995-1996$

$1994-1995$

$1985-1994$
Doktorarbeit in der Arbeitsgruppe Festkörper-NMR Spektroskopie (Dr. Marc Baldus) am Max-Planck-Institut für biophysikalische Chemie, Göttingen

Gewerbliche Tätigkeit als Fahrradrikschafahrer, Fa. Rikscha-Mobil, München Studium der Biologie und Physik an der Ludwig-Maximilians-Universität München; Schwerpunkte Neurobiologie, Physik, Genetik, Biochemie; Abschluß mit Diplom 08/2002 (Vordiplom Biologie 1998, Physik 2000)

Studium generale am Leibniz Kolleg Tübingen

Zivildienst am Behandlungszentrum Vogtareuth bei Rosenheim und FriedrichBaur-Institut des Klinikums Innenstadt, München

Finsterwalder-Gymnasium Rosenheim, Abitur 1994

\section{Auslandsaufenthalt}

2001-2002

Diplomarbeitsprojekt "Invariante Objekterkennung im visuellen Cortex und die Rolle von Aufmerksamkeit" bei Dr. Riesenhuber / Prof. Poggio, Center for Biological and Computational Learning, Massachusetts Institute of Technology, Cambridge, USA

\section{Wissenschaftliche Hilfskraft in der Forschung}

WS 1999/2000 Institut für Zoologie und Neurobiologie, Ludwig-Maximilians-Universität München (Dr. Lutz Wiegrebe)

\section{Stipendien}

2005-2008

2002

1996-2002
Promotionsstipendium des DFG-Graduiertenkollegs "Spektroskopie und Dynamik molekularer Knäuel und Aggregate"

DAAD-Kurzstipendium zur Anfertigung von Abschlussarbeiten im Ausland Förderung durch die Studienstiftung des deutschen Volkes sowie durch ein Stipendium nach dem Bayerischen Begabtenförderungsgesetz 